

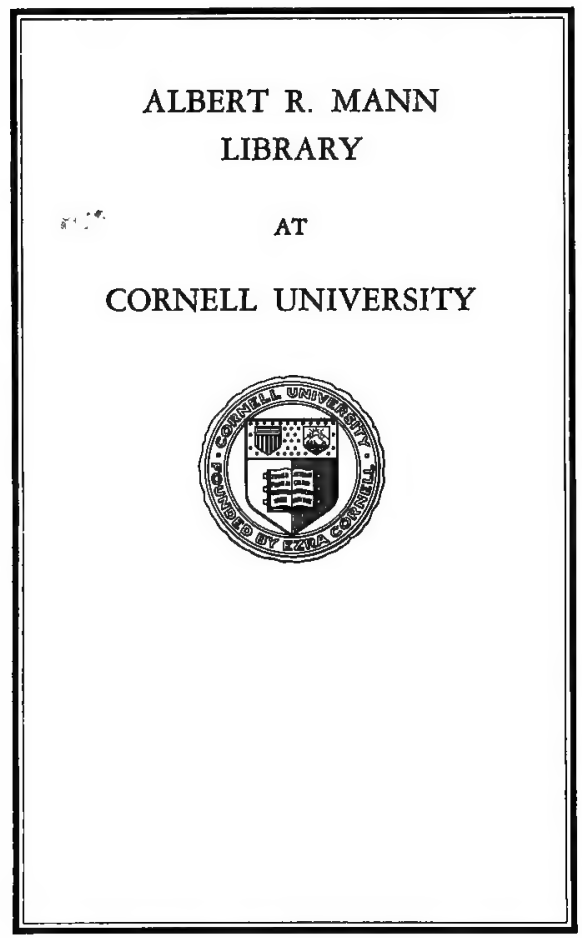




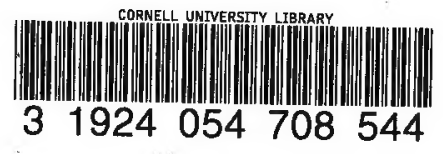




\section{Cornell University Library}

The original of this book is in the Cornell University Library.

There are no known copyright restrictions in the United States on the use of the text.

http://www.archive.org/details/cu31924054708544 





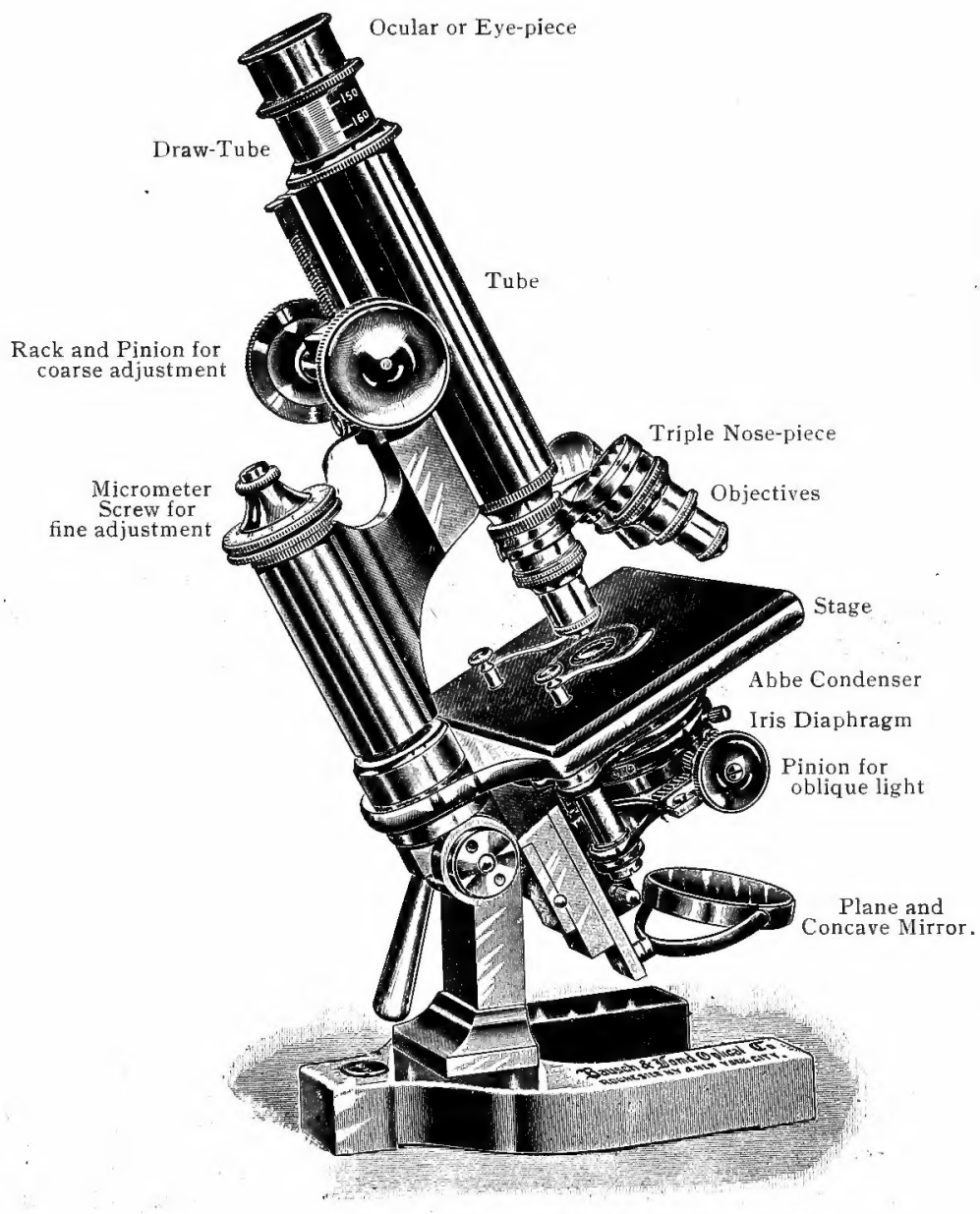




\title{
LABORATORY WORK
}

IN

\section{BACTERIOLOGY.}

\author{
BY \\ FREDERICK G. NOVY, Sc.D., M.D., \\ JUNIOR PROFESSOR OF HYGIENE AND PHYSIOLOGICAL CHEMISTRY; \\ UNIVERSITY OF MICHIGAN.
}

SECOND EDITION, REVISED AND ENLARGED, WITH FRONTISPIECE AND SEVENTY-SIX ILLUSTRATIONS.

ANN ARBOR:

George Wahr, Publisher.

I899. 
Copyright, 1899, by F. G. Novy. 
PREFACE.

A thorough course of laboratory instruction in bacteriology is absolutely essential to the proper education of the medical student of the present day. The practical knowledge thus acquired in the methods of handling bacteria, in the precautions necessary to the prevention of personal infection, and in the methods for the recognition and for the destruction of disease-producing organisms is fundamental and invaluable. Such information is directly useful as a means of diagnosis; it is necessary to the successful performance of antiseptic operations and is indispensable to the proper execution and understanding of the common hygienic measures for the prevention of communicable diseases.

It is therefore evident that the course in bacteriology should not be inferior, either in length or in the character of the instruction, to any other laboratory course offered in the medical curriculum. The student should be taught to work, not merely with a few harmless bacteria, but especially with all of the common pathogenic organisms. The exclusion of the latter organisms from a laboratory course on the plea of danger is an admission of weakness in instruction or in supervision. The danger in a laboratory is avoidable, and is not to be compared with that encountered 
in practice in connection with cases of actual disease. Accidents may occur, it is true, but they are extremely rare as compared with the numerous instances of infection incurred in post-mortems or in surgical operations.

Bacteriology, as an educational measure of the first importance, belongs in the first, or at the latest, in the second year of a medical course. The student is thus enabled to make use of his knowledge in connection with his clinical studies. The spirit of scientific investigation, and not mere book reading, must be fostered in the student from the outstart, since it is this that leads to progress in medicine and serves to distinguish the true physician from those bound down through blind faith, commercialism or ignorance.

This edition is thoroughly revised and greatly enlarged. It should be noted that it is a text-book of laboratory work for students. The arrangement of the subject matter is different from that usually met with in text-books, for the reason that it conforms as much as possible to the actual work as carried on, day by day, in the Hygienic Laboratory of the University of Michigan. During the past ten years three laboratory courses in bacteriology have been given annually. Each course covers a period of twelve weeks of daily work, to which the entire afternoon is devoted. Inasmuch as this laboratory work is required of all medical students, the number of students' ${ }^{\prime}$ hich annually take the course at times exceeds two hundred.

Since it is a beginner's guide and not a manual, it obviously is undesirable to introduce the numerous modifi- 
cations that can be found of almost every known procedure. The methods that are described are those which have stood the severe test of the practical instruction indicated above. Many of the methods, as well as some of the apparatus described, have originated in this laboratory. Illustrations of the various bacteria and of their cultural characteristics have been expressly omitted, inasmuch as the student is expected to sketch from observation the form of each organism and its peculiarities of growth in the colony, and in tube culture. Blank pages are provided for this purpose and for such additional notes as may be desirable.

More space has not been given to the consideration of the individual pathogenic bacteria, and of questions of immunity, for the reason that these and allied subjects are treated of in a general course of lectures which is wholly independent from the laboratory work. These lectures on general bacteriology are given daily and extend throughout a semester. From the standpoint of a teacher it is desirable that the two courses be kept distinct, and for that reason the treatment of the general subject should be published separately, if at all. An exception is made in the case of the first five chapters, which deal with the general properties of bacteria, and which, as such, are necessary for reading and reference in connection with the laboratory work.

The last two chapters are devoted to special methods which will be of value to advanced students. Several of the characteristic ingenious methods of the Pasteur school are given at length. Here, as elsewhere in the book, the 
attempt is made to supply the fullest details to the student in order to insure satisfactory results. A complete index will serve to render the text easily accessible.

It is hardly necessary to state that the various journals and the standard works on bacteriology have been freely drawn upon in the preparation and revision of these pages It has been deemed desirable to introduce illustrations especially, of such apparatus as is actually employed Thanks are due to the Bausch \& Lomb Optical Co., of Rochester, N. Y., also to Eimer \& Amend, and Stransky \& Co., of New York, for kindly supplying several of the cuts. F. G. NOVY.

ANN ARBor, Mich., April 15, 1899. 


\section{CONTENTS.}

\section{CHAPTER $I$.}

Page.

Form and Classification of Bacteria ..
Definition of bacteria.-Microscopic plants and animals, distinction. - Bacteria as plants. - Bacteria, fission fungi or schizomycetes; moulds, thread fungi or hyphomycetes; yeasts, budding fungi or blastomycetes. A Absence of a natural classification._Bacteria classified according to form; micrococcus, bacillus, spirillum.—Modifications; bacterium, vibrio, spirochæte.-Division into species. - Influence of environment on form and size. - - Involution forms. —-Vàriations due to methods of examination.-Plasmolytic changes.—-Pleomorphism. -Constancy of species. - Attenuation. - Origin of new species.

\section{CHAPTER II.}

Size and Structure of the Bacterial Cell

Bacteria as unicellular organisms.-Called the smallest of living beings._Existence of still more minute life.Micromillimeter or micron.- - Size of bacteria.

The cell-wall, composition, demonstration.-Plasmolysis. - Softening of the outer layer, the capsule.—Zooglea.

The contents of the cell, composition.-Existence of a nucleus._Appearance of contents; granulations, polar bodies, color, absence of chlorophyll._-Granulose reaction.

Motility._-Molecular or Brownian movement._-Real motion, flagella or whips.—Number and arrangement.Giant whips.

CHAPTER III.

The Life-history of Bacteria

Rapidity of multiplication._-Cell division: diplo-bacillus, threads; diplococcus, streptococcus, tetrad, sarcine, staphylococcus; vibrio, spirillum. 
Spores.—-Vegetating and reproductive forms.-Wndospore, arthrospore.-Sporulation._-Position of the spore; median, terminal, intermediate.-Resultant cell forms; clostridium, "drum-sticks."—Càuse of spore formation.—Asporogenic bacteria.—Germination.—-Spore structure.-Resistance of spores.—Spontaneous generation.

\section{CHAPTER IV.}

The Environment of Bacteria . . . . .

Conditions of growth-Wecessity of moisture.-Chemical composition of the cell.-Sources of carbon, nitrogen, hydrogen, oxygen, and other elements.-Reaction of the medium. - Distribution of bacteria in nature, where absent.

Classification according to habitat.- Saprophytic and parasitic bacteria.-Obligative and facultative forms.Classification according to oxygen requirements.-Aerobic and anaerobic bacteria.-Obligative and facultative forms. - Microbic association.

Temperature, minimum, maximum and optimum.-Thermophilic bacteria. - Effect of cold and heat on vitality.

Action of light, high pressure, electricity, —Chemotaxis.

\section{CHAPTER V.}

\section{The Chemistry of Bacteria}

The number and kind of products vary with each species. -Influence of environment. - Accumulation of waste-products.- Synthetic and analytic, primary and secondary products.

Bacterial proteins, tox-albumins.--Venoms, plant albumoses as abrin and ricin.-Toxins; synthetic products, elaborated within the cell, not bases or proteins.-Ferments, organized and unorganized.-Enzymes, their classification. - Ptomains._Alkalis._Acids.—Alcohols._-Gases.—Classification of bacteria according to function.

Fermentations. - Their cause, the nature of the chemical changes induced.-Diverse fermentations, alcoholic; acetic acid, vinegar, summer complaint; lactic acid, dental caries, stomach and intestinal disorder, souring of milk, koumiss, cheese; butyric acid, sauerkraut, ensilage, cheese, retting; viscous or slimy fermentations. - Fermentations of fats, flavor of butter.-Bydrogen sulphide and ammoniacal fermentations of urine.-Nitrification in water and soil, saltpeter.—Denitrification.-Indigo reduction.-Putrefaction, bacteria as scavéngers perpetuate life. 
Pigment production.—Phosphorescence.—Heat production.-Toxicogenic and pathogenic bacteria.

\section{CHAPTER VI.}

\section{The Microscope.-The Hanging Drop.-Simple}

\section{Staining}

Simple and compound microscopes. - Spherical and chromatic aberration. - Achromatic and apochromatic objectives. -Under-correction. - Magnifying power; equivalent focal distance.—Defining power-—Resolving and penetrating power. - Numerical aperture. Designation of objectives.

Huyghenian eye-piece.-Compensating eye-pieces.Designation of oculars.

Abbe condenser, iris diaphragm:-_Structural and colored images._-Rules for use of condenser, to secure illumination.

The stand, graduation of draw-tube.CCoarse and fine adjustment. - Nose-piece.—Care of microscope.

Measurement of an object._-Stage and ocular micrometers.-Micromillimeter or micron.-Micrometer value of an objective.

Slides and cover-glasses._Cleaning of the latter.Cover-glass forceps for bacteriological work.

Examination of living bacteria.- - The hanging-drop.Laboratory work.

Staining of bacteria.-Acid and basic anilin dyes.Stock solutions.—Dilute stains. - Simple staining.—-Laboratory work.

\section{CHAPTER VII.}

\section{Gelatin and Potato Media.-Cultivation of Bacteria .}

Preparation of the meat extract._Alkalization.Cleaning and plugging of tubes. - Dry and moist heat sterilization.-Fractional sterilization at $100^{\circ}$, at $60^{\circ}$. - Steam sterilizer.-The autoclave.

Preparation of potato media.--Dilution and mass cultures. - The pure culture._-Precautions in work. - Laboratory work.

Gelatin plate cultivation.-Advantages over potato and liquid media. -Glass plates:-Platinum wires. - Dilution in gelatin._-Pouring on plates._-Plating apparatus._- Moist chambers. Apparatus for constant low temperature.Laboratory work.

Modified gelatin plate method.—Petri dishes.-Esmarch roll-tubes._Laboratory work. 
Modified potato cultures.-Esmarch dishes.-Potato tubes.-Preparation of Roux tubes.—-Laboratory work.

Examination of colonies, macroscopic and microscopic.Transplantation of colonies.—Stab cultures. - The plan of study._Laboratory work.

\section{CHAPTER VIII.}

The Non-Pathogenic Bacteria .

Bacillus prodigiosus.-B. Indicus.-B. ruber of Kiel. -B. Fubidus.-B. violaceus.-B. fluorescens putidus.B. phosphorescens._-Orange sarcine._-Yellow sarcine.-B. subtilis.-B. mesentericus vulgatus. - B. megaterium.-B. ramosus. - Proteus vulgaris._Bacterium Zopfii.—_Spirillum rubrum.—B. acidi lactici.—B. butyricus.-B. cyanogenus.

\section{CHAPTER IX.}

Bouillon, Agar, Milk and Modified Media.-The Incubator and Accessories. . . . .

Advantages of bouillon, agar and milk.-Preparation of bouillon. - Preparation of agar._-Sterilization of milk.Modified media: peptonless agar, glycerin agar; glucose, lactose, litmus and serum media.-Laboratory work.

The incubator. - Thermo-regulators. - Gas-pressure regulator.__ Micro-burners.__ Koch's safety lamp._- Thermometers.

\section{CHAPTER $\mathrm{X}$.}

\section{Relation of Bacteria to Disease.-Methods of Infec-} tion and Examination

Infectious diseases. - Infection and intoxication. Rules of Koch.—Generation._-Attenuation.—Bacterial, fungous and protozoal diseases.-Unknown causes.

Methods of infection.-Cutaneous application.-Subcutaneous injection.—Animal holders.—-Intravenous injection. -Intraperitoneal._-Intrapleural. - Anterior chamber of the eye.—-Lymphatics._-Intracranial._-Infection along respiratory tract. - Alimentary tract.

Observation of infected animals. - Keeping of record.Food and drink.

Post-mortem examination. - Sterilizing case for instruments.-Preparation of the animal. - Examination of the sub-cutis._Peritoneal and pleural cavities.-Removal of 
portions of organs.- Drawing of heart blood.-Cover-glass preparations of tissues and organs, of blood.—Staining of the streak preparations.—Precautionary measures.

Laboratory work with anthrax animal.-Gelatin plates. Preparation of agar plates.--Impression preparation of colonies. - Hanging-drop examination of blood.- Simple stains of streak preparations._-Gram's method.—-Anilin-water gentian violet._-Spore staining.—Carbolic fuchsin.Phagocytes. - Summary.

CHAPTER XI.

The Pathogenic Bacteria

Bacillus anthracis.-B. anthracis symptomatici.-B. œdematis maligni._- B. odematis maligni No. II.—B. tetani. - Culture of anaerobic bacteria._- Staining of flagella. Giant whips._-B. lepræ._-B. tuberculosis._-B. mallei.B. diphtheriæ. - M. preumoniæ crouposæ.-B. pneumoniæ. -B. rhinoscleromatis.—Vibrio choleræ Asiaticæ.—Vibrio Deneke.—-Vibrio Finkler-Prior.—Vibrio Metschnikovi.— B. coli communis. - B. typhosus.-B. icteroides.-B. pestis bubonicæ.-B. influenzæ.-B. pyocyaneus.—-Streptococcus pyogenes._-Staphylococcus pyogenes aureus. - Micrococcus gonorrheæ.-M. tetragenus.-Spirillum Obermeieri.-B. choleræ gallinarum.-B. choleræ suis.—B. rhusiopathiæ suis. -B. muriepticus.

\section{CHAPTER XII.}

\section{Yeasts, Moulds and Streptotrices}

Yeasts, size and multiplication.-Saccharomyces.Torula or wild yeasts. - Pathogenic forms.

Moulds. — Mycelium. - - Hyphæ. — Eruit-organs. Characteristics of the mucor, aspergillus, penicillium, oidium, -Relation to fermentation.-Pathogenic forms.-Skin diseases.

Streptotrices.—-Relation to moulds and to bacteria.

Laboratory work. _-Preparation of bread flasks._- Examination of moulds.

Saccharomyces cerevisiæ.-S. glutinis.-Oidium lactis. -Monilia candida.-Mucor corymbifer.--M. rhizopodiformis.—Aspergillus niger._A. flavescens.-A. fumigatus. -Penicillium glaucum.-Achorion Schönleinii.-Dtreptothrix actinomyces._-S. Maduræ.—S. farcinica. 


\section{CHAPTER XIII.}

Examination of Water, Soil and Air. . . .

Water as a carrier of disease.-Limitations of a chemical analysis.- Recognition of typhoid bacilli.-Cholera vibrios.- + Method of analysis._- Counting of colonies, direct and by aid of microscope.-Number and kind of species.Detection of pathogenic bacteria by animal inoculation.Bacterial contents of snow, ice, rain-water, lakes, rivers, wells and sea-water.

The bacteria of the soil.-Their action.-Pathogenic forms. - Effect of rain and snow.-Filtering action of the soil.-Method of analysis.

The source of air bacteria.__ Effect of moist surfaces. Purification of air by sedimentation.-By rain and snow. Expired air.—-Number of organisms in the air.—Country and city air.-Pathogenic bacteria.—Methods of analysis.Laboratory work.

\section{CHAPTER XIV.}

\section{Special Methods of Work .}

Making of Pasteur pipettes.-Drawing of blood from animals, from man.—Oxalate blood and plasma.-Preparation of blood-serum. - Sterilization of serum by filtration.Fractional sterilization at $58^{\circ}$, at $75^{\circ}$, at $100^{\circ}$ : - Solidification of serum.-Modified serum media.-Filtration of bacterial liquids.—-Tuberculin._-Diphtheria toxin.—The minimum fatal dose. - Testing of antitoxin.--Immunization against diphtheria.—Antitoxic and anti-infectious serum.-Active and passive immunity.—- Pfeiffer's reaction.- Elsner's medium.—-Stoddart's medium.—-Hiss' tube medium.—Uschinsky's medium.—Preparation and use of collodium sacs.Inoculation for rabies.

\section{CHAPTER XV.}

\section{Special Methods of Work, Continued}

Serum agglutination.—Poisonous foods.—Purification of litmus. - The tubing of media._-The sealing and keeping of cultures. - - Thermal death point.—-Moist and dry heat.Testing of disinfectants.-Testing of antiseptics.-Room disinfection.-Hardening, imbedding and cutting of sections. -The staining of sections.-Simple, and Gram's method.Staining of anthrax, tubercle leprosy and typhoid bacilli, and actinomyces in sections.

List of apparatus and chemicals 


\section{LIST OF ILLUSTRATIONS.}

Frontispiece showing component parts of a microscope. PAGE.

Fig. 1.-Micrococcus, bacillus, spirillum.............. 17

2.-Bacterium, vibrio, spirochæte................ 18

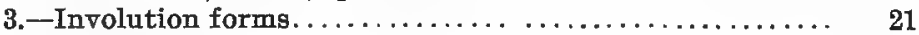

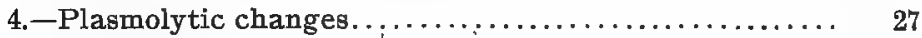

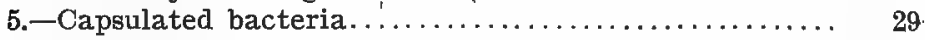

6.-Motile organs or whips on bacteria.............. 36

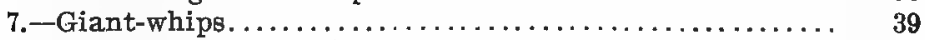

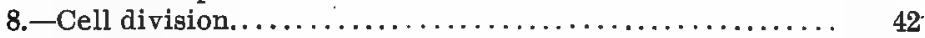

9.-Bacilli, single, in pairs and in threads.......... 43

10.-Division forms of micrococci .................. 44

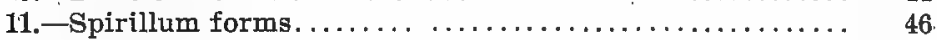

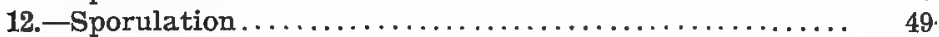

13.--Position of spores and resultant forms............ 50

14.-Spore germination......................... 54

15. - Virtual image, simple microscope (Carpenter)........ 123

16. - Real image (Carpenter) ................... 124

17.-Principle of the compound microscope (Carpenter).... 125

18. -Arrangement of lenses in an objective. Angle of aperture (Carpenter).......................... 130

19.-Structure and action of the Abbe condenser......... 133

20.-Cover-glass forceps (F. G. N.)................. 141

21.-The "hanging-drop "....................... 143

22.-Water-bath with hot iron plate (F. G. N.)......... 150

23.-Enamelled jar for making media................ 153

24. - Burettes for titrating media (F. G. N.) ........... 155

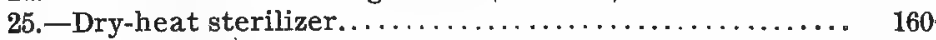

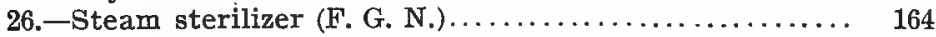

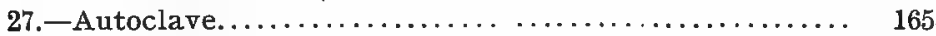

28.-Apparatus used in cultivation..................... 172

29. - Inoculation of a single tube................... 173

30. - Inoculation from tube to tube when diluting........ 174

31. - Ice apparatus for cooling plates................ 176

32. -Water apparatus for cooling plates (F. G. N.)........ 177

33. - Constant low temperature apparatus (F. G. N.)....... 179

34. - Potato tubes, ordinary and Roux form. ........... 184

35. --Apparatus for filtering agar (F. G. N.). ........... 237

36. -The incubator or thermostat................. 244 
37.-Thermo-regulator (F. G. N.).................. 246

38.-Murrill's gas pressure regulator................. 250

39. -Koch's safety burner........................... 251

40.-Adjustable syringe. . . . . . . . . . . . . . . . . . . . 262

41. - Syringe and holder, water-bath and radial burner..... 263

42. - Injection apparatus......................... 264

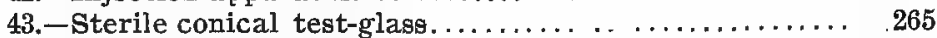

44. - Voges' cylindrical holder..................... 266

45.-Latapie's animal holder..................... 268

46. - Rat cage and forceps....................... 273

47.-Vaughan's cage for rabbits, etc............... 274

48.--Instrument sterilizing case and searing iron......... 275

49. -The Roux spatula and Nuttall needle............. 278

50. - Simple bottle for anaerobes (F. G. N.) ........... 308

51.-Bottle for tube culture of anaerobes (F. G. N.)....... 314

52.-Apparatus for plate cultivation of anaerobes (F. G. N.) 314

53.-Apparatus for plate cultivation of anaerobes (F. G. N.) 314

54. -Tube culture of tubercle bacillus on potato (F. G. N.).. 315

55.-Yeast cells with spores (Hansen).............. 387

56 . - Fruit-organs of moulds (Lehmann) . ............... 390

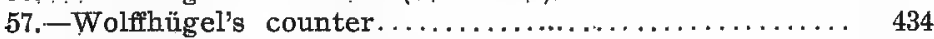

58.-Lafar's and Jeffer's counters .................. 436

59. - Esmarch's roll-tube counter................... 448

60.-Apparatus for the examination of air............ 454

61.-Drawn-out tube pipettes of Pasteur............. 457

62.-Sealing cultures in capillaries.................. 459

63.--Pipette used in drawing blood................. 460

64. - Roux water-bath for serum sterilization........... 466

65. -Koch's serum sterilizer..................... 467

66.-Apparatus for filtering bacterial liquids (F. G. N.). ... 469

67. $\rightarrow$ Connections for filtering apparatus (F. G. N.)........ 470

68-Berkefeld filter; globe receiving flask (F. G. N.)....... 471

69. -Roux flask for surface cultures................. 486

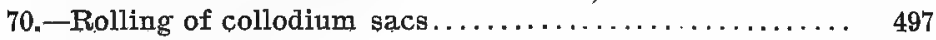

71.-Collodium sacs............................... 499

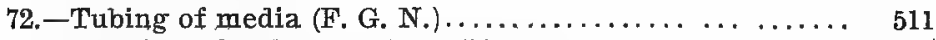

73. - Keeping of cultures (F. G. N.). ............... 512

74. - Filter for bacterial suspensions................. 514

75.- Determination of the thermal death-point (F. G. N.) .. 516

76. - Formaldehyde apparatus for room disinfection (F. G.N) 530 


\section{CHAPTER I.}

\section{FORM AND CLASSIFICATION OF BACTERIA.}

- Bacteria may be characterized as unicellular, microscopic plants. Their vegetable nature has been established only within comparatively recent years. From the time of Leeuwenhoek, who in 1683 discovered the first representative of this group, until 1854 these microscopic organisms were spoken of as animalculæ or minute forms of animal life. Indeed, it was not until about 1875 that the true relationship of bacteria to plants was definitely settled, largely through the labors of Cohn, Naegeli, DeBary and other botanists.

When studying the exceedingly minute forms of life observed under the microscope, one recognizes that it is not always possible to indicate the dividing line between the animal and vegetable kingdoms. There is no one characteristic which will serve for this purpose. From the very nature of things it must be expected that the lowest forms of animal and plant life will approach one another. The striking differences which are seen between the higher forms of animal and plant life gradually disappear as the comparisons are carried down the scale to the lowest forms. The latter merge into one another indicating a common origin in the remotest past. Under these conditions it is evidently impossible to characterize certain forms of life as plants or as animals.

Inasmuch as bacteria belong to the lowest and simplest forms of life, it cannot be expected that they will show any marked differentiation into plants. They are classified, however, among plants because of their evident relation. 
ship to certain well recognized types of plant life. In their characteristics of growth, multiplication and reproduction they resemble the group of algæ more than any other group of living beings, and it is this general relationship, rather than any one peculiarity, which has led to their being placed in the vegetable kingdom.

As will be indicated later, bacteria multiply by division or fission, and for this reason they are spoken of as fissionfungi or schizomycetes. The word germ or microbe is often employed to designate bacteria. It should be understood, however, that these two terms have a broader significance and include all microscopic life, whether animal or plant. Bacteria therefore constitute a definite group of germs or microbes.

In addition to their relationship to algæ, the bacteria resemble in many respects the moulds or fungi, and the yeasts. These two groups will be discussed more in detail in a subsequent chapter. It will be sufficient to indicate in this connection the more marked distinctions between the moulds, yeasts and bacteria. The former, as is well known, appear in velvety or cotton-like spreading growths which even the unaided eye can often resolve into a network of threads. The moulds are therefore spoken of as thread fungi or hyphomycetes. Growth results not by division, as in the case of bacteria, but by the lengthening of a cell or thread, that is to say by continuous end-growth. Moreover, the moulds are relatively higher forms of plantlife inasmuch as many species of this group possess special fruit-organs. The threads which make up the mass of a mould are much thicker than bacterial cells.

The yeasts are uni-cellular plants which differ from bacteria in size, method of multiplication and in other respects. The yeast cell may be considered as a giant in comparison with the minute bacterial cell. Moreover, yeasts multiply, not by fission, but by a process known 
as budding. They are designated by the term blastomycetes.

A great many elaborate attempts have been made at classifying bacteria. Owing to their extremely minute size it is, as a rule, impossible to follow out the life-history of each individual species. The characteristic development of the fruit-organs in higher plants affords a basis for a natural classification whereby the various species are grouped into genera and families. Such a classification is natural, because it brings together the various individuals which possess the same structure and development. Inasmuch as bacteria are unicellular it follows that they do not possess definite fruit-organs, and owing to their size very little indeed can be said of their structure. The various classifications proposed are based upon characteristics such as form, size, manner of division, presence of spores, motion, number and arrangement of whips, etc. It is evident, therefore, that all such systems of classification are more or less artificial.

For practical purposes it is sufficient to divide bacteria according to their external form into three groups. These are:

Micrococci, or spherical bacteria;

Bacilli, or rod-like bacteria;

Spirilla, or screw-shaped bacteria.
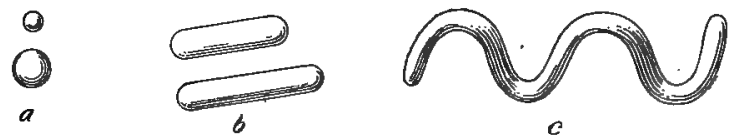

c

Fig. l. $a$-Micrococcus; $b$-Bacillus; $c-$ Spirillum.

In a few instances special names are applied to certain forms of one or another of these three primary types. Thus, the term bacterium is occasionally applied to a very short bacillus. It has the same significance as the word cocco-bacillus, which indicates that the organism may at 
times be almost spherical, at other times rod-shaped. Again, the term vibrio is applied to certain bacteria which may form spirals, but which commonly grow in segments of a spiral. They may therefore be considered as bent, twisted rods which appear under the microscope as commalike forms. The union of two of these "comma bacilli" gives an elongated $\mathbf{S}$ form, and when more of these elements unite thus they give rise to a spirillum. Long, slender, flexible spirals are frequently designated as spirochotes. Additional terms will be met with when describing the various characteristics of growth of bacteria.

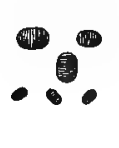

$a$

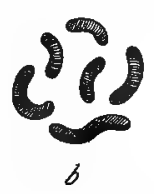

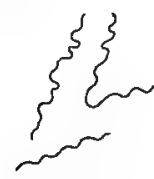

$c$

FIG. $a \quad a$-Bacterium; $b$-Vibrio; $c$-Spirochaete.

The above classification is based upon the external forms of bacteria, and is therefore artificial in character. A similar classification applied to the higher forms of life would lead to gross error. Thus, the worm, eel and snake, viewed at a distance, show the same general appearance, and yet they are wholly different, unrelated types of life. A close inspection at once reveals striking differences. In the case of bacteria, owing to the extremely small size and the simplicity of the bacterial cell, it is not possible to establish structural differences, and for that reason the form classification, imperfect as it may be, is of necessity adopted.

The various species of micrococci will show differences in their size, that is in their diameter, but otherwise they closely resemble one another. Occasionally, when micrococci grow in pairs they may show flattened, apposed surfaces, as if two biscuits were brought together. In other instances they may be elongated or lance-shaped (see fig. $10 a$ ). In the rod-shaped bacteria or bacilli greater differences will 
be observed in the form and size of various species. Thus, one species may be very short and thick, while another may be considerably longer and narrower, or longer and thicker. Again, in one species of bacillus the ends may be square, while in others they may be rounded or ellipsoildal. Among the spirilla marked variations in form or size will be observed. So much so that it is very doubtful at times whether certain species really belong to the group of bacteria.

While some species of bacteria show marked differences in form and size, it must not be supposed that this is always the case. On the contrary many undoubtedly distinct species of bacteria may have the same form and size, so that, as far as the microscope is concerned, they cannot be distinguished one from another. The division of micrococci, bacilli and spirilla into species is based, as a rule, not upon the mere microscopical appearance, but rather upon the sum total of the properties they possess. The characteristics of growth on various artificial media, the behavior to staining reagents, and the effect on the living animal must all be considered when an attempt is made to to distinguish one species from another. For this reason it is not possible, except in very few instances, to identify species among bacteria by mere microscopic examination. In the majority of cases the identification of species necessitates a careful, long and tedious study of all the properties possessed by that organism.

The higher forms of plant and animal life possess, as a rule, a constant form and size. Marked changes in the environment, such as temperature, and altitude, are necessary to produce type variations. Bacteria, however, are extremely liable to undergo alterations in form, size and other characteristics. Owing to their simple nature they are readily influenced, in some way or another, by the slightest change in their environment. A variation of a few degrees in temperature, a trifling difference in 
reaction or chemical composition of the soil, may profoundly affect the form and size of bacteria.

It is evident, therefore, that a given species of bacteria does not possess a constant form or size. Under certain conditions of environment it may be a large, thick bacillus, while at other times it may appear almost like a coccus. By the typical form of a given species is meant that form which is met with when the best conditions of temperature, soil, oxygen supply, etc., are provided. The slightest variation from these optimum conditions will, as indicated above, cause a deviation from the typical form.

Variations in form and size may be considered as arising from natural causes, the environment; and from artificial causes due to methods of manipulation.

When a perfectly pure specimen is examined under the microscope more or less marked differences in the size of the various cells will be observed. Such differences must be expected among living, actively growing forms. The small, young cells will always be present beside the large, old cells. Again, most of the bacterial cells in a given specimen may be single, but, now and then, some will be found forming threads, or filaments, many times longer than the single cell. These thread-like forms are not contaminations due to the presence of a different species, as might at first thought be supposed.

Another variation may be expected when the adult cell develops a spore or seed. Very characteristic forms result from the presence of a spore within the bacterial cell. These will be given special attention in a subsequent chapter.

The composition of the medium on which the bacteria grow will exert a marked influence upon their form characteristics. The same organism planted on solid media, such as coagulated blood serum, agar and potato, will show differences in form. The addition of small amounts of glycerin- 
glucose, or an acid, or alkaline reaction likewise may affect the appearance of the cell.

A comparison of the growths on solid and on liquid media will show peculiarities and modifications in the form and size of a given species. Thus, single cells or at most short threads may predominate on solid media, whereas in liquid media very long threads or filamentous growths may be found.

The temperature exerts a profound effect upon the form characteristics of bacteria. At low temperature the growth is slower, and hence the individual cell may attain an unusual size, whereas at a higher temperature multiplication results so rapidly that the cells are considerably smaller.

Under unfavorable conditions of soil or temperature certain bacteria will show remarkable variations from the normal type. What is ordinarily a perfect rod becomes distorted out of all semblance to the original form. Clubshaped, spindle-shaped, dumb-bell-like forms are produced. Sometimes they become twisted into irregular, spherical bodies. These peculiar, deformed cells are considered as degenerations. They are commonly designated as involution forms: The organism is struggling for existence under adverse conditions which, if they persist, will eventually cause its destruction. Transplantation to a favorable medium will promptly restore the typical form.

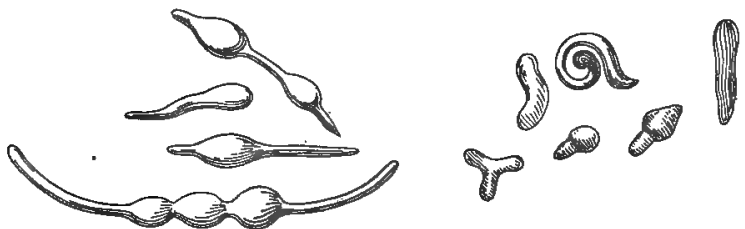

FIG. 3. Involution forms.

The alterations mentioned above are the result of environment. Whatever may be the variation, it is to be considered always as temporary, inasmuch as the typical 
form can be reproduced whenever the organism is transplanted and grows under its most favorable conditions of soil and temperature.

The methods of examination may apparently influence the form of a given species. This is very often seen in stained preparations. As a result of heavy staining, such as is resorted to in order to demonstrate flagella, the organism appears much larger and thicker than when stained in the ordinary way. The successive deposition of the dye, layer on layer, causes an apparent increase in the size of the cell. Again, in the method of double-staining tubercle and leprosy bacilli, the bacteria, after being heavily stained, are partly decolored with alcohol. Depending upon the extent of the decoloration, they may appear as relatively thick or as extremely thin rods.

As a rule the bacteria present in tissues, when stained, appear smaller and narrower than in the fresh material. This variation is in part due to the contraction of the protoplasm or contents of the cell by the alcohol employed to harden the tissue. It is also due to partial decoloration which is unavoidable when the bacteria are to be differentiated from the tissue in which they lie imbedded.

As a result of the action of various chemical substances the protoplasm of the bacterial cell may be contracted or drawn up into irregular masses or granules. When such forms are stained they may appear like minute micrococci, although the original form was that of a typical rod. This action on protoplasm is designated as plasmolysis. Exposure of bacteria to iodine is likely to result in plasmolytic changes. Similar alterations are met with as the result of overheating specimens in the process of preparation (Fig. 4).

From what has been said above with reference to variation in the form of a given species, it is evident that pleomorphism is very common among bacteria. Certain species are more prone to undergo modifications of this 
kind than others. Variations in the form and size of the individuals of a given species must be expected. The same form will be maintained only when all the conditions of environment are constant.

In view of the marked alterations in the form of bacteria, it has been supposed that there is a corresponding variability of species. Under certain conditions of environment a given species may cease to produce the pigment which under normal conditions it would elaborate. Again, similar unfavorable conditions may cause a disease-producing germ to lose its property of growing in the living body. In other words, not only the mere form and size, but also the physiological activities of the cell may be altered. This weakening of the functional activity of a bacterial cell is known as attenuation. The attenuated or weakened germ constitutes a variety of the original type, but it does not constitute a new species. On reversing the conditions of environment, that is rendering these more favorable, the attenuated form can be brought back to the full possession of its original properties.

Undoubtedly, species have originated in the past as a result of growth under altered conditions, and new species may even now be in process of formation. But under ordinary-conditions of observation this change of onespecies into another does not and cannot take place. The hay bacillus cannot be transformed into the anthrax bacillus, nor can the germ of pneumonia be converted into that of consumption. They may both be greatly modified, giving rise to varieties, but so long as these exist they still represent the original species. The typical form and the typical species can always be reproduced by restoring the most favorable conditions of growth. 
SIZE AND STRUCTURE OF THE BACTERIAL CELL.

Bacteria have been described as uni-cellular microorganisms. The fact that they consist of a single cell indicates at once their microscopic character. The microscope reveals a world of animate beings which differ enormously in size. Some of these organisms may be large while others are exceedingly small. The bacteria are usually spoken of as the smallest of living beings, the "infinitely small." Until very recently no form of life was known smaller than these. The discovery of the microbe of pleuro-pneumonia has revealed the existence of organisms considerably smaller than the bacteria. This new organism is so small that the very highest magnification of the microscope still leaves its form uncertain.

The size of microscopic objects is usually expressed in micro-millimeters. A micro-millimeter or micron may be defined as the thousandth part of a millimeter. Inasmuch as a millimeter corresponds approximately to of an inch, it follows that one micro-millimeter repre-

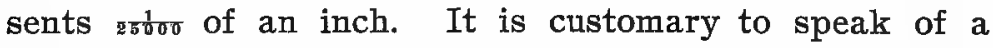
micro-millimeter as a micron and to designate it by the Greek letter $\mu$.

The largest micrococcus known has a diameter of $2 \mu$ or Istro of an inch. There are some micrococci that have a diameter only one-tenth as large as this. The common pus-producing micrococci have a diameter of about $0.8 \mu$ or IIto of an inch. Inasmuch as the diameter of a red blood cell is about $8 \mu$, or about so of an inch, it is evident that ten of these cocci placed in a row would correspond to the 
diameter of a blood cell. The average micrococcus has a diameter of about $1 \mu$, or इรั0 of an inch.

The width of the average bacillus is about $1 \mu$. The length will vary usually from 2 to $4 \mu$. The thickest bacilli, like the B. crassus, are said to have a width of $4 \mu$. Usually several bacilli must be placed end to end to correspond to the diameter of the red blood corpuscle.

From the dimensions given it is evident that an enormous number of bacteria may be present in a relatively small volume. One milligram of the pure cells of the golden pus-producing micrococcus will contain about $2,000,000,000$ jndividuals. One grain of this material would therefore contain $128,000,000,000$ cells.

In view of the extremely minute size of bacteria it is evident that they are very close to the limit of microscopic vision. It is manifestly impossible to make out much of any structure in such small organisms. The ordinary plant or animal cell can be readily shown to consist of a cell-wall, a protoplasm and a nucleus. It may therefore be expected that the bacterial cell will likewise consist of a cell-wall, containing the protoplasm and the nucleus.

It has been supposed in the past that the bacterial cell possessed a cell-wall which did not stain readily and which was composed of cellulose or woody fibre. There can be no question regarding the existence of an outer membrane or envelope, but its chemical composition is undetermined. Certain sarcines and a few bacilli have been said to give cellulose reactions, but these observations have not been confirmed by subsequent investigators. Some of the reactions employed in the detection of cellulose cannot be considered as characteristic. Thus, the production of a reducing substance on treatment with an acid may be due to cellulose, but it may also be due to the presence of a glyco-proteid. 
There is reason to believe that the cell-wall proper consists chiefly of protein substances. The cell-wall may be considered as a layer of hardened or condensed protoplasm. It is stained by anilin dyes and is not digested by proteolytic ferments such as pepsin and trypsin. It apparently responds to Millon's reagent, but is insoluble in Schweitzer's reagent, which, as is known, dissolves cellulose. It is quite possible that certain species, especially when growing on special media, acquire some cellulose-like substance. In other species dextrin-like products seem to be elaborated. Again, granulose, or a chitinous substance may be present at times in the cell-wall.

The existence of a cell-wall is indicated by various characteristics exhibited by bacteria. Thus, the constant form of a spirillum or of rods can only be accounted for by the presence of an outer hard envelope. Occasionally the contents of a cell may die out and disappear, in which case the empty shell remains and is easily distinguished from the normal cells, which stain perfectly.

The presence of a cell-wall is especially clearly demonstrated in certain cases. Thus, the contents of the cell contain, in addition to the protoplasm, more or less of a watery cell fluid. As a result of surface tension this may gather in minute droplets between the semi-solid protoplasm. Occasionally the protoplasm will show marked adhesion to the wall, and as a result the fluid is prevented from forming globules and causes a separation of the contents of the cell into discs. A disc of protoplasm will alternate with a disc of the cell fluid. On staining such an organism it will exhibit transverse bands.

The cell fluid holds in solution various mineral salts which exert a certain osmotic or internal pressure which is counteracted by the protoplasm and especially by the firm outer cell-wall. The normal internal pressure of the cell contents depends upon the composition of the fluid surrounding the cell. When the cell is placed in water which 
contains relatively less osmotic substances than the cell itself, there is a tendency for the cell fluid and the dissolved substances to pass out into the surrounding water. This is prevented by the outer layer of protoplasm, and hence the osmotic or internal pressure. When, however, the cell is placed in a liquid which is richer in osmotic substances, a 2.5 per cent. saltpeter or a 1 per cent. salt solution, the osmotic pressure of the outside liquid overcomes that of the cell fluid. A current is thus established into the cell. The protoplasm as a result retracts from the cell wall, and this retraction of protoplasm is known as plasmolysis.

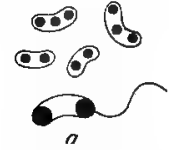

Fig. 4. Plasmolytic changes, after A. Fischer. vibrio; $b$-Typhoid bacillus; $c$-Spirillum undula.

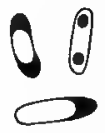

b

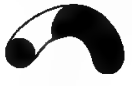

c

$a$-Cholera

In the above case the salts in the outer liquid, owing to the relatively impermeable wall, do not easily penetrate into the cell. If they did the internal pressure of the cell fluid would soon rise above that of the surrounding liquid, and as a result the protoplasm would expand and refill the cell. This actually does occur when a saltpeter solution of double the strength given is employed. When the plasmolyzed cell is placed in pure water the protoplasm returns. promptly to its original position.

In the case of the micrococcus the protoplasm contracts. to a small globular mass, while in the bacillus two round bodies form, one at each end. As a result the cell-wall between the polar bodies is rendered visible. These plasmolytic changes occur in the living cell. As will be seen later, the flagella or motile organs seem to arise from the outer layer of the cell-wall, in which case this membrane is functionally active and is not a passive structure, as in the case of the cellulose:wall of the ordinary plant cell. 
The presence of a cell-wall is also demonstrated by the action of iodine on the cells. An organism treated with iodine solution and then fixed and stained will show the cell membrane more or less distinctly. The protoplasm has contracted from the wall and a number of colorless globules or vacuoles will be seen, due to the cell fluid that has been squeezed out. Moreover one or more heavily stained roundish bodies, or "chromatin granules," can also be observed.

There is abundant evidence, therefore, which shows that a definite membrane is present in all the stages of the development of the bacterial cell. Moreover, plasmolytic experiments indicate that the protoplasm is not firmly united to this wall. The fact that strong solutions of salts do penetrate the interior of the cell indicates that the cellwall is permeable. This of course is necessarily so, since the nourishing material present in the surrounding fluid must pass through this wall in order to reach the protoplasm. Similarly the waste products of the cell must pass outward through a permeable cell-wall.

The bacterial membrane or cell-wall is usually very thin and colorless, and for this reason cannot be ordinarily seen. As indicated above, it probably consists of a protein and not of cellulose. Under special conditions the outer layer of the cell-wall takes up water and gelatinizes. In this case the cell becomes surrounded by a broad, colorless zone which does not'stain. This softened, expanded cellwall is known as the capsule. Owing to its soft, slimy character it causes the cells to stick together, thus giving rise to masses of cells which are, as it were, cemented together. Such a mass, when touched with a wire, can be drawn out into long, slimy threads. This massed condition of certain bacteria is designated as a zooglea.

Well marked capsule formation is met with in only comparatively few bacteria. In the majority of these organisms it is either very feebly developed or entirely 
absent. Capsulated forms are met with most often when staining the bacteria that may be present in the fluids of the animal body. Such forms are therefore met with occasionally in saliva, sputum and in blood. Certain species, however, may give rise to pronounced capsules, when grown on artificial media (Fig. 5 a).

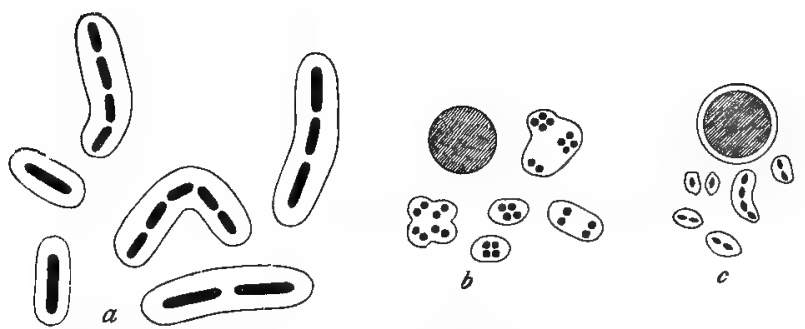

FIG. 5. Capsulated bacteria, drawn with camera lucida, Zeiss onetwelfth and ocular 2. a-Anaerobic bacillus isolated from human feces, capsules lormed on glucose agar; $b$-Micrococcus tetragenus with capsules, from blood of mouse-compared with red blood cell; c-Lanceolate diplococcus of pneumonia with capsules-above this a red blood cell showing false capsule, due to shrinkage in drying.

The chemical composition of the soil influences the formation of the capsule. Thus, the leuconostoc, when grown on sugar media, develops remarkably large capsules, whereas in the absence of sugar this gelatinization of the cell-wall does not take place. It is evident that the capsule is not a degenerative product, but a normal reaction induced by certain constituents of the medium.

While the presence of capsules gives rise to slimy growths, it must not be inferred that they are the invariable cause of a slimy consistence. Bacteria may actually secrete a mucin-like sticky substance, and as a result the liquid will be slimy although no capsules will be found surrounding the individual cells. Instances of slimy milk, beer, wine, etc., from causes of this kind are not uncommon.

The detection of the presence of a capsule is not always an easy matter. Frequently, as a result of manipulation capsule-like forms may be met with. This is invariably the case when the bacteria are present in an albuminous 
fluid like blood. The organic matter dries out on the coverglass first, whereas the bacterial cell dries later. In the process of drying it naturally shrinks somewhat in size, and hence a clear zone results between the dried organic matter and the retracted dried cell. On staining the specimen a colorless zone will surround each cell and may therefore be easily mistaken for a capsule (Fig. 5 c). The capsule, unlike the real membrane, does not stain easily with anilin dyes.

In certain organisms related to the bacteria, as the beggiatoa, a hardening rather than softening of the membrane takes place. This gives rise to a sheath or tube which surrounds the individual elements and may be considered as analogous to the cell-wall of higher plants. Such sheaths, however, are not met with among the true bacteria.

The contents of the bacterial cell. The relatively hard and more or less impenetrable cell-wall encloses the soft protoplasm which is the living portion of the cell. In it, therefore, are carried on the chemical and physical changes necessary to life. It contains, like all protoplasm, a relatively large amount of water. The solid constituents of the protoplasm are chiefly nitrogenous, that is to say protein, in character. Moreover, fatty substances are always present, and in some species, as the tubercle bacillus, they may make up a large percentage ( $30-40$ per cent.) of the total solids. There is reason to believe that at times a carbohydrate, like granulose, is present. The inorganic constitu. ents, or ash, constitute about 10 per cent. of the dried cells.

The composition of the bacterial cell will vary according to the soil on which it grows. Moreover, it is undoubtedly influenced by the age of the individual cell. In view of the different products elaborated by various species of bacteria, it is evident that their chemical composition must necessarily vary. 
The higher animal and plant cell always contains a nucleus within the protoplasm. The nucleus unquestionably plays a most important part in the division of the cell. So much so that it has been considered essential to reproductive changes. Inasmuch as bacteria possess the power of multiplication, it might be expected that they would contain, like higher forms, a well defined nuclear body.

The direct examination of a bacterial cell fails to reveal the presence of any form analogous to a nucleus. Usually, the cell appears perfectly homogeneous, and the closest examination will not bring out a structure. The question of the existence of nuclei in bacteria has been the subject of numerous extensive investigations which have led to very different conclusions. In fact, it may be said that the presence of a nucleus is not demonstrated.

There are some who consider bacteria as wholly devoid of nuclei. The minute granules which are not infrequently present, especially in older cells, are believed by some to be the first indication of the formation of a nucleus.

Some bave endeavored to show the existence of a central body which, while it is not a nucleus in the sense that this term is usually employed, nevertheless possesses certain properties of a nucleus, and may therefore be considered as a rudimentary form of that body. On the whole the opinion prevails that bacteria consist essentially of nuclear matter surrounded by a very thin protoplasmic layer and by the cell-wall. It is well known that in embryonic cells the nucleus almost fills the cell.

This view is especially strengthened by the behavior of bacteria to anilin dyes. The bacterial cells stain readily and intensely, like the nuclei of higher cells. This is assumed to be due to the presence of similar chemical substances such as nucleins. The presence of a nuclein compound in the bacterial cell is indicated by the fact that nuclein bases and even a protamin-like body have been isolated. 
As stated above, the cellular contents of most bacteria. appear perfectly homogenous. Certain species, however, show the presence of various sized granules. These are especially present in old cultures and seem to have a protein composition. They may be related to the chromatin of higher cells and, as pointed out above, they are believed by some to represent the earliest form in the development of a. nuclear body. These granules may be large or small, very numerous or apparently absent. It is quite possible that these are due to condensation of the protoplasm as a result. of plasmolytic changes. Similar bodies appear in the cell contents invariably previous to spore formation, and these have been termed sporogenic granules.

In the process of drying, fixing and staining bacteria, artificial changes not infrequently occur which may be considered as evidence of a structure which in reality does not. exist. For instance, a bacterial suspension is allowed to dry on a cover.glass. As the water evaporates the concentration of the salts present is increased, and as a result marked plasmolytic changes may result. The cell may then show marked. granules or polar bodies and vacuoles. The socalled spores of the tubercle bacillus may be due to changes of this kind.

Certain bacteria, such as the typhoid and chicken cholera group, on feeble staining show well stained ends separated by an almost colorless zone. This is spolien of as the bi-polar stain, and is supposed to be due to the presence of a vacuole or cell fluid in the middle of the cell. The protoplasm is consequently pushed to each side and, owing to its dense condition, readily takes up the stain. This may represent the normal conditions of the cell but, on the other hand, it may be due to the plasmolytic changes mentioned above. These polar bodies are certainly not spores, as has been at one time supposed.

The contents of the bacterial cell are, as a rule, perfectly colorless. This is true even though the organism. 
produces a brilliant pigment. In this case the pigment, or rather its antecedent, is made within the cell and is then excreted into the surrounding medium.

A few bacteria show a faint red coloration when exam. ined under the microscope. This is due to the presence of a red pigment known as bacterio-purpurin. This substance, in its microchemical reactions, appears to be identical with a similar coloring matter present in certain protozoa. It is believed to exercise a role similar to that of chlorophyll.

A green chlorophyll-like pigment has been observed to be present in a small number of bacteria. The absence of chlorophyll from the majority indicates that these organisms, unlike the higher plants, cannot assimilate carbonic acid. This, however, does not necessarily follow, since certain nitrifying bacteria are capable of assimilating carbonic acid even in the absence of light.

A number of bacteria have been met with in the mouth which show a yellowish or brownish tint. The exact nature of the pigment in either of these cases is unknown.

On contact with iodine, protoplasm in general takes on a light yellow color. A number of bacteria, however, give with iodine a blue or dark violet color. Inasmuch as this reaction is similar to that with boiled starch or granulose, it is spoken of as the granulose reaction. Such bacteria would seem to contain, therefore, a carbohydrate similar to soluble starch. This substance may be present only in scattered granules, or these may accumulate so that the entire cell is deeply stained. In some bacteria the substance is not present in the cell until just previous to spore formation. Bacteria giving this reaction are especially found in the mouth.

Motility of bacteria. When bacteria are examined in the living condition they will, as a rule, show motion. The movements observed may be apparent or they may be real. In the latter case the organism is in active motion, travel- 
ing from one place to another. The impulse that creates this real, active motion comes from within the cell, in other words from the living protoplasm. On the other hand, many bacteria will show an apparent motion. The cells move to and fro, trembling as it were, but do not actually change their relative position. The impulse in this case comes from without the cell, which itself remains passive.

The apparent motility of bacteria is known as Brownian, or molecular, or physical motion. All exceedingly minute objects, when suspended in the air or in a fluid, will show this peculiar swaying or pendulum movement. The molecules composing the air'or fluid are in constant motion, and consequently strike, again and again, the minute objects that may be in their path. This molecular bombardment of an otherwise motionless bacterial cell causes it to sway to and fro.

It may at times be very difficult to distinguish between Brownian and real motion. In that case it is necessary to resort to certain experiments. Brownian motion is obviously manifested by dead as well as by living cells. The organism may be destroyed by heating at $60^{\circ}$ for one hour, or it may be treated with a germicidal substance such as carbolic acid, or mercuric chloride. If the dead cell exhibits the same motion as the living cell, it is clearly due to purely physical causes. Again, by growing the organism at a constant low temperature of $15^{\circ}$ or less it will in many cases become larger than usual, and consequently will respond less readily to the impact of molecules. This procedure is especially useful in doubtful cases. Finally, as a rule, it is possible to distinguish between the two kinds of motion by demonstrating the presence or absence of the characteristic organs of motion. If these are present there can be no doubt of the true motility of the organism. On the other hand, failure to find these motile organs does not prove that they are absent, inasmuch as in some undoubt- 
edly motile bacteria it is very difficult to demonstrate their presence.

Bacteria exhibit real motion only in liquid or on moist media, and then only when in the actively growing condition. When in the seed or spore form they do not possess motion; neither do they possess active motion when floating about in the air as fine dust-like particles.

Real motion is observed in most of the spiral-shaped bacteria. The bacilli are, as a rule, motile. The micrococci are, on the other hand, usually non-motile. Two or three micrococci are known to possess motion. Two motile sarcines have also been studied.

The motion exhibited by bacteria will vary with the different species, and probably depends upon the number and arrangement of the organs of locomotion. Some rods show a slow, forward, wabbling movement. Others glide rapidly and steadily forward. In some the forward motion is accompanied by a rotation of the cell around its long axis, while in others a "somersault" movement is to be seen. Frequently the actively motile cell will suddenly reverse its motion and travel backward. It may come to a sudden stop, and then as suddenly, again begin to move.

The motility of a culture depends largely upon its age, upon the composition of the soil, and upon the temperature. In the case of anaerobic bacteria the presence of oxygen soon inhibits motion. The higher the temperature, as a rule, the more marked will be the movement. Thus, certain bacteria scarcely show real motion at the ordinary room temperature because of the presence of a slimy secretion. When placed, however, at the temperature of the body the motion becomes well marked.

Active motion is carried on by means of certain organs known as flagella or whips. The whips are very delicate, long, thin threads of protoplasmic substance. They are analogous to similar appendages on motile infusoria, on plants and on ciliated epithelium. Owing to their extreme 
delicacy they can only very rarely be seen in the living condition. Moreover, they do not color with anilin dyes in the same way as the mass of the bacterial cell, and for that reason they are not visible in the ordinary stained preparation. In order to demonstrate the presence of flagella or whips it is necessary to resort to a very special method of staining. They then appear as slender, wavy filaments. It is the lashing of these whips that propels the organism through the liquid.
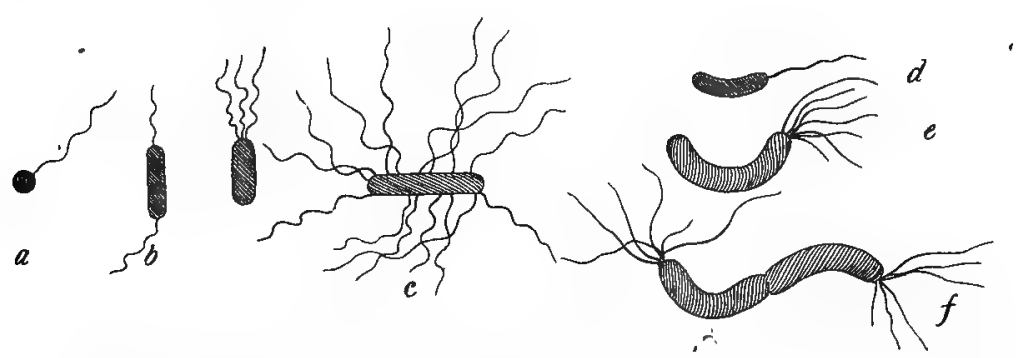

FIG.6. Motile organs or whips on bacteria, $a$-Micrococcus; b-Bacillus with terminal whips; $c-$-Bacillus with diffuse whips: $d$-Vibrio; $e-$ Single spirillum with bunch of whips; $f$-Spirillum after division with whips at each end.

The flagella will vary considerably in size in different species, and even at times in individuals of the same species. Their width is usually less than $\frac{1}{20}$ of the width of of the cell. Their length will usually be two or three times the length of the cell, but it is not uncommon to find some bacteria with whips that are ten or twenty times as long as the cell.

The flagella project from the outer border of the organism. They are supposed by some to be directly continuous with the protoplasm within the cell. In that case the protoplasmic threads are supposed to pass through minute openings in the cell-wall. Plasmolytic experiments, how. ever, do not indicate a protoplasmic continuity. On treatment with saline solutions, as indicated above, the protoplasm of the living cell withdraws completely from the cell-wall and gathers in one or two round masses. If 
the flagella are merely projecting threads of protoplasm it might be expected that in plasmolysis they would be with drawn within the cell and that motion would cease. This, however, is not the case. The plasmolyzed living cell continues to move the same as in the beginning. The flagella, therefore, are not directly connected with the inner protoplasm of the cell. They are given off by the cell-wall and chemically they would seem to be identical with the outer, softened layer of this structure.

As indicated above, the cell-wall is essentially protein matter and, unlike the cellulose wall of higher plant cells, it takes an active part in the life of the cell. The cell-wall receives its nourishment from the protoplasm and is itself, therefore, a living structure. The filaments or whips given off by the outer layer of this wall are also protein in nature and are also living. The flagella are unquestionably the organs of motion. By inducing movements in the liquid they renew continually the food supply. Moreover, it is possible that the protoplasmic whip is a means of absorbing nourishment for the cell. In the latter case it might be expected that flagella would also be present on non-motile bacteria. Flagella, however, are never found on strictly non-motile organisms. Pseudo-flagella, due to a mucouslike secretion, are sometimes met with in such cases.

The arrangement of flagella on a given species is fairly constant, but will vary with different species. In the vibrio of Asiatic cholera, and in the bacillus of green pus, there is usually only one whip present and that is attached to one end. At times, the cholera vibrio may have two, three or four whips at one pole, and again it may have none. In old cultures it may have a whip at each end (Fig. $6 d$ ).

The spiral forms, as a rule, have a bunch of whips at one end. When each end is equipped with a bunch of whips it is because there are really two cells present. The whips on the spiral forms are not as flexible and wavy as those on the bacilli. They appear rather stiff and are 
slightly curved, like an eye-lash. They can therefore be designated as cilia rather than as flagella (Fig. $6 e, f$ ).

In many bacilli the flagella are very numerous, and are diffuse, or distributed all over the surface of the cell. In such cases a perfect fringe of delicate wavy lines can be seen surrounding the organism. The Proteus vulgaris, typhoid bacillus and the anaerobic bacteria are especially well provided with flagella (Fig. $6 \mathrm{c}$ ).

Flagella are especially abundant on fresh young growths, about one day old. They disappear in old cultures by being torn off. Usually, they also disappear just before spore formation. This, however, is not the case with anaerobic bacteria. The formation of whips depends upon the composition of the soil. Thus, cultures of the cholera and typhoid bacilli which have been grown on the ordinary artificial media by the author for more than ten years show scarcely any motion. It has been proposed to employ the fact of the number and arrangement of whips as a means of identification of species, but for reasons indicated above this is not feasible.

In 1890 Löffler observed in stained preparations and in a living culture of the bacillus of symptomatic anthrax enormous spindle-shaped spiral bodies, which he believed to be formed like a braid of hair, by the twisting together of a large number of the ordinary whips. Three years later the author met with these same "giant-whips" while studying a new anaerobic bacillus, and was also able to repeatedly demonstrate their presence in three other anaerobic bacteria. In the same year Sakharoff met with these peculiar forms in gelatin cultures of the B. Asiaticus. Recently, A. Fischer has described giant-whips in several species, and Sames has found the same forms in cultures of a motile sarcine. The author, during the past year, has studied the development of these enormous spirals in cultures of the typhoid, coli, psittacosis, and icteroides bacilli. There is 
reason, therefore, to believe that all motile bacteria give rise to these so-called giant-whips. It is possible that the spirals observed in the intestinal contents of cholera, and those present in hospital gangrene, are not distinct organisms, but rather altered flagella. The author has found giant.whips in the bodies of animals (Fig $7 a)^{3}$.

Their size can be inferred from the fact that they can be seen in unstained specimens. The larger forms can be easily seen with a No. 3 objective. The author has repeatedly found giant-whips that were $70 \mu$ in length. In one instance the length was 132 : , or ${ }_{9 \frac{1}{8} \overline{6}}$ of an inch.

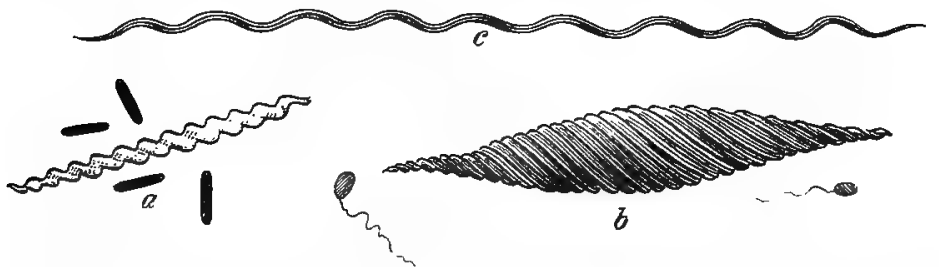

FIG. 7. Giant whips. $a$ and $b$ from photographs of Bacillus oedematis maligni No. II. $a$-Colorless spiral in streak preparation from peritoneum of a guinea-pigbacilli stained; $b$-Large spindle-shaped spiral, compared with ordinary whips; $c-$ Slender, long spiral form.

The giant-whips are invariably motionless and usually are spindle-shaped. In this case the borders are wavy and corresponding diagonal bands will be seen, resembling the twisted appearance of a rope. Sometimes a spindle seems to divide lengthwise, so that it appears as if two spindles diverged from a common point. The thick spindle is not the only form in which the giant-whip is met with. It may be a slender, wavy, very long spiral, without any enlargement or thickening. Such spirals may extend through the entire field. These thread-shaped giant-whips may be single, but they may also be bunched, proceeding, as it were, from a common point. The giant-whips are especially abundant in the water of condensation which is present in

1 Zeitschrift für Hygiene, 17, plates 1 and 2. 
a tube of freshly inclined agar. The method for their detection will be given in Chapter XI.

As mentioned above, Löfler, the discoverer of these strange forms, considered them to be woven masses of the ordinary whips, and this view has been quite generally accepted. It is doubtful, however, that this explanation of their origin is correct. If they result from a twisting process some motion should at times be observable, but such is not the case. The author has observed beautiful small spindles in cultures only eight hours old, but at no time could motion be observed. Moreover, the spindles, especially in the earliest stage, might be expected to be surrounded by the bacteria which have had their whips entangled. Usually, however, the whips stand out sharp by themselves. Furthermore, the braiding process does not satisfactorily explain the formation of the perfectly even and very thin spirals which frequently attain a length of $100 \mu$ or more. It has been supposed by some that the ordinary flagella, which, as pointed out, may be considered as living protoplasmic matter, when torn loose from the cell, may continue to move about for a short time, and in this way lead to the formation of giant-whips. There is reason to believe that the motile organs on certain flagellates, or animal organisms, are endowed with contractility and, for a short time at least, may live and move about after separation from the cell proper. It is possible, however, that these forms are unusually developed flagella, either as a result of involution changes, or because of a softening of the whip substance, corresponding to that observed in capsule formation. 
THE LIFE HISTORY OF BACTERIA.

Growth and multiplication is a characteristic of living organisms. As a rule the plant or animal cell, when it reaches the fully developed, adult stage, divides and thus gives rise to two new cells. The young bacterial cell, likewise, grows, attains its full size, and then multiplies by division or fission. Bacteria are, for this reason, designated as schizomycetes or fission-fungi.

The multiplication, or actual increase in number, of bacteria always results by the process of division whereby one cell forms two, and only two, new cells. There are instances, as will presently be seen, where apparently one cell gives rise to four or to eight cells, but in all such cases the division is consecutive and not direct. That is to say, the cell does not divide directly into fourths or eighths, but does form two halves which, subsequently dividing, yield four cells, and the next division yields eight cells.

Cell-division among uni-cellular plants and animals is completed in a very short length of time. This, perhaps, is especially true of the bacteria. Given a suitable soil, the rapidity of growth and multiplication of bacteria will depend upon the temperature. The nearer the temperature approaches the freezing point the slower will be the rate of multiplication. On the other hand a temperature of $30^{\circ}$ to $37^{\circ}$ gives the most rapid growth. Under such conditions the average bacterial cell will probably divide in less than a half an hour. This rate of multiplication cannot be maintained for any length of time, owing to the exhaustion of the soil and above all to the accumulation of waste products 
which retard and eventually stop growth. Assuming the continuance of favorable conditions, a single cell, dividing in 30 minutes, would be represented at the end of 12 hours by more than sixteen million descendants. In 24 hours the number would rise to more than two hundred and eighty billions.

The above figures will serve to impress the fact that bacteria multiply with extreme rapidity. Moreover, they will help to understand what marked changes may result in a comparatively short period of time when certain bacteria develop in milk, meats or in the living animal body. Small as bacteria are, owing to their rapid multiplication, they may give rise to poisonous substances in sufficient amount to cause in a few hours profound poisoning, or even death.
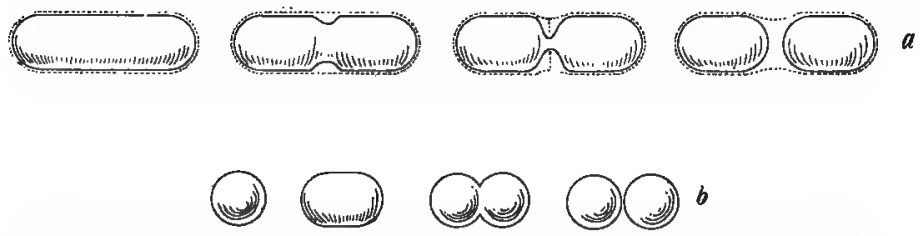

FIG. 8.-Cell division (diagrammatic), a-Bacillus, showing deflection of outer membrane; b-Micrococcus.

The process of division can best be observed in large bacilli. Owing to the absence of a definite nucleus, and of any cell structure, the change that takes place during multiplication is very simple. When the bacillus has attained the fully developed stage, a slight transverse constriction appears at the middle of the rod. The ringlike process of the cell-wall gradually extends toward the center of the cell until eventually the protoplasm becomes divided into two halves. Usually the first indication that cell division has taken place is the appearance of the clear, delicate transverse line. The division of bacilli and of spirals is always transverse, never longitudinal.

The wall that divides the cell into two is to be considered as an ingrowth of the cell membrane. Inasmuch as in 
stained preparations this dividing line remains colorless, it is evident that it largely consists of the same material as the outer layer of the cell membrane, namely, the softened, gelatinized mantle which, unlike the membrane proper, does not stain with anilin dyes. Obviously the entire cellwall, inner as well as outer membrane, is depressed at the zone of constriction (Fig. $8 \mathrm{a}$ ). When the ingrowing wall reaches the center the inner membrane coalesces and divides, leaving the space between the two new end walls filled with the gelatinized outer membrane. Continuous filaments showing no apparent division into cells are sometimes met with. In such cases division may have occurred, but is not visible, owing to the absence of the outer membrane.
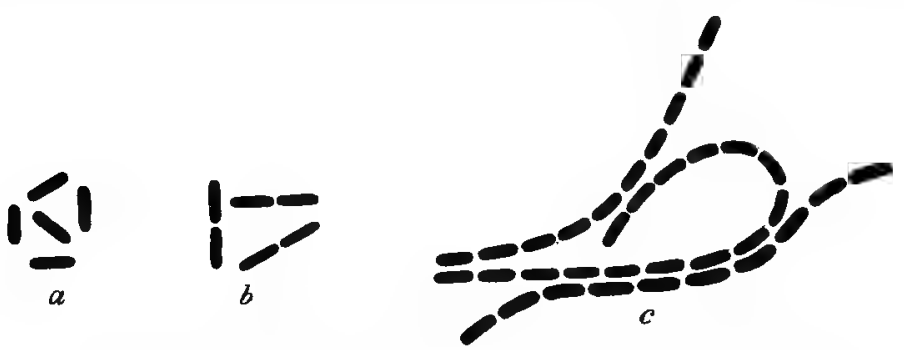

FIG. 9. Division forms of bacilli. $a$-single; $b$-in pairs; $c$-in threads.

As soon as the cell has completely divided, the two new cells may at once tear apart and lead a separate existence. Many bacilli are therefore characterized as growing singly. In some species there is a tendency for the two cells to remain attached owing to the firm union of the two cells, which are held together by the gelatinous connecting zone. The bacillus is then spoken of as growing in pairsdiplo-bacillus. In other species the cells are likely to remain attached even after repeated division. The individual rods remain attached, end to end, by the undivided outer layer of the cell-wall, and thus give rise to long filaments which are commonly designated as threads. A thread may be long or short; that is, it may consist of four or five, or fifty to one 
hundred or more cells. A thread is always composed of rod-shaped bacteria, bacilli, and may be compared to a row of bricks in a wall.

Micrococci multiply in like manner by division. The spherical organism is usually supposed to elongate somewhat just previous to fission (Fig. 8 b). The transverse constriction and dividing line then appear as in the case of a bacillus. According to some the micrococcus does not elongate first, but divides directly into two halves. It may increase in size previous to division, but its form remains spherical. On the other hand the bacillus always grows in length, and it would seem as if this fact could be utilized in distinguishing between a true micrococcus and a very short rod.
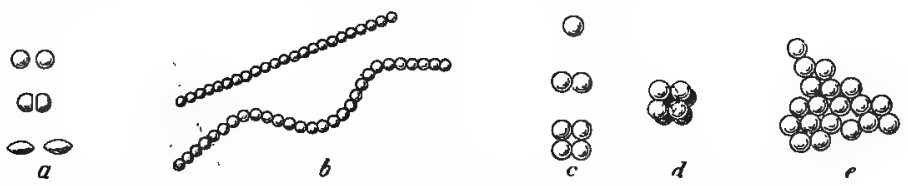

Fig. Io. Division forms of micrococci. $a$-diplococcus, perfect form-with flattened apposed surface (gonococcus), - lanceolate form (pneumonia); o-streptococcus; $c$-Consecutive division yielding a tetrad; $d$-Sarcine form resulting from division of tetrad $c$. $e-$ Staphylococcus.

The two half-spheres which result from the division of micrococcus may retain this form and remain attached by the undivided cell-wall. In -this case the two cells are spoken of as being biscuit-shaped and in pairs. The germ of gonorrhea presents this characteristic appearance. When micrococci grow in pairs, as in this instance, they are designated as diplococci (Fig. 10 a).

On the other hand, as soon as the division is complete, the two new cells may gradually round out and assume a spherical form. They may tear apart and grow singly; or, the two cells may remain attached by the narrow zone of undivided cell-wall, forming thus a diplococcus. If each of these two attached cells now divides in the same direction as the original one, a row of four spherical organisms will result. A continuation of this division in one direction 
eventually yields a long row or chain of attached micrococci. This exceedingly characteristic form is known as a streptococcus, and as indicated, its formation is analogous to that of a thread. It may be compared to the beads on a rosary (Fig. 10 b).

In the above instances the dividing membrane always forms in a plane parallel to the original plane of division. Consequently growth extends, as it were, along a line. The division is then said to occur in one direction of space. This does not hold true for all micrococci. Thus, after the original cell divides into two, each of these in turn may divide so that the line of division is at right angles to that in the original cell. The four cells which thus result are not arranged in a row, but form a tetrad, which may be compared, when stained, to the four spots on a die. Division has been consecutive and in two directions of space (Fig. $10 c$; also Fig. 5 b).

Again, each of the four cells of a tetrad may divide so that the plane of division will be parallel to the face of the tetrad. The result is a cubical mass of eight cells. This package-shaped mass of micrococci is known as a sarcine. Division has been consecutive and in three directions of space (Fig. $10 d$ ).

Lastly, micrococci may divide rather irregularly and, remaining adherent, eventually yield a mass of cells which resemble somewhat a bunch of grapes. Such forms are designated as staphylococci (Fig. $10 \mathrm{e}$ ).

Whatever may be the final form which results from the division of micrococci, whether the division occurs in one, two or three directions of space, it is always to be understood as consecutive. That is to say, one cell divides into two; these two, on division, yield four cells, and the latter in turn yield eight cells. One micrococcus never divides directly into quarters or into eighths.

The simple micrococcus, as shown above, may multiply so that it usually appears as a single cell. When the cells 
remain attached because of the incompletely divided cellwall, diplococci, streptococci, tetrads, sarcines and staphylococci may result. As a rule, a given micrococcus shows some one of these six forms as its characteristic form. Thus, a micrococcus that forms a streptococcus will not occur as a tetrad or as a sarcine.

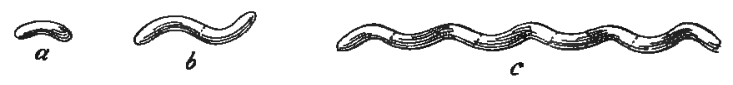

FIG. II. Division forms of the spirillum, $a-V$ ibrio, $b-D$ ju cell, form of elongated $\mathrm{S}$; $c$-Long spirillum, showing compsa cells.

The spiral or screw-shaped bacteria multiply by transverse division, as in the case of bacilli. When the organism grows single it forms a bent and twisted rod which, as ordinarily. seen, appears comma-shaped. The comma bacilli, or vibrios, are the individual cells which, when they remain attached, end to end, yield a spirillum (Fig. 11). "The spirillum, therefore, may be considered as analogous to the thread, and streptococcus. Certain spirilla, like those of the mouth and of recurrent fever, cannot be resolved into component cells, and are therefore to be considered as single individuals.

\section{Spores.}

It has been pointed out above that bacteria always multiply by division. In this process one cell, dividing into two halves, yields two new individuals. As long as the organism is growing and multiplying it is said to be vegetating and the form which it presents during this period of its life is spoken of as the vegetative form. This stage, therefore, may be compared to the growing, higher plant. The latter, however, when it reaches the adult condition develops reproductive organs and forms seeds. 
A somewhat similar condition is observed among many bacteria. The active growth stops and reproduction occurs. The organism in this case gives rise to what is called a spore which is the analogue of the seed of a higher plant. The object of either seed or spore formation may be said to be the perpetuation of the species. The plant, whether high or low, when in the vegetating condition is a relatively weak organism. It is readily destroyed by desiccation, heat, cold and other agencies. There is need. therefore, of some resistant form which will enable the plant to survive unfavorable external conditions. The flowering plant forms its seed, whereas the flowerless plant forms the spore.

It is evident, therefore, that bacteria may be either in the actively growing, vegetating form, or in the reproductive or spore form. The latter is sometimes spoken of as the resting, permanent or resistant form. As will presently be shown the bacillus, when it sporulates or forms spores, almost invariably gives rise to a single spore. The latter, in turn, when it sprouts or germinates gives rise to a single bacillus. Consequently it is not proper to speak of bacteria as.multiplying by spore formation. When the parent cell gives rise to but one spore which in turn develops into one young cell the process cannot be considered as a multiplication or an increase of species. Bacteria reproduce, perpetuate themselves by means of spores but they multiply by cell division. The vegetating cell may divide, or may form a spore, whereas the spore can only germinate.

The formation of spores has been observed in some spirals, in many bacilli but not among micrococci. The vast majority of bacteria, therefore, have not been seen to possess spores. From this it by no means follows that these bacteria always remain sporeless. The conditions of spore formation are as yet but imperfectly understood and it is quite probable that many, if not all, of the bacteria in which spores have not been found can give rise to these bodies under the conditions which prevail in nature. The 
conditions under which bacteria exist in the laboratory are far from being the best.

The spore is always formed within the cell. It may therefore be spoken of as an endospore. The botanist DeBary attempted to make use of this fact as the basis of a natural classification of bacteria. He divided the group into endospore and arthrospore bacteria. In arthrospore formation it was supposed that the entire cell converted itself into a spore-like resisting form. That is to say, the cell-wall of the individual organism would thicken, harden and become impenetrable. In this condition the cell, like a spore, could survive unfavorable conditions and could in turn germinate or multiply. All bacteria which were not known to produce endospores were therefore placed in the arthrospore group. As a matter of fact there is no good and sufficient reason to believe in the existence of arthrospore formation.

Sporulation.-Owing to the extremely small size of bacteria it is manifestly impossible to follow out the process of spore formation in its minutest detail. This much, however, can as a rule be observed. The contents of the cell are at first homogeneous and the first indication of the beginning of sporulation is the appearance of very fine granules in the protoplasm. These are sometimes spoken of as sporogenic granules. Some of these are larger than others. One of these located at a certain place in the cell gradually increases in size probably because the other granules gather or flow together at this point. The result is a roundish or ellipsoidal, bright body which at first has no definite envelope or wall. Presently, however, a distinct spore-wall does form which may.be due to a condensation of the protoplasm of the cell around the central body. At all events the protoplasm of the cell disappears, as can be shown by plasmolysis, and in part at least makes up the substance of the spore. Occasionally a very small resi- 
due of the protoplasm may remain on the outside of the spore.

The spore, therefore, may be considered as the condensed cell contents. It contains all the proteins of the parent cell, and, when completed, lies surrounded by an aqueous liquid inside the otherwise empty shell or cell membrane. This original cell wall soon softens and dissolves and the spore is thus set free. Free spores are frequently met with in about 24 hours, when the growth occurs under the most favorable conditions. Usually, however, they are not liberated for several days. It not infrequently happens that all the cells, composing a thread, form spores at the same time. In that case the bright, highly refracting spores are present, one in each cell, and may be compared to a string of beads. With the exception of two or three doubtful cases, it may be stated as a general rule that a bacillus gives rise to but one spore. Inasmuch as the protoplasm of the parent cell goes to make up the spore it follows that the former ceases to exist as soon as the spore is fully developed.
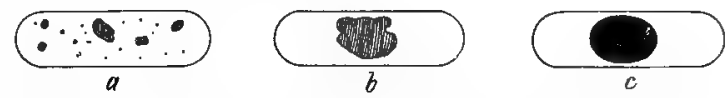

FIG. I2. Sporulation. $t$-First stage showing sporogenic granules; $b$-Incomplete spore; $c$-Fully developed spore.

In a given species the spore nearly always develops in the same relative position within the cell. Thus, in some species the spore forms in the middle and occupies, therefore, a median position. In others it develops at the very end and this position may be designated as terminal. Again, the spore may be located between these positions, in which case it is said to be intermediate (Fig. $13 a, b, c$ ).

The form of the parent cell is very often unchanged by the development of the spore on the inside. At other times a slight enlargement may result in that portion of the cell occupied by the spore. Sometimes this enlargement is very 
marked and characteristic forms result. Thus, in the case of a median spore a marked enlargement of the central portion of the cell gives rise to a spindle-shaped form which is known as a clostridium (Fig. $13 a$ 2). When the spore is terminal, a corresponding spherical enlargement of the end gives rise to what is known as the "drum stick" bacillus (Fig. $13 c 2$ ).

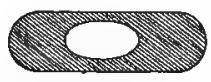

$a$

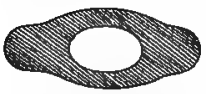

FIG. I3. Position of spores; resultant forms (diagrammatic). $a$-Median spores: $b$-Intermediate spores; $c$-Terminal spores

$2 a, b, c-C h a n g e$ in form of cell due to the presence of the spore; a $a$-Clostridium; $2 a-"$ Drum-stick" form.

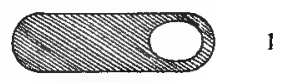

$c$
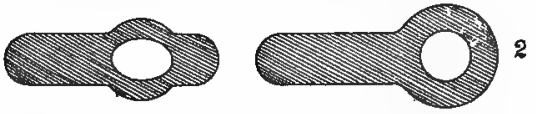

In the case of motile bacteria spore production is usually accompanied by a loss of motion. This is especially true of the aerobic species. On the other hand the anaerobic bacteria may continue to move about for some time after the spore has fully developed. Flagella can be demonstrated on these motile spore-bearing rods. The persistence of motion in such cases may be due to the remnant of protoplasm which is left outside of the spore and which lines the cell wall.

Spore formation has been supposed to take place whenever the culture medium became exhausted. This view, however, can be easily shown to be incorrect. The nourishing material of the soil is by no means consumed when a given organism begins to form spores. There may be times, however, when this factor may come into play. Under the artificial conditions of the laboratory it is more likely that the accumulation of the waste-products of the organism and the action of these products on the living cell cause it to pass into the spore form. As long as the 
organism is growing under the best possible, conditions it will not give rise to spores. If, for instance, it is transplanted every few hours in order to avoid the formation of waste products it can be maintained indefinitely in the vegetating condition. It is for this reason that bacteria growing in the blood of a living animal do not form spores.

The temperature is an important factor in spore production. The formation of spores has not been observed to occur below $16^{\circ} .{ }^{1}$ The optimum temperature is at $30-35^{\circ}$. Certain bacteria will not form spores in the absence of oxy'gen, whereas other bacteria require very little or no oxygen.

The composition of the soil has much to do with sporulation. The presence of calcium salts and the absence of pepton favor spore production. On the other hand, the presence of minute amounts of carbolic acid, or of mercuric chloride will so alter an organism as to give rise to a sporeless variety. Asporogenic bacteria may also result from prolonged artificial cultivation on the ordinary laboratory media. The anthrax bacillus which has been cultivated for some years never gives rise to spores when grown on the ordinary media. The failure in spore production in such instances is similar to the loss of motion of certain bacteria under like conditions (p. 38). The asporogenic varieties may be compared to the highly cultivated, seedless flowering plants.

In its earliest stage, the spore appears within the cell as a bright, oil-like, refracting body. This appearance becomes well marked when the spore is fully developed, that is to say, when the spore-mass has surrounded itself with its characteristic spore-wall. It is especially marked in the free spore. The spore, as a rule, possesses the same width as the parent cell. It is a short oval or roundish body and can, as a rule, be readily recognized by its microscopical appearance and by its behavior to stains.

${ }^{1}$ Unless otherwise indicated the temperatures given in this work are Centigrade. 
Owing to the peculiarly dense character of the sporewall the anilin dyes do not readily stain the spore. On staining, therefore, it appears as a colorless body imbedded in a stained cell, or rather within the stained cell-wall. The presence of a bright, refracting body within an unstained cell, or the presence of a colorless, non-staining body in a stained cell does not prove that it is a spore. Such spore-like bodies, which may be termed pseudo-spores, have been frequently observed, and mistaken for spores. Thus, the so-called spores of the tubercle bacillus are due to differences in the density of the protoplasm which contracts into beads or balls, thus leaving vacuoles or clear spaces filled with a cell fluid. The small bright polar bodies which are frequently seen in glanders, diphtheria, typhoid and other bacilli are to be considered as small masses of condensed protoplasm since they stain more deeply than the surrounding contents. The resistance to destruction is a valuable criterion of a spore-like body, but even this does not prove its spore character. The true character of a spore can only be established by observing its germination.

Spore germination. - The spore which is formed in a given culture medium does not germinate until after it has been transplanted to a good new soil. The original medium is unfit for this purpose largely because of the presence of various chemical products, which were elaborated by the vegetating or growing organisms. Moreover, it was the presence of these products which stimulated the cells to form spores. In a suitable soil, and under proper conditions, the spore gives rise to a young, growing cell, which rapidly increases in size, reaches what may be called the adult state, and multiplies. Eventually, this progeny of vegetating cells gives rise to new spores.

Like the seed of higher plants, the spore itself does not multiply. Under no condition will one spore divide and give 
rise to two or more spores. All that it can do is to reproduce the type from which it originally developed. The germinating spore gives rise to one young cell, and, inasmuch as a fully developed cell produces only one spore and then dies, it follows that spore production or germination is in no wise a means of multiplication. The spore is therefore solely a reproductive or perpetuating form.

The process of germination of spores is not the same for all species. It will vary, more or less, with different types, but there is "reason to believe that it is always constant for a given species. The mode of germination, consequently, might be made use of in classifying bacteria but, unfortunately, it requires a very careful and prolonged observation. For this reason, the study of the germination of spores is very rarely resorted to. The process has been followed out in only a relatively small number of bacteria, probably in not more than 10 or 12 species.

The first change observed when the spore is about to germinate is that it swells up and lengthens. This increase in size is probably due to imbibition of moisture. As the spore enlarges it becomes less bright. The marked refraction disappears and gives place to a dull appearance. The altered spore now gives rise to a young cell in one of three ways.

1. Direct germination.-The spore gradually changes, by elongating and growing, directly into a new cell. In this case no shell or spore-wall is thrown off, as such, but it may possibly be softened and dissolved during the process of conversion. Bearing in mind the development of the spore, as a condensation of the cell protoplasm, it would seem that this method of germination was the reverse of sporulation. It has, however, been observed in but one or two cases (Fig. $14 a$ ).

Usually the spore-wall splits open at some one place on the surface and through the opening thus produced the young cell makes its appearance. The opening in the spore-wall may be at one or both ends, that is to say polar, 
or it may be across the middle, in which case it is designated as equatorial.

2. Polar germination.-The elongated spore opens at one pole and the young cell thus passes out. In this case the long axis of the young cell is parallel to the long axis of the spore. The anthrax spore germinates in this way (Fig. $14 b$ ).

3. Equatorial germination.-The lengthened spore opens as the result of a split across the middle. In some forms, as the B. megaterium, the spore-wall is divided into two, and the two halves are pushed asunder by the young cell. In the case of the $B$. subtilis the cleft is incomplete. In such instances the young rod leaves the spore-wall either by doubling up, forming a horse shoe as it were, or it rotates and passes out at right angles to the long axis of the spore (Fig. $14 c, d, e)$.

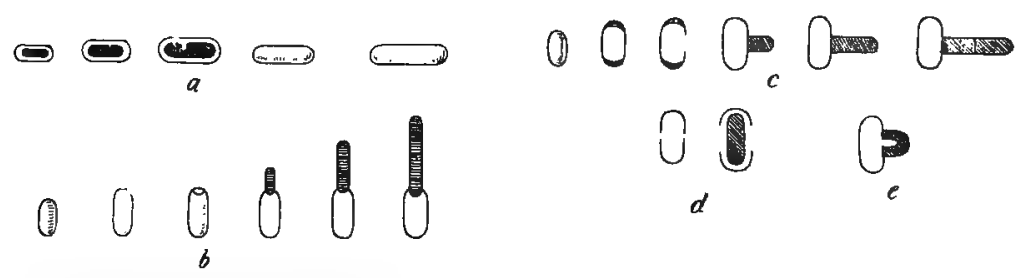

FIG. I4. Spore germination. a.-Direct conversion ef a spore into a bacillus without the shedding of a spore-wall (B. leptosporus); $b$-Polar germination of B. anthracis; $c$.Equatorial germination of B. subtilis; $d$.-Same of B. megaterium; $e$.-Same with" "horseshoe" presentation.

In addition to a suitable soil, the germination of a spore is markedly influenced by the temperature. The process will take place slowly at a low temperature, and more rapidly at or near the temperature of the body. In the latter case it may require from 3 to 5 hours. When a mass of spores is present, the germination of some may be greatly delayed as compared with others. This fact must be taken into account in the sterilization of culture media.

Structure of the spore.-A rather dense, impenetrable envelope or membrane invests the contents of the spore. 
The prime object of this spore-wall is to protect the protoplasmic contents against conditions which would otherwise destroy the life of the organism. The exact chemical com. position of the spore-wall is not known, but it is assumed to be protein in nature. During germination it usually softens or gelatinizes, and in some cases becomes thicker at the poles (Fig. 14 c).

The contents of the spore, as seen from the method of formation, are essentially those of the parent cell, less water. Consequently the spore substance, which may be considered as condensed protoplasm, contains all the proteins of the original cell. Inasmuch as the bacterial cell contains more or less fat it follows that the spore will likewise possess fatty compounds. The bright, highly refractive appearance of the spore is highly suggestive of the presence of an oil-like body. When spores are treated with ether no fat can be extracted, but this does not justify the assumption that fat is absent. When ether is shaken up with milk it will not remove the fat which is present in the milk globules.

Spores are, as a rule, perfectly colorless. In a few fluorescing bacteria red spores have been observed. In several other instances markedly green spores have been met with.

The anilin dyes do not stain spores as readily as the vegetative form. This is commonly believed to be due to the dense spore-wall, which hinders the penetration of the dye. Undoubtedly, the contents of the spore will take up the stain very slowly owing to the almost total absence of water. For the same reason, the contents, when once stained, can be decolored only with difficulty. The method of double staining spores will be described in Chapter X.

Spores are characterized by their extreme resistance to destruction. The vegetating form is readily killed by mere desiccation, whereas the spore can be kept in the dry condition for years. Again, the vegetating form is killed in a 
few minutes by exposure to moist heat of about $70^{\circ}$, but the spore form may resist for hours. Steam heat, which, as a rule, instantly kills the vegetating form will require 5 to 10 minutes, and in some instances as many hours, to destroy the spore. A similar difference between the vegetating. and spore form will be observed in the action of various chemical substances.

Spores will resist the action of dry heat more readily than that of steam heat. A dry heat of $140^{\circ}$ may require an hour or more to destroy spores which would be killed in a few minutes by exposure to steam. Heat and moisture are therefore more destructive than heat alone. This is equally true for the higher forms of life. Thus, in a very dry climate man can endure without discomfort a temperature of $130-140^{\circ} \mathrm{F}$. whereas a much lower temperature in the presence of moisture is prostrating.

Steam heat under pressure will destroy spores more readily than will ordinary steam heat. The spores of a certain potato bacillus, which resist steam for 5-6 hours, are destroyed in 10 minutes by an exposure to steam under pressure at a temperature of $120^{\circ}$.

The spores of different species possess different degrees of resistance. Thus, the anthrax spores are more easily destroyed than those of the hay bacillus. Again, the several varieties of a given species may produce spores which will show extreme variation in resistance. There are anthrax spores which are readily destroyed by 5 per cent. carbolic acid within 24 hours, whereas other anthrax spores may not be affected by an exposure of 40 or 50 days to this solution.

The extreme resistance of some spores, as briefly indicated above, accounts for the theory of spontaneous generation which at one time was quite universally accepted. According to this theory it was supposed that the lower forms of animal and plant life could develop without 
the agency of other organisms; in other words, that life could originate directly from dead matter. This view was apparently substantiated by the fact that when an infusion of wheat or barley was boiled in a closed flask for some minutes and then set aside various bacteria would develop in the liquid. The exposure to the temperature of boiling water was assumed to be sufficient to destroy all living matter and consequently any new life that might subsequently develop must come from dead matter. Such was the theory of spontaneous generation which, formulated by Needham in 1747 , persisted as a dominant idea for more than 100 years.

This theory existed because, in the first place, it was not known that bacteria were to be found almost everywhere in nature. In the second place, the existence of spores and the remarkable degree of resistance which they possessed was not dreamed of. The researches of Pasteur (1861) first demonstrated the wide distribution of bacteria in the air and elsewhere, and, above all, clearly proved the existence of highly resistant forms of bacteria, the spores. A moderate amount of boiling will kill all vegetating forms and some feeble spores. The highly resistant spores are not destroyed and hence subsequently germinate. The beginner in the lab. oratory will invariably meet with instances of so-called spontaneous generation whenever the culture media have not been properly sterilized. The smallest of living beings, like the highest forms of life, are always descended from antecedents of their own kind. While it may not be true to say that all life comes from the egg, it is true that "all life comes from life." 
THE ENVIRONMENT OF BACTERIA.

Although bacteria are present almost everywhere upon the surface of the globe it must not be supposed that they are capable of growing wherever they may be found. The air always contains a greater or less number of bacteria, but these organisms are not multiplying. They are present merely as extremely minute particles picked up, and wafted about by currents. The conditions met with in the air are far from being favorable to their development. On the contrary the desiccation or drying, to which the floating bacteria are subjected, is directly injurious to these organisms. The sporeless bacteria are probably speedily destroyed as a result of desiccation and of the action of sun-light.

Inasmuch as bacteria are specifically heavier than air they tend to settle together with the coarser, floating matter. The dust which therefore deposits on the floor, windows, or upon the clothes, hair, or skin is rich in various bacteria, yeasts and moulds. But even when thus deposited these organisms will not grow and multiply. In order that they shall grow it is necessary that they reach a suitable nutrient medium and an essential condition of a nutrient soil is that it shall contain moisture.

Moisture, consequently, is absolutely necessary for the development of bacteria. More than that, it is necessary to the well being of every form of life, whether high or low. This is seen in the fact that water enters largely into the composition of living, growing protoplasm. The tissues of higher plants and animals contain from 70 to 80 
per cent. of water. The organisms which grow in water, like algae and fish, contain a relatively higher per cent. As indicated above bacteria live only in liquids and on moist surfaces. They are, therefore, essentially aquatic and, as such, they hold within their cells a large amount of water.

The chemical examination of various actively growing bacteria shows that they contain about 85 per cent. of water. The proteins, which are necessary constituents of protoplasm, make up about 10 per cent. The amount of fatty substances present will vary with different species. On an average they may be said to contain about one per cent. of fat. Certain bacteria, like those of glanders and tuberculosis, are very rich in fats, which may constitute as much as 40 per cent. of the dried cells. The carbohydrate group, represented by cellulose, granulose or dextrin-like compounds, is at times present. The inorganic or mineral constituents make up about one per cent. of the living organism.

In addition to the above components of the bacterial cell other substances may at times be present. The diseaseproducing bacteria elaborate within their cells the specific poison or toxin. All bacteria, moreover, contain one or more soluble ferments or enzymes.

Carbon. - The contents of the living cell are made out of the food on which the organism lives. The majority of bacteria differ from the higher plants in one marked respect, and that is, that they do not contain chlorophyll. It is by the aid of chlorophyll, in the presence of sun-light, that the higher plant assimilates the carbonic acid of the air. The carbon is retained by the plant while the oxygen is, as it were, exhaled and returned to the atmosphere. Consequently, the source of the carbon, present in the higher plant as protein matter, cellulose, starch, fats, etc., is the simple, inorganic compound, the carbonic acid 
of the air. Owing to the absence of chlorophyll the majority of bacteria cannot utilize this.source of carbon They are, as a rule, dependent upon organic compounds. That is to say, the more or less complex carbon compounds elaborated by higher plant and animal life constitute the food out of which the bacteria can appropriate the carbon and build up their own protoplasm. To a very large extent, therefore, bacteria depend for their food upon dead animal and vegetable matter. In this respect they resemble animal life. As is well known the animal cannot utilize the carbonic acid of the air but obtains its carbon from the preformed carbon compounds in the animal or vegetable food. The proteins, carbohydrates and fats supply the necessary carbon to the growing animal and also to the vegetating bacteria. The latter may also obtain their carbon from more simple organic compounds such as glycerin, or lactic, or tartaric acids. It may be interesting to note that bacteria, like animals, instead of assimilating actually give off carbonic acid.

The above is true, undoubtedly, for the majority of bacteria. There are, however, certain bacteria which can live on wholly inorganic matter. The interesting group of nitro. bacteria, although they do not contain chlorophyll, are nevertheless able to assimilate carbonic acid even in the absence of sun-light. Organisms of this type may well be consid. ered as belonging to the earliest inhabitants of the globe.

Nitrogen.-Carbon is a characteristic and essential constituent of protoplasm. There are other elements equally important, and among these nitrogen deserves especial attention. Although the air contains nearly 80 per cent. of free nitrogen, the higher plant cannot obtain this element from this source. All the nitrogen which enters into the composition of the higher plants, with certain exceptions presently to be mentioned, is derived from various nitrogen compounds present in the soil. The ammonia, nitrous and 
nitric acids present in the earth are absolutely essential to the growth and development of most of the higher plants. This form of life, therefore, obtains all of its nitrogen from inorganic compounds. The animal organism, on the other hand, cannot utilize these compounds as food. The nitrogen which is necessary to the building up of protoplasm in the animal cell is derived from preformed, organic, nitrogen containing substances. Moreover, only certain nitrogenous, organic compounds can serve as food. These are the protein or albuminous substances which have been made by the living plant or animal cell. Animal life is therefore dependent upon plant life for its supply of nitrogen.

Bacteria, like animal organisms, may obtain the nitrogen necessary for their growth from organic, nitrogenous substances. The proteins present in dead animal or vegetable matter constitute, therefore, an important source of nitrogen for these lower forms of plant life. It must not be inferred, however, that the proteins are the only source, for such is by no means the case. Many bacteria can obtain their necessary nitrogen from compounds that are vastly more simple than the proteins. Thus, the amido acids, such as asparagin, constitute an excellent food in this respect for certain bacteria. Furthermore, many bacteria may obtain their nitrogen from wholly inorganic compounds, such as ammonium chloride. There are other species which can thrive better on nitrates than on other forms of nitrogen.

The nodules or tubercles which are found upon the roots of leguminous plants, such as the pea, lupine, etc., are essentially masses of certain bacteria, or bacteroids, which possess the remarkable property of assimilating the free nitrogen of the air and in transmitting this element to the growing plant. A vigorous, healthy growth of such plants is directly dependent upon the presence of these parasitic bacteria.

It is evident, therefore, that bacteria can obtain their nitrogen from various sources. This element may be appropriated from the complex 'orgà'nic protênn mólecule, or from' 
the simpler amido acids, or from strictly inorganic compounds, such as ammonium salts, nitrates or, in rare cases, free nitrogen. The fact that some species can thrive better than others upon one of these sources of nitrogen can be made use of in differentiating one form from another. Thus, the typhoid bacillus cannot utilize the nitrogen in ammonium salts, whereas the colon bacillus can. Artificial culture media (see Uschinsky's medium) can be prepared which contain chiefly inorganic constituents and only simple organic compounds, such as lactic, or tartartic acids, or glycerin, or glucose. The latter compound is especially useful as a source of carbon under these conditions of growth.

The hydrogen which enters into the composition of pro. toplasm is probably derived along with the carbon from the organic food constituents. Whether it can be obtained from inorganic compounds, such as ammonia and water is not known. Water, as such, is taken up by the cell from the surrounding medium and, moreover, may be formed, in part, like carbonic acid, by oxidation changes within the organ. ism.

In addition to carbon, nitrogen and hydrogen every living organism must be supplied with oxygen. The higher animal obtains its oxygen from the air, whereas higher plants are obliged to depend upon the oxygen contained in water, nitrous and nitric acids. The majority of bacteria can probably utilize the oxygen of the air, which is seen in the fact that they grow only in the presence of air. Other bacteria, as will presently be seen, thrive only in the ab. sence of air. In such cases the necessary oxygen is undoubtedly obtained from organic compounds such as proteins, or carbohydrates.

Certain inorganic salts or mineral constituents are likewise necessary to the well-being of living organisms. Bacteria may obtain their sulphur and phosphorus from preformed organic compounds such as the proteins. They can, however, 
appropriate these elements from inorganic sulphates and phosphates. The various chlorides found in nature furnish the necessary chlorine, and incidentally such metals like sodium and potassium. The traces of calcium, iron, and other metals present in various liquids are sufficient, as a rule, to meet the requirements of an organism.

The amount of mineral constituents necessary to support bacterial life is exceedingly small. As shown above, the ash constitutes only about one per cent. of the growing bacteria. It has been estimated that $1 \mathrm{mg}$. of living bacteria contains about $30,000,000,000$ cells, in which case this large number of organism will yield only ro $\mathrm{mg}$. of ash. Inasmuch as the organic constituents of bacteria make up less than 15 per cent. of the whole it will be seen that the amount of organic matter necessary as food is likewise exceedingly small. This is shown by the fact that many bacteria can multiply not only in ordinary water, but even in distilled water.

The reaction of the nutrient medium exerts an important influence upon the growth of bacteria. An acid reaction is not as favorable as an alkaline one. Thus, an acidity corresponding to 20 or $30 \mathrm{c}$. c. of normal acid per liter will inhibit the growth of many bacteria, whereas the same organisms will thrive in a medium, the alkalinity of which corresponds to $50 \mathrm{c}$. c. of normal alkali per liter. It is customary, therefore, to cultivate bacteria upon a neutral or slightly alkaline soil. The moulds, on the other hand, seem to thrive best upon an acid medium.

Organic matter derived from dead animals or plants can be met with almost everywhere. The simple food requirements of bacteria are, therefore, widely distributed in nature and for that reason these organisms will be found almost universally present upon the surface of the globe. There are but few places where bacteria are absent. The air at high 
altitudes and that in mid-ocean; the air exhaled from the lungs; the deeper layers of the soil and the water coming from such depths; the internal fluids, tissues and secretions of healthy, normal animals and plants are practically the only places in nature where bacteria are not present.

As pointed out on p. 58, the ordinary air always contains bacteria which, as minute dry particles, are carried about by the movements of the atmosphere. In a perfectly tight room, free from currents, the suspended organisms will readily settle to the floor, owing to the fact that they are specifically heavier than air. A similar tendency exists in the open air and as a result the lower layers of the atmosphere will contain more bacteria than the higher ones. Moreover, the air is purified by the falling rain or snow, which drag down to the earth the suspended solid particles. It is evident, therefore, that the atmosphere on the tops of high mountains will be practically free from bacteria.

The air in mid-ocean may also be considered as free from suspended particles, including bacteria. This is due to the washing, as it were, of the winds or currents of air in their passage over the water. The bacteria in the air on coming into contact with a moist surface are held back. This fact holds true not only in the case of the air which passes over large surfaces of water but also in the case of expired air. No matter how many hundreds or thousands of bacteria may be present in the air which. is drawn into the lungs the expired air is always practically free from organisms. The latter adbere to the moist, mucous membrane of the mouth, nose, throat and bronchi, and consequently, the expired air contains very few or no bacteria.

It may be stated as a general and most important rule that the bacteria present in liquids or on moist surfaces cannot leave such places and enter the air. The most deadly organism can be studied in the laboratory, as it grows on moist culture media, without any danger in this respect. For the same reason; the' breath of a consumptive is free from 
the dreaded tubercle bacillus which can only leave the mouth, with the sputum, or in the small particles of liquid which may be scattered about in a fit of violent coughing.

The surface layers of the soil are exceedingly rich in bacteria. These organisms decrease rapidly in numbers with the depth, and eventually disappear entirely. The soil at, or below, a depth of 8 or 10 feet can be regarded as practically free from bacteria. This is due, in the first place, to the fact that the earth acts as a filter retaining most of the organisms at, or near, the surface. It is also due, in part, to the unfavorable conditions, such as low temperature and insufficient supply of oxygen. The rain, or melting snow, percolates through the soil and eventually becomes wholly free from the suspended organisms. It is clear, therefore, that the water which comes from the deeper layers of the earth is free from bacteria. The water of a spring, or of a tubular, or artesian well may contain some organisms, but these are due to accidental contamination at, or near, the surface.

The higher animal inhales every day an enormous number of bacteria. Moreover, these organisms are constantly being introduced into the alimentary canal with the food. The intestinal contents, as a result, are extremely rich in bacteria. The surface of the body may also harbor a large number of these organisms. In spite of the fact that the body is thus besieged on all sides by countless bacteria, it is nevertheless free from them. In a healthy individual the blood and Iymph, the various internal organs and tissues are wholly free from bacteria. It follows, therefore, that the secretions, such as the urine and milk, of a normal individual are perfectly germ free. 


\section{Saprophytic and Parasitic Bacteria.}

Bacteria are grouped, according to their habitat, into saprophytic and parasitic. The saprophytic bacteria are those which live on dead animal, or vegetable matter. The vast majority of bacteria belong to this group. Only a relatively small number of organisms possess the power of growing in the living animal or plant. These are, therefore, designated as parasitic bacteria. Because the latter grow and thrive in the living body it must not be inferred that they subsist entirely on living matter. The waste products of the living, or of the dead cells may furnish the necessary food supply. The parasitic bacteria include many harmless forms in addition to the disease-producing organisms.

The majority of the bacteria present in the mouth, stomach and intestines cannot be regarded as parasitic. They live on the dead matter in the alimentary tract and can live equally well outside of the body. They are to be consid. ered as saprophytic bacteria. A few of the mouth bacteria, such as the vibrios and spirals, cannot be made to grow on artificial media. They are, apparently, dependant upon the living organism. This is equally true of the leprosy bacillus and to a certain extent of the germs of gonorrhea and of tuberculosis. It is customary to designate those bacteria which are compelled to live in the living body as obligative parasites.

On the', other hand, among the saprophytic bacteria there are those which are unable to thrive in the living body. They are compelled, as it were, to live on the dead matter found in nature. Consequently, they are known as obligative saprophytes. It is evident that the obligative parasites and the obligative saprophytes constitute two extreme groups. Numerous bacteria occupy an intermedi- 
ate position between these extremes. There are bacteria which may primarily be considered as saprophytic, but which, at times, may lead a temporary, parasitic existence. These are designated as facultative parasitic bacteria. Again, certain bacteria which are primarily parasitic may be able to thrive somewhat, outside of the body, on dead matter. They elect, so to speak, a saprophytic life and hence are known as facultative saprophytes.

The transition from one extreme to the other can per, haps be seen best from the following arrangement of the several groups:

Obligative saprophytic bacteria, Facultative parasitic bacteria, Facultative saprophytic bacteria, Obligative parasitic bacteria.

The parasitic as well as saprophytic bacteria are unable to utilize carbonic acid as a food. They cannot build up protoplasm out of wholly inorganic substances. In other words, they are dependent upon organic compounds which were made by animal or plant life. The group of nitro-bacteria (p. 61) constitutes an exception to this rule, inasmuch as these can exist on wholly inorganic matter. Consequently they do not, strictly speaking, belong in the group of saprophytic bacteria.

The parasitic bacteria, as mentioned above, include the various disease-producing organisms. The saprophytic bacteria include those forms which thrive on dead matter, and consequently the bacteria which produce fermentation and putrefaction fall under this head. 


\section{Oxygen Requirements.}

It was at one time generally accepted that all living beings required free oxygen. In 1861, Pasteur described as the cause of butyric acid fermentation a bacillus which could be cultivated only in the absence of oxygen. $\mathrm{He}$ designated those organisms which required oxygen for their development as aerobic; whereas those which lived in the absence of oxygen were designated as anaerobic. The "vibrion butyrique" was the first known anaerobic germ. In 1877, Pasteur demonstrated that an experimental disease, "septicémie" or, as it is more commonly known, malignant edema, was due to an anaerobic bacillus. Since then about fifty anaerobic bacteria have been described.

An examination of the various anaerobic bacteria will show that some are absolutely dependant upon the total exclusion of oxygen. The presence of a small amount of air will promptly stop the growth of such organisms. These remarkable forms can be designated as obligative anaerobes since they are obliged to live under strictly anaerobic conditions. On the other hand, there are aerobic bacteria which live only in the presence of an abundance of oxygen. As soon as the amount of air, or oxygen is diminished growth stops. They are obliged to live in the presence of air and, for this reason, they are termed obligative aerobes.

The obligative aerobes and the obligative anaerobes constitute the two extremes as far as oxygen requirements are concerned. Most of the bacteria show an adaptability to one or the other of these conditions, and as a result the two obligative groups are conuected by a series of species which can, more or less readily, accommodate themselves to the presence or absence of oxygen. A given organism which thrives best in the presence of air may grow, though perhaps less abundantly, in the absence of oxygen. Such 
an organism is designated as a facultative anaerobe. On the other hand, a germ which may grow in the presence of air, but which grows best under anaerobic conditions, is known as a facultative aerobe. The transition from one extreme to the other can be seen from the following arrangement of the groups:

Obligative aerobic bacteria, Facultative anaerobic bacteria, Facultative aerobic bacteria, Obligative anaerobic bacteria.

The ability of an organism to grow in the tissues and fluids of the body indicates that it can thrive in the more or less complete absence of free oxygen. Consequently, most of the disease-producing bacteria are facultative anaerobes. When grown, however, under artificial conditions they may show an almost obligative aerobic character. This is especially true of the cholera vibrio.

Although many bacteria can live in the absence of free oxygen it must not be supposed that they are wholly independent of that element. As stated heretofore, living protoplasm always contains proteins and these compounds contain oxygen as one essential constituent. Consequent$1 y$, anaerobic bacteria require oxygen in some shape or other. The fact that they obtain oxygen from some source is seen not only in the chemical composition of the cell, but also in the fact that anaerobes, like otber bacteria, exhale carbonic acid. Apparently, anaerobic bacteria obtain the necessary oxygen from various organic compounds. The proteins can be utilized for this purpose. The carbohydrates, especially glucose; are valuable in this respect. It is customary, therefore, to grow anaerobic bacteria on media to which glucose has been added.

Obligative anaerobic bacteria are commonly met with in the soil. They are present, to some extent, in the intestinal contents. Only one organism of this group has been 
found in the mouth, in a decaying tooth cavity. It would appear to be somewhat contradictory to find anaerobic bacteria growing in the soil apparently in the presence of air. Nevertheless, such is the case. The explanation of this curious fact is very simple and can be very easily demonstrated in the laboratory. If, for example, an obligative anaerobic germ is planted in beef tea, in the presence of air, it will not develop. If, however, an aerobic germ is planted at the same time as the anaerobe both organisms will develop. It would seem as if the aerobic germ consumes the oxygen in the immediate neighborhood of the anaerobe and thus enables the latter to grow. This favor. ing action on the part of certain bacteria is known as microbic association, and it is undoubtedly because of such assistance that obligative anaerobic bacteria are able to multiply in the soil. Moreover, it is an association of this kind which enables certain pathogenic members of this group, such as the tetanus bacillus, to develop in the body of animals.

With but one or two exceptions, all the known obligative anaerobic bacteria are bacilli. An obligative anaerobic micrococcus has been isolated from pus. This group is characterized by the production of large quantities of gas. Moreover, butyric acid is a very common product and may be detected by the odor, not only in the artificial cultures, but at times even in the body of an animal infected with certain organisms of this group. The obligative anaerobic bacteria, therefore, exhibit a marked ability to produce fermentation.

The methods of cultivating anaerobic bacteria will be described in connection with these organisms, (Chapter XI). 


\section{Temperature.}

In addition to a suitable nutrient soil and a proper supply of oxygen, bacteria require a certain temperature for their development. As with other forms of life, each bacterial species has a minimum, optimum and maximum temperature at which it will develop. In some species the range of temperature at which they can grow is very limited. Thus, in the case of the tubercle bacillus and the gonococcus the maximum and minimum temperatures may not be more than five degrees apart. On the other hand, a few species are known which can grow anywhere from 15 to $68^{\circ}$.

The above instances, a narrow limit of five degrees and a very wide limit of fifty degrees, may be considered as exceptional. In general it may be said that bacteria do not grow, or but very poorly, below $10^{\circ}$ and above $40^{\circ}$.

The outimum temperature should especially be sought for when cultivating bacteria. It may be said to vary with each individual species. In the case of the parasitic bacteria, those which grow in the living body, the temperature of the host may be considered as best adapted for their development. Consequently the optimum temperature for the parasitic group of bacteria may be placed at 35 to $40^{\circ}$. On the other hand, the saprophytic bacteria, those which grow in nature on dead matter, find the warm summer temperature to be most favorable for their growth. As is well known, fermentative and putrefactive changes are favored by a warm, and retarded by a low temperature. Hence the optimum temperature for saprophytic bacteria may be said to range from 25 to $30^{\circ}$. From what has been said, it is evident that, as a rule, pathogenic bacteria should be cultivated in an incubator at, or near, the temperature of the body $\left(37.5^{\circ}\right)$; whereas, non-pathogenic bacteria are grown best at the ordinary room temperature. 
Although, as a general rule, bacteria do not grow below $10^{\circ}$, yet a number of notable exceptions are known. Several phosphorescing bacteria grow and emit light even at $0^{\circ}$, the temperature of melting ice. Nearly a score of organisms have been studied which can multiply at, or near, the freezing-point. As is well known, meat kept in cold storage may appear perfectly normal, but when taken out of storage it will decompose much more rapidly than ordinary fresh meat. This difference is due to the fact that the bacteria in the preserved meat slowly multiply, in spite of the prevailing low temperature.

The temperature of $40^{\circ}$ is usually considered as the the maximum temperature for the growth of bacteria. The majority of bacteria cannot grow above this limit. Moreover, a temperature of 45 to $50^{\circ}$ will rapidly weaken, and eventually kill all the ordinary forms of bacteria. There are, however, certain bacteria which thrive exceptionally well at such abnormal temperatures. Indeed, some forty or more bacterial species have been isolated which grow at 58 to $60^{\circ}$. A few species have been observed to multiply at $68^{\circ}$ and even at $74^{\circ}$.

The bacteria which can thus grow at unusually high temperatures are non-pathogenic, and, are designated as thermophilous. When it is remembered that the ordinary vegetating bacteria are promptly killed, in a few minutes, by a temperature of $60-70^{\circ}$ the existence of this group of organisms will appear all the more remarkable. The albumin which is present in the white of an egg, or in blood serum will coagulate at a temperature of $65-70^{\circ}$. The destruction of bacteria by this temperature is due, therefore, to the coagulation of the albuminous constituents of the protoplasm. Evidently, the thermophilous bacteria possess a markedly different chemical composition.

This interesting group of organisms is chiefly represented by bacilli. Only a few micrococci have been found to grow under these conditions. The thermophilous bac- 
teria have been especially isolated from garden soil. Strange to say, some have been found in the soil, even at a depth of 12 feet. They are present in the dust of rooms and a few have been obtained from water, especially in the case of hot springs.

In the latter instance the necessary temperature is furnished by the hot water It is more difficult, however, to understand how the organisms of this group can obtain their required high temperature when present in the soil. The heat of the sun will not warm up the soil to this extent. A considerable amount of heat may be generated by the fermentative changes that occur on the surface of the earth. The heat from this source may at times favor the development of these organisms. It has been shown that these bacteria, under anaerobic conditions, will grow at considerably lower temperatures than when cultivated in the presence of air. Thus, certain species which required a temperature of $50-70^{\circ}$ under aerobic conditions could be grown as anaerobes at $34-44^{\circ}$. The anaerobic conditions, as shown on p. 70, are readily supplied in the soil as a result of microbic associations. This explains very satisfactorily how the thermophilous bacteria can live in nature. The growth of these organisms at temperatures which are rapidly fatal to ordinary bacteria can only be due to the presence of difficultly coagulable proteins.

As shown above, a temperature of $0^{\circ}$, or less, stops the multiplication of bacteria. These organisms, however, may be exposed to extreme cold without loss of vitality. In the case of bacteria, cold arrests the functions of protoplasm but does not destroy its vitality. This is true not only of the spore condition but also of the vegetating form. The typhoid bacillus may remain frozen in ice for months and yet be, apparently, uninjured. The weakest individuals, of course, will die out first. Moreover, some species are more susceptible to cold than others. Alternate freez- 
ing and thawing tends to destroy bacteria. It is evident, therefore that cold cannot be employed for the purpose of destroying bacteria.

The action of heat is very different from that of cold. Thus, many pathogenic bacteria when grown at $41-43^{\circ}$ for some time become so weakened that they are unable to grow in the living body. In other words, they become attenuated. When this abnormal condition persists it eventually causes the death of the organism.

The higher temperatures act in a similar way. They first weaken and then destroy the organism. A temperature of $60-70^{\circ}$ will destroy the ordinary vegetating bac. teria in a few minutes. In such cases the heat probably causes a coagulation of the protoplasm. As pointed out on p. 56, spores may resist the action of steam-heat for some minutes, and even for some hours, whereas the vegetating form is destroyed instantaneously. Heat coagulation results in death because of the marked alteration in the protein constituents of the cell. In cold coagulation, on the other hand, the water within the cell is frozen but the protoplasm is not altered. It is evident from what has been said above, that cold acts as an antiseptic, whereas heat is a germicide.

When cultivating bacteria it is very important that the temperature at which they develop shall be as nearly constant as possible. In the case of pathogenic bacteria this condition is usually observed, inasmuch as the organisms are grown in an incubator which maintains a fairly constant temperature. When saprophytic bacteria are cultivated, or whenever gelatin is employed, this is done at the socalled room temperature, which may vary considerably. At night it may be 10 or $15^{\circ}$ below the temperature that prevails during the day. The thermal oscillations of this kind, alternately hastening and checking the growth of bacteria, are not without effect upon the form of the organisms, of the colonies and upon other cultural character- 
istics, such as pigment production, thickness of the growth, etc. A constant low temperature of $16-18^{\circ}$ is as desirable as a constant high temperature in an incubator. It can readily be obtained by the apparatus shown in Fig. 33, Chapter VII, or by a properly constructed ice-chest.

\section{Light.}

The higher plants, in the presence of sun-light, are able to assimilate carbonic acid. The relatively few, colored bacteria are likewise favored by an exposure to sun-light. Apart from these few organisms it may be said that direct sun-light exerts an unfavorable action on all bacteria. Naturally, some species will be less affected than others. Moreover, the vegetating form will, as a rule, be more easily destroyed than the spore form. A few hours' exposure to the direct sun usually destroys vegetating bacteria.

The action of sun-light may be exerted upon the medium as well as upon the organism. This is seen in the fact that sterile bouillon, or urine, after an insolation of some hours, will not permit a development of certain organisms. The change that has taken place in the soil seems to inhibit the germination of spores more readily than a multiplication of the organism itself. Clearly, therefore, a prolonged exposure of a given medium to sun-light changes, in some way, its chemical composition. Similar alterations will be met with when strongly alkaline media are heated in an autoclave for some time at $120^{\circ}$. The action of sunlight, or of heat, may cause an oxidation of the fats or sugars present giving rise to acid products which change the reaction of the medium and consequently render it useless. Moreover, oxidation products may form, like formic acid, or formaldehyde, which exert a marked antiseptic action. Apparently the most important antiseptic that 
may'be formed in the presence of sun-light is hydrogen peroxide. Insolated urines not only become sterile but remain so, as a result of the formation of this substance.

The action of direct sun-light is especially marked in the presence of air. It must not, however, be inferred that oxygen is necessary. Insolation will kill bacteria which are kept in a vacuum, though more slowly than if air.was present. Oxygen, therefore, favors or assists the action of sun-light.

Diffuse sun-light has very little or no action on ordinary bacteria. Some pathogenic bacteria, like the tubercle bacillus, are weakened by mere exposure to day-light. By far the best results are obtained when bacteria are grown in the dark.

Sun-light, the same as heat, first weakens or attenuates the organism and finally destroys it. Thus, any one of the bright pigment-producing bacteria on exposure to sunlight becomes so altered physiologically that it will nolonger produce a pigment. Colorless varieties can thus be readily obtained. In the case of disease-producing bacteria the weakened condition is seen in the fact that the insolated organisms will no longer kill animals.

\section{High Pressure.}

When bacteria are grown under a high pressure they develop under unfavorable conditions. A weakening or attenuation results especially if a number of successive generations are made under such conditions. Thus, the anthrax bacillus, when grown for several generations under a pressure of 9 atmospheres, became so attenuated that it had no effect upon the most susceptible animal, the white mouse. That bacteria can live under considerable pressure is seen in the fact that certain species are known to grow on the ocean's bottom at a depth of several thousand feet. 
Moreover, direct experiment has shown that many bacteria can multiply under a pressure of several hundred atmospheres. Of course a pressure of several hundred atmospheres to the square inch becomes reduced, owing to the extremely small size of bacteria, to but a few milligrams for each individual cell.

\section{Electricity.}

It is very difficult to demonstrate a direct action of electricity on bacteria. This is due to the fact that an electric current induces chemical and physical changes in the liquid. The chemical changes are especially marked in solutions of various salts. Thus, if common salt is present it may not only decompose into acid and alkali but it may also give rise to free chlorine and to hypochlorites. The bacteria suspended in a liquid containing salt may be destroyed as the result of the passage of an electric current, but the immediate cause of death is the formation of the germicidal products mentioned above. Moreover, hydrogen peroxide, and especially ozone, may form and destroy the bacteria present. The purification of polluted river-water by means of ozone generated by powerful currents of electricity has been carried on successfully.

In addition to the chemical changes caused by an electric current a considerable rise in temperature may be observed. Obviously, the heat thus generated will not be without effect upon the organisms in suspension.

It is not possible to overcome, or to do away with the action of the chemical products and of heat when studying the action of an electric current. It would seem as if the latter possessed little or no direct action. A feeble galvanic current has little or no effect, whereas à strong current, especially if allowed to act for some hours, will destroy the organisms present. That this result is due to an alter- 
ation of the medium and the presence of germicidal substances can be easily demonstrated. Such a medium when subsequently inoculated with a fresh culture will remain sterile.

The effect of X-rays on bacteria has been the subject of considerable study. These rays, as in the case of the electric current, can be said to be without any direct action.

\section{Chemotaxis.}

The lower forms of life exhibit a singular behavior with reference to certain chemical substances. If, for instance, a minute capillary tube is partly filled with a solution of pepton or meat extract and the end of the tube is placed in a drop of water which has been inoculated with a small number of motile bacteria it will be found that, in a few minutes, all the bacteria will have gathered around the tube. This attraction of the bacteria by the chemical substance in the tube is known as chemotaxis. The various chemical substances will show different degrees of attraction. Moreover, the concentration of the solution has much to do with the result. Other compounds, such as acids, alkalis and alcohol do not attract bacteria and, indeed, may be said to exert a repellant action. It is customary, therefore, to speak of positive and of negative chemotaxis.

The phenomenon of chemotaxis, which was first observed on higher plants, and then on lower forms, has been utilized to explain the behavior of the cells of the body in the presence of invading bacteria. It should be remem. bered that there is no satisfactory explanation of the phenomenon itself. 


\section{OHAPTER V.}

\section{THE CHEMISTRY OF BACTERIA.}

All living cells take in food, build up protoplasm and throw off waste-or metabolic products. In a multi-cellular organism, such as the higher plant or animal, it is not pos. sible to trace each of the various chemical compounds which are given off back to the cell which made them. In the case of the uni-cellular organisms this can, as a rule, be easily done. Although a number of different species may be growing together in a liquid it is possible to separate the species from one another and to obtain thus pure cultures. The chemical products of each species can then be studied, unmixed with the products of other organisms.

A large number of bacterial species may grow upon one and the same nutrient medium. The chemical products, however, will vary with each species. Thus, one organism growing upon a given medium may produce an acid whereas another species growing upon the same medium may give rise to an alkaline reaction. In some instances differences of this kind are very marked and are utilized for the purpose of distinguishing one species from another. Thus, the colon bacillus produces acid and gaseous products and indol, whereas the typhoid bacillus, which otherwise resembles the former very much, does not elaborate either one of these products.

A given micro-organism always produces a considerable number of different chemical compounds. Thus, the yeast plant is said to produce alcohol, but it must not be inferred that this is the only substance which it elaborates. On the contrary it produces gases, several kinds of alcohol, a num- 
ber of acids, ferments and the like. The composition of the nutrient medium will, of course, influence the kind of products. Thus, grape-sugar is readily fermented by the yeast-plant yielding alcohol, carbonic acid and other products whereas milk-sugar is not affected.

The several chemical compounds elaborated by a given species are subject to variation depending upon the health and vigor of the organism. If the latter has been weakened by an abnormally high temperature, by sun-light, or by poisonous chemicals it will be found that this change in the phy. siological condition of the cell will be evidenced by altered waste-products. Thus, a normal species may give rise to a bright-red pigment whereas the attenuated or weakened variety may yield a perfectly colorless growth. Again, a certain organism may normally curdle milk in a few hours, but when altered it will do so very slowly or not at all. Numerous other instances might be cited to show that the kind of products formed depends not only upon the composition of the soil, but also upon the physiological condition of the cell.

In the study of the chemistry of bacteria and allied forms it will frequently be found that a given chemical compound is produced by several distinct species. Alcohol is said to be the product of the yeast-plant. More than a score of species of the yeast-plant, however, are known to possess the power of making alcohol. One species may, of course, produce a much larger amount than another. Moreover, alcohol is produced by many bacteria and by moulds. What has been said of alcohol is equally true of acetic, lactic, butyric acids and other compounds. In other words, entirely different species of organisms may give rise to a given chemical compound, though in different amounts. It is evident, therefore, that alcoholic, acetic, etc., fermenta. tions are not necessarily due to one species.

When bacteria grow in a tube or fiask the various wasteproducts which they elaborate remain in the liquid in direct 
contact with the cells that produced them. The accumulation of waste-products eventually stops the growth of the organism, and as pointed out on p. 50, in many species it will induce spore formation. Prolonged contact with these chemical products is injurious and may result in the death of the organism. If the bacteria are grown in a tube, the walls of which are permeable, as in the case of a collodium sac, the waste-products will diffuse through the wall into the surrounding liquid, and, as a result, growth will continue as long as nutrient substances are present. A similar diffusion, or rather dilution, enables these organisms to grow, more or less continuously, in various waters.

The accumulated waste-products under ordinary experi. mental conditions soon stop the growth and multiplication of the germs present. Inasmuch as the chemical products of one organism are more or less different from those of another, it will be evident that in some instances the inhibition will be more marked than in others. Thus, in lactic acid fermentation the process stops when about 0.8 per cent. of free lactic acid has formed. On the other hand, in acetic acid fermentation the growth may not be inhibited by 10 per cent. of the free acid. Similarly, the yeast-plant may continue to grow until about 15 per cent. of alcohol has accumulated. Certain bacteria give rise to phenol or carbolic acid which is a well marked antiseptic even when considerably diluted.

Although bacteria are small in size and simple in structure they, nevertheless, elaborate a great variety of chemical compounds. The changes which these minute organisms induce are exceedingly complex. In the first place it must be borne in mind that the cell builds up its protoplasm and other cell constituents out of the food supplied. Such a chemical change is obviously carried on within the cell. It is intracellular and synthetic. The bacterial proteins, toxins and ferments are undoubtedly formed in this way. 
On the other hand, the waste-products are formed in the cell and are excreted. They result from a tearing-down, or cleavage of more complex bodies and, therefore, are to be considered as analytic products.

Inasmuch as the compounds mentioned are formed within the cell they may be designated as primary products. These should be distinguished, as far as possible, from the secondary products which are formed wholly on the outside of the cell. Thus, the ferment produced within the cell may be eliminated and may then act upon suitable material on the outside. The pepsin of the gastric juice is secreted by certain cells of the stomach and, whether in the stomach or in a test-tube, it will digest or split up albumin into simpler bodies such as albumose, or pepton. In like manner, the ferment produced by bacteria may act upon various substances and give rise to cleavage products. Again, certain bacteria produce compounds which unite with the oxygen of the air to form bright-colored pigments. In either case, the products are not made directly by the cell and for this reason they may be spoken of as secondary.

Bacterial proteins. - The albuminous substances which are formed within the cell, and which constitute an integral part of the cell protoplasm, are known as the bacterial cellular proteins. Very little is known in regard to their exact chemical composition. The cellular proteins of some bacteria possess a marked action upon the animal body. They may cause suppuration, or may possess a decided poisonous action.

Secondary bacterial proteins are formed outside of the cell, in the culture medium, by the action of the soluble ferments secreted by the organisms. Various albuminous substances may consequently be found in the liquid in which bacteria are growing. Thus, the coagulated white of an egg, or ordinary blood-fibrin, may be dissolved and changed to an albumose. If the ferment is very active the 
albumose may in turn be split up into pepton. It is evident, then, that a bacterial liquid may contain in solution, albu. min, globulin, albumoses, or even pepton. These protein substances will be precipitated from such culture fluids by the addition of several volumes of absolute alcohol. If harmless, non-poisonous bacteria were cultivated in the liquid the precipitated proteins will produce no effect when injected into an animal. On the other hand, in the case of injurious, disease-producing bacteria the precipitate will be highly poisonous. In this way a tox-albumin was obtained from cultures of the diphtheria bacillus; a tox-albumose from those of the anthrax bacillus, and a toxo-pepton from those of the cholera vibrio.

The fact that the precipitated protein is poisonous does not prove that the protein itself is actually poisonous. There is every reason to believe that the protein substances present in a bacterial liquid are non-poisonous. When, however, they are thrown out of solution they mechanically drag down the real bacterial poison and, as a result, the precipitate is toxic. If a precipitate of calcium phosphate or of aluminum hydrate is produced in such a liquid it will be likewise poisonous for the reason given.

Although the "toxalbumins," etc., are not the real bacterial poisons as was at one time supposed, it nevertheless remains true that certain albuminous substances, elaborated by higher plants and animals, may be intensely poisonous. There is reason to believe that the venom of serpents owes its marked poisonous action to certain proteins, such as pepton and globulin. Again, the jequirity seed contains an albumose body, known as abrin, which is very toxic. When injected intravenously, abrin is fatal in a dose of $0.00001 \mathrm{~g}$. per $\mathrm{kg}$. body weight. In other words, $1 \mathrm{~g}$. of abrin is capable of killing 200,000 guinea-pigs, each weighing one pound. The calculated fatal dose for a man weighing 130 pounds would be sor grain. A somewhat less poisonous albumose, ricin, bas been obtained from the castor bean. 
Toxins.-It has been pointed out (p. 79) that a given micro-organism produces a considerable number of chemical compounds. It is possible for several of these products to be more or less poisonous. In the case of the diseaseproducing bacteria each one elaborates a highly toxic substance, which has been designated by the term toxin. It is certain that these toxins, the specific bacterial poisons, are made within the cell, by synthetic processes. Thus far, no one has succeeded in isolating toxins in a strictly pure condition, and consequently their chemical composition is undetermined. While it is not known what the toxins are, it nevertheless has been definitely shown that they are not basic substances or ptomains, and that they are not proteins.

Obviously the toxin of the diphtheria bacillus is different from that of tetanus, or of cholera. Each pathogenic organism, therefore, makes a toxin which is characteristic for that particular species. In the case of the tetanus and diphtheria bacilli, the specific toxins readily pass out of the cell into the surrounding liquid. Consequently, the filtered cultures of such organisms may be intensely poisonous. It is possible, for instance, to obtain a filtered diph. theria culture which will kill a guinea-pig in a dose of 0.002 c. c., or even less. The toxin, of course, makes up probably only a small fraction of one per cent. of this dose. The purified tetanus toxin was fatal to a $15 \mathrm{~g}$. mouse in a dose of $0.000,000,05 \mathrm{~g}$. In other words, a mouse weighing half an ounce is killed by тrв $\frac{1}{800}$ of a grain of the purified toxin. The calculated fatal dose for a man weighing $\mathbf{1 5 5}$ pounds is 28 grain. It is evident that these toxins are by far the most powerful poisons known to man.

In the instances cited, the specific toxin readily passes out of the cell into the surrounding liquid. This, however, is not always the case. As a matter of fact, the toxin is usually retained within the cell and consequently such cultures, when filtered, are but very sligbtly poisonous. The toxin which is stored up within the organism may be 
liberated on the death of the cell. Moreover, the outward diffusion of the toxin is favored by certain conditions which are, as yet, but imperfectly understood.

The toxins are very unstable chemical compounds. They are weakened, or destroyed by heat, light, exposure to oxygen and by various chemical reagents.

Ferments.-As a rule, a substance in order to be utilized as a food must be brought into solution. The starch present in bread, or in the potato, is insoluble in water and consequently would be valueless to an animal if the latter did not possess some means of splitting up the starch molecule and thus bringing its constituents into solution. The saliva and the pancreatic secretion contain soluble, starch-splitting ferments which act upon starch and convert it into sugar. The sugar can now be readily absorbed and utilized by the animal organism. A hard boiled egg, or a piece of meat, consists chiefly of protein or albuminous matter, which is practically insoluble. As long as it is in this insoluble condition it cannot be absorbed by the living organism and utilized as food. It is well known, however, that the hardest egg and toughest meat can be readily dissolved by the aid of the protein-splitting ferments contained in the gastric and pancreatic secretions. The fats can be absorbed directly, when in a very finely divided condition. The formation of an emulsion is necessary and this is brought about by the fat-splitting ferment of the pancreatic juice.

It is evident, then, that in the case of the animal the cells of the salivary gland, of the stomach, and of the pancreas produces certain soluble substances known as ferments or enzymes. These ferments are necessary to the preparation of the food for absorption and without their aid, it is safe to say, the animal could not exist. What is true of the higher form of life is equally true of lower, forms. When bacteria are planted on coagulated egg albumin, or 
on blood-serum they cannot utilize this material as food unless some of it is first dissolved, and in this condition only can it be absorbed through the cell-wall. Bacteria must therefore produce proteolytic ferments analogous to those made by the stomach and by the pancreatic gland.

Many bacteria are characterized by the rapidity with which they digest or dissolve albuminous matter. The liquefaction of gelatin by certain bacteria is due to the formation of peptonizing ferments. Many bacteria do not liquefy gelatin and this would seem to indicate the absence of this class of enzymes. There is reason to believe that even these organisms produce such ferments which, however, do not readily diffuse into the surrounding medium. The tubercle and typhoid bacilli do not peptonize coagulated blood-serum and yet the cell contents in each case will show a distinct, though slow proteolytic action. It has been shown above (p. 84) that certain bacteria elaborate toxins, which in some species, may readily pass out of the cell, whereas in others they are apparently retained within the cell. The same is apparently true of the bacterial ferments, which in some cases easily diffuse outward (liquefying bacteria), whereas in others they tend to remain within the cell.

Bacteria, clearly, cannot absorb the insoluble starch. Like the animal cell they can make use of starch as food only after it has been dissolved and converted into sugar. They, therefore, secrete starch-splitting or amylolytic ferments. In some bacteria this action on starch is quite marked, whereas in others it is apparently absent. The action of bacteria on fats, likewise, is largely due to the presence of a soluble ferment.

It has been customary to divide the so-called ferments into two groups:

1.) Organized, formed, living, or insoluble ferments;

2.) Unorganized, formless, non-living, or soluble ferments. 
The yeasts, bacteria and moulds were designated as organized ferments because they possess a cell structure, and act on organic matter like the unorganized or soluble ferments secreted in the animal body. There can be no doubt but that the ferment action of living cells, such as yeasts, bacteria, etc., is due to soluble ferments or enzymes which diffuse outward, more or less readily. The uni-cellular organism requires soluble ferments or enzymes, just as do other forms of life. The sprouting seed contains such ferments which are necessary to the nourishment of the embryo, enabling it to utilize the starch and other stored up foods. Malt, for instance, contains not only an amylolytic ferment, diastase, but also a proteolytic ferment. The yeast cell contains a ferment, which changes glucose into alcohol and carbonic acid. It also contains a peptonizing ferment. Papain, a vegetable ferment, shows a marked proteolytic action.

Soluble ferments or enzymes are, therefore, produced by plants as well as by animals and are primarily necessary to the absorption of certain foods.

It will be seen from the above that the soluble ferments or enzymes differ in their action. The more common enzymes may be grouped or designated according to their characteristic behavior, as:

Diastatic or amylolytic,

Peptonizing or proteolytic,

Fat-splitting,

Inverting,

Rennet-like.

The diastatic ferments are designated thus because they resemble in their action the well known ferment of malt,diastase. Like diastase they split up starch, first into dextrin, and this in turn is changed into sugar. The saliva and the pancreatic juice contain amylolytic ferments. These compounds are frequently met with in higher plants, espe- 
cially in sprouting seeds. Malt, or sprouting barley, is rich in diastase. Similar ferments are elaborated by bacteria.

The proteolytic enzymes act on albuminous substances and convert these into albumose and the latter into pepton. The pepsin of the gastric juice and the trypsin of the pancreatic secretion will serve as types of this class of ferments. As indicated above, similar enzymes are met with in the higher as well as in the lower forms of plant-life. The liquefaction or the peptonizing of gelatin is due to similar ferments.

The fat-splitting ferment breaks up neutral fats into glycerin and fatty acids. A ferment of this kind is present in the pancreatic secretion. Many bacteria possess a similar action which may be carried on in the intestines, or otherwise. The rancidity of fats, especially butter-fat, is frequently due to such organisms.

Inverting ferments change cane-sugar into glucose. They are elaborated by plants as well as by animals.

Rennet, as is well known, coagulates or curdles milk. The gastric secretion of all vertebrate animals contains a rennet ferment. The mucous membrane of thè calf's stomach, is utilized for this reason in the preparation of curd or cheese. A rennet-like action is exhibited by many bacteria.

Ptomainns. - These are organic, basic products resulting from the action of bacteria on albuminous matter. They all contain $\mathrm{C}, \mathrm{H}$, and $\mathrm{N}$, and some contain $\mathrm{O}$. They are alkaline substances which, when neutralized by acids, yield crystallizable salts. Most of the ptomains are not poisonous, or but very feebly so. Only a few can be said to be very toxic.

The discovery of, more or less, poisonous basic substances among the bacterial products led to the belief that bacteria produced disease by giving rise to ptomains. This 
view was largely based upon analogy. Thus, it is well known that many of the poisonous higher plants owe their injurious action to the presence of the so-called vegetable alkaloids. Strychnin, morphin, atropin, nicotin, etc, are examples of this kind. It soon became evident, however, that the ptomains could not be considered as the chief poisons elaborated by bacteria. In the first place, the amount of ptomaïns present in a filtered bacterial liquid was too small to account for the intensely poisonous action of such a liquid. Moreover, certain bacteria, like the diphtheria bacillus, do not give rise to ptomaïns. Clearly, therefore, the real weapon possessed by bacteria is of a different chemical nature.

It has already been shown that the specific poisons of the pathogenic bacteria are known as toxins. They are certainly not basic substances or ptomains, and they are not protein compounds. The ptomains, consequently, are of only secondary importance as factors in the production of disease. They are simple, waste-products which result from the breaking down of albuminous matter.

Alkalis.-Many bacteria impart an alkaline reaction to the medium in which they grow. As a rule, certain nitrogenous substances must be present in order that alkaline products may form. The protein compounds are of this kind. In the bacterial decomposition of urine the urea is decomposed into ammonia and carbonic acid. Ammonia is the most common alkali made by bacteria. It represents the final, inorganic form to which the nitrogen of dead protoplasm is eventually reduced. An alkaline reaction may result from the oxidation of sodium acetate and similar salts. In such instances sodium carbonate would form.

Certain organic derivatives of ammonia are frequently met with. These are known as amines and result from the introduction of an organic group into the ammonia 
molecule. This can be understood from the followingformulæ:

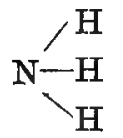

Ammonia.

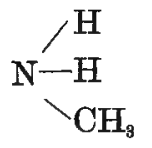

Methylamin.

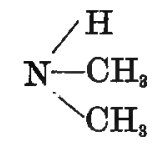

Di-meth ylamin.

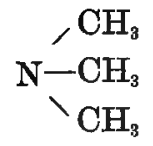

Tri-methylamin.

The amines possess a fish- or herring-like odor. Compounds of this kind are present in herring-brine and are frequently formed in the decomposition of protein matter. Inasmuch as they are organic and basic in character they belong to the group of ptomains.

Acids.-Products of this kind are very common. They usually result from the oxidation of carbohydrates. Thus, glucose is readily split up into acid products by a large number of bacteria. One organism may convert it into. lactic acid, whereas another may produce butyric acid. Hydrogen sulphide, an acid product, will, of course, be formed only out of certain sulphur containing compounds, such as the proteins. These compounds, moreover, may. give rise to various fatty acids.

It must be understood that a given organism may produce acids and alkalis at the same time. The final reaction in that case will depend upon which of these compounds is. present in excess. It not infrequently happens that an alkaline medium turns acid and then, after a few days, again becomes alkaline.

The acid products of bacteria are very numerous. The fatty acid series is usually represented by formic, acetic, propionic and butyric acids. The higher fatty acids, such as stearic and palmitic acids, will form in the decomposition of proteins, or of fats. Lactic acid is very common. Oxalic and succinic acids may also be formed. Carbonic acid and hydrogen sulphide are likewise to be 
mentioned among the acid products. Amido-acids, such as leucin, tyrosin, etc., may be formed in protein decomposition.

The fact that some bacteria readily give rise to acids, whereas others do not, can be made use of for the purpose of recognition. The reaction can best be observed by coloring the medium with some litmus solution. When acids are produced the litmus colored medium will assume a red color, otherwise it remains blue. The bacillus of tetanus, for example, does not redden glucose gelatin, whereas that of malignant edema does. Again, the typhoid bacillus does not produce an acid when grown on media which certain milk-sugar, whereas the colon bacillus does.

Alcohols.-In the decomposition of carbohydrates by bacteria several alcohols may form, but the amount is usually very small. Ethyl and butyl alcohols have been frequently met with. Glycerin is produced in small quantity by the yeast-plant. Phenol or carbolic acid, which is liberated in the putrefaction of proteins, belongs under this head.

Gases.-The gaseous products of bacterial growth are of considerable interest. It has already been indicated that the different bacteria give off, or exhale, variable amounts of carbonic acid. It may happen in such cases that no visible evolution of gas occurs. Usually, however, the presence of bubbles in the liquid or solid media indicates the active greneration of gaseous products. An increased formation of gas is observed when glucose is added to the culture medium.

The carbohydrates, in general, are very prone to give rise to gaseous products when acted upon by micro-organisms. Carbonic acid represents the final form to which all organic substances are eventually changed. It is, conse- 
quently, a very common product. Marsh-gas is found wherever cellulose is undergoing decomposition. It is sometimes an important constituent of the intestinal gases. The volatile amine compounds have been already referred to. Carbon monoxide may possibly occur among the bacterial products.

Free nitrogen and hydrogen are sometimes met with. Sulphur occurs frequently in the gaseous condition as hydrogen sulphide. Phosphorus may likewise appear as phosphuretted hydrogen. It is evident, therefore, that proteins, as well as carbohydrates, give off gaseous decom. position products.

The formation of gas bubbles is made use of in differentiating one species from another. Thus, the colon bacillus produces gas in abundance, whereas the typhoid bacillus does not. The anaerobic bacteria possess especially marked aerogenic properties.

As a result of abnormal fermentations in the stomach, or in the intestines, a considerable amount of gas may be produced. Moreover, in certain bacterial diseases, as symptomatic anthrax, a marked accumulation of gas may occur in the subcutaneous tissue. In such cases a distinct crackling sensation is felt when the fingers are passed over the skin. An interesting pathological phenomenon is met with in the so-called "foaming liver." On the cut surface of this organ gas bubbles may appear and form a scum which rapidly increases in thickness. When this foam is removed a new one will form. This peculiar condition is, of course, met with only when certain markedly aerogenic bacteria are present. Inasmuch as the gases produced frequently contain marsh-gas and hydrogen they will burn on contact with a lighted match.

The various bacteria can be designated, or grouped, according to the characteristic action which they induce. 
The following terms are frequently employed in this connection:

Zymogenic, or fermentation bacteria, Saprogenic, or putrefaction bacteria, Chromogenic, or pigment bacteria, Aerogenic, or gas producing bacteria (p. 91), Photogenic, or light producing, phosphorescing bacteria,

Toxicogenic, or poison producing bacteria,

Pathogenic, or disease producing bacteria.

It must not be supposed from the above grouping of bacteria that a given species is necessarily confined or limited to one or the other of these groups. It should be remembered that the work done by a given organism depends upon the medium and on the surrounding conditions, or environment. A given species may produce a typical fermentation when grown in a medium containing sugar, and, when transplanted to albuminous material it may cause an equally typical putrefaction. Moreover, the same organism may produce gas, or when introduced into the animal body may give rise to disease. The above terms, therefore, are to be taken as referring to the most pronounced action or function exhibited by an organism under certain conditions.

\section{Fermentations.}

The change brought about by an organized ferment (bacteria, yeasts, moulds, etc.), whereby complex organic bodies are broken up into relatively simpler compounds, may be designated as a fermentation. It is evident, therefore, that fermentations are vital phenomena, the result of the activity of micro-organisms. From what has been said heretofore, it is clear that these organisms induce fermentative 
changes largely, if not wholly, by means of the soluble ferments or enzymes which they elaborate.

Diverse fermentations result, depending upon the kind of organism at work, and, the material that is acted upon. It has been customary to restrict the term fermentation to those phenomena where carbohydrates undergo cleavage. The word fermentation (meaning, to boil) was originally applied to those changes which were accompanied by the active evolution of gas, in which case the liquid appeared to be boiling. This conception must be enlarged to accord with the action of bacteria in general. It matters not whether the substance that is acted upon is a carbohydrate, fat, or protein; whether gaseous products are, or are not evolved; the change, if due to a micro-organism, is one of fermentation.

In the decomposition of albuminous substances disagreeable products are evolved. Putrefaction can, consequently, be designated as putrid fermentation. The chemical composition of the protein molecule accounts for the different products thus obtained as compared with those which result in the decomposition of carbohydrates. The latter contain only $\mathrm{C}, \mathrm{H}$ and $\mathrm{O}$, and, on fermentation, yield diverse alcohols and acids which are not disagreeable. On the other hand, the protein molecule usually contains in addition to the elements mentioned, N, S and P. Hence, in putrefaction various $\mathrm{N}$ compounds form such as ammonia, amines, indol, skatol, etc., which possess a more or less offensive odor. Similarly, the $\mathbf{S}$ may appear as hydrogen sulphide, mercaptans, etc., which are likewise disagreeable.

It is evident that not only the material which is acted upon, but also the particular species at work will influence the kind of fermentation. Thus, when glucose is acted upon by the yeast, it is changed to alcohol, whereas another organism can convert it into lactic, or into butyric acid. Moreover, it should be understood that a given organism which induces a typical fermentation when grown in a sugar 
solution, may produce an equally typical putrefaction when allowed to develop on albuminous matter. The distinction between saprogenic and zymogenic bacteria is, therefore, not very marked. Furthermore, well known pathogenic bacteria may give rise to a typical fermentation, or putrefaction according to the nature of the material on which they are grown.

While it is customary to speak of alcohol, acetic, butyric, lactic acid, etc., fermentations, it must not be supposed that each one of these fermentations is due to a single specific organism. It will presently be shown that alcoholic fermentation may be caused by a large number of species of yeasts, by many moulds and even by bacteria. Similarly, acetic acid fermentation is brought about by any one of a large number of species. The same is true of the other common fermentations.

The chemical changes observed in fermentations may result in one of several ways. By far the most common change is that of hydration. In this case, the elements of water are introduced into the molecule which then breaks up. This is seen in the following equations which reprerent the inversion of cane-sugar, and the fermentation of urea.

$$
\begin{aligned}
& \underset{\text { Cane-sugar. }}{\mathrm{C}_{12} \mathrm{H}_{22} \mathrm{O}_{11}}+\mathrm{H}_{2} \mathrm{O}=\underset{\text { Dextrose. }}{\mathrm{C}_{4} \mathrm{H}_{12} \mathrm{O}_{6}}+\underset{\text { Levulose. }}{\mathrm{C}_{6} \mathrm{H}_{12} \mathrm{O}_{6}} . \\
& \underset{\text { Urea. }}{\mathrm{CO}\left(\mathrm{NH}_{3}\right)_{2}}+\mathrm{H}_{2} \mathrm{O}=\mathrm{CO}_{2}+2 \mathrm{NH}_{3} .
\end{aligned}
$$

A second way in which cleavage may result is by halving. Thus, in lactic acid fermentation, glucose is apparently changed direct into two molecules of lactic acid.

$$
\underset{\text { Glucose. }}{\mathrm{C}_{6} \mathrm{H}_{12} \mathrm{O}_{6}}=\underset{\text { Lactic acid. }}{2 \mathrm{C}_{2} \mathrm{H}_{6} \mathrm{O}_{3} .}
$$

A third and very important change observed in certain fermentations is that of oxidation. The best illustration of 
this type of fermentation is that seen in the conversion of alcohol into acetic acid.

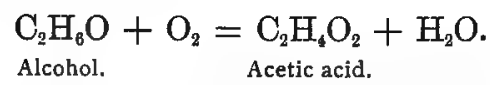

The oxidation of $\mathrm{NH}_{8}$ to $\mathrm{HNO}_{2}$ and to $\mathrm{HNO}_{3}$ is another illustration.

Lastly, the fermentation process may be largely one of reduction. Thus, nitrates may be reduced to nitrites and even to ammonia. Glycerin may be changed in this way into ethyl alcohol. Reduction changes can be easily recognized if the medium has been previously colored with. litmus. The medium, in that case, becomes colorless.

Alcoholic fermentation.-This fermentation is of the utmost practical importance, inasmuch as all the alcohol of commerce is thus obtained. The yeasts are especially endowed with the power of producing alcohol. It should be remembered that this group of plants differs from the bacteria in many important respects (p. 16, also Chapter XII). Moreover, there are a large number of species of yeasts which differ not only in the amount of alcohol to which they can give rise, but also in the amount and character of other by-products. It is the presence of these secondary products which influences to a large extent, the appearance, odor and taste of beer and other alcoholic liquors. In this fermentation, as it has been carried on since ancient times, it was always possible for diverse yeasts and bacteria to develop, and thus, more or less, alter the final product. This uncertainty in the process has been overcome by the employment of a pure culture of a yeast of known power. The work in large breweries has thus been placed on a scientific basis: The pure yeast which is used must be free from other species of yeasts as well as from bacteria. In this way a constant product is assured. 
In the production of wine, the yeast-plant is not added to the grape-juice. It is present upon the surface of the grapes, and hence, passes into the liquid when the grapes are crushed. As yet, puré cultures of yeast have not been employed in the manufacture of wine.

The best known species of the yeasts is the saccharomyces cerevisice. Some species will produce more alcohol than others. Beer, as a rule, contains less than 5 per cent., but in other sugar solutions, as in wines, the amount of alcohol may rise to 10 or 15 per cent. This may be considered as the maximum limit of alcohol formation, inasmuch as this amount tends to inbibit the growth of the yeast. The so-called "wild-yeasts" or torulæ do not yield more than about one per cent. of alcohol.

The production of alcohol is not restricted to the yeast family. There are many bacteria which can convert glycerin into alcohol. Friedländer's bacillus of pneumonia when grown on sugar media produces alcohol and acetic acid. The same is true of the typhoid bacillus. A number of bacteria which are present in the mouth and in the intestines of normal persons are capable of producing alcohol. Many moulds, especially mucors, possess the power of forming alcohol out of sugar, and at least one of these organisms is utilized on a commercial scale for the preparation of alcohol.

In alcoholic fermentation alcohol is the chief, the characteristic product. A large number of secondary or by-products are always present. Among these may be mentioned, carbonic acid, aldehyde, the higher alcohols such as propyl, butyl and amyl, which are commonly known as fusel oil. Glycerin, acetic and succinic acids are likewise always present.

The yeast-cell when destroyed by a process of grinding yields a soluble ferment, an enzyme, which can convert sugar solutions into alcohol and carbonic acid. This ferment is made and stored up within the cell. It may possi- 
sibly diffuse into the surrounding liquid, and cause there the characteristic cleavage of sugar jnto alcohol. It has been usually held that the sugar was absorbed by the cell and then split into alcohol and carbonic acid. While the yeast decomposes most of the sugar into alcohol and other products, it utilizes a small portion for the purpose of building up its own cell contents. Cellulose and glycogen may be formed direct from the sugar, whereas the fats and proteins result indirectly.

The yeast-plant cannot convert starch, or cane-sugar directly into alcohol. These substances must first be converted into glucose. The diastase present in malt is employed to convert the starch of a grain into sugar. In Japan and China certain moulds which possess a marked diastatic action are employed for this purpose. On the other hand, maltose and cane-sugar are changed by a ferment produced by the yeast-cell, invertin, into glucose which is then converted into alcohol and carbonic acid.

$$
\begin{gathered}
\underset{\text { Cane-sugar. }}{\mathrm{C}_{12} \mathrm{H}_{22} \mathrm{O}_{11}}+\underset{\text { Dextrose. }}{\mathrm{H}_{2} \mathrm{O}=} \underset{\text { Levulose. }}{\mathrm{C}_{6} \mathrm{H}_{12} \mathrm{O}_{6}}+\underset{\mathrm{C}_{6} \mathrm{H}_{12} \mathrm{O}_{6} .}{\mathrm{C}_{6} \mathrm{H}_{12} \mathrm{O}_{6}}=\underset{\text { Invert-sugar. }}{\mathrm{C}_{2} \mathrm{H}_{6} \mathrm{O}}+2 \mathrm{CO}_{2} . \\
\text { Alcohol. }
\end{gathered}
$$

As is well known, the yeast is also utilized in the making of dough. It has been mentioned above that the yeast cell is unable to act directly upon starch. It lias been supposed that the bacteria present invert the starch and thus enable the yeast to carry on an alcoholic fermentation. As a result of the formation of carbonic acid the mass is distended.

Acetic acid fermentation.-Alcoholic liquors such as wine, beer, cider, etc., on exposure to air undergo acetic acid fermentation. The alcohol is oxidized to acetic acid and the resulting liquid is commonly known as vinegar. The free 
access of oxygen is necessary to this change, which is represented by this equation:

$$
\underset{\text { Alcohol. }}{\mathrm{C}_{2} \mathrm{H}_{6} \mathrm{O}}+\underset{\text { Acetic acid. }}{\mathrm{O}_{2} \mathrm{H}_{4} \mathrm{O}_{2}}+\mathrm{H}_{2} \mathrm{O} \text {. }
$$

It was supposed by Liebig that this was a purely chemical reaction, but the studies of Pasteur showed that living organisms were necessary to effect the change. The liquid undergoing this kind of fermentation becomes covered with a slimy scum, which in reality is a zooglea, or mass of various bacteria. Inasmuch as experience has taught that the conversion of alcoholic liquids into vinegar was hastened by the addition of some of this scum, the latter came to be known as "mother of vinegar." It was subsequently named mycoderma aceti. This term, however, must not be understood to designate a single bacterial species.

The production of acetic acid is not limited to any one organism. From the mother of vinegar various kinds of bacteria have been isolated. As yet, pure cultures have not been employed for the preparation of vinegar. Among those which have been studied may be mentioned the M. aceti, Bacterium aceti, Bacillus aceticus, B. Pasteurianus, etc. These organisms exhibit a marked tendency to give rise to involution forms, especially, when grown at about $40^{\circ}$ (Fig. 3, p. 21).

Acetic acid fermentation does not occur in liquids which contain more than 15 per cent. of alcohol. Moreover, it is not possible to obtain by direct fermentation a vinegar which will contain more than about 14 per cent. of acetic acid. A temperature of $30-35^{\circ}$ is most favorable to the change. When the nutrient material becomes exhausted the bacteria present may attack the acetic acid which they produced, and, may oxidize this to carbonic acid and water.

$$
\mathrm{C}_{2} \mathrm{H}_{4} \mathrm{O}_{2}+2 \mathrm{O}_{2}=2 \mathrm{CO}_{3}+2 \mathrm{H}_{2} \mathrm{O} \text {. }
$$


It has been indicated above that acetic acid fermentation is not a specific process, due to a single organism. Several bacteria have already been mentioned as being capable of producing acetic acid. In these cases the acid is made out of alcohol. In some instances, it can apparently be made direct from sugar. This is true of the pheumococcus. Moreover, it is a frequent product in the anaerobic putrefaction of proteins. The common intestinal bacteria, such as B. lactis aerogenes and B. coli communis, produce acetic acid together with formic and lactic acids and other products. These acids, when formed in the intestines of infants, are unquestionably intense irritants, and may, therefore, be considered as factors in the production of infant diarrhea. Consequently, the summer complaint of infants is essentially, an abnormal fermentation in the intestines.

Lactic acid fermentation.-An unusually large number of bacteria are capable of producing lactic acid, as the chief product, in the decomposition of sugar and other carbohydrates. As in other fermentations various products are present, though in small amounts. Carbonic acid is usually present, accompanied by traces of alcohol, acetone, formic and acetic acids.

Owing to the wide distribution of lactic acid bacteria this type of fermentation will be frequently met with. Thus, in the souring of milk, of sugar-beet juice, in pickles, sauer-kraut, ensilage, etc., lactic acid is a characteristic product. It plays an important part, moreover, in certain affections of the mouth, stomach and intestines.

A temperature of $30-35^{\circ}$ is most favorable to lactic acid fermentation. The latter ceases when about 0.75 per cent. of lactic acid has formed. The material usually acted upon is glucose, or lactose, but lactic acid may be formed from various other compounds. The transformation 
of glucose into lactic acid may be expressed by the equation:

$$
\mathrm{C}_{6} \mathrm{H}_{12} \mathrm{O}_{6}=2 \mathrm{C}_{3} \mathrm{H}_{6} \mathrm{O}_{3} \text {. }
$$

This equation is not strictly correct, inasmuch as small amounts of by-products are always present. Sometimes considerable amounts of carbonic acid and of hydrogen may form.

The lactic acid which usually forms in this kind of fermentation is optically inactive. It does not rotate, either to the right or left, a ray of polarized light. Some organisms, however, give rise to sarco- or para-lactic acid whereas others form levo-rotatory lactic acid. The union of the latter with sarco-lactic acid, which is dextro-rotary, results in the formation of the ordinary inactive lactic acid. It is interesting to note in this connection that under cerditions the colon bacillus, which ferments lactose as well as glucose, produces dextro-lactic acid, whereas the typhoid bacillus ferments glucose but not lactose, and gives rise to levo-lactic acid. Depending upon the kind of sugar acted upon, the colon bacillus may give rise to levo-, dextro-, or inactive lactic acid.

In dental caries, lactic acid fermentation is ascribed an important rôle. The organisms present in the mouth are supposed by Miller, to elaborate lactic acid out of the remnants of starchy food, left between the teeth or at the edge of the gums. This acid then unites with the calcium of the teeth and thus causes decalcification. This first stage is followed by a second, wherein the organic matter of the tooth is softened and dissolved, by the peptonizing bacteria that are present.

In abnormal fermentations in the stomach (dyspepsia) lactic acid is a common product. These decompositions are apt to occur when the amount of free hydrochloric acid is diminished, or when the food remains too long in 
the stomach. In some instances, acetic, or butyric acid may predominate in the stomach contents, and, at other times considerable gas may be given off. The production of lactic and acetic acids in the intestines has already been referred to, in connection with infant diarrhea.

The most common illustration of lactic acid fermentation is met with in the souring of milk. This secretion, as elaborated in the gland, is strictly sterile, and if collected under suitable precautions it will keep indefinitely. In ordinary milking an enormous number of bacteria are introduced into the milk, from the air and through unclean hands, containers, etc. Given a suitable temperature, these organisms rapidly multiply and convert the milk-sugar into lactic acid. As a result, the reaction of the milk, which was neutral or alkaline in the beginning, becomes acid. Inasmuch as the casein is insoluble in the presence of an acid, it forms a precipitate which mechanically drags down the fat. The curdling of milk may result from the action of a rennet ferment, but, as a rule, it is due directly to the acid reaction which develops in the milk.

It is evident that some bacteria will coagulate milk, whereas others will not. This fact is made use of, at times, in differentiating bacteria. Thus, milk is coagulated by the colon bacillus but is not altered by the typhoid bacillus.

In eastern Europe and in Asia, the milk of the cow, ass, or camel is subjected to an alcoholic, as well as lactic acid fermentation. The resultant beverage is known as kephyr or koumiss. The fermentation is induced by adding to the milk the so-called kephir grains, which consist essentially of bacteria and yeasts.

The lactic acid bacteria favor the development, in the body, of anaerobic bacteria, such as the tetanus bacillus. Microbic associations of this kind play an important part in many diseases.

It may be well to briefly allude in this connection to the so-called ripening of cheese. Bacteria are to a very large 
extent, if not wholly, the cause of this change. As a result of gas production, vacuoles or spaces form in the mass. The characteristic flavor and odor is due, as in the case of butter, to bacterial products. Abnormal fermentations in a cheese may give rise to poisonous products, or to marked alteration in the appearance of the cheese.

Butyric acid fermentation. - This rather common fermentation is usually the result of the action of anaerobic bacteria. A considerable number of obligative anaerobes have been described as producers of butyric acid. The patho. genic organisms of this class may form butyric acid, even in the animal body. A number of aerobic bacteria can likewise give rise to butyric acid.

Many of the anaerobes mentioned, give a granulose reaction on contact with iodine. They are usually motile, and in those species which develop spores, there is always a corresponding enlargement of the cell. Hence, either the clostridium, or drum-stick forms are met with in such cases.

In this, as in other fermentations, various additional products are encountered. Among these may be mentioned, butyl alcohol, acetic and carbonic acids, hydrogen, etc. The material acted upon may be a carbohydrate, such as glucose, or it may be a protein, or a fat. It is evident that butyric, like lactic acid, is a very common product in the decomposition of organic matter. The transformation of glucose into butyric acid is usually represented by the equation:

$$
\underset{\text { Glucose. }}{\mathrm{C}_{6} \mathrm{H}_{12} \mathrm{O}_{6}}=\underset{\text { Butyric acid. }}{\mathrm{C}_{4} \mathrm{H}_{8} \mathrm{O}_{2}}+2 \mathrm{CO}_{2}+2 \mathrm{H}_{2} .
$$

Butyric acid is ordinarily prepared by allowing milk, to which calcium carbonate has been added, to undergo lactic acid fermentation. The lactic acid unites with the lime to form calcium lactate. Eventually, this salt is acted upon by other organisms and butyric acid forms. 
In the fermentation of cellulose, anaerobic bacteria play a most important rôle. The cellulose is first converted into sugar, which in turn yields butyric acid, marsh-gas, and diverse other products. The changes that take place in the production of sauer-kraut, ensilage, etc., are similar to that just Imentioned. The cellulose is softened, and partially fermented with the production of butyric and lactic acids. In the ripening of cheese, similar decomposition products are formed out of the casein. The retting of hemp and flax is a change analogous to the fermentation of cellulose. The fibres are held together by a cementing substance, calcium pectate, which is removed by allowing the material to soak in water. The intercellular material is not dissolved by the water, but is digested, or dissolved by the action of butyric acid bacteria.

Viscous or slimy fermentation.-It not infrequently happens that milk, urine, beer, wine and dilute sugar solutions take on a slimy or ropy consistence. This is due to the presence and development of certain bacteria. As might be expected a very large number of wholly different organisms are capable of imparting a slimy character to these several liquids. It may happen that such bacteria will develop in sausage, bread, or in biscuits. In such cases, on breaking some of the material and slowly drawing the pieces apart, they will be seen to be connected by numerous, fine, cobweb-like threads. The slimy covering of the tongue, teeth, etc., in many fevers, is probably due to organisms of this kind.

The slimy character may be due to one of two causes. In the first place, the organism may secrete, or throw off a slimy product. This material passes out into the surrounding liquid and is no longer connected with the cell. This condition is analogous to the secretion of mucin by the cells of the mucous membrane. On the other hand, the sliminess may be due to the formation of capsules. It has been 
pointed out (p. 28) that the cell-wall of many bacteria may soften or gelatinize, giving rise to the so-called capsule. The organisms, in such cases, tend to stick together, forming zoogleæ.

Several of these fermentations are of interest, because the gummy substance which is produced, known as viscose or dextran, is a carbohydrate allied to dextrin. Its formula, $\mathrm{C}_{6} \mathrm{H}_{10} \mathrm{O}_{5}$ is the same as that of dextrin. Among the by-products in this case may be mentioned carbonic and lactic acids, and mannite. The latter probably results by the action of nascent hydrogen on glucose. The Leuconostoc mesenterioides is a common cause of such fermentations in molasses, etc., in sugar refineries. This organism, when grown in sugar solutions, develops enormous capsules, which are 10 or 20 times the width of the cell itself. On the usual nutrient media it appears as an ordinary streptococcus. Under favorable conditions it may develop with extreme rapidity, converting the sugar solution into a jelly, or even into a cartilaginous mass. In one instance, 80 barrels of molasses became converted into a gelatinous mass within 12 hours. A temperature of $30-35^{\circ}$ favors this fermentation.

Fermentation of fats. - The various animal and vegetable fats or oils are essentially neutral compounds of glycerin and one or more fatty acids. A strictly pure fat cannot be acted upon by bacteria, inasmuch as it contains only $\mathrm{C}, \mathrm{H}$ and $O$. It may, however, become rancid by exposure to the action of air and light. The change that results consists in the splitting of the fat into its components, glycerin and fatty acids. If, on the other hand, the fat is not pure but contains nitrogenous and other matter it will be possible for bacteria to grow in such a mass. In that event, a more or less extensive fermentation, or cleavage of the fat will occur.

The decomposition of fat by means of bacteria is a constant occurrence in the intestines. The change which 
these organisms induce is analogous to that produced by the fat-splitting ferment of the pancreatic juice.

Butter, as is well known, is apt to become rancid, especially, if it has been imperfectly worked. In that case, more or less casein and other milk constituents remain in the mass of butter-fat and furnish the necessary soil for the development of bacteria. Inasmuch as butter contains, among other fats, a small amount of a butyrate, the latter on decomposition will, of course, yield butyric acid. The odor of strong butter is largely due to this acid. It may be well in this connection to mention that the aroma of a good butter is largely due to volatile etheread products made by bacteria. Certain organisms, which possess the power of improving the flavor, when planted in the cream will render an excellent butter out of what otherwise might have been a poor grade article.

Fatty acids are not only liberated in the decomposition of fats, but they may also form in the cleavage of proteins. The fatty acids in the free state are not readily split up into simpler products by bacteria. When they unite with metals to form salts they are more prone to fermentation. In that case they are oxidized and form carbonic acid and other compounds. Thus, calcium formate yields calcium carbonate, carbonic acid and hydrogen, whereas calcium acetate will give rise to the same products except that the hydrogen is replaced by marsh-gas. The calcium salts of the higher fatty acids are split up into a variety of products. The glycerin, likewise, undergoes fermentation, forming butyric, lactic and carbonic acids.

Hydrogen sulphide fermentation.-In many instances, when certain sulphur compounds are present in a fermenting liquid they are converted into hydrogen sulphide. In minute quantity, this gas is formed by nearly all of the pathogenic bacteria, and, inasmuch as it is a highly poison- 
ous substance, it-may not be without effect when formed in the tissues, in disease conditions.

An interesting fermentation of this type is occasionally met with in urine. Bacteria, when introduced into the bladder, either as the result of an injury, or by the use of instruments or otherwise, may develop and give rise to fermentative changes. Usually an ammoniacal decomposition results, but at times the urine remains strongly acid and possesses a marked odor of hydrogen sulphide. This condition, or hydrogen sulphide fermentation of the urine, is known as hydrothionuria. A number of bacterial species have been isolated by the author and by others from such urines, and when inoculated into sterile, normal urine they promptly produce this gas. The so-called "neutral" sulphur compounds of the urine, and not the sulphates, are acted upon. Hydrogen sulphide is a common product in the decomposition of protein substances. The odor of rotten eggs is due to this gas.

Ammoniacal fermentation of urine.-Normal urine is perfectly free from bacteria for reasons heretofore given (p. 65). When bacteria are introduced into the bladder, in some way as mentioned above, they usually induce a marked ammoniacal decomposition. The urea undergoes hydration, forming ammonia and carbonic acid, which, of course, unite to form ammonium carbonate. The same fermentation will almost invariably occur when normal urine is allowed to stand for some hours at a warm temperature. The change corresponds to the equation:

$$
\underset{\text { Urea. }}{\mathrm{CO}\left(\mathrm{NH}_{2}\right)_{2}}+\mathrm{H}_{2} \mathrm{O}=\mathrm{CO}_{2}+2 \mathrm{NH}_{3} \text {. }
$$

Pasteur designated the cause of this fermentation as the Micrococcus ureo. The change, however, is not specific, due to a single organism, but can be induced by a 
host of aerobic as well as anaerobic bacteria. These are found everywhere in nature, in the air, water and soil.

Ammonia, like hydrogen sulphide, is a very common product formed in the putrefaction of protein substances, whether of animal or vegetable origin. Diverse bacteria and moulds take part in this transformation.

Nitrification.-As indicated above, the albuminous substances present in the dead plant or animal, are acted upon by bacteria and decomposed into the simplest inorganic products. The nitrogen eventually appears as ammonia, although a small amount may be returned to the air as the free element. The ammonia, thus formed, is essential to the growth of higher plants. In order to be assimilated it must be oxidized. This change is again effected by certain bacteria, which oxidize the ammonia first to nitrous, and finally to nitric acid.

Organisms of this kind are widely distributed in the soil and in water. Many of the ordinary, pathogenic and nonpathogenic bacteria, are capable of forming nitrites and nitrates. This change can be readily observed in urine, which ordinarily contains only traces of these compounds. As soon as bacteria begin to develop in the urine, nitrites and nitrates can be detected. It is for this reason that the chemist, when examining water suspected of being polluted, tests for nitrites and nitrates, as well as for ammonia.

The formation of nitrites and nitrates is, therefore, taking place wherever dead, nitrogenous, plant or animal matter is present. The water, and especially the soil, contains bacteria which are capable of effecting this conversion. A suitable temperature, moisture, and a proper amount of oxygen are, of course, likewise essential. It is in this way that the dung-heaps of India furnished the world's supply of saltpeter. The soda salt-peter which exists in vast deposits in Chili and Peru, likewise, owes its origin to the action of bacteria on the guano, or excrement of birds. 
It may be of interest to note that nitrites are almost always present in the saliva, as a result of the action of the mouth bacteria upon the food constituents. Nitrates do not seem to be present.

Among the nitrifying organisms there are some which differ markedly from all other known bacteria. Unlike the latter, they do not require organic matter inasmuch as they are able to assimilate carbonic acid. Without the aid of chlorophyll, and without light, they can utilize the carbonic of the air as food. Not only is organic matter unnecessary, but it is even injurious, and retards the growth of these bacteria. Hence, special methods, wholly unlike those ordinarily employed when cultivating bacteria, must be resorted to, in order to isolate these remarkable organisms. They can be grown best in pure water to which 0.1 per cent. of ammonium sulphate and of potassium phosphate, and some basic magnesium carbonate has been added. They can also be isolated by growing upon a nitrite-agar, or upon a mineral-gelatin which is essentially silicic acid.

In view of the cultural requirements of these organisms it will be seen that they belong to the simplest and earliest forms of life. They exist on wholly inorganic matter, since the carbonic acid of the air supplies the necessary carbon, while ammonia yields the nitrogen essential to the formation of protoplasm. These organisms convert the ammonia, by process of oxidation, first to nitrous acid and then to nitric acid. These changes can be indicated by the equa. tions:

$$
2 \mathrm{NH}_{3}+3 \mathrm{O}_{2}=2 \mathrm{HNO}_{2}+2 \mathrm{H}_{2} \mathrm{O} \text {. }
$$

$$
2 \mathrm{HNO}_{2}+\mathrm{O}_{2}=2 \mathrm{HNO}_{3} \text {. }
$$

While there are nitrifying organisms which can change ammonia into nitrates, there are many others which can produce nitrites, but not nitrates. Other organisms, in that case, complete the process, converting the former com- 
pounds into the latter. Hence, the nitrite-forming or nitroso-bacteria must be supplied with an ammonium salt, whereas the nitrate-producing forms, or nitro-bacteria require a salt of nitrous acid.

The formation of nitrites and of nitrates in the soil is, therefore, as Pasteur pointed out in 1862, a process analogous to acetification. The production of acetic acid and of nitric acid is due to oxidation changes, which are not purely chemical, as was at one time supposed. These changes are dependent upon the presence of certain organisms. Nitrification is, therefore, a fermentation analogous to that of acetic acid. It is not one organism but rather a large number, or group of organisms, which possess the power of inducing these fermentative changes.

A striking contrast to the action of these organisms is that of the de-nitrifying bacteria. The latter possess remarkable reducing powers. They convert the nitrates into nitrites, and continue the reduction until all, or nearly all, of the nitrogen has been set free, as such. This apparent waste of nitrogen may be considered as being balanced by the activity of the bacteria which are present in the root nodules of leguminous plants. These organisms áre able to assimilate, or fix the atmospheric nitrogen, and transfer it to their host. There are, therefore, four groups of bacteria according to their action on nitrogen and its inorganic compounds.

1.- Those which cause a fixation of free nitrogen.

2.-Those which produce nitrites out of ammonia.

3.-Those which produce nitrates out of nitrites.

4.-Those which reduce nitrates to nitrites and to free nitrogen.

A further illustration of the reducing action of bacteria is seen in the manufacture of indigo. The indigo does not exist, as such, in the plant, but rather as a glucoside. 
The cut plants are placed in water and allowed to ferment, whereby the glucoside is split up into its constituents, sugar and reduced or white indigo. The latter passes into solution, and, on subsequent agitation with air, becomes oxidized to the insoluble indigo-blue.

\section{Putrefaction.}

The decomposition of albuminous substances is a fermentative change analogous in every respect to the changes which carbohydrates and fats undergo. The cleavage of dead organic matter is always brought about by the activity of micro-organisms. A given organism may be capable of splitting up carbohydrates, and thus, inducing a typical fermentation when planted on a soil rich in these compounds. The same germ, when grown on albuminous matter, may produce a typical putrefaction. The difference between fermentation and putrefaction is, therefore, principally due to the different chemical composition of the material that is acted upon.

While the carbohydrates and fats contain merely $\mathrm{C}, \mathrm{H}$ and $\mathrm{O}$, the protein substances contain, in addition, $\mathrm{N}, \mathrm{S}$ and at times $\mathbf{P}$. Consequently, cleavage products containing the latter elements will be met with in putrefaction, which can not be present in ordinary fermentation. These products may possess a more or less intensely disagreeable odor, and hence, putrefaction may be spoken of as putrid fermentation. Among the compounds thus formed may be mentioned ammonia, and the amines, which possess a fishlike odor; also, indol and skatol which are characteristic products of intestinal putrefaction and the cause of the fecal odor. These substances contain the nitrogen which was present in the protein molecule. The sulphur of the protein molecule may appear as hydrogen sulphide, mercaptans, etc. 
In the fermentation of carbohydrates, or of fats, as might be expected, various organic acids form. The reaction of the fermenting liquid is, therefore, acid and the odor may be considered as pleasant. On the other hand, in the fermentation of proteins the ammonia and amines that form may impart an alkaline reaction to the medium, and, as indicated above, disagreeable odors are usually present.

Without the agency of micro-organisms the dead animals and plants would accumulate upon the earth's surface. The action of atmospheric oxygen, and of light, would be wholly insufficient to accomplish the change of "dust to dust." The removal of dead matter may be considered as the special function of microscopic life. In this respect, the bacteria are to be looked upon as performing a most important part-that of nature's scavengers. The wastematter of the living, and the lifeless remains themselves are in a short time converted into the simplest inorganic chemical compounds. The carbon present in the starch, cellulose, or protein molecule is returned by the agency of these organisms to the inorganic world as carbonic acid. In like manner the hydrogen becomes converted into water. The nitrogen present in the cell protoplasm, which is by far the most complex chemical substance known, is brought back to its simplest form ammonia, nitrous, and nitric acids.

Bacteria are, unquestionably, the most active agents in this resolution of dead matter. Other forms of life, however, may also induce similar changes. The moulds and yeasts, and even the infusoria, are deserving of mention in this connection.

The removal of dead matter, as indicated above, has one important consequence and that is the perpetuation of life. The ordinary plants derive their food from the carbonic acid of the air and from the nitrates of the soil. The starch, sugar, fats and protein matter which they elaborate, in turn, 
serve as the sole food for the herbivorous animal. The carnivorous animal derives its food from the latter, and man, as an omnivorous being, is consequently, directly or indirectly, dependant upon the vegetable kingdom for food. The nitrogen which as ammonia, nitrites and nitrates was present in the soil, and the carbon, which as carbonic acid was present in the air, have been built up into the complex protoplasm of the animal cell. On the death of the plant or animal, these elements would remain indefinitely in this combination were it not for the action of micro-organisms. These induce the decay or putrefaction, which results in the complete cleavage of these complex chemical substances, so that the elements are transformed into the simple compounds in which they originally existed, in the soil and air.

The carbonic acid, which is thus liberated in the fermentation or putrefaction of dead matter, is utilizable by a growing plant. As long as the carbon was present in the cellulose, starch, fat or protein molecule it was as useless to new plant life as that contained in the limestone deposits of the earth. The same is equally true of the nitrogen stored up in the protein molecule. It is only when brought back to the inorganic form that it can serve as plant food. The microscopic workers, therefore, in their rôle of scavengers prevent the accumulation of dead animal and plant matter, and, at the same time, maintain a cycle of life in which they themselves form an important link, and thus justify the axiom "Death is Life."

The bacteria which produce putrefaction have been designated as saprogenic. These same organisms, however, when grown on media containing sugar or other carbohydrates may give rise to fermentations, so that in the latter case they would also be designated as zymogenic. It is evident, therefore, that putrefaction is not a specific change due to a single organism, or even to a special group of organisms. It is induced by any one of a large number of bacteria, whenever these are grown in solutions of protein 
substances. As might be expected, the products will differ according to the kind of organism at work, the temperature and the amount of oxygen, etc. The bacterium termo of the older writers, is to be considered as a group name covering a number of saprogenic bacteria, rather than any one individual organism.

\section{Pigment Production.}

The various bacteria which give rise to pigments are said to be chromogenic. The activity of these organisms is not limited to the production of pigment. Thus, the wellknown golden, pus-producing micrococcus not only produces a pigment, but is also pathogenic, and may, indeed, induce typical fermentations. It is evident that the pigment is to be considered as one of the many chemical products elabor ated by the bacterial cell.

The number of chromogenic bacteria probably exceeds one hundred. All shades of color may be found among the different individuals of this group. It has been shown, heretofore, that the majority of bacteria are perfectly colorless. In only a very small number of bacteria do the cells contain a pigment. The contents of the cell, in such cases, may be colored a light pink or purple, green, yellow or brown. In the first case the pigment, known as bacterio-purpurin, may be considered as allied to chlorophyll, inasmuch as the organisms which contain it, unquestionably, give off oxygen in the presence of light. The green pigment, present in a few species may, in like manner, be possibly related to chlorophyll.

With the exception of the instances just mentioned, the cells of the chromogenic bacteria are perfectly colorless. It is clear that in these cases, the pigment, or.its antecedent, is made within the cell, and is excreted or passed out as rapidly as it is made. Moreover, there is reason to be- 
lieve that, in most of these cases, the pigment is not a primary, but rather a secondary product. That is to say, the bacterial cell elaborates a colorless, or leuco-product which, on contact with the oxygen of the air, becomes converted into the pigment. Almost invariably, pigment production will be observed on the surface of the culture media, where there is free access of oxygen.

In a few instances, as in the case of the Spirillum rubrum, the pigment is formed in the absence of air. This would indicate that in the particular case, the pigment was a primary product, made directly by the cell.

The pigment production in a given species is subject to considerable variation. At one time the growth may possess a very bright color, and again, at another time it may be very pale or colorless. The chromogenic property is, therefore, easily influenced by even trivial conditions. Under no condition, however, does a given organism give rise to different pigments, although, in a few instances, several pigments may be formed at the same time. The temperature, reaction and composition of the medium, and the oxygen supply are factors especially deserving attention.

As a rule, pigment production is diminished, or suppressed, when the cultures are grown at about the body temperature, $37-39^{\circ}$. The most brilliant pigments are obtained when the cultures are grown at about $15^{\circ}$. Prolonged cultivation, at a high temperature, may result in the produc. tion of permanent, colorless varieties.

Exposure to sun-light has a similar effect as a high temperature. In many instances, an insolation of a few hours may give rise to colorless varieties.

The reaction of the medium has a considerable influence upon pigment production. This will especially be pointed out in connection with the bacillus of blue milk. As to the composition of the medium, it should be said that a starchy surface, such as that of a potato, seems to be most favorable to pigment production. 
After the lapse of a few days, the brightest pigment fades, and may even disappear. This alteration may be due to the action of the air, although the bacterial products, especially the enzymes, may assist in the change.

Although the bacterial pigments have been the subject of numerous investigations, their exact chemical nature is still unsettled. In some instances, the pigment would seem to be a fatty compound (lipochrome); in another instance, it would appear to be a basic substance, allied to the ptomains (pyocyanin); and again, in other cases, it appears to be a protein compound.

The various pigments may be divided into two large groups according to their solubility in water. The fluorescing bacteria, and the bacillus of blue milk afford the best examples of soluble pigments. These pigments form on the surface of the medium, where there is an abundance of oxygen, and gradually. diffuse downward into the medium. In the other group the pigment is insoluble in water, and soluble in alcohol. The Bacillus prodigiosus, violaceus, etc., are good examples of this kind. In these instances the pigment exists as granules on the outside of the cells. In only two organisms does the pigment seem to be insoluble in water and in alcohol.

It is possible for various articles of food such as meat, milk, cheese, bread, etc., to develop growths of pigment producing bacteria. The B. prodigiosus and allied forms have been frequently met with in such "food epidemics" "The miracle of the "bleeding host," as well as "bloody milk," imputed in a former age to witch-craft, find an easy explanation in the sudden development of chromogenic bacteria.

\section{Phosphorescence.}

The extremely interesting, and rather mystical phenomenon of phosphorescence is common to the sea-water in all parts of the globe. Salt-water fish and oysters, when 
kept in a dark place, will not infrequently phosphoresce. When such fish are kept in meat-shops, it may happen that veal, pork and beef will, likewise, develop this strange property. This phenomenon is met with especially in seacoast towns. The river and lake waters do not phosphoresce, nor do the fish taken from such waters. Instances of phosphorescence are met with inland, in the well-known fox- or punk-fire which is observed in the woods at night, on the surface of old stumps. The fire-fly or glow-worm affords another illustration.

Phosphorescence, like pigment production, or fermen. tation, is a vital phenomenon. It has been known for a long time that certain protozoa in the sea-water emit light. Moreover, this property has been observed in some algæ and the ordinary fox-fire is due, unquestionably, to certain moulds. It is only within comparatively recent times that bacteria have been shown to possess this strange power. Pflüger's observation, in 1874, rendered it probable that certain bacteria were the cause of the phosphorescence observed on fish and meat, but it was not until 1887, when Fischer obtained pure cultures, that the cause was demonstrated. The mysterious glow on fish and meats like the pigmented milk, meat or starchy food is, therefore, due to the presence and growth of bacteria. In both cases, the microscope has revealed the cause which in the past was but too often ascribed to sorcery, or to supernatural powers.

At the present time about 25 species of phosphorescing bacteria are known. It is quite likely that some of these may be mere varicties. They are without exception inhabitants of salt-water. In large rivers, such as the Elbe, phosphorescing vibrios have been found which, otherwise, resemble the vibrio of Asiatic cholera. It is possible that these vibrios are derived from the sea. All the phosphorescing bacteria known are aerobic and, with possibly one exception, are non-pathogenic. They are, with the exception of one micrococcus and the vibrios mentioned, all rod- 
shaped bacteria. In order to give expression to their characteristic physiological property, they have been grouped together under the generic term photobacterium.

The property of phosphorescing is even more unstable than that of pigment-production. Nearly all of the organisms readily lose their phosphorescence when grown for some time upon artificial media. This is chiefly due to the reaction and composition of the ordinary media. As indicated above, the natural habitat of these organisms is the sea, and they require, consequently, a large amount of salt. About 4 per cent. of common salt should be added to the medium, which is still further improved by the addition of small amounts of glycerin and asparagin. The formula for such a medium will be found under B. phosphorescens. On this medium, the phosphorescence develops to a remarlsable degree. Some species which have been grown in the laboratory for some years and apparently have lost all power of phosphorescing, promptly develop this property when grown on such media.

It is quite possible that bacteria will be found inland which ordinarily do not phosphoresce but may do so when developed on media which are rich in salt. The author has observed in Ann Arbor a splendid phosphorescence on kidneys. The composition of these organs might favor the development of such otherwise non-phosphorescing bacteria. Again, the glow observed at times on manure heaps may possibly be due to similar forms favored by the large amount of saline constituents.

Absence of oxygen and an acid reaction are unfavorable to the production of light. The temperature of the body is likewise unfavorable. The very best phosphorescence is, as a rule, obtained when the growth takes place at about $15^{\circ}$. Gelatin tubes and plates under these conditions will phosphoresce for more than a month. The phenomenon of phosphorescence is observed at $0^{\circ}$, or even at a lower temperature. On a cold night the colonies on a plate 
shine like the stars in the heavens and when very numerous a diminutive "milky way" will be seen.

The character of the light emitted by these bacteria varies somewhat for the various species, but not enough to be of use in distinguishing between them. Moreover, the light will vary according to the composition of the soil. It may be whitish, bluish or greenish. The intensity of the light, at times, is such that a streak culture, on inclined fish-gelatin, is sufficient to reveal the time, on a watch. Indeed, photographs of a watch, fish, etc., have been made by means of such light. When examining cultures for phosphorescence it is essential that the eye should be accustomed to darkness, in order to perceive the delicate bacterial light. At night time the phosphorescence can be recog. nized, at once, by any one. In the day time, the transition from bright day-light to total darkvess is so sudden that it will be necessary to wait for some minutes before the phosphorescence will be perceived.

Very little that is positive can be said regarding the mechanism of phosphorescence. The commonly accepted view is that the emission of light is the result of intracellular activity. As long as the protoplasm of the cell is at work it liberates energy. This is ordinarily set free as heat, but in these organisms it is supposed that the energy or wave motion is, in part at least, that which corresponds to light. On the other band, the striking similarity between pigment production and phosphorescence should not be overlooked. Thus, free access of oxygen is essential to both phenomena; high temperatures are unfavorable and even give rise to atypical species or varieties; low temperatures are especially favorable to the production of pigment and of light. In the latter case, both may remain bright and unaltered after the lapse of many weeks. It would seem, therefore, that phosphorescence, like color, is associated with the production of certain bacterial products 
which are made within, but eliminated from the cell. The fact that filtered cultures of phosphorescing bacteria fail to give off light does not disprove this view. The phosphorescing substance, like most of the bacterial pigments, may be insoluble in water. It undoubtedly is very unstable since any chemical treatment yields inert products. Vari. ous aldehyde derivatives are known to phosphoresce in alkaline solutions, and it is possible that similar products are elaborated by certain bacteria.

\section{Heat Production.}

The heat of the animal body is ascribed to the oxidation or combustion of the assimilated food. In the cleavage of complex bodies heat is almost invariably generated. Inasmuch as in the various fermentations, the process is essentially one of cleavage, it follows that an evolution of heat must be expected. It matters little, whether the fermentative change is induced by bacteria, yeasts, or by moulds, in either case heat will be produced. Several instances may be mentioned to show to what extent thermogenic bacteria may raise the temperature of the medium in which they grow.

In ordinary alcoholic fermentation, due to the yeastplant, the temperature of the liquid may rise to an appreciable extent. It is well known that turnips, potatoes, etc., especially if moist, when placed in large heaps in a closed room will warm up to 50 or $60^{\circ}$, as a result of fermentation. A strong odor of tri-methylamin will be recognized and many of the tubers will show an evolution of gaseous products. Similar spontaneous heating is observed at times in masses of hops and the same fish-odor is present.

Another interesting case is met with in the curing of tobacco. After a preliminary drying the leaves are packed in large masses and subjected to a fermentation. The tem- 
perature in the interior of the mass rises and, in a day or two, may reach $50^{\circ}$ or more. As a result of this fermentation a special flavor or aroma is imparted to the tobacco. Inasmuch as this fermentation is due to certain bacteria, it has been proposed to utilize pure cultures of certain of these organisms in order to obtain good grades out of otherwise poor material. In the preparation of snuff, the tobacco is, likewise, subjected to a fermentation, in which the temperature rises even higher than mentioned above.

In the preparation of sauer-kraut, fermented hay, and ensilage, similar phenomena take place. The material is thoroughly stamped, or compressed, in large masses, and allowed to ferment. Not infrequently, the temperature on the inside, will rise to $70^{\circ}$. The material becomes acid, due largely to the presence of lactic, and butyric acids. At the same time it becomes soft, and acquires a more or less, characteristic, not unpleasant odor.

The high temperature which develops in the interior of large masses of green hay, may, if air is suddenly admitted, lead to spontaneous combustion. This may also occur with masses of moist cotton, and the fires in cotton laden ships are not, infrequently, due to such causes.

\section{Toxiçogenic and Pathogenic Bacteria.}

In fermentation and putrefaction, more or less complex, dead animal and vegetable substances are acted upon by diverse organisms, such as bacteria, moulds and yeasts. They are transformed into relatively simpler compounds, and eventually into inorganic forms, such as carbonic acid, ammonia, nitrites, nitrates, sulphates, phosphates, etc., which, as shown heretofore, are then utilizable by living plants. Hence, lifeless remains are indispensable to new life, and, the micro-organisms which accomplish this 
change, are absolutely essential to the continued existence of higher plants and animals.

The products, elaborated by the majority of bacteria, are practically harmless, if introduced into the living body. It follows, therefore, that the vast majority of bacteria are unable to produce poisoning, or disease, in man or in animals. A relatively small number of bacteria give rise to poisonous products, and are also able to grow in the living body. In such cases they live at the expense, and to the injury of the host, and hence induce disease. Such organisms are, therefore, designated as pathogenic. These will receive special consideration in subsequent chapters. There are bacteria which produce poisonous products, but cannot grow in the living body. When such organisms develop in foods they may give rise to poisonous products, which will cause intoxication when the food is consumed. The subject of poisonous foods is briefly touched upon in Chapter $\mathrm{XV}^{1}$.

The term toxicogenic applies to all bacteria, which produce poison, irrespective as to whether they are able, or unable, to grow in the living body.

${ }^{1}$ Additional information will be found in Vaughan and Novy, Ptomaïns and Leucomaïns, 3rd ed. 
THE MICROSCOPE.-THE HANGING DROP.-SIMPLE STAINING.

In view of the fact that many of the students begin the study of bacteriology without any previous experience in the use of a microscope, it is very desirable to describe this instrument, and the manner in which it should be employed.

It is customary to speak of simple, and of compound microscopes. The former consist, usually, though not necessarily, of a single lens, as in the case of an ordinary reading glass. In the simple microscope, the rays of light which enter the eye come directly from the object, and a virtual image is produced. Fig. 15 illustrates the action of a simple mi. croscope. It should be observed that the object is between the principal focus $P$ and the lens. This figure

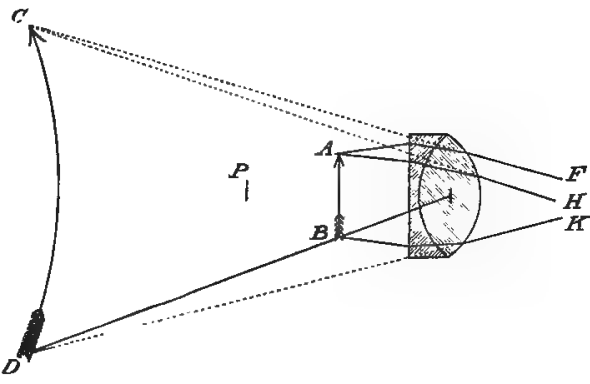

FIG. I5. Virtual image, simple microscope (Carpenter). also illustrates the action of the eye-piece in the compound microscope.

If the object is placed beyond the principal focus $(P)$, as in Fig. 16, a real image will result, and can be received on a screen. This corresponds to the action of the objective in the compound microscope.

The compound microscope consists of two set of lenses, the objective and the eye-piece. The former is placed near the object, and gives rise to a real image (Fig $17 \mathrm{~A} \mathrm{~B}$ ). This 
image, inverted and reversed, lies inside of the principal focus of the eye-piece, and the rays of light leave it as if they came from a real object. If a screen was placed at this point, it would show an image. Since this image lies. inside of the principal focus, it becomes magnified by the eye-piece $(E)$, and the virtual image $(C D)$ is produced. It has been, not inaptly, said that " a compound microscope is a simple microscope applied, not to the object, but to its image already magnified by the first lens."

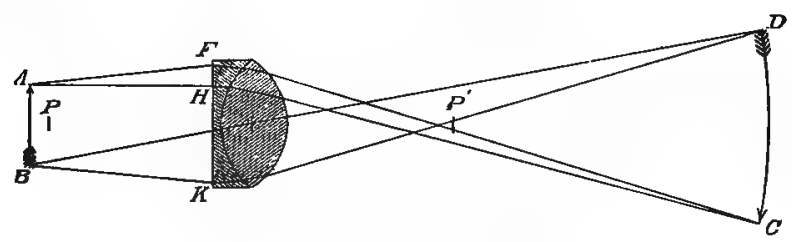

FiG. I6. Real image (Carpenter).

The single lens naturally represent the earliest form of the microscope. The English monk, Roger Bacon, in 1276, appears to have been the first to recognize the peculiar properties of a lens. It was he who applied the new knowledge to the construction of spectacles. It was not, how. ever, till the beginning of the 17 th century, that the microscope was sufficiently perfected to bring about the discovery of the existence of microscopic life. The discovery of the compound microscope may be said to have been made by Galileo in 1610, although it is probable that others may have antedated him by a few years. The early compound microscope consisted of a single lens for an objective, and of another single lens for an eye-piece. Such an instrument, necessarily gave very imperfect results.

The image produced, by a single lens, is not a perfect reproduction of the original object. All the rays of light do not meet in the same plane, and hence, the image has a spherical appearance. This fault of a lens is known as spherical aberration. Again, the lens, acting as a prism, decomposes some of the rays of light which pass through it. 
The violet component is bent most, and hence, is brought to a focus at a different point from the red ray, which is bent the least. The result is a fringe of colors. This is designated as chromatic aberration.

It is necessary, therefore, to correct the chromatic and spherical aberration, in order to obtain the best optical results. The spherical aberra. tion is partially corrected by means of stops or diaphragms, which hold back the peri. pheral rays, and allow only the central ones to pass through, since these give rise to an almost flat image. Such diaphragms are shown in Fig. 17. The use of flint glass still further, serves to correct this error.

The chromatic aberration is more difficult to correct. The first successful attempt at the correction of chromatic aberration in an objective, was made by an Italian, Marzoli, in 1811. In other words, 200 years elapsed between the discovery of the compound microscope and the correction of the most serious defect in the working of the

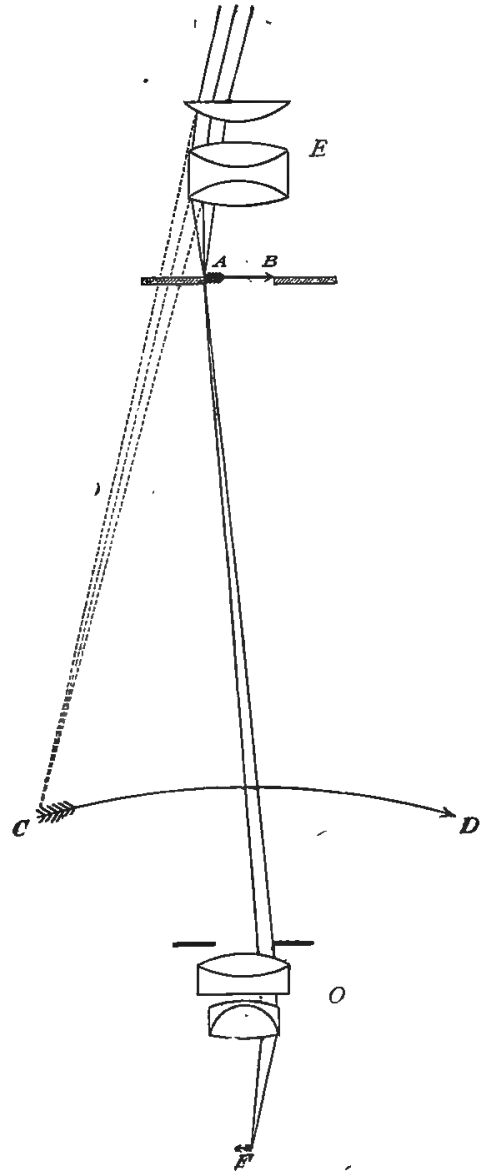

FIG. 17. Principle of the compound $\mathrm{mi}$ croscope (Carpenter). F-Object in focus above this an objective with diaphragm; AB-Real image of $F$, in opening of diaphragm; above this a compensation ocular which magnifies the real image $A B$, thus forming the virtual image CD. objective. This early work, however, seems not to have attracted much attention, and Chevalier, of Paris, was given 
credit for introducing, in 1825, the method of correcting chromatic aberration. The real value of a microscope, as an aid in scientific research, dates from this period. These improved objectives, since the color defects were done away with, were designated as achromatic objectives. This achromatism was obtained by the combination of two glasses, crown and flint.

The dispersive power of flint glass is almost double that of crown glass, and hence, a crown bi-convex lens is fitted into a plano-concave lens of flint glass. The effect of this combination will be understood, if a prism of crown glass, having an angle of $50^{\circ}$, be placed by the side of an inverted one of flint glass, having an angle of $25^{\circ}$. The ray of light passing through the crown glass is decomposed into its components, the colored rays of the spectrum. These then enter the inverted flint glass prism, and are reconstituted to white light. The concave flint lens is virtually an inverted prism, with reference to the convex, crown glass lens. Refraction is thus obtained without dispersion,

Even the best achromatic objective gives an image that is not wholly free from color. This is due to the fact that the two kinds of glass employed do not disperse the several rays in the same proportion. For instance, the green ray may occupy the exact middle of the spectrum produced by one glass, whereas, in that produced by the other, it may lie to one side, possibly only one-third of the distance from the red to the violet. If, therefore, the red and violet rays are neutralized, the green will still remain. Consequently, there is always a certain amount of color present around the image of an achromatic objective. This is known as the secondary spectrum.

About 10 years ago Abbe and Zeiss prepared special kinds of glass, the so-called borate and phosphate glass, by means of which it was possible to do away with the secondary spectrum. These objectives, which represent 
the highest optical attainment at the present time, are designated as apochromatic. The lenses, entering into the composition of these objectives, are made of the special glasses mentioned, and in addition, there is present a lens made of fluorite.

The employment of a cover-glass, between the object and the objective, disturbs the correction of the latter. The rays of light, passing from the object through the cover-glass, are refracted, so that the peripheral rays which enter the objective, seem to come from a point nearer the objective than do the central rays. This condition is overcome by making an under-corrected objective, which will focus both these points at once. In objectives provided with a "collar" under-correction is obtained by turning the collar so as to bring the back lenses nearer to the front lens. The correction collar is placed only on the dry, high power objectives. In the absence of a collar, the necessary correction for a thick cover-glass can be obtained by shortening the tube-length, thus causing the eye-piece to approach the objective. Objectives are usually made without a collar, and are corrected for a cover-glass having a thickness of $0.17 \mathrm{~mm}$. Markings on an object, which are easily seen when a cover-glass of, this thickness is employed, disappear when an appreciably thicker, or thinner cover-glass is used. Unlike the dry lenses, the oil immersion objectives are largely independent of the thickness of the cover-glass.

It is evident that the objective is the most important part of the microscope. Consequently, in purchasing a microscope, special attention should be given to the quality of the objectives. A good microscope means, above all, good objectives. It is a common error for the student. to believe that the best microscope is the one that magnifies the most. This is far from being true. It is not the power of magnification, but the ability to show up objects in their 
minutest details which makes an instrument truly valuable. The properties which a good objective should possess are as follows:

1.-A proper magnifying power.-In the case of a single lens the magnifying power may be said to depend upon its focal distance. The shorter the focal length the higher the magnification. Therefore, a lens with a one-fourth inch focus will magnify more than one having a focal distance of one inch. In the case of the objective, we have to deal not with one lens, but with a combination of lenses, and it is customary then to speak of the "equivalent focal distance." This represents what would be the focal distance of a simple lens having the same magnification as the objective. When, therefore, a lens is designated as a onefourth inch objective, it does not mean that the distance from the object to the front lens is one-fourth of an inch. On the contrary, this "working distance," as it is called, would be much less than a fourth of an inch. The one-fourth inch objective, however, does possess the same magnification as a single lens having a focal distance of one-fourth of an inch.

A table showing the magnifying power of the several objectives and eye-pieces is usually supplied by the instrument maker. The magnifying power, of a given objective and eye-piece, can be obtained by the following simple method. - A stage micrometer, or object of known length, is necessary. The micrometer is a glass slide on which an accurate scale has been cut. The scale may consist of one mm. divided into 100 parts. The micrometer is adjusted on the stage of the microscope. The draw-tube of the latter should be drawn out, vertically, so that the upper surface of the eye-piece is 10 inches from the table. On looking into the microscope, both eyes kept open, the image of the micrometer will be seen to be projected on the table. The limits of the projected scale can be marked on the table, and the distance then measured. If, for exam- 
ple, one $\mathrm{mm}$. is magnified so as to measure $100 \mathrm{~mm}$., it is evident that this magnification of the objective and eyepiece is 100 .

The student should distinguish between linear and superficial magnification. The former is always meant in scientific work, whereas, the latter is employed for popular purposes. As an example, corresponding to the above, a one $\mathrm{mm}$. square would be magnified to a $100 \mathrm{~mm}$. square. The linear magnification would be 100; the superficial, or area magnification would be 10,000 .

2.-A good defining power.-It is obvious that a good objective should give as flat a field as possible. Perfect flatness of the field is not attainable, but a great deal can be done by reducing the spherical aberration. The definition of an objective is likewise increased by properly correcting the chromatic aberration. Moreover, the perfect centering of the lenses is necessary to the good performance of an objective. The flatness of field can best be tested by a stage micrometer, whereas the definition proper can be tested by means of stained bacteria, such as the tubercle bacillus.

3. - A good resolving and penetrating power. $\rightarrow$ By the resolving or delineating power is meant the ability of a lens to show up fine markings and delicate structures.) A normal, unaided eye may recognize divisions on a scale which are sह่ of an inch apart. Another eye may fail to resolve these lines, whereas a third may be able to recognize even a smaller fraction of an inch. Again, on account of "acuteness of vision", one person may make out, with the unaided eye, a double star, which another person may fail to do. The resolving power, therefore, and not its magnifying power, is what imparts value to an objective.

The resolving power of an objective depends primarily upon the amount of light which it admits. The light that enters is included in the angle made by the two extreme 
rays, which leave the object (when in focus) and enter the front lens. This angle, known as the angle of aperture, is shown at $a$, in Fig. 18. This figure shows, in cross-section, the lens system employed in the construction of an oil immersion objective. The rays of light diverging from the object enter the front of the small lens. It is obvious that the more light admitted by an objective the more distinct the image. The angle of aperture,
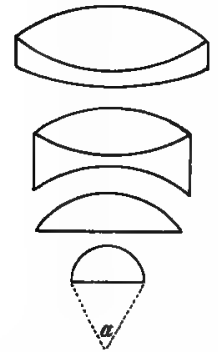

Fig. 18. Arrangement of lenses in an one-twelfth inch oil immersion objective (Carpenter). $\alpha-A n-$
gle of aperture. therefore, conditions the resolving power of an objective.

In actual practice, the rays of light, after they leave the object, pass into the cover-glass (a denser medium) and from this into the air, which is less dense. The result is refraction, and, more or less, loss of light by reflection. If the air is replaced, by a denser medium, there will be less refraction and hence, it is possible to utilize some of the light that would otherwise be lost. These considerations led to the introduction by Amici, of the water immersion objective. In this objective a drop of water was placed between the cover-glass and the front lens. The ray of light passing from the cover-glass into the water suffered less refraction than it would if it passed into air. Since the index of refraction of water is 1.0 and that of crown glass is 1.52 , it is evident that there was still room for improvement. In 1878, Stephenson suggested that the water in the immersion objectives be replaced by an oil having the same index of refraction as crown glass. Abbe and Zeiss, thereupon, introduced the homogeneous oil immersion objective. The cedar oil, which is placed between the cover-glass and the front lens of the object, has the same index of refraction as crown glass (1.52). Consequently, a ray of light after it once enters the cover-glass passes in a straight line directly into the objective. The 
angle of aperture is utilized to its widest extent, and, as a result, the resolving power is increased.

(The resolving power of an objective, therefore, depends upon the angle of aperture and on the index of refraction of the medium, which is between the cover-glass and the front lens. Abbe has reduced these factors to a mathematical expression, which is known as the numerical aperture. (The numerical aperture of an objective corresponds to the sine of half the angle of aperture, multiplied by the index of refraction of the medium that lies between the front lens and the objective. It is usually expressed as,

$$
\text { N.A. }=n \text { sine } u \text {. }
$$

$n$ represents the index of refraction, and $u$ represents half the angle of aperture. ;

It is well to repeat, that, the value of an objective depends not upon its magnification, but upon its resolving power, and that this is directly proportional to its numerical aperture. Of the lenses having the same magnifying power, the one that has the highest numerical aperture is the most valuable and the most expensive.

For ordinary work, it is not desirable to have an oil immersion objective of more than about 1.30 N.A. since above this, on account of their peculiar construction, they are very liable to suffer injury. Zeiss has succeeded in making objectives possessing a N.A. of 1.50 , where the theoretical limit is 1.52. By means of fluorite lenses, and a special immersion fluid, mono-brom naphthalen, Zeiss has been able to produce a lens having a N.A. of 1.63 , which represents the highest achievement in the construction of objectives.

The penetrating power, or depth of focus, is expressed by

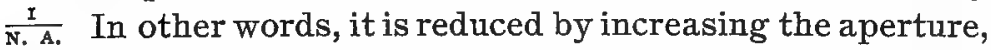
as well as the magnification. Just as the resolving power is necessary, in order to bring out the structure, or lines in a given plane, so a penetrating power is desirable, in order to perceive depth in an object. For ordinary work, therefore, it is preferable to employ a low objective, with a mod- 
erate aperture. The high objective, with a wide aperture, is to be used only as the special occasion requires.

In England and America, it is the custom to designate objectives by their equivalent focal distance. On the Continent, however, no such designation exists and some makers mark the objectives with numerals, others with letters. Leitz, for example, marks his objectives with numbers from 1 , which corresponds to a $1 \frac{8}{4}$ inch, to 10 which is a water immersion objective. Zeiss, on the other hand, designates the achromatic objectives with letters, while the apochromatics are designated by the equivalent focal distance expressed in mm. The oil immersion lenses are designated after the English method $\frac{1}{10}, I^{2}$, $i^{\frac{1}{6}}$ inch. The objectives which have met with most favor in bacteriological studies are the 3, 7 and $\frac{1}{7}$. The No. 3 corresponds to a $\frac{3}{4}$ inch, and to Zeiss' A. The No. 7 is about the same as a inch, while the Zeiss $D$ is a $\frac{1}{6}$ inch.

The eye-piece, or ocular, serves to magnify the image made by the objective. The form commonly employed, is that known as the Huyghenian. It consists of two lenses, a lower or field-glass, and an upper or eye-glass. Both lenses are plano-convex. The lower, broad lens serves to refract the rays of light, thus bringing the image within the focus of the eye-glass.

When apochromatic objectives are employed, it is necessary to use special oculars, known as the compensation eye-pieces. The eye-piece represented, in section, in Fig. 17, p. 125 is of this type.

The designation of eye-pieces is subject to variation, as in the case of objectives. Some designate by letters, others by numbers. In this country, they are designated in the same way as the objectives, that is, according to their equivalent focal distance. Thus, a 2 -inch eye-piece would magnify the same as a single lens of 2 -inch focus. The eyepieces of low power are spoken of as "shallow," while those of high power are called "deep." The compensating eye-pieces of Zeiss are designated by their amplification. "Thus, a 2 eye-piece doubles the initial magnification of the objective; an 18 eye-piece magnifies the image 18 times. 
It is well to have three eye-pieces, having an equivalent focal distance of 50,35 and $25 \mathrm{~mm}$. respectively. This corresponds to numbers 0,2 and 4 of Leitz, and $2,1 \frac{1}{2}$ and 1 inch eye-pieces of this country. As a rule, the low eyepiece should be used for work. . The higher eye-pieces may be used, when it is desired to increase the magnification. It should be remembered, however, that the eye-piece magnifies the image, and hence, any imperfections that may exist in the image. On account of eye-piece magnification, the field becomes darker and less distinct. It is preferable, therefore, to obtain an increased magnification by changing the objectives, rather than by the use of deep oculars. When apochromatic objectives, are used, it is possible to employ even the deepest compensation oculars.

The Abbe condenser.-This is a most important accessory to a microscope, and, indeed, it should not be wanting on an instrument intended for bacteriological work. The usual Abbe condenser is chromatic, although for special purposes, as in micro-photography, an achromatic condenser is employed. The ordinary condenser is made of two lenses, as shown in Fig. 19 , and has a numerical aperture of 1.20. By the introduction of a third lens, the aperture is in-

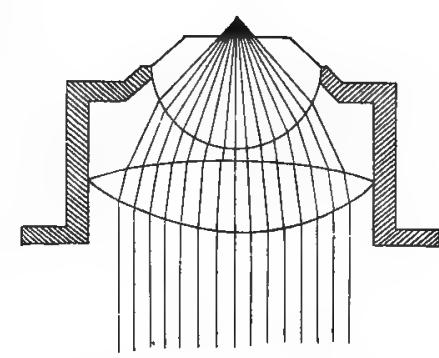

FIG. I9. Cross-section of the Abbe condenser, showing convergence of the rays of light. creased to 1.40 .

The aim of the condenser is to illuminate the object as much as possible. Without a condenser, given a plane mirror, an object will be illuminated by a pencil of light corresponding to its own diameter. This may be sufficient for a low power, but when high powers are used, it is evident that more light must be thrown on the object in order to see distinctly. By means of the condenser, the 
broad pencil of light, corresponding to the diameter of the front lens of the condenser, is brought to a focus at about $2 \mathrm{~mm}$. above the upper spherical lens. An object, therefore, placed in this focus will receive all the light that enters the condenser. In order to obtain the best results, the condenser should always be focussed on the object. When parallel light is employed, the reflected light from a white cloud, the focus will lie nearest to the plane face of the upper lens. When artificial light, as that of a lamp, is employed, the rays that are brought to the condenser are divergent, and hence, the focus will be farther away from the upper face of the condenser. The correct adjustment of the Abbe condenser should, therefore, receive the same attention as the proper focussing of an objective. (The plane mirror should always be used with the condenser, since the concave mirror, if employed, would give off converging rays. These would be brought to a focus so close to the upper face of the condenser, that, owing to the thickness of the glass-slide, it would not be possible to bring the object into this focus.)

The Abbe condenser is ordinarily employed with its optical axis in line with that of the microscope. It thus gives central illumination. The iris diaphragm on the more expensive instruments can be placed in an excentric position by means of a thumb-screw, and can be rotated in this position about the optical axis. Hence, oblique light can thus be admitted from any desired direction.

The condenser is always accompanied by an iris diaphragm. This enables one to regulate the amount of light which enters the condenser. If, for instance, an unstained section of a liver is placed under the microscope, and carefully focussed, it will be seen perfectly, provided the diaphragm is partly closed. Now, if while looking at the section through the microscope, the diaphragm be thrown wide open the object will at once become invisible. The excessive amount of light coming from the unrestricted condenser 
renders the object invisible - it destroys the so-called structural image. An uncolored object is rendered visible, because of inequalities in the reflection, or refraction of the light, as it strikes the object. On the other hand, a stained object is visible because of the color. The color image, unlike the structural image, is practically unaffected by the light admitted through an open condenser.

The following rule, therefore, should govern the use of the condenser: When examining unstained preparations, as a hanging-drop, the diaphragm should be restricted. When examining stained preparations, the diaphragm should remain open. In the former case, the diaphragm should be restricted to such an extent, that on looking into the microscope with the objective in, or near the focus, it gives a dim twilight. The diaphragm is contracted so that, at most, a pin-head opening exists. When the specimen is stained, the diaphragm remains open. It is not advisable to open the diaphragm to the widest possible limit, since a stained object is rendered more distinct if the diaphragm is slightly con. stricted.

In order to secure the best illumination of an object, it is necessary (1) to set the plane mirror at the best angle for reflection of the light, as it comes from a white cloud; (2) to adjust the iris diaphragm, according, as the object is stained, or colorless; (3) to focus the condenser on the object. Direct sunlight should never strike the mirror, or the stage of the microscope.

The stand.-The various parts of the stand will be understood best by reference to the frontispiece. The drawtube slides up and down in the body of the microscope. It is graduated, and, when the instrument is about to be used, the draw-tube should be raised to the line marked 17, provided no nose-piece is present. The upper face of the eye-piece is then $170 \mathrm{~mm}$. distant from the shoulder of the objective. 'This position should always be maintained 
when at work, because the lenses have been corrected for this distance. A deviation from this distance will cause deterioration in the image perceived. Usually, the microscope is provided with a nose-piece, which is $10 \mathrm{~mm}$. thick. When, therefore, the nose-piece is attached to the microscope, the draw-tube should be raised to the line marked 16 (160 mm.)

The objective is always moved to, or from, the object by means of the coarse adjustment. This is a rack and pin. ion, and is used to bring the objective near the focus. It is not used for "focussing," except when the low objective (No. 3) is used. When the high power objective is brought as near to the focus as possible, by means of the coarse adjustment, it is then focussed exactly, by means of the fine adjustment. The latter is a fine micrometer screw, by means of which the entire body of the microscope is raised or lowered.

For bacteriological purposes, the microscope should as a rule be kept in a vertical position. It is manifestly inconvenient to examine plates, hanging-drops, etc., with an inclined instrument. Although a triple nose-piece is not necessary, yet it is a very desirable, time-saving addition to a microscope.

Care of the microscope.-There is no instrument placed in the hands of the student which requires as careful attention. An untidy microscope at once characterizes the work of the individual. The following general directions should be closely observed in taking care of the instrument:

1.-The microscope should always be taken up and carried about by means of the pillar below the level of the stage. A chamois skin should be employed, in order not to soil or remove the lacquer. It should never be lifted by taking hold of the fine adjustment tube, or by the barrel.

2.-All unnecessary contact of the fingers with the lacquered parts should be avoided. If finger-marks have been 
left, they should be removed by gentle breathing on the spot, and then slightly rubbing with clean, washed linen, or with a piece of chamois skin. Alcohol should never be applied to the lacquered surface, since it will remove the lacquer.

3.-The milled parts on the objective, eye-piece, drawtube, etc., should be handled and not the neighboring smooth parts.

4.-The stage of the microscope should be kept clean and dry. The glass slide, or plate, should never be placed on the stage, unless the under surface is known to be dry.

5. - The mirror should be kept clean and free from dust. The same is true of the Abbe condenser.

6. - The front lens of the objective must be kept perfectly clean. The No. 3 and No. 7 objectives are $d r y$ lenses, and should never be allowed to touch oil, water, gelatin and the like. If oil has touched the lens, it should be carefully removed by means of a cloth moistened with a drop of alcohol. Gelatin and agar can best be removed by touching with a cloth dipped in clean, warm water. The ra inch objective is an oil immersion lens, and is never used dry. A drop of cedar oil is placed between the front lens of this objective and the clean, dry, upper surface of the coverglass. At the close of the day's work, the cedar oil must be removed from the front lens by gently touching several times with a well washed piece of old linen, or with a soft tissue-paper.

7.-The eye-pieces should be kept clean, free from moisture and dust.

8.- At the close of the day's work, the microscope should be carefully inspected, cleaned wherever it is necessary, and placed in its locker.

Measurement of an object.-In this connection, it will be well to describe the method pursued in measuring the size of an object. It is necessary to have a stage micrometer 
aud an ocular micrometer. The former is a glass slide on which a scale of $1 \mathrm{~mm}$. is subdivided into 100 parts. The ocular micrometer is placed inside the eye-piece on the diaphragm, by unscrewing the eye-lens which is then replaced. It lies, therefore, in the same plane as the image made by the objective. If this is not the case the diaphragm should be raised or lowered till the plane of the ocular micrometer coincides with that of the image. It is necessary, first, to ascertain for each objective the value of a division of the ocular micrometer.

The stage micrometer is focussed, first, with the No. 3 objective. It will probably be found that 10 divisions on the scale in the eye-piece cover 15.5 divisions of the stage micrometer. 1 division of the ocular micrometer corresponds, therefore, to 1.55 divisions on the stage micrometer. Since, each of the latter represents rto mm., it follows that 1 division on the scale in the eye-piece corresponds to $\frac{1.55}{100}=0.0155$. The robo th part of a mm., as explained on p. 24 , is known as a micron, and is designated by the letter $\mu$. The micron is approximately 500 th of an inch. Therefore, each division on the ocular micrometer, used in connection with this same eye-piece and objective, corresponds to $15.5 \mu$. If, now, an object is placed under this No. 3 objective in place of the stage micrometer, and it corresponds to 10 divisions on the scale of the ocular micrometer, it is evident that the object measures $155 \mu$.

The "micrometer value" of the No. 7 objective is ascertained in the same manner. Thus, if 50 divisions on the ocular scale correspond to 18 divisions on the stage micrometer, 1 division of the former will correspond to 0.36 divisions of the latter $=\frac{.88}{100}=.0036 \mathrm{~mm} .=3.6 \%$ Again, in case of the 1 inch objective, 50 divisions on the eye-piece micrometer correspond to 8.6 divisions of the stage micrometer. Hence, 1 division of the former corresponds to $\frac{8 \cdot 6}{60}=$ 0.172 of one division of the latter. Since each division of the latter represents ro of a mm., one division of the eye- 
piece micrometer represents $\frac{172}{100}=.00172 \mathrm{~mm} .=1.72 \mu$. If, therefore, a bacillus is examined with this objective, and occupies three divisions on the eye-piece scale, it is $1.72 \times 3$ or $5.16 \mu$ long.

When the micrometer value of an objective is once ascertained, in the manner described, it becomes a very easy matter to determine the exact size of an object by means of the ocular micrometer. It should be remembered, however, that this micrometer must always be placed in the same eye-piece with which the original measurements were made. Likewise, only the particular objectives tested should be employed in such measurements. The draw-tube, it is understood, should be drawn out when measuring an object, to the same distance as when the micrometer value was determined (160 mm.)

When an ocular screw-micrometer is employed, owing to its height, the draw-tube must be lowered, so that there is a distance of $170 \mathrm{~mm}$. between the upper surface of the ocular and the shoulder of the objective. The value of a division on the screw is ascertained, for each objective, by means of a stage micrometer in the manner described above.

The approximate size of an object can be determined without the use of a micrometer, by dividing the length of the image, as projected on the table (p. 128), by the magnification of the objective and eye-piece used. The latter is obtained in the manner described on p. 128 , or from the table supplied by the maker of the instrument.

Drawing an object.-The reproduction of objects seen under the microscope, constitutes a most important training. of the student. The object should be drawn, so as to have the size of the image reproduced. This can only be done by projecting the image on the drawing paper, and then sketching in the outline. The paper is placed on the table, on the right side of the microscope. On looking into the 
microscope, with the left eye, the image of the object will appear on the paper, and can be readily outlined. The relative size of different objects, seen under the microscope, can thus be faithfully reproduced. With a little care and patience, very satisfactory results will be obtained in a short time.

Various optical contrivances have been devised to facilitate the drawing of microscopic objects. The camera lucida of Abbe is especially useful for this purpose.

\section{Cover-Glasses.}

The slides and cover-glasses, employed for microscopic work, must be rigidly clean. The usual method of cleaning cover-glasses, is to immerse them in alcohol, and then, to wipe with a clean cloth till dry. Frequently, coverglasses thus treated, although apparently clean, will not permit the spreading of a drop of water on their surface. This thin layer of fatty matter often cannot be removed, even by treatment with sulphuric, or with acetic acid. The cover-glass may be passed rapidly, five or six times, from above downward, through a Bunsen flame, in which case the heat destroys the organic matter on the cover-glass. When thus treated, a drop of water, placed upon that surface of the cover-glass, which was in direct contact with the flame, can be spread evenly over the entire surface. It will not gather into small droplets, as is usually the case. The objection to this method is, that many of the coverglasses crack during heating.

The following method of preparing cover-glasses will give perfect satisfaction. The cover-glasses are immersed in alcohol, and are then wiped dry, and as clean as possible. They are then placed in an Esmarch or Petri dish, and heated in a dry-heat sterilizer at $170-180^{\circ}$, or higher, for one hour The organic matter is thus subjected to de- 
structive distillation; the cover-glasses are, as a result, perfectly clean, and they remain thus, as long as they are kept in the covered dish. It is advisable to clean up a box of cover-glasses at a time. The Fresenius iron drying plate, with its six cups, is especially convenient for student's use. The temperature of this plate can easily be raised to $200^{\circ}$. Only very rarely, will this procedure for obtaining clean cover-glasses, fail. In such cases, an additional passage of the cover-glass, two or three times, through the flame, will give a suitable surface. The droplet of water, or the bacterial suspension, should be placed on that side of the cover-glass which touched the flame.

The cover-glass should always be handled with a pair of forceps. The ordinary, slender, narrow-pointed forceps, are well adapted for this purpose. In staining, however, they have one serious disadvantage. The weak blades compress on the slightest pressure, and form a capillary along which the staining reagent readily drains from the cover-glass. Under these conditions, the hands of the student stain more readily than does the specimen.

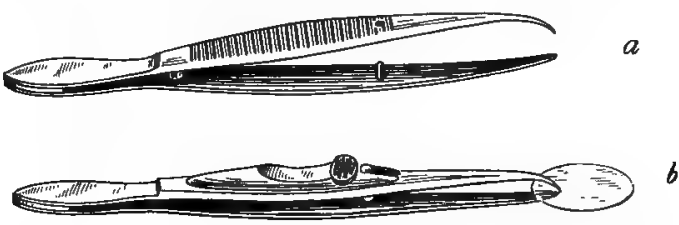
FIG. 2o. Cover-glass forceps of the author. a-Simple
form; $b$-Forceps provided witb clasp.

The forceps devised by the author, and shown in Fig. 20, are extremely convenient in handling cover-glasses. The lower blade is flat and has a broad end ( $2 \mathrm{~mm}$. wide), with a thin, sharp edge. The upper blade is narrow, bent, and terminates in a point, which, when the forceps are shut, rests close to the end of the lower blade. The advantages possessed by these forceps are, that it enables one to pick up 
cover-glasses from a flat surface, and that, in staining, no copillary drainage exists. Moreover, owing to the pointcon'iact, the specimen can always be washed perfectly clean, without leaving the unsightly spot so often caused by a broad pointed pair of forceps.

The above forceps can be obtained, supplied with a clasp (Fig. 20 b), as in the case of Ehrlich's forceps. In this form, it will be found to be superior to the awkward, heavy Cornet forceps, or its several modifications.

\section{Examination of Living Bacteria."}

This is intended to show the bacteria in their natural condition. The study of an organism is manifestly incomplete, if the examination of the living form is omitted. A great deal of information can thus be acquired, which might otherwise be overlooked. Care should be bestowed especially upon the form and grouping of the cells, the presence, or absence of motion, the appearance of the protoplasm, whether colored or granular, the presence of sporogenic granules, and of spores. Obviously, the process of cell division, spore formation, and germination can only be observed on living organisms.

The simplest method for examining living bacteria is as follows: A little of the growth is picked up on the end of a sterile, cooled platinum wire (Fig. $28 \mathrm{e}$ ), which is then touched, several times, to a drop of water on a glass slide. The drop of water should, as a result, show a visible cloudiness. It is then covered with a glass slip, and examined under the microscope. When this procedure is followed, it is desirable that the drop of water taken be so small that it will not float the cover-glass.

The examination of the living organism on an ordinary glass slide, in the manner indicated, is not always satisfac-

${ }^{1}$ The examination of hanging-drops and the staining of bacteria follows the work which is given in the next chapter. 
tory, because the liquid soon begins to evaporate and, as a result, rapid currents are established. To overcome this evaporation, it is customary to examine the material in a "hanging-drop." For this purpose, a thick glass slide, hav. ing a concave well in the middle, is made use of. The ordinary "concave," or hollow slides, usually on the market, are so shallow that the drop of water will touch the bottom. This should not occur.

The "hanging-drop" is easily made in the following manner: Place a small drop of water on a $3 / 4^{\prime}$ inch, clean cover-glass which is placed on the table or on a short board. The drop must be small enough so that it will not run, if the cover-glass is placed on edge. The growth from the potato, or other medium, is then touched off into the drop of water, by means of a sterile platinum wire. A ring of vaselin is placed around the edge of the well on the upper side of the concave slide, by means of a brush or match stick. The slide, with its ring of vaselin, is then inverted over the cover-glass and gently pressed down The coverglass now adheres to the slide, which is then inverted. Care should be taken to see that the vaselin is continuous around the edge of the well. If such is the case, no evaporation of the drop of water can take place, and hence, the hanging-drop can be examined at leisure and without the presence of annoying currents in the liquid. Fig. 21 indicates the hanging-drop in longitudinal cross-section.

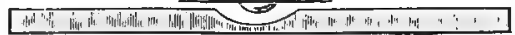

FIG. 2I. The "hanging-drop," showing concave slide and suspended drop.

The Ranvier slide is very useful for certain purposes. The central portion of the slide is ground down so that it is about Ir mm. below the surface. This shallow well is surrounded by a deeper groove. The drop of water, instead of being convex as above, is, in this case, flattened out into a thin layer. The edge of the well is ringed with vaselin as above. 
Examination of the hanging-drop.-Place the slide, with the cover-glass uppermost, on the stage of the microscope and find the edge of the drop with a low power-No. 3, or $\frac{2}{3} \mathrm{rd}$ inch objective. If too much light is present, constrict the diaphragm. The edge of the drop should be seen as a sharp line passing through the field of the microscope. Holding the slide between the thumb and forefinger of the left hand, slowly move it so that the edge of the drop constantly remains in the field. In this way the entire edge, or circumference of the drop, should be examined, chiefly for the purpose of practice in moving a slide under the microscope. Owing to the minute size of bacteria, they cannot be seen under this magnification. At most, fine granules can be detected. To observe the individual cells, therefore, recourse must be had to a higher power-No. 7 , or $\frac{1}{8}$ inch objective, or to the ${ }^{1} \mathrm{t}$ th inch homogeneous, oil immersion objective.

Examination with the No. 7 objective.-Having found the edge of the drop with the No. 3 objective, replace this with No. 7 , by rotating the nose-piece. Then lower the tube of the microscope by the coarse adjustment, till the objective almost touches the cover-glass. The field of the microscope is now, usually, very dark; hence open the diaphragm a trifle to admit enough light, so as to see distinctly. . With the fine adjustment now raise the microscope tube, till the edge of the drop is brought out distinctly. By focussing the edge carefully, the bacteria will be readily detected. Now move the slide, as mentioned above, so as to examine the entire edge of the drop, and also the center. Study the characteristics of the micro-organisms present, especially with reference to form, structure and motility.

In working with a high power, while focussing, it is desirable to constantly hold the slide between the thumb and forefinger of the left hand, imparting to it a slight motion. If this motion is arrested, it is due to pressure of the objective, which has been lowered too far, and unless the pressure is promptly relieved, damage may result. The 
student should acquire, as soon as possible, the habit of keeping both eyes open, when using the microscope.

Examination with the roth inch homogeneous oil immersion objective.-Having studied the bacteria in the hanging-drop with the No. 7 objective, replace the No. 3 objective, and again find the edge of the drop. Now raise the tube of the microscope, by means of the coarse adjustment, and bring the $\frac{1}{1}$ th inch objective into position. Place a drop of cedar oil on the center of the cover-glass, and lower the tube till the objective touches the oil. As the field is now very dark, open the diaphragm slightly. Focus the edge of the drop with the fine adjustment, holding the slide between the fingers of the left hand. Carefully study, in the manner already indicated, the bacteria which are present at the edge, and in the different parts of the drop.

Laboratory work.-The student will make hanging-drops of the several chromogenic bacteria, which have been grown on potatoes, according to the directions given in the following chapter. Too much time cannot be devoted to this method of examination, inasmuch as the practice thus obtained is indispensable to the easy and successful manipulation of the microscope in the subsequent work. A delicate touch, and a quick perception of the smallest detail, can only be acquired by repeated effort.

When the examination of a specimen of living bacteria is completed, the cover-glass should be carefully removed, and placed in boiling water for 10 or 15 minutes, or in mercuric chloride solution (1-1000) for some hours. If, in removing the cover-glass, the suspension touches the slide, the latter should likewise be at once disinfected.

\section{Staining of Bacteria.}

One of the most important conditions when staining bacteria is to have perfectly clean cover-glasses. The 
latter should be so clean that, when a drop of water is placed on any of them, and spread over the surface by means of a platinum wire, it will remain spread out as a thin film. If it gathers in minute droplets, which follow the wire and refuse to spread out, the cover-glass is not clean, and is not suitable in that condition for staining pur. poses. The thin layer of fatty matter must be removed, and this is often impossible when the ordinary procedure is followed. The method of obtaining absolutely clean coverglasses has been described on p. 140 .

Anilin dyes are employed for staining bacteria. It is customary to speak of acid and basic anilin dyes. The acid dyes do not stain bacteria, or but feebly, and are, therefore, useful as contrast colors. Eosin belongs to this group, and its value will be seen in the double staining of pathogenic bacteria. The basic anilin dyes stain the nuclei of cells and bacteria equally well. They are, therefore, invariably used when staining bacteria. The basic anilin dyes, most frequently used, are fuchsin, gentian violet, methyl violet, and methylene blue. Occasionally, vesuvin or Bismarck brown, is employed.

The fuchsin and gentian violet are to be preferred, and used for all ordinary purposes. They stain rapidly and deeply, and the preparations, when properly mounted, will not appreciably alter, even after many years. Methylene blue stains slowly, and is excellent for special purposes. It is not, however, a permanent stain, and such preparations are likely to fade after a short time.

The dyes mentioned above are dissolved to complete saturation, in absolute, or in strong alcohol. These concentrated solutions are never used as such, but serve as a stock, from which the dilute stain which is employed can be readily prepared.

If the alcoholic solution of the stain is diluted too much, the dye will tend to precipitate, and may yield unsightly deposits on the cover-glass. It is advisable, there- 
fore, not to dilute jtill the liquid is transparent. If a cloudiness forms after a few days, the stain should be discarded and a fresh solution prepared. The best results are obtained when the dilute stain contains but little alcohol. If the stock solution is incompletely saturated it will, on dilu. tion, yield a transparent, feeble stain.

The dilute anilin stain is prepared as follows: Some of the saturated, alcoholic solution of the dye is placed in a one-ounce bottle, and then diluted with three or four parts of water. The bottle should be provided with a cork through which passes a glass tube, the lower end of which is slightly drawn out. This then serves as a pipette (Fig. 22, p. 150). A drop of stain, as it leaves the pipette, should be opaque. If this is not the case it is due, either, to the use of an unsaturated stock solution, or, to excessive dilution.

Simple staining.-Place a small drop of water on a cover-glass which is held between the thumb and forefinger of the left hand. The drop of water should not be much larger than a good sized pin-head. By means of the sterile platinum wire, a portion of the growth is transferred to the droplet of water. The latter should now be distinctly cloudy. Sterilize the wire again by heating in the flame, and, when cold, spread out the droplet of "water over the surface of the cover-glass. The water "evaporates rapidly, if the drop is small, and leaves a perfectly even residue distributed over the entire surface. If the drop is large, it will dry slowly and will leave unsightly "shore lines:"

The evaporation of the water may be hastened by waving the cover-glass, to and fro, a few inches above a flame. Care must be taken not to transfer too much material to the drop of water, for in such a case the cover-glass on subsequent staining will be found to be one mass of bacteria.

Instead of making the suspension on the cover-glass as indicated above, the beginner will find it better to proceed as follows: Place one 
or two large drops of water on a slide and touch the wire, laden with the growth, into this water until a cloudy suspension results. Touch a straight sterile wire to this liquid and transfer the droplet that adheres to a cover-glass. The thin film dries almost immediately and is not likely to contain too many bacteria. Any number of preparations can be made from the original suspension.

A sliminess of the liquid, and a tendency of the bacteria to clump, can be overcome by gently heating the suspension over a flame.

As soon as the water has evaporated the material is "fixed" to the cover-glass by means of heat. For this purpose the cover-glass is taken up in the forceps, specimen side up, and is touched, from above downward, once or twice, to a Bunsen, or alcohol flame. This little operation requires judgment and care. The cover-glass must become heated sufficiently to fix the specimen, so that it will not wash off when subsequently treated with the dye and with water. On the other hand, too much heating will destroy the organism and render it incapable of taking up the dye. The best rule to follow is to touch the cover-glass, once or twice, to the flame and then to bring it immediately into contact with the end of the finger. If the cover-glass is so hot that the finger must be withdrawn at once, it indicates that the specimen is fixed and that it is not necessary to heat any more.

The cover-glass, with the specimen side still turned up, is held in the forceps and a drop or two of water is placed on the specimen. Then, two or three drops of the dilute gentian violet, or fuchsin are added, and allowed to act for for one-quarter to one-half minute. The cover-glass is then washed perfectly clean of the dye, by being held under a tap, or by rinsing in one or two glasses of water. It is then touched edgewise to a piece of filter or blotting paper in order to drain off the excess of water, and is then placed on the paper with the specimen side turned up. By gentle rotation of the cover-glass, the lower surface becomes perfectly dry. This can be easily done if the paper is supported on the tip of the index finger. 
The cover-glass is now inverted, with the moist specimen side downward, on a clean glass-slide. Or, the latter may be brought down over the cover-glass till it touches. The specimen should be examined to see if the upper surface of the cover-glass is perfectly dry. Furthermore, just sufficient water should be present between the cover-glass and slide to keep the former in position. The specimen should nẹver be examined in the dry condition. If there is insufficient water, a drop should be placed near the edge of the cover-glass. Too much water must be avoided, since it would float the cover-glass, in which case the examination with the oil immersion objective is quite impossible. Excess of water can be drained off by touching the edge of the cover-glass with a piece of filter-paper. The water under the cover-glass should be colorless, which will be the case if the specimen was properly washed.

The slide, thus prepared, is placed on the stage of the microscope and examined first with the No. 7 objective and subsequently with the th homogeneous oil immersion objective, in the same way as was done when examining hanging-drops.

A good specimen should show the bacteria deeply stained, not in masses, but separated from each other, and evenly distributed over the entire cover-glass. If the stained bacteria are seen to move about, it is due to insufficient fixing in the flame.

Failure to take the stain properly may be due to overheating while fixing the specimen, or to a weak dye, or to a too short exposure to the dye. By repeated trials, the exact conditions necessary can be ascertained, and then followed without any difficulty.

In the method as described above, the dye is not added direct to the cover-glass, but to a drop of water which is first placed upon it. This little deviation from the process, as ordinarily described, serves to prevent over-staining and deposition of coloring matter. 
The color can be forced into the specimen, thus making it stand out more sharply than would otherwise be the case, by holding the glass-slip, covered with the dye, over a low Bunsen, or alcohol flame till vapors begin to rise. The specimen is then washed as before.

Instead of heating the dye on the cover-glass, it may be heated before use. For this purpose the bottle of dye is placed on an iron plate, and this is beated by a low flame. Fig. 22 shows such an iron plate, as used in the author's laboratory, slipped under the flange of the ordinary waterbath. This plate is $3.5 \mathrm{~mm}$. thick, $7.5 \mathrm{~cm}$. wide, and 15 $\mathrm{cm}$. long.

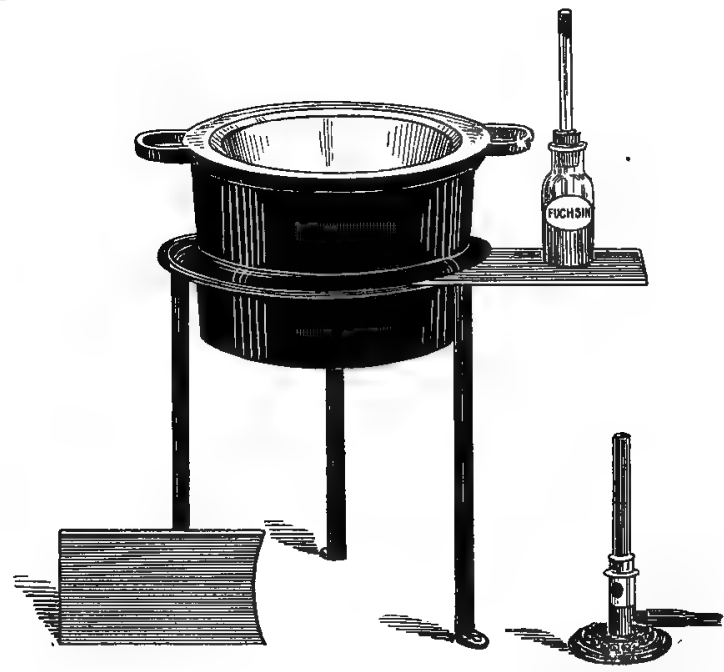

FIG, 22. Water-bath with iron plate for heating dyes, coverglassses, etc.

When it is desirable to preserve a stained specimen, this should be floated off the slide by the addition of a drop, or two, of water to the edge of the cover-glass. If cedar oil has been used it should be carefully removed by rotating the cover-glass on a piece of filter paper. The preparation should then be placed, specimen side turned up, under a watch-glass until perfectly dry. Or, the drying may be 
hastened by waving the cover-glass over a flame. A drop of Canada balsam is then placed on the center of a clean slide and the thoroughly dried cover-glass is inverted and brought down on the balsam, with the specimen side turned down. On gentle pressure, aided by slight heating of the slide, if need be, the balsam will spread out evenly under the cover-glass. If the cover-glass bas not been properly dried, the specimen will be hazy, and may even show droplets of water. In either case, the stain will rapidly fade.

The entire process of simple staining can be briefly summarized as follows:

Clean cover-glass.

Spread specimen.

Dry in air.

Fix in flame.

Add drop of water.

Add dilute dye ( $\frac{1}{4}-\frac{1}{2}$ minute).

Wash in water.

Examine in water.

Dry in air.

Mount in balsam.

Laboratory work. -The student should practice staining cover-glass preparations made from the different potato cultures (p. 170). The sereral dyes mentioned above should be employed. An early mastery of this truly simple process of staining will save much time during the subsequent work. Preparations should also be made from the white matter on the teeth, near the edge of the gums, and these should be examined for micrococci and bacilli, and especially for "comma bacilli," spirals, and leptothrix threads. 


\section{GELATIN AND POTATO MEDIA.-CULTIVATION OF BACTERIA.}

The study of bacteria is not limited to the mere observation of these organisms under the microscope. Indeed, as long as this was of necessity the case bacteriology was far from being an exact science. A drop of blood in anthrax, or a drop of a putrid liquid, may teem with bacteria. If the examination is limited to the use of a microscope, no positive conclusion can be formulated as to the relation of these organisms to the condition in which they occur. An exact knowledge of their function was possible, when methods were discovered for artificially cultivating these organisms.

The first requirement, in order to accomplish this object, is the preparation of suitable, sterile, nutrient media. The soil on which the bacteria in question are to be planted, must be absolutely free from other forms of bacterial life. Otherwise, foreign adventitious organisms may develop and outgrow the particular form under observation. The use of sterile infusions, or nutrient liquid media was introduced. at an early date by Pasteur.

When the material mentioned above is planted in a sterile, liquid medium, it is evident that each of the different kind of bacteria that may be present will multiply. The result is a "mixed culture." With liquid media, it is extremely difficult to separate the several bacteria that may be present in a mixture. The introduction of nutrient gelatin by Koch, in 1881, rendered this a relatively easy task. The remarkable progress in bacteriology during the past two decades, is largely the result of this simple transforma- 
tion of a beef-tea into a transparent, solid, and easily liquefiable medium.

\section{Preparation of Nutrient Gelatin.}

Place $500 \mathrm{~g}$. of chopped, lean beef in an enamelled jar, such as is shown in Fig. 23. This has a capacity of $1 \frac{1}{2}-2$ liters. Now ald 1000 c.c. of tap-water and stir thoroughly. Immerse the jar in a waterbath, and warm gently, till the temperature of the meat suspension reaches $55-60^{\circ} .^{1}$ Maintain this temperature for $\frac{8}{4}-1$ hour, stirring frequently. The soluble constituents are thus brought into solution. Special care should be taken not to allow the temperature to rise above $60^{\circ}$, inasmuch as the albuminous substances would then coagulate. By keeping Frc. 23. Jar for these in solution at this stage, they will subsequently assist in clarifying the final product, and hence, the addition of a white of an egg will be unnecessary.

When the digestion is completed, strain the liquid through muslin and thoroughly squeeze the residue. The filtrate is dark red in appearance and should measure 1000 c.c. 'If this is not the case, add water to make up this volume. To 1000 c.c. of the filtrate returned to the clean jar, now add

$100 \mathrm{~g}$. of gelatin,

10 g. of dry pepton (Witte's),

$5 \mathrm{~g}$. of common salt,

and warm at $60^{\circ}$, in the water-bath, with constant stirring, till the gelatin has completely dissolved. The next step is to render the liquid slightly, but distinctly alkaline.

1 A thermo-regulator may be used to maintain a constant temperature in the water-bath (see Chapter IX, also Fig. 75). 
The neutralization is usually accomplished by the cautious addition of a saturated solution of sodium carbonate. After each addition of 1 to 2 c.c. of the alkali, the liquid is well stirred, and a drop is taken out by means of a glass rod and touched'to a blue litmus paper. If the reaction is acid, this paper will turn red. The addition of alkali is continued until the blue paper retains its color, and the red litmus paper turns slightly blue.

In the hands of the beginner, this procedure not infrequently fails, because of the difficulty in judging the end-reaction. Moreover, even the practised eye cannot establish the same degree of alkalinity in two separate preparations. For these reasons, some workers prefer to titrate the solution with an alkali of known strength, using phenol-phthalein as an indicator. The latter is a most delicate indicator when mere aqueous solutions of acid and alkali are to be tested. In the presence of organic matter, ammonium salts and carbonic acid it ceases to be a sharp indicator. Moreover, the neutral point, as obtained with phenol-phthalein, does not correspond with the neutral point obtained with litmus. Indeed, gelatin neutralized thus, is intensely alkaline to litmus. Consequently, it has been found necessary to deduct 20 or 25 c.c. from the total amount of alkali necessary to neutralize a liter of the medium. The amount thus subtracted is so arbitrary that the resulting reaction cannot be duplicated, except approximately, in another batch of the same or of other media.

The following method determines, with reference to litmus, the neutral point of any medium, whether gelatin, bouillon, or agar to a degree of exactitude that leaves nothing to be desired. The beginner, with no previous knowledge of quantitative analysis, can impart any desired degree of alkalinity (or acidity) to a given medium.

In order to neutralize the gelatin solution, it is necessary to prepare two solutions of sodium hydrate.

1.-One that will contain $40 \mathrm{~g}$. of this base in one liter. This is known as normal $(\mathrm{N})$ sodium hydrate. To prepare this solution so that it will have exactly this strength requires some experience in methods of quantitative analysis. ${ }^{1}$

1 For the preparation of exact normal and deci-normal solutions the student is referred to the author's Laboratory Work in Physiological Chemistry, 2nd ed., pp. 220-239. 
For practical purposes it is sufficient to dissolve $40 \mathrm{~g}$. of sodium hydrate in distilled water, and to dilute this solution to one liter. This will give an approximately normal solution.

2.-One that will contain $4 \mathrm{~g}$. of this base in one liter of water. This is known as a deci-normal ( $(\mathrm{N})$ sodium hydrate solution. It is prepared by taking 100 c.c. of the normal solution, and diluting this with distilled water to one liter. It is evident that this solution has one-tenth the strength of the former. These two solutions are placed in the apparatus shown in Fig. 24. This consists of two burettes, each of 50 c.c. capacity, graduated in is c.c., and connected with bottles or flasks which contain the normal and decinormal solutions, respectively.

FIG. 24. Burettes for titrating nutrient
media. a-Connected with stock of $\mathrm{A}$ NaOH
Titration of the gelatin. - By solution. $b$-Connected with stock of means of a pipette measure

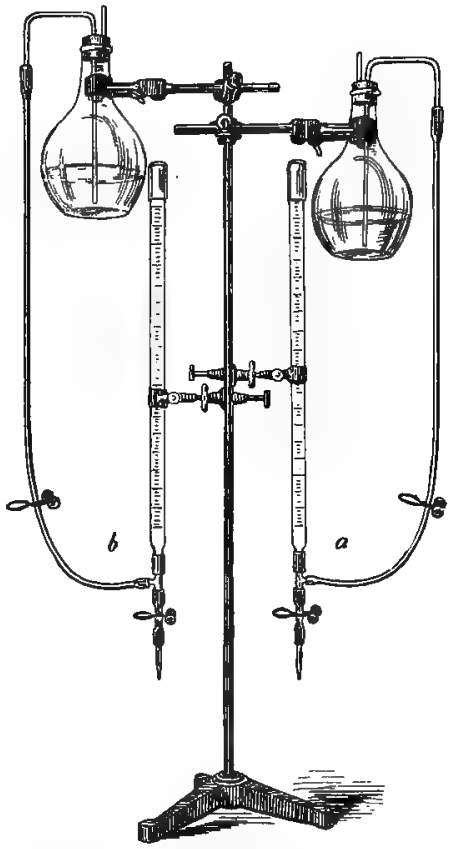
$\mathrm{N} \mathrm{NaOH}$ solution (F. G. N.). out 10 c.c. of the gelatin solution into each of four testtubes, and label these $1,2,3$, and 4 .

To tube 1 add 2.5 c.c. of the $\mathrm{N}$ NaOH solution. To tube 2 add 2.8 c.c. of the $\frac{\pi}{10} \mathrm{NaOH}$ solution. To tube 3 add 3.2 c.c. of the $\mathrm{NaOH}$ solution. To tube 4 add 3.5 c.c. of the $\frac{5}{10} \mathrm{NaOH}$ solution.

Heat each tube in the flame till the liquid boils, is order to expel carbonic acid, and to precipitate phosphat and albumin. Then, drop into each tube a strip of blue 
and one of red litmus paper. These should be immersed in the liquid. Again, heat to boiling and set aside for one minute. By means of a glass rod draw out the papers, side by side, upon the wall of each tube and, when cold, compare the colors of the papers, by holding the tubes before a window, over a white surface. What was originally the blue paper in tube 1, will probably have a slight red color. This shows that 2.5 c.c. of ${ }^{N} \mathrm{NaOH}$ is not suffiuent to neutralize 10 c.c. of the gelatin solution. On the other hand, in tube 4 , both papers are blue, which indicates that 3.5 c.c. of the alkali is more than is necessary to neutralize 10 c.c. of the liquid. The neutral point, that is, where the blue and red paper retain their color side by side, lies therefore between these two extremes. Tube 2 , apparently, shows a slight acid reaction, whereas tube 3 , is faintly alkaline. The neutral point lies, therefore, between 2.8 and 3.2 ; it probably is about 3.0. Hence, measure out into a test-tube 10 c.c. of the gelatin, and add 3.0 c.c. of the $\mathrm{NaOH}$. Then boil and add litmus paper, and examine as above. In this way it is possible to determine the neutral point to within 0.1 or 0.2 of a c.c., and this corresponds to a probable error of 1 or 2 c.c. of $\mathrm{N} \mathrm{NaOH}$ per liter of gelatin.

The above experiment has shown that 10 c.c. of the gelatin requires 3.0 c.c. of $\mathrm{NaOH}$ for neutralization. In order to ascertain the amount of alkali necessary for the neutralization of all of the remaining gelatin, the latter must be measured in a cylindrical graduate. The amount left corresponds, for example, to 950 c.c. The amount of If alkali necessary to neutralize this quantity is ascertained from the following proportion:

$$
10: 3:: 950: x \quad x=285 .
$$

That is to say, in order to neutralize the 950 c.c. of gelatin, it would be necessary to add 285 c.c. of $\mathrm{NaOH}$. Since this corresponds to 28.5 c.c. of $\mathrm{N} \mathrm{NaOH}$, the latter is 
added in preference to the large volume of alkali, which would unnecessarily dilute the liquid.

Bacteria grow best on slightly alkaline media. It is, therefore, desirable to add an amount of alkali, over and above that necessary for mere neutralization. An excess of 10 c.c. of $\mathrm{N} \mathrm{NaOH}$ per liter imparts a desirable alkalinity. Hence, to the 950 c.c. of gelatin there will be added 28.5 c.c. of $\mathrm{NaOH}$ for neutralization, and 9.5 c.c. for alkalinity-a total of 38 c.c. of $\mathrm{N} \mathrm{NaOH}$.

After the addition of the necessary amount of alkali to the remaining gelatin in the jar, the latter is then placed in the water-bath. The water is now raised to boiling, and is maintained at this point for $3 / 4-1$ hour. The albuminous substances, which are present in the meat extract,.coagulate in flakes, and clarify the liquid so that on subsequent filtration it will be perfectly clear.

The liquid is then filtered through a plaited filter. These may be obtained ready made. Schleicher and Schüll's No. 580 is particularly well adapted for filtering gelatin. It is advisable to pass about 200 c.c. of boiling water through the filter, before beginning the filtration. If the first portion of the filtrate is cloudy, it should be returned to the filter. The filtrate should be (1) perfectly clear; (2) should be neutral or slightly alkaline in reaction; (3) should not become cloudy or coagulate when boiled in a test-tube for 1 to 2 minutes; (4) should solidify when cooled.

If the filtrate is cloudy and strongly alkaline, correct the reaction by the addition of a few drops of dilute acetic acid. If it becomes cloudy, or coagulates when warmed, continue heating in the water-bath. A cloudiness can be cleared up by the addition of a white of an egg dissolved in a little water. The commercial, dry egg-albumin is convenient for this purpose. In either case, the albumin solution should not be added to the boiling solution, but this should first be cooled to about $60^{\circ}$. The temperature should 
then be raised slowly to the boiling point, and the liquid stirred constantly. By following the directions given in the preparation of the meat extract it will not be necessary to resort to clarification with egg-albumin. If the gelatin fails to solidify on cooling it is because it has been heated too long. In that case more gelatin should be added (50 g.), the liquid then neutralized and treated as before.

If the filtered gelatin answers the above requirements it is ready to be "tubed", that is, filled into sterile tubes. This should be done by means of a small glass funnel ${ }^{1}$ and the tubes should be filled to a depth of $1 \frac{1}{2}$ inches. The large tubes will then contain about 8 c.c. of gelatin. The utmost care should be taken to avoid touching the neck of the tube with the gelatin, since otherwise the cotton will adhere to the tube. The tubes, filled with gelatin, are placed in the copper sterilizing pail (Fig. 26, p. 164).

The tubes employed are 15 or $16 \mathrm{~mm}$. in diameter and $150 \mathrm{~mm}$. long. Although the tubes may be new and apparently clean, they should be invariably washed with hot water, preferably slightly acidulated, in order to remove any alkali that may remain from the manufacture. They should then be rinsed in cold water, and allowed to drain till dry. The tubes are then plugged with cotton. The simplest way of doing this is to place a piece of cotton, $1 \frac{1}{2}$ to 2 inches square, on the mouth of the tube and then pushing down the middle by means of a narrow glass tube, rod, or matchstick. The best and most solid plugs are obtained, by folding over a piece of cotton into thirds and the rolling up from the end. A firm, cylindrical plug is thus obtained without any frayed out border. The plug should be 1 to $1 \frac{1}{4}$ inches long and should project out of the tube as little as possible. The nutrient medium can be filled into the tubes which have not been sterilized. The subsequent exposure to steam will bring about sterilization of the walls of the of tubes.

${ }^{1}$ The globe funnel shown in Fig. 72 can be used for the rapid filling 
tube, of the plug, and of the contents. It is customary, however, to subject the tubes, before they are filled with culture media, to sterilization by means of dry-heat. It is desirable to do this owing to the presence of highly resistant spores in the cotton, or in the tubes. The tubes are placed erect in baskets made of heavy wire gauze.

\section{Dry-heat Sterilization.}

By sterilization is meant the total destruction of all living forms, in or about the object exposed to the process. Heat is the most effective agent in securing this condition. It may be used as dry-heat or as moist-heat. Each of these has its special advantages. Dry-heat is used only, for the sterilization of glass and metal instruments or articles. Owing to the high temperature employed, $150-170^{\circ}$, culture media and other organic substances cannot be thus treated, since charring would promptly result. For such substances moist, steam-heat is usually employed.

The dry-heat sterilizer, or oven, is made of sheet-iron and is double-walled, thus permitting the circulation of hot air around the inner compartment. The heat is applied by means of a good Bunsen burner. As usually constructed, the heat is directed against the bottom of the inner compartment. In time, this bottom burns out and permits the flame to enter. The apparatus, in that case, cannot be readily repaired. This defect in construction can be readily obviated by attaching, to the bottom, a loose plate, which is held in position by a pin, or nut. This plate, when burned out, can be replaced at a merely nominal expense. The sterilizer can be still further improved by placing the two openings, which are on top, in the rear corners. Through each of these openings is passed a brass tube, freely perforated on all sides (see Fig. 25). These tubes are intended to receive a thermometer and a thermo-regulator, respectively. These instruments are thus protected against accidental injury and do not interfere with the placing in, or the taking out of baskets and other apparatus. Fig. 25 shows a dry-heat sterilizer which, however, is twice the usual length. The thermo-regulator in- 
dicated is of the author's own construction (see Fig. 37). As a rule, a regulator is not necessary when using the dry-heat sterilizer.

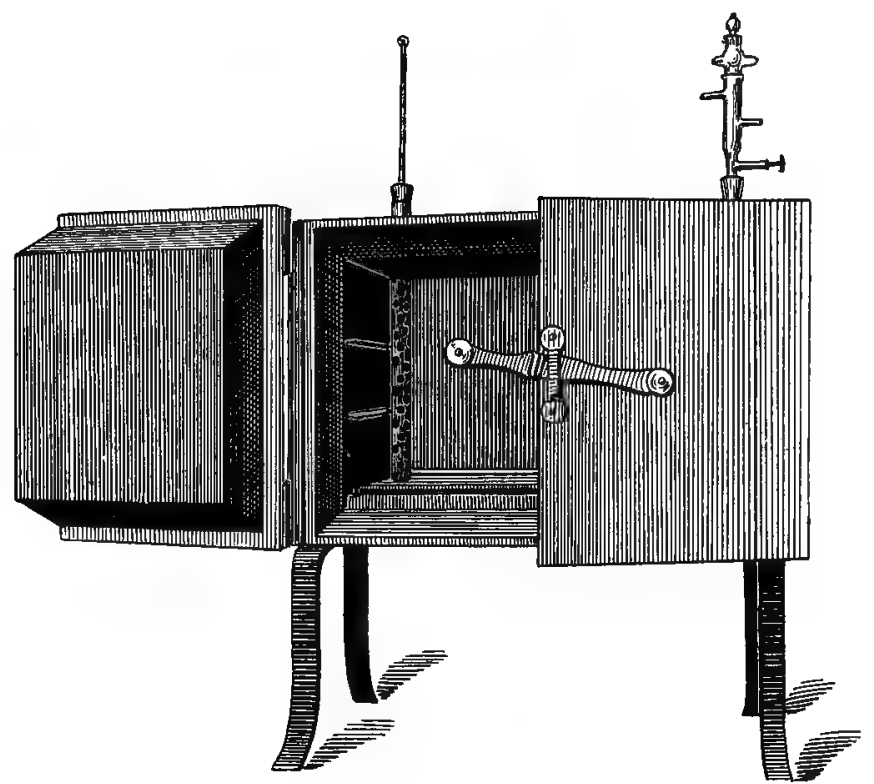

FIG. 25. Dry-heat sterilizer, double width. In the corners, perforated brass tubes to protect the thermometer and the thermoregulator.

The time necessary to effect sterilization in the dryheat oven will, of course, depend upon the temperature. The ordinary laboratory articles are quickly sterilized at $150^{\circ}$. When the thermometer indicates $150^{\circ}$, the time should be noted, and this temperature should then be maintained for $1 / 2$ to $3 / 4$ of an hour. Glass tubes and dishes are, therefore, to be sterilized in the dry-heat oven at a temperature of $150-160^{\circ}$ for $1 / 2-3 / 4$ of an hour. A temperature of $170^{\circ}$, maintained for 15 minutes, will be suffcient to effect sterilization. Another procedure is to allow the temperature to rise till it reaches $195^{\circ}$, when the light is turned out. The door of the oven remains closed until the temperature falls below $100^{\circ}$. The cotton plugs in the 
tubes, which have been subjected to sterilization, should show a light yellow tinge.

\section{Steam Sterilization.}

When the gelatin is filled into tubes, these must be again subjected to sterilization. If this is not done, or done improperly, bacteria will promptly develop in the gelatin and render it worthless. Such instances of "spontaneous generation." are the result of insufficient exposure to steam-heat. As indicated above, cultural media, such as gelatin, are never sterilized in the dry-heat oven. The destruction of all the living forms that may be present, is accomplished by the aid of moist heat.

It will be remembered that bacteria may exist in two stages - the actively vegetating or growing condition, and the resting or spore condition. When in the regetating state, these organisms, as a rule, are readily destroyed by moist heat. A temperature of $65-70^{\circ}$ may do this in 10 or 15 minutes, whereas steam-heat $\left(100^{\circ}\right)$ may be said to destroy the growing cell instantaneously. On the other hand, the spore, or seed, is exceedingly resistant to destruction. Moist heat of $65-70^{\circ}$ has little or no action, and, even steam-heat may require some time. In order to sterilize nutrient media it is customary, therefore, to employ steamheat. They may be sterilized by one prolonged exposure to steam at $100^{\circ}$. Thus, the gelatin tubes might be sterilized by steaming for one hour. This procedure, indeed, is sometimes resorted to when it is desired to employ the medium on the same day on which it is prepared.

Prolonged steaming tends to alter a medium; it may render the latter acid, and, in the case of gelatin, may soften this to such an extent that it will not congeal on subsequent cooling. By taking advantage of the difference in the resistance offered by the vegetating and spore forms, it is possible 
to sterilize without materially altering the physical or chemical character of the medium. An exposure of 15 minutes to. steam will surely cause the destruction of all the vegetating organisms that may be present. The spores, however, survive. If the medium is now set aside at the ordinary room temperature for 24 hours, all or nearly all of the spores will germinate, and thus give rise to the growing, vegetating type. The highly resistant spore has transformed itself into a feebly resistant organism. An exposure, on the second day, to steam for 15 minutes will, therefore, result in the destruction of all these young cells. The material is set aside for another 24 hour period, in order to give any remaining spores an opportunity to germinate. A third exposure to steam for 15 minutes, on the third day, will render the medium sterile.

This method is known as fractional sterilization, and is the one commonly employed in rendering cultural media sterile. The exposures of 15 minutes each, as just described, are sufficient in the case of gelatin. When the medium is in bulk, or if, as in the case of agar, it does not melt readily, it will be necessary to lengthen the exposure to at least 30 minutes on each day. The gelatin tubes are to be exposed to steam for 15 minutes, as soon as the tubes are filled. Then on the second and on the third day, this exposure to steam for a like interval is repeated. Nutrient gelatin is steritized, fractionally, by exposure to steam-heat for 15 minutes on each of three consecutive days.

Sometimes, bacteria develop in tubes which have apparently been subjected to proper sterilization. Spores, if clumped together in a mass, will not all develop at the same time. Moreover, the spores in the interior of such a mass will resist the action of heat for a considerable length of time. Thus, if a growth of the potato bacillus, rich in spores, be gathered into a little mass, the size of a grain of wheat, and placed in a tube of bouillon, it can be exposed to active steam for 8 or 10 hours, or even longer, without destroying all the organisms present. The same material stirred up so as to 
form a very fine suspension, can be sterilized in two to three hours. Delay in the germination of spores, because of low temperature, or because of shelter, may give rise to a contamination of the medium.

In very warm weather, as in mid-summer, failure to obtain perfect sterilization may be due to another cause. The spores that may be present, after the first heating, will germinate but the young cells, owing to the prevailing high temperature, multiply rapidly and eventually form new spores. Consequently, there may be as many spores present after the second heat, as after the first. Under these conditions, the medium can be steamed on each of six or eight days, and yet not become sterile. Sterilization, however, can be accomplished in such a case by steaming at intervals of 12 to 15 hours, or by keeping the material at a low temperature $\left(18-20^{\circ}\right)$.

Failure to secure sterilization may, in exceptional cases, as pointed out by Smith, be due to the presence of spores of anaerobic bacteria. Such spores are unable to germinate in the presence of air, and consequently may survive fractional sterilization. The medium will be apparently sterile, and it is only when aerobic bacteria are planted, that the spores of the anaerobe are enabled to germinate.

Some substances cannot be heated to $100^{\circ}$ without profound change. Blood-serum, for instance, will coagulate when heated above $70^{\circ}$. The principle of fractional sterilization, however, can be applied to serum. Since the temperature cannot exceed even $60^{\circ}$, it must be allowed to act for at least one hour, on each day. This temperature is sufficient to kill most of the vegetating forms. The spore resists perfectly, and it is only after germination that the organism can be destroyed.

The steam sterilizer.-The apparatus usually employed in Germany, is known as the Koch steam sterilizer. It is essentially a large cylinder, the lower part of which contains water. A grating, at about one-fourth the distance from the bottom, serves to support the objects to be sterilized. The water is raised to boiling by means of a large burner, and the steam escapes from the opening in the lid. Owing to the large amount of water to be heated, much time is lost in waiting for the appearance of active steam. If, perchance, the gas pressure is low, it may be very difficult to obtain any steam. In this country, the Arnold steam sterilizer is used extensively. 
In this laboratory the Koch sterilizer has been entirely supplanted by the simple sterilizer shown in Fig. 26. It may be considered as a modification of the former. It consists of the ordinary Hoffmann iron water-bath, which has an internal diameter of $18 \mathrm{~cm}$., and a copper pail $(20 \times 20 \mathrm{~cm}$.) which is provided with a perforated bottom. Two perforated rings, on the inside, allow the passage of steam, and prevent the cotton of the tubes from resting against the side of the pail.

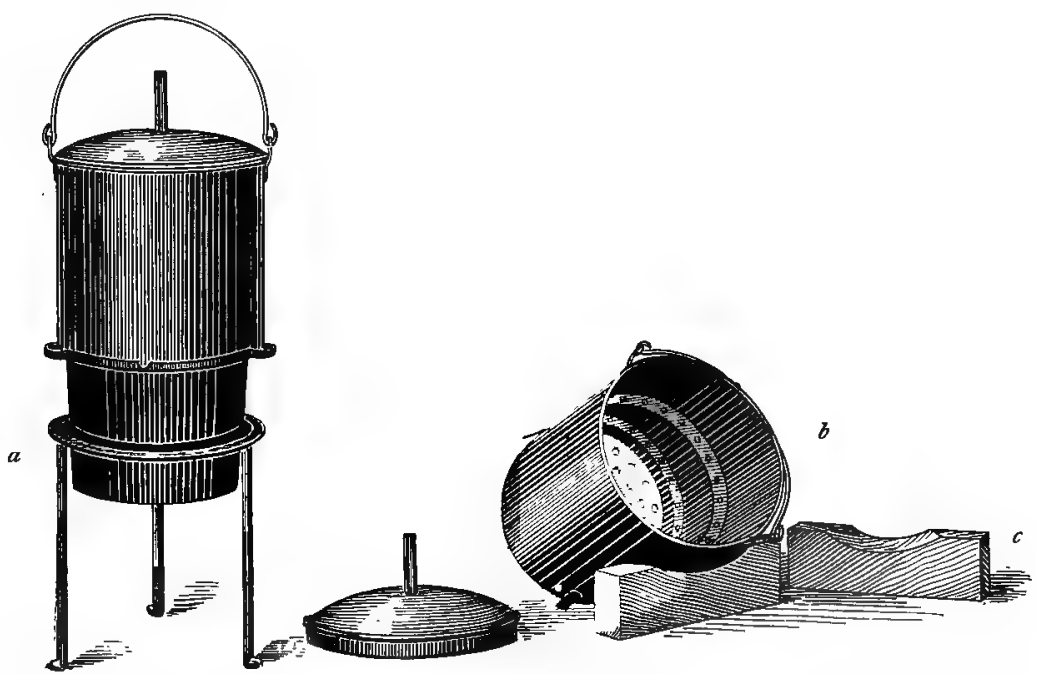

Fig. 26. The author's steam sterilizer. $a$-An ordinary Hofmann's iron water-bath, $18 \mathrm{~cm}$. in diameter; $b$-Copper sterilizer; $a$-Support blocks.

The copper pail is filled with the gelatin tubes, and is placed on the water-bath, in which the water should be actively boiling. In from five to seven minutes steam will issue rapidly, from the tube in the cover, showing that the temperature in the interior has reached $100^{\circ}$. The steaming is then continued for an additional 15 minutes. This process is now repeated, on each of the following two days. By means of this simple sterilizer, the student is enabled to perform all the necessary sterilization at his own table. Practically no time is lost in waiting for the generation of

${ }^{1}$ Centralblatt für Bakteriologie, Bd. 22, p. 340, 1897. 
steam, or for a sterilizer, as is often the case, when the large Koch apparatus is employed.

The autoclave.-As indicated heretofore, the spore, which is the most resistant form encountered, may resist the action of steam even for several hours. Steam escapes from the ordinary Koch sterilizer or its several modifications, at the ordinary atmospheric pressure, and hence, the temperature never exceeds $100^{\circ}$. If, however, the steam is not allowed to escape, but remains confined in an apparatus, the temperature of the steam will rise with the increase in pressure. In this way, steam having a temperature of $110^{\circ}, 120^{\circ}$

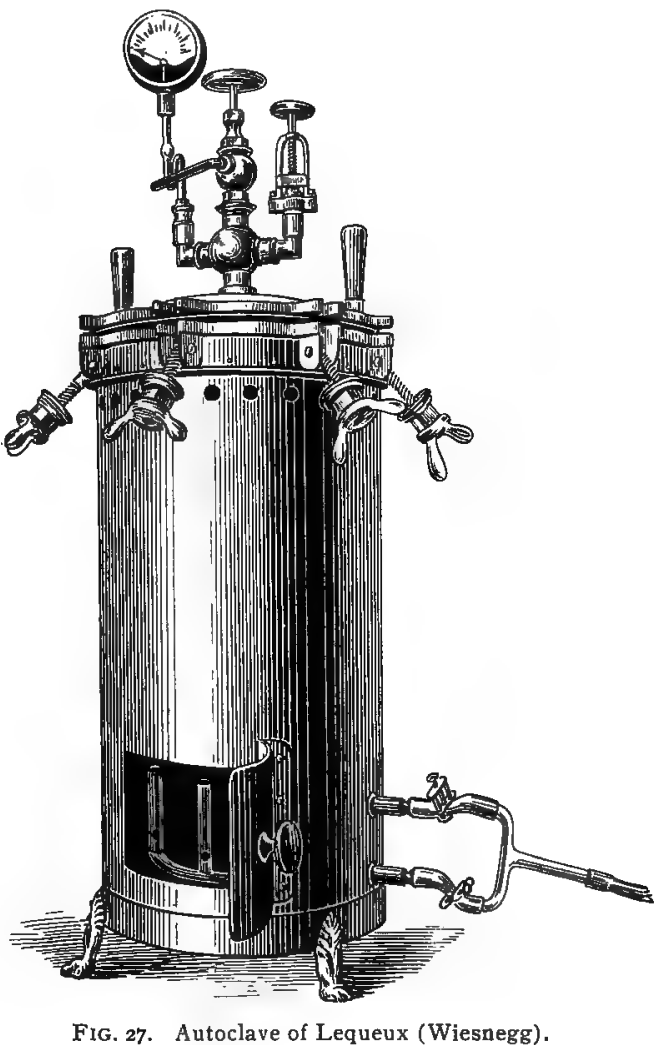
or $130^{\circ}$ can be readily obtained. The higher the temperature of the steam, the more rapidly will the resistant spores be destroyed. Steam at $130^{\circ}$, under pressure, will destroy instantaneously, spores which are able to withstand 3 or. 4 hours' exposure at $100^{\circ}$. The Pasteur school recognized, at an early date, the value of steam under pressure as a means of effecting sterilization and, for that reason, in 
France, the autoclave (Fig. 27), is used almost exclusively. The apparatus, although expensive, and requiring careful, intelligent usage, is by no means difficult to handle. By means of the autoclave it is possible to have a sterile medium in about half an hour after this has been prepared. No well equipped laboratory should be without this apparatus.

A temperature of $120-130^{\circ}$, if allowed to act for 15 minutes or longer, may render the medium acid, or may give rise to decomposition products, which will inhibit the subsequent growth of bacteria. This is especially true of media which contain sugar, as for instance milk. In either case the medium becomes worthless. A temperature of $110^{\circ}$ has very little effect on the media, and, if allowed to act for 15 minutes will sterilize the same.

The following directions should be closely observed when using an autoclave:

1.- See that sufficient water is present.

2.-Place the lid in position and fasten tight, by hand. The wrench should not be used, if possible.

3.-Open the steam-valve, and light all the burners. See that they do not "shoot back."

4.-After the steam has issued violently for about 1 minute close the steam-valve. The steam is allowed to escape in order to expel all the air that is present in the autoclave.

5. -The pressure now rises, and when the gauge indicates $110^{\circ}$ the supply of gas to the inner ring of burners is shut off, and that to the outer ring is turned down as low as possible. A few trials will enable one to turn down these burners so that the temperature of $110^{\circ}$ will continue constant.

6.-Maintain a temperature of $110^{\circ}$ for 15 minutes, then turn off all gas.

7.-When the temperature has fallen to $100^{\circ}$, or less, the steamvalve can be opened, but not before. If the latter is opened when the temperature is above $100^{\circ}$, the sudden release of pressure will cause the liquid in the tubes or flasks to boil over. After the steam-valve has been opened the lid of the autoclave may be loosened and removed, without waiting for the apparatus to completely cool. 
8.-The safety valve should be set to open at about $125^{\circ}$. The noise caused by the escape of steam when this limit is reached will call attention to the apparatus in case it has been overlooked.

The autoclave can be used, by leaving the steam-valve open, for fractional sterilization at $100^{\circ}$.

The student should distinguish between steam under pressure, as employed above in an autoclave, and superheated steam. A current of steam, passing through a tube, may be heated by a lamp to $130^{\circ}$ or higher. Owing to the expansion, since the pressure remains normal, the steam will not have the destructive action of that which has the same temperature, but is under pressure. Indeed, superheated steam may be said to possess but little advantage over a corresponding degree of dry heat.

\section{Preparation of Potato Cultures.}

Select three sound potatoes and clean them thoroughly, under the tap, with the aid of a brush. By means of a knife remove any bad spots, or depressions that may exist, since these frequently harbor bacteria which are highly resistant to destruction. In so doing, avoid cutting off the skin more than is necessary. Place the potatoes, thus prepared, in a solution of mercuric chloride $(1 \text { to } 1,000)^{1}$ for $\frac{1}{2}$ an hour; then transfer to a steam sterilizer and steam for $\frac{3}{4}$ of an hour. The potatoes should be well cooked. Allow the potatoes to remain in the pail till partially cool.

A "moist chamber" (Fig. 28 b) is prepared by placing a round filter-paper on the bottom of the lower dish, and moistening it with mercuric chloride, the excess of which is allowed to drain off. Three potato knives are then sterilized. This is done by heating the blade in the flame till

'A stock solution of mercuric chloride is first prepared by dissolving $200 \mathrm{~g}$. of the salt in $100 \mathrm{c}$.c. of concentrated commercial HCl. 5 c c. of this solution added to 1000 c.c. of water will give a 1:1000 'solution. 
the edge begins to redden. The knives are then set aside to cool, with the edges turned up, on a block or over the edge of the table, care being taken that the blade does not touch anything.

The partially cooled potato is now picked up with the left hand, which previously has been dipped in mercuric chloride, and cut into halves by a horizontal section with a sterilized knife. Each half of the potato is carefully placed in the moist chamber, with the cut surface turned upward.

The cut surface must not come into contact with the fingers, paper or glass, since such contact is very likely to result in the planting of bacteria on the potato. The moist chamber should not be allowed to remain uncovered, even for a minute. The cut potatoes remain in the dish until perfectly cool. If the subsequent transplantation is carried on with a hot knife, or to a hot potato, it may result in the killing of the organism to be planted. The potatoes, while cooling, give off aqueous vapor which condenses on the lid and may, eventually, drop down and thus cause contamination. The condensed water should, therefore, be repeatedly removed from the cover, by means of a piece of filter-paper.

When cold, the potatoes are ready to be inoculated. A small portion, the size of a pin-head, of the bacterial growth is transferred by means of a sterilized and cooled wire, or knife to one of the above pieces of a potato, and then thoroughly spread over the surface. Care shoutd be taken to avoid the outer inch. The potato is held in the fingers of the left hand, which has been dipped in mercuric chloride. The number of bacteria which is thus transferred to the surface of the potato is usually so great that when they develop the entire surface is covered with a continuous growth. Such a growth is spoken of as a mass culture.

The object in planting the bacteria on the potato is not so much to obtain a mass growth, as it is to spread a minute portion over as large an area as possible. When, 
therefore, as the next step, a pin-head portion is removed by means of a sterile knife from the surface of this first potato. (No. 1) it will contain a very small fraction of one per cent. of the total number of bacteria spread over the surface of that potato. This relatively small number of bacteria is now spread, or distributed, as thoroughly as possible, over the surface of a second potato. In doing this the same precautions are taken as when potato No. 1 was inoculated. When the bacteria that are thus planted on the second potato (No. 2) develop they may still be so numerous as to give rise to a mass culture. Should only a few bacteria be present they will be scattered over the surface, separated" by an appreciable distance, and, when they develop, they will give rise to isolated growths or colonies. Inasmuch as the result with the second potato is uncertain it is customary to take, by means of a sterile knife, a small portion of material from the surface of potato No. 2, and, to spread this over that of a third potato (No. 3). The latter will probably have planted on its surface some 10 or 20 bacteria. These may be $\frac{1}{4}$ to $\frac{1}{2}$ an inch apart. The bacterial cell, wherever deposited, grows and multiplies, and eventually forms a visible, pin-head growth. This isolated growth is. known as a colony, and, inasmuch as it is derived from a single cell, it is a pure culture of that organism.

By resorting to dilution cultures, in the manner indicated, it is possible to isolate the various bacteria that may be present in the original material. To illustrate the dilution that takes place it may be assumed that $10,000,000$ cells. were planted on potato No. 1. The small portion taken from this may have planted 10,000 bacteria on potato No. 2 . Similarly, the small amount taken from the second and planted on the surface of the third potato may not contain more than 10 cells. In 36 to 48 hours, if the temperature is. favorable, these cells will have multiplied to such an extent as to give rise to visible growths or colonies.

The simple method of dilution, as just described, is the. 
basis of all bacteriological work. The potato was the first medium employed for isolating bacteria. In time, other media were introduced, but the fundamental principle, that of physical dilution, is the same, regardless of the medium employed.

In this, and all subsequent work, successful results and freedom from personal danger depend upon the rigid sterilization of all articles used. Attention to the smallest detail is necessary in order to prevent contaminations. The training, thus acquired, will be invaluable, in time, in the intelligent prevention of disease. The following rule cannot, therefore, be emphasized too strongly: Steritize all instruments, wires, etc., immediately before use and immediately after use. Instruments are sterilized immediately before use in order to avoid contaminations; and immediately after use, in order to prevent personal danger. This rule should be rigidly adhered to. Careless manipulations, acquired while engaged in the study of non-pathogenic bacteria, may be unconsciously resorted to when studying disease-producing organisms. A careless student is a source of danger, not only to himself but also to his neighbors. The sterilization of all instruments should be attended to, before they are placed back on the table.

Certain additional personal precautions should be observed, while engaged at work in the laboratory. Pencils, pens, glass rods and labels should never be introduced into the mouth. The tumblers, or glasses in the desk should not be used for drinking purposes. If any material drops on the table or floor, it should at once be rendered harmless by covering it with the mercuric chloride solution. Invariably, at the close of the day's work, the table should be washed with this solution and, at the same time, the hands should be disinfected.

Laboratory work.-Make a dilution culture, as described (on. three potatoes), of the Bacillus prodigiosus. 
Make a mass culture (single potato) of each of the following: Orange sarcine, Red bacillus of water, Violet bacillus of water.

The inoculated potatoes are kept in the moist chamber, which should be set aside where the sun will not strike. When moisture accumulates on the under side of the lid, it should be removed at once, in the manner indicated. On the second day, the cultures are examined in hanging-drops, and on the day following they are used for staining purposes.

For modifications of the above method of making cultures on potato see p. 182.

\section{Gelatin Plate Culture.}

It has been mentioned that cultivation on potatoes was resorted to at a very early date. The far-reaching studies of Pasteur led to the introduction of various liquid media, such as beef, veal, or chicken broth. Nutrient solutions, containing various mineral salts, were likewise employed. In order to isolate bacteria, which might be present in a mixture, it was necessary to resort to excessive dilution, and, even then, it was far from certain that a pure culture was really obtained. One of the greatest aids to the advancement of bacteriology was supplied by Koch, when he introduced the use of gelatin. By the addition of gelatin to beef tea, a transparent medium was obtained which was solid at ordinary temperature, and which could be. liquefied without any difficulty. This transformation of a liquid medium into a solid one rendered it possible to isolate bacteria in a condition of absolute purity. The uncertainty regarding the action of bacteria, whether they themselves produced certain changes, or whether it was due to something else that accompanied them, was thus easily set aside.

The object of the gelatin plate method, as with the dilution potato culture already made, is to isolate the several kinds of bacteria that may be present in a mixture. The isolated organisms developing in a solid, transparent medium, form colonies which are easily perceived, and 
from which transplantations can be readily made. Colonies of bacteria which would escape detection when growing on the surface of a potato, because they are invisible, are easily seen in the solid, transparent layer of gelatin. Pure cultures, of the different kinds of bacteria, are thus obtained.

Method.-First, sterilize six glass plates $(13 \times 10 \mathrm{~cm}$. $)$ by placing them in an iron box, and heating this in the dryheat sterilizer, at a temperature of $150-175^{\circ}$, for $1 / 2-3 / 4$ of an hour. A temperature of $170^{\circ}$ maintained for $15 \mathrm{~min}$ utes, will be sufficient (see p. 160). Remove the box, and let it cool in the room at the ordinary temperature.

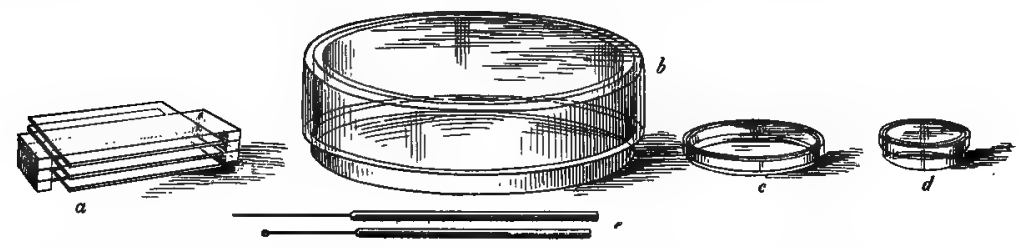

FIG. 28. Apparatus used in cultivation. $a$-Glass benches and plates; $b$-Moist chamber for plates; $c$-Petri dish; $d$-Esmarch dish; $e$-Platinum wires, straight and with loop, fused into the ends of glass rods.

In the meantime, prepare the necessary platinum wires. These should be about $5 \mathrm{~cm}$. ( 2 in.) in length, and in thickness should correspond to about gauge number $22(0.5 \mathrm{~mm}$.). The end of a glass rod, about $18 \mathrm{~cm}$. (7 in.) long and $6 \mathrm{~mm}$. (1/4 in.) wide, is heated in a Bunsen flame, or better still, in a blast lump, till it has become quite soft. The platinum wire is then pushed into the soft end. If a depression occurs around the wire, where it enters the glass, this can be easily remedied by gently pulling on the wire, while the glass is still soft. A glass tube should never be used for mounting platinum wires.

In order to prevent sudden, uneven cooling, it is well to partially anneal the glass, by allowing a small luminous flame to deposit a layer of lamp-black on the hot end. This is then wiped off when the glass is perfectly cool. It is well to have on hand three mounted, platinum wires. The wire should be kept perfectly straight, not crooked. When plating or doing similar work, the end is bent into a small loop which has a clear diameter of about $2 \mathrm{~mm}$. (Fig. $28 \mathrm{e}$ ). 
The platinum wires are sterilized by holding them in the flame, till incandescent. The end of the glass rod should always be thoroughly heated, and, if any organic matter is present, such as gelatin, it should be burned off. The wires should be kept in a conical test-glass (Fig. 43), in the bottom of which is some cotton.

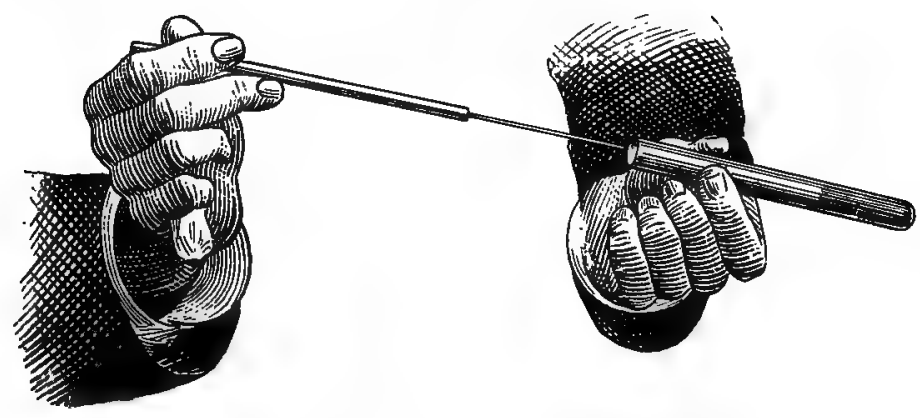

F1G. 29. The inoculation of a single tube.

Place three of the sterilized, gelatin tubes in a waterbath which has been warmed to about $30-35^{\circ}$. When the gelatin melts, the tubes are ready for inoculation. With a sterilized, cooled platinum wire, pick up a minute amount, of the growth on potato, of the Bacillus prodigiosus. Place one of the liquefied gelatin tubes between the thumb and index finger of the left hand, so that it is almost horizontal. It is held in this position in order to prevent foreign matter from dropping into the tube. The neck of the tube with its plug, as well as the palm of the left hand, is turned to the right. In this position the entire length of the tube is before one's eyes (Fig. 29). While still holding the platinum wire in the right hand, grasp the cotton plug with the little finger of that hand, and, remove it by slight rotation. If any tufts of cotton are adherent to the neck of the tube, touch it for a moment to a Bunsen flame. Otherwise, the platinum wire might carry in some of this material, and thus cause unnecessary contamination. As a matter of routine, it is well to always flame the neck of a tube before insert- 
ing the wire. Now pass the inoculated wire into the tube, and thoroughly mix the bacteria thus introduced, with the gelatin. Then withdraw the wire, replace the cotton plug, and sterilize the platinum wire in a flame.

With a colored wax pencil, mark the tube thus inoculated, 1. Likewise, mark another liquefied gelatin tube, 2. Place tube 1 in the left hand, in the same position as before, and then place next to it tube 2 (Fig. 30). Remove the cotton plug of tube 2 , and place it between the adjoining index and middle fingers. Then remove the cotton plug of tube 1, and place it between the ring and little finger. Touch the flame of a burner to the necks of the tubes, in order to burn off any loose cotton. Now, with a sterilized, cooled platinum wire, the end of which is provided with a small loop, transfer a loopful of gelatin from tube 1 to tube 2, and mix well. Return the same platinum wire to tube 1 , and again transfer a loopful of gelatin to tube 2. Repeat this once more, so that all told, three transfers of inoculated gelatin have been made. Replace the cotton plugs in their respective tubes, sterilize the platinum wire and set the tubes in a tumbler, the bottom of which is covered with a layer of cotton.

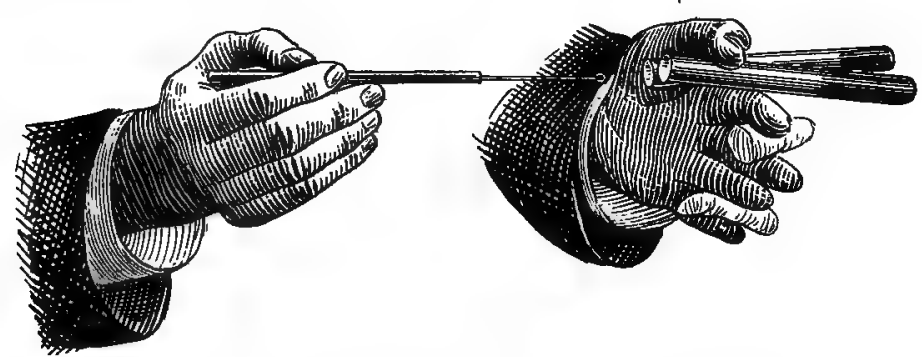

FiG. 30. Inoculation from tube to tube when diluting.

Mark another liquefied gelatin tube, 3. Then place 2 in the position in which No. 1 was just held, and next to it place tube 3. Remove the cotton plugs, place in their respective positions, and flame the necks of the tubes as 
before. With a sterilized, cooled platinum wire, make three successive transfers of gelatin from tube 2 to tube 3. Return the cotton plugs to their tubes, sterilize the wire, and set the tubes aside in the tumbler.

Each of the three gelatin tubes has now, been inoculated. Tube 1 usually has a very large number of bacteria, while tube 2 has less and tube 3 should have but a small number, so that, subsequently, when colonies develop these should be separated from one another by an appreciable distance. This successive dilution, it will be observed, corresponds to that performed when making potato cultures. It is necessary to take a very minute amount of material for the inoculation of tube 1 in order to to obtain good dilutions. Moreover, working as the class does with pure cultures, it will be sufficient to make transfers of only one or two loopsful of gelatin, instead of three.

In transferring gelatin from one tube to another, care must be taken to prevent the wire from coming into contact with the neck, or wall of the tube. Unnecessary contact of the end of the glass rod with gelatin should be avoided, and, if gelatin does cover the end it should be burned off completely.

The ice-plating apparatus should now be prepared for use. Broken ice is placed in the glass dish, which is then filled with water, so that when the plate is placed in position it rests directly on the ice water. No air-space should be present. The apparatus should be levelled and is then ready for use (Fig. 31). Instead of the ordinary plating apparatus, one similar to that shown in Fig. 32 can be employed. It is made of galvanized iron or of copper, and is $15 \mathrm{~cm}$. $(6$ in.) high. The inflow tube $(1 \mathrm{~cm}$.) extends along the bottom to the farther end of the box. The wide outflow tube $(2 \mathrm{~cm}$.) does not extend inward. Tap-water is allowed to flow into the apparatus. It runs the entire length of the box and thus reduces the temperature of the surface plate to that of the water (about $15^{\circ}$ ). The box is $68.5 \mathrm{~cm}$. (27 in.) long, $28 \mathrm{~cm}$. (11 in.) wide, and $7.5 \mathrm{~cm}$. (3 in.) deep.

Remove a sterilized glass plate from the iron box, by grasping the edges with two fingers; place it upon the 
ground plate of the ice apparatus, and cover with the belljar. As soon as the plate is cool, it is ready to receive the gelatin. Before pouring the contents of a tube upon a plate; it is necessary, as a matter of precaution, to sterilize the neck of the tube. This is easily accomplished, in the following manner: Remove the cotton plug from the tube (No. 1), and rapidly rotate the neck of the tube in the flame. This is thus sterilized, and the contents of the tube can now be poured, without coming into contact with foreign organisms. In about a minute, the neck of the tube is sufficiently cool to proceed. Raise the bell-jar somewhat, shielding the plate as much as possible from draughts of air, and pour the gelatin on the center of the plate. With the lip of the tube, rapidly spread the gelatin over as much of the surface as possible, avoiding, however, the edges of the plate. The plate is now allowed to remain

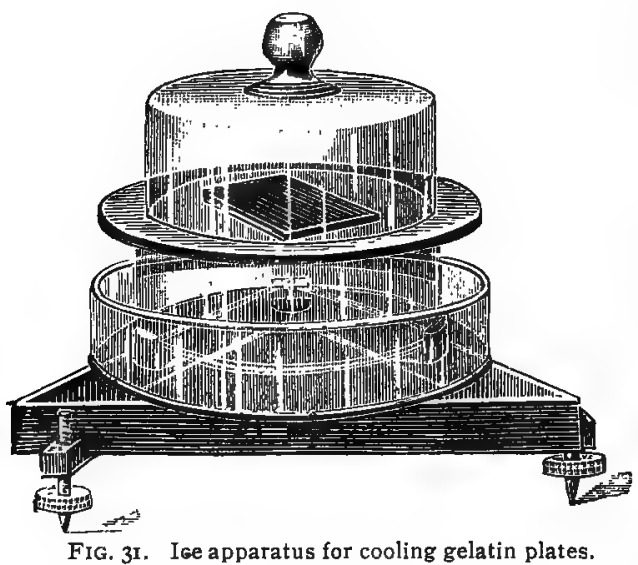

exposure to boiling water or to steam.

While the gelatin on the plate is becoming solid, a "moist chamber" is prepared in the same way as for potato cultures (p. 167). It is not necessary to sterilize, either the moist chamber, or the glass benches on which the plates are to be placed. Instead of mercuric chloride, tap-water till the gelatin becomes solid. The empty gelatin tube should not be placed on the table but should be set in a tumbler. When through plating, all the empty tubes should be sterilized, either by immersion in mercuric chloride, or better by

under the bell-jar 
may be used to moisten the paper which covers the bottom of the dish. On three small pieces of paper, write the name of the germ or material, the number of the plate, and the date. This label may be written on the glass bench (Fig. 28 a). Now, place a glass bench on the bottom of the moist chamber, and, on it the label for plate 1. Transfer the gelatin plate from the ice apparatus to the bench. Pour the contents of the remaining gelatin tubes on plates, in the same manner as described; and, when cool, transfer to the benches which are arranged one above the other, in the moist chamber. In doing this, the utmost. care should be taken to avoid touching the gelatin, either with the fingers or with a glass bench. Each chamber can, or should hold a stack of six plates.

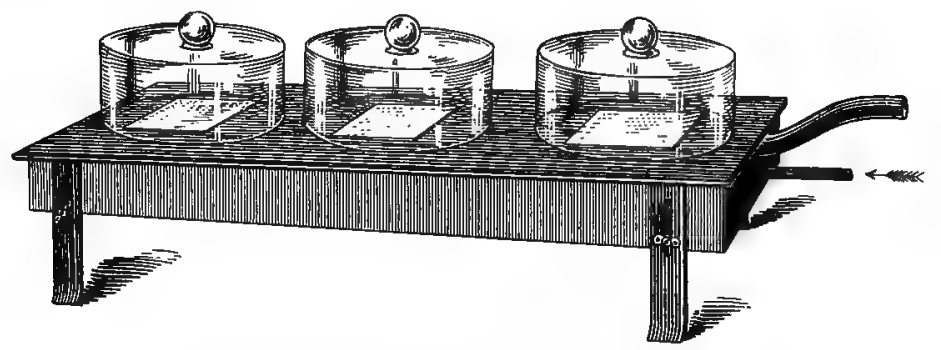

FIG. 32. Water apparatus for cooling plates (F. G. N.).

The moist chamber is now set aside. It should not be placed near the steam coil, or where the sun-light may possibly strike it. The rapidity with which colonies develop in the gelatin will depend upon the temperature of the room, and, to a certain extent, upon the organism itself. The best temperature for the development of a growth on gelatin plates is about $18-20^{\circ}$. Under this condition, excellent colonies will form, as a rule, in from 36 to 48 hours. The lower the temperature the slower will be the development. If the temperature exceeds $20^{\circ}$ the growth will be more rapid, but, in that case, there is always danger of melting the gelatin and thus spoiling the outcome. The ordinary, 10 per cent. 
gelatin melts at about $25^{\circ}$. In warm weather, as in summer, gelatin plates cannot be developed at the prevailing room temperature. In order to keep the gelatin solid, it is customary in that case to set the plates in an ice-chest. Inasmuch as the temperature in the ice-chest is about $10^{\circ}$, the growth of the colonies will be materially retarded, and this at times may lead to annoying delays.

The nutrient gelatin, as commonly prepared, contains 10 per cent. of gelatin. Unless otherwise specified, this is assumed to be the composition of the medium when "gelatin" is mentioned. Occasionally a nutrient medium containing 15 per cent. of gelatin is employed. This melts at a. higher temperature than does the ordinary gelatin, and hence, is specially made use of during warm weather. It should be borne in mind, however, that the cultural characteristics of an organism may vary considerably with the hardness of the medium in which it is grown. Atypical cultures will not infrequently be obtained when an organism is grown on hard gelatin.

The apparatus shown in Fig. 33 is employed in this laboratory, to cultivate bacteria at a constant low temperature, regardless of that which prevails in the room. It will be found as useful for this purpose, as the incubator is for higher temperatures. It has the merit of being simple and inexpensive. The apparatus is made of galvanized iron or of copper. The outer box is $22 \mathrm{~cm}$. high, $68 \mathrm{~cm}$. long and $35 \mathrm{~cm}$. wide, while the inner compartment is $18 \times 53 \times 27 \mathrm{~cm}$. The narrow tube $(1.2 \mathrm{~cm}$. diameter) to the left stops short, on the inside of the box. It is connected directly with the water-supply pipe, and not by means of a narrow faucet. Next to this inflow is the wide outflow tube $(2 \mathrm{~cm}$. diameter), which passes along the bottom of the box to the opposite end, where it is turned upward. A short rubber tube is slipped over the end of the upright tube, and, by moving it up or down, the height of the water can be regulated at will. The bent wide tube on the side is a safety over-flow. The inner box is held in place and kept from floating, by means of two stout rods. The water flows into the large box, under and around the inner compartment, and then leaves by the outflow and overflow tubes.

The temperature in the inner compartment will depend upon the rate of flow of the water, and on the temperature of the water. With 
a maximum flow the temperature in the inner compartment will be the minimum attainable. In this laboratory the temperature of the water during summer, as it comes from the ground, is $14-15^{\circ}$. This represents, then the lowest constant temperature attainable. By diminishing the flow of the water, heat is absorbed, and a higher temperature can be obtained. The inner compartment can thus be maintained at $18^{\circ}, 20^{\circ}$ or $22^{\circ}$. In the apparatus, as actually used, the flow is such that a temperature of $18-20^{\circ}$ is maintained throughout the summer months. It is well to keep inside of the apparatus a. Kappeler maximum and minimum thermometer.

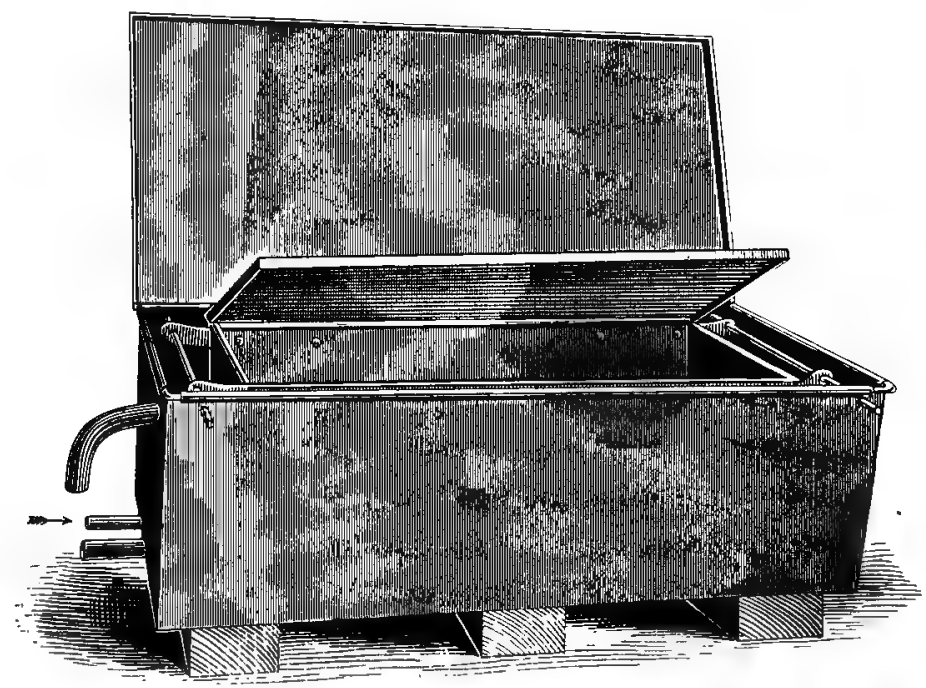

FIG. 33. The author's constant, low temperature apparatus.

Laboratory work.-The student will make plate cultures of the Bacillus prodigiosus and of Bacillus Indicus. The potato cultures made heretofore will supply the material for inoculation.

\section{Modified Gelatin Plate Cultures.}

The original plate method of Koch, as described on p. 172, has been subjected to several modifications, some of which may be considered as decided improvements. The iron box, plates, benches, moist-chambers and levelling ap- 
paratus, are more or less expensive, and are too cumbersome to be transported from place to place, in the case of out-door investigations. Even in the laboratory, the latter apparatus requires time to prepare and to level it, and, not infrequently, ice may be lacking. Furthermore, the moistchamber when placed in an incubator, or in a cooler, takes up an unnecessary large amount of space. In the plate method as given, more or less contamination may result from exposure to the air, while the plates are on the ice apparatus, or subsequently, when they are kept in the large moist chamber. Whenever it is desired to examine the growth on the plates, it is necessary to expose these freely to the air. Even if but one plate is to be examined, it necessitates the exposure of all to contamination from the air. Furthermore, it may happen that the gelatin on an upper plate liquefies, either by heat or by the growing bacteria, and then drips over the edge of the plate on those which are below it. To overcome these difficulties, Petri introduced the use of the shallow dishes (Fig. $28 \mathrm{c}$ ) which bear his name. The cover to the dish should be $10 \mathrm{~cm}$. in diameter, and the bottom dish should be as flat as possible, and should not be more than $1 \mathrm{~cm}$. deep. A greater depth interferes in the subsequent examination with the microscope.

Petri dish culture. - The Petri dishes (Fig. 28 c) are placed in a wire basket, and sterilized in the dry-heat oven (Fig. 25), and then allowed to cool. If the dishes are, to be kept for some time before use, or are to be transported, they should be wrapped in paper, and then sterilized. Three gelatin tubes are inoculated with the organism to be plated, the dilutions being made in exactly the same manner as described in connection with the ordinary plate method (p. 174). The contents of each tube are then poured into one of the cooled, sterile Petri dishes. The precaution of flaming the neck of the tube (p. 176) before pouring out the gelatin must, of course, be observed. 
When pouring the gelatin, the cover of the Petri dish should not be raised any more than is absolutely necessary. The cover is then replaced, and the dish is gently tilted, from side to side, so as to cause the gelatin to spread over the entire bottom. The dishes are then placed on a flat surface, and, when the gelatin has solidified, they are set aside in order to allow the colonies to develop.

In order to make a preliminary examination of the colonies, the dish is inverted on the stage of the microscope. The colonies can thus be studied almost as well as if the top was removed. In order to make transplantations from the colonies, it will be necessary, of course, to remove the top, and to place the bottom upright on the stage of the microscope.

In this method, each dish constitutes a plate and a moist chamber. The progress in development can be observed without exposing the gelatin to the air, and without any risk to the other plates. Consequently, the chance of contamination is reduced to a minimum. There is little, or no danger of the gelatin dripping on the floor, table, or stage of the microscope. Moreover, the use of the ice apparatus, of plates, benches, boxes, etc., is done away with. This method of obtaining colonies is to be used, whenever possible, in preference to the original Koch method.

Esmarch roll-tube method.-In this the advantages of the plate method are secured without the use of any extra apparatus, such as plates or dishes. The inoculated gelatin, instead of being poured out on sterilized plates, or into dishes, is solidified in a thin film on the inside wall of the test-tube. Another advantage of this method is, that it is well adapted for those organisms which grow very slowly, and require a week or two to form distinct colonies. Desiccation of the gelatin can be readily prevented in the rolltube, whereas this is more difficult to do in plate, or in dish cultures. 
Three gelatin tubes are inoculated in the usual manner (p. 174) with the material to be plated. It is advisable to select very wide tubes. The cotton projecting from the tube is then cut off, and the neck of the tube is rotated rapidly in a flame in order to insure sterilization of the outer layer of cotton, since this may subsequently, come into contact with the gelatin. The cotton plug is then pushed in a trifle, and the tube is closed by means of a rubber cap. A boiled cork or rubber stopper may be used for this purpose. It is then immersed in cold, or better, in icewater, and is rotated slowly in an almost horizontal position. The gelatin should solidify in a thin, even layer, over the inner wall of the tube. During the process of rolling, care should be taken to prevent the gelatin from coming into contact with the cotton plug. It will-not only cause the cotton to adhere to the glass, but may prevent access of air, and thus bring about partial anaerobic conditions within the tube.

A better method of rolling the tube is to do this on a block of ice. A very slightly inclined groove can be made in the ice by means of a test-tube filled with hot water. In this case it is unnecessary to heat the cotton, or to use a rubber cap or a stopper. The roll-tubes should be set aside in a cool place $\left(15^{\circ}\right)$, to prevent collapse of the gelatin. The colonies are then examined under the microscope and transplantations can be readily made from these, especially if the tube is wide. For transplanting, a platinum wire should be used, the end of which is bent at right angles.

Laboratory work. - The student will make Esmarch roll tubes of Yellow sarcine, and Petri dish cultivations of the Orange sarcine.

\section{Modified Potato Cultures.}

The method of making potato cultures, as described on p. 167 , is open to many of the same objections which were made in connection with the ordinary gelatin plate method. 
It has, consequently, given place to two modifications which give excellent results. One or the other of these should, therefore, be employed whenever it is desired to obtain strictly pure cultures on potatoes.

Esmarch potato cultures.-The cover of the Esmarch dish (Fig. $28 d$ ), is about $5 \mathrm{~cm}$. in diameter, and the bottom is 2 cm. in height. These dishes, like those of Petri, are sterilized in the dry-heat oven (Fig. 25, p. 160), and then allowed to cool.

A small, sound potato is selected, and held with the thumb and forefinger of the left hand. The outer edge of the potato is pared circularly, by means of a clean potatoknife which is held upright. Two horizontal sections, above and below, are now made. The clean, sound core of the potato, which is thus obtained, is slipped into a sterilized Esmarch dish. Each dish is thus supplied with a clean potato section. The dishes are then placed in a steam sterilizer and steamed for $\frac{3}{4}$ to 1 hour. The potatoes will then be sterilized and cooked. It is not necessary to heat the potatoes on three successive days. The potatoes will become more or less dark, unless they are heated immediately after they are cut.

Laboratory work.--The student will prepare three of the Esmarch potato dishes, and then make dilution cultures of the Bacillus prodigiosus. As little of the material as possible should be transferred, each time, and it should be spread over the entire surface. The method of inoculation is the same as that described on p. 168, except that the potatoes are not taken out of the dishes. When dilution cultures are not desired, a single mass culture may be made, or several parallel streaks may be made by means of a platinum wire. In the latter case, the organism will develop along the streaks thus made. Inasmuch as each potato is in a small sterilized dish by itself, the risk of contamination, with careful and rapid manipulation, is very small.

Test-tube cuitures on potato.-In this method, the special dish of Esmarch is done away with and its place is taken 
by a test-tube. The method was introduced about the same time by Bolton in this country, Globig in Germany, and Roux in France. It is the method which is most often employed when studying the cultural characteristics on

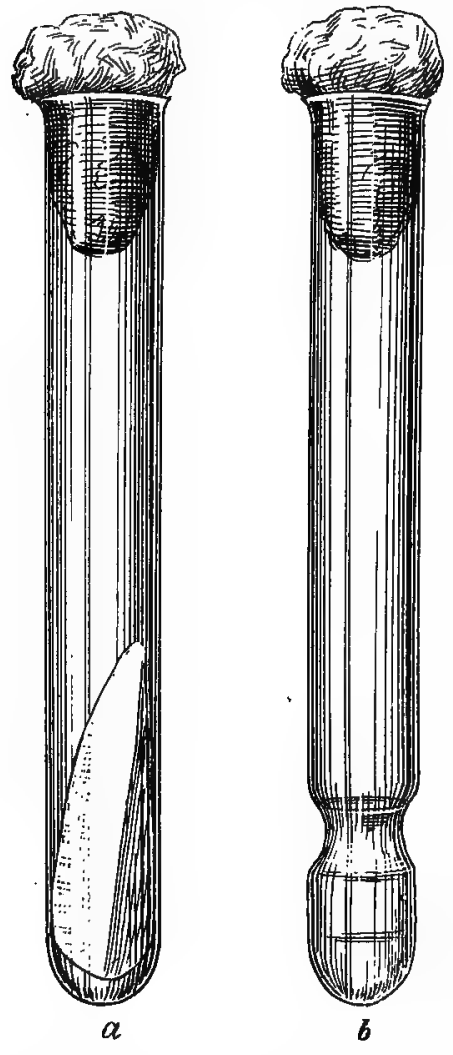

FIG. 34. Tubes for cultivation on potato. $a$-Ordinary form; $b$-Roux form. potato, of an organism. This is because it is convenient and simple in execution, and, like all tube cultivations, almost entirely free from danger of contamination.

The large test-tubes $(15 \times 150$ $\mathrm{mm}$.) should be employed. These are cleaned, plugged, and sterilized in the usual way. A corkborer should then be selected which will easily pass into these tubes. In the absence of a corkborer, the student can easily improvise one out of a narrow testtube. For this purpose a sharp file-mark is made, about threefourths of an inch from the end. The end of a glass rod, which has been heated in the flame of a burner, or blast-lamp, till redhot, is then touched to the edge of the file-mark. The glass cracks, and leaves a clean cut end. A piece of ignited charcoal is better than the glass-rod since, by gently blowing, it can be kept red-hot, while being held against the glass.

Several large potatoes are brushed, under the tap, and then steamed, or immersed in boiling water for 20 minutes. A number of cylinders are then punched out of the cooled potatoes. These cylinders (4-5 cm. long) should be placed 
on a clean piece of paper; the ends should then be trimmed off at right angles, and finally the cylinders should be cut diagonally in two. The potato wedges, thus obtained, are now slipped, with the wide end downward, into sterilized tubes (Fig. 34a). The cut surface of the potato is inclined, and hence can be readily inoculated by means of a platinum wire. The potato tubes, thus prepared, can be submitted to fractional sterilization in steam, being heated for about 20 minutes each day on three successive days. Or, they may be given a single steam sterilization of $3 / 4-1$ hour.

The method, as given above, will yield potato cylinders which are pure white, and will remain so. If a raw potato is punched or cut, the cut-surface, as a result of oxidation, will soon take on a light pink tinge and, on subsequent heating, this turns to a dirty gray. This alteration in the appearance can be avoided by at once submitting the cut potato to the action of steam. If the potato has been cooked before hand, it will not oxidize on subsequent exposure.

Instead of the ordinary test-tube, Roux employed one which was provided with a slight constriction near the end (Fig. 34 b). The wedge of potato, in this case, does not rest on the bottom of the tube, but on the constricted portion. The space below this may be filled with water, or with any desired liquid. The surface of the potato can, therefore, be moistened, whenever it is desirable to do so. When a five per cent. solution of glycerin is employed, the potato becomes an excellent soil for the growth of the tubercle bacillus.

The Roux potato tube is consequently very useful. It can be prepared by the student without the least difficulty. The clean, sterile, wide test-tubes should be used. A narrow fiame from the blast-lamp should be directed horizontally, against the test-tube, at about one inch from the bottom. The tube should be held vertically, and rotated slowly, so that the point of the flame just strikes it. The glass gradually softens and the constriction results. 
The inoculation of the sterilized potato tubes is easily done. If it is desired to obtain dilution cultures, that is, to say, colonies, this can best be accomplished by making several parallel streaks on the surface of the potato with the end of a straight platinum wire. The same wire, un sterilized, is then streaked repeatedly over the surface of a second potato, and then over that of a third. The latter will undoubtedly have but a few cells planted upon its surface, and, when these multiply, they will yield isolated colonies.

When transplanting a pure culture, such as a portion of a colony, a single streak should be made along the middle of the inclined potato. The characteristics of the resultant streak should be carefully noted.

Laboratory work. - The student will prepare 20 or 30 potato tubes according to the directions given above. Streak cultures will be made of each of the various organisms studied.

\section{Examination of Colonies.}

If the temperature is about $22^{\circ}$ the gelatin plates will probably show signs of growth in 24 hours. As a rule, however, 2 or 3 days must be allowed for the development of colonies. These become visible to the eye, first as mere white points; eventually, they enlarge and attain the size of a pin-head, or become even larger. A careful study of the colonies on the several plates should first be made with the unaided eye. This macroscopic examination is very important. The eye should be taught to detect the first sign of a liquefaction, the early appearance of pigment, the various differences in the form, size, color and structure of the colonies. In this way important information may be gained which will enable the subsequent work to be done to advantage. The various characteristics can often be recognized at quite an early period. 
Especial attention should be given to the form, and to the appearance of the colonies; the presence, or absence of pigment; liquefaction, or non-liquefaction of gelatin, etc. It should be remembered, however, that a given organism may give rise to at least two kind of colonies which some times are quite different in appearance. Thus, we may have surface as well as deep colonies. The former, developing on the surface of the gelatin, are unhindered in their development, and may, therefore, spread out and thus acquire peculiar characteristics. Moreover, having ready access to oxygen, pigment formation and liquefaction will be first seen in connection with these surface colonies. "The deep colonies, on the other hand, are surrounded on all sides by solid gelatin, and hence, much the same resistance to growth will exist in all directions. The result is that the deep colonies of various bacteria may be very much alike in appearance. The deep colony is usually round or oval, with sharp edges, and its contents are slightly granular and yellowish.

In the study of the various bacteria, considerable variety in the form of the surface and deep colonies will be met with. In many cases the growth is so characteristic that it is of great value in the recognition of that organism. It should be remembered, however, that the cultural characteristics of many bacteria are subject to more or less variation, depending on the temperature at which the growth occurs, as well as on the reaction and consistency of the gelatin. The colonies on a hard, 15 per cent. gelatin will be different, to some extent, from those that develop on a soft, 10 per cent. gelatin.

Plate 1 will usually have a cloudy or milky appearance. On examination with a low power-No. 3 objective- the cloudiness will be found to be due to countless numbers of minute, round colonies. This will be the case when the material which is employed for the inoculation is rich in bacteria. Only exceptionally will plate 1 have a sufficiently 
small number of colonies to be of value for further study. It is, therefore, advisable to destroy this plate as soon as. it has been found to be of no value. It should be placed in the solution of mercuric chloride, for at least over night.

The object of the plate method, it will be remembered, is to secure a perfect separation of the various bacteria that may be present. The farther apart these organisms. are, at the time the gelatin solidifies, the better it will be for subsequent study. On a good plate the colonies will be 5-10. mm. apart. They can be then transplanted without any risk of touching a neighboring colony.

Plate No. 2 will sometimes show a successful dilution. This will often be the case, if but one loopful of gelatin is transferred. At other times, the colonies on this plate may be so numerous as to make it of but little value.

Plate No. 3 should show perfectly distinct and well separated colonies. When studying and transplanting these growths, care should be taken not to pick out an accidental colony which owes its origin to some organism that dropped down from the air. Such a foreign colony is usually large, and grows rapidly; moreover, it is likely to be the only one of its kind on the plate. A single large colony, especially if unlike the others in appearance, should always be avoided. This is true when working with pure cultures. When, however, some blood or a portion of an organ is used for making plates the single colony in that case may be of great importance.

After having made a careful study of the various colonies with the unaided eye, the plates should then be given a careful microscopic examination. The plate should be placed upon the stage of the microscope and the colonies. closely studied under a low-power, the No. 3 objective. Further characteristics can thus be brought out which have escaped the eye.

A study of the micro-organisms which compose the colonies should now be made. This is done by making 
hanging-drop examinations and stained preparations according to the directions already given.

\section{The Stab Culture in Gelatin.}

The object in making plate cultures is to obtain colonies which, since they are derived from a single cell, are pure cultures of that organism. To perpetuate and keep up the pure culture thus obtained, it is necessary to resort to transplantation. For this purpose, the colony to be transplanted is touched with a sterilized and cooled, straight platinum wire. A portion of the colony will adhere to the end of the wire, and can be transferred to a tube of sterilized gelatin. The wire is usually pushed down the center of the solid gelatin, in which case we have what is known as a stab or stich culture. The operation of touching the colony is one that requires the greatest care to prevent contamination with foreign colonies, or with other material, since this would vitiate the pure culture. For this reason it is always carried out under a microscope, and so far as patience is concerned, it certainly is not inaptly called " fishing."

The gelatin plate is placed on the stage of the microscope, and, with the No. 3 objective, a suitable colony for transplantation is selected. It is desirable to have but one colony in the field of the microscope. A straight platinum wire, previously sterilized and cooled, is held in the right hand in the pen position. The hand is supported by resting the little finger on the right corner of the stage. The platinum wire is then inserted, about midway, between the front lens of the objective and the surface of the gelatin. It is held steadily in this position, and on looking into the microscope an indistinct shadow is seen. The wire is slowly drawn back till the end of the shadow, or indistinct wire is directly over the colony. Should the wire 
in doing this, touch the objective or the gelatin, it must: be sterilized at once and the operation repeated. When the end of the wire has been brought over the colony, gradually lower the point till it touches, or cuts the colony into two.

Now carefully remove the wire, without touching the microscope or some other portion of the gelatin. A tube of solid gelatin is held in the left hand in an almost horizontal position, the plug is then removed by grasping it with the little finger of the right hand (Fig. 29). The mouth of the tube should be flamed in order to remove any adherent cotton. The platinum wire, which has a small portion of the colony attached to it, is now slowly forced down the center of the gelatin, to the bottom of the tube. The cotton plug is at once replaced, the wire sterilized and the tube set. aside.

Transplantation can, of course, be made direct from a colony provided it is very far removed from any adjoining one. A careful microscopic examination should be made of the surrounding gelatin looking out especially for the presence of exceedingly small colonies.

The $s t a b$ culture thus made is a pure culture, and should now be labelled with the name of the organism and date, and set aside to develop. In a few days development takes place along the line of inoculation, and, a more or less characteristic growth results.

The manner of growth should be observed daily. Hanging-drop and stained preparations can be made, if it is desired, from the tube cultures. When the gelatin is very old, and hence too solid, it tends to split as soon as the platinum wire is forced into it. This is remedied by melting the gelatin and allowing it to re-solidify. Occasionally, the liquefied gelatin is placed in an inclined position, till it solidifies. A streak culture can then be made by drawing the wire over the surface. 
When gelatin tubes, and other plugged glass-ware have been kept for some time, more or less dust settles upon the cotton. There is, therefore, always danger of contamination when removing a plug. This risk can be reduced to a minimum, if the cotton is touched to a flame before it is withdrawn. It is advisable, as a matter of routine, to scorch every cotton plug before it is removed.

Laboratory work.-The student will make a careful macroscopic and microscopic examination of the various plates. Stab cultures are to be made of each organism studied. Hanging-drop examinations and permanent stained preparations should be made, either from the colonies or from the stab cultures. The operation of fishing should be practised until it can be done readily and successfully. It will be well to have another person watch the operation, to call attention whenever the wire touches either the lens or a part of the plate, other than that intended. Should the wire touch anything but the colony intended, it must be immediately sterilized before repeating the attempt.

The line of study of the Bacillus prodigiosus, Bacillus Indicus and of the various bacteria to be presently taken up consists, first, in making plate cultures. Colonies are thus obtained, the characteristics of which are thoroughly studied. Hanging-drop examinations, and stained preparations are next made, in order to become familiar with the organism itself. Stab cultures in gelatin are then made, and also streak cultures on potato. Later on, when the study of the pathogenic organisms is taken up, cultures are also made on agar, in bouillon and in milk. Finally drawings are made showing the form of the colony, the form of the organism, the appearance of the stab culture, etc.

In order to economize on gelatin, one-half of the class makes plates of one set of organisms, and, the next time, the other half of the class does the plating. Each student, however, is expected to examine and study every organism which is given out. 
Summarized, then, each organism is to be studied by making:

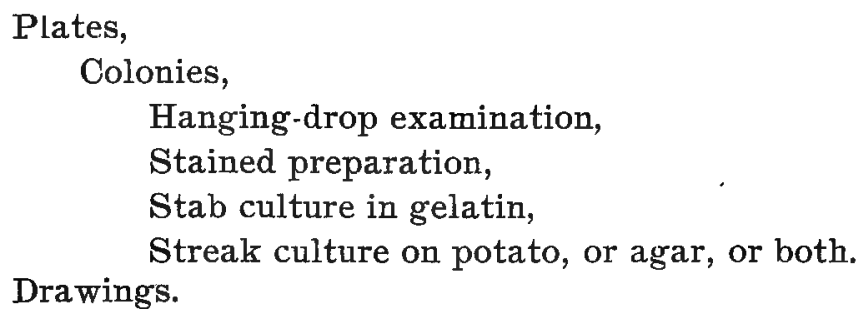

The laboratory work, during the first few weeks, is carried on as nearly as possible according to the following schedule:

1st day.-Cleaning up, and plugging of tubes.

2nd day.-Preparation of gelatin; sterilization and filling of tubes.

3rd day.-Dilution and mass potato cultures; sterilization of gelatin.

4th day.-Sterilization of gelatin; use of microscope; hangingdrop work.

5th day.-Hanging-drop work, continued: simple staining.

6th day.-Gelatin plates (prodigiosus and Indicus); simple staining, continued.

7 th day.-Study of colonies, stab cultures, etc.

8 th day.-Gelatin plates (red anc violet bacilli); Petri dishes (orange sarcine); Esmarch rolls (yellow sarcine).

9th day.-Study of colonies, stab cultures, etc.

10th day.-Gelatin plates (Kiel and fluorescing bacilli); Esmarch dilution potato (prodigiosus); potato tube culture.

The plating of two organisms, on alternate days, is continued till the non-pathogenic bacteria have been covered. The class then takes up the work on yeasts and moulds, as given in chapter XII. This is followed by the examination of air, water and soil, according to the directions laid down in chapter XIII. The preparation of bouillon and agar completes the first half of the course.

The class then enters upon the study of pathogenic bacteria, beginning with the work on anthrax animals. The study of the anaerobic bacteria is followed by that of the remaining organisms. These are supplied as pure cultures, or in infected animals. 


\section{CHAPTER VIII.}

THE NON-PATHOGENIC BACTERIA.

BaCILlUS PRODIGIOSUS.-B. INDIOUS.-B. RUBER OF KIEI.-B. RUBIDUS.-B. VIOLACEUS.--B. FLUORESCENS PUTIDUS.-B. PHOSPHORESCENS.-ORANGE SARCINE.-YELLOW SARCLNE.-B. SUBTILIS.-B. MESENTERICUS VULGATUS.-B. MEGATERIUM.-B. RAMOSUS.-PROTEUS VULGARIS.-

BACTERIUM ZOPFII.-SPIRILLUM RUBRUM.

-B. ACIDI LAOTICI.-B. BUTYRICUS.- B. CYANOGENUS. 


\section{Bacillus Prodigiosus.}

MONAS PRODIGIOSA, of Ehrenberg. MicRococuUs PRODIGIOSUs.

OrIGIN.-Found on starchy substances, rice, potatoes, moist bread; also on meat, albumin, milk, etc. May cause at times local "epidemics," infecting foods as bread, meat, and sausages, which assume a pink or red color. "Bleeding" bread or wafers (p. 116).

FORM - A very short rod, slightly longer than its width. May form short threads, especially in old cultures or in slightly acid media. Usually single or in pairs.

Morility.-Ordinarily shows no motion other than a marked Brownian movement. In acid or very dilute media the slimy character of the growth decreases and, as a result, a slight motion is observed; whips have been demonstrated.

Sporulation.-Has not been observed. It possesses, however, marked resistance to desiccation.

ANILIN DYES.-Stain readily.

GrowTH.-Very rapid.

Gelatin plates.-Deep colonies, round or oval, with sharp border and light brown color. Surface colonies, irregular, rough border, granular, with reddish center, and surrounded by clear, liquefied gelatin.

Stab culture.-Rapid, funnel-shaped liquefaction, extending along the entire line of inoculation. A red scum forms on the surface of the liquid; this eventually settles and the entire contents of the tube are colored bright red.

Streak culture.-On agar, it forms an abundant, moist, spreading growth, having an intense red color which is non-diffusible. On potato. the growth is especially rapid, and slimy, with marked pigment production. The pigment, when old, has a metallic, fuchsin-like luster, Odor of trimethylamin. On blood-serum, growth as on agar, with liquefaction.

Milk.-Grow th takes place and the pigment is held in solution by the fat globules. Coagulation results.

OXYGEN REQUIREMENTS.-It is a facultative anaerobe.

TEMPERATURE.-Grows best at ordinary room temperature. In the incubator it ceases to form pigment, and may temporarily lose this property, i. e., becomes attenuated.

BEHAVIOR TO GELATIN.-Rapidly liquefies as the result of the formation of a soluble peptonizing ferment. This liquefying property may be diminished, or temporarily lost by growth in acid media.

AERoGFNesis.-Strong odor of trimethylamin on potatoes. It ferments sugar solutions.

PIGMEN' ' PRODUCTION.--On various media a bright red pigment forms. This is soluble in alcohol, ether, chloroform, etc. It is formed only in the presence of air, and at ordinary temperature; not at $37^{\circ}$.

PATHOGENESIS. - It is not pathogenic. Its soluble products in large amounts may have a toxic action. The cellular proteins may induce suppuration. Animals insusceptible to malignant edema are rendered susceptible by an injection of this bacillus. Rabbits inoculated with anthrax are saved by the injection of Bacillus prodigiosus. 



\section{Bacillus Indicus, Koch.}

B. RUBER INDICUS.

OrIGIN.-Isolated in India from the contents of the stomach of a monkey.

Form.-Small, narrow, very short rod with rounded ends.

Motility.-Actively motile.

Sporulation.-Not definitely observed.

ANILIN DYEs. - Stain readily.

Growth.-Is rapid.

Gelatin plates. - Deep colonies are yellowish, with a wavy contour. Surface colonies grayish yellow, finely granular, with fibrillated borders. Show movement of contents, rapidly liquefy and may show a light pink color.

Stab culture.-Rapid liquefaction along line of inoculation. Dense flocculent growth settles on the bottom, and is grayish or light pink in color. A delicate scum forms on the surface and is colored from a light pink to a brick red.

Streak culture. - On agar, it forms a low, moist, spreading growth which usually is faint pink in color. On potato, the growth is low, not slimy as that of $B$. prodigiosus, and the color is more marked than on other media. On blood-serum, liquefaction results with, or without pigment production.

OXYGEN REQUIREMENTS. - It grows best in the presence of air, but is a facultative anaerobe. The pigment production depends upon the presence of oxygen.

Temperature. - The optimum is about $35^{\circ}$. The pigment is absent in cultures that develop in the incubator. Splendid pigment when growth occurs at $15^{\circ}$.

Behavior to gelatin. - It liquefies very rapidly.

Pigment PRoduction.-Varies greatly. The pigment may be grayish to bright brick-red; lacks the violet tinge of B. prodigiosus. Usually it is light pink, so that the ordinary cultures may be considered as attenuated.

Pathogenesis. - It has a marked toxic action, and when injected in large amounts into the abdominal cavity, or into the veins of rabbits and guinea-pigs, it proves fatal. Rabbits develop marked diarrhea and die in from 3 to 20 hours. On post-mortem the intestines show a severe inflammatory condition of the mucous membrane; at times, ulcerations are present. 
DRAWINGS. 


\section{Bacillus Ruber of Kiel, Breunig.}

B. RUBER BALTICUS.

\section{OrIGIN. - Drinking water of Kiel.}

Form.-Thick rods two to three times as long as wide; at times, may be much longer.

MотіLiтy. - It is somewhat motile, and the motion depends on presence of oxygen.

Sporulation. - This has not been observed.

AnILIN DYes. - Stain readily.

Growth. - Rapid and abundant.

Gelatin plates.-Deep colonies are oval, pale yellow, with wavy or even border. The surface colonies are blood-red in color, spread rapidly, and have a sinuous border; are surrounded by a clear zone, and liquefy gelatin.

Stab culture.-Growth and liquefaction take place along the line of inoculation. The fluid becomes strongly colored, and gas may form in the deeper layers.

Streak culture.--On agar, at $30-35^{\circ}$, the thick, slimy growth is at first a pale rose, and later becomes a brick-red. On potato, at $30-35^{\circ}$, it develops rapidly, forming a purple red growth. At lower temperatures the color is very marked; at first it is orange, later carmine red.

Milk.-At $35^{\circ}$, coagulation takes place in 24 hours, without a trace of coloration, due to rapid growth and production of acidity. At ordinary temperature the coagulation takes place slowly, and the fluid gradually colors.

OXYGEN REQUIREMENTS. - It is a facultative anaerobe, but requires oxygen to form the pigment.

Temperature.-Grows from 10 to $42^{\circ}$. The optimum is $30-35^{\circ}$, and above this the growth ceases to be colored. Direct insolation kills it in five hours. An exposure of three hours does not kill, but alters it so that it no longer produces the pigment, i. e., becomes attenuated.

BEHAVIOR TO GELATIN. - It liquefies gelatin quite rapidly. Aerogenesis. - Gas bubbles form in the gelatin.

PIGMENT PRODUOTION.-This weakens considerably on cultivation, and almost colorless varieties are met with. The color may be restored by successive culture on potato, and by growth at $15^{\circ}$. It is soluble in alcohol, but not in chloroform. The pigment is turned red by acids, and is decolored by alkalis. It is discolored by zinc dust and glacial acetic acid-distinction from that of B. prodigiosus. 


$$
\text { mansmeace }
$$




\section{Bacillus Rubidus.}

B. RUBER BEROLINENSIS.

ORIGIN.-Water supply of Berlin.

FORM.-Long, narrow rod; forms threads.

Motility.-Actively motile.

SPORUlation.--No spores observed.

Anilin Dyes. - Stain readily.

Growth._Fairly rapid.

Gelatin plates.-Small, yellow, finely granular colonies, with irregular border. It liquefies gelatin.

Stab culture.-Gelatin slowly liquefies along line of inoculation. A yellowish mass of bacteria settles to the bottom, and a thin folded scum forms on the surface.

Streak culture.-On agar, it forms a thin, irregularly bordered, slightly folded, yellowish growth. On potato, the most characteristic growth forms; it spreads, and has a bright brick-red color. On bloodserum, liquefaction takes place and a red pigment forms.

OXYGEN REQUiREMENTS, - It is aerobic.

Temperature.--It does not grow at the temperature of the body.

BEHAVIOR TO GELATIN.-Liquefies.

Pathogenesis. - No effect observed.

An interesting red pigment-producing bacillus, allied to the $B$. prodigiosus and to the Kiel bacillus, has been found on freshly packed oil sardines, and also in the suppurating felons on the fingers of the packers. In the latter case it was associated with an anaerobe.

About ten additional species or varieties of bacilli are known, that are characterized by the production of a red pigment. Moreover, a red pigment can be produced by various micrococci, sarcines, spirilla and yeasts. 
DRAWINGS. 


\section{Bacillus Violaceus.}

B. VIOLACEUS BEROLINENSIS. VIOLET BACILLUS OF WATER.

OrIGIN.-Water of the river Spree at Berlin, and of the Thames at London; also in well water.

Form.-A slender rod about two to three times as long as wide; it forms threads, but is usually in pairs.

Motility.-It is actively motile.

Sporulation.-Forms median spores.

ANILIN DYES. - React readily.

Growth.-This is moderately rapid.

Gelatin plates.-The colony is irregular with loose fibrillated border. The center shows quite early a violet color. It liquefies.

Stab culture.-Funnel-shaped liquefaction along the entire line of inoculation. A violet sediment collects on the bottom, while the liquefied gelatin above is perfectly clear. A violet ring may adhere to the wall of the tube on the surface of the liquid.

Streak culture.-On agar, it forms a smooth, thin, moist, bright violet covering. On potato, the growth is somewhat slow but very characteristic, forming a bright violet, eventually dark covering. On blond-serum, a violet color forms and liquefaction takes place.

OXYGEN REQUIREMENTS. - It is a facultative anaerobe. Oxygen is necessary to pigment formation.

TEMPERATURE. - It does not grow at higher temperatures.

Behavior to gelatin.-Liquefies.

Pigment production.-This depends upon the presence of oxygen. The color is soluble in alcohol, insoluble in ether and in chloroform. It is changed to a green by mineral acids.

Pathogenesis. - It has no effect.

About ten additional violet or blue pigment producing bacilli are known. Some of these, however, may be mere varieties. 



\section{Bacillus Fluorescens Putidus, Flügge.}

FLUORESCING BACILLUS OF WATER.

Origin.-Putrid media, water.

Form.-Short, small rods, with rounded ends.

Motility. - Very actively motile.

SPORUlation.-No spores observed on ordinary media. On althea, or on quince-jelly splendid spores form (Migula).

ANILIN DYES. - Stain readily.

GrowTH. -Rapid.

Gelatin plates.-The deep colonies are small, round, and finely granular. The surface colonies spread rapidly and form at first a very thin plaque, with irregular, wavy horder which shows markings. Later, a bluish green color diffuses through the surrounding gelatin. Odor of trimethylamin. No liquefaction.

Stab culture.-No growth in the lower part of the tube. The surface of the gelatin is covered with a grayish white growth. The fluorescing pigment gradually diffuses downward into the gelatin. The gelatin itself becomes yellowish.

Streak culture.-On agar, a moist, spreading growth. The agar becomes greenish, but later on the color fades. On potato, a thin grayish or brownish, moist growth forms.

OXYgen Requirements.-Aerobic.

Temperature. - Ordinary room temperature is best.

Behavior to gelatin. - It does not liquefy.

Pigment production. - This occurs only in the presence of air. Sulphur and phosphorus seem to be necessary. The coloring matter is soluble in water, and hence, diffuses into the surrounding medium. It possesses fluorescing properties; in other words it exhibits one color by transmitted light (yellow), and another (bluish-green), by reflected light. The pigment is destroyed by acids and is favored by alkali, such as ammonia. Colorless varieties can be obtained, by insolation and otherwise, as in the case of other pigment bacteria.

Pathogenesis. - It is without action on animals.

About a score of species or varieties of liquefying and non-liquefying fluorescing bacteria have been isolated from water, air, soil and various sources. The green diarrhea of children, green sputum, etc., may in some cases, be due to organisms of this class. 
DRAWINGS

205 


\section{Bacterium Phosphorescens, Fischer.}

PHOTOBACTERIUM PHOSPHORESCENS.

ORIGIN.-Found in the water of the harbor of Kiel, alsoon dead sea-fish, oysters, etc. From these it may spread to. meat in butcher shops.

Form.-Short, thick bacillus, with rounded ends; sometimes almost a coccus. It is usually in pairs, but may form threads. Involution forms soon develop.

Motility.-No motion.

Sporulation.-This has not been observed.

ANILIN DYEs. -Stain readily; so does Gram's method.

Growth. - Is moderately rapid. The cultures show in the dark a marked bluish-green phosphorescence (see p. 116).

Gelatin plates.-Show small, white, glistening colonies, which do not liquefy. The border is sharp, irregular, and the contents are granular, and show several concentric rings.

Stab culture.-A slight granular growth along the line of inoculation. Most abundant on the surface, where it forms forms a thin grayish white covering. Eventually, the gelatin is colored a yellowish brown.

Streak culture.-On aqar, potato, etc., growth is limited to the line of inoculation. It grows well on fish, beef, bread, fats, etc.

OXYGen Kequirements. - It is a facultative anaerobe. The production of light depends upon the presence of oxygen, and is, therefore, most marked on the surface growths. The intensity of the light may diminish, and, may become lost-attenuation. It may be restored by growth on suitable media, such as fish. ${ }^{1}$

Temperature. - Does not grow in the incubator, but may grow at $0^{\circ}$.

BeHavior to gelatin.-It does not liquefy gelatin, but can ferment sugar.

Pathogenesis. - No effect on animals. One phosphorescing bacillus is said to produce a disease in certain crustacea.

${ }^{1} \mathrm{~A}$ good medium for the growth of these organisms is prepared in the same manner as ordinary gelatin, by adding $500 \mathrm{~g}$. of chopped fish to $1,000 \mathrm{c}$.c. of water. The material is digested, and strained. To the filtrate $100 \mathrm{~g}$. of gelatin, $40 \mathrm{~g}$. of salt, $5 \mathrm{~g}$. of glycerin, and $5 \mathrm{~g}$. of asparagin are added, and the mixture is rendered slightly alkaline (p. 155). The liquid after heating is filtered, placed in tubes and sterilized. 



\section{Sarcina Aurantiaca.}

ORANGE SARCINE.

Origin. -From air, also from weiss-beer.

Form.-Small, spherical cocci, grouped as diplococci or tetrads, and at times in package-shaped masses. The latter are especially constant in hay infusions.

Motility-None.

Sporulation.-None.

ANILIN DYES.-Stain very easily, and are likely to overstain.

GRowTH.-Rather rapid.

Gelatin plates.-Show round, sharp-edged colonies, which are granular and of an orange-yellow color. These soon liquefy the gelatin.

Stab culture.-The gelatin is liquefied along the entire line of inoculation. Eventually, an orange-colored deposit of bacteria forms on the bottom, and the liquid above becomes clear.

Streak culture.-On agar, it forms a thick, orange-colored growth. On potato, the pigment is excellently developed, and the growth is thick.

OXYGEN REQUIREMENTS. - It is aerobic.

Temperature. - A high temperature is unfavorable.

BEHAVIOR TO GELATIN. - It liquefies rapidly.

AERogenesis. - Not observed

Pathogenesis. - It has no effect on animals.

A red sarcine has been found in so-called "red milk" -a condition which at times may be due to $B$. prodigiosus, or to red yeasts. A somewhat similar micrococcus has been found in "red sweat." It is interesting to note that the sarcine commonly found in the lungs is said to form spores. Another red sarcine, isolated from an ascitic fluid, possessed active motion. A motile sarcine with giantwhips has been observed. 


$$
\text { Daromeses }
$$




\section{Sarcina Lutea, Schröter.}

YELLOW SARCINE:

ORIGIN.-Air.

Form.-Larger cocci than the orange sarcine, and moreover, it forms more perfect package-shaped masses.

Motility.-None.

SPORUlation.-None.

AnILIN DYEs.-React readily and are likely to overstain. The characteristic division of the cells is then lost. GrowTH.-Very slow.

Gelatin plates.-Colonies develop very slowly as minute yellowish spots, which show an irregular oblong form and are markedly granular. The colonies do not liquefy gelatin.

Stab culture.-Growth is especially developed on the surface and extends but slightly down the line of inoculation. Lower half of tube is usually free from growth, or at most, contains a few, isolated, spherical colonies. The color is bright yellow and in very old tubes liquefaction slowly shows itself, so that eventually a bright yellow deposit forms on the bottom while the supernatant liquefied gelatin is perfectly clear.

Streak culture.-On agar, it forms a very thick, moist, bright yellow covering. On potato, the growth is slow, with production of the same thick growth and color.

OXYGEN REQUIREMENTS.-It is an aerobic organism.

TEMPERATURE. - It may grow in the incubator.

BeHAvior to gelatin. - A very slow liquefaction is observed after a lapse of several weeks.

Pathogenesis. - None.

The sarcine form was first observed in 1842 in vomited stomach contents and was designated as $\mathbf{S}$. ventriculi. Within recent years a stomach sarcine has been isolated in pure culture and found to correspond, in many respects, to the yellow sarcine. More than one species, however, may exist in the contents of the stomach. 
DRAWINGS.

211 


\section{Bacillus Subtilis, Ehrenberg.}

HAY BACILLUS.

ORIGIN.-In air, water, soil, feces, putrid fluids, and in infusions of hay.

Form.-Large, rather thick rods, 3-4 times as long as wide, with rounded ends. Usually in pairs, often in threads.

Motility.-Active snake-like motion. 8-12 lateral flagella.

SPORULATION.-It forms large, oval spores at or near the middle, without enlargement. They are highly resistant and can be readily double stained. Germination, p. 54.

ANILIN DVES. - Stain readily; so does Gram's method.

GrowTH.-Very rapid. At $21^{\circ}$ cell division has been observed to take place in $75 \mathrm{~min}$; and at $35^{\circ}$ in $20 \mathrm{~min}$.

Gelatin plates.-The surface colonies liquefy gelatin rapidly and extensively, and present a striking appearance. The central portion appears as a grayish yellow, irregular mass, which on close examination can be seen to be made up of moving cells. This central portion is surrounded by a lighter, granular zone. The border of the colony is quite characteristic. It consists of a dense zone of bacilli and threads, radially arranged, so that the ends project outward, thus presenting a peculiar appearance-the so-called "ray crown."

Stab culture.-Very rapid, funnel-shaped liquefaction takes place along the entire line of inoculation. White flocculent masses accumulate at the bottom while the liquid above, at first turbid, becomes clear. On the surface a dense white scum or zooglea usually forms.

Streak culture.-On agar, it forms a dull grayish white, thick, folded scum. On potato, it develops excellently, and forms a moist, thick, yellowish-white covering, which at first is velvety in appearance but later becomes dry and granular, and contains spores as well as involution forms. On blood-serum, it also forms a folded scum and liquefies.

OXYGEN REQUIREMENTS. - It is aerobic.

Temperature. -Grows from 10 to $45^{\circ}$. Optimum about $30^{\circ}$.

BEHAVIOR TO GELATIN.-It liquefies rapidly and extensively.

Pathogenesis. - It has no pathogenic power. When a large number of spores are injected into the blood they soon disappear and are taken up by the liver and spleen. They may be stored up in these organs for 60 to 70 days and yet preserve their vitality (Wyssokowitsch).

A large number of bacilli resemble the above B. subtilis to a marked extent. It is customary, therefore, to speak of the group of hay bacilli. 
DRA WINaS.

213 


\section{Bacillus Mesentericus Vulgatus, Flügge.}

\section{POTATO BACILLUS.}

Origin.-Widely distributed in the soil, on the surface of potatoes, in feces, putrid fluids, water, milk, etc.

Form. -Small, thick rods, with rounded ends, usually in pairs, may form threads.

Moтility.-Actively motile; flagella numerous.

Sporulation.-It readily forms large median, roundish spores. The spores of one variety of "potato bacillus" described by Globig, showed enormous powers of resistance, withstanding the action of steam-heat for five to six hours.

ANILIN DYes. - React easily; so does Gram's method.

GrowtH. - Is very rapid, and in many respects resembles that of the hay bacillus.

Gelatin plates.-Show yellowish white, slightly granular colonies, with irregular borders. They liquefy rapidly and extensively.

Stab culture.-Growth occurs along the entire line of inoculation, but liquefaction is more energetic in the upper part. The liquefied gelatin remains turbid for some time and a thin, grayish, folded scum forms on the top.

Streak culture.-On agar, it forms a dull white or grayish, folded growth. On potato, the most characteristic growth develops. The surface is rapidly covered with a thick, white, strongly folded, coher. ent growth. Later, the growth becomes dirty brown or red in color.

Milk. - Casein is coagulated, and peptonized. Starch is inverted.

OXYGEN REQUIREMENTS. - It is aerobic.

TEMPERATURE. - It grows at ordinary, as well as at higher temperatures.

BeHavior to Gelatin. - It liquefies rapidly.

Pathogenesis. - No effect observed.

Several varieties of "potato bacilli" have been met with. Some form a brown, while others give a reddish growth on potatoes. The spores of the potato and hay bacilli are extremely resistant. When the material is in a small mass, not in a fine state of suspension, it may require an exposure to steam of 10 hours or more, in order to insure sterilization (p. 162). 
DRAWINGS. 


\section{Bacillus Megaterium, De Bary.}

OrIGIN.-Originally found on boiled cabbage leaves, but may be present on other vegetable matter; also found in the air and in soil.

Form.-Large cylindrical rods, with granular contents, three to six times as long as broad, with rounded ends. They are usually slightly bent, are in pairs, and may form threads. Involution forms are very common. Capsulated cells are especially found in the slimy growths.

Mоті.гту.—Slow, creeping motion. 6-8 lateral flagella,

SPORULation.- - Itforms median spores.

ANILIN DYES. - Stain readily, though irregularities due to granular protoplasm may be seen.

GrowTH. - Rapid.

Gelatin plates.-Colonies are at first irregular, small, yellowish masses, but subsequently show marked radiating or branching forms, which soon liquefy the gelatin. Kidney-shaped colonies may be met with.

Stab culture.-Rapid growth and liquefaction along the line of inoculation. May show threads of bacteria penetrating outward into the solid gelatin. Eventually, the gelatin is wholly liquefied and a flocculent mass accumulates on the bottom; the supernatant liquid clears up without formation of scum on top.

Streak culture.-On agar, it forms a dull white or grayish covering. On potato, it grows rapidly as a thick, slimy, grayish white mass, which is rich in spores and involution forms.

OXYGEN REQUIREMENTS. - It is aerobic.

Temperature. - The optimum is at about $20^{\circ}$. It may, however, grow in the incubator.

BeHAVIOR to gelatin. - It liquefies rather slowly.

Pathogenesis. - No effect observed. 
DRA WINGS.

217 


\section{Bacillus Ramosus.}

ROOT OR "WURZEL" BACILLUS.

ORIGIN.-Very common in earth; occurs also in river and in spring water.

FORM. --Rather large rods, thicker than the Hay bacillus; with slightly rounded ends. Threads are common.

Motility.-It is slowly motile.

Sporulation. - Large median spores occur.

Anilin dyes. - It stains well.

Growth.-Rapid.

Gelatin plates.-The colonies present a characteristic appearance, resembling somewhat fine branching rootlets, hence the name. At first the colonies are round, dark and with bristly borders. Subsequently the colonies branch and ramify throughout the gelatin which is slowly liquefied.

Stab culture.-This is also characteristic. Growth develops along the line of inoculation and from this threads penetrate or radiate into the surrounding gelatin. The growth is more rapid at the top than in the lower parts of the tube so that the appearance of an "inverted pine tree " results. Later the gelatin is liquefied completely. The bacterial growth accumulates on the bottom while the liquid above becomes clear and has a thin scum on the surface.

Streak culture.-On agar it forms a grayish growth, spreading outward from the streak so that the appearance often is not unlike that of a centipede. On potato, a slimy, whitish growth develops, which is rich in spores.

OXYGEN REQUIREMENTS. - It is aerobic.

TEMPERATURe. - It grows at ordinary temperature, and also in the incubator.

BeHAVIOR to GELATIN. - It liquefies slowly.

Pathogenesis. - It is without effect, even in very large doses. 
DRA WINGS. 
ORIGIN.-It is very widely distributed and is commonly present in the putrefaction of animal proteins (p. 111). It has also been met with in water, in meconium, in purulent abscesses, and in the blood and tissues of several cases of fatal putrid infection of the intestines.

Form.-Rods, of varying length, from short oval forms to those which are 2 to 6 times as long as wide. It is usually bent and grows in pairs; may also form twisted, interwoven threads. Roundish involution forms are common.

Moriliry.-It is actively motile. Flagella are very numerous (60-100), and are arranged all over the surface.

Sporulation.- Not observed, though cultures are resistant to desiccation and retain vitality for many months.

ANILIN DYes. - It stains readily; not by Gram's method.

Growth. - Very rapid.

Gelatin plates.-Rapid and extensive liquefaction of the gelatin. The colonies are yellowish brown, with bristly borders, and in soft gelatin tend to spread over the surface and assume peculiar figures. Detached portions of colonies can be seen to move about-"swarming islets." A disagreeable odor and an alkaline reaction is present.

Stab culture.-Rapid liquefaction along entire line of inoculation. so that in a few days the entire contents are liquefied. The fluid is at first diffusely cloudy, but later clears up and a flocculent sediment settles on the bottom, while on the top a grayish white layer is formed.

Streak culture.- On agar, it forms a grayish, slimy, rapidly spreading growth. On potato, it forms a dirty colored, sticky covering.

OXYGEN REQUIREMENTS. - It is a facultative anaerobe.

Temperature. - The optimum lies between 20 and $24^{\circ}$. It grows excellently in the incubator.

BEHAVIOR TO GELATIN. - This is rapidly liquefied.

Aerogenesis. - It forms hydrogen sulphide.

Pathogenesis. - Small doses have no effect. The injection of large quantities of living, or filtered cultures produces in rabbits and guinea-pigs toxic effects, and death may even result. It is therefore toxicogenic. At times, it may even take on a pathogenic character.

This and several related species are included in the Bacterium termo of the older writers. 


$$
\text { nomsuase. } \quad \text { an }
$$


ORIGIN.-Isolated from the intestines of chicken; also from water and feces.

Form.-Rods, two to five times as long as wide. It forms threads, which in gelatin are often peculiarly bent or twisted, resembling spirals. In old cultures coccus-like involution forms are abundant.

Motility.-It is actively motile.

Storulatron.-Spore-like bodies are formed, which are said to resist desiccation, but are readily destroyed by heat, and are readily stained by anilin dyes. These in reality are not spores, but involution forms.

ANILIN DYES. - It is stained easily.

GROw:H.-Rapid.

Gelatin plates.-The colonies ${ }^{1}$ form delicate cloudy patches of radiating threads, and show, under the microscope, in addition to the network of threads, numerous rounded little masses or bunches of cells.

Stab culture.-Marked growth in the upper part of the tube, but absent from the lower part. It shows fine radiating lines which, at or near the surface, penetrate deepest into the surrounding gelatin.

Streak culture.-On agar, it forms a very thin, dry, grayish growth.

OXYGEN REQUIREMENTS. - It is an obligative aerobe.

Temperature. - It grows best at ordinary temperature. It can grow at $37-40^{\circ}$, but tends to develop involution forms and to die out.

Behavior to gelatin.-No liquefaction. No indol.

Pathogenesis. - No effect has been observed on animals.

This organism resembles in some respects certain species of the Proteus group and, indeed, is considered by some to be identical with the P. Zenkeri.

${ }^{1}$ When surface colonies, as those above, present some special characteristic they can be reprinted on cover-glasses. To make such an impression or "Klatsch" preparation, select a suitable spreading colony, with the aid of a No.3 objective, then raise the tube of the microscope and carefully drop a clean cover-glass on top of the colony. Apply gentle pressure with a pair of forceps, then grasp the edge of the cover-glass and carefully remove; allow to dry in the air; fix and stain in the usual manner.

In making the reprint only the growth should adhere to the cover-glass. Considerable gelatin, solid or liquid, on the cover-glass, is undesirable and interferes. 


$$
\text { numsome }
$$


Spirillum Rubrum, Esmarch.

OrIGIN.-From the putrefied cadaver of a mouse.

Form.-Clear, transparent, thick cells, which are usually single, appearing as large bent rods or comma bacilli (vibrio). It may form spirals of three or four, or even forty windings. Involution forms are common in old cultures.

Motility. - It is actively motile. Each end of a spiral has one wavy flagellum.

Sporulation. - True spores have not been observed.

AnILIN DYEs. - These stain slowly but well, especially if the dye is slightly warmed.

Growth._-This is extremely slow.

Gelatin plates.-Owing to the very slow development of colonies ordinary plates cannot be used. In roll-tubes, colonies develop in from seven to ten days, and at first are minute and grayish; later the center of each colony becomes tinged with pink and eventually becomes red. The edge is smooth and the contents are finely granular.

Stab culture.-This is the most characteristic. Growth takes place along the entire line of inoculation, forming a row of colonies. The growth spreads slightly on the surface, and is colored a light pink. The pigment formation is most marked along the line of puncture-where oxygen is absent. It passes through a light pink to a beautiful dark wine-red color. Ordinary bacterial pigments are formed only in the presence of air, and are secondary products, whereas this pigment is formed in the absence of air and is, therefore, a primary product.

Streak culture.-On agar, it forms moist, thick, non-spreading patches, which, when old, possess a light pink or red color, especially near the center. On potato, it develops slowly, forming minute deep red colonies. On blood-serum, the growth is much the same as on agar.

Milk.-In fluid media, milk, bouillon, etc., it forms long spirals which show little or no motion.

OXYGen Requirements. - It is a facultative anaerobe.

Temperature.--It grows between $16^{\circ}$ and $40^{\circ}$. The optimum temperature is about $37^{\circ}$.

Behavior to gelatin. - It does not liquefy.

Pathogenesis. - It has no effect on animals. 
DRAWINGS. 


\section{Bacillus Acidi Lactici, Hueppe.}

ORIGIN.--It was obtained from sour milk.

Form.-Short, thick rods, two to three times as long as wide; these are usually in pairs; rarely in chains or threads.

Motility.-No real motion. Marked Brownian movement.

SPORULATION.-Round, terminal bodies have been observed, but these are not spores.

ANILIN DYES. - It stains readily; also by Gram's method.

GrowTh.-Abundant and rapid.

Gelatin plates.-The deep colonies are round or oval, yellow, sharp bordered, finely granular. The surface colonies spread, forming thin plaques, with irregular, wavy borders. The outer zone of the colony is at first almost transparent and shows markings resembling the venation of leaves.

Stab culture.-Slight growth along the puncture, but on the surface it is considerable and spreads rapidly as a thin, dry, pearly-white covering. In old cultures bundles of crystals form along the line of inoculation, especially at or near the surface.

Streak culture.-On agar, it forms a grayish white, moist, spreading growth, which offers no special characteristics. On potato, it forms a brownish yellow, slimy covering.

Milk.-In sterilized milk it converts the lactose or milk-sugar, in part, into lactic and carbonic acids. The acid reaction thus produced causes a precipitation of the casein or curd. This change occurs only in the presence of air. Old cultures do not alter milk-attenuation.

OXYGEN REQUIREMENTS. - It is a facultative anaerobe.

TEMPERATURE. - It grows between $10^{\circ}$ and $45^{\circ}$. The optimum is at about $30^{\circ}$.

Behavior to GeLatin. - The latter is not liquefied.

Aerogenesis. - Gas is produced in milk. Carbon dioxide and alcohol are formed.

Pathogenesis. - No effect. 0.75 per cent. lactic acid stops the growth. Production of lactic acid in the mouth (dental caries); abnormal fermentations in the stomach, in the intestines. Lactic acid bacteria favor the growth of anaerobic bacteria (see p. 100).

The cultural characteristics resemble those of certain colon bacilli and it may, therefore, be regarded as a variety of the $B$. aerogenes. It may be considered as a common cause of the souring of milk, but the production of lactic acid is common to a large number of bacteria, and hence, such organisms will also coagulate milk. 
DRA WINGS. 


\section{Bacillus Butyricus, Hueppe.}

OrIGIN.-Milk.

FonM.-Long, narrow rods, with rounded ends, frequently in pairs, may form threads.

Motility. - It is actively motile.

Sporulation. - At about $30^{\circ}$ it forms bright, oval, median spores.

Anilin Dyes.-React well.

Growth._Rapid.

Gelatin plates.-The deep colonies form yellowish masses, whereas the surface ones liquefy rapidly and then form grayish-brown, granular patches with fibrillated borders.

Stab culture.-Slow liquefaction along the entire line of inoculation. The gelatin becomes colored yellowish and on the surface a thin, folded, grayish-white scum forms. The liquid remains cloudy for some time, but later the growth settles to the bottom.

Streak culture.-On agar, it forms a thick, grayish or yellow, sticky covering. On potato, a light brown, transparent grow th results which sometimes becomes folded.

Milk.-Without change in the amphoteric reaction the casein gradually coagulates, as with rennet. Subsequently, after about 8 days, the casein is redissolved or peptonized with formation of pepton, leucin, tyrosin, ammonia and bitter products. From hydrated milk sugar and lactates it forms butyric acid.

OXYGEN REQUIREMENTS. - It is aerobic.

Temperature. - It can grow at the ordinary temperature, but its optimum is at 35 to $40^{\circ}$.

Behavior to gelatin. - It liquefies gelatin.

AERogenesis.---Butyric acid is formed.

Pathogenesis. - It has no effect on animals.

A large number of aerobic and anaerobic bacteria give rise to butyric acid fermentation (see p. 103). The vibrion butyrique of Pasteur was the first anaerobe discovered (1861). 
DRAWINGS. 


\title{
Bacillus Cyanogenus, Fuchs (1841).
}

\author{
BACILLUS OF BLUE MILK.
}

ORIGIN.-It was obtained from blue milk.

Form.-Small, rather narrow rods, with slightly rounded ends, 2 to 3 times as long as wide. It frequently grows in pairs, very rarely in threads.

Motility.-Very actively motile. Bunch of whips at one end.

SPORUlation.-The small terminal spore-like bodies observed by some are probably involution forms. True spores have been observed to form on althea or quince jelly.

ANILIN DYES.-It stains easily.

Growth.-Rapid.

Gelatin plates. - The deep colonies are round with sharp, smooth border, and yellowish granular contents. The surface colonies are moist, elevated, convex masses, which are round, finely granular and dark colored. At times the colonies may be thin, spreading and with an irregular border.

Stab culture.-Little or no growth in the lower part of the puncture. It spreads over the surface as a thick, moist, dark-gray covering. A dark steel-blue color diffuses downward into the gelatin. The shade of color varies with the reaction of the medium. In neutral or acid media it is quite blue, whereas in very alkaline media it is dark, or even black. The culture when old becomes dark colored.

Streak culture.-On agar, it forms a dirty gray, thick, moist covering, and the medium becomes dark colored. On potato, it likewise forms a thick, raised, slimy growth, which rapidly spreads and becomes colored, On blood-serum, no color is formed.

Milk.-In sterilized milk it produces no acid or coagulation, but the liquid becomes colored a slate-gray which with acids turns blue. In unsterilized milk, that is in presence of lactic acid bacteria, the color is sky-blue. The color is developed from casein, not from lactose. In bouillon or milk containing 2 per cent. of glucose it forms a splendid blue color and lactic acid. It does not convert lactose into an acid.

\section{OXYGEN REQUIREMENTS.-Aerobic.}

TEMPERATURE. - It grows best at the ordinary temperature; to less extent in the incubator. The pigment is most marked when growth occurs at low temperatures, $15-18^{\circ}$.

BeHavior to Gelatin.-This is not liquefied.

Pathogenesis. - It has no effect on animals.

The production of a blue color in milk is frequently observed, and, as in the case of red milk, this is due to the development of certain species of bacteria. 
DRAwINGS. 
BOUILLON, AGAR, MILK AND MODIFIED MEDIA-THE INCUBATOR AND ACCESSORIES.

The work, thus far, has enabled the student to acquire familiarity with the fundamental methods of bacteriology. The various non-pathogenic bacteria have been isolated by means of gelatin plates; their characteristics of growth in the colony, in stab culture, and on potato, have been studied. A special bread medium has been employed in cultivating the several important moulds. The gelatin, potato and bread media are, however, by no means the only ones employed in growing micro-organisms.

While the nutrient gelatin is invaluable for the isolation of certain bacteria, especially those of the saprophytic group, it is not so generally useful in connection with the pathogenic bacteria. Many of these grow only, at or near the temperature of the body $\left(37.5^{\circ}\right)$, and, inasmuch as the ordinary gelatin melts at about $25^{\circ}$, it cannot obviously be employed to obtain colonies, or stab cultures, of such organisms.

It is, therefore, desirable to have a substance which, to a certain extent, can take the place of gelatin; one which will make a medium such that it will not melt, even at the temperature of the body. The sea-weed agar-agar, which is gathered off the coast of Asia, and about the islands of the Pacific, answers this requirement. A more than two per cent. solution of agar is not made, and is not desirable. The prepared agar medium melts at the temperature of boiling water $\left(100^{\circ}\right)$, and becomes solid again when the temperature is reduced to about $40^{\circ}$. This medium, there- 
fore, is extremely useful in the study of pathogenic organisms. Unlike gelatin, it is not liquefied by bacteria except perhaps in a very few rare cases, and hence, it can not supplant gelatin in the study and differentiation of bacteria.

In addition to the solid media, it is desirable to have a liquid medium. The beef-tea or bouillon, prepared according to the following directions, is extremely useful for this purpose. Indeed, before the introduction of solid media, it was the chief medium employed. The growths in beef-tea are frequently very characteristic, and materially assist in the recognition of various bacteria.

The meat extract employed in the preparation of gelatin, agar and bouillon, is usually made out of beef. The Pasteur school, almost invariably, use veal for the preparation of these media. The reason for this lies in, the greater amount of extractive substances contained in veal. For special purposes, bouillon etc., is made out of chicken, pork, or other flesh. The commercial meat extracts can be employed for making bouillon and the other media (see p. 243).

Milk constitutes another important liquid medium. On account of the presence of lactose and casein, milk is especially valuable in differentiating certain bacteria. Some bacteria will decompose the milk-sugar and cause the evolution of gas-bubbles, whereas others will not do so. Again, some will cause coagulation, as is seen in sour milk, while other bacteria produce no appreciable change.

Consequently, before taking up the study of pathogenic bacteria, the student will prepare, in addition to the gelatin, and potato media, already on hand, the necessary bouillon, agar, and milk media (see p. 243). 


\section{Bouillon.}

The meat extract is prepared according to the directions given under the preparation of gelatin (p. 153). To 1 liter of the meat extract, $10 \mathrm{~g}$. of dry pepton (Witte's) and $5 \mathrm{~g}$. of common salt are added. The liquid is then warmed at $55-60^{\circ}$, with constant stirring, till the pepton dissolves. It is then rendered alkaline according to the directions given on p. 154. The portions of 10 c.c. of the solution in test-tubes should receive $0.4,0.6,0.8$ and $1.0 \mathrm{c.c}$. of the $\mathrm{NaOH}$, respectively. The neutral point is usually reached by the addition of about 0.5 c.c. $\mathrm{N}$ NoOH per 10 c.c. The remaining liquid is measured and amount of $\mathrm{N} \mathrm{NaOH}$ necessary to neutralize the entire amount is then calculated. This calculated amount, plus 10 c.c. of $\mathrm{N} \mathrm{NaOH}$ per liter, in order to impart a slight alkalinity, is then added. The liquid is then placed in the enamelled jar (Fig. 23, p. 153), and immersed in a boiling water-bath, or boiled directly over the flame for $\frac{1}{2}$ an hour. Inasmuch as there will be a considerable loss of water by evaporation, it is advisable to mark the level of the liquid, before boiling. At the close of the operation sufficient distilled water should be added to bring the liquid back to the original volume: $A$ better procedure is to weigh the liquid, before and after boiling, and for the number of grams lost in weight to add a corresponding number of c.c. of distilled water.

The liquid is then filtered through a wet filter. The filtrate is usually perfectly clear and transparent, but it not infrequently happens, when it is placed in tubes and sterilized, that a fine amorphous precipitate will form. Usually this is unimportant, but, at times, it is desirable to have a bouillon which will remain perfectly clear, free from the slightest deposit. To obtain this result, the filtered bouillon is returned to the clean, enamelled jar and subjected to more heat. It can be placed in the autoclave and 
heated for $\frac{1}{2}$ an hour at $110^{\circ}$, or, what is even more preferable, it is concentrated over a flame to less than $\frac{1}{2}$ its original volume. The vessel is finally weighed, and the distilled water necessary to bring back the bouillon to the original weight is added. The bouillon is again filtered and the filtrate is then filled into tubes. The small testtubes $(12 \times 150 \mathrm{~mm}$.) are, as a rule, desirable for this purpose. These are plugged and sterilized in the usual manner.

The bouillon is then sterilized according to the directions given under gelatin (p. 162); that is to say, it is exposed to steam-heat for 15 minutes on each of three consecutive days.

The bouillon prepared as above should be (1) perfectly clear; (2) should be slightly alkaline in reaction; and, (3) should not cloud on heating.

\section{Agar-Agar.}

One liter of bouillon is prepared as above, except that the second heating is omitted. $20 \mathrm{~g}$. of agar ( 2 per cent.) are cut up into as small pieces as possible, and these are then added to the bouillon which has been returned to the clean jar. The jar and contents are weighed ${ }_{3}$ and then placed over a free flame to boil. The heat shopld be just sufficient to gently boil the liquid, otherwise the liquid is liable to froth and run over. The liquid is boiled for $\frac{1}{2}-\frac{3}{4}$ of an hour, or until the agar has completely dissolved. This point can be readily ascertained by drawing up the liquid into a clean glass tube or pipette. No solid, translucent lumps should be visible. The jar and contents are now weighed, and distilled water is added to replace that lost by evaporation.

The jar is then placed in the steam sterilizer; or, better, immersed in a water-bath at a constant temperature of 
about $50^{\circ}$. In an hour or two, the finely divided, insoluble matter gathers into masses and settles to the bottom, leaving above a clear, transparent liquid.

The filtration of 2 per cent. agar through paper is usually a very slow, tedious and unsatisfactory operation. Consequently, it is usually filtered either through a layer of cotton, or else, after sedimentation, the agar is allowed to solidify and then the clear portion is cut off while the bottom layer, containing the impurities, is discarded. By employing the following method, a liter of agar can be filtered perfectly clear, or as clear as is necessary for all purposes, in from 3 to 5 minutes.

The filtering arrangement is shown in Fig. 35. The receiving flask ( $1 \% / 2$ l. capacity) is connected with a Chapman aspirator. The neck receives the rubber stopper, which is slipped over the end of a funnel. A Witte's porcelain, perforated plate $(10 \mathrm{~cm}$. in diameter $)$ is placed in the funnel, and steadied in position by the glass rod which passes through the center. A disc of muslin having the same diameter as the plate (or a trifle less) is placed on the latter. Then a circle of absorbent cotton, about $12 \mathrm{~cm}$. in diameter, is placed on top, and finally another layer of muslin. A second Witte's plate is now placed on top, as a weight, but it remains there only during the preliminary warming up of the funnel. About balf a liter of boiling water is then poured into the funnel. The pump is set into action, and the hot water rapidly passes through the filter. It is returned to the funnel, several times in succession, thus warming up the funnel, filter and flask. During this operation, care should be taken to see that the cotton closes tight over the edge of the porcelain, and that no openings exist. While the pump is still active, the upper plate is removed. The suction of the pump draws down the filter, and hence the weight is no longer necessary.

${ }^{1}$ It is advisable to place the jar in a water-bath which is provided with a thermo-regulator (see Fig. 75). 
While the filter is still hot, and the pump active, the well sedimented agar (p. 236) is slowly poured on the center of the filter. The liquid passes through as rapidly as it strikes the surface. The impurities are brought last upon the filter. If the filtrate is not perfectly clear, it can be passed through the filter a second or a third time. The operation of filtration requires but a few minutes. The resulting filtrate, when filled into tubes and sterilized, is usually wholly free from a deposit, and is perfectly transparent. At most, a few floccules will separate during the subsequent sterilization.

In order to facilitate the filtration through paper many workers prefer to make a 1 or $1 / 2$ per cent. solution of agar. While it is true that such solutions filter more readily, yet this

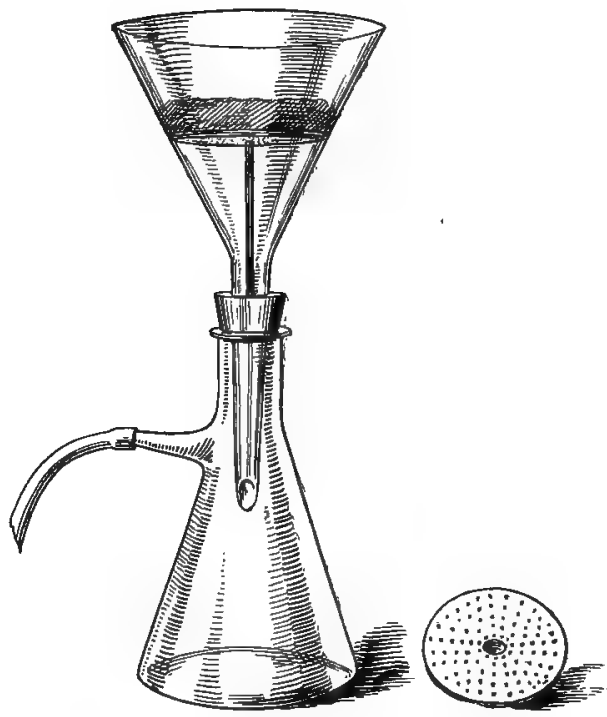

Fig. 35. Apparatus for filtering agar (F. G. N.). advantage is more than offset by the softer consistency of the agar. The two per cent. agar prepared as above is hard, and though it is not filtered through paper, it is, nevertheless, perfectly clear.

The above method of making agar would seem to be long and tedious, but in reality, it is no more difficult than the preparation of gelatin. While the pump is very useful, in the above filtration, it is by no means necessary.

In the method as described, the agar is not added to the albuminous meat extract, but to the ready-made bouil- 
lon. If it were added to the former, the agar would dissolve much more slowly than in clear bouillon, and, moreover, the coagulated albumin would materially interfere with the subsequent filtration. As it is, only the impurities in the agar are to be filtered off. The agar is neutral in reaction, and hence, it is added to the already alkalized bouillon.

The filtered agar is filled, by means of a small funnel, into plugged, sterilized test-tubes, and these are then sterilized by steaming for 30 minutes, on each of three successive days. As many tubes as are necessary for immediate use are inclined by placing on a thick glass rod, or narrow strip of wood, or on the gas tubing. The small test-tubes $(12 \times 150 \mathrm{~mm}$. $)$ are preferable for agar. The remaining tubes are kept in the upright position, and are inclined whenever it is necessary. Agar may be used, either, in the inclined position, for streak cultures, or in the upright position, for stab cultures. It may be mentioned incidentally that inclined gelatin may often be employed to advantage.

The agar solidifies in this inclined position. A large surface can thus be obtained, which can be used for making a streak culture, or even for the isolation of colonies. The streak culture is made, as in the case of those on potato (p. 186), by drawing the inoculated wire along the middle of the inclined surface. In describing the use of the potato tube (p. 186) it was shown that these could be used for obtaining isolated colonies. The same method can be applied to the inclined agar. It enables one to obtain isolated colonies in less than twenty-four hours, and that, without the use of any special plating apparatus. A very small amount of the material is taken up, on a platinum wire, and spread over the entire inclined surface of an agar tube. The same wire, without sterilization, is then rubbed over the inclined surface of a second tube, and then in like manner over a third and fourth. The tubes are then placed in an incubator to develop. 
If the material used is a solid growth, it is advisable to transfer a minute amount of this to a tube of bouillon (10 c.c.). With a sterilized wire, a loopful of this suspension is transferred to a second tube of bouillon. The wire is again sterilized, and, a loopful of this second dilution is transferred to a tube of freshly inclined agar and carefully spread all over the surface. The same wire, not sterilized, is then rubbed over the surface of a second tube of inclined agar. The inoculated agar tubes should be placed in a horizontal position in the incubator for 18 to 24 hours. Perfectly isolated colonies will thus be obtained.

The method of isolating colonies by means of agar plates will be described in the next chapter. Esmarch roll-tubes (p. 181), can be made with agar in the same manner as with gelatin. The tubes should be rolled in ordinary tap-water and not in ice-water. They should then be set aside in a cool place, in a horizontal position, in order to allow the agar to adhere firmly to the glass.

\section{Milk.}

This should be obtained as fresh as possible, before the reaction has become acid. It is then filled into sterile tubes and sterilized by exposure to steam for 30 minutes on each of three successive days. It can be sterilized in the autoclave at $110^{\circ}-115^{\circ}$ for $10-15$ minutes. Too much heat should be avoided, since this may cause an alteration of the milk. It should be remembered, however, that milk is difficult to sterilize because of the presence of resistant organisms, and, above all, because of the protection afforded by the fat. Hence, it is advisable to place the milk tubes in the incubator at $37^{\circ}$ for 1-2 days in order to make sure of the sterility of the medium,

In the method as described, whole milk is employed, and hence, on standing, a thick layer of cream rises to the surface. This can be avoided by employing centrifugated 
milk; or, if that cannot be obtained, the milk should be skimmed. In order to avoid fermentative changes, the milk should be steamed for at least 15 minutes. It can then be set aside in a cool place for the cream to rise. By means of a 100 c.c. bulk pipette the thin milk can be drawn off from the bottom and filled into tubes.

Milk is used in order to ascertain whether an organism can coagulate casein, and whether it can decompose the lactose thus giving rise to gas bubbles, or to acid products. The first two changes can be observed in the ordinary sterile milk. To detect the presence of acid products it is necessary to color the milk with blue litmus. This can be done by adding a concentrated litmus solution to the milk before sterilization; or, by adding the sterile litmus solution to the sterilized milk, by means of a drawn out pipette (Fig. 61).

\section{Modified Media.}

The ordinary nutrient agar, bouillon, or gelatin, prepared according to the directions heretofore given, may be greatly improved as nutrient media for certain organisms by the addition of glycerin, glucose, lactose, litmus, etc.

Peptonless agar.-This is ordinary agar minus the pepton. It is especially useful when it is desired to obtain spores, as in the case of anthrax.

Glycerin media.-Usually 5 per cent. of glycerin is added to bouillon, or to agar. In the case of Roux potato tubes (Fig. 34 , p. 184), the lower bulb may be filled with a 5 per cent. aqueous solution of glycerin. The addition of glycerin renders these media especially useful for the cultivation of the tubercle bacillus. "The streptococci, and the bacteria of diphtheria, glanders and pneumonia likewise thrive exceed- 
ingly well on glycerinated media. The manner in which glycerin favors the growth of the tubercle bacillus is not known, but inasmuch as this organism stores up within its cell a large amount of fat, it would seem as if the glycerin was directly utilized for the making of this compound.

Glucose media. - The addition of 2 per cent. of glucose to bouillon, gelatin, or agar greatly improves these as media for the cultivation of anaerobic bacteria. Glucose is a powerful reducing substance, taking up oxygen from its neighborhood. In this way, it is supposed to favor the growth of these bacteria. Again, certain bacteria readily ferment glucose, giving rise to acid, and to gaseous products. Hence, the addition of glucose is of great value in differentiating species. Sometimes litmus is added to glucose media in order to indicate the reaction produced by the organism. Litmus glucose gelatin is especially useful for cultivating anaerobic bacteria, with or without a special apparatus. Moreover, the vitality of these organisms are greatly prolonged in this medium.

Lactose media.-Instead of glucose, 2 per cent. of milksugar may be added to the several media. Usually, the lactose media are colored at the same time with litmus. Certain bacteria, like the typhoid bacillus, cannot ferment lactose, and hence the reaction remains alkaline and no gas bubbles are given off. On the other hand, some bacteria like the colon bacillus, do ferment lactose, in which case the litmus turns red and gas bubbles appear. Lactose media are therefore valuable as a means of differentiating organisms.

Litmus media. - The commercial litmus usually contains: a large admixture of red pigment which interferes, more or less, with the delicacy of the reaction. It is advisable, therefore, to purify the litmus. This can be done according 
to the method given in Chapter XV. Litmus is usually added to the glucose, or lactose media, or to milk in order to ascertain whether a given organism produces acid products. The medium should be colored decidedly blue. A very concentrated aqueous solution of litmus should be employed in order not to dilute the medium too much. Excessive sterilization of sugar media, which contain litmus, results in the decoloration of the latter. The color may, in part; return on standing. It is advisable, therefore, not to steam for more than 15 minutes at a time; or better still, to sterilize the litmus solution by itself and then add this, by means of a sterile pipette (Fig. 61), to the sterile nutrient medium.

Serum media.-The preparation of blood-serum, and of serous exudates will be described later. In this connection it will be sufficient to state that the addition of blood-serum from animals, or of highly albuminous exudates obtained from man, makes an agar or bouillon specially valuable for the cultivation of certain bacteria.

Thus Loffler's blood-serum which is an excellent medium for the diphtheria bacillus, consists of 3 parts of bloodserum and 1 part of a 1 per cent. glucose bouillon.

The addition of 1 part of ascitic fluid to 2 parts of agar gives a medium well adapted for cultivating the gonococcus. The fluid, must of course, be collected under sterile conditions, otherwise it should be filtered through porcelain (Fig. 66). Such a mixture can be used for plating purposes, in which case, however, the agar should be first melted and allowed to cool to about $50^{\circ}$. The ascitic fluid, or bloodserum, previously warmed to this temperature, should then be added to the liquid agar. The mixture can now be inoculated, dilutions made and Petri dishes poured as in the case of ordinary agar cultures.

Laboratory work.-One half of the class will prepare agar and bouillon according to the methods just given. One liter of bouillon is prepared first, and from this the agar and the modified media are pre- 
pared in the quantities indicated below. The other half will prepare the same media using, however, commercial meat extract instead of fresh beef. The method is as follows:

To 1000 c.c. of water add 10 g. of dry pepton (Witte's), $5 \mathrm{~g}$. of common salt, and 2.5 g. of Liebig's meat extract, ${ }^{1}$ warm till solution re. sults, then alkalize according to the directions given (p. 234). Boil for half an hour and replace the water lost by evaporation, then filter.

Take $100 \mathrm{c}$ !c. of the bouillon and fill into the small sterilized testtubes, using a small funnel, to a depth of $1 \frac{1}{2}$ inches = ordinary bouillon.

To 100 c.c. of the bouillon, add 2 per cent. of glucose and warm till dissolved. Then fill this into test-tubes $=$ glucose bouillon.

To the remainder ( 800 c.c.), add 2 per cent. of finely cut agar and boil over a flame till it dissolves. Then sediment and filter according to the directions already given (p. 236).

To 100 c.c. of the filtered agar, add 5 per cent. of pure glycerin and mix thoroughly. Test the reaction in order to make sure that it is alkaline. Finally, fill into small, sterilized test-tubes $=$ glycerin agar.

To 100 c.c. of the filtered agar, add 2 per cent.' of glucose and warm till it dissolves. Then fill into tubes to a depth of about 2 inches $=$ glucose agar.

Transfer the remainder of the agar to sterilized test-tubes $=o r$ dinary agar. The several bouillon and agar media are then fractionally sterilized (p. 162).

\section{The Incubator.}

In cultivating pathogenic organisms it is advisable to grow these, whenever possible, at the temperature of the body. In this way, they are supplied with one of the conditions under which they live in nature. A few organisms, notably the tubercle bacillus and the gonococcus, will show no growth at the ordinary temperature. In such instances, it is absolutely'necessary to use a higher temperature in or-

${ }^{1}$ Most any of the commercial meat extracts will answer equally well. A nutrient gelatin can be prepared with such extracts in the same way as agar. 
der to obtain results. The apparatus employed for this purpose is known as an incubator or thermostat. It is made of heavy copper and has double walls. The space between these, which should be as large as possible, is filled with distilled water. In form the thermostat may be oblong; square or oval. Fig. 36 shows an excellent thermostat of oval form.

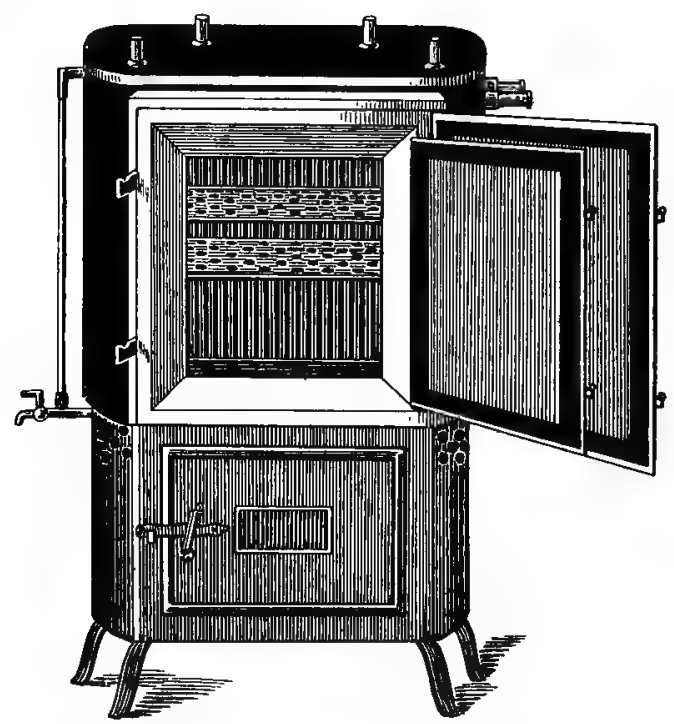

FIG. 36. The incubator or thermostat.

It is desirable that the incubator should have as much available space as possible for dishes, flasks, tubes, etc. The apparatus as ordinarily made is quite expensive. Any one with a little ingenuity can plan out a double-walled box and have an incubator made by a tinsmith which will answer all ordinary purposes. It should have a double door or lid, and it is advisable, though not necessary, that the side and top should be covered with asbestos or felt. The top should be provided with an opening which communicates with the water compartment and serves to hold a thermo-regulator. 
Another opening serves to communicate with the interior of the incubator and receives a thermometer.

A large, well-equipped laboratory should have, in addition to a number of the ordinary thermostats, at least one incubating room. That is to say, a room which is maintained at a constant body temperature. This can be heated by means of a small cylindrical stove, which is heated by a gas burner and, from which hot air pipes radiate to different parts of the room, A more constant temperature can be maintained by passing hot-water pipes around the walls of the room. The water is heated on the outside of the room by means of a suitable burner.

Thermo-regulators. - In order that a thermostat shall maintain a constant temperature, it is necessary that it shall be provided with a good thermoregulator. This instrument automatically regulates the supply of gas. The bulb of the instrument, containing alcohol or mercury, is immersed in the water between the walls of the incubator. When the temperature rises, the alcohol or mercury expand and shut off the supply of gas. In order to prevent the gas from being cut off completely, a minute opening is made in the delivery tube, thus permitting the passage of a small amount of gas; which is sufficient to maintain a small light.

The simplest mercury thermo-regulator is that of Reichert. As ordinarily made, it is the exception to find a Reichert regulator that will work properly. The minimum opening is invariably too large, and will, therefore, allow the passage of more gas than is necessary to maintain the the desired temperature. In order to obviate this difficulty, a number of modifications of the Reichert instrument have been made, and some of these answer the purpose very well. Unfortunately, the diameter of the delivery tube, as usually made, is narrow and hence such a regulator can be used only for low temperatures. 
The author's thermo-regulator ${ }^{1}$, shown in Fig. 37, is intended to overcome the difficulties mentioned. Although, apparently, more complicated than the ordinary regulator, it nevertheless is easily adjusted and is extremely sensitive. The gas tubing is attached to the strong, wide inflow and outfiow tubes ( $\alpha^{\prime}$ and $a^{\prime \prime}$ ) and is

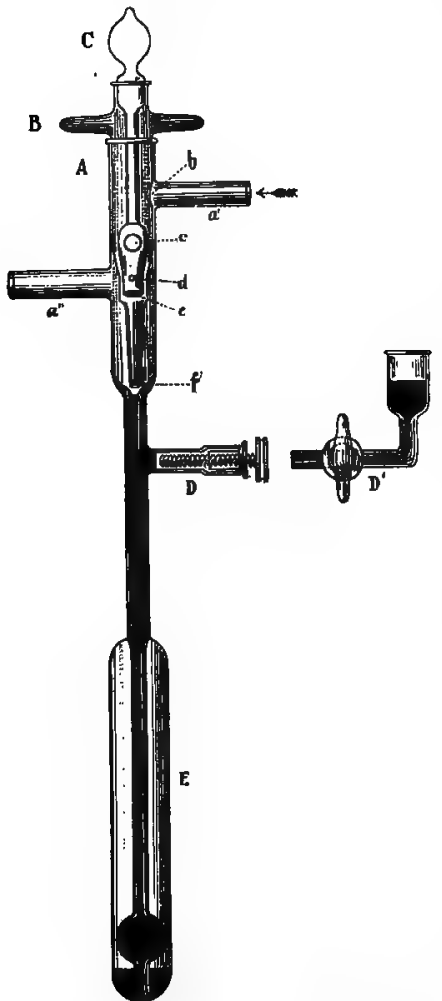

FIG. 37. The author's thermoregulator. thus independent of the regulating parts. The upper portion consists of the three parts A, B and $C$, the arrangement and working of which is readily understood from the illustration. The gas enters the inflow tube $a^{\prime}$, and passes through the opening $b$, into the interior of $B$. On account of the stopper $C$ the gas cannot leave except by passing through the opening $c$ into the narrowed portion of $B$. It leaves this delivery tube at the lower end $(f)$ and through the opening $d$. The latter supplies the minimum amount of gas necessary to keep the lamp burning. This supply can now be regulated with absolute precision. The opening $d$ is made through the parts B and C. By turning $\mathrm{C}$, the opening through this part becomes excentric to that of $B$, and hence, the minimum supply of gas is thus diminished.

The delivery tube $f$ should not be too narrow and should almost touch the bottom of $\mathrm{A}$. The first droplet of

1 Centralblatt für Bakteriologie, 23, p. 1054, 1898. This instrument can be obtained of Greiner and Friedrichs, Stützerbach i. Thüringen. 
mercury as it issues from the capillary will therefore cut down the supply of gas. The instrument is thus rendered more sensitive.

By turning part $B$ the inflow of gas can be.regulated. The instrument, therefore, permits regulation of the maximum inflow and minimum outflow of gas. Owing to the large connecting tubes ( $\alpha^{\prime}$ and $\left.a^{\prime \prime}\right)$ it is possible to have a large supply of gas, and hence, the apparatus can be used for regulating high, as well as relatively low temperatures.

The ordinary regulator, provided with a narrow cylinder or bulb filled with mercury, is not as delicate and as responsive as might be desired. This is due to the fact that the volume of mercury in the bulb is relatively small and hence the rise and fall of the liquid in the capillary, or upper tube is not marked, when only a slight variation in the temperature occurs. Consequently, it is not possible to maintain a very constant temperature with a plain mercury regulator. A variation of one or two degrees, or even more, must be expected. Moreover, the mercury in the side-arm, since it is exposed to the air, will tend to counteract the action of the remaining mercury. This will especially be the case, when there is a marked fluctuation in the temperature of the room.

The most delicate regulator is that which contains a large volume of alcohol. An increase of a fraction of a degree in the temperature will, in that case, cause a linear expansion of the mercury in the capillary tube which will be sufficient to shut off the gas supply. This fact is utilized in the construction of the lower, or bulb portion (E) of the author's regulator. As shown in Fig. 37, the capillary tube is prolonged so as to almost touch the bottom of the glass cylinder. The lower portion of the tube is provided with a small bulb which serves to prevent air from entering the cylinder when at any time the regulator is disconnected. 
Mercury fills the capillary and covers the bottom of the cylinder to a depth of about $1.5 \mathrm{~cm}$.

The filling of the regulator is not difficult. For this purpose parts $B$ and $C$ are removed, and the end of $A$, and one of the side arms $\left(a^{\prime \prime}\right)$ is stoppered air-tight. The other arm, or gas inflow tube $\left(a^{\prime}\right)$, is connected with a Chapman aspirator. The rubber tubing, leading to the aspirator, should be provided with a clamp or stopcock. The adjusting screw $D$, is then removed, and replaced by a short rubber tube provided with a clamp. The rubber tube connects with a short narrow glass tube, which dips into a beaker of absolute alcohol.

The pump is now set into action. The clamp on the regulating arm $\mathrm{D}$ is slightly opened, till the alcohol begins to pass into the tube, after which it is closed. When the air has been evacuated, as much as possible, the clamp on the tubing that leads to the pump is closed, while that on regulating arm is slowly opened. The alcohol rapidly fills the cylinder. If the evacuation is incomplete, the regulator should be inverted, and the air again exhausted, after which alcohol can be admitted as before. A few bubbles of air that may persist can be disregarded, inasmuch as they will be absorbed by the alcohol.

The next step is to introduce the necessary amount of mercury. For this purpose, the alcohol bulb of the regulator is immersed in a water-bath which has been heated to about $70^{\circ}$. The alcohol expands and fills the lower portion of $\mathrm{A}$. When $\mathrm{A}$ has been nearly half filled with the expanded alcohol, mercury is then added, and the apparatus is allowed to cool. Additional mercury is added, if need be, to prevent the entrance of air into the cylinder. The regulating arm is likewise filled'with mercury, and the screw inserted about onehalf.

At ordinary room temperature the mercury in the capillary tube should not be higher than the regulating arm. The ground surfaces of the regulator should be lubricated very slightly with vaselin, or better with a mixture of bees-wax and olive oil (1:4). The inflow tube $a^{\prime}$ is then connected with a gas-pressure regulator, or with the gas-supply direct. The out-flow tube $a^{\prime \prime}$ is connected with a microburner. A suitable cork, or rubber stopper, is slipped over the tube of the regulator, which is then placed in the thermostat, so that the bulb is immersed in the water. The adjusting screw $D$ is turned outward, if necessary, so as to allow the gas to escape from $f$. The gas is turned on and lighted. As the temperature, rises the alcohol ex- 
pands and forces up the mercury, which tends to close up $f$. The adjusting screw should be again turned outward, if need be, to prevent this from happening. When the temperature in the incubator has reached the desired point, for example, $37^{\circ}$, the adjusting screw should be turned in till the mercury just closes the opening $f$, of the delivery tube. The minimum opening now supplies the gas to the burner. This minimum supply should now be reduced, as much as possible, by turning $\mathrm{C}$, without causing the flame to shoot down inside of the burner. As soon as the temperature drops, the mercury contracts, and thus allows gas to escape from $f$. In a few minutes the increase of heat causes the mercury to again shut off the main supply of gas.

Inasmuch as alcohol boils at $78^{\circ}$, the alcohol regulator should not be used to maintain a temperature above $70^{\circ}$. For temperatures above this point the bulb should be filled entirely with mercury, or the alcohol may be replaced by air or by anilin, which boils at $185^{\circ}$. The ordinary adjusting screw cannot be used when a high temperature is to be maintained. For such purposes the regulator should be provided with a glass stop-cock, which connects with a small cup filled with mercury (Fig. $37 \mathrm{D}^{\prime}$ ). When the gas-supply is turned off, as in the case of a water-bath or oven, this stop-cock should be opened.

The adjustment of the ordinary mercury regulator is similar to that just mentioned. Frequently, the thread of mercury is broken and air is thus introduced into the capillary tube. This can be removed by inserting a thin platinum wire, or a drawn out capillary glass-tube, and gently turning it about. Care should always be taken to remove the air that may be present in the arm $D$.

Obviously, a continual fluctuation in the temperature of the incubator will exist. A good alcohol regulator, however, should not allow a variation of more than one or two-tenths of a degree. The greater the volume of water in an incubator the more constant will be the temperature. The latter, in that case, will be likewise more independent of any variation in the pressure of the gas. Frequently, the pressure of the gas is so variable that it is not possible to maintain a perfectly constant temperature in an incubator, even when this is provided with the best form of regulator. This difficulty can be obviated by employing a gas-pressure regulator. 
Gas-pressure regulator.-The apparatus shown in Fig. 38 was designed by Murrill ${ }^{1}$ and is used in this laboratory. In cheapness, simplicity and effectiveness it excels any of the ordinary gas-pressure regulators.

As indicated, the gas enters through a valve which is connected with a gas cylinder or float. The gas passes

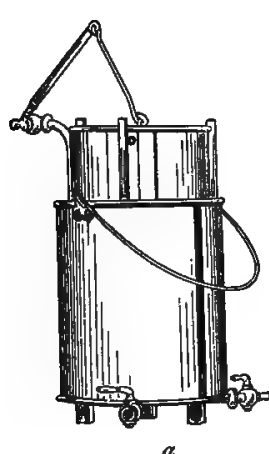
into the interior and raises this cylinder. The greater the pressure of gas the higher will the cylinder rise. As it does so, it turns off the valve, more or less. completely. The cylinder then falls, and hence gas is readmitted. The pressure at which the gas is delivered can be regulated at will by placing suitable weights. on the cylinder. In this laboratory, the float is weighted till the gas is delivered at a pressure of $40 \mathrm{~mm}$, as observed in a
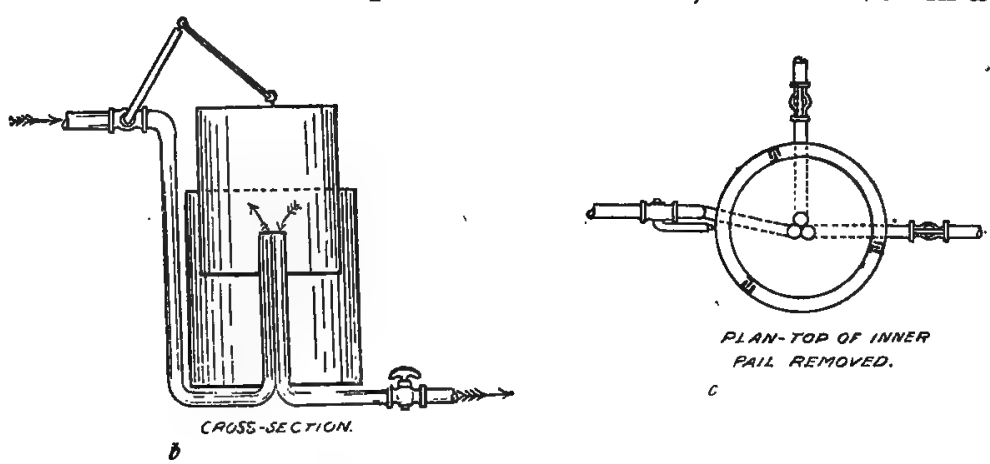

FIG. 38. Murrill's gas-pressure regulator.

$\mathrm{U}$ tube containing water. The cylinder may be floated in water, but, inasmuch as this is liable to evaporate, it is better to use liquid paraffin. The apparatus is provided with two outflow tubes, which are to be connected with thermo-regulators. It is of convenient size, being only 6

1 Journ. of Applied Microscopy 1, p. 92, 1898. Centralblatt für Bakteriologie 23, p. 1056, 1898. 
inches in diameter. By means of the gas-pressure and temperature regulators, which have been described, it is pos. sible to maintain a constant warmth even if the incubator is placed in a room where the ambient temperature is liable to considerable variation.

Micro-burners. - The outflow tube of the thermoregulator should be connected with a suitable burner placed underneath the incubator. An ordinary Bunsen burner may be used in some instances, but, as a rule, it gives off too much heat, and, moreover, the flame is' liable to "shoot back" when the supply of gas is low. A very good micro-burner can be obtained by unscrewing the tube of the Bunsen burner. The gas now burns with a luminous flame, but this does not interfere with its usefulness. A cylinder of copper wire-gauze may be placed around the flame to prevent currents of air from extinguishing it.

The small narrow Bunsen burner, so-called microburner, can be used to better. advantage than the ordinary form. Small jet, luminous microburners can also be used, but they

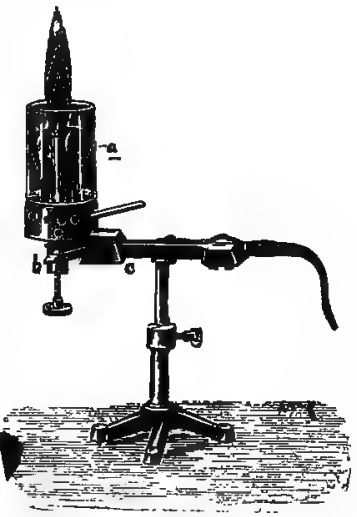

FIG. 39. Koch's safety burner. $a-$ Metal spiral, one on each side of flame; $b$-Arm moved by the spirals; $c-$ Weighted lever arm of stop-cock, supported by $b$. possess no advantage over that obtained in the manner described above.

The lamp usually employed under an incubator is known as the safety burner. This is intended to shut off the flow of gas, if for any reason the light should go out. The gas is thus prevented from escaping into the room, and danger of subsequent explosion is thus avoided. Several forms of the safety burner have been devised, but the one which is used perhaps the most often is that of Koch. Obviously, the safest procedure is to place the incubator, or 
other apparatus which is heated day and night, within a good hood.

The Koch safety burner is shown in Fig. 39. It is a small Bunsen burner provided with two steel springs. These are heated by the flame and expand. The motion, thus induced, is communicated to a small arm which can be set, so that, when the burner is lighted, it just supports the weighted lever. The latter is connected with a valve. When the light goes out the springs cool and draw the small arm from under the weighted lever. This then falls and shuts off the flow of gas. The tube of the Koch burner is not infrequently wider than it should be, because the minimum supply of gas from the thermo-regulator is too small to keep the light burning above the burner. In that case, the flame drops down into the tube. This defect can be easily remedied by inserting a short piece ( $/ / 4 \mathrm{in}$.) of brass tubing just inside of the end of the burner. The flame is thus made narrower and is, therefore, less liable to drop down.

Thermometer.-Any kind of a glass thermometer can be used in connection with a thermostat, but it is preferable to have one which would indicate fractions of a degree. The thermometer ustually employed is graduated from 0 to $60^{\circ}$ and reads tenths of a degree. A Kappeler maximum and minimum thermometer is, at times, very useful to ascertain the limits of variation in the temperature. 
CHAPTER X.

RELATION OF BACTERIA TO DISEASE, METHODS OF INFECTION AND EXAMINATION.

By the application of the gelatin plate method, or its several modifications, it is possible to readily separate a given organism from the other forms which may be present, and to obtain thus a pure culture. The isolated colony as. it develops on a plate, furnishes the first pure cultivation, since it is derived from a single micro-organism. Transplantations made from a colony, if made with proper precautions, in turn yield pure cultures, or growths containing but a single species. Tube cultures can thus be made in gelatin, bouillon, agar, blood-serum, potato, etc., and where it is desired, as in the study of the chemical products of bacteria, flask cultures can be made.

\section{Relation of Bacteria to Disease.}

It is evident that in order to demonstrate that a given bacterium is the cause of a certain fermentation, or of the production of a pigment, or of phosphorescence, etc., it is necessary that it should, first, be isolated and obtained in a pure culture. And that, second, a pure culture of the organism grown under the same or under similar conditions, should give rise to the original phenomenon-the production of the same fermentation, pigment, phosphorescence, etc. Having thus demonstrated that a given organism is the cause of a certain change, it does not follow that this organism has the exclusive power to do this. 
Thus, in alcoholic fermentation, the yeast plant is commonly said to be the cause, but, as pointed out on p. 96, a large number of different species of yeasts are known which have this power. Moreover, many bacteria and moulds possess similar properties. Again, a considerable number of bacteria have been shown to be capable of inducing acetic, lactic, butyric acid fermentations, the ammoniacal and hydrogen sulphide fermentations of urine, the phosphorescence of sea-water, etc.

The most that can be said of a given organism which induces a certain change, therefore, is that it is the cause in that particular instance. The possibility of other organisms giving rise to the same change, or effect, or to the same chemical products must be conceded, and the demonstration of the relation of an organism to such a change rests with the proof that it is $a$ cause.

Just as there are organisms which induce changes in dead animal or vegetable matter, there are others which are capable of inducing similar changes in living animals and plants, thus living at the expense, and, frequently, to the detriment of the host. The infectious diseases in man, animals and plants, possess as an essential characteristic the property of transmissibility. They are the result: first, of infection that is the entrance of a specific micro-organism; and second, of intoxication due to the poisonous products elaborated by that micro-organism.

The poisonous chemical compounds, which are thus made, may produce the symptoms and the changes observed in the infectious disease. They are the cause of those symptoms and changes, in other words, of the resulting intoxication. But, they are not the cause of the disease itself, since the symptoms and changes thus obtained, are not transmissible from one individual to another. Chemical substances have no power of multiplication and the effect observed is, therefore, directly proportional to the amount of the chemical compound introduced. Micro-organisms, however, 
have the power of multiplication, and the introduction of a minute amount, even a single cell, may bring about entirely disproportionate results. The invading organism is, therefore, the cause of the disease, since it imparts the characteristic property of transmissibility, and, through the action of its chemical products, produces the symptoms and effects of that disease.

In order to positively demonstrate the causal relation of a micro-organism to a given disease, it is necessary to meet the following requirements, commonly known as the four rules of Koch:

1.-The organism must be present in all cases of that disease.

2.-The organism must be isolated and obtained as an absolutely pure culture.

3.-The pure culture of the organism when introduced into susceptible animals must produce the disease.

4.-In the disease thus produced, the organism must be found distributed the same as in the natural disease.

To these four requirements, a fifth may be added, namely: That the chemical products of the organism must produce the characteristic symptoms and effects of that disease.

The demonstration of the constant presence of an organism in a disease is accomplished by making hangingdrop examinations of the fluids or exudates of the body; by making stained cover-glass preparations, or by stain. ing sections of the tissues and organs. Frequently, the direct detection of the organism is difficult, owing either to its scarcity, or to the absence of definite characteristics. In such cases artificial culture, or the animal experiment, will usually prove the presence of the organism. In the advanced stages of some diseases the germs 
may be supplanted or outgrown by other organisms-secondary infection.

The value of artificial culture, in suspected cases of disease, is seen in diphtheria. On the other band, the pus, sputum, or pleuritic fluid from a diseased person may not show, on direct microscopic examination, any sign of the tubercle bacillus. Yet, if such material is injected into a guinea-pig, it may produce tuberculosis and death. The microscope failed to detect the few bacilli present but the living body afforded these same cells an excellent medium for growth, and, as a result, they rapidly multiplied and eventually brought about the death of that animal. An examination of the altered tissue, in such an animal, will reveal the presence of myriads of tubercle bacilli. Since living cells are derived only from cells of their own kind, it follows that these organisms were present in the original diseased condition.

The mere fact that an organism is constantly present in a given disease, does not prove that it is the cause. It certainly is strong presumptive evidence that the organism does bear a causal relation, but at the same time the possibility must be admitted that it may be an accompaniment, or even a consequent of that disease. The latter view, it is scarcely necessary to note, would imply the existence of spontaneous generation. To complete the chain of evidence, it is necessary, therefore, to obtain the organism in an absolutely pure culture, free from any other organism or foreign chemical poison. Subsequent inoculation of susceptible animals with such cultures must reproduce the disease. Whenever possible, an animal should be selected which is naturally susceptible to the disease. An animal that is not subject to the disease can hardly be expected to reproduce the typical affection on inoculation with the organism.

The isolation of the organism and the preparation of pure cultures, is accomplished by means of the gelatin 
plate method, or by its modifications. The isolated colony which develops on a plate, is derived from a single cell and is, therefore, a pure culture. Transplantations from such a colony, when properly made into tubes of gelatin, agar, or bouillon, in turn give rise to pure cultures. Subsequent transplantations, from tube to tube, can be made as often as may be desired, or as may be necessary. Each growth thus obtained is called a generation.

In many cases, as in tuberculosis, anthrax, and hog cholera, the organisms have thus been carried through several hundred consecutive generations without impairment of pathogenic properties. In other instances, as in glanders, the organism does not find in our artificial media the conditions favorable for its growth, and, as a result, it undergoes a physiological alteration so that the cultures become less and less active, till finally they cease to have any effect on animals. This change in the physiological properties of an organism-known as attenuation -is frequently accompanied by a corresponding decrease in the vitality of the growth, so that, when the virulence is wholly lost, the culture soon dies out. Sometimes, however, the organism adapts itself to the artificial media, and continues to grow, although with diminished pathogenic properties. The phenomenon of attenuation, it should be understood, is not confined to the pathogenic bacteria. Corresponding changes in the activities of many non-pathogenic bacteria are constantly being met with. Thus, a phosphorescing bacillus may emit a strong light when first obtained, but in a short time it may lose this power. The B. ruber of Kiel usually give an intense red growth on potato, but it is not uncommon to meet with it as a colorless growth. Again, a given jorganism may rapidly coagulate milk, but after it has been cultivated on artificial media it may lose, more or less completely, the power of altering the composition of milk. These illustrations teach, that when bac- 
teria are under the very best conditions of environment they possess a maximum physiological activity. They may give rise to intense poisons, pronounced pigments, or other characteristic chemical products. When, however, these conditions are altered and the environment becomes unfavorable, the bacteria are likely to respond at once by a corresponding lessening of vitality. The chemical products which were formed, when the organism was in a vigorous, healthy condition, are now diminished in amount and may even be replaced by others. Attenuation, therefore, is a phenomenon which is common to all bacteria, although the term was first employed in connection with the loss of virulence of certain pathogenic organisms.

The above four rules have been fully complied with in a large number of infectious diseases. In others the first two rules are satisfied but the third is not, owing to the difficulty of obtaining a susceptible animal. Again, the first rule may be the only one complied with, as in leprosy, where the isolation of the organism has not, thus far, been unquestionably successful. And again, a large number of infectious diseases remain (such as small-pox, scarlet fever and measles) in which even the presence of a specific organism has not been definitely shown.

It is evident that while a great deal of information has been gained during the past 15 years in regard to the causation of disease, there still remains a great deal of work to be done. Enough facts have been gathered to clearly demonstrate the importance of the new science of bacteriology to medicine, surgery and hygiene. What was once called the "germ theory of disease" has ceased to be a theory since it has been reduced to incontrovertible facts. That micro-organisms are the causes of disease has been demonstrated.

Although many of the infectious diseases have been shown to be due to bacteria, it must not be forgotten that 
other low forms of plant and animal life possess similar properties.

Certain fungi are known to live as parasites on the higher animals. Thus, some skin diseases of man are due to this class of organisms. These may be grouped together under the head of fungous diseases.

Again, certain uni-cellular animal organisms, belonging to the group of protozoa, invade the body and give rise to disease. Malaria is due to one of these animal parasites. The diseases due to these organisms can be designated as protozoal.

The bacterial, fungous and protozoal affections are included in the large group of infectious or microbic diseases. It has been stated that the cause of certain diseases, like measles and scarlet fever, is as yet wholly unknown. The cause, when discovered, may belong to one of these three groups, but not necessarily so. It is customary to consider the bacterial cell as the smallest living mass of matter. It is difficult, indeed, to conceive of the existence of organized beings smaller than the "infinitely small bacteria." And yet, the fact that all known methods of study have failed to discover the cause of certain diseases would indicate that we must look to an undiscovered group of microbes which must perforce be smaller than any known bacteria.

The first step has already been taken in the direction of opening up this new field of study. By means of the collodium sac method, which will be described in Chapter XIV., it has been shown that the cause of pleuro-pneumonia in cattle is an organism so small, that, with the best microscope and a magnification of 2000 diameters, it is still impossible to make out its form. Evidently, this is the first of a new group of microbes.

Pathogenic micro-organisms are of first importance in so far as they produce disease in man and in the lower animals. The fact, however, should not be lost sight of that similar organisms produce disease in plants. A large 
number of bacterial and fungous diseases of plants are known at the present day. The various species of the vegetable kingdom, just as man and the lower animals, are subject to their peculiar infectious diseases.

\section{Methods of Infection.}

As indicated in the preceding part, the detection of an organism in every case of a given disease does not, in itself, constitute a proof that it is the cause of that disease. It is necessary to carry the investigation farther in order to furnish an indubitable proof. The second rule of Koch requires that the suspected organism shall be isolated, in other words, obtained in a condition of absolute purity. If the pure culture of the suspected organism, isolated according to the methods heretofore described, on inoculation into susceptible animals reproduces the characteristic symptoms and pathological lesions of that disease there can be no escape from the conclusion, that the organism tested is the causal factor of the disease in question.

Extreme care must be taken in order to obtain an absolutely pure culture. Likewise, the utmost care must be employed, when making the inoculations of animals, to prevent the accidental introduction of foreign organisms. Careless inoculation may give wholly misleading results. All operations on animals, even the most trivial, should be carried out under as nearly sterile conditions as possible. Every instrument employed should be sterile, and, while it is not possible to render the hands of the operator and the skin of the animal as free from organisms as in the case of an instrument, yet nothing should be left undone to decrease the number of bacteria present and to lessen thus the danger of mixed infection.

The experimental methods of infection are numerous. Some are exceedingly simple, whereas others entail a more 
or less difficult surgical operation. They include, moreover, all the known avenues of infection whereby man and animals contract disease. The several methods employed will now be described as briefly as possible. The guineapig, rabbit and white mouse are the animals employed most often. The white rat, house mouse, pigeon, chicken, dog and others are used less frequently.

1.-Cutaneous application.-Bacteria cannot penetrate into the interior of the body through an unbroken skin or mucous membrane. Nevertheless, a person may become infected, without any visible injury, as sometimes occurs when making operations on the living, or when making post-mortem examinations of the dead. In such cases, the existence of microscopic abrasions or fissures, or an uncon. scious puncture is looked upon as an explanation of the infection. The organism to be tested in this manner is rubbed into the skin as much as possible with the aid of some fat or vaselin. In the study of pus-producing organisms results have been obtained by this method which are strictly analogous to conditions observed in man. The production of boils or felons has thus been brought about experimentally.

2.-Subcutaneous application or injection.-By application is meant, in this case, the introduction of the organism through an opening in the skin: This is made by means of a lance, or pair of scissors. Through the small nick, thus made, the platinum wire or other instrument, laden with the organism, is inserted into the subcutis and the material is spread about as much as possible. This method may be considered as analogous to the ordinary wound infection in man and animals. A puncture with a sharp piece of wood, glass or metal enables the introduction of the organisms that may be present on such a surface. Tetanus, or lockjaw, is a striking illustration of this method of infection. 
In the above procedure, the hair is removed from over the desired area by means of scissors. The spot is then rubbed thoroughly, with a cloth or sponge soaked in mercuric chloride (1-1000) or lysol (2 per cent.). This skin is then raised by the fingers, or with forceps, and the small nick or incision is made. This should extend through the skin. It is advisable to insert the lance or a blade of the scissors under the skin, thus making a pouch. Guinea-pigs and rabbits are inoculated over the side. The animal is held down on the table by an assistant. Mice and rats are inoculated over the root of the tail. The tail of the animal is seized with a pair of rat or crucible forceps (Fig. 46 b, p. 273) and drawn over the edge of the jar. The weighted wire-gauze cover, of the battery jar in which it is confined, is moved slightly to one side so as to allow the tail and lower portion of the body to be drawn out. The tail is held by the left hand while the opening and subsequent inoculation are made with the right.

Subcutaneous injection is practised by means of a sterile syringe. For this purpose the Koch syringe has been used extensively, but it cannot be

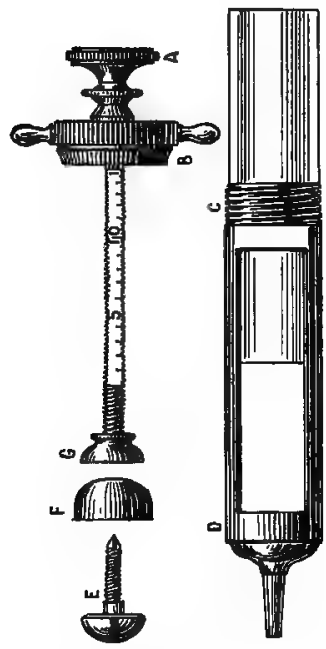

FIG. 40. Adjustable syringe. $\mathrm{F}-\mathrm{Rubber}$ piston. said to possess any marked advantage over a good hypodermic syringe, and, it certainly is less convenient. It is advisable to employ a syringe with an adjustable rubber piston. The stem of the piston should be graduated to indicate c.c., and should have a set-screw, thus enabling the operator to inject the amount desired with the least trouble or uncertainty. The RouxCollin syringe, or one such as is shown in Fig. 40, is the most durable and useful of its kind.

The syringe should be sterilized in boiling water in a water-bath. An enamelled stewing-pan heated by a Fletcher radial burner (Fig. $41, a, b$ ) is an extremely useful accessory in the laboratory. The syringe should not be placed direct in the boiling water. The latter should be drawn up into the syringe and expelled several times in 
succession. In this way, the syringe is heated up evenly. It is then partially filled with water, placed on the syringe holder (Fig. $41 \mathrm{c}$ ), and immersed in the boiling water. An exposure for three to five minutes under these conditions will be sufficient. The holder with the syringe is then removed and the latter is allowed to cool, after which the contained water is expelled. The bacterial fluid is now drawn up into the syringe through the needle. If any air is present this should be expelled, by holding the syringe in a vertical position and applying gradual pressure to the piston. Before doing this, it is advisable to pass the needle through a piece of sterile filter paper, slipped over the end of a wide test-tube. The latter receives the few drops of liquid that are expelled at the same time with the air.

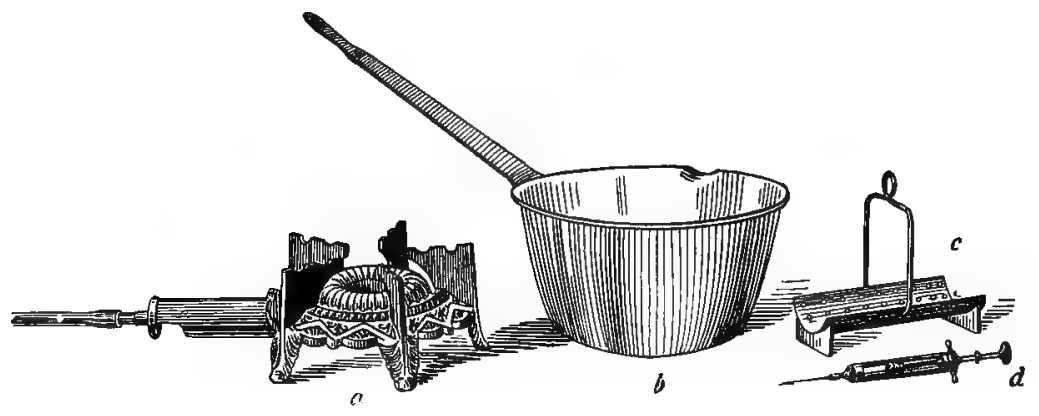

Fig. 4I. $a$-Fletcher radial burner; $b$-Enameled stew-pan or water-bath; $c-$ Syringe holder; $d$-Syringe.

After using the syringe, it should be rinsed with boiling water in the manner mentioned, and then immersed in the boiling water and sterilized. In order to prevent rusting of the needles, these should be kept in a 10 per cent. solution of borax.

When it is desirable to inject a relatively large quantity of liquid into an animal, or to inject a number of animals in succession with the same liquid (as in the immunization of horses), then an arrangement similar to that shown in 
Fig. 42 is made use of. This apparatus can be readily improvised out of an ordinary 250 c.c. measuring cylinder.

Usually, bouillon cultures are employed for the purpose of injection. Occasionally, however, the bacterial growth is on a solid medium, like agar or potato. In that case, bouillon or sterile water should be introduced into the tube by means of a drawn out tube pipette (Fig. 61). The

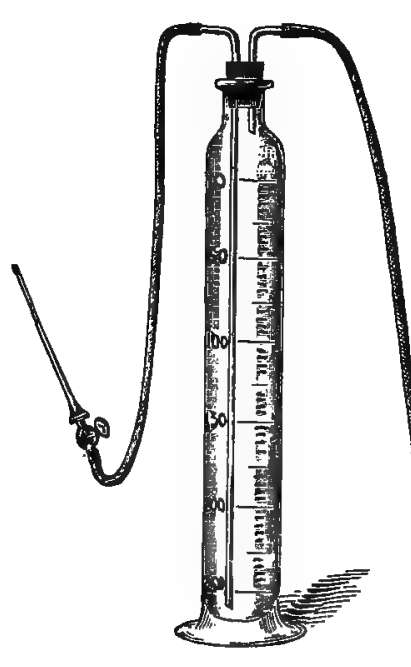
growth is then thoroughly stirred up in the liquid by means of the end of the pipette. The suspension is then drawn up into the tube, and transferred to a sterile Esmarch dish, or to a small sterile conical test-glass (Fig. 43). In the case of a bouillon culture, this is transferred directly to the sterile vessel. The neck of the tube, after withdrawal of the cotton

FIG. 42. Apparatus for injecting large quantities of liquid.

plug, must, of course, be heated in the flame before pouring out the culture.

When injecting small animals care must be taken that the needle does not pass through the abdominal wall into the peritoneal cavity. A fold of the skin should be raised, and the needle inserted while the syringe is held in a position almost parallel to that of the body. A successful subcutaneous injection will show a swelling over the place of inoculation.

The injection is made most conveniently over the abdominal wall, inasmuch as the skin is softer there than elsewhere. The rabbit 
or guinea-pig should be held on its back by an assistant. The head and front legs should be held in the palm of the left hand, while the hind legs are held down firmly with the right. A very convenient way of holding a rabbit is for the assistant to take it by the ears and hind legs and stretch it over his hip or knee.

Guinea-pigs may be inoculated without the aid of an assistant by placing these within an ordinary large graduate cylinder. A cylindrical holder of copper, as described by. Voges, is preferable to the use of a glass one. Fig. 44 illustrates the manner of using these holders. It is well to have 2 or 3 of these cylinders of different diameters, so as to fit guinea-pigs of various size. The length should be about 18 to $20 \mathrm{~cm}$. The diameters may be 6 and $8 \mathrm{~cm}$., respectively. The longitudinal slit should be about $1 \mathrm{~cm}$.,

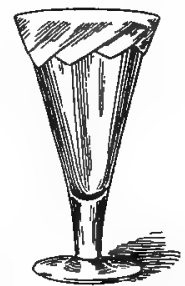

FIG. 43. Sterile test-glass. wide and $10 \mathrm{~cm}$. long. Equally useful holders can be made by boring holes, of the size given, in a block of wood.

The mouse or rat is usually picked up by the tail with a pair of rat forceps (Fig. $46 \mathrm{~b}$ ) and set on the table. A folded piece of cloth is dropped on its head, which is then seized between the thumb and finger. The tail and hind legs are held by the other hand. When holding the animal thus, the pressure should be applied to the head and not to the neck or chest. A better procedure than the above, which, moreover, dispenses with the need of an assistant, is to grasp the animal by the nape of the neck in a Collin pressure forceps. The forceps are now suspended from a hook or from a retort stand, and the tail and hind legs grasped in the fingers of the left hand. The animal is thus placed on a slight stretch and the inoculation is made with the right hand.

3.-Intravenous injection.-The direct introduction into the circulation of a bacterial suspension or toxin is frequently practised. This method is especially valuable in the immunization of animals. Inasmuch as the liquid is introduced directly into the blood current, and is thus rapidly distributed throughout the body, it follows that the results 
obtained by this procedure are more rapid and are far more dangerous than a subcutaneous injection.

The injection is made by means of the syringe or injection apparatus, described above. Special care must be

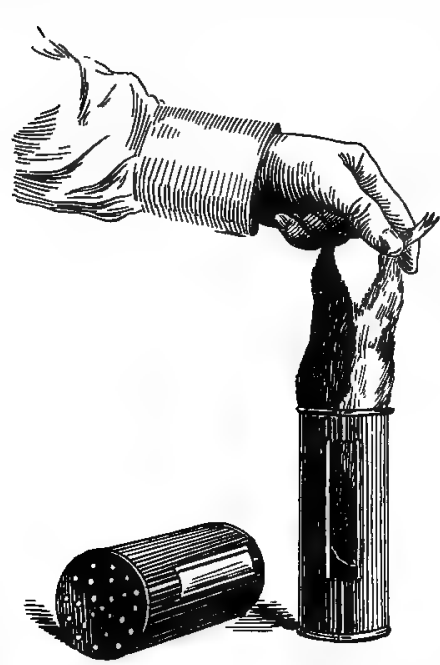
FIG. 44. The Voges cylindrical holder, practice the operation can be for guinea-pigs. taken to see that all air-bubbles are expelled from the syringe before it is used. Air in itself is not dangerous, provided it is not injected rapidly and in large bubbles. It is well, therefore, to begin the injection as slowly as possible.

The rabbit is the animal employed most often for this method of injection. This is due to the fact that the marginal branch of the posterior auricular vein is easily entered with the needle. With a little done almost as expeditiously as an ordinary subcutaneous injection. It is well to select an animal with white ears, and in which the marginal vein is fairly large. Occasionally this vein is very narrow and it is then extremely difficult to enter. It is advisable, moreover, to use a sharp new needle.

The animal is placed on the table before a window. The assistant holds the animal down gently but firmly. One hand rests over the pelvis, the other covers the head and holds the front legs. There is really but one vein in the rabbit's ear which can be used for the purpose of injection. The large, middle veins of the ear are imbedded in loose connective tissue, and hence, readily roll away from the needle. The posterior marginal vein is narrow, but it is imbedded in such a manner that it cannot move away. Hence, little difficulty is experienced in penetrating this vein although it may seem to be narrower than the needle.

By means of a pair of scissors, preferably bent, the hair is removed from the surface over this marginal vein. A piece of cotton, 
or cloth, dipped in warm water, is then rubbed over this surface. This usually makes the vein more prominent. A small clamp can be applied over the lower part of the vein to cause it to distend, but this if by no means necessary. The ear is then bent over the index finger, thus supporting that portion of the vein which it is desired to enter. The syringe is held almost parallel to the course of the vein. $\Lambda$ s the needle enters the tissue one meets with the expected resistance, but the moment it enters the vein, this resistance disappears. After a very few trials, the student is able to tell whether or not the needle has entered the blood-vessel. It should now be inserted into the vein about a quarter of an inch, and the thumb then applied over the point of entrance. The needle and ear are thus held between the thumb and forefinger. Care must be taken to hold the needle parallel to the course of the vein. The liquid can now be gradually injected, removing first of all the clamp from the base of the ear, if one has been used.

As the liquid flows into the vein it drives the blood before it, and hence, only a colorless stream will be seen. If there is any resistance to the pressure on the piston, and, especially if a swelling forms at the point of inoculation, it is evident that the vein has been missed. The needle should therefore be withdrawn, and the attempt repeated a trifle lower down the vein. It is well always to make the first attempt at penetrating a vein as high up as possible. When the needle is withdrawn, the place of inoculation should be held a few minutes between the fingers, or a clamp should be applied to prevent hemorrhage.

When the amount of the liquid to be injected is large, it is better to make the injection in that case into the jugu. lar vein. This method requires a little more time, and greater precaution than the preceding. For this purpose the animal must be fastened down on some form of a holder and anesthetized.

The rabbit holder of Czermak is frequently employed for fastening down rabbits and guinea-pigs. The Latapie holder shown in Fig. 45, is admirably adapted for laboratory purposes. It can be used equally well for rabbits, guineapigs, chicken or pigeons. The flexed joint is placed over the cross-piece of the clamp, and the ring is then bent over till it is held by a spring catch. 
The hair is removed from the neck by means of a pair of bent scissors. The part should then be shaven clean and washed with an antiseptic. A median incision is made along the neck with a sterile scalpel, and the skin is loosened from the subcutis by using the handle of the knife, or the fingers. The external jugular vein which lies on the side, covered by subcutaneous tissue, is now exposed as carefully as possible. The size of the vein is such that no difficulty will be experienced in finding or entering it. A ligature should then be applied to the vein and the wound in the skin, sewed up as neatly as possible. A few tufts of cotton should be spread over the incision, and then moistened with collodium. When this has dried the animal can be released.

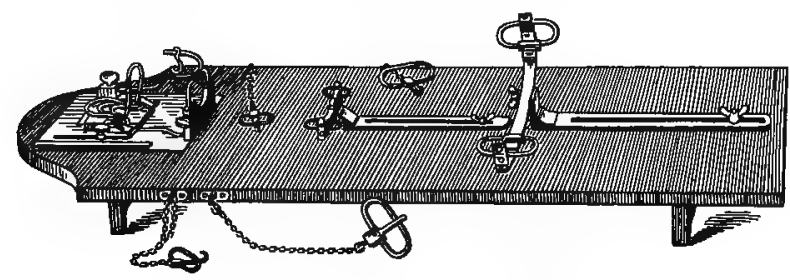

FIG. 45. Latapie's animal holder.

In the above method of inoculation, every possible precaution must be taken to prevent accidental infection. The hands, as well as the neck of the animal, must be thoroughly disinfected. All instruments, ligatures, etc., must be sterile. The instrument sterilizing case, shown in Fig. 48 , p. 275 , is used for this purpose.

Intravenous injection is practised frequently during the immunization of horses against diphtheria, pest, etc. The operation as made on the horse is relatively as easy as the injection into the veins of the ear of a rabbit. An attendant should compress the jugular vein with the thumb, while the operator cuts away the hair over the vein. This cut area is then thoroughly rubbed with lysol, or some other disinfectant. The skin is first pierced with a lance, and then the trochar is inserted as nearly parallel to the vein as possible. After withdrawal of the trochar, the opening should be compressed by a finger, and eventually it may be coated with collodium. 
4.-Intra-peritoneal injection.-This method of experimental infection is employed as frequently as the subcutaneous one already described. When organisms are introduced directly into the peritoneal cavity, the results of infection rapidly follow. The animal may die in 12 hours or less. Moreover, it should be noted that many bacteria, which will not kill when injected subcutaneously, are invariably fatal when introduced into the peritoneal cavity. A good example of this is seen in the colon bacillus.

The animal to be injected is placed on its back, or, in the case of the mouse or rat, it is suspended in the manner described on p. 265. A fold of the skin is raised and the needle introduced at an angle of about 45 degrees. The fact that the needle enters the peritoneal cavity is recognized by the absence of resistance to the needle and by the absence, at the point of inoculation, of a local swelling which would indicate that the fluid has entered the subcutaneous tissue. As a rule, there is very little or no danger of injuring the intestines. In making injections it is advisable to cut away the hair over the point of inoculation, thus marking as it were, the place of operation. It is hardly necessary, as a rule, to attempt a thorough disinfection of the skin previous to making an injection.

Intra-peritoneal injection into a large animal, as the horse, is made by means of a trochar. The animal is held in the standing position and the trochar introduced through the abdominal wall, after previously.cutting the skin with a lance. A point 4 or 5 inches in advance of the anterior iliac spine is selected as the site of inoculation.

In certain cases it is desirable to observe the disease process in the peritoneal cavity, or to introduce solid masses into this cavity. In that event it is necessary to make a laparotomy, in which case the utmost precaution to prevent accidental infection must be observed. This operation is resorted to whenever the collodium sac method 
is employed. The necessary directions will be found under that head (Chapter XIV).

5.-Intra-pleural injection.-This method is sometimes employed, though rarely. Great care must be taken to prevent injury to the lungs or heart. The introduction of a large quantity of liquid is in itself dangerous. The needle is introduced into the right pleural cavity at some distance from the median line. The results of infection are brought on rapidly, as in the case of intraperitoneal injection.

6.-Infection of the anterior chamber of the eye.-This method has been followed with excellent results in the study of tuberculosis. The pathological changes induced can be observed from day to day. The operation itself is simple. The eye is first anesthetized with cocain. An incision is made, by means of a very narrow scalpel, at the juncture of the cornea and sclera. Through the opening thus made, the material to be tested is inserted into the anterior chamber. Instead of making an incision the bacterial fluid can be injected by means of a syringe.

7.-Infection of the lymphatics. - This is accomplished easily by injecting directly into a testicle. The infection spreads rapidly through the neighboring lymphatics and as a result marked alterations result in a short time.

8.-Intra-cranial injection. - This is the method employed by Pasteur and his pupils in producing rabies in rabbits and other animals. The procedure will be described in Chapter XIV.

9.-Infection along the respiratory tract.-This may be brought about (1) by the inhalation of the finely divided organisms; (2) by injection into the trachea. The former method is analogous to the usual mode of infection in 
tuberculosis. An atomizer is employed to bring the organisms into a finely divided condition. It need hardly be stated that this method is extremely dangerous to the operator. In fact, it is the most dangerous procedure in the entire bacteriological technique, and, for that reason, it is but rarely resorted to.

The intra-tracheal injections have been extremely valuable in the study of diphtheria. The method is simple, and is carried out in much the same way as when making injections into the jugular vein. The animal is fixed on its back and anesthetized. After removal of the hair, and after local disinfection, a median incision is made. The trachea is then gradually exposed, making use of a grooved sound. When fully exposed, it is well to pass the sound under the trachea, thus placing this on the stretch. The needle is then easily inserted between the cartilaginous rings. A few scratches should be made with the needle on the inside of the trachea, in order to give the organism a foot-hold. The amount of fluid injected should be relatively small. Frequently, the material is introduced into the trachea by means of the platinum wire. In that case, one or two tracheal rings are cut and the mucous membrane is slightly injured by scratching, or by means of a hot wire. The wound is then closed, employing the usual precautions against accidental infection.

10. - Infection of the alimentary canal.-A large number of diseases of man and animals are due to infection along this tract. Hence, in establishing the relation of suspected organisms to such diseases, it is desirable to do so by following, as nearly as possible, the natural mode of infection.

The usual procedure is to administer the bacterial fluid with the food or drink. When the animal refuses to partake it is necessary to employ a stomach-tube. A rubber tube, about five mm. in diameter, will answer this purpose. It should be connected with a small funnel. In order to 
prevent the animal from biting the tube, this should be passed through a perforated piece of wood, or through a cork-borer which is held firmly in the mouth. Any desired amount of liquid can thus be administered without difficulty.

In experiments with the bacteria of cholera and typhoid fever, it has been the practice to first introduce into the stomach some sodium carbonate solution. This was done in order to neutralize the free hydrochloric acid of the stomach, which otherwise might destroy the bacteria introduced. Again, it was customary to inject opium into the peritoneal cavity, in order to retard or paralyze the peristaltic action of the intestines. The bacteria were thus given every opportunity to develop in the intestinal canal.

In his classical studies on cholera, Metschnikov showed that the above precautions can be done away with, and better results obtained by feeding milk suspensions of the germs to new-born rabbits or guinea-pigs. The intestines of the new-born animal are free, at least for a short time, from bacteria. The experimental organism, when introduced under these conditions, is enabled to develop unhindered. The intestinal flora of the adult individual may, in some cases, favor the growth of the invading organism whereas, in other cases, it may exert a positive inhibiting action-microbic association.

Occasionally, it may be desirable to avoid passage of the material through the stomach. In that case the material can be injected directly into the intestines, but to do this properly necessitates a laparotomy. Such intraduodenal injections are very rarely made.

\section{Observation of Infected Animals.}

The inoculated animal should be placed by itself, in a suitable cage or jar. Obviously, where a number of animals, as guinea-pigs, are injected with the same material, they may be placed in the same cage. They should be 
numbered and designated by their color, by their curly or smooth hair, etc., and, if need be, by painting the nose, back or flank with anilin dyes. The student should never burden his memory with details as to dose, time of inoculation, etc. A complete record should be made at the time the work is done, and in this way all uncertainty is done away with.

The experimental animals, as a rule, should be weighed before infection, and every day or two afterward. Each weighing should be done, as nearly as possible, under the same conditions as the first one. That is to say, the animal should have plenty of food and water before weighing. In warm weather the need of water is especially imperative. An experimental animal may show a difference of 200 or $300 \mathrm{~g}$. between the two weighings, and this difference may be wholly due to the withholding of water. Unless the precautions mentioned are observed the weighing results will have no value whatsoever. When properly carried out, it affords the best possible index

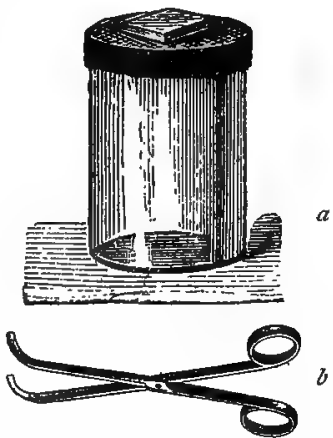

FIG. 46. $a-$ Rat cage with leaded, wire-gauze top; $b$-Rat or crucible forceps. of the physical well-being of the animal. A steady, though slight decrease in weight, is a sure indication of a chronic, wasting disease. On the other hand, a steady increase in weight is, as a rule, a reliable index of health.

The temperature of the animal should be taken before beginning the experiment, and subsequently on each successive day at the same hour. The ordinary clinical thermometer should be inserted well up into the rectum. Previous to insertion, the bulb should be covered with vaselin. The variations in temperature are especially important when studying the action of bacterial poisons on the animal body. 
The inoculated animals should be kept in glass jars, or in wire cages which can be readily sterilized. It is undesirable and not safe to keep such animals in wooden boxes. White mice or rats can be kept in battery jars. These should be provided with a wire-gauze top, loaded down with a mass of lead. Fig. 46 shows such a jar and also the crucible or rat forceps ordinarily used. Rabbits, guinea-pigs, etc., can be kept in wire cages similar to the one shown in Fig. 47. By removing the four thumb-screws on the upper

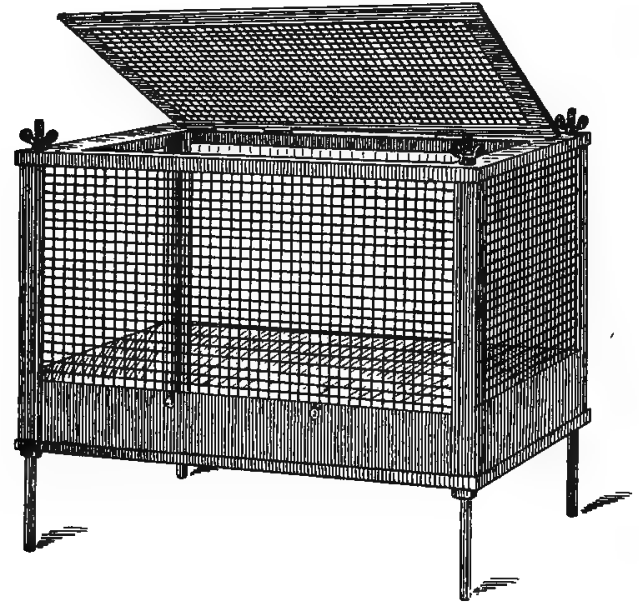

FIG. 47. Vaughan's cage for rabbits, guinea-pigs, etc.; sterilizable.

side, the ends, sides and top collapse. Several cages can thus be arranged on top of each other and then placed in a large, dry-heat sterilizer. The cage proper is $30 \mathrm{~cm}$. high, 38 deep and 54 wide. The feet are $12 \mathrm{~cm}$. high.

\section{Post-Mortem Examination of Infected Animals.}

When the infected animal dies it should be examined as soon as possible. After the lapse of a few hours, especially in warm weather, the body is liable to become invaded by 
the common putrefactive bacteria from the intestinal canal. Consequently, in delayed examinations, impure cultures are apt to be met with. Moreover, an uncertainty will exist as to whether the secondary invasion occurred during life, or after death. Hence, in case the animal cannot be examined at once, it should be placed in a suitable vessel and kept on ice.

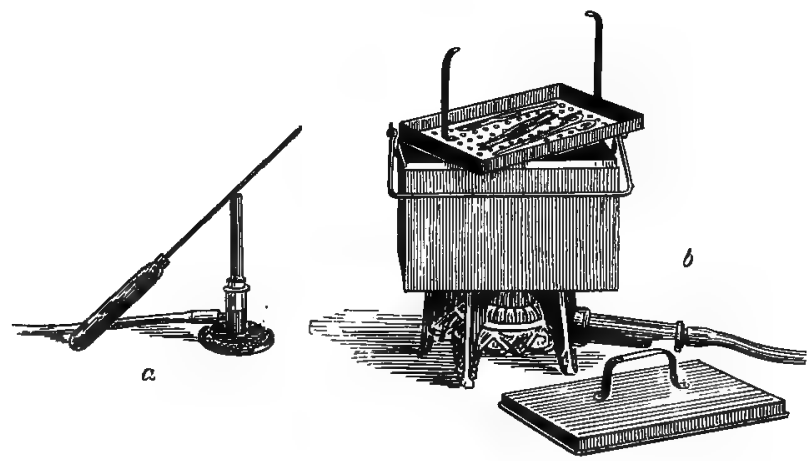

FIG. 48. $a-$ Searing iron; $b-$ Instrument sterilizing case.

All the necessary instruments should be sterilized before use. This may be done by heating the instruments, directly in the flame. Inasmuch as this procedure destroys the temper it should be avoided as much as possible. The best method of sterilizing instruments, is to place these on a perforated tray which is then immersed in a copper or enameled iron box. The box should contain an almost saturated solution of borax (10 per cent.). The borax is to be preferred to sodium carbonate, since it prevents tarnishing as well as rusting of the instruments. The latter can be kept constantly in the borax solution. When it is desirable to sterilize these the box is placed on a Fletcher radial burner. In a few minutes the liquid will boil actively. After 3 to 5 minutes of boiling, the flame can be turned off. This instrument sterilizer is shown in Fig. $48 \mathrm{~b}$.

A searing iron, also shown in Fig. 48, several sterile drawn out pipettes (Fig. 61), and several tubes of gelatin, 
agar and bouillon should be placed on the table. A number of clean cover-glasses (p. 140), or glass slides should also be available.

The animal should be nailed or tacked to a board. This should be about $34 \mathrm{~cm}$. wide and $54 \mathrm{~cm}$. long. It is well to surround it with a raised border, and to fill any crack that may be present with melted paraffin. The board, thus prepared, will not leak and hence can be thoroughly disinfected after use. Instead of a board, a galvanized iron or zinc tray can be used. In the latter case the animal is fastened by means of strings tied to the legs and slipped through holes on the side.

The animal is placed on its back, on the board or tray, and its feet, extended as much as possible, are fastened with nails or cord as the case may be. The hair over the abdomen and thorax is thoroughly moistened with a cloth soaked in mercuric chloride. With a pair of sterilized forceps, the skin over the lower part of the abdomen is raised and a slight transverse nick is made with sterilized scissors. Into the opening thus made, the lower blade of the scissors is introduced and an incision is made along the median line to the neck. While making the incision, the skin is kept raised by means of the forceps to avoid cutting through the abdominal or thoracic walls. At each end of this incision, lateral cuts are made in the direction of the extremities and the two flaps of skin, thus prepared, are carefully reflected, thus exposing the entire abdominal and thoracic walls. The condition of the subcutaneous tissue, of the abdominal walls, of the blood-vessels and the presence or absence of pus, edema, gas, etc., should be noted.

Before proceeding further, it is well to prepare several cover-glasses from the subcutaneous tissue. The cover-glass is placed on the desired spot. It is then seized at the edge, by the forceps, and carefully drawn off, not lifted up. If much edematous fluid is present it will be better to take up some of this on a looped wire and then spread it over 
the cover-glass. Cultures, as a rule, are not made from the subcutaneous tissue inasmuch as they can be obtained in a more pure condition from the peritoneal cavity, and especially from the heart-blood.

With another sterilized pair of forceps the lower part of the abdominal wall is raised and a slight nick made with sterile scissors. While the abdominal wall is kept stretched the lower blade of the scissors is introduced into the cavity and an incision is made as far as the diaphragm. The diaphragm is then cut close to the ribs. The costal cartilages on both sides are now cut, if need be, with sterile bone forceps. The triangular piece of the thoracic wall, the sternum and ribs, is then wholly removed. The lower end of the abdominal incision is extended toward the ex. tremities. The abdominal walls can now be turned back.

The entire abdominal and thoracic cavity is thus opened for inspection. In making the incisions, special care must be taken to avoid cutting into the intestines, or internal organs.

The condition of the abdominal and thoracic cavities should be carefully observed. The quantity, consistence and color of the pleural or peritoneal exudate, if any, is to be noted. The appearance of the peritoneum, and the possible presence of minute miliary tubercles should be considered. The color, size and consistency of the liver, spleen, and kidneys should be observed. Likewise the lungs, pericardial sac and the heart, whether it is in diastole or systole, should be examined. Attention should be given to the presence of abcesses, tubercles or necrotic areas.

The peritoneal fluid, if it is found on examination to be rich in bacteria, can be drawn up into a syringe and injected into another animal. Or, the fluid may be drawn up into a sterile, drawn-out tube pipette (Fig. 61). This is 
then sealed in the flame and the material can thus be preserved for future use. At times, there is very little exudate although the bacteria may be present in abundance. In that case, if it is desired to inoculate direct into another animal, some bouillon should be transferred by means of a drawn-out pipette to the abdominal cavity. By rubbing the pipette over the surface of the peritoneum and drawing up the liquid into the pipette, several times in succession, a good bacterial suspension can be obtained. One of the best methods of increasing the virulence of an organism is by successive passage through animals.

With sterile forceps and scissors, the spleen, liver and kidneys can be transferred to sterile Esmarch dishes and subsequently examined. Pieces of these organs can be hardened and sectioned (Chapter XV).

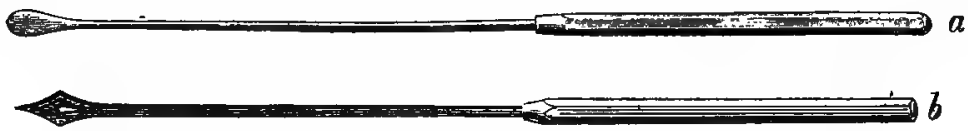

FIG. 49. $a$-The Roux spatula of nickeled steel; $b$-Nuttall's platinum needle.

If it is desired to make transplantations from any of these organs, the surface should first be seared with a hot knife or with the searing iron (Fig. $48 a$ ). Portions of the organ can then be cut out with sterile instruments and transferred to tubes. The sterile pipette can also be used for removing a portion of the pulp to the culture media. The spatula employed by Roux to remove diphtheritic membranes is well adapted to pick up pieces of tissues. These can then be thoroughly smeared over the surface of the medium, or squeezed against the walls of the tube. Nuttall's platinum needle (Fig. 49 b) is likewise very useful in transferring bits of tissue to the culture tube.

The heart-blood, as a rule, contains the specific organism in the purest condition. A large amount of blood can be drawn from the heart, especially if this is done immediately 
after death, before coagulation has set in. This can be easily accomplished in the following manner: With sterile scissors the pericardial sac should be opened and the heart exposed. The apex of the heart is grasped in a pair of broad pointed forceps, preferably arterial forceps, and the surface of the heart is burned with a red-hot glass rod or searing iron (Fig. 48). The point of a sterile, drawn out tube pipette, is then broken or cut off. The end of the pipette is sterilized by passing through the flame several times, and, when cool, it is passed through the burned surface into the right ventricle. The heart should be held on a stretch. Suction is now applied to the mouth of the pipette, and the blood is thus drawn up into the tube. The culture media can be inoculated direct by means of the pipette, or the latter may be sealed and the contents thus preserved. It may be incidentally remarked that the organisms thus kept in blood preserve their vitality and virulence for a greater length of time than on ordinary media.

In many instances, it is desirable to examine the mucous membrane of the stomach and intestines for hemorrhagic infiltrations and necroses. The digestive tube in that case should be slit up longitudinally and washed, preferably in a dilute formaldehyde or in mercuric chloride solution.

Cover-glass preparations should be made from the peritoneal surface of the abdominal wall, or from the sur. face of the intestines, liver, lungs, etc. These are easily prepared by placing the clean cover-glass on the selected surface, and then drawing it off by means of a pair of forceps. A thin layer of material adheres to the cover-glass.

Similar preparations should be made from the cut surfaces of the various organs. For this purpose, the coverglasses are placed on a mounting board ${ }^{1}$ and held in place

${ }^{1}$ Two boards are supplied. The large one is $25 \mathrm{~cm}$. square; the other $7 \frac{7}{3} \times 20 \mathrm{~cm}$. 
while the cut organ is drawn over the surface of the glass. The piece of tissue should be held by a pair of wide-pointed forceps. Not infrequently, the cut surface of an organ is very soft and pulpy, and if applied to the cover-glass will leave a thick daub of material. This can be avoided by first touching the tissue to a clean slide, or to the bottom of a Petri dish. In this way the excess of fluid is removed, and it becomes possible to spread a very thin, even film over each cover-glass.

Cover-glass streaks should also be made from the heartblood. A good preparation of the blood should show the corpuscles spread out in a single layer and separated from one another. This cannot be done if a platinum wire is employed to spread the material. A minute drop of the blood should be placed on the glass-slip and covered with another one. The two slips should then be drawn apart, when a thin layer will be left on each cover-glass.

An easier procedure is to place the droplet of the blood on a cover-glass. A large square cover-glass $(20 \mathrm{~mm}$.) should be cut into halves. The edge of one of these should be touched to the droplet of blood and then drawn across the cover-glass. The same result can be obtained with a glass-slide. The drop of blood, placed on one of these, is touched with the edge of another slide which is then drawn along the surface. The slide is then covered with alcohol, for a minute or two. This is then washed away and the stain applied for a few seconds. The slide is then washed and examined. For this purpose, a cover-glass can be dropped upon the moist slide. Or, the latter may be dried between filter-papers, and a drop of cedar-oil can then be placed directly on the specimen. If a permanent mount is desired the slide is freed of water or oil by means of paper; a drop of balsam is then added and covered with a glass-slip.

The fixing of the blood preparations requires special care in order to prevent alteration of the corpuscles. A minimum of heat should be used. The best specimens are obtained by immersing, for a few minutes, in absolute alcohol or in a mixture of equal parts of absolute alcohol and ether. When fixed in this way the organisms 
appear larger than usual, and really beautiful specimens may be obtained.

In anthrax and other diseases the specific organisms are present in large numbers in the blood, and tend to accumulate in the narrow capillaries. Thin sections of the internal organs and other tissues, on staining, will reveal the presence of these bacteria. Sometimes beautiful demonstrations of the crowding of bacteria into the capillaries can be obtained by making preparations of the mesentery. For this purpose a large animal, rabbit or guinea-pig, is desirable. A portion of the intestine should be stretched so as to expose the mesentery. A cover-glass is dropped on the clean mesentery, and the intestine is then laid over the glass-slip. With a pair of broadpointed forceps the cover-glass is firmly seized and turned over so that the membrane covers the entire upper surface. The mesentery is then cut loose with a pair of sharp scissors. The cover-glass now is covered on one side by a thin piece of the mesentery. It is placed in an Esmarch dish and covered with absolute alcohol, to which a few drops of formaldehyde are added. The specimen is thus fixed in a few hours, after which it is washed with water and stained by Gram's method, if this can be used. The minute branching capillaries will often be found plugged full of bacteria. Appearances of this kind gave rise, at one time, to the hypothesis that bacteria produced disease by mechanical interference with the circulation.

The cover-glass preparations made from the animal body, in the manner indicated, contain a relatively large amount of organic matter. Consequently, care must be taken to prevent over-staining of the back-ground, inasmuch as this will interfere with a satisfactory examination of the bacteria that may be present. The following procedure will enable one to deeply stain the bacteria, without scarcely coloring the back-ground. The importance of this will be recognized when but a few organisms are present on the cover-glass.

The cover-glass preparation is fixed with a minimum amount of heat. The directions for fixing a specimen have been given on p. 148 .

When the "fixed" glass-slip has cooled, a drop or two of water should be added so as to cover the material. A 
drop or two of the fairly strong solution of the dye (gentian violet, p. 147), is then added, and the specimen is gently swayed for a few seconds (5-10). The cover-glass is then washed as rapidly as possible, and, after drying the clean under surface, it can be examined. Scarcely any color will be seen by the eye, but under the microscope the bacterial cells will be seen deeply stained, with hardly any colored back-ground. Hanging-drop examinations of any exudate that may be present, and of the heart-blood should be made.

The necessary material for cultivation should be placed in culture tubes, which can be then set aside until the microscopic examination is completed and the animal disposed of. Gelatin or agar plates are then made from this material. The material should also be planted in bouillon and should be streaked over the surface of an inclined agar tube. Isolated colonies may be obtained by successively streaking a number of agar tubes in the manner described on p. 238. The agar and bouillon tubes should then be set aside in an incubator and allowed to develop.

In the post-mortem work proper, the greatest care is taken to prevent the introduction of foreign organisms, since these might give misleading results. Throughout the work, however, the operator must be extremely careful to prevent personal infection. The rule to sterilize every instrument shortly before and immediately after use, before it has left the hands, must be strictly attended to. Direct contact of the hands with infectious matter must be carefully avoided, and when such contact has taken place prompt disinfection must be resorted to. It may be well in this connection to call attention to the precautions emphasized on p. 170. When blood or pieces of tissue adhere to the instruments, the latter should not be placed at once into the flame, inasmuch as the sudden heating may cause the material to spurt and scatter about. To avoid this, the ma- 
terial should first be dried by holding the instruments close to the flame. This precaution should also be observed when sterilizing wires which are covered with gelatin.

When the examination is completed, the animal is placed in a jar and covered with mercuric chloride or other disinfectant. Eventually, it should be cremated. If this cannot be done, the jar and contents should be placed in a sterilizer and steamed for at least one hour.

All instruments employed should be returned to the borax solution and boiled for about 5 minutes. The glass pipettes which have been used, are sealed at the lower end, then strongly heated in a flame; after which, they are plunged direct into the water-bath. The tube breaks into fragments, and hence can now be effectively sterilized by boiling. The nails should be sterilized in the flame and the board should be treated with a strong mercuric chloride solution (1-500).

\section{Laboratory Work with Anthrax Animals.}

A guinea-pig or a rabbit is inoculated subcutaneously with a small amount of an agar growth of the anthrax bacillus. When the animal dies, in $1 \frac{1}{2}-2$ days, a postmortem examination is at once made. The material thus obtained is used for the following experiments.

Isolation of the bacillus in pure culture.-The bacillus of anthrax which is present in the blood, tissues and organs of the guinea-pig, must be isolated and obtained in pure culture. This can be readily accomplished by the gelatin plate method. For this purpose a small piece of liver, about half the size of a grain of wheat, is cut off with a pair of sterilized scissors. The piece of tissue is placed on the loop of a sterilized platinum wire and transferred to a tube of liquefied gelatin. By rubbing the piece, with the wire, against the walls of the tube the blood can be 
squeezed out, and the organisms present can thus be spread throughout the gelatin. From this tube, which is No. 1, transfers are made in the usual manner to tube No. 2, and from this to tube No. 3. Gelatin plates (Petri dishes) are are then made in the usual manner and set aside for two or three days to develop.

The form of the colonies should then be carefully studied, inasmuch as this is very characteristic. If possible, impression preparations of the surface colonies should be made and stained with methylene blue.

Since the colony is a pure culture of the anthrax bacillus, transplantations to tubes in turn will yield pure cultures. A stab culture in gelatin and a streak culture on in. clined agar, and on two potatoes in tubes, should be made. The inoculation of the inclined media is made by simply drawing the end of the platinum wire over the middle of the surface of the agar or potato. The agar tube and one potato tube are placed in the incubator at 37 to $39^{\circ}$, for one or two days, then removed and examined for threads and spores. The other potato tube is allowed to develop at a temperature of $15-18^{\circ}$. Under these conditions spores are not produced, but instead, marked involution forms will be found.

A tube of peptonless agar should be inoculated at the same time as the above and allowed to develop in the incubator. This growth, unlike that on the ordinary agar, will be extremely rich in spores. The material obtained in this way will be utilized, subsequently, for the staining of spores. The ordinary agar does not yield a good supply of spores, inasmuch as the anthrax bacillus employed has been cultivated artificially for many years, and, as a result, shows an asporogenic tendency. If $1 / 2-1$ drop of calcium hydrate is added to the tube of ordinary agar, and this then sterilized, it will yield an abundance of spores. The absence of pepton and the presence of calcium favor spore formation. 
Agar plate culture.-The gelatin plates, prepared as above, do not always yield the most characteristic colonies. Moreover, owing to the liquefaction of the gelatin, it is difficult to obtain satisfactory cover-glass impressions. The agar plates, on the other hand, give excellent colonies from which impression preparations can be easily made. The method of making agar plates is as follows:

Three agar tubes, each of which contains about 8 c.c. of agar, are selected and placed upright in boiling water. When the agar has become perfectly fluid, the burner is then removed from under the water-bath, and the water with the immersed tubes is allowed to cool slowly until a temperature of $50^{\circ}$ is reached. Tube 1 is then inoculated with a piece of the liver, or other organ, in the manner described above when making gelatin plates. The usual dilutions to tubes 2 and 3 , are then made as rapidly as possible. After withdrawal of the cotton plug, the neck of each tube is flamed and the contents poured into sterile, Petri dishes. The plates are then set aside in the incubator at $37^{\circ}$ for $12-18$ hours.

The nutrient agar solidifies at about $40^{\circ}$. Consequently, rapid work is necessary in order to inoculate the tubes and pour the contents before solidification takes place. If this does occur, the experiment must be repeated with new agar tubes. Ice-water must not be used to congeal the agar plates. They will solidify without employing any cooling apparatus.

When developed, the colonies should be compared with those on gelatin; they should be sketched and used for making impression preparations.

Hanging-drop examination.-Take a clean $\frac{8}{4}$ inch coverglass and sterilize by passing it several times through the flame. Transfer a small drop of sterile bouillon to the cover-glass and then add to it, with a sterilized wire, a min- 
ute amount of the heart-blood. Apply the concave slide, ringed with vaselin, and examine the hanging-drop with the No. 7 objective. Study the characteristics of the anthrax bacillus as it exists in the blood, and compare its size with that of the blood-cell. Then label the slide and set aside in the incubator for 24 hours. Examine the same on the following day and observe the formation of threads, of sporogenic granules and possibly of spores.

Owing to the rapid flow of blood, the anthrax bacillus cannot give rise to long threads in the living body. In a quiet liquid, as in the drop, the tendency to form threads is favored. Stained preparations can be made from this hanging-drop culture. The cover-glass should be moved to one side, just sufficiently to permit the forceps to pick it up. It is then carefully raised, avoiding contact with the vaselin. A portion of the drop culture, transferred to a droplet of water on a clean slide, is spread out, dried, fixed and stained in the usual way.

Stained preparations. - Place about 18 clean cover-glasses on the small mounting board $\left(7 \frac{1}{2} \times 20 \mathrm{~cm}\right.$.), or on the lid of a slide-box. Pick up a piece of the spleen, kidney or liver in a pair of forceps, and, while holding the cover-glass down with another pair, lightly pass the cut surface of the organ over the cover-glass. A very thin and even film is desirable (see p. 280). In this way streak all the coverglasses; some with spleen, others with liver and the remainder with kidney tissue. Keep each set distinct. Then allow the specimens to dry in the air and fix cautiously by passing once through the flame. Special care must be taken not to over-heat; inasmuch as the anthrax bacillus in that case will not stain readily. If, on subsequent trial, a cover-glass is found to be fixed imperfectly the others can be given an additional passage through the flame. These cover-glasses are commonly known as streak preparations. 
Stain some of the fixed cover-glasses with simple anilin dyes, such as gentian violet or fuchsin, observing the sug. gestions made on p. 281. Examine and study the specimens carefully and make permanent preparations. Note the organ in which the bacilli are most abundant. The remainder of the cover-glasses will serve for double-staining by Gram's method.

Stained preparations of the heart-blood (p. 280), should also be made.

Gram's method.-This excellent method of demonstrating the presence of certain bacteria in the fluids and tissues of the body is based upon the fact that the protoplasm of the bacterial cell, when stained with anilin-water gentian violet and then treated with iodine, forms a difficultly soluble compound. By proper exposure to a solvent the dye can now be removed from the entire cover-glass, but not from the bacterial cell. The deeply stained violet bacteria lie on a colorless back-ground which on treatment with a contrast color, as eosin or picro-carmin, becomes stained a light pink. The method is as follows:

A solution of anilin-water gentian violet is first prepared. Anilin oil is placed in a test-tube to a depth of about half an inch. The tube is then filled with water, closed with the thumb, and thoroughly shaken in order to obtain a saturated aqueous solution of anilin. The liquid is then passed through a small filter and collected in another test-tube. The filtrate should be perfectly clear, not cloudy. To the anilin water thus obtained a saturated alcoholic solution of gentian violet is added till the fluid is deeply colored, and opaque. This result is obtained when $0.3-0.5$ c.c. of the saturated gentian violet is added to 10 c.c. of the anilinwater.

Some of the anilin-water gentian violet thus prepared is poured out into a watch-glass, or better, into a wide Esmarch dish. The fixed cover-glass is placed between the 
thumb and forefinger, with the specimen side down, and. then carefully dropped upon the surface of the stain. It is allowed to float on the dye for 3-5 minutes. Sometimes, it is necessary to stain for a longer period, or to warm the dye on the radiator or on an iron plate (Fig. 22, p. 150), in order to obtain a rapid and intense stain. The best results are obtained when the specimen is treated with a strong dye for a few minutes. The longer the dye acts, the more difficult it will be to subsequently obtain a good decoloration.

The cover-glass is then picked up with the forceps, thoroughly washed with water, and immersed in a solution of iodine in potassium iodide. This is made by dissolving 2 $\mathrm{g}$. of potassium iodide and $1 \mathrm{~g}$. of iodine in 300 c.c. of distilled water. The specimen is allowed to remain in the iodine solution for 3 to 5 minutes. Care must be taken not to expose the specimen too long to the action of iodine, inasmuch as this tends to cause the protoplasm to contract into granules. The cover-glass is then removed from the iodine solution, washed with water, and placed in 95 per cent. or in absolute alcohol. This can be kept in a watchglass or in an Esmarch dish, which should be tilted from time to time to facilitate the decoloration. In the case of greatly over-stained specimens, a drop of dilute acetic acid may be added to the alcohol.

From time to time, the cover-glass should be washed with water and examined with the No. 7 objective in order to ascertain the progress in decoloration. If the material is spread over the cover-glass in a perfectly thin, even layer the decoloration will be rapid and thorough. On the other hand, if thick masses are present, it will not be possible to obtain complete decoloration without also decoloring many of the bacteria. When, therefore, the greater part of the back-ground has been decolored, the treatment with alcohol should be discontinued.

The cover-glass is then washed with water and stained 
with dilute eosin for $\frac{1}{4}$ to $\frac{1}{2}$ minute. The eosin is an acid anilin dye and therefore stains the protoplasm of cells, nuclei, etc., but not bacteria (p. 146). Care must be taken not to overstain the preparation with eosin, as it would tend to diminish the sharp contrast that is desired. Moreover, the eosin is liable to decolor the violet bacilli. After staining with eosin, the cover-glass is thoroughly washed with water and examined under the microscope. It should show the deeply stained violet bacilli on a light pink background. If the specimen is satisfactory it can then be floated off the slide, dried in the air and finally mounted in baisam.

Weigert's picro-carmin solution, or Bismarck brown can also be used for contrast colors. The Gram-Weigert's fibrin stain, as given in Chapter XV, can be used to advantage where sometimes the ordinary Gram's method fails. As will be seen, many important organisms are not stained by this method.

The gentian violet employed in Gram's method may be substituted by other para-rosanilin dyes, such as methyl violet, or Victoria blue. The rosanilin dyes, such as fuchsin, methylene blue, and vesuvin will not react with iodine.

Gram's method is applicable to many pathogenic bacilli and to most of the micrococci. A notable exception among the latter is the gonococcus.

The following organisms are stained by Gram's method:
B. anthracis.
B. tetani.
B. anthracis symptomatici (p. 298).
B. tuberculosis.
B. diphtheriæ.
M. pneumoniæ crouposæ.
B. lepræ.
M. tetragenus.
B. murisepticus.
Moulds.
B. œdematis maligni (p. 300).
Staphylococci.
B. œdematis maligni, No. II.
Streptococci.
B. rhusiopathiæ suis.
Streptothrix actinomyces. Yeasts. 
The following are not stained by Gram's method:

B. anthracis symptomatici (p. 298). B. pneumoniæ.

B. choleræ gallinarum.

B. rhinoscleromatis.

B. choleræ suis.

B. typhosus.

B. coli communis.

M. gonorrheæ.

B. icteroides.

B. influenzæ.

B. mallei.

B. cedematis maligni (p. 300).

B. pestis bubonica.

Spirillum Obermeieri.

Vibrio choleræ Asiaticæ.

V. Deneke.

V. Finkler-Prior.

V. Metschnikovi.

The following synopsis of the staining methods for streak preparations will be of service:

Cover glass preparation.

Dry in air.

Once through flame.

Simple stain:

Add drop of water.

Add dilute dye (1/4-1/2 min.).

Wash in water.

Examine in water.

Dry in air.

Mount in balsam.

\section{Gram's stain:}

Anilin-water gentian violet ( 3 to 5 min.; if hot, 1 to 3 min.).

Wash in water.

Iodine in potassium iodide (3 to 5 min.).

Wash in water.

Decolor in absolute alcohol.

Wash and examine in water.

Contrast color(dil.eosin, few sec.).

Wash and examine in water.

Dry in air.

Mount in balsam.

The above examinations of hanging-drop and stained preparations serve the purpose of demonstrating the presence of the anthrax bacillus in the different organs and tissues of the body. The form, size, etc., of the bacillus found under these conditions should be compared with the characteristics of the organism when grown in pure culture on the different media. For this purpose make hangingdrop examinations and permanent simple stains of the 
bacillus grown in stab culture in gelatin, in bouillon, on ordinary agar, and on peptonless agar. The preparation of impression cover-glasses of the anthrax colonies and simple stains of the bouillon hanging-drop culture have been mentioned.

Double staining of spores. - The growth of the anthrax bacillus on peptonless agar, or on potato at $37^{\circ}$, when examined in the hanging-drop, will show the presence of an abundance of bright, highly refracting oval bodies or spores. These may be observed free and also within the parent cell. Simple stains of this material with fuchsin, etc., will show the bacilli deeply stained, whereas the spores remain colorless. This is undoubtedly due to a special composition of the spore contents, as well as to the dense impenetrable wall which surrounds the spore and prevents the dye from passing into the interior (p. 55). By proper treatment with strong anilin dyes it is possible to force the stain into the spore. Once within the spore, it is as difficult to remove the dye as it was to cause it to enter. By suitable decoloration it is, therefore, possible to remove the stain from everything on the cover-glass, except from the spores. Then, on the application of a contrast color the specimen will show a bright red spore within a blue bacillus. The method of double staining spores is as follows:

The cover-glass preparation from the peptonless agar is dried in the air and fixed in the usual manner. The cover-glass is held in the forceps in the left hand, with the specimen side up, and covered with a solution of hot car. bolic fuchsin. This is held over a Bunsen flame, so that vapors are given off from the liquid. Active ebullition should be avoided. From time to time the liquid which is lost by evaporation is replaced by a fresh addition of the carbolic-fuchsin, and under no condition should the dye be allowed to dry down on the cover-glass. The best results in heating are obtained when the flame is turned low, so that 
it is not over two inches high. After heating the specimen in this manner for two or three minutes, the stain is thoroughly washed off with water and the cover-glass examined with the No. 7 objective. Colorless spores should no longer be visible, but everything should be stained a deep red. If the spores are not colored the heating with carbolic fuchsin is repeated until they become stained.

The cover-glass may be floated on hot carbolic fuchsin in an Esmarch dish for $1 / 2$ to 1 hour. The carbolic fuchsin, whether in the bottle or in the Esmarch dish, can be heated on the iron-plate as shown in Fig. 22, p. 150. Anilin-water fuchsin can be prepared in the same way as the stain which is employed in Gram's method (p 287), and can be used in place of carbolic fuchsin. It will give excellent results.

The cover-glass with the deeply stained spores is then placed in dilute alcohol and gently moved about. From time to time, it should be washed with water and examined with the No. 7 objective. As soon as the bacilli are decolored, the washing in alcohol is discontinued. The specimen then shows bright red spores within cells that are almost or wholly colorless. The cover-glass is then stained for a short time with methylene blue, washed with water and examined. The spores should be stained deep red, while the bacillus itself should be light blue.

Spores may be readily simple stained by passing the cover-glass, after it has been fixed, 8 or 10 times through the flame. The specimen is then heated for one to two minutes with carbolic fuchsin, washed with water and exam. ined. The spores are deeply stained, but the cell proper is not. This excessive heating disintegrates or weakens the spore-wall, and thus the dye is enabled to enter. Unfortunately, the cell-wall proper is destroyed and hence will not stain.

The carbolic-fuchsin (Ziehl's solution), is prepared by adding $1 \mathrm{~g}$. of fuchsin and $13 \mathrm{c}$.c. of absolute alcohol to 100 c.c. of 5 per cent. carbolic acid. The liquid is heated on 
the water-bath until everything dissolves, and the solution has a clear, deep bright-red color.

The carbolic fuchsin prepared as above will keep for a considerable length of time. It should always be warmed on the iron-plate in order to bring the constituents into solution. Eventually, an insoluble deposit forms and the solution becomes more transparent, weaker, and hence stains less energetically than in the beginning. Instead of preparing a large amount of carbolic fuchsin it is better to dissolve the constituents separately and to bring these together in small quantity, whenever desired.

For this purpose $8 \mathrm{~g}$. of fuchsin are dissolved by heat in 120 c.c. of absolute alcohol. A deposit may form on cooling, hence before measuring out the liquid it should be warmed till complete solution results. 15 c.c. of this solution contain $1 \mathrm{~g}$. of fuchsin. The addition of 7.5 c.c. of the liquid to 50 c.c. of 5 per cent. carbolic acid will, on warming, give an excellent Ziehl solution. Tubercle bacilli which will not stain with old carbolic fuchsin will promptly react with a fresh solution prepared in the manner described.

The following summary will be of assistance when staining spores:

Cover-glass preparation.

Dry in air.

$\begin{array}{ll}\text { Simple: } & \text { Double: } \\ \text { Pass } 12 \text { times or less through } & \text { Pass once through flame. } \\ \text { flame. } & \text { Carbolic fuchsin (hot } 2 \text { to } 5 \text { min.). } \\ \text { Carbolic fuchsin (hot } \frac{1}{2} \text { min.). } & \text { Wash and examine in water. } \\ \text { Wash in water. } & \text { Wash in dilute alcohol. } \\ \text { Examine in water. } & \text { Wash and examine in water. } \\ \text { Dry in air. } & \text { Contrast color (methylene blue, } \\ \text { Mount in balsam. } & \frac{1-\frac{1}{2} \text { min.). }}{} \\ & \text { Wash and examine in water. } \\ & \text { Dry in air. } \\ & \text { Mount in balsam. }\end{array}$

Phagocytes.-According to Metchnikoff's cellular theory of immunity, the white blood cell is endowed with the power of taking into itself, and ultimately destroying, the invading organism. Phagocytic action can be readily demonstrated in frogs inoculated with anthrax. For this 
purpose a pure culture of the anthrax bacillus is injected into the dorsal lymph sac of a frog, and at the of 12 or 18 hours the animal is killed with chloroform.

Cover-glass preparations should be made with the fluid in the dorsal lymph sac and stained, some with simple anilin dyes and others by Gram's method.

Animal inoculations.-A pure culture of the anthrax bacillus, isolated from the dead animal, can be used to inoculate a white mouse, a white rat or a rabbit. In the animal thus inoculated the organism can in turn be detected and isolated. The rules of Koch can be easily demonstrated with reference to the anthrax bacillus, thus showing conclusively that it is the cause of the disease.

\section{Summary of Laboratory Work with Anthrax.}

From the guinea-pig make :

Gelatin plates.

Colonies.-Impression preparations.

Stab culture in gelatin.

Agar streak culture.

Peptonless agar streak.

Potato tube culture.

Potato tube at $\mathbf{1 5}^{\circ}$.

Bouillon tube culture.

Examine each of these in the hanging-drop, and make permanent mounts.

Agar plates at $37^{\circ}$.-Impression preparations.

Hanging-drop culture of heart-blood.-Permanent mounts.

Streak preparations of blood, spleen, liver, kidney, lung, stained

by simple and by Gram's method.

Inoculate a frog with a pure culture of anthrax and examine the lymph fluid by the simple and by Gram's method for phagocytes.

Simple and double staining of spores, developed on peptonless agar and on potato at $37^{\circ}$.

Involution forms on potato at $15^{\circ}$.

Sketch characteristic growths and forms.

Inoculation of other susceptible animals, thus proving that this organism is the cause of a disease. 


\section{CHAPTER XI.}

\section{THE PATHOGENIC BACTERIA.}

B. ANTHRACIS.-B. ANTHRACIS SYMPTOMATICI.-B. EEDEMATIS MALIGNI.-B. EDEMATIS MALIGNI NO. ㅍ.-B. TETANI.-B. TUBERCULOSIS.

-B. LEPRA.-B. MALLEI.-B. DIPHTHERI 左.-M. PNEUMONIA CROUPOSA.-B. PNEUMONIA.-B. RHINOSCLEROMATIS.-VIBRIO CHOLERAE ASIATICAS.-VIBRIO DENEKE.-VIBRIO FINKLERPRIOR.-VIBRIO METSCHNIKOVI.-B. COLI COMMUNIS.-B. TYPHOSUS.-B. ICTEROIDES.-B. PESTIS BUBONIC.E.-

B. INFLUENZ两.-B. PYOCYANEUS.-STREPTOCOCCUS PYOGENES. - STAPHYLOCOCCUS PYOGENES AUREUS. - MTCROCOCCUS GONORRHEAE. - M. TETRAGENUS. - SPIRILLUM OBERMEIERI.

- B. cholere galliNarum. - B. CHOLERA SUIS. - B. RHUSIOPATHIA SUIS. - B. MURISEPTICUS. 


\title{
Bacillus Anthracis, Davaine, Pollender (1849).
}

\author{
ANTHRAX, SPLENIC FEVER (in cattle): WOOL-SORTER'S DISEASE, \\ MALIGNANT PUSTULE (in man); MILZBRAND (Germ.); \\ CHARBON, SANG DE RATE (Fr.).
}

ORIGIN.-Found in the blood and tissues in anthrax.

Form.-Large, clear, homogeneous rods, with slightly rounded ends; size varies with different media, but the length is less than the diameter of a blood cell. Occurs in blood in short threads of 2-4-6 cells, which may show slightly swollen ends. In bouillon and on agar it forms long threads. Involution forms develop on potato at $16^{\circ}$.

MoTILITY.-It has no motion.

SPORULATION.-Forms median, oval spores, without enlargement of cell. After long cultivation it may lose the property of forming spores-asporogenic variety. In such cases, growth on peptonless agar, or the addition of $\frac{1}{2}-1$ drop of $\mathrm{Ca}(\mathrm{OH})_{2}$ to an agar tube, favors spore formation. Optimum temperature, $30^{\circ}$. Not formed below $16^{\circ}$ or above $42^{\circ}$, or within the body. Spores possess variable resistance.

ANILIN DYES. - It stains readily, also by Gram's method.

GrowTh.-Is rapid.

Gelatin plates.-Deep colonies form round, granular, yellowish-brown masses, with irregular borders. Surface colonies are very characteristic, and according to the consistency of the gelatin the border is fibrillated or shows very wavy strands of threads-Medusa head. This typical colony is readily obtained on agar plates grown at $37^{\circ}$. Liquefaction.

Stab culture. - Short threads radiate from the line of inoculation into the surrounding gelatin, imparting a brush-like appearance. Cup-shaped liquefaction forms on top and gradually extends till the contents are wholly liquefied. The mass of bacteria settles to the bottom and leaves a perfectly clear solution above, without scum.

Streak culture.-On agar, it forms a dry, grayish-white, slightly folded growth. On potato, the growth is abundant, white, cream-like and rather dry, and is rich in spores $\left(37^{\circ}\right)$; or in involution forms $\left(16^{\circ}\right)$.

In bouillon, a thick gelatin ring forms on the surface. Milk is coagulated, the casein is then peptonized. Acid production on lactose media.

OXYGEN REQUIREMENTS. - It is aerobic, but can grow in the body as a facultative anaerobe.

TEMPERATURE.-Growth at $12-45^{\circ}$. The optimum is about $37^{\circ}$.

BEHAVIOR TO GELATIN, - Liquefies slowly.

Attenuarion.-By heating for 10 minutes at $55^{\circ}$; 1 minute at $100^{\circ}$. By growing at $42.5^{\circ}$ for four weeks. By action of chemicals as mercuric chloride, carbolic acid, etc. By insolation. By growth under pressure. In the body of immune animals, as frogs.

IMMUNITy,-Obtained with attenuated cultures, 1st and 2nd vaccine of Pasteur; with sterilized cultures; with purified toxin. Blood of anthrax animals heated to $55^{\circ}$ protects. The blood-serum of an animal vaccinated against the bacillus is preventive, and to some extent curative.

PATHogenesis.-White mice, guinea-pigs, rabbits, sheep, cattle, horses and man are susceptible. Dogs, old white rats, cats, Algerian sheep, birds and frogs are insusceptible. Young animals, as dog or rat, are more susceptible than old ones. Subcutaneous application kills guinea-pigs in 24-48 hours. Post-mortem examination shows subcutaneous edema and enlarged spleen. Bacilli everywhere; leave body in bloody discharges from the nose, intestine, urine, etc.

INFECTION.-(1) Through the food, presence of spores.-Intestinal anthrax in sheep and cattle. (2) Through wounds, - Inoculation anthrax in man (malignant pustule). (3) 'Through the air,--Lung anthrax in man, the wool-sorter's and possibly rag-picker's disease.

Diagnosis. - Microscopical examination of spleen, blood, or of contents of pustule; isolation of pure culture. Characteristics of the latter and effect on white mouse or guinea-pig. It does not appear in the blood until shortly before death. It is present in the "rusty" or dark-colored sputum of the "wool-sorter's disease." 
DRAWINGS.

297 
SYMPTOMATIC ANTHRAX, BLACK LEG, QUARTER EVIL; CHARBON SYMPTOMATIQUE ( $F r_{\text {. }}$; RAUSCHBRAND (Germ.).

ORIGIN.-In the subcutaneous tissue, muscles, serous exudate, etc., of symptomatic anthrax.

FORM.-Rather large, narrow rods, with distinctly rounded ends; almost invariably single, may form in pairs. About three times as long as wide. Involution forms appear in old cultures-swollen in the middle or at the ends.

Motility.-Actively motile. Spore-bearing rods eventually lose their motion. Shows lateral flagella: giant whips are common.

Sporulation.-Spores develop readily as bright oval bodies, near one end which is enlarged. Not formed in body till after death.

ANILIN DYES.-Stain readily. Gram's method is applicable if a strong dye acts for some time. The spores are readily double stained. GrowTH. - Rapid, and gives off a strong butyric acid odor. Acid or alkaline glucose media are best. Requires anaerobic conditions.

Plates.-On gelatin, forms irregular masses surrounded by a dense whorl of threads. Liquefies. On agar, the colonies vary. Usually appears as a dense mass of threads.

Stab culture.-In glucose gelatin development takes place in the lower part of the tube; the contents are liquefied and gas is produced. Energetic growth and gas production in glucose agar. The contents of the tube are torn into several parts. Giant whips. common (Novy).

Streak culture.-On glucose agar, in hydrogen, a whitish spreading film forms. On blood serum good growth; giant whips (Löffler).

Bouillon.-Becomes cloudy; gas bubbles accumulate on the surface; after several days the growth settles to the bottom, forming a compact, adherent sediment. Liquid above remains cloudy for several days.

Glucose gelatin colored with litmus.-Develops growth in incubator under ordinary conditions. The color of the litmus disappears (reduction), then changes to a wine-red, showing formation of acids. Heavy flocculent sediment on the bottom. Milk. - The casein is quickly coagulated. Starch is not inverted. Grows on potato.

OXYGEN REQUIREMENTS. - Is an obligative anaerobe. Grows in vacuum, hydrogen, carbonic acid, etc. Also in glucose litmus gelatin in air.

Temperature. - Grows slowly at room temperature. Best at $37-38^{\circ}$. BeHAVIOR TO Gelatin. - Liquefies.

AERogenesis. - Energetic production of gas, having a disagreeable odor; is inflammable and consists of marsh-gas, hydrogen, etc.

Attenuation.-Bouillon cultures soon lose virulence, but maintain their vitality. Attenuation takes place at $42-43^{\circ}$. Dry spore bearing material heated to $80^{\circ}$ or $100^{\circ}$ becomes attenuated. Virulence restored by inoculating animals, and at the same time injecting some lactic acid. Virulence maintained in solid media.

IMMUNITY._-Obtained (1) by inoculating small amounts of virulent germ; (2) by intravenous injections; (3) by injecting heated cultures, $100^{\circ}$ or $80^{\circ}$; (4) inactive old cultures; (5) filtered cultures.

PATHogenesis. - Young cattle, sheep, goats, guinea-pigs, mice, are highly susceptible. Horse, ass, white rat are less so; while hogs, dogs, cats, ordinary rats, rabbits, doves, ducks, chickens, are almost immune. Subcutaneous injection in guinea-pigs produces death in 24-48 hours. An extensive subcutaneous bloody edema is present. The muscles are dark, infiltrated, and gas is present.

INFECTION.- Takes place naturally by inoculation through deep wounds; very rarely through the food. Poisoned arrows used in fishing in Norway.

Diagnosis.-Especially a disease of young cattle, not of man. Difficult to distinguish from bacillus of malignant edema. Inoculation of the rabbit negative; absence of threads; tendency to involutions. Distinguished from anthrax bacillus by form, motility, position of spores, cultural properties, and by its distribution in the body. 298 


$$
\text { numsases } \quad \Rightarrow
$$




\section{Bacillus Edematis Maligni, Pasteur (1877).}

VIBRION SEPTTQUE (of Pasteur). MALIGNANT EDEMA; SEPTICÉMIE GANGRENE GAZEUSE ( $F r$.); MALIGNES EDEM (Germ).

Origrn.-From animals inoculated with garden soil; from infected horse and from man. In putrid liquids, and in intestines.

Form.-Rods about three times as long as wide, with rounded ends; usually single, but may form threads especially in the body. In size, etc., resembles the bacillus of $\mathrm{S}$. anthrax; is narrower than the anthrax bacillus.

Moti.iTy.-Actively motile. Lateral flagella; giant whips (Novy).

SPORULATION.--In bouillon and agar, spores appear in 24 hours. The best temperature is about $37^{\circ}$. The spores are median or nearly :so, with corresponding enlargement of the parent cell.

ANILIN DYES.-React readily. It is stained by slightly modified Gram's method. Spores double stain readily.

GrowTH.-Is very rapid, especially on glucose media. Requires anaerobic conditions.

Plates.-On gelatin, colonies develop in 2-3 days, and under the microscope resemble those of the Hay bacillus. As they become larger gas bubbles form. On agar plates at $37^{\circ}$ the colonies appear as an irregular, dense net-work of threads.

Stab culture.-In gelatin, growth occurs in the lower part of the tube; the gelatin is liquefied, gas given off and the growth settles to the bottom. Agar cultures are torn into several parts by the gas which is formed. In the liquid on the bottom of the tube, giant whips can be found by staining.

Streak culture.-On agar, offers no special characteristics. Grows on potato without forming a scum.

Bouillon.-Becomes cloudy, and in I-2 days the growth settles to the bottom as a low, adherent sediment, and in a few days the liquid becomes clear.

Glucose gelatin. colored with litmus.-In air at $37^{\circ}$ is liquefied and litmus first reduced, then in presence of oxygen it becomes red-acid production.

Milk.-Develops a good growth; a part of the casein is precipitated. Starch is not changed to sugar.

OXYGEN REQUIREMENTS.-Is an obligative anaerobe. Grows in vacuum, hydrogen, carbonic acid, etc.

Temperature.-Growth is best at the temperature of the body.

Can grow at ordinary temperature.

BeHAVIOR TO GELATIN. -Liquefies.

Aerogenesis. - On glucose media, especially when distinctly alkaline, it gives rise to the production of gas.

ATTENUATION.-Bouillon cultures retain virulence for months. In general the virulence varies greatly. Virulence increased in mixed cultures (prodigiosus, proteus, etc.).

IMMUNITY.- One attack of malignant edema does not protect against a second. 100 c.c. of heated or filtered cultures injected into guinea-pigs in three portions confers immunity; 6-8 c.c. of the serous exudate accomplish the same result.

PATHOGENESIS. - Rabbit susceptible-distinction from symptomatic anthrax. The horse, hog, dog, cat, chicken, dove, guinea-pig, mouse and man, are susceptible. Cattle are immune. Subcutaneous inoculation in guinea-pigs of $1 / 2$ c.c. or more of bouillon culture produces death in about 24 hours. Marked subcutaneous, spreading, reddish edema; muscles dark. Bacilli present, single or in threads, in subcutaneous tissue, on serous surfaces as peritoneum, etc.; scarce or not present in the blood. 25-30 c.c. of the filtered bouillon culture, injected subcutaneously, kills guinea-pigs.

Infection.--Takes place by inoculation through wounds. Poisoned arrows of the New Hebrides. Rag-picker's disease, supposed to be due to inhalation of this bacillus; is probably anthrax.

Diagnosis. - To be distinguished from anthrax. The distribution. form, motility and cultural properties, will enable differentiation. 
DRAWINGS, 


\section{Bacillus Edematis Maligni, No. II, Novy (1893).}

OrIGIN.-From guinea-pigs inoculated with milk nuclein obtained from casein by digestion with artificial gastric juice.

Form.--In the animal body it occurs usually in single rods, 4-5 times as long as wide; may also occur in short threads. On artificial media it develops as straight or bent rods, sometimes forming peculiarly twisted threads. The contents are often granular, and show a bright body at one end.

Morility.-Possesses a slight swaying motion, which is often absent. Has lateral flagella, and in pure culture, as well as in the animal, it gives rise to giant whips which may attain a length of 40-50-72 microns (Fig. 7, p. 39).

SPORULarion.--Spore formation not observed.

ANILIN nyes.-Stain readily. Gram's method applicable.

Growth.-Depends upon the vitality of the organism. When taken from an animal it grows rapidly.

Plates. - On glucose agar good colonies develop in 2-3 days at $37^{\circ}$. Show a very irregular, fibrillated border, and often give rise to gas bubbles. May contain giant' whips.

Stab culture.-Develons only in the lower part of the tube. In glucose agar having proper alkalinity, it develops rapidly forming a plainly visible growth along the line of inoculation; the agar is soon torn into several parts by the gas that is produced. Cultures soon die out.

Streak culture. - Growth on glucose agar only when oxygen is completely excluded. It forms a white film which spreads over the surface. On acid agar involution forms develop.

Bouillon.-An excellent growth develops which in 24 hours settles to the bottom as a loose, flocculent sediment; the liquid above becomes clear-distinction from $M$. edema and S. anthrax bacilli.

Glucose gelatin, colored with litmus. - Is liquefied and acid is produced-the litmus is reduced and then turned red.

OXYcien Requirements. - Is an obligative anaerobe. Grows in vacuum, hydrogen, nitrogen, carbonic acid, illuminating gas.

Temperature. - Does not grow below $25^{\circ}$. Optimum temperature about $39^{\circ}$. Can withstand freezing for 24 hours.

Behavior to GELATIN.-Liquefies.

Aerogenesis. - In alkaline media gives rise to gases. Volatile acids, as butyric acids, etc., are formed in artificial culture and also in the body of rabbits.

Attenuation. -Cultures left in hydrogen, or exposed to light, lose their virulence. It is not attenuated when kept in the dark or when frequently passed through animals. Lost virulence can be reconstituted by inoculation with a "mixed" culture containing Proteus vulgaris.

IMMUNITY.-Not conferred by a non-fatal inoculation, or by old, weakened cultures, or by the serous exudate of the pleural cavity.

Pathogenesis.-Subcutaneous injection of $\frac{1}{\text { c.c. }}$ of hydrogen bouillon cultures kills guinea-pigs, rabbits, white rats, white mice, doves, in 12-24 hours. Marked subcutaneous edema present; serous exudates in thoracic and abdominal cavities. Cover-glass preparations made from the subcutaneous tissue or serous surfaces, as peritoneum, shows usually enormous numbers of bacilli, and frequently giant whips are also present. The latter are visible as colorless spirals.

Diagnosis. - The morphological characteristics distinguish it readily from malignant edema and from symptomatic anthrax. 
DRAWINGS

303 


\section{Bacillus Tetani, Nicolaier (1884).}

TETANUS, LOCK-JAW; WUNDSTARRKRAMPF (Germ.); TETANOS (Fr.).

ORIGIN.-Found in animals that die of tetanus after inoculation with earth; in traumatic tetanus of man and animals; in headtetanus; tetanus of new-born. Present in intestines.

Form.-Large, narrow rods with rounded ends; may form threads. Motility.-Is motile. May show long spirals or giant whips.

Sporulation.-Occurs rapidly, in 24-48 hours at $37^{\circ}$. Forms terminal spores, with enlargement-drum-sticks.

ANILIN DYES.--Stain rapidly. Gram's method is applicable.

Growth.-This is slow.

Plates.-At ordinary tem perature colonies develop in gelatin in $4-7$ days, and resemble those of the Hay bacillus or of Proteus. The gelatin is slowly liquefied and gas produced. On agar plates the colonies appear as faint clouds which, under the microscope, are seen to be oval and partly surrounded by a whorl of threads which are finer than those of otber anaerobes.

Stab culture.-Development restricted to the lower part of the tube. Cultures in glucose gelatin tubes show along the line of inoculation a cloudy growth, radiating outward into the surrounding gelatin; resembles that of the Root bacillus. Eventually the gelatin is liquefied. Gas bubbles present. In glucose agar at $37^{\circ}$ the growth is sometimes indistinct and shows radiations.

Streak culture.-On glucose agar the growth is rapid and is practically invisible.

Bouillon.-At $37^{\circ}$ becomes diffusely cloudy and remains so for several days; eventually the growth settles to the bottom, forming a scarcely visible sediment-distinction from the preceding anaerobes.

Glucose gelatin, colored with litmus.-At $37^{\circ}$ becomes permanently liquefied; a very small sediment forms, and the culture remains blue, showing absence of acid formationdistinction from preceding.

Milk.-It grows well in milk without inducing any change. Does not invert starch. The growth on potato is invisible.

OXYGEN REQUIREMENTS.-It is an obligative anaerobe and grows in vacuum, hydrogen, nitrogen, and carbonic acid.

TrmperatuRE. - No growth below $16^{\circ}$. The optimum is about $38^{\circ}$.

Behavior to gelatin.-Liquefies.

Aerogenesis.-Gaseous products; disagreeable odor; $\mathrm{H}_{2} \mathrm{~S}$.

Atrenuation.-Partial loss of virulence by culture.

ImMUNITY.-Iodine trichloride; thymus bouillon cultures; injection of filtered culture; of purified toxin; blood-serum of artificially immunized rabbits, horse, sheep, dog; milk of immunized goat. The nucleohiston from the thymus gland destroys the tetanus toxin.

Pathogenesis.-Man, horse, sheep, guinea-pigs, young cattle, goats, white mice and white rats, are susceptible. Rabbits and dogs are less susceptible. Ducks and chickens are immune. It is not in the blood but is present at the point of inoculation, although in small numbers; at times it may be wholly absent. Intensely poisonous products. The filtered bouillon culture in a dose of $0.0002 \mathrm{c}$.c. may kill a mouse, and 0.002 c.c. may kill a guinea-pig. The strictly pure tetanus spores cannot produce the disease. Mixed infection.

Infection.--Occurs through wounds. Poisoned arrows of the New Hebrides contain tetanus and malignant edema spores.

DiAgNosis. - The detection of the bacillus is difficult because of its scarcity, and because of the presence of other anaerobic and aerobic bacteria. The pus should be removed from the wound with a sterile drawn-out glass tube pipette and transferred to glucose. litmus gelatin. A loopful of this dilution should be transferred to each of 8 or 10 tubes of liquefied glucose agar. These are then poured into Petri dishes and developed in hydrogen. The characteristic colony is oval, one end of which is surrounded by a whorl of threads.

The original glucose litmus gelatin is developed at $35^{\circ}$ and a portion is then injected under the skin of a guinea-pig, or a white mouse.

A portion of the pus should be stained direct and examined for the long slender tetanus bacilli and for "drum-sticks." 
DRAWIngs. 


\section{The Culture of Anaerobic Bacteria.}

Obligative anaerobic bacteria, those which grow only in the absence of oxygen, require special conditions for cultivation. Their growth is favored by the addition of 1 to 2 per cent. of glucose to the nutrient medium, such as gelatin, bouillon or agar. Freshly prepared media are, as a rule, best adapted for cultural purposes.

The numerous methods which have been proposed for obtaining growths of anaerobic bacteria can be classified under the following heads:
1.-Exclusion of oxygen.
4.-Displacement of air.
2.-Exhaustion of air.
5.-Cultures apparently in air.
3.-Absorption of oxygen.
6.-Microbic association.

Exclusion of oxygen.-Several different methods can be grouped under this head. Thus, the earliest method employed was to cover the liquid with a layer of oil. Although not perfect, yet this method of Pasteur can at times be employed to advantage. Later on, Koch endeavored to obtain colonies under anaerobic conditions by placing a thin mica sheet on the surface of a gelatin plate. Better results are obtained by covering the gelatin or agar plate with another sterile glass plate (Sanfelice).

The well-known method of Liborius, culture in deep layers of gelatin or agar, depends upon the exclusion of air. The method is simple and very convenient. The tubes should contain glucose agar or gelatin, and the medium should be about 2 inches deep. Stab cultures are made in the usual manner. Growth develops along the line of inoculation in the lower two-thirds of the medium, while the upper third (about $\frac{1}{2}$ inch), serves to exclude the air. In order to insure complete exclusion of oxygen, the contents of an ordinary agar or gelatin tube can be liquefied and 
then, with proper precautions against contamination, poured on top of the inoculated medium and quickly cooled. This extra layer is, as a rule, unnecessary.

Isolated colonies can be obtained by the method just given. The liquefied glucose agar or gelatin tubes are inoculated in the usual manner for making plates. The contents of the tubes are then solidified and an extra layer of the medium is poured into each tube.

The method of Roux deserves mention under this head. The inoculated medium is drawn up into a sterile glass-tube pipette (Fig. 61), which is then sealed above and below the liquid by means of a flame. A deep layer is thus obtained with little or no air above. By cutting the tube a colony can be easily reached.

Exhaustion of air.-Pasteur in his studies on the bacillus of malignant edema employed special tubes which were connected with an air-pump, and, when a vacuum was reached, they were sealed in the flame. The method bas been simplified by Gruber and is easy of execution. Special test-tubes, with a constriction below the cotton plug may be obtained. After the medium is inoculated a vacuum is produced in the tube which is then sealed in the flame. If desired, colonies can be obtained by "rolling" the sealed tube.

Absorption of oxygen. - If a solution of pyrogallic acid is rendered alkaline it will immediately become dark, then brown, and finally black, due to the rapid absorption of oxygen. This reaction has been utilized in a variety of methods. Other chemicals may be used for absorbing oxygen, but they have no advantage over that mentioned. Buchner's method consists in placing the inoculated tube inside of a larger one, which contains on the bottom some pyrogallic acid. Caustic alkali is added to the acid and the tube is then closed at once with a rubber stopper. 
Displacement of air. - This can be accomplished by passing through the tube or apparatus a current of inert gas. Hydrogen is employed most often for this purpose. Carbonic acid has been employed by Pasteur and others, but it is not as indifferent a gas as hydrogen. If employed, it should be washed by passage through a solution of sodium carbonate. Illuminating gas may be used with fair results.

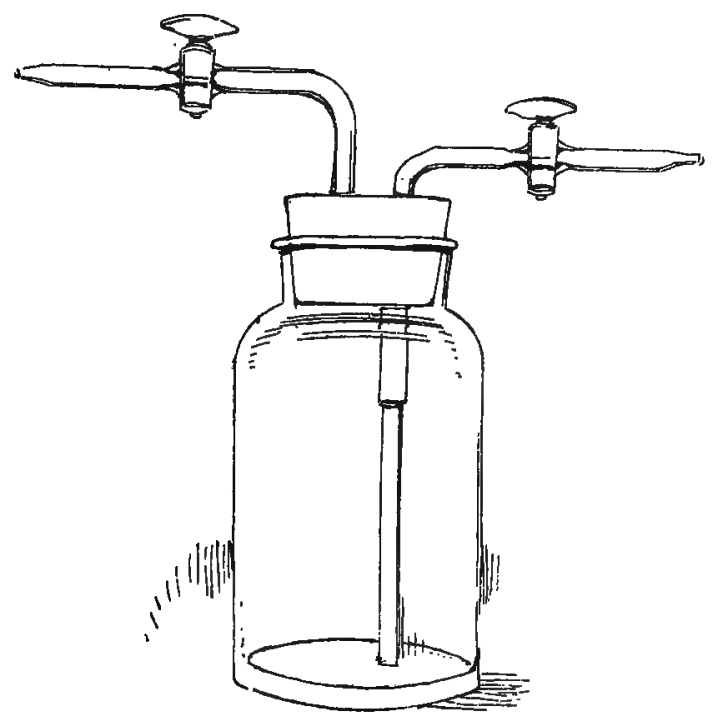

FrG. 5o. Simple bottle for anaerobes (F, G. N.).

The Liborius tube which is most often mentioned in this connection is a test-tube with a constriction below the neck. At a distance of about 2 inches from the bottom a narrow side-tube is attached, and is continued on the inside almost to the bottom of the test-tube. After the medium is inoculated a current of hydrogen is passed into the tube, through the side-tube, until the air has been displaced. When this result is attained the tube is sealed below the cotton plug and finally the side-tube is likewise sealed. Various modifications have been proposed, but although they render the 
method less expensive, they do not materially simplify the procedure.

Fig. 50 shows an apparatus which was employed at one time by the author. It can be constructed by any one and is simple, inexpensive, and will give excellent results. Ordinary test-tubes are employed instead of the expensive tubes of Liborius. Moreover, a large number of tubes can be placed in one apparatus. The directions for use as given in connection with the author's special apparatus (p. 312), are applicable to this bottle. The stop-cocks serve to seal the apparatus.

A great variety of apparatus has been described for the purpose of obtaining colonies. Some of these, like Botkin's, enable one to make use of the ordinary Petri dishes. More often, however, they consist of a special dish, such as that of Kitasato.

Cultures in the presence of air.-This method, from the nature of the organisms under consideration, would seem to be impossible. Nevertheless, as the author has shown, good cultures can be obtained without the use of any special apparatus, and these cultures are made with apparently free access of air. When a tube of glucose agar is liquefied, and then allowed to become solid, a drop or two of water will separate out on the surface. If a stab culture is now made, for instance of symptomatic anthrax, it will show a good growth not only along the line of inoculation in the deeper layers of the agar, but also in the liquid on the surface. This liquid is apparently in direct contact with the air. It is possible, however, that the carbonic acid and other gases given off by the organism displace the air from the tube, and allow thus a growth to take place. It may be remarked in this connection that this growth in the water of condensation is extremely rich in those peculiar spirals known as giantwhips (p. 39). 
A better and more useful procedure than the above is to employ gelatin containing two per cent. of glucose and colored with litmus. The ordinary test-tubes containing this material are inoculated and placed direct in the incubator at $37^{\circ}$.

Although the gelatin melts and apparently there is free access of air, yet abundant growths of all the anaerobic bacteria can thus be obtained. Similar, though not as constant results have been obtained with glucose bouillon containing two per cent. of gelatin. The viscosity of the liquid undoubtedly prevents the penetration of the air. The cultures grown in litmus colored glucose gelatin pre. serve their vitality better than on any other medium. Moreover, they possess another advantage in this that the growth is readily accessible for transplantation or for study. For these reasons it is the author's usual method for keeping stock cultures of anaerobes.

Microbic association.--The soil seems to be the natural habitat of the anaerobic bacteria. And yet, of all places this would seem to be the least adapted for their growth owing to the abundance of oxygen. When a strongly aerobic organism, such as the B. prodigiosus or Proteus vulgaris, is inoculated into a tube of bouillon, and, if at the same time an anaerobic organism is planted, it will be found that both bacteria will develop. Apparently the aerobic form consumes the oxygen in the immediate neighborhood of the anaerobic organism, and thus allows the latter to develop. Such cultures are intensely virulent. Conditions of this kind not only favor the growth of anaerobic bacteria in the soil, but are known to bring on disease. Tetanus or lock-jaw is induced in this way, as a result of such microbic associations.

Of the several methods touched upon in the preceding summary two deserve especial attention owing to their practical usefulness. In these the ordinary test-tubes are 
employed and no apparatus is necessary. The methods referred to are: stab culture in deep layers of gelatin or agar, and cultivation in glucose litmus gelatin.

The various forms of apparatus indicated above and described fully in most of the text-books, are far from being satisfactory. The use of special tubes or of special plates, where a large number are to be used, is a matter of considerable expense. The treatment of each tube by itself and the subsequent sealing involves a waste of time, and is not altogether free from danger. At least one death is recorded as the result of endeavoring to seal a hydrogen culture of the tetanus bacillus. It is obviously desirable to make use of the ordinary test-tubes and ordinary Petri dishes. This can be done by means of the special apparatus ${ }^{1}$ shown in Fig. 51-53. During the past six years this apparatus has been in constant use in this laboratory, and the fact that frequently several hundred cultures are made by the students at one time will indicate its practical usefulness.

The bottle shown in Fig. 51 is intended for tube culture. It is made in two sizes, for large and for small tubes. The small bottle has an internal diameter of $8 \mathrm{~cm}$., and the inside beight to the neck should be $16 \mathrm{~cm}$. The large bottle has a corresponding diameter of $10 \mathrm{~cm}$. and a depth of 20 $\mathrm{cm}$. It is provided with a hollow stopper which should be not less than $4 \mathrm{~cm}$. in diameter. There are openings in the glass stopper corresponding to the two tubes in the neck of the bottle. One of these openings has a glass tube attached which extends down to within a short distance of the bottom. After the gas has been passed for some time, the stopper is turned through an angle of $90^{\circ}$, thus effectually sealing the bottle.

Ordinary test tubes containing glucose bouillon, gelatin, agar or potato are inoculated in the usual manner. The projecting part of the cotton plug is cut off close to the

${ }^{1}$ Centralblatt für Bakteriologie 14, p. 581, 1893; 16, p.566, 1894. 
mouth of the tube, and, the plug is slightly raised with sterilized forceps in order to facilitate diffusion of the gas. The inoculated tubes are then placed in the bottle by means of a pair of forceps (Fig. 46), and the apparatus is now connected with a Kipp's hydrogen generator. The current of hydrogen shonld be passed, first, through an alkaline solution of lead acetate, then through a six per cent. solution of potassium permanganate, and finally through a solution of silver nitrate. After passing through the apparatus the gas passes through a small wash-bottle containing water, which serves as a valve. A rapid current of gas is passed through the bottle for 1 to 2 hours. The bottle is then sealed by turning the stopper, and is set aside in the incubator.

The hydrogen is generated from ordinary granulated zinc by means of commercial sulphuric acid. The stopper should not be greased with vaselin, but with a mixture of bees-wax and olive oil (1-4). Before applying the lubricant, it is advisable to spread a layer of cotton over the bottom of the flask. Rubber bands should be slipped over the stopper and around the lateral tubes. This is to prevent the stopper from being raised by the pressure of the confined gases when the apparatus is placed in the incubator.

When the cultures have developed they should be taken out of the bottle and preserved the same as ordinary bacteria. Care should be exercised when removing the stopper so as to avoid breakage. It should first be turned so as to allow air to enter. At the same time the rubber bands are removed. The thumb and forefinger of the left hand should rest firmly on the shoulder of the stopper, while this is gradually worked to and fro with the right hand. This little precaution will prevent the sudden jerking out of the stopper.

The pyrogallate method can be used in connection with this bottle. 2-3 g. of the acid can be placed on the bottom, the inoculated tubes then inserted, and finally the necessary concentrated alkalican be delivered from a pipette. The stopper should then be inserted at once and turned at right angles.

Figs. 52 and 53 show two forms of apparatus for obtaining plate cultures. The former is provided with a stopper like that in the bottle just described. This apparatus can 
be used for the gas or for the pyrogallate method. The apparatus shown in Fig 53 is provided with a slightly different stopper, and can be used for the gas, pyrogallate or vacuum method. In the latter case the wider end of the stopper should be provided with a piece of rubber tubing, which should be clamped tight. The apparatus provided with the ordinary stopper (Figs. 51-52), should never be used for vacuum work since the atmospheric pressure will cause the stopper to wedge so firmly that it will be almost impossible to turn.

The lower half of the apparatus should have an internal diameter of $12 \mathrm{~cm}$. and an inside height of $12 \mathrm{~cm}$. The apparatus can hold 6 or 8 Petri dishes. It can be used for small flasks, or for a large number of tube cultures. The total height of the apparatus should not exceed $24 \mathrm{~cm}$. Each flange should be $2 \mathrm{~cm}$. wide and $\mathrm{cm}$. thick. The outer circumference should be ground vertically so that a rubber band can be employed to effectually seal the apparatus. It is necessary, therefore, that the diameter of the top and bottom should be exactly the same. The unground surfaces of the flanges should be parallel or nearly so in order that the clamps will not slip off.

The gelatin or agar Petri dishes are placed in the lower jar. It is unnecessary and undesirable to remove the tops. The upper part is then placed in position and the wide rubber band is slipped over the circumference. Three clamps are then applied to the flanges. A slit piece of rubber tubing should be slipped over the jaws of the clamps. ${ }^{1}$ Hydrogen is then passed through the apparatus in the manner described on p. 312. Finally the stopper is turned and the apparatus set aside for the organisms to develop.

The pyrogallate method can be used with excellent results. $3-5$ g. of pyrogallic acid are placed in a glass dish, which is about $10 \mathrm{~cm}$. in diameter and about $3 \mathrm{~cm}$. high. This is placed on the bottom of the lower jar and covered with a strip of glass about $5 \mathrm{~cm}$. wide. The Petri dishes are then stacked on top. The upper half of the apparatus

${ }^{1}$ No. 1. Amateur vise made by the Phoenix Hardware $\mathrm{Hnfg.} \mathrm{Co.,}$ Phoenix, N. Y. 
is placed in a position so that only a narrow slit remains

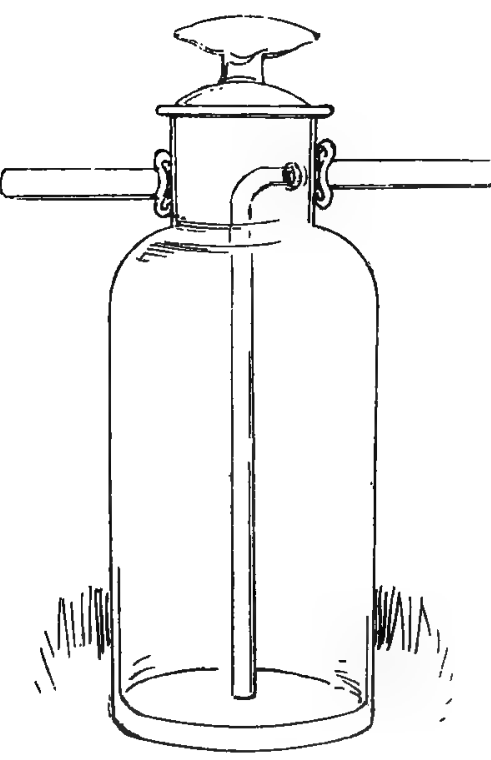

FIG. 5 I.

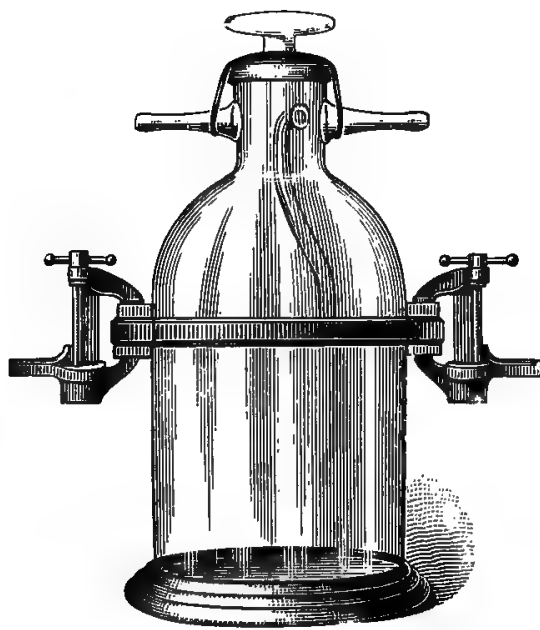

FIG. 52 open. By means of a pipette or other arrangement 25 c.c. of concentrated potash solution $(1: 4)$, are added as rapidly as possible. The top is then closed completely and the rubber band and clamps applied as before.

Laboratory work. - Liquefy four glucose agar tubes by heating in the water-bath; then allow to solidify in an upright position. When cool make deep stab cultures of the 4 pathogenic anaerobic organisms and then place the tubes at $37^{\circ}$.

If the agar in the tube is less than one inch deep, it will

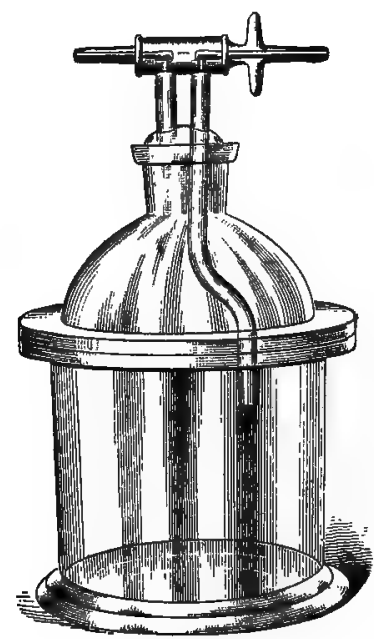

FIG. 53.

FIGS. 51-53. The author's apparatus for the culture of anaerobic bacteria. FiG. 5I. Bottle for tube cultures. Fig. 52. Apparatus for Petri dishes or tubes, - gas or pyrogallate method. FIG. 53. Apparatus for plates or tubes,-gas, pyrogallate or vacuum method. 
be necessary to pour on top, after inoculation, the contents of another agar tuhe, taking care to sterilize the mouths of both tubes. The drop or two of liquid which sometimes accumulates on the surface of the agar, and invariably on the bottom of the tubes can be stained for ordinary flagella and for giant-whips.

Likewise inoculate litmus glucose gelatin tubes and place at $37^{\circ}$.

The four organisms are also planted in glucose bouillon and these tubes are then placed in bottles in hydrogen at $37^{\circ}$. In about 3 days the tubes can be taken out and examined for spores. It is best to remove the bacterial deposit from each tube by means of a sterile drawn-out pipette (Fig. 61). The growth can then be placed in a small Esmarch dish, or salt cellar, and after dilution with water it can be used for making cover-glass preparations. When preparations are made direct from the bouillon they are likely the give a bad back-ground owing to the organic matter present. The spores can be readily double-stained.

Inclined glucose agar should also be inoculated with the four organisms, and the culture should be developed in hydrogen at $37^{\circ}$ for 24 hours. The growths should then be examined carefully in hanging-drops for motion, spores, giant-whips, etc. The latter will be found especially abundant in the liquid of condensation on the bottom of the tube. With this material cover-glass preparations will be made, as presently described, and employed for demonstrating the presence of flagella or whips.

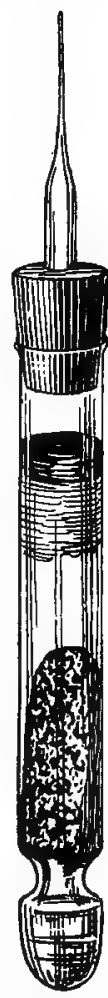

FIG. 54. Tube cultivation, on potato, of the tuber-

Glucose agar Petri dishes should also be prepared. open capillary. Some of these should be placed in the plating apparatus (Fig. 52) and cultivated in an atmosphere of hydrogen. The pyrogallate method (p. 313) should be used to develop another set of the plates. A third set of plates should be grown in a vacuum (Fig. 53) which is brought about by means of a Chapman aspirator.

Make the following cultures at the same time as the preceding:

1.-Streak cultures of the tubercle bacillus on inclined glycerin agar and on glycerin potato (Roux tube, Fig. 54). A pure culture, or 
a tubercle from a guinea-pig inoculated with tuberculosis should be used. The material should be thoroughly spread over the surface of the medium.

2.-Streak cultures of the aviary tubercle bacillus on inclined glycerin agar and on glycerin potato as above.

3.-Streak culture of the Achorion Schönleinii (the fungus of favus) on ordinary inclined agar.

4-Streak culture of Actinomyces (the fungus of lumpy-jaw) on ordinary inclined agar.

5.-Streak culture of the fungus of Madura-foot on inclined agar or glycerin potato.

After these inoculations have been made the cotton plug of each tube is cut off close to the mouth of the tube. The end of the tube is then rapidly turned in a flame till the cotton changes color. The tube is then sealed either with a rubber cap (previously soaked in mercuric chloride), or with sealing-wax, or with paraffin of a high melting point, $56^{\circ}$. Ordinary corks can be used to advantage provided they are first immersed in mercuric chloride solution and steamed for at least $\frac{1}{2}$ hour. The heated, slightly charred cotton plug is pushed into the tube by means of sterile forceps. The softened, sterile cork is then inserted. The sealed tubes are then placed in the incubator at $39^{\circ}$ for several weeks.

Instead of sealing the tubercle cultures air-tight, it is advisable to employ a cork through which passes a drawn-out capillary tube (Fig. 54).

The Staining of Flagella.

The locomotive organs of bacteria, as described on $p$. 35, are extremely thin, wavy whips or flagella. These cannot be seen in the unstained preparations, although under favorable conditions they can be demonstrated by photography. Only very rarely can motion be observed in the liquid immediately surrounding the organism. In simple stained preparations the flagella are likewise invisible, inasmuch as they do not readily take up the dye. The presence of whips or flagella can, therefore, be satisfactorily demonstrated only by the aid of special staining methods. The procedure as employed below is essentially that of Löffler. 
In order to obtain good stains of flagella special care must be given to the preparation of the cover-glasses. These should be cleaned according to the method given on p. 140, and when spread must contain as little organic matter as possible in order to prevent the formation of a dirty precipitate on the cover-glass. Excellent cover-glasses can be made by resorting to dilution. For this purpose, a small loopful of the turbid fluid found on top of the agar stab culture, or at the bottom of the inclined agar culture of the edema bacillus No. II, or of the symptomatic anthrax bacillus, is transferred to a large drop of distilled water on a glass slide.

By means of a straight platinum wire, three transfers are made from this drop to another drop of distilled water on the same slide. This second drop will now contain only a small number of bacteria and very little foreign matter. By means of a platinum wire, with a very small loop, not much larger than a pin-head, transfers can now be made to six or eight clean wide cover-glasses. Each small loopful is spread at once over as much of the surface of the coverglass as possible. The thin film of liquid evaporates almost immediately, and the cover-glass can then be fixed by passing it once through the flame. Over-heating the coverglass is very likely to destroy the slender flagella. This can be prevented if the cover-glass is held between the thumb and index finger as it is passed through the flame.

The cover-glass, with the specimen side up, is held in a pair of forceps and covered, by the aid of a pipette, with the hot mordant solution. The cover-glass is then held over the flame for about a minute. The fiame should be turned down till it is less than $1 \mathrm{I} / 2-2$ inches high. The liquid should be warmed so as to give off vapors, but should not be actually boiled. As fast as evaporation takes place fresh mordant solution should be added, and at no time should it be allowed to dry down on the coverglass. 
After one or two minutes of careful heating the mordant is then thoroughly and completely washed off the cover-glass by a jet of water. This can be easily done if the specimen has not been over heated. If the edge has dried down, it should be loosened with a pin or knife and then washed off. To still further clean the cover-glass, it may be dipped for a few spconds in absolute alcohol and again washed with water.

The excess of water is drained from the cover-glass which is then covered with a hot saturated solution of anilin-water fuchsin. The specimen is then gently and slowly heated over the flame for one to two minutes, avoiding actual ebullition. It is then heated to boiling for about half a minute, and finally it is thoroughly washed with water and examined with the 19 inch oil immersion objective. A number of slender flagella can be seen surrounding each cell.

Summary for staining flagella:

Dilution cover-glass preparation.

Dry in air.

Once through flame.

Mordant, hot (1 to 2 .min.).

Wash in water (and clean).

Dip in alcohol (few seconds).

Rinse in water.

Anilin-water fuchsin, hot (1 to $2 \mathrm{~min}$.).

Wash in water and examine.

Dry in air.

Mount in balsam.

The mordant employed is a slight modification of that originally proposed by Löffler. It has the following composition (A. Fischer):

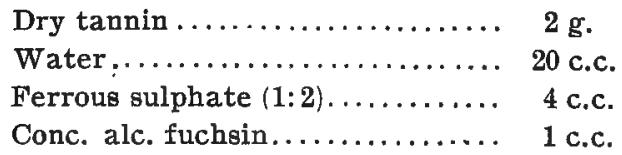

The tannin should be kept in a desiccator. It is dissolved in the stated amount of water by the aid of gentle heat. The two other 
solutions are then added and the liquid is filtered at once. A voluminous precipitate must remain on the filter, otherwise the mordant is useless. The mordant can be used at once, and will keep for six weeks or more. It should be kept in the dark. The mordant is said to give excellent results with all motile bacteria.

The stain employed is made by adding 4 to $5 \mathrm{~g}$. of fuch. sin to 100 c.c. of anilin water, to which 1 c.c. of a 1 per cent. $\mathrm{NaOH}$ solution has been added. Hot, freshly prepared carbolic fuchsin, or an aqueous 1 per cent. solution of fuchsin, may be used. Both mordant and stain should be kept warm while in use. The iron plate shown in Fig. 22 (p. 150), will be found useful for this purpose.

Giant-whips.-These interesting forms are especially abundant in the few drops of condensation water which accumulate at the bottom of the freshly inclined agar. They are met with under these conditions in cultures of most of the known motile bacteria. Owing to their extremely large size they can readily be seen in the hanging-drop. The author's method is to place on a coverglass a drop of blood-serum, which is then inoculated with the turbid liquid mentioned. In about 15 minutes, more or less agglutination of the bacteria results and the large spiral bodies are then plainly visible. They may be broad, spindle-shaped, or very long, slender, wavy lines. They have been observed to exceed $100 \%$ (g so inch) in length (see p. 38). 


\section{Bacillus Lepræ, Hansen (1879).}

\section{LEPROSY BACILLUS.}

ORIGIN. - It is found in enormous numbers in the leprous nodules of the skin; to less extent in the mucous membrane, lymphatic glands, liver, spleen, marrow, etc. It usually occurs in masses within the so-called leper cells. It may be present in the blood in small numbers.

Form.-Small, narrow rods, which resemble the tubercle bacillus.

Motility.-It is non-motile.

Sporulation.-Bright bodies, are frequently observed within the cell, but as in the case of the tubercle bacillus, these are not spores.

ANILIN LYES. - In fresh material the bacilli can be readily stained with the ordinary anilin dyes. They can likewise be easily demonstrated in such material by means of the method employed for staining the tubercle bacillus (p. 324). The latter stain fails when the tissue has been kept in alcohol for some time. In that case Gram's method will give excellent results (Chapter XV).

GrowrH. - This has not been obtained on artificial media with certainty. It is to be considered as a typical obligative parasitic organism.

Pathogenesis. - The constant presence of this bacillus in enormous numbers, in leprous tissue justities the prevailing view that it is the cause of that disease. Nevertheless, it should be remembered that as yet unquestioned pure cultures have not been obtained, and hence, successful inoculations are impossible. Direct infection with leprosy tissue has given but few, if any, positive results. The monkey, cat, dog, goat, hog, chicken, guinea-pig, rabbit, etc., are apparently not affected.

INFECTION.--Leprosy, as in the case of tuberculosis, may be congenital. As a rule, however, it is acquired but the exact mode of infection is as yet unknown. It would seem that leprosy, like glanders, attacks first of all the nasal mucous membrane. The leprosy bacillus leaves the body with the secretions of the nose and mouth.

Diagnosis. - The bacillus of leprosy is to be distinguished from that of tuberculosis. The inoculation of a guinea-pig will decide the presence of tubercle bacilli. An examination of the nasal secretion, and of sputum should be made in suspected cases. 
DRAWINGS.

321 


\title{
Bacillus Tuberculosis, Koch (1882).
}

\author{
TUBERCLE BACILLUS.
}

ORIGIN.-In tuberculosis of mammals; in lupus vulgaris. The bacillus of chicken tuberculosis is distinct from that of mammals.

FOR.M. - Very narrow, rather long rods which are smaller than the diameter of a red blood cell. They may be beaded. The ends are distinctly rounded and the bacillus itself may be straight, or more frequently is slightly bent or nicked. Occurs usually single, but may form short threads of 3-6 cells. In the sputum, tissues etc., it is frequently found in small bunches. Rarely, it occurs in branching form and with club-shaped ends.

Motility.-Has no motion.

Sporulation.-Frequently shows a number of bright bodies within the cell, but these cannot be considered as true spores (p. 32). The bacillus itself possesses a relatively high power of resistance to heat, desiccation, acids, putrefaction etc.

ANILIN DYES. - It stains very slowly and difficultly with simple anilin dyes; readily with hot carbolic fuchsin, or anilin-water fuchsin or gentian violet. When once stained it is difficult to decolor, whereas ordinary bacteria do so readily-distinction from ordinary bacteria. Gram's method applicable, but requires a long exposure to the dye.

GrowTH.-Takes place very slowly, so that usually several weeks elapse before it becomes clearly visible. Furthermore, a special temperature, at or near that of the body, and special media as bloodserum or glycerin-agar, etc., are necessary. Access of air is also essential. The cultures have a characteristic yeast-like odor. It is possible to accustom the tubercle bacillus to grow on ordinary bouillon and agar, even at or near the room temperature-attenuation.

Plates,-No growth has been obtained on plates. Colonies can be readily obtained by making successive streaks on glycerin-agar or blood-serum. Colonies obtained direct from the sputum are round, white, opaque, and raised, resembling colonies of white yeast. On subsequent culture the colonies form dry, grayish scales. Under the microscope they appear as interwoven, twisted strands of threads.

Stab culture.-Can be obtained on glycerin-agar. The growth is restricted to the upper part of the tube. It spreads over the surface as a thick, raised plaque which at first is white, but later becomes yellowish.

Streak culture.-On glycerin-agar or blood-serum it develops an abundant dry, granular, raised growth. which at first is grayish, but later takes on a light yellow tinge.

Potato.-In Koux tubes containing 5 per cent. of aqueous glycerin (Fig. 54, p. 315) the growth is thick, yellowish and in ridges. Requires at least 2 weeks at $39^{\circ}$.

Bouillon.- One which contains 5-6 per cent. of glycerin is necessary. A dry folded growth covers the surface; the liquid remains clear and a granular sand-like deposit forms. The material planted must float on the surface of the liquid, otherwise the culture will not develop. or but very poorly. Such bouillon cultures, filtered and concentrated, constitute the so-called tuberculin.

OXYGEN REQUIREMENTS. - Free access of oxygen is necessary for growth. It is a facultative anaerobe (Fränkel).

Temperature. - Optimum at $37-39^{\circ}$. Slight variations above or below this stop the growth. It cannot grow at room temperature. BEHAVIOR To GELATIN.-No growth at ordinary temperature. It does not peptonize blood-serum.

ATTENUATIN.-Prolonged artificial cultivation results in partial attenuation. This is especially marked when grown on potato and similar media. Passage of the bacillus through doves or frogs yields an attenuated form which may grow at ordinary room temperature. 'The tubercle bacillus as found in man possesses a variable virulence.

Pathogenesis. - See p. 325. Diagnosis. - See p. 324.

INFECTION.-Takes place most frequently along the respiratory tract-Inhalation tuberculosis. May occur through wounds-Inoculation tuberculosis, and also through food-Intestinal tuberculosis. In the latter case the bacilli introduced into the intestines may localize in distant parts of the body. Ilacental infection may occur. 
DRAWINGS.

323 


\section{Recognition of the Tubercle Bacillus.}

Ziehl-Neelsen method.-A large loopful of the sputum is transferred to a wide cover-glass and thoroughly spread over the surface. It is then allowed to dry in the air or by moving it to and fro, over the flame, after which it is fixed in the usual way. The cover-glass is held in the forceps, specimen side up, and covered with carbolic fuchsin solution. It is warmed over a low flame for 1 to 2 minutes, avoiding actual ebullition. The dye should not be allowed to dry down on the cover-glass. A drop or two of the stain should be added, from time to time, to prevent this from happening. The excess of dye is then washed off with water and the specimen is placed in dilute nitric acid for 10 to 15 seconds. This dilute acid solution is prepared by adding 3 or 4 drops of the concentrated acid to a watchglass full of water. When the color disappears it is at once transferred to dilute alcohol (60 to 70 per cent.), where it is moved about till it is almost decolored. After this it is washed with water and stained for a few seconds with methylene blue. The latter is washed off with water and the specimen is then examined with the 1 th inch oil immersion objective. It should show the bright red tubercle bacillus on a light blue back-ground. The ordinary bacteria that may be present are stained likewise blue. It is evident, therefore, that the ordinary bacteria are readily decolored by treatment with acid and alcohol and in this respect differ from the tubercle bacillus. This is due to a difference in the chemical composition or to a concentration of the protoplasm of the tubercle cell, rather than to the presence of a denser cell-wall.

Heavily stained preparations can be obtained by float. ing the prepared cover-glass on slightly warmed carbolic fuchsin solution for 15 to 30 minutes, then decoloring as above. 
Summary of the method given:

Cover-glass preparation.

Dry in air.

Fix in flame.

Carbolic fuchsin, hot, (1-2 min.).

Wash in water.

Dilute nitric acid (10-15 seconds). Mount in balsam.
Dilute alcohol (60 per cent.).

Wash in water.

Methylene blue (1 $-\frac{1}{2}$ min.).

Wash in water and examine.

Dry in air.

The sputum should be collected in the morning immediately after rising. inasmuch as at this time it is likely to be rich in bacteria. It may be necessary at times to instruct the patient that sputum, and not saliva, is what is wanted. The material should be poured into a wide glass dish, such as that of Petri, and examined for the presence of yellowish particles. These are portions of the caseous matter from the lungs and are likely to be rich in bacteria. The sputum should be examined also for elastic fibers, and the presence of streptococci and of other bacteria should be noted (mixed infection).

Suspected urine should be centrifugated and the deposit examined in the same way as indicated above. In the case of milk, it is advisable to centrifugate and then to inject the combined cream and sediment into guinea-pigs.

Occasionally sputum, pus, or other products of disease will not reveal the tubercle bacillus by this method. Either the germ is present in a spore-like condition, or it is present in very small numbers and thus escapes detection. In such a case it is necessary, as with urine or milk, to resort to an animal experiment in order to establish the diagnosis. For this purpose some of the material is injected into the peritoneal cavity of a guinea-pig. In three or four weeks the animal is killed, and if, on postmortem examination, tubercular nodules are found in the abdominal cavity they should be examined for the tubercle bacillus. A portion of the cheesy matter from the inside of the nodule should be used for the examination. Cultures may be made as indicated on p. 315 .

Pathogenesis.-Man, monkey, cattle, horse, ass, hog, goat, parrot, guinea-pig, field mouse, rabbit, and cat are susceptible. White 
mice, rats, canaries, and dogs are somewhat insusceptible. The pigeon, sparrow, chicken, and cold blooded animals are immune. Inoculation of pure cultures produces tuberculosis in susceptible animals. The bacilli are usually very abundant, but at other times are scarce and difficult to find. Guinea-pigs are extremely susceptible to the intraperitoneal injection of the tubercle bacillus, whereas rabbits are somewhat less susceptible. There is a marked loss in weight and death may result in 10-14 days. If the dose is small this will not occur for from 4 to 6 weeks, or later. The peritoneum may be studded with fine sand-like tubercles. Larger tubercles are present in the liver, spleen and lungs. Very large tubercles are usually present on the omentum. These have a soft, caseous center and are rich in bacilli.

The diagnosis of tuberculosis in cattle is effected by the injection of a small dose of tuberculin. A marked rise in the temperature occurs, and may persist for a day or more.

The staining reaction as given above will usually be sufficient to establish the presence of the tubercle bacillus. In doubtful cases, the animal experiment must be resorted to. It should be borne in mind, however, that a few bacteria are known which resist decoloration by an acid and hence may be mistaken for the tubercle bacillus. Moreover, the mere presence of nodules or tubercles in an animal does not imply the presence of the tubercle bacillus since pseudo-tuberculoses are known which are due to several different kinds of bacteria. These possible contingencies may be briefly alluded to.

1.-The leprosy bacillus stains like the tubercle bacillus. It can be stained with the ordinary dyes. Moreover, it exists.in masses and cannot be transferred to animals, nor can it be grown artificially. The recognition of tuberculosis in a leper can be effected by means of the animal experiment. Bacilli resisting decoloration with acids have been grown from leprosy material.

2.-The 'smegma bacillus which may be found in the smegma of the prepuce or of the vulva, stains the same as the tubercle bacillus. While it is resistant to 
acids, it is readily decolored by absolute alcohol. The smegma bacilli of diverse origin show unequal resistance to decoloration with acids. It is not transferable to animals and is very difficult to cultivate. The animal experi ment will again demonstrate the tubercle bacillus. The smegma bacillus should be expected, whenever urine or secretions of the genito-urinary apparatus are examined. The same or a similar organism has apparently been found in the mouth, and in lung gangrene.

To differentiate from the tubercle bacillus the specimen which has been stained with carbolic fuchsin should be decolored by immersion for some minutes in a saturated absolute alcoholic solution of methylene blue; or by an acid alcoholic solution (alcohol 97, HCl 3) for 10 minutes. Another procedure is to treat it with absolute alcohol for at least three hours, then with five per cent. chromic acid for at least 15 minutes, after which it is stained with carbolic fuchsin, decolored by exposure to dilute sulphuric acid for 2-3 minutes, and finally stained with concentrated methylene blue.

In order to cultivate the smegma bacillus it is advisable to inoculate a tube of liquefied agar, cooled to $50^{\circ}$, with the suspected material. By means of a sterile syringe about 2 c.c. of blood should be drawn from the vein in the arm (see Chapter XIV), and this is then mixed with the agar. The blood agar mixture is poured into a sterile Petri dish, which is then set aside for a day or two at $37^{\circ}$. The colonies should be examined for bacilli which resist decoloration by acids.

3.-From milk, butter, timothy hay, cow-dung, etc., bacteria have been isolated which stain like the tubercle bacillus, and have indeed been mistaken for this organism. Guinea-pigs, as a rule, survive injection whereas rabbits appear to be immune. The inoculated guinea-pigs may die in a month or two. Numerous small nodules or tubercles are present in the mesentery, peritoneum, liver, lungs, etc. Cultures made from these tubercles develop in three or four days and apparently attenuate rapidly. The tubercles do not show giant cells, or caseation. It is evident, therefore, that in some instances it is necessary to supplement the cover-glass examination by a histological study of the suspected tubercles. Bacteria 
resisting decoloration with an acid have been found in certain eye affections.

4.-Pseudo-tuberculoses. - The preceding case may be said to fall under this head. There are, however, bacteria which give rise to tubercles or nodules but otherwise are easily distinguished from the tubercle bacillus. They are easily cultivated and stain with the ordinary dyes, but do not give the double stain like the tubercle bacillus. Pseudo-tuberculoses are rare in man, though rather common in animals (sheep, chicken, rabbits, etc.).

5.-Aviary tuberculosis. - The bacillus present in the ordinary bird tuberculosis stains like the tubercle bacillus. It grows more rapidly on various media and forms a moist soft growth, which unlike that of the tubercle bacillus, will easily form a cloudy suspension when stirred into water. Guinea-pigs when injected subcutaneously react with a local affection, whereas intraperitoneal injection into a chicken yields general tuberculosis. The rabbit is susceptible to both organisms. The tubercles do not show giant-cells. The bacillus can grow at a higher temperature than the tubercle bacillus, but otherwise the growths on the various media are very much alike. The aviary tubercle bacillus may naturally infect man, rabbits, and other animals. It has always been believed to be a variety of the mammalian tubercle bacillus. Cultures in collodium sacs'seem to prove that the aviary bacillus can be transformed into that of mammals.

6. - Fish tuberculosis. - This organism was isolated from a small tumor on a carp. The presence of giant cells and the histological structure pointed to tuberculosis. The bacillus present stained like that of tuberculosis, but was culturally different. It grows best at $23-25^{\circ}$ and does not grow at $39^{\circ}$. It would seem as if the mammalian tubercle bacillus on passage through cold-blooded animals (fish and frogs) gave rise to a variety, as in the case of bird tuberculosis. 


\section{Glanders.}

Diagnosis.-The glanders bacillus is extremely difficult to detect in old, and eren in new diseased tissue. Consequently, direct detection, as in the case of tuberculosis, cannot be made with any degree of certainty. The failure in staining, and even in direct cultivation, renders it necessary to resort to the animal experiment in doubtful cases. For this purpose two procedures are available.

1.-Method of Straus. - The suspected matter or secretion is diluted with sterile water, or bouillon, and injected intraperitoneally into male guinea-pigs. The reaction is manifested in a few days by a marked swelling of the testicles. The scrotum becomes red or violet and adherent, so that the testicles cannot be pushed back into the abdomen. Death occurs in a week or two. Bacilli may be isolated by making agar streaks from the spleen. They are especially abundant in the altered testicles. The marked swelling of the organs, which is observed within three or four days, is taken as confirming the diagnosis of glanders.

2.-Injection of mallein.-The heated and filtered bouillon culture of the glanders bacillus, when injected into a healthy animal, produces no rise of temperature, and at most a slight swelling which disappears within 24 hours. In a glandered animal the local swelling is extensive and painful; the lymphatic vessels which lead from this part are likewise enlarged and painful. The swelling increases for 24-36 hours, lasts for several days, and finally gradually subsides in about 8 or 10 days. The general condition of the animal is likewise markedly changed. A marked stupor and profound prostration is observed. The temperature rises $1.5-2.5^{\circ}$ and reaches its maximum between the 10th and 12th hour. A diagnosis can thus be established within 48 hours.

The two tests should always be carried out together, inasmuch as each one by itself is subject to fallacy. Thus, the Straus reaction is obtained in ulcerative lymphangitis of the horse. This disease resembles farcy but is due to a different organism. The diseased animal is not affected by mallein. On the other hand, an injection of mallein may give rise. to fever in non-glandered animals. In such cases, however, the local reaction is absent. It is evident, therefore, that a positive Straus reaction and negative mallein test; or, a negative Straus reaction and increased temperature with mallein, would .exclude glanders. 


\section{Bacillus Mallei, Löffler and Schütz (1882).}

GLANDERS; MORVE (Fr.); ROTZ (Germ.); MALLEUS (Lat.).

Origin.-Found in the nodules, ulcers, discharges, etc., of 'glanders or farcy.

FORM.-Rods with rounded ends, straight or slightly curved, shorter and thicker than the tubercle bacillus. Usually single; may grow in pairs or in short threads.

Motility.-It shows very marked Brownian motion.

SPorulation.-Bright bodies are frequently found in the cells, as in the tubercle bacillus; are considered by Löffler as the first indication of degeneration. Real spores are unknown. The bacillus itself is not very resistant to desiccation.

ANILIN DYEs. - It is stained unevenly and decolors rapidly. Carbolic fuchsin, or alkaline anilin gentian violet, or anilin fuchsin stain well, especially when warmed. It is not stained by Gram's method.

GRowTH.-This occurs best at a relatively high temperature. Growth is rapid. Glycerin agar is the best medium.

Plates.-As a rule, colonies cannot be obtained with gelatin. On glycern agar at $37^{\circ}$ excellent colonies form in a day or two. These are round, grayish and glistening in appearance, with granular contents and smooth sharp borders.

Stab culture.-Can be made in glycerin agar; in gelatin it develops very slowiy.

Streak culture.-On glycerin agar forms a thick, moist, slimy, semi-transparent growth. On potato the growth is very characteristic. At first it forms a thin, transparent, honey or amber-colored growth which later becomes reddish-brown. On blood-serum it forms yellowish, transparent spots which eventually fuse together and yield a slimy, whitish growth.

Bouillon.-In this it grows readily and abundantly; diffuse cloudiness with slimy ring on the surface. Mallein is the filtered bouillon culture of the glanders bacillus. It is, therefore, analogous to tuberculin.

In milk it produces an acid reaction.

OXYGEN REQUIRLMUNTS. - It is a facultative anaerobe.

Temperature.-It does not grow below $25^{\circ}$ very readily, or above $42^{\circ}$. The optimum is about $37^{\circ}$.

Behavior to GeLATIN.-Scarcely any growth at first. Eventually, may become accustomed to growth at the ordinary room temperature.

AtTenuation.-This takes place rapidly when grown on artificial media. The bacillus must be frequently passed through an animal, otherwise the virulence is lost and the organism may die out.

ImMUNiTy. - Small amounts of a bouillon culture injected intravenously into dogs confer immunity.

Pathogenfsis. - Man, horse, ass, guinea-pigs, field mice, cats and goats are highly susceptible. Ordinary and white mice, cattle and hogs are immune, while dogs, rabbits and sheep are but slightly susceptible. White mice become susceptible when fed with phloridzin. Susceptible animals on inoculation develop typical glanders. In guinea-pigs death results in 4 to 6 or 8 weeks. Field mice die in a few days. Enlarged lymphatics, nodules in liver, spleen, etc. Bacilli present.

INFECTION.-This may occur through wounds-inoculation glanders. In one instance a man was accidentally and fatally inoculated with a pure culture. Probably, the usual source of infection in horses is along the respiratory tract. 
DRAWINGS. 


\title{
Bacillus Diphtheriæ, Klebs, Lơffler (1883).
}

\author{
BAOILLUS OF DIPHTHERIA.
}

ORIGIN.-Found in diphtheric pseudo-membranes, and in very small numbers in the spleen, liver, etc., of diphtheria; rarely in the throats of healthy children.

FORM. Rather large, thick rods which are straight or slightly bent or wedge-shaped and have rounded ends. The form is subject to considerable variation, and rods with swollen, club-shaped ends are frequently met with; likewise branching forms-involutions. Usually single; length variable.

Motility.-It has no motion.

SPORULation.-Spores have not been observed. The bacillus is very susceptible to desiccation, or to heat of $50^{\circ}$ and above.

ANIIIN DYES. - Simple anilin dyes react poorly. Round polar bodies and transverse bands. Can be stained best with carbolic fuchsin, or with Löffler's alkaline methylene blue. ${ }^{1}$ It is also stained by Gram's method.

GROWTH. - Very rapid, especially on glycerin agar and blood-serum.

Plates.-On gelatin plates kept at about $24^{\circ}$ it forms very small round, white colonies, which have granular contents and irregular borders; do not liquefy gelatin. On glycerin agar plates, kept in the incubator, excellent colonies form in 24 hours. The deep colonies are round, or oval, coarsely granular. The surface colonies are flat, grayish white, glistening, with irregular borders; coarsely granular contents.

Stab culture.- In gelatin the growth spreads over the surface while along the punc ture only a very limited, scarcely perceptible growth of small, round, white dots occurs. Marked involution forms present. Vitality is retained best in gelatin.

Streak culture.-On glycerin agar a thin, grayish, spreading, adherent film, which is quite characteristic. On potato the growth is invisible or forms a dry, thin glazeirregular forms of the bacillus are numerous. On blood-serum it forms a thick white, opaque growth; branching forms may be present, especially if the medium is soft

Bouillon--May, or may not be diffusely clouded; the growth rapidly subsides, forming a granular deposit on the sides of the tube and on the bottom. A pellicle usually forms on the surface and the liquid becomes perfectly clear. The reaction becomes acid, owing to the presence of muscle sugar; in a few days becomes alkaline. No gas or indol is produced. Milk is not altered.

OXYGEN REQUIREMENTS.-Facultative anaerobe; grows best in air.

Temperature.- Very slight growth at $20^{\circ}$. The maximum is about $42^{\circ}$ and the optimum is at $35-37^{\circ}$

BEHAVIOR TO. GELATIN. - It does not liquefy.

ATtenuation,-Cultures isolated direct from membranes show marked variation in virulence. By artificial culture, especially if transplanted infrequently, the virulence is still further diminished. Attenuated by growth at $40^{\circ}$ in a current of air; by desiccation.

IMMUNITY. -This is produced by filtered bouilion cultures heated to $60-70^{\circ}$. or unheated, Also by injections of thymus bouillon cultures previously heated to $65-70^{\circ}$. Partial results with $\mathrm{ICl}_{8}$. Living cultures can be used. Serum of artificially immunized animals is antitoxic.

PATHOGENESIs.-Mice and rats are wholly immune. Finches, sparrows, doves, chickens, rabbits, guinea-pigs and cats are susceptible. Likewise, horses, cattle, dogs and goats. Subcutaneous inoculation in guinea-pigs usually produces death in 24-48 hours. Pseudo-membranous masses form at point of inoculation; an extensive hemorrhagic edema forms under the skin and exudates occur in the pleural cavity. Inoculation in the trachea of cats, chickens, doves, rabbits etc., is followed by pseudo-membrane formation, and by death. In some animals as rabbits, typical diphtheric paralysis of the extremities can be observed. Local swelling and scar, in prolonged cases. The filtered bouillon culture contains a highly poisonous toxin (p. 84).

INFECTION.-This undoubtedly occurs through the air, or by contact with infected articles. Dracnosis. - See p. 334.

1 This is prepared by adding 30 c.c. of conc. alc. solution of methylene blue to rooc.c. . of a 0.01 per cent. solution of potassium hydrate. 
DRAWINGS.

333: 


\section{Diphtheria.}

Diagnosis.-It must not be supposed that all cases diagnosed as diphtheria contain the Loffler bacillus. It may happen that diphtheria-like conditions, due to other organisms, will present themselves. This, so-called false diphtheria is frequently due to streptococci; at times, to staphylococci or to various bacilli. A correct diagnosis of true diphtheria, that due to the Loffler bacillus, can therefore be made only by the aid of the microscope. Furthermore, a case of diphtheria may be a source of danger, even after complete recovery, because of the continued presence of Loffler bacilli in the mouth. It is important, therefore, not only to recognize the disease as early as possible, but also to recognize the time when the infected individual ceases to harbor the bacillus in question.

In order to carry out a bacteriological diagnosis of diphtheria it is necessary to have some cotton swabs and some tubes of solidified, inclined blood-serum. The swab can be prepared by twisting a little absorbent cotton around the end of a stout wire ( $2 \mathrm{~mm}$. wide and $14 \mathrm{~cm}$. long). The swabs are placed in plugged test-tubes and sterilized in a dry-heat sterilizer (p. 160). The culture medium, usually employed, is known as Löfler's blood-serum. It is prepared according to the directions given in Chapter XIV. If necessary, ordinary blood-serum or even glycerin-agar may be used.

The sterile swab is thoroughly rubbed over the affected area, and, if possible, a portion of the false membrane should thus be removed. The swab is then streaked over the surface of one or two of the sterile, inclined serum tubes. These are then set aside in the incubator at $35-39^{\circ}$ for $18-24$ hours. The use of a disinfecting solution, previous to the swabbing of the throat, should be avoided.

A direct microscopical examination can be made by rubbing the swab over one or more cover-glasses. These are then fixed and stained with Löffler's methylene blue (p. 332). As a rule, however, the most satisfactory results will be obtained by examining and staining the colonies that develop on the blood-serum. On this medium, the Löffler bacillus outgrows the other bacteria that may be present, and 
hence forms large colonies which have a very characteristic appearance.

The diphtheria colonies, as they appear on serum within 24 hours, are relatively large, round in outline, grayish and moist in appearance. The center is thicker and more opaque than the outer zone which is surrounded by a slightly wavy border. Coverglass preparations made from these colonies and stained with Löfller's methylene blue, will reveal the characteristic diphtheria bacillus. Care must be taken not to over-stain the specimen which, moreover, should be examined with the oil immersion objective. The presence of irregular, club-shaped or swollen rods, among otherwise normal bacteria, is an important mark of recognition. Moreover, many of these rods will show irregularities in staining. Thus, transverse, alternately dark and light bands may be seen. In many cells, one or more bright blue, deeply stained, roundish bodies will be observed. These characteristics are, as a rule, sufficient to establish the identity of the organism in question. It is well to ascertain the kind of organisms which are associated with the Löfler bacillus.

In doubtful cases, Gram's stain and cultivation on agar at low temperature may be resorted to. The subcutaneous injection of a guinea-pig with $1 / 2$ c.c. of a $24-48$ hour bouillon culture will serve to distinguish virulent from nonvirulent forms, and from the pseudo-diphtheria bacillus.

The pseudo-diphtheria or xerosis bacilli are found in the mouth; nose, and on the conjunctiva of healthy persons, and consequently may be met with in various throat and lung affections. They grow better than the Loffler bacillus on agar and impart to it a dark color. One species of this group forms spores. The absence of pathogenic properties is an important distinction from the diphtheria bacillus. It must be borne in mind, however, that attenuated diphtheria bacilli may be met with. Indeed, Roux and his coworkers even at the present time, regard this organism as a modified, weakened diphtheria bacillus. An important, though not absolute distinction, is the change in the reac. tion of litmus-colored bouillon cultures. The diphtheria bacillus promptly produces an acid reaction, whereas the pseudo-diphtheria bacillus does not. Again, the diphtheria, like the tubercle bacillus, does not readily form a homogeneous suspension when touched into a drop of water, whereas the pseudo-forms do. 
Apparently, a useful method of distinguishing the Löfller bacillus from the pseudo-diphtheria bacillus is that of Neisser. The cultures are developed for 10-20 hours on the Löfller's serum at a temperature not exceeding $35^{\circ}$. The cover-glass preparations are treated for 1-3 seconds with an acetic acid methylene blue solution. This is prepared as follows: $1 \mathrm{~g}$. of Grübler's methylene blue is dissolved in 20 c.c. of 96 per cent. alcohol, and to this 950 c.c. of distilled water and $50 \mathrm{c.c}$. of glacial acetic acid are added. The specimen is rinsed in water, and then treated for 3-5 seconds with aqueous Bismarck brown. The latter is made by dissolving $2 \mathrm{~g}$. of vesuvin in 1 liter of boiling water. By this process the isolated polar granules are rendered manifest in the case of the true diphtheria bacillus, but are not to be seen in the pseudo-diphtheria bacilli. According to Fraenkel, an organism which fails to show these double stained polar granules is not a true diphtheria bacillus. On the other hand, pseudodiphtheria bacilli may, though very rarely, show slight polar bodies. In such cases the production of acidity in bouillon and the effect on guinea-pigs should be tested.

\section{Pneumonia.}

The Diplococcus lanceolatus is of extreme interest because of its peculiar biological properties, and because of its frequent occurrence in various inflammatory diseases. Thus, it is by far the most common cause of croupous or fibrinous pneumonia, and fully one-half of the cases of cerebrospinal meningitis are due to it. Many cases of bronchopneumonia are likewise ascribed to the Fraenkel diplococcus. The lungs and the meninges are, therefore, especially suitable for its development. The serous surfaces, moreover, furnish a favorable location and hence it is that this diplococcus is a frequent cause of pleurisy, pericarditis, endocarditis and peritonitis. It is, likewise, met with in inflammation of the middle ear (otitis media), and in abscesses.

The fact that it is frequently present in the healthy mouth, nose and throat, indicates that the natural resistance of the body must be overcome or lowered before the organism is capable of inducing a disease. Invasion may occur through the blood or through the lymph spaces. The virulence of the germ, the way in which it enters, and the 
place of least resistance, are important factors. As a result, the disease may be acute or chronic, wide-spread or localized. Infection from the mouth or nose may lead to otitis or to meningitis, especially in early life; whereas pneumonia is more common in old age.

AtTenuatron.-Cultures from different sources show marked difference in virulence. Cultures isolated from pneumonia in the early stage are more virulent than those obtained later. When grown on artificial media it rapidly attenuates and soon dies out, unless it is passed through a susceptible animal, as a rabbit, every few weeks. Attenuation in a few days at $42^{\circ}$. The virulence and vitality can be best preserved by drawing up the heart-blood of an infected rabbit into sterile tube pipettes (Figs. $61 d, 62 a$ ). These are then sealed so as to leave as little oxygen as possible in the tube. The blood-serum of rabbits is well adapted for growing the germ and for maintaining its virulence.

Diagnosis.-The fact of the frequent presence of the diplococcus in the normal saliva must be borne in mind when examining the sputum of suspected pneumonia. Moreover, it may happen that the diplococcus, which is very abundant in the early stages of pneumonia, may be rare or even absent from the later stages. The white mouse, or the rabbit, may be given an intra-peritoneal injection of the suspected material. Or, the latter may be injected subcutaneously into the ear of a rabbit. In this case ordinary sputum will not kill, whereas that of pneu monia will prove fatal in two or three days, or later.

A microscopic examination of the sputum or exudates: in man, or of the heart-blood of the animal should show the typical capsulated, lance-shaped diplococcus, which can be easily stained by Gram's method. Glycerin agar plates should be developed at $20^{\circ}$ and at $37^{\circ}$. The Fraenkel diplococcus will not grow, or only very exceptionally, at $20^{\circ}$.

Meningitis may be induced by other organisms than the pneumococcus. Among these, especially deserving attention, is the Diplococcus intracellularis meningitidis, which in many respects resembles the gonococcus. The biscuit-shaped, flattened diplococci, which occur in groups or within cells, do not stain by Gram's method. Unlike the gonococcus, they can be readily cultivated on agar at $37^{\circ}$. 


\title{
Micrococcus Pneumoniæ Crouposæ,
}

\author{
Sternberg, Pasteur (1880). Fraenkel (1886).
}

FRAENKEL'S DIPLOCOCCUS, D. LANCEOLATUS, D. PNEUMONI AE, MICROBE OF SPUTUM SEPTICEMIA.

ORIGIN.-Occasionally in the saliva and nose of healthy persons; especially frequent in the "rusty" sputum of pneumonia. The same organism, or scarcely distinguishable varieties, may be present in cerebro-spinal meningitis, pleuritis, peritonitis, pericarditis etc.

FORM.-Occasionally round, usually oval, barley, or lance-shaped diplococci. It may form chains of $4-6$ cells, and hence may resemble a streptococcus. Owing to its oval form it is sometimes regarded as a bacillus. In the animal body, it is surrounded by large capsules: (Fig. 5, p. 29). These are not present on artificial media.

Motility.-It has no motion.

SPORULA'TION.-Unknown.

ANILIN DVES. - Stain readily; so does Gram's method. The capsules remain colorless.

GrowTH.-Takes place somewhat slowly and only at higher temperature, and on alkaline media. The ordinary media are far from being favorable for its growth. This is seen in the more or less marked change in form and size, and especially in the rapid loss of virulence and vitality. 5 per cent. glycerin agar makes an excellent medium. 2-3 per cent. of glucose is also beneficial. It should be transplanted every two or three weeks.

Plates.1-On gelatin plates kept at $24^{\circ}$, small, round, sharply defined, slightly granular, whitish colonies develop slowly. On agar plates in the incubator, in 48 hours, the surface colonies appear as delicate, glistening transparent drops which, under the microscope, are round, sharply bordered, finely granular, and usually possess a dark center.

Stab culture. - In gelatin a row of small, white granules develop along the line of inoculation. Titality preserved for some time; acid reaction. Slight growth on the surface. Does not liquefy; resembles in this respect the streptococci.

Streak culture.-On agar in the incubator, the growth develops as a thin layer of delicate, glistening, almost transparent drops. It dies out rapidly. On blood-serum it forms a transparent film of dew-like drops. No growth on potato.

Bouillon.-Slight diffuse growth which soon settles yielding a very slight deposit.

Milk.-Is a favorable culture medium; it may, or may not coagulate.

OXYGEN REQUIREMENTS. - It is a facultative anaerobe.

Temperature. - Growth occurs only betwéen 24 and $42^{\circ}$. Its optimum is about $37^{\circ}$. After repeated cultivation it may adapt itself to a temperature of about $18^{\circ}$.

BEHAVIOR TO GELATIN,-Does not liquefy.

Attenuation.- See p. 337.

IMmUNITy. - This can be obtained by intravenous injection of a very small amount of the virulent culture; by injections of filtered cultures, especially when heated to $60^{\circ}$; blood serum of immune animals has a questionable value. Blood-serum from pneumonic patients has been said to immunize rabbits against the pure culture, but this is doubtful. Injections of nuclein immunize rabbits.

PATHOGENESAS. - In rabbits a subcutaneous injection of $0.1-0.2$ c.c. of recently isolated bouillon cultures produces death in 24-48 hours. The diplocoscus is found in the blood and internal organs and is sur. rounded by a capsule. Tracheal injections in rabbits produce true pneumonia. Mice and rabbits are highly susceptible; guinea-pigs, sheep, dogs and cats are less susceptible. Chicken and doves are immune.

1 Make glycerin agar Petri dishes from the peritoneal exudate in a rabbit. Make cover-glass preparations from the peritoneum, surface of intestines and from the heartblood, and stain by the simple, and by Gram's method. 
DRAWINGS. 


\title{
Bacillus Pneumoniæ, Friedländer (1883).
}

\author{
FRIEDLÄNDER'S PNEUMOCOCCUS, OR PNEUMO-BACILLUS.
}

ORIGIN.-It is frequently found in normal saliva; also in the lungs and in the "rusty" sputum of pneumonia. May occur elsewhere as in otitis. It has been found in the air and in water.

Form.-In the exudates from the body it may appear as an ova] coccus, but in reality it is a short, thick rod, which may grow in pairs and even in short threads. In the animal body it is enveloped by a well-marked capsule. This is not present in artificial cultures.

Motility.-It has no motion.

Sporulation.-Spores have not been observed. Cultures retain their vitality for many months.

ANILIN DYEs. - The cell is stained readily but the capsule remains colorless. The bacillus is not stained by Gram's method-distinction from Fränkel's diplococcus.

Growtн.-Is rapid and abundant.

Plates.1-On gelatin plates it develops rapidly. The deep colonies are round or oval, sharply bordered; finely granular and yellowish. The surface colonies are quite characteristic and appear as thick, moist, glistening, white masses which do not tend to spread but rather tend to become convex and raised. This appearance is more marked when grown at $15^{\circ}$ than when developed at $25^{\circ}$. In the latter case, they may be flat and irregular. No liquefaction.

Stab culture.-Growth takes place along the entire line of inoculation and is especially developed on the surface forming a "nail-shaped" culture. As the culture becomes old the gelatin near the surface becomes brownish in color and small gas bubbles may form. Streak culture.-On agar, it forms a thick, mucus-like, whitish or transparent, moist, slimy growth. On blood-serum develops as a grayish, slimy mass. On potato it forms a thick, yellowish, sticky growth, which shows gas bubbles. A diastatic ferment is present. Bouillon is rendered diffusely cloudy.

Milk is not coagulated, or but rarely. Indol is not produced.

OXYGEN REQUIREMENTS. - It is a facultative anaerobe.

Temperature. - It grows rapidly even at low temperatures, $16-20^{\circ}$; best growth in the incubator.

Behavior to gelatin. - It does not liquefy.

AEROGENI:SIs. - Abundant production of gas in 4 per cent. gelatin. Potato cultures grown in the incubator also give rise to gas.

PATHogenesis. - It is pathogenic for mice and young rats. Guineapigs, rabbits and dogs are less susceptible. The virulence varies considerably.

The Friedländer bacillus is unquestionably capable of inducing pneumonia. However, it is only in a relatively small percentage of cases that it may be looked upon as the cause. It is frequently associated with the diplococcus pneumonix, thus giving rise to a mixed infection. This organism is, moreover, an etiological factor at times in other inflammatory diseases, such as angina, pleuritis, pericarditis, endocarditis, otitis, suppurative peritonitis, etc.

Diagnosis. - The bacillus pneumoniæ resembles very closely that of ozena, and of rhinoscleroma. Moreover, it must be carefully distingushed from certain varieties of the colon bacillus (B. aerogenesi. Glycerin agar plates, grown at $37^{\circ}$, should be resorted to for the detection of the Friedländer and Fränkel germs in suspected material.

1 Make gelatin plates and cover-glass preparations from the lungs and blood of a young rat which has received an intrapleural injection. 
DRAWINGS. 
Bacillus Rhinoscleromatis, Frisch (1882).

RHINOSCLEROMA.

OrIGIN. - In the tumors of rhinoscleroma, a rather rare disease which occurs in Austria and Italy. Very rare in this country. These growths may appear on the mucous membrane of the nose or throat.

Form.-Short, thick rods with rounded ends resembling the Friedländer's pneumobacillus and the B. ozænæ; may form short threads. They are likewise surrounded by a colorless capsule. These capsules, when masses of cells are present, coalesce to form the so-called cells of Mikulicz.

Motility.-It has no motion.

Sporulation.-This has not been observed.

Anilin Lyes. - Stain readily, and show a colorless capsule. The bacillus is not stained by Gram's method.

GrowTH.-This resembles in almost every respect that of the. Friedländer bacillus. The colonies, stab and streak cultures agree so closely as to be scarcely distinguishable.

OXYGEN REQUIREMENTS. - It is a facultative anaerobe.

Temperature.-It grows rapidly at the ordinary temperature. The optimum is about $36-38^{\circ}$.

Behavior to gelatin.-This is not liquefied.

Aerogenesis.-Gas is produced when grown on potato at a high temperature.

Pathogenesis. - The relation of this bacillus to rhinoscleroma has not been positively established. It has less virulence than the Friedländer bacillus. It is pathogenic for mice and guinea-pigs. Rabbits are less susceptible. The bacilli appear in the blood in small numbers.

Diagnosis. - The constant presence of the bacillus and the ease with which it can be isolated from the diseased tissue is of some diagnostic value. As indicated above, the bacteria occur usually in groups. 
DRAWINGS. 


\title{
Vibrio Choleræ Asiaticæ, Koch (1884).
}

\author{
CHOLERA SPIRILLUM, COMMA BACILLUS; BACILLE VIRGULE (Fr.).
}

Origin.-In the excreta of cholera patients, also in the intestinal contents after death. Found several times in the water supply.

FORM.-Usually appears as a short, rather thick rod with rounded, narrowed ends, and with a more or less decided bend or twist. It may, therefore, vary from apparently a straight rod to one bent in form of a half circle. Usually the bend is such as to resemble a comma, hence the name comma bacillus. When two cells remain attached the elongated " $\mathrm{S}$ " form results. Grown in liquid media under unfavorable conditions it may form long spirals. The bent rod is a segment of a spirillum and is designated as a vibrio (Fig. 11, p. 46). Peculiar involution forms develop in old cultures.

Motility.-It is actively motile and usually has at one end a single flagellum (Fig. 6 d, p. 36). Hanging-drop cultures (p. 286) should be developed at $37^{\circ}$-motion, spirals and involutions.

Sporulation.-No resistant form known. Arthrospores (p. 48).

ANILIN DYES. - Stain slowly. Carbolic fuchsin is excellent. It is not stained by Gram's method.

GrowTH.-Is fairly rapid at the ordinary temperature.

Plates.-On gelatin plates kept at $22^{\circ}$, characteristic colonies develop in $15-20$ hours. The colonies appear as small, white points, which gradually reach the surface and produce a rather slow liquefaction so that funnel-shaped depressions form. After several days the plate becomes wholly liquefied. Under the microscope. the colonies show an irregular, rough border; have a white or pale-yellow color and the contents are coarsely granular, as if made up of broken glass. A faint rosy hue surrounds the border. On agar plates, at $37^{\circ}$ the large colonies have a peculiar, bright, grayish-brown, transparent appearance, quite distinct from that of the common bacteria present in water and in feces.

Stab culture. - Growth occurs, in gelatin. along the entire line of inoculation. At the surface a funnel-shaped liquefaction forms with an air space above, while the lower part contains the subsided growth. The lower part of the puncture gradualiy widens by liquefaction: growth settles to bottom, and eventually entire contents of tube are liquefied.

Streak culture.-On agar it forms a bright, glistening, whitish growth. Blood-serum is slowly liquefied. On potato, kept in the incubator, it forms a grayish or yellowish brown thin and rather transparent layer, which resembles somewhat that of the glanders bacillus. At ordinary temperature, no growth unless a mixed culture is used.

Bouillon.-Growth takes place rapidly, especially in the incubator, and a scum or pellicle forms on the surface. Cultures, 12-24 hours old, on addition of sulphuric acid show a reddish-violet color - the indol reaction 1 -due to formation of indol and nitrous acid.

... - Milk.-It grows abundantly in sterile milk, without much change; also in sterile water.

OXYGEN REQUIREMENTS. - Artificial cultures require oxygen.

Temperature.-Grows at $15^{\circ}-42^{\circ}$. Optimum at $37^{\circ}$. Killed at $50^{\circ}$.

BeHAVIOR TO GELATIN.-Liquefies slowly; especially old cultures.

IMMUNITY.-Subcutaneous or intra-peritoneal injections of the dead or living vibrio yield an anti-infectious serum; injections of the soluble toxin yield an antitoxic serum. The cell contents of the cholera vibrios immunize. Pfeiffer's reaction with the serum of convalescents, or that of immunized animals or man (Chap. XIV).

PATHOGENESIs. - Intravenous injection into rabbits kills rapidly. In guinea-pigs intra-duodenal injections or introduction of cultures into the previously alkalized stomach produce death with choleraic effects (p. 272). The intraperitoneal injection of agar culture is extremely fatal to guinea-pigs, - rapid fall of temperature. Subcutaneous injection of pigeons is not fatal-distinction from V. Metchnikovi. Ingestion of cultures produces typical cholera in man. Feeding of cultures to new-born guinea-pigs and rabbits is usually fatal (p. 272).

INFECTION.-Takes place along the alimentary canal, through the water, food, contact with freshly soiled matter, etc. The organism grows in the intestines and the soluble poisons which are elaborated there induce the characteristic symptoms of intoxication.

Diagnosis.-See p. 346.

1 Dissolve $\mathrm{I}$ g. of pepton and $0.5 \mathrm{~g}$. of $\mathrm{NaCl}$ in $100 \mathrm{c.c}$. of ordinary tap-water (Dunham's solution). Fill into tubes and sterilize by steam. Then inoculate with the cholera and other vibrios, and place in the incubator over night. The next day add to each culture I-2 drops of sulphuric acid and note the pink color-the indol reaction. 
DRAWINGS.

345 
Vibrio Deneke, Deneke (1885).

SPIRILLUM TYROGENUM; SPIRILLUM OF DENEKE; CHEESE SPIRILLUM.

ORIGIN.-From old cheese.

Form.-Short, slightly bent rods: may form spirals. Resembles somewhat the cholera vibrio but it is smaller and the comma-form is less pronounced.

Moril.ry.--Very motile. Single flagellum.

Sporulation.-Not known.

ANILIN DYES. - Stain readily.

GrowTH.-Is quite rapid. but less than that of the Finkler-Prior vibrio. On some gelatin media it liquefies very slowly or not at all. It tends to die out rapidly.

Plates.-The colonies which develop on gelatin plates are yellowish, liquefy and the plate as a whole may resemble somewhat that of the cholera vibrio, but the liquefaction is more rapid. Under the microscope they are seen to be circular, sharply bordered and coarsely granular, the center is yellowish-green and later becomes dark yellow.

Stab culture.-Growth takes place in gelatin tubes along the entire line of inoculation; funnel-shaped liquefaction. The mass of bacteria settles to the bottom while on the surface a yellowish scum or layer forms.

Streak culture-On agar a thin yellowish growth develops along the line of inoculation. On potato, in the incubator, it forms a delicate, yellowish covering which frequently contains well-formed spirals. Frequently, no growth. On blood-serum, it soon produces liquefaction.

Bouillon cultures are cloudy but form no scum. They do not give the indol reaction unless a nitrite is added.

Milk is slowly coagulated.

OXYGEN REQUIREMENTS. - Is a facultative anaerobe.

Temperature.-Grows at ordinary temperature, also at $37^{\circ}$.

Behavior to Gelatin.-Liquefies more rapidly than the cholera vibrio, but less than that of Finkler-Prior. The power of liquefaction is subject to very great variation. On prolonged cultivation it may almost disappear-attenuation.

AERogenesis.-Not observed, even on glucose media.

Pathogenesis.-It is less pathogenic to guinea-pigs than the Finkler-Prior vibrio. It is to be considered as a saprophyte.

Diagnosis of Asiatic cholera.-When making microscopic examinations of intestinal contents it should be remembered that comma bacilli and spirals, which are derived from the normal mouth, may be present. A positive recognition of the cholera vibrio can only be effected by cultural methods such as are given in Chapter XIII. This procedure should be employed not only to effect a diagnosis, but alsoto ascertain and confirm the absence of the vibrio from the intestines of convalescents. Although they usually disappear in about a week they may, however, persist for 4 or even 7 weeks, in which case precaution must be taken to prevent the spread of the infection. Again, in cholera times apparently healthy persons may contain the comma bacillus. In such cases active immunity, or unfavorable microbic associations may prevent development of this organism. 
DRAWINGS. 


\title{
Vibrio Finkler-Prior, Finkler and Prior (1884).
}

\author{
VIBRIO PROTEUS, FINKLER-PRIOR'S SPIRILLUM.
}

OrIGIN.-In the dejections of cholera nostras which had been kept fourteen days. It is not the cause of cholera nostras. Apparently the same organism has been found in a tooth cavity (V. Miller), and also in normal intestines.

FORM.-It resembles the cholera vibrio but is longer and thicker; occasionally forms short spirals. Marked tendency to involution forms, hence the name $V$. proteus. These develop especially in gelatin which contains glucose or glycerin.

MoriLrty.-It is actively motile and has a single flagellum at one end.

SPORULATION.-This has not been observed.

ANILIN DYES.-Stain readily.

GRowTH.-Is much more rapid than that of the cholera vibrio, from which it can be easily distinguished.

Plates.-The colonies develop rapidly on gelatin plates and produce extensive circular liquefactions which are diffusely clouded. Under the microscope they appear as yellowish brown, finely granular masses, the contents of which can be seen to possess marked motion. The edge is dark and is beset with short delicate fibrils. Concentric rings may be present.

Stab culture.-In gelatin tubes, rapid growth and liquefaction along the entire line of inoculation-the so-called stocking-shaped liquefaction. In a few days the entire contents are liquefied, and a thin film usually forms on the surface.

Streak culture.-On agar it forms a thick, moist, slimy, spreading growth. On potato growth occurs rapidly, even at ordinary temperature, forming a grayish-yellow, slimy, glistening, spreading mass. Distinction from the comma bacillus which does not grow on potato at the room temperature. On blood-serum, rapid development with liquefaction.

Milk.-It can grow in milk, which it coagulates; in water it soon dies"out. Abundant growth in bouillon. No indol unless nitrite is added.

OXYGEN REQUIREMENTS.-It is a facultative anaerobe.

Temperature.-It grows well at ordinary temperature; likewise, in the incubator.

BeHaVIOR To Gelatin. - This is liquefied rapidly and extensively.

Aerogenesis. - No gas observed. Disagreeable odor evolved.

Pathogenesis. - As a rule, it is fatal to guinea-pigs if the bouillon cultures are introduced into the previously alkalinized stomach. The intestines are pale and contain watery contents. Intra-peritoneal injections are less fatal than those of the comma bacillus. 
DRAWINGS.

349 


\title{
Vibro Metchnikovi, Gamaleïa (1888).
}

\author{
SPIRILLUM METCHNIKOFF.
}

Origiv. - From the intestinal contents, blood and organs of chickens afflicted with a disease resembling chicken cholera. The disease exists in Russia during the summer months and is due to this organism. Also found in the Spree at Berlin (Vibrio Nordhafen).

FORM.-Occurs as a bent rod which bears a marked resemblance to the vibrio of Asiatic cholera, although it is somewhat shorter and thicker and more decidedly bent. In the animal body it is very short, almost a coccus. Typical spirals may form in old cultures.

Motility.-Very actively motile. It possesses a long, slender whip at one end.

Sporulation.-Has not been observed. It is readily destroyed, like the cholera vibrio, by heat, desiccation, acids, etc.

ANILIN DYES.-Stain very well, especially if warmed. Bi-polar stain may be seen if the dye is weak. Gram's method is not applicable.

GRowTH.-In the hanging-drop and in stained preparations this organism can scarcely be distinguished from the cholera vibrio. The cultural properties show some differences, and especially is this seen in the pathogenic action on animals. The rapidity of growth is greater than that of the cholera vibrio and less than that of the Finkler-Prior vibrio.

Plates.-The colonies on gelatin plates may resemble those of the cholera vibrio and also those of the Finkler-Prior. They are circular, coarsely granular, and yellowish. Rapid liquefaction.

Stab culture.-In gelatin tubes the growth resembles that of the cholera vibrio, which is about twice as old. Eventually they are both alike.

Streak culture.-On agar the growth resembles that of the cholera vibrio. It is fairly thick and yellowish. On potato in the incubator it forms a moderate yellowish brown covering.

Bouillon--Abundant, diffuse growth and a whitish scum forms. It gives a more pronounced indol reaction than does the comma bacillus.

Milk.-Is slowly coagulated and acid products form.

OXYGEN REQUIREMENTS.-Same as cholera vibrio.

Temperature.-Same as cholera vibrio.

Behavior to GrLatin.-Grows and liquefies more rapidly than does the comma bacillus.

AEROGENESIS.-No gas is produced in glucose media.

IMMUNITY.-Is conferred on pigeons and guinea-pigs by injection of sterilized cultures. Such animals are not immune to the comma bacillus.

Pathogenesis. - It is very infectious for guinea-pigs, pigeons, and chicken; rabbits are also susceptible. Guinea-pigs and pigeons, after subcutaneous injection of minute amounts, die within 24 hours-distinction from the cholera vibrio, which is not as pathogenic and does not induce a septicemia as in the above case. The vibrios are abundant in the blood, in the internal organs, and in the serous fluid which permeates the muscles. Old sterilized cultures are very toxic and cause a rapid fall in temperature. 
DRA WINGS.

351 


\title{
Bacillus Coli Communis, Escherich (1885).
}

\section{BACTERIUM COLI COMMUNE; THE COLON BACTLLUS; EMMERICH'S}

\author{
BACILLUS; B. NEAPOLITANUS.
}

OrIGIN. - It is very common and constant in the intestinal contents of man and animals, especially in the colon; occurs in the discharges of healthy infants, also in summer diarrhea. It is frequently present, accompanying the comma bacillus, in the discharges of Asiatic cholera, and in later stages may be the only organism present. Not infrequently it is present in pus (B. pyogenes fotidus). It is apparently the most frequent cause of appendicitis. In many respects it resembles the typhoid fever bacillus.

FORM.-Short, narrow rods; may vary in length from oval or coccus-like forms to rods 4-6 times as long as wide. Usually grows in pairs; occasionally in short threads.

Motility.-Exceedingly variable, depending upon the temperature, age and the medium. Diffuse flagella and giant-whips present.

SPORULATION.-Not observed.

ANILIN DYes. - It stains readily, but not by Gram's method. Bipolar stain frequently met with; likewise plasmolytic changes, as in potato cultures.

GrowTH.-Is more rapid than that of the typhoid bacillus.

Plates,-On gelatin plates the surface colonies are flat, spreading, aniso-diametric, and have a dull-white color. The border is irregular and markings are present in the outer zone. The deep colonies are yellowish, round or oval; frequently divided, forming lobulated masses. The deep circular colonies usually show a yellow granular center which is surrounded by a colorless, homogeneous ring (dish appearance). No liquefaction. Strong amine and indol order. Moreover, the gelatin owing to the ammoniacal reaction, deposits a cloudy precipitate between the colonies, and along any scratches that may be on the glassplate. These characteristics are not given by the typhoid bacillus.

Stab culture.-Rather energetic growth along the line of inoculation, while on the surface it spreads as a white film with wavy border.

Streak culture.-On agar, it forms a moist, white, spreading growth; old cultures may show needle-shaped crystals. On potato, it forms an abundant, yellowish, moist, slowly spreading growth. necessary.

Milk.-Is coagulated in one or two days, though at times a week or more may be

Uschinsky's fluid gives a good growth; likewise a solution of ammonium chloride and glycerin-distinction from the typhoid bacillus.

Bouillon becomes very clouded. The sediment is heavy and a thick ring may adhere to the glass at the surface of the liquid. At times a broken pellicle may form. Indol reaction is marked. species.

Hiss' and Stoddart's media yield diffuse growths according to the motility of the

OXYGEN REQUIREMENTS.-Is a facultative anaerobe.

Temperature,-Grows well at ordinary temperature. Its optimum is about $37^{\circ}$.

Behavior to Gelatin.-Does not liquefy.

AERogenesis.-Carbonic acid and hydrogen is produced in abundance when glucose is present. Unlike the typhoid bacillus it can form acid and gas in lactose media.

Pathogenesis. - Guinea-pigs are very susceptible; rabbits less so; mice insusceptible. Small quantities injected intravenously or into the abdominal cavity produce diarrhea, collapse and death in 1-3 days. The small intestine is hyperemic: more or less intensely inflamed; serous exudates may be present. The bacilli are abundant in the blood and organs and on the peritoneum. Subcutaneous injection is usually non-fatal and produces only a local abscess.

DIAGNOSIS.--See p. 364. 
DRAWIngs.

ss 


\section{Bacillus Typhosus, Eberth, Koch (1880).}

\section{BACILLUS OF TYPHOID FEVER; KOCH-EBERTH'S BACILLUS.}

Origin.-First obtained from the spleen and lymphatic glands of typhoid fever cadavers; present in the blood, though in small numbers; also in the feces and urine of typhoid patients.

FORM.-Good sized rods, 3-5 times as long as wide, with rounded ends. The length varies greatly with the nature of the medium on which it grows. 'Thus, on agar it appears as very short rods, while on the potato it may grow into long threads. Involution forms.

MотіLITY.-Is very actively motile; on prolonged artificial culture it may show little or no motion. It has numerous lateral whips. Excellent giant-whips.

SPORULATION.- - Terminal, round or oval bodies are found in potato and agar cultures, which are grown in the incubator for several days. They do not double stain and the bacilli containing these are very susceptible to heat. They are not therefore true spores, but rather little masses of condensed protoplasm (see p. 27). The bacilli are very resistant to desiccation and may retain their vitality for months.

ANILIN DYEs. - It does not stain as well with ordinary anilin dyes as do most bacteria: carbolic fuchsin stains excellently. It does not stain by Gram's method. Excellent bi-polar stain when grown on potato; no plasmolysis (Migula).

GROWTH. - Is less rapid, but in its cultural properties it greatly resembles the preceding organism. Slow at $16-18^{\circ}$.

Plates.-The deep colonies on gelatin plates are small, round, or oval, sharply bordered finely granular and yellowish. They may show a central dark portion or ring. Frequently, a protuberance or swelling will be seen on the border of the colony. At times delicate fibrils may surround the border (see Elsner's medium). The surface colonies (craterlike) spread freely as an almost transparent film, which has an irregular, wavy border, and is delicately marked with branching lines. No liquefaction. For appearance of colonies on Elsner's or on Stoddart's medium, see Chapter XIV.

Stab culture. - Abundant growth along the entire line of inoculation, and especially so on the surface where it spreads as a thin, grayish white covering. Gelatin eventually becomes cloudy, due to the production of acids. See also stab culture in Hiss' medium.

Streak culture.-On agar and on blood-serum it forms a moist, white growth, without any special characteristics. On potato, as a rule, the growth is very characteristic. It covers the surface as a moist, invisible layer-distinction from preceding organism. On alkaline potato, the growth is yellowish and no longer characteristic. For litmus lactose agar see p. 24 I.

Bouillon.-Slight diffuse: cloudiness, much less than with the colon bacillus. Very little deposit and scarcely any ring or film. Remains clouded for a long time. Unilke the colon bacillus, it will not grow in bouillon which contains $20 \mathrm{ccc}$. of $\mathrm{N} H \mathrm{HCl}$ or $50 \mathrm{c}$.c. of $\mathrm{N}$ $\mathrm{NaOH}$ per liter. No indol is produced. No growth in Uschinsky's medizem.

Milk.-Is not coagulated. No gas in glucose media, nor acid in lactose media.

OXYGEN 'REQUIREMENTS. - It is a facultative anaerobe.

Temperature. - Grows well at ordinary temperature. Optimum at $37^{\circ}$. Exposure of a few minutes to moist heat of $60^{\circ}$ kills.

AEROGENESIS. - No acid or gas production on lactose media.

BEHAVIOR TO GELATIN.-Does not liquefy.

IMMUNITY. - As in cholera, injections of dead or living cultures yield an anti-infectious serum, whereas injections of the toxin yield an antitoxic serum. The serum in the former case will give Pfeitfer's reaction with the Eberth, but not with the colon bacillus.

PATHOGENESIS, - Intravenous injections usually kill rabbits. It is usually fatal to guinea-pigs, when introduced into the previously alkalized stomach, or when injected into the duodenum or into the peritoneal cavity. Subcutaneous injections are also fata'l go guineapigs - distinction from the colon bacillus. The same method of infection will produce abscesses in rabbits and dogs. At times it produces abscesses in man. Cultures killed with chloroform, or by heating at $54^{\circ}$ for one hour are fatal to guinea-pigs in a dose of $3-4 \mathrm{mg}$. per $100 \mathrm{~g}$. body-weight.

INFECTION, Diagnusis.--See p. 382. 
DRAWINGS. 


\section{Bacillus Icteroides, Sanarelli (1897).}

Origin.-In the blood and organs in yellow fever. Although present in small numbers and isolated only in 7 out of 13 cases, it is regarded by Sanarelli as the cause of the disease.

'Form.-Small, very slender rods; two to three times as long as wide. It is usually single or in pairs; short threads are occasionally found in bouillon. In the body and on agar it is almost coccus-like in appearance, whereas in bouillon large rods are met with. Bright polar bodies.

Mo'tuITy.-Extremely motile, especially in the water of condensation on agar. It bears 4-8 long whips. Splendid giant-whips (Novy).

Sporulation.-Not observed in cultures of the typical bacillus.

ANILIN DYES.-Stain readily. Gram's method is not applicable. At times, a bi-polar stain may be obtained.

GRowTH.-Is rapid and on some media very characteristic. In general, it resembles quite closely that of the typhoid bacillus. Rapidity of growth is like that of $B$. typhosus and less than that of the colon bacillus. No growth on distinctly acid media.

Plates.-The colonies on gelatin plates are extremely characteristic. To obtain typical colonies it is necessary to maintain a constant temperature of. 16-18. (See Fig. 33. p. I79); moreover, the gelatin should not be too hard (Io per cent. or less) and should be alkalin (ep, 157). Under proper conditions, as indicated, characteristic colonies can be obtained from cultures which have been kept in the laboratory for several years (Novy). Otherwise, aty pical colonies will result.

The deep colonies are perfectly circular and sharp bordered. At first they are light yellow, almost homogeneous, wax-like in appearance. Later they become dark or perfectly black. They may show at the center slight, irregular radiating lines. Aty pical colonies are those which do not become black; those which are lobular or are surrounded by a fringe of fibrils. The latter two forms are rather rare. The former develop on acid gelatin.

The surface colony appears to the eye like a droplet of boiled starch or mucus. It is thick and convex. It may be perfectly circular, but frequently will show a distinct kidney-shape. An opaque, yellowish white' nucleus can, as a rule, be seen at or near the center; or, in the case of the kidney-shaped colony at the hilum. Under the microscope the border appears colorless, or with a central, light-brown tinge. It is finely granular and the border is sharp perfectly smooth. The nucleus is opaque and may be round, but more often is hat-shaped, and has even been compared to Saturn with its rings. In the kidneyshaped colony the crown of the hat projects into, or is turned toward the hilum. On hard or acid gelatin only atypical colonies form. These are small, raised points usually without the nucleus, and the kidney shape is absent

Isolated colonies on inclined agar (p. 239), developed at $39^{\circ}$, are thin, flat, grayish and circular. If at the end of 24 hours the tubes are placed at $I 6^{\circ}$, growth at the edge of each colony will continue, but it will take on a thick, slimy character. In two or three days the colonies present a peculiar appearance-a thick, opalescent, slimy ring surrounds a flat thin, transparent area. When several colonies are close together the slimy growths may coalesce and give rise to rivulets. Eventually, the outer slimy border becomes transparent. and the original colony is seen as an opaque body imbedded in it.

Stab culture.--It grows slowly along the line of inoculation and, unless kept at about $25^{\circ}$, does not tend to spread on the surface.

Streak culture.-At $39^{\circ}$ on agar, like the B. typhosus it forms a thin, transparent growth. At I6 it forms a thick, moist slimy growth, which is homogeneous and mucouslike in appearance, and is not unlike that of Friediander's bacillus. On potato, it yields a moist, colorless, invisible growth. On blood-serum a scarcely visible, transparent film forms. Boullon. - No ring or pellicle as a rule, forms on the surface. The liquid is clouded and remains so for some time. Very little sediment in the tube. Involution forms.

Milk.-Is not coagulated. Indol reaction is not given, or but very faintly.

On glucose media it produces gas, also an acid reaction. No growth in Uschinsky's or in Elsner's medium. On Stoddart's medium it forms a thin, transparent, rapidly spreading film. Similar diffusion in Hiss's tube medium.

OXYGRN REQUIREMENTS. - It is a facultative anaerobe.

TEMPERATURE.-Has a marked effect upon the character of the growth. Grows at $14-40^{\circ}$. The optimum is at about $37^{\circ}$.

BEHAVIOR To GELATIN. - It does not liquefy.

ATTENUATION.- It is not affected by freezing for some days.

ImMUNITy. - An antitoxic serum has not been obtained. Normal

blood agglutinates.

PaTHOgEnesis, Diagnosis.-See p. 382. 
DRAWINGS

35 


\title{
Bacillus Pestis Bubonicæ, Yersin, Kitasato (1894).
}

\author{
BLACK OR BUBONIC PLAGUE; BLACK DEATH: PEST.
}

Origin.-In the enlarged or suppurating glands or buboes; in sputum in the pneumonic form of the disease. Also present in the urine and feces. In severe cases may be present in small numbers in the blood.

Form.-A short, thick bacillus with rounded ends (cocco-bacillus). At times, may be almost a coccus and may form short chains, of 6-8 cells. It is subject to great variation in form, and not infrequently is surrounded by capsules. Involution forms are frequent, especially in the presence of mercury, salt and other antiseptics.

Mótiliry.-It is said to be non-motile. May have 1 or 2 flagella (Gordon). Brownian motion is marked.

SPORULA'Tion.-No spores observed. It is a relatively weak organism; a few minutes at $58^{\circ}$ kills.

ANILIN DYES. - It stains readily; Gram's method is not applicable. At times, a bi-polar stain may be observed and in the case of short threads the resulting appearance is not unlike that of the "beaded" tubercle bacillus. Abnormal forms do not stain well.

GRowTH.-Is slow and not abundant. The cultures die"out readily. They are usually slimy.

Plates.-The colonies on agar are small, transparent and white, with iridescent edges. On gelatin the colonies are very small, circular and sharply bordered; eventually they become brown and coarsely granular.

Stab culture.-Shows a very slight white growth along the line of inoculation, and spreads but little on the surface. At times, minute branches extend into gelatin from the puncture, the resulting appearance resembles somewhat an anthrax stab culture.

Streak culture.-On agar it forms a rather thin, whitish, slim growth which usually is made up of discrete colonies. The old cultures show large coccus-, or torula-like forms and but very few typical ovals or short rods. Glycerin agar yields a better growth." It grows well on serum; poorly on potato.

Bowillon:-In this the growth is very characteristic and resembles that of the streptococci. The liquid usually remains clear while the granular or slightly flocculent, moderate growth develops on the walls and bottom of the tube. Old cultures show a ring or "collaret" on the surface of the liquid. A bouillon with 2 per cent. each of pepton and glycerin furnishes the best medium. On staining, various bizarre forms are met with. Short threads of 6-8-ro cells like streptococci are present.. Good rods may be present but the size of the cells varies greatly.

Milk.-Is not coagulated. Acid is produced in bouillon. An indol reaction results on the addition of a nitrite.

OXYGRN REQUIREMENTS.-It can grow as a facultative anaerobe, but only in gelatin.

TEMPERATURE.--It grows well at the ordinary room temperature. Prolonged exposure to $37^{\circ}$ is unfavorable. Cold has no effect.

BEHAVIOR TO GELATIN. - It does not liquefy.

AEROGENESIS.-Not observed.

AtTenuation.-Takes place readily. Cultures from convalescents may be non-virulent, Other growths rapidly lose their virulence on artificial culture. By successive passage through animals or by the collodium sac method (Chapter XIV), the virulence may be greatly increased. A culture of maximum virulence obtained by passage through mice is feeble with reference to rabbits, and vice versa.

IMMUNITY.-Can be obtained by means of agar cultures. The injections, at first, are made with cultures heated at $58^{\circ}$ for 1 hour. Subsequently, living cultures are injected intravenously. The rabbit and horse can be thus readily immunized, whereas guinea-pigs are difficult. The serum of the immunized horse is anti-infectious and to less extent antitoxic. As usually tested 0.1 c.c. of the serum should protect a white mouse against $10 \mathrm{mg}$. of the toxin which has been precipitated by $\left(\mathrm{NH}_{4}\right)_{2} \mathrm{SO}_{4} .2 .5 \mathrm{mg}$. of this toxin will kill a control mouse in about 12 hours. Vaccination by repeated injection of bouillon cultures, heated to $70^{\circ}$ (Haffkine).

PATHOGENESIS, InfECTION AND DIAGNosis.--See p. 383. 


$$
\text { naxumear }
$$




\title{
Bacillus Influenzæ, Pfeiffer (1892).
}

\author{
INFLUENZA; LA GRIPPE (Fr.).
}

ORIGIN.-In the greenish-yellow, purulent sputum of the disease; present in masses in the nasal and bronchial secretions; rarely in the blood. In the early stage they are free, whereas later on they are included within pus cells.

FORM.-Extremely small, rather plump rods; usually in pairs, very rarely in threads. Involutions may be present in old cultures. Branching forms observed. The pseudo-influenza bacillus forms decidedly larger rods which have a marked tendency to form threads.

Motility.-It has no motion.

Sporulation.-This has not been observed. It is very sensitive.to desiccation.

ANII.IN DYES. - Stain with some difflculty. Löffler's methylene blue and carbolic fuchsin are useful. Gram's method is not applicable. At times, a bi-polar stain may be observed.

GrowTH.- Is slight and requires a special medium (see below) and the body temperature. Moreover, the culture should be transplanted every 3 or 4 days. No grow th on ordinary agar or serum. It is favored by association with staphylococci. The addition of defibrinated blood to agar at $100^{\circ}$ yields a good medium (Voges). Plates. - The colonies on agar-blood appear as isolated, minute, glassy drops which,
under the microscope, appear colorless and homogeneous. Later on, the middle of the colony may show a yellowish or brownish color. They remain discrete and are usually so small that a lens may be necessary to reveal them.

Stab culture.-Very slight growth along the puncture in hämatogen-agar. 2-3 weeks.

Streak culture.-Isolated, dew-like colonies on agar-blood. Vitality may persist for

Bouillon.-Blood must be added to the medium which moreover, must be shallow. Delicate white floccules form. The cultures retain their vitality for 2-3 weeks. In sterile tap-water it dies out in about 24 hours.

OXYGEN REQUREMENTS.- - It appears to be an obligative aerobe.

Temperature.-Optimum at $37^{\circ}$. May grow at 26 to $42^{\circ}$.

ATTENUATION. - - It is readily destroyed by mere desiccation, and is even more sensitive in this respect than the cholera vibrio.

IMMUNITY. - Has not been established in guinea-pigs even after prolonged treatment with living and dead cultures.

PATHOGENESIs. - The effects on animals are not very characteristic. Dead or living cultures injected intravenously in rabbits produce fever, weakness and, at times, death; subcutaneously they induce abscesses. Mice and guinea-pigs are less susceptible. Monkeys react with a fever. The disease is primarily one of the respiratory passages from whence toxic products are absorbed. A somewhat similar organism, Bacillus conjunctivitidis, is found in a catarrhal eye disease. The latter is present in large numbers in the pus cells and can be cultivated on agar-blood medium (Diplo-bacillus of Weeks).

DIAGNOS18. - In the early stage a microscopical examination will show large numbers of the characteristic rods. Otherwise it is necessary to isolate the organism. This is done as follows: Agar is poured into Petri dishes and allowed to solidify. Some human or rabbit blood (Chap. XIV) is then spread over the surface of the plate. Finally the material itself, from a fresh acute case, is rubbed up in sterile bouillon and is streaked over the plates, thus prepared, which are then set aside at $37^{\circ}$. Obviously inclined agar tubes may be also employed. The material should also be streaked over the surface of plain agar in which case failure to obtain growth would confirm the nature of that which developed on agar-blood. 
DRA WINGS.

361 


\section{Bacillus Pyocyaneus, Gessard (1882).}

\section{BACILLUS OF GREEN OR BLUE PUS.}

ORIGIN.-In green pus. The color forms on exposure to air. Several varieties have been described. It is very widely distributed in nature. - It has been found on the skin and in the mouth, nose, stomach and intestines. Not infrequently, it is found in the lungs, blood and internal organs of men and animals; in dust, soil and in water. In many respects it resembles the liquefying, fluorescing bacillus.

Form.-Small narrow rod like that of blue milk. At times almost a coccus. The ends are tounded. It is usually single, may form short threads of 4-6 cells, or even longer, spiral-like filaments. Motility.--Actively motile. Single polar whip.

Sporulation.-Has not been observed.

ANILIN DYES.--It stains easily; also by Gram's method.

GrowTH.-Is rapid and abundant. Oxygen is necessary to the formation of pigment.

Plates.-On gelatin plates a green fluorescing pigment develops quite early. The surface colonies at first tend to spread; then produce funnel-shaped liquefactions. The deep colonies appear as round, coarsely granular masses with serrated borders. They are yellowish and may show radial markings.

Stab culture. - In gelatin tubes funnel-shaped or cylindrical liquefaction results. The upper layer is at first green but later the entire contents are colored. In very old cultures the color changes to a brownish black. A scum forms on the surface.

Streak culture.-On agar a moist, slimy, yellowish growth develops and the medium itself becomes bright green. When very old the agar becomes dark colored and the growtb has a peculiar scaly, metallic appearance: On potato a yellowish green or brownish slimy growth forms.

Milk,-Grayish yellow spots form on the surface; the casein is precipitated (rennet action); subsequently it is peptonized with production of ammonia.

Bouilion.-Becomes very cloudy and a heavy deposit forms. A thick white scum forms on the surface. The upper layer of the liquid is greenish. Indol is produced.

OXYGEN REQUIREMENTS. - Is a facultative anaerobe. No growth under mica plates, but can grow in the body. The presence of air is necessary to the production of the pigment, pyocyanin (p. 116).

TEMPERATURE.-Grows at ordinary temperature; best at $37^{\circ}$.

BEHAVIOR TO GHLATIN.-Liquefies rapidly. Under anaerobic conditions this property may be lost.

AEROGENESIS. - Produces a characteristic aromatic odor. Hydrogen sulphide, mercaptan, butyric acid, hydrogen, carbonic acid, etc. attenuation.-Artificial cultures diminish in virulence. Moreover, variation in pigment production will be met with. Even, colorless varieties have been obtained.

IMMUNITY.-Injection of small amounts of the culture, or of sterilized cultures induces immunity.

Pathogenesis. - Subcutaneous injection, in guinea-pigs and rabbits, of about 1 c.c. of a fresh bouillon culture produces a rapidly spreading edema, purulent inflammation and death. In case of recovery a local abscess forms. Intraperitoneal injections produce purulent peritonitis and death. Bacilli are numerous in the tissues, blood, organs, etc. Small amounts produce less marked results and recovery. Virulent cultures will kill rabbits in about 2 days in a dose of ${ }_{10}$ c.c. or less, subcutaneously. Cure of animals infected with anthrax by inoculation with $B$. pyocyaneus. This bacillus was formerly considered as a harmless form, accidentally introduced into wounds. While, in general, it is not markedly pathogenic for man, yet there are times when it may take on toxic properties.

DIAGNOSIS. - It is to be distinguished from the ordinary fluorescing bacteria. When an agar culture of $B$. pyocyaneus is whipped up with chloroform, the latter becomes blue. Isolated colonies in deels gelatin liquefy, whereas those of the fluorescing bacilli do not. 


$$
\text { nearmeses }
$$




\title{
Streptococcus Pyogenes, Rosenbach (1884).
}

\author{
STREPTOCOCCUS ERYSIPELATIS, Fehleisen (1883).
}

ORIGIN.-In abscesses, pyemia, puerperal fever, erysipelas and many other diseases as indicated below. In erysipelas it is found in the lymphatic vessels of the diseased skin, and only very rarely in the blood and internal organs. Streptococci are found in the mouth and sputum; on the mucous membranes of the nose, urethra, vagina, etc.; frequently present in mixed or secondary infections.

Form.-Small, spherical cells which may grow in pairs or in short chains of 6-8; not infrequently, as when grown in bouillon, the chains may consist of a hundred or more cells.

Motility.-Hias no motion.

Sporulation.-None.

ANILIN DYEs. - Stain readily; Gram's method is applicable.

GrowTH.-Is readily obtained on various media, even at ordinary temperature, but the growth is slow and limited. Alkaline reaction is necessary. Glycerin agar is very useful. 1 part of ordinary bouillon and 2 parts of human blood-serum yields the best medium. In this the virulence is unaltered. 2 parts of bouillon and 1 part of ascitic fluid is useful though not as good as the preceding.

Plates.-On gelatin the colonies develop rather slowly, forming minute oval or round, yellowish-brown, finely granular colonies, which are sharply bordered and usually show concentric rings. Surface colonies remain small. No liquefaction. On agar plates, developed in the incubator, it forms delicate, grayish, translucent, drop-like colonies.

Stab culture. - In gelatin the growth is quite characteristic. Along the line of inoculation a row of minute colonies forms, which usually remain separate, but may fuse together, giving rise to a continuous line, Scarcely any growth forms on the surface.

Streak culture.-On agar or blood-serum, it develops as minute, scarcely visible, round colonies which resemble minute dew-drops. They do not tend to spread.

Bouillon.-At $37^{\circ}$ becomes diffusely clouded and a slight, whitish sediment forms. As a rule, however, the liquid does not cloud but remains clear, in which case the growth occurs on the walls and bottom of the tube.

Milk is coagulated. Acid is produced. Growth on potato is doubtful

OXYGEN REQUTREMENTS -Is a facultative anaerobe.

TEMPERATURE. - Grows slowly at room temperature; best at 30-37 .

BeHavior to gelatin.-Does not liquefy.

Atrenuation.-The virulence of the organism is subject to con. siderable variation, even when taken directly from a case of the disease. Artificial cultures soon become attenuated and will die out unless transplanted every few weeks. The virulence and vitality can be preserved best by sealing the heart-blood of an infected rabbit in tube pipettes (see p. 279). Vitality is also well preserved in gelatin stab culture; or on agar to which human blood has been added. By successive passage through animals, or by the sac method, the virulence can be increased so that virtually a single cell is fatal to a rabbit. Thus, cultures have been obtained of which one hundred millionth of a c.c. was invariably fatal; whereas, a one hundred thousand millionth would kill 1 or 2 out of 4 rabbits. The virulence can be also increased by injection together with Proteus vulgaris.

IMMUNITY, PATHOGENESIS.-See p. 383.

INFECTION.-Undoubtedly through wounds or injuries of the skin.

Diagnosis. - The detection of streptococci in blood can be effected by simple or by Gram's stain, supplemented by culture on glycerin agar. It is better to add 2 c.c. of the fresh blood to 4 c.c. of agar and to make Petri plates with this mixture $\left(37^{\circ}\right)$. Intra-peritoneal injection into white mice; intravenous into rabbits. 
DRAWINGS. 


\section{Staphylococcus Pyogenes Aureus, Rosenbach (1884).}

\section{GOLDEN PUS PRODUOING COCCUS.}

OrIGIN.-One of the most common organisms in pus-in about 80 per cent. Found on the skin, in saliva, air, water, dust and soil.

Form.-Small cocci, arranged in irregular groups (Fig. 10 e,p. 44); may grow single or form diplococci. Size varies with the medium. Motility.- Has no motion.

Sporulation.-No spores observed. Possesses a high degree of resistance to desiccation, heat, chemicals, etc.

ANILIN DYES. - Stain readily; so does Gram's method.

GrowTH.-Is rapid.

Plates.-On gelatin plates the colonies are round, with sharp smooth borders, very granular and of a dark-brown or yellow color: The gelatin is liguefied somewhat rapidly. The yellowish colony lies in the center of a broad liquefied disc. On agar the surface colonies are bright yellow in color.

Stab culture. - In gelatin development takes place along the entire line of inoculation forming a finger-shaped liquefaction. 'The growth settles to the bottom as a yellowish deposit while the liquid above remains clouded for some time. Peculiar acid odor.

Streak culture.-On agar it forms a moist, glistening orange-yellow covering. On potato the growth is excellent, forming a thick, moist yellow mass. Peculiar odor present.

Bouillon. -A slight cloud permeates the liquid and eventually a yellow sediment forms. Lactic and other acids develop.

Milk.-Coagulation results and the casein is then slowly peptonized.

OXYgen requirements. - Is a facultative anaerobe. Pigment formation depends on presence of oxygen.

TEMPERATURE.-Grows at ordinary temperature: best at $30-37^{\circ}$.

BEHAVIOR TO GELATIN.-Liquefies rapidly.

ATtEnUATION.-The virulence is rapidily decreased on artificial media. The vitality is not decreased, even in very old cultures.

ImMUNITy.-Repeated injections of small doses of dead or living cultures immunize. The serum of such animals seems to protect.

PATHOGENESIs.- Pure cultures applied to the unbroken skin in man produced'suppuration and carbuncles (p. 261). Subcutaneous application in mice, rabbits, and guinea-pigs induces local abscesses. Intraperitoneal and intravenous injections produce fatal results with formation of minute abscesses in the different organs and tissues-pyemia. Purulent peritonitis may result and the cocci are present in the leucocytes in enormous numbers. The organism is present in the blood as well as in the internal organs. Intravenous injection of potato cultures induces ulcerative endocarditis. Osteomyelitis results when the bones of the leg are first fractured. It is especially pathogenic for man. The virulence of the culture and the avenue of infection are of great importance. The cells contain the active toxin which is not destroyed by heat. This is seen in the fact that sterilized cultures induce suppuration.

The golden staphylococcus is by far the most common cause of pus formation. It may occur alone, or may be associated with the white or lemon staphylococcus, or with streptococci and other bacteria. It is, therefore, to be expected, as a rule, in acute abscesses and boils; also in angina, empyema, otitis and osteomyelitis Its presence in endocarditis and pyemia has been referred to.

INFECTION.-Usually through scratches and wounds. May penetrate the uninjured skin.

DIAGNosis.-A microscopical and cultural examination of the pus will reveal the characteristic organism if present. In suspected pyemia 2 c.c. of the blood may be plated (p. 364).

In suppuration other staphylococci may be found, as the $S$. pyogenes albus and the $\boldsymbol{S}$. pyogenes citreus. These perhaps are less frequent and less virulent. The cultural properties are practically the same with the exception of the difference in pigment. 
DRA'ं'INGS.

367 


\title{
Micrococcus Gonorrhœæ, Neisser (1879).
}

\author{
GONOCOCCUS, DIPLOCOCCUS OF GONORRHEA.
}

Origin.-Constant in gonorrheal discharges. The disease may affect any mucous membrane: urethra, bladder, rectum, conjunctiva, uterus, etc. The organism is present in ophthalmia neonatorum, gonorrheal ophthalmia, gonorrheal rheumatism and salpingitis. It may even cause endocarditis and general septicemia.

Form.-_" Biscuit-shaped" micrococci which are usually in pairs, with the flattened surfaces facing each other (Fig. $10 a$, p. 44). The diplococcus is usually grouped in masses of 20-40 or more cells. The pus cells are frequently invaded and filled by the gonococci.

In pure culture the typical diplococcus form is rarely present. Rounded cubes in irregular masses are frequent.

Motility.-Has no real motion.

Sporulation.-Not known.

ANILIN DYEs.-Stain readily. It is decolored by Gram's method, especially if the specimen is not washed in water. Excellent double stains can be obtained if the specimen is first treated with dilute eosin or safranin and then with Löffler's methylene blue, or with methyl violet. Mathylene blue is best adapted for staining coverglasses of gonorrheal pus.

Growtн.-Behaves as a strict parasite, and hence can be grown only under very special conditions of soil and temperature. Requires an albuminous medium (see p. 384).

Plates.-No growth on gelatin or agar plates. On agar-serum plates (p. 384 ) the colonies appear in 24 hours as transparent, finely granular points, which show an indented border. They may become yellowish with coarsely granular center.

Stab culture,-In agar-serum a thin delicate growth forms along the line of inoculation. A similar film spreads over the surface. Ascites-agar $(\mathrm{I}: 2)$ is excellent.

Streak cubture.-On inclined agar-serum it yields a glistening, grayish white growth. On blood-agar it forms small transparent discrete colonies which resemble those of the influenza bacillus. On rabbit serum the colonies are minute, transparent, roundish with a raised center; slimy character.

Ascitic fluid and ordinary bouillon ( $: 3$ ) gives at $36^{\circ}$ an excellent growth. At first it becomes cloudy and a fine deposit forms; eventually, a slight creamy or viscous film forms on the surface; long filaments descend into the liquid. The culture dies out in about a week.

OXYGEN REQUIREMENTS. - It is an aerobic organism.

Temperature.-Growth at $32^{\circ}$ to $38^{\circ}$. A temperature of $35^{\circ}-37^{\circ}$ should be maintained, otherwise it will soon die out. Above $40^{\circ}$ it is easily killed. Likewise in a few hours at $15^{\circ}$.

Attenuation.-Dies out very rapidly on some media. May live on rabbit serum at $36^{\circ}$ for 3-4-8 weeks.

Pathogenesis.-Pure cultures of the gonococcus produce typical gonorrhea when introduced into the healthy urethra. The toxin has a like effect. Gonorrheal pus may be inoculated on the mucous membranes of animals without the least effect. Intraperitoneal injection into mice produces a non-fatal purulent peritonitis. The gonococcus is, therefore, pathogenic only for man.

Diagnosis.-See p. 384. 
DRAWINGS. 


\section{Micrococcus Tetragenus, Gaffky (1881).}

Origin.-First obtained from the contents of a tubercular lung cavity; present in normal saliva (26 times out of 111 cases, Miller), rather common in sputum of tubercular persons. Has been found in a few instances, as the only organism present in acute abscesses. A similar non-pathogenic organism which, however, cannot be grown artificially, may be present in the mouth.

FORM.-Large cocci, which in pure cultures on artificial media are either single or in pairs, or in irregular groups. In the animal body it forms perfect tetrads, which are surrounded by a wide colorless capsule (Fig. 5 b, p. 29).

MoTiltTy.-None.

Sporulation. - None.

ANILIN Dyes.-Stain readily. Gram's method is applicable.

GrowTH.-Is rather slow.

Plates.-The colonies which develop on the gratin plate are round or oval, slightly granular, yellowish and sharp bordered. No liquefaction. The surface colonies are white, elevated and thick.

Stab culture.-Along the line of inoculation, in the gelatin tube, the growth develops either as a row of white dots or as a continuous white line. On the surface a characteristic moist, white, thick mass forms.

Streak culture.-On agar it usually develops as discrete sharply defined, round, white colonies. At times the growth may be confluent. On potato it forms a thick, slimy covering, which can be drawn out into long threads.

OXYGEN REQUIREMENTS. - It is a facultative anaerobe.

TEMPERATURE.-Grows well at ordinary temperature; better in the incubator

Behavior to gelatin.-Does not liquefy.

AtTenuation.-Cultures grown for yearis on artificial media eventually become attenuated.

PATHogenesis.-White mice and guinea-pigs are susceptible. House and field mice are usually insusceptible, while rabbits and dogs are immune. A subcutaneous application or intraperitoneal injection kills white mice in from 3-10 days. The blood-vessels of the kidney, spleen, liver, lungs, etc., are full of the tetrads which are invested by capsules. Subcutaneous injection into guinea-pigs produces usually a local abscess; whereas an intraperitoneal injection is followed by a purulent peritonitis. The exudate in this case is rich in capsulated forms. The frequent presence of this organism in tuberculous cavities indicates that it plays, as well as streptococci and other bacteria, an active part in the destruction of the lung tissue. 
DRAWINGS. 


\title{
Spirillum Obermeieri, Obermeier (1873).
}

\author{
SPIRILLUM OF RELAPSING OR RECURRENT FEVER; \\ SPIROCHAETE OBERMEIERI.
}

OrIGIN.-It is always and exclusively present in the blood of relapsing fever patients, and, is especially found during the febrile paroxysms at which time it may be present in large numbers. A similar, closely related spirillum is found in the Trans-Caucasus in the blood of geese affected with a disease which resembles somewhat the recurrent fever of man ( $S$. anserini, Sakharoff).

Form.-Delicate, flexible, long, wavy spirals. May have 10-20 windings. Length is usually from 16 to $30 \mu$. They are much narrower than the comma bacillus.

Motility.-Actively motile. Flagella have not been demonstrated. It preserves its motility at the ordinary temperature for many days.

SPORulatron. - This has not been observed.

ANILIN DYES. - It is stained rapidly and intensely by the ordinary dyes (p. 281). Gentian violet is useful for this purpose. Gram's method is not applicable.

"Growth.-It is an obligative parasite. It has not been successfully cultivated outside of the living body. They may live in the blood of a leech for some days.

IMMUNITY. -One attack does not protect against another.

Pathogenesis. - Inoculation of healthy individuals with blood which contains these spirals produces typical relap. sing fever, which is accompanied by the presence of the characteristic spirals. The disease can also be transmitted to monkeys, but the animal usually recovers. In monkeys from which the spleen has been removed the spirilla develop in enormous numbers in the blood, and death results'. Although it has not been isolated and grown in pure culture, yet the constant presence of the organism in this disease leads to the accepted belief that it is the cause. This is also true of the leprosy bacillus, the plasmodium of malaria and the ameba coli of dysentery.

Diagnosis. - The spirillum is easily detected if present in large numbers. Otherwise it will require a very careful examination. The blood should be examined during the attack.

${ }^{1}$ Consult Soudakewitch, Annales de l'Institut Pasteur, 5, 1891, p. 545. Plates XIV-XVII. 
DRAWINGS. 


\title{
Bacillus Choleræ Gallinarum, Perroncito, Pasteur (1880).
}

\author{
CHICKEN OR FOWL CHOLERA; CHOLERA DES POULES (Fr.); \\ HÜHNER-,GEFLÜGEL-CHOLERA (Germ.).
}

OrigrN.-In the blood, organs and excreta of chickens which have the disease. A somewhat similar disease of chickens, occurring in Russia, has been shown to be due to the Vibrio Metchnikovi which resembles very closely the vibrio of Asiatic cholera. A similar, if not identical organism has been isolated by Koch from water and designated as the $B$. of rabbit septicemia.

ForM. - Small short rods which have rounded ends and are frequently in pairs, rarely in long threads. At times the form is almost that of a coccus.

MoTility.-It has no motion.

Sporulation.-Spores have not been observed. Nevertheless, it possesses considerable power of resistance and can withstand the acidity of the gastric juice.

ANIIIN NYES. - Usually stain the ends first while the center remains uncolored-bi-polar stain. The appearance then is that of a diplococcus. On more intense staining the entire rod becomes colored. Gram's method is not applicable.

GrowTH.-- Is rather slow.

Plates.-Colonies appear in a few days on gelatin plates as ininute white dots which, under the microscope, are seen to be roundish plates with sharp, smooth borders. The contents are finely granular, show concentric rings and are yellowish in color. No liquefaction. ,

Stab culture-Forms in gelatin, a delicate white line or row of dots along the line of inoculation. On the surface it forms a delicate whitish growth which spreads very slowly.

Streak culture.-On agar it develops as a thick, glistening, grayish-white mass. No growth on potato at ordinary temperature, but in the incubator in a few days it gives rise to a yellowish-gray. transparent wax-like covering.

Bouillon.-Diffuse cloudiness and a slight deposit. Indol is formed.

Milk,-Is slowly coagulated-acid production.

OXYGEN REQUIREMENTS. - Is a facultative anaerobe.

Temperature.-Grows at ordinary temperature and also in the incubator.

Behavior to gelatin.-Does not liquefy.

At Tenuation.-Artificial cultures soon lose their virulence. The virulence can be increased remarkably by successive passage through animals, and by the collodium sac method. In sealed tubes (Figs. 61, 62) the virulence is preserved.

IMMUNITY.-Is produced in chickens and pigeons by inoculation with first and second vaccines. It was in connection with this disease that vaccination by means of attenuated cultures was discovered (Pasteur, 1880).

Pathogenesis.-Chickens, geese, pigeons, sparrows, mice and rabbits are susceptible to subcutaneous inoculation. Guinea-pigs, sheep, horses are less susceptible and only local abscesses form. Dogs and cats are immune. After death the bacilli are found distributed throughout the body-a true septicemia.

INFECTION.-Usually results in chicken through the food. It may possibly occur through scratches and wounds.

DIAGNOSIS. - Stained preparations of the blood (p. 281) will show the typical short rods in enormous numbers. Absence of motion, the behavior to milk, etc., and the animal experiment will distinguish it from similar organisms. 
DRAWINGS 


\section{Bacillus Choleræ Suis.}

HOG CHOLERA; SWINE-PLAGUE of Billings; SCHWEINEPEST (Germ.); CHOLERA DU PORC, PNEUMO-ENTÉRITE (Fr.).

OrigrN.-In the blood, organs and intestinal contents of swine that die of, hog cholera.

FORM.- Short, small rods, like those of chicken cholera. On some media, as gelatin, it may form long rods. Occurs single or in pairs.

Morility.-It is actively motile and has several long, wavy flagella. Shows no motion in serum or in blood.

SPORULATION.-Not observed.

ANILIN DXes.-At first impart a bi-polar stain, but on sufficient exposure the entire rod is colored. Not stained by Gram's method.

GrowTH.--Is fairly rapid.

Plates, - In a couple of days colonies develop on gelatin plates. The deep colonies are very small, yellowish-brown and spherical. The surface colonies spread slightly. No liquefaction.

Stab culture.-Shows along the line of inoculation a white line or row of colonies, while on the surface of the gelatin a thin, very slowly spreading growth forms.

Streak culture.-On agar forms a moist grayish-white growth, without any special characteristics. On potato a straw-yellow growth develops, resembling somewhat that of glanders.

Bouillon.-Diffuse cloudiness of the liquid; a partial film forms on the surface. Indol and phenol are not formed. Milk.-Is not coagulated.

OXYGEN REQUIREMENTS.-It is a facultative anaerobe.

Temperature.-Grows well at ordinary temperature. Best at $37^{\circ}$.

BEHAVIOR TO GELATIN.-Does not liquefy.

Aerogenesis.-Glucose is fermented.

Attenuation.-Artificial cultures retain their virulence quite well. The virulence can be readily increased "by repeated passage through rabbits. It is then very fatal to pigeons.

Immunity.-Can be produced experimentally by inoculation with filtered cultures; with repeated small doses of blood, previously heated to $54-58^{\circ}$, from infected rabbits (?). Blood serum of immunized animals protects.

Pathogenesis.-Hogs, mice, rabbits and guinea-pigs are highly susceptible; pigeons are less susceptible, while chickens, sheep and calves are immune. I/4 c.c. of bouillon culture injected subcutaneously into rabbits kills in about four days. Bacilli distributed every. where. White necrotic areas in the liver. Hemorrhagic infiltrations are common.

Infection.-Results through the food. The hog is the only animal that naturally contracts the disease.

Diagnosis. - As a rule the bacillus can be isolated from the heartblood and organs. In chronic cases, because of secondary infection, diverse bacteria may be present. Isolation may be facilitated by - inoculating a rabbit with the suspected material. 
manmone

377 


\section{Bacillus Rhusiopathiæ Suis, Pasteur (1882).}

\section{HOG ERYSIPELAS; ROUGET ( $\left.F r_{.}\right)$; SOHWEINEROTHLAUF (Germ.).}

with the disease.

Form.-In the body it occurs as very small, narrow rods resembling needle-shaped crystals. On some media, as glycerin agar, the slender rods may be quite long, and may show a slight bend. Are usually single, but may occur in pairs and even in threads.

Motility.-It has no motion.

SPORUlation.--Spore formation is not known.

ANILIN DYEs. - Stain readily. Gram:s method, excellent results.

GRowTH. - Is rather slow but extremely characteristic.

Plates,-On gelatin plates the colonies are very characteristic and appear as diffuse cloudy patches which are sometimes difficult to see. Little or no surface growth. No liquefaction.

Stab culture.-In gelatin is likewise very characteristic. The growth develops along the line of inoculation as a delicate, cloud-like radiating column. As the culture becomes old a depression forms at the top, due to slow liquefaction and corresponding evaporation. Sometimes liquefaction can be observed. The vitality is maintained longest in gelatin cultures.

Streak culture-On agar and on blood-serum it forms a scarcely visible thin film or group of colonies. Glycerin agar is best and on this the vitality is prolonged. No growth on potato except under anaerobic conditions.

Bouillon.-A very delicate diffuse cloudiness forms which can best be seen on slight agitation. Resembles the bouillon culture of the tetanus bacillus. No indol is formed.

OXYGEN REQUIREMINTS. - Is a facultative aerobe. Best growth under anaerobic conditions, which, moreover, preserve the virulence.

Temperature.--Grows slowly at ordinary temperature. Best at $36^{\circ}$.

Behavior to gelatin.-Does not perceptibly liquefy gelatin.

Aerogenesis.-Produces hydrogen sulphide in pure cultures, and in the body. This gas is also produced by the anaerobic bacteria and to a less extent by nearly all pathogenic bacteria.

AtTenuation.- Old cultures become attenuated; this result can also be obtained by growing the virulent germ at high temperatures, about $42^{\circ}$, for some time (Pasteur). Passage through rabbits attenuates; whereas passage through pigeons increases the virulence with reference to swine.

IMмUNiTY.-By inoculation with attenuated culture-first and second vaccine of Pasteur-perfect immunity can be produced. One attack of the disease confers immunity. The serum of immunized. animals is anti-infectious.

Pathogenesis.-Swine, rabbits, pigeons, white mice, rats and house mice are susceptible, Death in 3 to 5 days. Field mice, guineapigs, dogs, cats, chickens and ducks are insusceptible. Bacilli distributed throughout the organism (septicemia). They are very numerous in the blood; single or in pairs; "very often can be seen to be enclosed in leucocytes.

InFECTION.-Occurs naturally in swine through the food. The bacillus is always present in the feces.

DIAGNosis.-The bacillus can be readily detected in the blood and especially in the spleen by Gram's method. The cultural characteristics will distinguish it from all other organisms except that of mouse septicemia. Inoculation of mouse or rabbit. 
DRAWINGS. 


\title{
Bacillus Murisepticus, Koch (1878).
}

\author{
MOUSE SEPTICEMIA; MÄUSESEPTIKÄMIE (Germ.).
}

OrIGIN.-From mice after inoculation with putrid blood. It is widely distributed in water, soil, etc.

FORM.-The rods are narrower and thinner than those of the rouget bacillus, but otherwise resemble the latter very much.

Motility.-Appears to possess motion, but is really non-motile.

SPORUlation.-Round, glistening bodies form within the cells as in the case of rouget. They have nothing to do with spores.

AnILIN Dyes.-Stain rapidly." Gram's method is applicable.

GrowTH.-Is rather slow and resembles very closely that of the rouget bacillus.

Plates.-The colonies on tbe gelatin plate resemble those of the rouget bacillus, except that they spread [somewhat more rapidly and are more delicate and transparent in appearance.

Stab culture.-Shows this distinction in growth" quite sharply. While the cloudy growth of the rouget bacillus is dense and somewhat limited to the line of inoculation, that of the mouse septicemia bacillus is lighter and spreads readily tbroughout the entire gelatin. This difference is clearly seen in young cultures.

Streak culture.-On agar the growth is scarcely to be distinguished from that of the rouget bacillus. Glycerin agar is most suitable.

Bouillon.-The bacillus develops a growth similar to that of the bacillus of rouget.

OXYGIN REQUIREMENTS. - It is a facultative aerobe and hence grows best when air is excluded.

Temperature. - It grows well at the ordinary temperature. Optimum about $35-37^{\circ}$.

Behavior to gelatin.-Ordinarily no liquefaction can be observed. Sometimes, however, the gelatin gradually softens.

Aerogenesis.-Produces less hydrogen sulphide than the rouget bacillus.

AtTenuatron.-Old cultures possess diminished virulence.

IMMUNITY.-Rabbits that recover after one inoculation with the pure culture are rendered immune against subsequent inoculation. They are at the same time immune against the rouget bacillus.

Pathogenesis.-White mice, house mice, pigeons, sparrows and rabbits are susceptible. Chickens, guinea-pigs and field mice are wholly immune. After death the bacilli are distributed throughout the body, single or in pairs, and are frequently inclosed in leucocytes.

DiAGNosis. - In cultural, morphological and pathogenic properties it resembles very closely the preceding organism. So much so that it is commonly considered to be an attenuated form of the rouget bacillus. 
DRAWINGS.

$\cdot 381$ 


\section{Colon Bacillus (p. 352).}

Diagnosis.-The motility of the colon bacillue distinguishes it from the aerogenes group of bacteria, which includes well known intestinal bacteria, such as the $B$. lactis aerogenes, and the $B$. coli immobilis. The latter forms tend to produce convex raised growths on the surface of gelatin in plate and stab cultures, whereas the motile colon bacillus is more likely to give a thin spreading growth. The variable motility of the colon bacillus, the influence of temperature, and the consistency of the gelatin influence the cultural characteristics to such an extent as to make this distinction of but little value.

The colon bacillus is to be especially distinguished from the typhoid and similar bacilli. The distinctions are given at length in Chapter XIII. Acid, gas and indol production as well as coagulation of milk are important characteristics.

\section{Eberth Bacillus (p. 354).}

INFECTION.-Commonly takes place through the mouth by means of water, food, contact with soiled articles, etc. Air transmission, as fine dust, is possible. Danger from flies and other insects. In later stages other organisms as streptococci frequently appearmixed infection.

Diagnosis.--The differentiation of the Eberth from the colon bacillus is difficult and necessitates a comparison of all known reactions (See Chapters XIII and XIV). The recognition of the disease is based primarily upon the serum reaction (Chapter $X V$ ) and to less extent, as yet, upon the isolation of the bacillus from urine or feces.

\section{Bacillus Icteroides (p. 356):}

Pathogenesis. - It is very pathogenic for white mice, rabbits, guinea-pigs. Dogs, monkeys, goats, sheep and horses are also susceptible. In guinea-pigs 1 c.c. of a 24 hour bouillon culture, introduced subcutaneously or into the peritoneal cavity, produces death in from 4-7 days. Virulent cultures, obtained by frequent transplantations, will kill in less than 24 hours. In the latter case the bacilli are extremely numerous on the serous surfáces, in the blood and internal organs-septicemia. When death is delayed they are very scarce, and little or no peritoneal effusion exists. 
Diacinosis. - The organism may be found, at times, in the blood of yellow fever patients. 2 c.c. of blood should be drawn (Chapter XIV), added to liquefied agar or gelatin and plated. It mayalso be streaked over the surface of inclined agar. Suspected colonies should be replated, under conditions of temperature, already emphasized, in order to obtain typical growths on gelatin and on agar. In the absence of characteristic growth the other cultural and morphological properties should be tested.

\section{Black Plague (p. 358).}

Pathogenfsis:-It is fatal to white mice or rats in 1-3 days: to guinea-pigs in 2-5 days; to rabbits in 2-7 days; to monkeys in $2 \frac{1}{2}-5$ days. The horse, pigeon, field mouse, hog, cat and frogs are somewhat refractory. Doves, chickens, geese, dogs and cattle are said to be immune. Abscesses are frequently produced by the bacillus or by its toxin. On post-mortem the animals show an extensive rose-colored edema, enlarged lymphatic vessels and glands and hemorrhagic condition of the abdominal walls. The minute rods are numerous in the blood and internal organs-septicemia. They may be englobulated in leucocytes. Accidental inoculation with pure cultures has proven fatal to man.

INFECTION.-The disease naturally attacks mice, rats, hogs, buffaloes, flies and man. Rats and flies are important as a means of spreading the disease. It may be contracted through wounds-inoculation form; or by inhalation-pneumonic form; or through the foodintestinal form.

DIAGNosis.- The pus from the bubo will show enormous numbers of the short, oval rods. In the pneumonic form they can be detected in the sputum. The cultural and/morphological characteristics will be necessary to complete the identification. The involution forms,

- according to Hankin, are especially marked when the pure culture of the organism is planted on agar to which about 3 per cent. of salt has been added. In 24-18 hours at $37^{\circ}$ large spherical or pear-shaped involutions can be found. Pest serum causes agglutination.

\section{Streptococci (p. 364).}

IMMUNITY.-Can be established in horses and other animals by repeated injection of filtered or of virulent cultures. The blood-serum in that case is not protective against all varieties of streptococci. This 
would indicate the existence of diverse species although morphologically they may be almost alike.

Pathogenesis.-Man, rabbits and mice are susceptible. Typical erysipelas results in man from inoculation with pure cultures, as in cases of inoperable carcinoma, etc. Infection in young rabbits frequently gives rise to local suppuration, or to severe general symptoms and death. Like the Fränkel diplococcus, it is a widely distributed engenderer of inflammatory processes. It may occur in connection with other diseases as diphtheria, scarlet fever, typhoid fever, pneumonia, tuberculosis, etc. (secondary infection). Or, it may cause a primary infection, in which case the results will depend largely upon the virulence of the germ and the avenue of infection. Thus, it is the common cause of puerperal fever, erysipelas, otitis, endocarditis, pericarditis, pleurisy, peritonitis and pseudo-diphtheria; of a simple abscess or of general sepsis (pyemia).

Gonococcus (p. 368).

Pure human serum was used at first for cultivating this organism. Better results, however, are obtained with human serum or blood added to agar or bouillon (1:2). The serum or blood may be replaced by ascitic fluid; 1 part of latter to 2 or 3 parts of agar or bouillon. Coagulated rabbit serum is said to be as good as human blood. For its preparation see Chapter XIV.

To isolate the gonococcus the pus should be stirred into liquefied agar $\left(45^{\circ}\right)$ and dilutions in agar should be made in the usual way. The tubes should each contain about 4 c.c. of agar. 2 c.c. of freshly drawn human blood (see Chapter XIV) should then be added to each tube, and the mixture poured into Petri dishes. These should then be kept at $35-37^{\circ}$. The organism may also be isolated by streaking the pus over agar plates or inclined agar on which human blood has been spread. Or, the material may be spread thoroughly over inclined rabbit. serum. The cultures should be placed at once in the incubator.

DiAgnosis.-Cover-glasses should be prepared from gonorrheal pus while it is fresh, and before it has dried down. It is advisable to dilute the drop of pus with a drop or two of water. The cover-glasses should not be over-heated. They should be stained with simple, or with Löfller's methylene blue. The characteristic form, grouping, presence in pus cells, and the fact that it does not stain by Gram's method will serve to distinguish it from other organisms. It can be detected in blood by the method given for streptococci (p. 364). 
YEASTS, MOULDS AND STREPTOTRICES.

The study of micro-organisms as the cause of diverse fermentations and of disease would be incomplete if limited to the group of bacteria. While it is true that the latter are the most frequent causes of such conditions, it must be remembered that there are other organisms entirely distinct from bacteria which can induce similar changes. The yeasts, moulds and the streptothrix forms are extremely important in this respect. Furthermore, in practical laboratory work yeasts and moulds are frequently met with as contaminations of plate or tube cultures. Consequently, it is advisable to understand the general characteristics of these organisms in order to facilitate their recognition.

\section{Yeasts.}

These are microscopic unicellular plants which differ from bacteria in size and in manner of multiplication. They are usually about $6 \mu$ thick; in other words, they are about as large or even larger than the red blood cell. Consequently, a very simple examination will be sufficient to distinguish the yeasts from the bacteria.

While bacteria multiply by dividing in two equal parts the yeasts increase in number by a process of budding. That is to say, at some point on the surface of the cell a minute protuberance develops which gradually enlarges and. forms a daughter cell. This budding may occur at several places in the same cell and the young cells, thus formed, 
may remain attached to the original one. It is because of this peculiarity that yeasts are known as budding fungi or blastomycetes.

The yeast-cells are large, oval or roundish bodies, but under special conditions, as when grown deep in gelatin, they may elongate and give rise to thread-like growths. These pseudo-mycelial threads, as they are called, indicate a certain relationship to the group of moulds. The yeast cells by their size and by the presence of budding cells can be readily distinguished from other microscopic forms. They are readily stained by simple anilin dyes and by Gram's method.

When actively multiplying, the contents of the yeastcells appear perfectly homogeneous but later on granules of various size appear and surround small, clear portions or vacuoles. The contents of the cell on contact with iodine take on a brownish violet color which disappears on heating and reappears on cooling. This is due to the presence of a starch-like or glycogen compound. Occasionally a gelatinous envelope or capsule may form.

Yeast colonies, when they develop on a gelatin plate, can be readily recognized even with a low power. Owing to the large size of the cells the colony has a peculiar, coarsely granular appearance. They do not liquefy gelatin, although they do contain within their cells a proteolytic ferment.

It is customary to divide the yeasts into two groups according as to whether they, form spores or not. The spore producing yeasts are designated as saccharomyces, whereas those forms that do not produce spores are usually brought together under the group name torula.

The saccharomyces as indicated above give rise to endogenous spores. Usually several of these are formed within a cell as shown in Fig. 55. The spores can be double stained like those of bacteria or like the tubercle bacillus. 
Most of the saccharomyces can give rise to alcohol and are, therefore, of great industrial importance. Certain species induce the alcoholic fermentation at a temperature of 14 to $18^{\circ}$ and are known as the top or upper yeasts, whereas other forms are active at a lower temperature, 4 to $10^{\circ}$, and are hence commonly designated as bottom or lower yeasts.

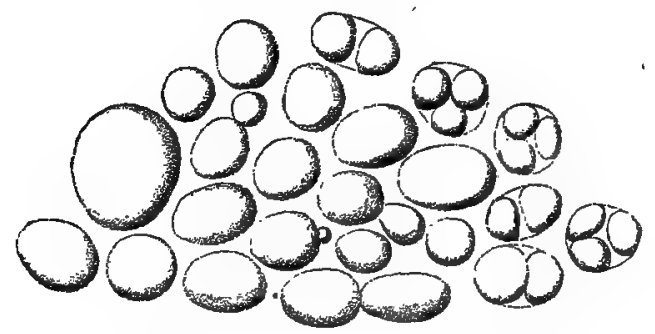

FIG. 55. Yeast cells with spores (Hansen).

The common Saccharomyces cerevisiæ is a typical upper yeast. It is used in brewing and in baking. The ordinary compressed yeast contains this organism mixed with more or less starch. It forms large, round or oval cells. Not infrequently, in brewing, this organism is contaminated with other yeasts or even with bacteria, and the products elaborated by these foreign organisms may greatly alter the composition and general characteristics of the beer. As a result of the studies of Pasteur and of Hansen, on these so-called diseases of beer, it has become customary in large establishments to employ only pure cultures of yeasts.

The most common cause of the spontaneous fermentation of grape and other fruit juice is the Saccharomyces ellipsoideus. Secondary fermentations or "diseases" may occur in wine as in beer. A group of species which frequently show sausage-like cells are known as the $\mathbf{S}$. Pastorianus.

The torula group contains the so-called wild yeasts. These rarely give rise to thread-like forms, and never produce spores. As a rule, they cannot produce more than 
about one per cent. of alcohol, and some species are wholly unable to ferment sugar. A considerable number of species have been described, and among these may be especially mentioned several forms which are widely distributed in the air. The so-called red-yeast is not one species but rather a group name for a number of species or varieties. Red and white torulæ are frequently deposited from the air

- on gelatin plates. Occasionally a black yeast is met with.

Until very recently the yeasts have been considered as being wholly non-pathogenic. When introduced in large quantity they may induce a catarrhal condition in the stomach and in the intestines. When the ordinary yeasts are injected subcutaneously apparently no bad results follow. Rabinowitsch, however, isolated seven varieties of pathogenic yeasts. Monilia candida, the cause of thrush in children, is a yeast-like organism which is very pathogenic for mice and rabbits. Mice were very susceptible; rabbits less so, and guinea-pigs were refractory to the several yeasts. Gram's method can be used to advantage for the detection of the yeast cells within the animal body.

- Recently yeast-like forms have been described as occurring in certain malignant new growths, such as sarcoma and cancer. They are presumed to be identical with the so-called inclusion cells.

\section{Moulds.}

This group of organisms, known as thread fungi or hyphomycetes, cannot be defined with the same degree of precision as is the case with the bacteria and the yeasts. It includes a large number of plants which differ greatly in form, size, shape and color. As a rule the vegetative form consists of threads or filaments which intertwine or inter- 
lace, giving rise to a felt or cotton-like-growth known as the mycelium.

The individual filaments or threads which go to make up the mycelium are known as mycelial threads or as hyphoe. While the bacterial cell is usually about $1 \mu$ in width the mycelial thread will'vary from 2-5-7 $\mu$ in width. In other words, the width of the mycelial thread is greater than that of bacteria, and may be the same as that of the yeasts.

The threads may be very short, clinging to the surface on which the growth is developing; or, they may be very long, so that the resulting mycelium may have a height of $1 / 2-1$ inch or more. The color of the mycelium will vary considerably in the different species. It may be pure white, yellow, green, pink or black.

Many of these organisms, especially the true moulds, at a certain stage in their development give rise to reproductive cells, and eventually spores or conidia form. Usually a stalk or thread known as the fruit-hypha rises upward and bears on its end the fruit organ. The latter is so characteristic for the different groups or families that it is utilized as the basis of a natural classification. It is thus possible to divide the large group of moulds into well defined genera and even species. In certain forms, intermediate between the true moulds and the bacteria, the fruit organs are wanting or are imperfect, and, in such cases, the classification is based on general characteristics such as habitat, size, cultural properties, etc.

In general, the true moulds can be divided into two groups according as the spores produced are contained in a sac or sporangium, or are free and arranged in rows on the ends of modified hyphæ.

A large number of families or groups of moulds are met with accidentally in the ordinary routine bacteriological work. The most important of these are the Mucor, Aspergillus, Penicillium and Oidium. 
The mucor group is characterized by the presence of spherical sacs or sporangia on the ends of the vertical fruit hyphæ. The contents of these sacs are at-first homogeneous but, eventually, differentiation into round or oval spores results. When fully ripe the sac bursts and the spores are set free. The end of the fruit hypha is enlarged or club-shaped and projects into the sporangium, forming what is known as the columella (Fig. 56 a). When the sporangium bursts the membrane disappears or dissolves with the exception of a small ring around the base of the columella.
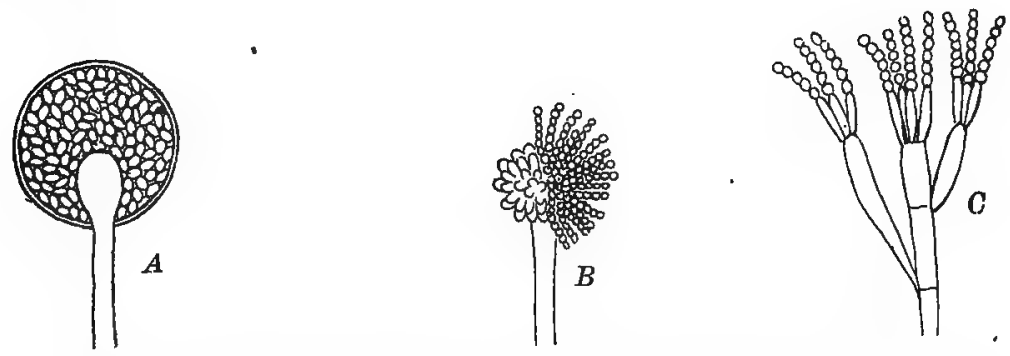

FIG. 56. Fruit-organs of moulds, after Lehmann. A.-Sporangium of mucor, tlled with spores, showing columella; B-Aspergillus with sterigmæ and spores; C-Penicillium with basidia and spores.

The mucors also give rise to zygospores which result by the union of the ends of two mycelial threads. The mycelial thread up to the fruiting stage is unseptate, and represents, therefore, a continuous tube. When grown in liquids the thread character gives place to yeast-like cells. 3 or 4 species of this group are capable of inducing experimental pathogenic effects. In very rare instances mucor mycoses have been met with in man.

The aspergillus group has no sporangia but, instead, the ends of the fruit-hyphæ are enlarged or club-shaped, the enlarged end, or columella, is covered with radially arranged, minute bottle-shaped bodies-the intermediate spore bearers or sterigmo-from which chains or rows of spores extend outward. Additional fruit organs, perithecia 
and sclerotia, are sometimes present: The mycelial threads branch freely, but the fruit hyphæ are not divided into cells (Fig. 56 b).

The penicillium has fruit organs resembling somewhat those of the preceding group. The fruit hyphæ, however, are septate and divide, and each branch ends in a characteristic brush-like form. The end of each branch is not enlarged as in the case of the mucor or aspergillus type, but like the latter it is covered by a number of bottle-shaped intermediate spore-bearers, known as basidia. Each of these in turn bears a chain or row of spores or conidia. As many as 8 spores may be held in a row on the end of the bottle-shaped basidium (Fig. $56 \mathrm{c}$ ).

- The oidium does not possess a definite fruit organ such as has been described in connection with the preceding forms. The mycelial threads branch and are usually made up of short thick cells. From the ends of the thread or from the ends of the cells, chains of large, oval conidia are given off. On sugar media it will slowly give rise to alcohol. The odor of limburger cheese may be largely due to the Oidium lactis.

The moulds, to a certain extent, induce fermentative decompositions(pp. 93, 97). They are of especial interest as the causes of certain diseases in plants, in lower animals and in man. At the beginning of the century, certain botanists held that the blight of wheat and of other plants was due to a microscopic parasite. The first actual demonstration of the relation of a fungus to a diseased organism was supplied in 1837 by Bassi, who showed that the silk-worm disease, known as muscardine, was due to a mould -Botrytis Bassiana. In the same year the relation of the yeast plant to alcoholic fermentation was for the first time clearly indicated. The possibility of microscopic moulds 
being the cause of disease in plants and animals lead the investigators of that period to make thorough studies of such affections. This led to the discovery during the fourth decade of a large number of moulds which were shown to be pathogenic for man, animals and plants.

Several of these fungous diseases may be briefly mentioned. The blight on potato leaves is due to the Peronospora infestans. The mildew of grapes and other plants is due to various species of Oidium. The ergot of rye and other grasses is due to the Claviceps purpurea. The smut on corn and other plants is due to several species of the fungus Ustilago. Bunt, the blight which attacks the grain of wheat and spelt forming a black fetid powder or mass of spores, is due to another mould, the Tilletia. The rust or mildew on grains is due to várious species of the Puccinia.

The parasitic mould which can be seen on dead flies is known as the Empusa muscae. The silk-worm disease, muscardine, as stated above is due to the Botrytis Bassiana.

The skin affection in man, known as Herpes, is due to a group of fungi, commonly designated as Tricophyton tonsurans, which, by some is placed in the Botrytis group. The fungus of thrush in children is known as the Monilia candida and by some is placed in the Oidium group. Favus is a skin disease of man due to the Achorion Schönleinii. Similar affections are known to exist among birds, mice and other animals. Another cutaneous disease, pityriasis is ascribed to the fungus Microsporon furfur which, however, has not as yet been successfully cultivated.

\section{Streptotrices.}

- Under this name are included certain saprophytic, and, at times, pathogenic organisms which show some resemblance to the moulds as well as to the bacteria. In other words, they are to be considered as intermediate forms. 
Like the moulds, they consist of cylindrical cells which branch dichotomously and form radially arranged masses or mycelia. Moreover, fruit-hyphæ develop and bear chains of roundish spores or conidia. Many of these forms when growing in the animal body or in pure culture form hard masses which have a radial structure.

The resemblance to the bacteria is especially evident under the microscope. The filaments, unlike those of the moulds, are extremely narrow-about the same width as the average bacterial cell. These threads branch freely and are perfectly homogeneous, without any transverse division into cells as in the case of the moulds. As the threads be. come old they may break up into rod-, or coccus-like forms not unlike bacteria. Even spiral forms may thus result. "This fragmentation of the filament is to be distinguished from the segmentation process whereby the round spores or conidia form on the ends of the fruit-hyphæ.

The spores of these organisms have not the resistance of those of bacteria. Thus, in the case of the actinomyces they are destroyed in 5 minutes at $75^{\circ}$. Moreover, they are stained readily by the simple anilin dyes and by Gram's method.

The diphtheria and tubercle bacilli undoubtedly belong to this group, although their predominant characteristics are those of bacteria. Nevertheless, branching forms have been observed in both germs. The fragmentation of the cells into rod-, or coccus-like forms is analogous to that of the true streptothrix. The club-shaped rods are'involution forms similar to those observed in actinomyces. Moreover, the necrotic action or the production of new growths by these bacilli differs in no wise pathologically from similar changes induced by the true streptothrix.

Under the term streptothrix are to be classed: the fungus of lumpy-jaw, of which there are perhaps several species; that of farcy in cattle, and that of Madura-foot in man. Moreover, many forms of pseudo-tuberculosis in 
man and animals are due to these organisms. Other pathogenic species have been found, though but rarely, in man and in the lower animals. Many of the streptotrices. are found in the air and in water. The members of this group, like the moulds and yeasts, are stained by Gram's method.

Laboratory work. - The student will inoculate ordinary gelatin, or better glucose gelatin, with the several yeasts. The Saccharomyces cerevisiæ, or the white yeast is to be poured into Petri dishes, while the red or black yeasts are to be used for making Esmarch rolltubes.

Ordinary baker's or brewer's yeast should be examined in the hanging-drop and in stained preparations. If compressed yeast is employed it will be well to distinguish the budding yeast-cells from the very large starch grains that are present.

The yeasts are fixed on the cover-glass and stained in the same way as bacteria.

The moulds grow best on slightly acid media. They can, therefore, be grown to advantage on the surface of potatoes or on moist bread. The addition of about 2 per cent. of cane-sugar or of glycerin to agar renders this a very good medium. The Oidium lactis should be grown on gelatin plates.

\section{Preparation of Bread-flasks.}

A bread powder is usually first prepared. For this purpose the bread is cut up into slices and heated in a dry-heat over till over-toasted. It is then finely crushed and preserved in a bottle. The dry powder can be kept indefinitely in a well stoppered bottle.

Six small, 30-50 c.c. Erlenmeyer flasks are cleaned, plugged and sterilized. The dry, powdered bread is filled into each flask to a depth of about $\frac{1}{4}$ inch, and water is then added so as to render the mass thoroughly moist. The bread-flasks are now steamed for half an hour on each of three successive days. 
The sterile bread-flasks are inoculated with the follow. ing moulds:

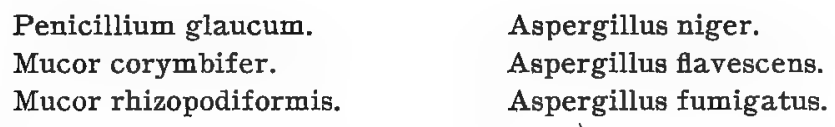

All these flasks, except the first, should be placed in the incubator at about $37^{\circ}$, for 24 to 36 hours. They should then be examined for the characteristic fruit-organs and for spores. A portion of the growth for this purpose is transferred to a watch-glass containing some 50 per cent. alcohol, to which a drop or two of ammonium hydrate has been added. When the growth becomes moist, it should be transferred to a drop of glycerin on a slide. The specimen is thoroughly and carefully teased with needles or pins. It is finally covered with a glass-slip and examined with the No. 7 objective.

If the specimen is satisfactory it may be made permanent by placing a ring of asphalt, with the aid of a turntable, around the edge of the cover-glass. Although the moulds stain readily by the simple and by Gram's method, it is not advisable to prepare dried specimens owing to the alteration which results during desiccation.

In this laboratory it is customary to study the yeasts and moulds at the close of the work on non-pathogenic bacteria. The parasitic skin moulds and the streptothrix forms are studied in connection with the pathogenic bacteria (see p. 316). 


\section{Saccharomyces Cerevisiæ.}

ORIGIN.-Beer or bakers' yeast; at times in the air.

COLOR. - White.

Form.-Cells spherical or egg-shaped 8-10 $\mu$ broad. The cells are colorless and when actively growing have a homogeneous protoplasm. Later, granules and vacuolès develop. Owing to a gelatinous exudate it may form zoogleal masses. The cells may be single or may have several buds; at times long branching forms may be found, especially if the temperature is about $30^{\circ}$.

Motility.-None.

SPORUlation.-Usually several spores form. Can be double stained. Spores develop between 11 and $37^{\circ}$.

AnILIN Dyes. - Stain readily; so does Gram's method.

GrowTH. - Thick white growth, especially abundant on glucose media and in wort.

Gelatin plates.-Small, opaque, white, circular colonies which are very coarsely granular and slimy.

Stab culture. -The growth is confined to the upper portion of the tube and spreads over the surface. It is thick and opaque white.

Streak culture.-On agar and on potato it forms a thick, somewhat raised white growth,

Temperature. -As an "upper yeast" the most rapid fermentation takes place between 14 and $18^{\circ}$.

Behavior to Gelatin. - Does not liquefy gelátin.

Aerogenesis. - It gives rise to a ferment (invertin) which changes cane-sugar into glucose. The latter is then changed by another ferment, zymase, to carbonic acid and alcohol (4-6 per cent.). It does not ferment lactose.

Painogenesis. - It has no effect on animals. A large amount may produce a catarrhal condition in the alimentary tract. 
DRAWINGS.

397 


\section{Saccharomyces Glutinis.}

RED YEAST.

ORIGIN.-Very common in the air, from which several distinct kinds of red yeast have been obtained.

COLOR.-Red or pink.

Form.-Round or oval cells with granular protoplasm which stains irregularly. Cells single or in pairs, budding.

Motility.-None.

SPorulation. - None.

ANILIN DYES. - It stains readily, also by Gram's method.

Growrr.-Abundant, though somewhat slow.

Gelatin plates.-Colonies are small, round, elevated, moist and pink-colored and coarsely granular.

Stab culture. -Growth absent from the lower part of the tube. It spreads slowly over the surface, forming a thick, moist, bright red covering.

Streak culture.-On agar, it develops in a few days as a thick, slimy, spreading, pink-colored growth. On potato, it forms the same pigment.

Temperature.-Grows best at ordinary temperature.

Behavior to gelatin.-Does not liquefy.

Aerogenesis.-Does not change glucose to alcohol.

Pathogenesis. - No effect on animals.

A white yeast is frequently deposited from the air. The cells are usually smaller than those of the red yeast.

A black yeast, $S$. niger, forms a brownish or black growth. The size of the cell is about the same as that of the red yeast. It grows very slowly. 


$$
\text { mumsana }
$$




\section{Oidium Lactis.}

OrIGIN.-Almost invariably present in milk and in butter; also in sugar solutions of various kinds, and in brewer's yeast.

Form. -A delicate, white mycelium of forked, wavy threads. No special fruit-organ is present. The conidia or spores are very irregular in size and form; they may be oblong, round or oval.

AnILIN Dyes.-React readily. The specimen shrinks on drying.

GRowtн.-Is rapid, especially on acid media.

- Gelatin plates.-Delicate white stars form, which rapidly enlarge, and spread on the surface as dry, flat, whitish masses. Under the microscope the colonies show radiating, branched hyphæ and chains of conidia.

Stab culture.-Growth takes place along the entire line of inoculation, and is most abundant at or near the surface. A branching network of threads extends outward into the solid gelatin. On the surface a grayish white, dry, low growth forms. In old cultures only the upper layer of gelatin shows the radiating lines.

Streak culture.-On agar, it forms a grayish white, thin growth.

Milk.-Growth occurs without altering the composition.

Temperature.-Grows best at ordinary temperature. It can grow in the incubator.

Behavior to gelatin.-Does not liquefy.

Pathogenesis. - It has no effect on animals. A somewhat similar organism, the Oidium Tuckeri, produces a vine, disease. 
DRAWINGS 


\section{Monilia Candida, Robin (1847).}

THRUSH FUNGUS; OIDIUM ALBICANS, SACCHAROMYCES ALBICANS; MUGUET (Fr.); SOORPILZ (Germ.).'

Origin.-Found in thrush on the mucous membrane in the mouths of infants and of grown persons; also in air, in milk, in barns; occurs as a white growth on cow-dung. It may occasionally be found on mucous membrane other-than that of the mouth.

Form.-Occupies an intermediate position between the moulds and yeasts. On gelatin plates and on sugar media it forms yeast-like cells (conidia), whereas in the deeper part of a stab culture it forms mycelial threads. The mycelial threads are developed best in the absence of sugar and at a high temperature.

AnILIN Dyes. - Stain readily.

Growth.-Is rapid and abundant.

Plates.-Snow-white, coarsely granular colonies form on gelatin plates and no liquefaction takes place.

Stab culture. - In gelatin show a slight growth along the line of inoculation, while on the surface a milk-white, thick mass forms.

Streak culture.-On agar, forms a glistening, moist, thick, white growth. On potato, it grows rapidly as a thick, white, yeast-like mass.

OXYGEN ReQUirements. - It is an aerobe.

'Temperature. - Grows at the ordinary temperature; also in the incubator at $40^{\circ}$.

Behavior to gelatin. - It does not liquefy.

Pathogenesis. - Intravenous injection in rabbits produces death in 1-2 days; with very small doses death may not result for a week or two. The internal organs are sometimes permeated with a growth of long mycelial threads. Under rare conditions it has been found in the internal organs of man. When inoculated into the throat of doves and chickens it produces a typical thrush-membrane. Subcutaneous application of a few mg. will produce death in white mice in 1-3 days. It has no effect on guineapigs. The identity of Monilia candida with thrush cannot be. said to be fully established.

It ferments cane-sugar and maltose without previous inversion, and in time may give rise to about 5 per cent. of alcohol. 
DRAWINGS

403 


\section{Mucor Corymbifer, Lichtheim.}

ORIGIN.--Is of rare occurrence, and was found as a contamination on bread-gelatin plates and on white bread which was kept at the body temperature. It has been found in the ear-passages of man. It is probable that this same species has been found in a case of generalized mycosís.

Color. - The mycelium, spores and sporangia are colorless.

Mrcelium.-Loose, wavy, branching, slender mycelial threads which form a white cotton-like mass an inch or more in height.

FrUIT-ORGans.-The fruit.hyphæ branch forming clusters or corymbs which terminate in spherical or pear-shaped sporangia. Within these are the colorless, oval or elongated spores, which are about $3 \mu$ long and $2 \mu$ wide.

Growth.-Rapid and extensive.

Bread-flasks-In the incubator it forms a white, elevated, cottonlike growth which soon fills the flask. On potato a similar cotton-like, tall growth develops.

Temperature. - Grows slowly at the ordinary temperature; best at $37^{\circ}$.

Pathogenesis. - Intravenous injection of the spores into rabbits produces death in 3 to 4 days. The kidneys, mesenteric glands and Peyer's patches contain mycelial masses. The Peyer's patches are swollen and ulcerated. Intraperitoneal injections produce the same results, Dogs are immune. 
DRAWINGS

405 
Mucor Rhizopodiformis, Lichtheim.

OrIGIN.-White bread kept at $37^{\circ}$.

CoLor. - At first white, but later becomes grayish.

Mycelium.-The mycelial threads are colorless, later on brownish, and thicker than those of the preceding mucor. They are not jointed or divided. At first the growth is white, then becomes grayish.

FrUIT-organs.-The fruit hyphæ occur in groups or bunches, which adhere to the nutrient medium by means of special root tufts. The large dark sporangia on the ends of the hyphæ contain colorless rounded spores which are larger than those of the preceding organism $(5-6 \mu)$.

Growtr.-Is rapid and has a pleasant odor.

Gelatin plates.-Development is best when the gelatin is made with bread infusion. It forms a coarse grayish-black mass which liquefies the gelatin.

Bread flasks. -The growth is lower than that of M. corymbifer, and is grayish, owing to the dark colored sporangia. An ethereal or aromatic odor is present. at $37^{\circ}$.

Temperature.-Slow growth at 12 to $15^{\circ}$; develops best

Behavior to gelatin.-Liquefies.

Pathogenesis. - It has a similar effect as M. corymbifer, but is more pathogenic.

The mucors are characterized by marked fermentative powers. They convert dextrose and maltose into alcohol. Only one species, however, can invert cane-sugar. Some species may produce 7-8 per cent. of alcohol and many of them give rise to diastatic ferments. The most common species is the $M$. mucedo which is relatively frequent on the excreta of herbivorous animals. The $M$. racemosus is likewise common and its spores are found in the air. 
DRAWINGS.

407 
OrIGIN.-In putrid substances; in the lungs of birds.

COLOR.-Black or dark brown.

Mycelium.-This is low and at first white, then brownish or black.

FruIT-organs. - The fruit hyphæ are spherical, or flask-, or club-shaped at the end, and this enlargement is covered with radially arranged, minute bottle-shaped bodies-the intermediate spore bearers or sterigmoe-fróm which rows of spores extend. The sterigmæ are divided. The spores are black or brownish and spherical, and are 3-5 $\mu$ in diameter.

Growrh.—Slow.

Bread flasks. -It forms a low growth which becomes very black.

Temperature. - The optimum is about $35^{\circ}$.

PATHOGENESIS. - Intravenous injection of spores in rabbits is not followed by as malignant results as with the next two forms. It gives rise to diastatic, inverting and other ferments.

Aspergillus Flavescens, Wreden.

OrIGIN. - White bread.

Color.-At first whitish, eventually pale yellow or yellowish green.

Mycelium.-The mycelial threads and spores are smaller than those of $\mathrm{A}$. niger.

FrUIT-ORgans. - The club-shaped ends of the fruit hyphæ are covered with sterigmæ, from which extend rows of spores, as in A. niger. The spores are yellowish or brownish in color and are 5-7 $\mu$ in diameter.

Growth.-Rapid.

Bread flasks.-Grows best on bread where it forms a yellowish, low growth.

Temperature.-The optimum is about $28^{\circ}$, but it grows well in the incubator.

Pathogenesis. - It is more pathogenic than A. niger, and less than $\mathrm{A}$. fumigatus. 
DRA WINGS. 
Aspergillus Fumigatus, Lichtheim.

OrIGIN.-White bread; in the air passages and lungs of birds; also met with in man.

CoLoR.-Greenish or bluish green growth, resembling very much that of penicillium.

Mrcelium.-About the same as that of the preceding.

FRUIT-ORGANS. - Like the preceding, but the spores are only about one-half as large and are usually colorless. The sterigmæ do not divide.

Growry. - Is best on bread and is rapid.

Bread flasks.-The growth is low and at first bluish green, but when old is grayish.green and resembles that of Penicillium.

Temperature. - The optimum is $37-40^{\circ}$. It can grow at the ordinary temperature, but not below $15^{\circ}$.

Pathogenesis. - Intravenous injections of millions of spores in rabbits and dogs produced death in a few days. Mycelia were found in the kidneys, heart and other muscles, and occasionally in the liver.

Infection of doves and other birds by inhalation of the spores produces a pneumonic or pseudo-tuberculous disease. Natural affections of this kind are frequently observed among birds. Occasionally, they are met with in horses and in cattle, and at times in man.

In mycoses of man, the lungs, ears, eyes or nose are subject to inviasion.

The Japanese utilize the growing A. Oryzæ as a diastatic ferment, like malt. It converts rice grains into sugar and dextrin. This liquid is then subjected to fermentation and yields the national drink, Sake, which contains about 14 per cent. of alcohol. Taka-diastase is a ferment derived from an aspergillus like that mentioned. 
DRAWLNGS.

411 


\section{Penicillium Glaucum.}

ORIGIN. - Widely distributed in the air, water and soil. 60 per cent. of the mould contaminations in the laboratory are said to be due to this organism.

CoLor.-Is at first whitish, then becomes bluish.green.

Mrcelium.-Consists of horizontally arranged, straight or slightly wavy, jointed mycelial threads from which the fruit hyphæ rise vertically.

FrUIT-ORGans. - The ends of the septate fruit hyphæare forked, and are covered with the intermediate spore bearers, or sterigmæ, also 'sometimes called basidia. Each of these in turn bears a row of 8 spores or conidia, so that the appearance of the whole is that of a brush. The spores are about $3.5 \mu$ wide.

Growth.--Is rapid.

Gelatin plates. -The colonies form whitish floccules which rapidly increase in size, and at the same time the center colors green. The gelatin is liquefied quite early. A low objective will show the above characteristics of growth.

Bread flasks.-Show a low, finely flocculent covering, which at first is white but soon changes to a distinct green.

Temperature.-The optimum temperature is from 22 to $26^{\circ}$. It does not grow at the temperature of the body.

Behavior to gelatin. - Slowly liquefies.

Pathogenesis. - It has no effect on animals. I It frequently develops on grapes and causes a marked alteration in wine. It gives rise to inverting and to diastatic ferments. This organism is said to be used in the preparation of Roquefort cheese. 
1

DRAWINGS.

413. 
Achorion Schönleinii, Schönlein (1839).

THE FUNGUS OF FAVUS.

OrIGIN.-Found in the scaly accumulations on the skin of persons afflicted with favus. Similar, if not identical organisms occur in the favus of the dog, cat, rabbit, mouse, chicken, etc. It is closely related to the Oidium lactis.

FORM.-Apparently belongs to the moulds that produce oidia. The mycelium when actively developing consists of stellate threads which are not septate. The individual hyphæ vary considerably in thickness and usually fork. The ends are swollen and, moreover, peculiar yellowish lateral buds or corpuscles are seen. When the culture becomes old the threads divide into oval elements (oidia) which remain attached. In the deeper layers of the culture medium, moss-like ramifications are found.

FRUIT-ORGANS. - No true fruit-organs observed, but on special media, as on blood-serum at $30^{\circ}$, conidia or spores are said to form.

ANILIN DYES. -Stain well; so does Gram's method.

GRowTH.-Is rather slow and requires a week or more. It is at first grayish-white, then yellowish. On the usual media it tends to grow below the surface and only a feeble development occurs in contact with the air.

Plates.-On gelatin, the colonies grow slowly and form whitish, stellate masses which liquefy the gelatin. No conidia present.

Stab culture.--Growth is very poor in the lower part of the gelatin tube. On the surface it forms a white covering, the lower side of which is light-yellow. On potato it forms thick scales.

Streak culture.-On agar it forms a closely adherent, dry, folded, whitish mass.

Temperature. - Dies out at the ordinary temperature. The optimum is about $30^{\circ}$.

BeHAVIOR TO GELATIN. - Slowly liquefied; becomes reddish.

Pathogenesis. - Inoculation with a pure culture produces typical favus in man. It is usually localized on the scalp.

The favus fungus is closely related to that of Herpes tonsurans-the Tricophyton tonsurans (1845); and to that of Pityriasis versicolor-the Microsporon furfur (1846).

In order to isolate the organism in pure culture it is advisable to crush the scales in a sterile mortar with sterile sand. This finely divided powder is then used to make dilution cultures on ordinary agar. As many as nine different varieties of Achorion have been described. 
$i$

DRAWINGS.

415

- 
ACTINOMYCES BOVIS; RAY-FUNGUS; STRAHLENPILZ (Germ.).

OrIGIN.-Occurs in actinomycosis or lumpy-jaw in cattle, hogs, horses and in man. It probably leads a saprophytic existence on plants, etc.

FORM.-It gives rise to nodules which consist of a whorl of mycelial-like,-multiple branched threads. These radiate outward from a central point and become clubshaped. In pure cultures only slenider, wavy threads are formed. The club-shaped or swollen ends which are usually present in tissues are lacking; unless the organism is growing deep in gelatin or in blood-serum. The club-shaped ends are the result of degenerative changes. In other words, they are involution forms due to a gelatinization of the cell-wall.'

ANILIN DYes.-It stains readily with carbolic, fuchsin; also by Gram's method.

Growtr.-Develops somewhat slowly, requiring several days in the incubator. It can, however, grow on various media even at the ordinary room temperature. Although usually yéllowish it may take on a brick-red color.

Streak culture.--On agar, the growth begins as minute, isolated colonies which slowly enlarge, forming thick, convex, glistening, yellowish, opaque masses. The colonies are exceedingly hard and for examination should be crushed between two glass slides, previously sterilized by passing several times through the flame. Cover-glass preparations are then made and stained in the usual manner. the liquid.

In bouillon the growth develops on the bottom and does not cloud

OXYGEN REQUIREMENTS.-Said to grow best in the absence of air, but grows very well on the surface of agar. body.

TEMPERATURe.-It grows best at or near that of the

PATHOGENESIS. - In rabbits, intraperitoneal injection of the pure culture is said to produce typical actinomycotic nodules on the peritoneum, mesentery, intestinal walls, etc.

The disease is recognized by the presence of small yellowish granules in the pus which is derived from the tumors. The granules when examined with a No. 3 objective will show the typical stellate growth, the hyphæ of which have club-shaped ends.

Infection results from the plant food on which the organism is growing. Several varieties of actinomyces have been described; can be distinguished only by culture. 



\section{Streptothrix Maduræ, Vincent (1894).}

Origin.-Occurs in Madura Foot, or Mycetoma, a disease which is endemic in India. It has been met with in Italy, Africa and several cases have been studied in this country. The discharge in somecases contains minute, black, gun-powder-like grains; in others, grayish or yellowish granules are present. These granules are due to mycelial growths and resemble those found in actinomycosis.

Form.-It consists of slender, wavy threads, 1-1.5 $\mu$ wide, which branch and show a radial growth, as in actinomyces. Club-shaped ends, however, are absent or very feebly developed. Fragmentation is frequently observed.

ANILIN DYEs.-Stain readily; so does Gram's method.

GrowTH.-Is rather slow, requiring several days to form appreciable colonies. It grows best on unneutralized potato, or in hay infusions.

Stab culture.--In gelatin it shows a slight white growth along the line of inoculation and on the surface.

Streak culture.-On ordinary agar the growth is feeble. On glucose glycerin agar it forms splendid, raised, rounded, smooth colonies which are slightly yellowish, and later develop frequently a rose or bright red color. These colonies adhere to the surface and are very hard, as in the case of actinomyces.

Milk.-Is slowly peptonized, but is not coagulated.

- Potato.-It forms, on about the 5th day, small whitish colonies which slowly develop, forming a nodular growth. The center of each nodule is depressed. In old tubes, especially on acid potatoes, a pink or deep red color may develop. A similar color develops when grown on carrots.

Bouillon.-After a lapse of 2 or 3 weeks small, whitish balls form on the bottom. Vegetable infusions are better than ordinary bouillon. The growth then develops in 4 or 5 days, as small floccules, which later become as large as a pea. The liquid does not become cloudy. May grow on the surface of bouillon forming a growth like that of the tubercle bacillus.

Resistance - Vitality unimpaired after drying on paper for 9 months, or being kept as a potato culture for 21 months. The ovoid spores form on the surface, in contact with air, and are easily colored with simple stains, or by Gram's method. They resist heating at $75^{\circ}$ for 5 minutes.

OXYGEN REQUIREMENTS. - It is aerobic and does not grow in vacuo, in $\mathrm{CO}_{2}$ or in illuminating gas.

Temperature.-The optimum is at $37^{\circ}$, but it may grow at ordinary temperature.

Behavior to gelatin.-It does not liquefy.

Pathogenesis.-Pure cultures of the original grains injected subcutaneously or intravenously, or into the peritoneal cavity are without effect in the guinea-pig, rabbit, dog, sheep, pigeon and chicken.

The above organism was isolated from the yellowish caseous granules mentioned above. Recently, Wright has succeeded in growing a streptothrix from the black granules. In this case the hyphæ were $3-8 \mu$ in diameter. Fragmentation was present, and in old cultures black granules or sclerotia developed. It was likewise nonpathogenic to animals. 
DRAWTINGS

419 


\section{Streptothrix Farcinica, Nocard (1888).}

ORIGIN.-It is present in the pus from the subcutaneous nodules, also in the pseudo-tubercles in the lungs and other internal organs in the disease known as cattle-farcy ( farcin du boeuf). The disease is very rare in France; apparently quite common on the Island of Guadeloupe.

FORM.-The organism in diseased tissue and in pure culture forms slender filaments which in width are comparable to the rouget bacillus. The filaments are densely interwoven, forming masses. They branch dichotomously.

ANILIN DYEs.--It does not stain like the tubercle bacillus. In Gram's method, if treatment with alcohol is continued it decolors. It is stained by the Gram-Weigert method (decoloration in anilin oil).

GrowTH.-Develops readily on solid and liquid media at the body temperature. Acid media are not favorable. Cultures kept at $40^{\circ}$ retain their vitality.for months. No growth in the absence of air.

Streak culture.-On agar it forms irregular, raised, yellowish masses which unite and form a thick, coarsely folded membrane, in appearance not unlike that produced by the tubercle bacillus.

On potato, it forms raised, dry, yellowish scales and the growth as a whole resembles that of the tubercle bacillus. On blood-serum, the growth is slow and has the same characteristics as that on potato.

Bouillon.-Cultures show irregular masses which usually fall to the bottom. When, however, the growth develops on the surface it presents the same characteristics as the tubercle bacillus. It does not coagulate milk or alter its reaction.

Resistance.-Old growths on the surface of glycerin bouillon are said to contain extremely small ovoid spores which resist staining. These may, however, be the result of fragmentation. 10 minutes heating at $70^{\circ}$ destroys the organism.

Temperature.-Does not grow at ordinary temperature, but does grow at 30 to $40^{\circ}$.

OXYGEN REQUIREMENTS.-Is aerobic; will not grow in vacuo or in $\mathrm{CO}_{2}$.

Pathogenesis. - The guinea-pig is the most susceptible animal, then come cattle and sheep. The rabbit, dog, cat and horse seem to be refractory. Intravenous and intraperitoneal injections produce in from 9 to 20 days miliary pseudo-tubercles which cover the serous surfaces, and, in the former case, they are also present in the various organs. When inoculated subcutaneously in guinea-pigs or other susceptible animals, a local abscess forms, which discharges and secondary ones form slowly. This infection, like the natural one, rarely produces death. 


$$
\text { mammese }
$$


CHAPTER XIII.

EXAMINATION OF WATER, SOIL AND AIR.

A pure water-supply is of first importance in the conservation of public health. That impure water is the cause of many diseases is, to-day a well recognized fact. It has ceased to be a theory. Cholera and typhoid fever are striking examples of water-borne diseases. It should not be inferred, however, that these diseases are always conveyed through drinking-water. The specific germs may, at times, be introduced into the body by means of various articles of food or by direct contact of the mouth with infected articles. Nevertheless, the most common vehicle by which these organisms enter the body is the drinking-water.

A pure water, therefore, is essential to the prevention of these and other diseases. Until very recent times an opinion as to the purity of a water was based wholly upon a chemical examination. 'In some instances, the water may be injurious because of the presence of poisonous metals or other injurious compounds. Thus, the presence of arsenic or lead, or of large amounts of copper or zinc would certainly render the water impure and highly dangerous to health. In such cases the chemical examination will directly reveal the presence of the poisonous metal. As a striking illustration of this, may be mentioned the fact that Vaughan detected relatively large amounts of arsenic and antimony in the river water of a Western mining region. The water had been used by animals, a large number of which, as a result, died from arsenical poisoning.

The real danger in water, however, does not lie in the presence of poisonous chemical substances. Intoxications, 
such as mentioned, are extremely rare. On the other hand, infections through impure water are unfortunately but too common. By infection is meant the introduction into the body of a specific, pathogenic micro-organism. When impure water is spoken of, it does not follow that the water is unsightly, malodorous or repulsive to the taste. The water may be perfectly clear, sparkling in character and a chemical examination may show that it contains minimal amounts of organic and inorganic constituents, and yet, that water may be impure because of the presence of pathogenic bacteria.

A chemical examination will not detect the presence of bacteria, much less assist in their identification. It can, however, detect the presence of certain chemical substances from the relative amounts of which an inference may be drawn as to the existence of pollution with human or animal excreta. A pollution of this kind once established - indicates that danger from infection may be expected whenever the pathogenic germ is actually present in such excreta. From a chemical standpoint a good water should not contain more of the several constituents, in parts per million or mg. per liter, than what is shown in the following table:

\begin{tabular}{|c|c|}
\hline al residue... & Nitric anhydride \\
\hline Earthy bases..........180-200 & Nitrous acid........ traces \\
\hline Sulphuric anhydride. . 80-100 & Albuminoid aminonia. \\
\hline
\end{tabular}

Moreover, it should not consume more than 8 to 10 parts, per liter, of potassium permanganate.

It should not be understood from these requirements that a water containing an excess of these constituents above the limits given will necessarily be dangerous. The chlorine, nitrates, nitrites and ammonia are in themselves harmless and can be taken with impurity in relatively large doses. The data given, however, are supposed to represent the average composition of good water. An 
excess of chlorine and nitrogenous matter would therefore indicate the presence of animal excreta. An increase, for instance, in the amount of chlorine might be due to the presence of sodium chloride derived from urine. Similarly, large quantities of free and albuminoid ammonia are usually taken to indicate the presence of urea. Unchanged urea would be represented by albuminoid ammonia. But, as is known, urea readily undergoes ammoniacal fermentation and yields ammonia and carbonic acid. Other bacteria attack the ammonia and convert the nitrogen present into nitrous and nitric acids. For these reasons an increase in free and albuminoid ammonia, and in nitrates and nitrites is taken as indicating a pollution with organic, nitrogenous, waste matter.

The assumption of the existence of a pollution is not always justifiable whenever the limits, as given above, are exceeded. Thus, in Michigan it is not uncommon, on account of the peculiar geological formation, to meet with a . marked increase in the amount of chlorine or salt. Moreover, in many, especially low lying sections, the water will be rich in nitrogenous constituents which in this case are derived from vegetable and not animal matter.

It is evident from what has been said that a chemical analysis may, or may not, indicate the presence of pollution with animal excreta. Furthermore, the important fact should not be overlooked that a specific organism may be present, and hence render a water extremely dangerous without any appreciable increase in the chemical constituents of such water. A chemical analysis of water is useful and it should always be carried out, but the results obtained should, as a rule, be subordinated to those obtained by a bacteriological examination.

A bacteriological examination of water is intended to reveal the number and kind of bacteria that may be present. As a rule, the smaller the number of bacteria in a 
given volume, the more likely is it to be free from injurious forms. On the other hand, if the number of bacteria is subject to considerable fluctuation and is usually high, it indicates conditions favorable to decomposition. The bacteria in a given specimen of water may be extremely numerous, and yet they may be mere harmless saprophytes. The fact that such water is a good nutrient medium should make it suspicious inasmuch as intestinal bacteria, if once introduced, will be favored in like manner. 'They may indeed be present but, masked by the large number of common water bacteria, they may escape detection.

The chief interest is attached to the kind of bacteria present. The recognition of certain well known intestinal bacteria in a given water may be taken as a good indication of the existence of pollution by animal excreta. Thus, the Bacillus coli communis is invariably present in the intestinal contents of man and animals, and hence its detection in water, especially in appreciable numbers, points to the source of the contamination. The fact, however, should not be overlooked that this or related species are widely distributed in nature. It makes its appearance in the intestines of the new-born within a few hours after birth and this fact in itself indicates the wide prevalence of the colon bacillus. Consequently, the detection of the colon bacillus in a water should arouse suspicion and cause a thorough examination of the premises with reference to the close presence of polluting material. The finding of the colon bacillus, therefore, does not indicate more than the possibility of the existence of pollution, which, as a rule, will also be indicated by a chemical examination.

The recognition of a specific pathogenic germ in a water is of prime importance inasmuch as it demonstrates actual pollution as well as the possibility of infection. The typhoid bacillus is the one most frequently looked for. Inder exceptional conditions the cholera, anthrax and 
pus-producing germs are included in the scope of a water examination.

Typhoid bacillus.-The typhoid bacillus when once introduced into a water-supply does not necessarily remain in such water for a considerable length of time. The results of many experiments made by different observers would indicate that the typhoid bacillus when introduced into ordinary water may remain alive for about a week. This is about the limit when they are placed in very pure water kept at a low temperature, i. e., under conditions very unfavorable to the vitality of the organism. This applies to natural as well as distilled water, whether sterilized or unsterilized.

When the temperature is above $10^{\circ}$, and especially, when the water contains an appreciable quantity of organic matter, the typhoid bacilli may persist for a much longer period. The organisms under these conditions may actually increase in number and retain their vitality for weeks and months. Hence an impure water, as indicated by a chemical analysis, is to be regarded as suspicious because it may favor the growth and persistence of typhoid germs when once introduced.

The recognition of the typhoid bacillus in water is by no means an easy matter. Usually an examination is called for after an outbreak of the disease has taken place. That is to say, the period of incubation, which extends from 1 to 2 weeks, is allowed to pass before a search is instituted for the organism. This time is sufficient, under conditions indicated above, for the typhoid germ to disappear, and, consequently, the examination in such cases will be negative. Moreover, even in water which in the beginning was favorable to the growth of this organism, the detection is still difficult. At the time of the examination, the germs - may already be dying out and decreasing in number. The small volume of water which is necessarily taken for analysis may, or may not, be a perfect sample of the whole. 
Chemically speaking, it is a good sample because the soluble constituents in one drop are present in relatively the same amounts as in a liter or barrel of the water. This is not true with reference to the suspended bacteria. These are solid particles, and as such tend to settle or subside. The so-called water purification in many instances. may largely consist of mechanical sedimentation. Although, therefore, the mass of the water may still contain some typhoid bacilil, the small quantity taken for examination (1-10-20 drops), may, or may not, contain one or more of these organisms. The few specific germs are easily masked by the hundreds and thousands of common water bacteria that are usually present. If necessary, the number of organisms taken for an examination may be increased by prolonged centrifugation of the water. Moreover, the separation may be favored by the addition of typhoid serum or of histon.

- The typhoid-like or pseudo-typhoid bacilli that may be present require careful and prolonged study. Recent investigations have shown that typhoid bacilli, or at all events organisms that cannot be distinguished from these by any known test, may be present in water, in soil; also in the feces of healthy persons who have never had typhoid fever. Thus, Lösener isolated 5 such organisms from tap-water, field soil, normal pus, from a hog cadaver buried 4 weeks, and from a typhoid spleen buried 96 days. Pfeiffer and Kolle have pronounced these organisms to correspond exactly to the typhoid bacillus, even to the application of Pfeiffer's reaction, and hence their typhoid character cannot be questioned. Remlinger and Schneider, employing Elsner's medium and working under the direction of Vaillard at the Yal-de-Grâce hospital at Paris, isolated a bacillus indistinguishable from that of Eberth:

8 times out of 36 samples of water;

6 times out of 10 samples of soil;

3 times out of 8 samples of normal feces. 
Of the 18 pseudo-typhoid bacilli isolated, 12 killed guinea pigs when injected intraperitoneally in doses of 2 c.c., whereas the remaining 6 had no effect. Anti-typhoid serum was said to be efficacious in preventing infection by these organisms. A mention may be made, moreover, of the fact that Ohlmacher isolated a bacillus, indistinguishable from that of Eberth, from the Cleveland tap-water. In cadavers buried for $1 \frac{1}{2}-2$ years only typhoid-like bacteria are said to be present.

The typhoid bacillus cannot be identified by any one characteristic. The suspected culture must give most, if not all, of the known reactions of the typhoid germ. These reactions may be grouped together in the following summary:

1.-Appearance of colonies.

2.-Active motion.

3.-Large number of whips, including giant-whips.

4.-Gram's stain negative.

5.-No gas production.

6. - No coagulation of milk.

7.-No indol reaction.

8.-No acid production (lactose media).
9.-Invisible growth on potato.

10.-Colonies on Elsner's medium.

11.--Transparent diffusion on Stoddart's medium.

12.-No growth in Uschinsky's fluid.

13.-Agglutination by diluted typhoid fever serum.

14.-Pfeiffer's reaction with antiinfectious typhoid serum.

15.-Pathogenic effects.

The above characteristics will be found described under their respective heads. The ubiquitous colon bacillus is especially ruled out by the last 11 tests. The colon bacillus is less pathogenic and rarely kills guinea-pigs when injected subcutaneously.

Plate cultures can be made with ordinary gelatin, with Elsner's medium or with a 3-5 per cent. urine gelatin (see Chapter XIV). The suspected typhoid colonies are then transplanted to other media and subjected to the tests given above. It is advisable to transplant 20 or more suspicious looking colonies into bouillon tubes which are then placed at $39^{\circ}$ to develop. A drop or two of each culture 
that now develops is placed, by means of a drawn-out tube pipette (Fig. 61), into sterile milk tubes. The inoculated milk tubes are then placed at $39^{\circ}$ for $1-3$ days. As a control of the sterility of the milk a number of the uninoculated tubes should likewise be placed in the incubator. Moreover, it is advisable to inoculate a like number of milk tubes with a known typhoid bacillus in order to eliminate a possible error (p. 163), which might arise if spores of anaerobes were present. The milk tubes that coagulate can be discarded at once, together with the bouillon cultures from which they wete inoculated. The milk tubes that do not coagulate may, or may not, contain the Eberth bacillus. The bouillon cultures from which these tubes were inoculated can now be used for the supplementary tests.

The procedure as outlined is preferable to direct inoculation into milk from the suspected colonies. The transplantation in the latter case may sometimes fail to develop or does so very slowly. When bouillon cultures are grown, the inoculations can be made with liberal amounts of the organism and the original material is saved for subsequent tests.

At times it may be more advantageous to make a surface touch folony on Stoddart's medium contained in a large flask or Esmarch dish. The transparent border growth can then be plated on gelatin or on Elsner's medium, and the suspicious colonies can be tested as in the manner indicated above. The addition of a drop of a bouillon culture to a liter of tap-water can be detected quite readily in this way. The methods mentioned above are also resorted to when isolating the Eberth bacillus. from the urine or feces of typhoid patients.

Cholera vibrio.-This organism has been repeatedly found in water during times of cholera epidemics. As in the case of the Eberth bacillus, the detection of the comma 
bacillus is not an easy matter. The cultural and morphological properties are subject to considerable variation. Moreover, closely similar organisms may exist in river water. These water vibrios phosphoresce, but this property as in the case of other photogenic bacteria may disappear on cultivation. It is doubtful whether the true cholera vibrio can phosphoresce.

The recognition of the cholera vibrio in water or in suspected discharges is based upon the following characteristics:

1.-Microscopical appearance.

2.-Cultures in Dunham's solution.

3.-Appearance of colonies on gelatin plates.

4.-Appearance of colonies on agar plates.
5.-Agar and gelatin tube cul- tures.
6.-Positive indol reaction.
7.-Inoculation of guinea-pigs.
8.-Pfeiffer's reaction.
9. Agglutination.

In the case of suspected intestinal contents, the microscopical examination may in a few minutes justify a diagnosis of cholera, but a positive diagnosis can only be obtained by confirming all the characteristics of the organism. Under favorable conditions this may be accomplished in less than 24 hours. A drop of the intestinal liquid should be spread over the cover-glass, or better still, one of the numerous flakes present in the rice-water discharge should be thoroughly rubbed over the cover-glass. By staining with carbolic fuchsin, the presence of comma-shaped organisms can be readily established if they are present in large numbers.

It should be remembered, however, that the cholera vibrio may disappear from the intestines after about the fifth day. In other instances, only a few cholera vibrios may be present and these may escape detection, owing to the large number of other organisms that occur in the material.

Even when only a few cholera germs are present in the discharge or in the water, they can be brought to light by a peculiar method of "accumulation." Thus, when planted in 
Dunham's solution the vibrio grows rapidly, and on account of its extreme aerobic tendency it accumulates or gathers on the surface of the liquid forming a broken pellicle. Dunham's solution is water to which 1 per cent. pepton and 0.5 per cent. salt has been added (p. 344). A modification by Metchnikoff contains 1 per cent. each of pepton and salt, and 2 per cent. of gelatin.

About 100 c.c. of the liquid are placed in each of several Erlenmeyer flasks. These are then inoculated and set aside at $37^{\circ}$. In from 10-20 hours the surface growth is examined. A loopful can be used for staining and for hanging-drop examination. At the same time gelatin and agar plates should be made from this surface pellicle.

The gelatin plates should be developed at as high a temperature $\left(22-24^{\circ}\right)$ as possible, without melting the gelatin. This can be done very satisfactorily by keeping the plates in the water-cooler (Fig. 33, p. 179). The agar plates are not prepared in the ordinary way. Koch's procedure consists of pouring the agar into Petri dishes where it is allowed to solidify and remain for several days. This is to allow the water of condensation to evaporate. The surface of the agar plates, thus prepared, is repeatedly streaked with a platinum wire. The organisms are thus planted on the surface and herice good isolated colonies can be obtained. The agar plates are placed at $37^{\circ}$ and in urgent cases they can be examined in about 8 or 10 hours.

The characteristic colonies on gelatin or on agar are transplanted to Dunham's solution in tubes, and to agar and gelatin tubes. The first two media are then placed at $37^{\circ}$. In about 10 hours the indol reaction may be applied to the culture in Dunham's solution. A loopful of the growth (2 mg.) on inclined agar can be removed at the end of about 20 hours, suspended in 1 c.c. of bouillon and this then injected into the peritoneal cavity of a guinea-pig. The temperature and the weight of the animal should be taken before the injection. The former should again be taken every 
2 hours after the injection. In a few hours the animal becomes sick, the temperature drops gradually to $30^{\circ}$ and. death eventually results.

In the case of water which contains but a very few cholera vibrios, instead of adding a small amount to the Dunham's solution, it is better to add the necessary amount of pepton and salt to 300 c.c. or more of the water, thus converting the suspected water into Dunham's solution. These constituents can best be introduced by adding the calculated amount of a sterile solution containing 20 per cent of pepton and 10 per cent. of $\mathrm{NaCl}, \mathrm{i}$. e., 5 c.c. per 100 c.c. of water. It should then be placed in a large flask, so as to have as large a surface as possible, and allowed to develop at $37^{\circ}$. The subsequent examinations are the same as those outlined above.

It must not be expected that the cholera vibrio which has been isolated will agree in every respect with the classical description of this organism. On the contrary, varieties must be expected inasmuch as pleomorphism is more marked in the case of the cholera germ than in any other known. species. Usually it is a short, thick, bent rod, but it is possible to have long, slender, almost straight varieties. Usually it possesses but one whip, but some have been shown to possess as many as four flagella. Moreover, although it is usually exceedingly motile, varieties may be found that are motionless. Some varieties will coagulate milk, others will not. Again, as a rule, the liquefaction of gelatin is slow, whereas some liquefy this medium very rapidly. The indol reaction and virulence; which are especially relied upon in an identification, are likewise subject to extreme variation. Pfeiffer's phenomenon is described in Chapter XIV. It affords as good a means of differentiation as any known procedure.

The cholera vibrio may retain its vitality in water for a considerable period. It has been kept in sterile tap-water for more than a year. It dies out rapidly in water which is kept at or near the freezing-point. Certain varieties, however, can resist actual freezing for many days. As a rule, 
they will live much longer in water having the ordinary room temperature of about $20^{\circ}$. The interesting studies of Hankin have shown that the water of certain rivers in India will destroy the cholera germ in 3 hours; whereas, if the water is previously boiled it will have no such effect. Similar germicidal substances may be present at times in the water of other localities and thus explain, at least in part, the so-called local immunity.

\section{Water Analysis.}

A bacteriological analysis of water consists: (1) in the determination of the number of bacteria; (2) the identification of the several species; (3) the recognition of the pathogenic bacteria present.

Number of bacteria. - The water to be examined should be received in a sterilized bottle or flask and thoroughly protected against subsequent contamination. Furthermore, in view of the rapid multiplication of bacteria, a given sample of water should be examined as soon as possible after collection. The method commonly employed in the determination of the number of bacteria present is as follows:

Several 1 c.c. pipettes, graduated in $\frac{1}{10}$ c.c., are placed in a pipette box and sterilized in the dry-heat oven in the usual way. 3 gelatin tubes are then liquefied and marked. By means of a sterilized, cooled pipette 1 c.c. of the water is transferred into tube No. 1. In like manner $\frac{1}{2}$ c.c. and 1 drop are placed into tubes 2 and 3, respectively. The contents of the tubes are gently agitated to secure complete mixture. The gelatin is then poured on sterilized glass plates or into Petri dishes, observing the usual precautions in making plate cultures ( $\mathrm{p}: 175$ ). The gelatin plates thus obtained are set aside for two or three days at $18-20^{\circ}$ and and the colonies which develop are then counted. 
Inasmuch as the bacteria are liable to multiply rapidly, especially if the water is taken from a cool source and is then kept at ordinary temperature, it is advisable to plate the water at the time it is collected. Under these circumstances, instead of plates or Petri dishes, flat flasks or bottles can be used. These contain the requisite amount of sterile gelatin which is inoculated with the water as soon as it is drawn. The flask is placed on its side, and, when the gelatin solidifies, it can be taken back to the laboratory.

If only a small number of colonies are present they can be counted with the unaided eye, but when, as it frequently happens, the number is very large, it is desirable to make use of a counting apparatus. Fig. 57 shows the counting apparatus of Wolffhügel, which is usually employed when ordinary glass plates are used. The gelatin plate, on which the colonies are to be counted, is placed on the black glass base and covered with a glass plate ruled into squares. The number of colonies under each square can thus be easily determined. When possible, the number of colonies under each square should actually be counted. As a rule, however, it is customary to count the number of colonies found under each of 6,8 or 10 squares selected at random from over the surface of the plate. The average number present in one square is then ascertained.

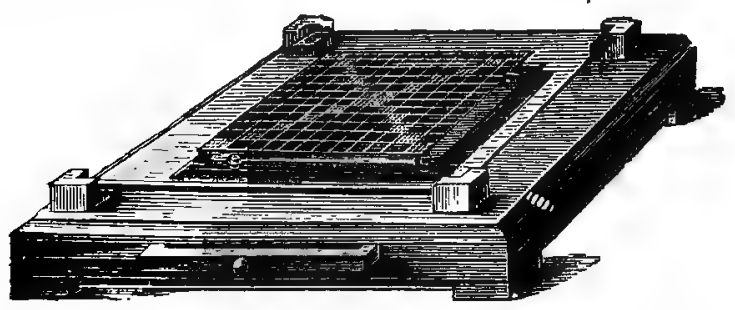

F1G. 57. Wolffhügel's apparatus for counting colonies.

The total number of colonies on the plate is found by determining the number of square centimeters which the gelatin on the plate covers, and multiplying this figure by the average number of colonies per square. Since each colony 
is derived from a single cell this figure then represents the number of bacteria present on the plate examined. If this plate is made from the tube to which 1 c.c. of water was added then the results are expressed, at once, as so many bacteria per c.c.

In case the plate is made with the gelatin that received $\frac{1}{2}$ c.c. of water the result is multiplied by 2 . In order to express the results obtained with the plate containing 1 drop of water, it is necessary to know how many drops the particular pipette employed will discharge from 1 c.c. of water. Drops vary a great deal in size but, on an average, 1 c.c. will yield 20 drops. As indicated above, the results should always be expressed as so many bacteria per c.c.

Wolffhügel's apparatus can also be employed to count the number of colonies in a Petri dish. The latter, if possible, should be inverted and the ruled plate then brought into contact with the bottom of the dish. The average number of colonies per square are determined as above. Since each square is actually $1 \mathrm{~cm}$. square, it is necessary now to determine the number of square centimeters covered by the gelatin in the dish. The area of a circle is $\pi R^{7}$. Hence, on multiplying the square of the radius by 3.1416 we obtain the area of the circle expressed in square $\mathrm{cm}$. This, multiplied by the average number of colonies per square, will give the number of colonies on the plate.

Several modifications of the above apparatus have been devised especially for use in connection with Petri dishes. That of Lafar (Fig. 58) is wideiy used. It consists of 5 concentric rings which are divided by 18 radii. Each of the several spaces, thus resulting, has an area of $1 \mathrm{sq}$. cm. Three of the sectors are still further subdivided in order to facilitate the counting of colonies when very numerous. The Petri dish, unlike the ordinary.gelatin plate, is not strictly flat. The center is almost invariably slightly raised, and, as a result, the medium over the center will be thinner than that near the edge. Obviously, the number of colonies in a square $\mathrm{cm}$. over the center will be considerably less than in one farther removed from this point. It is, therefore, not advisable to count at random the number of colonies in a square cm., as in the case of the Wolffhügel apparatus. The counting should be done by sectors. The lines are etched on a glass plate which is fixed in a brass collar. The bottom of the Petri 
dish, the diameter of which must not exceed $9.5 \mathrm{~cm}$., is placed in this collar and is then wedged, so as to be immovable. A somewhat similar counter (Park or Jeffer) ruled on paper can be obtained at very little expense, and is to be preferred to the glass plate of Lafar.

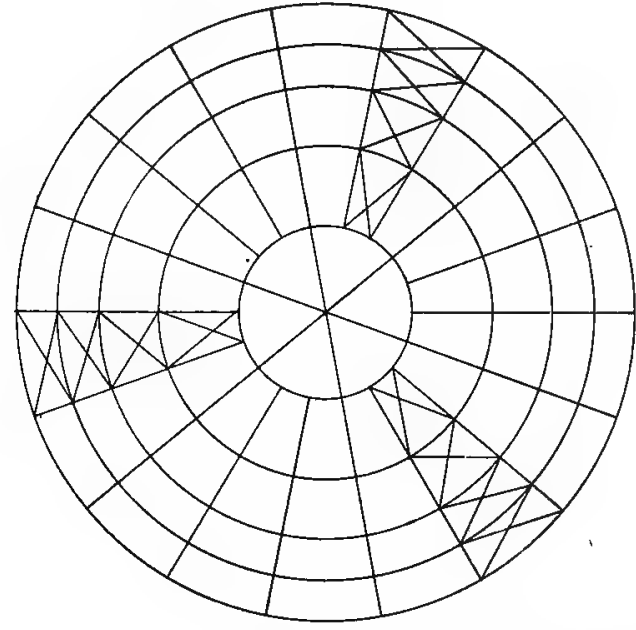

$a$

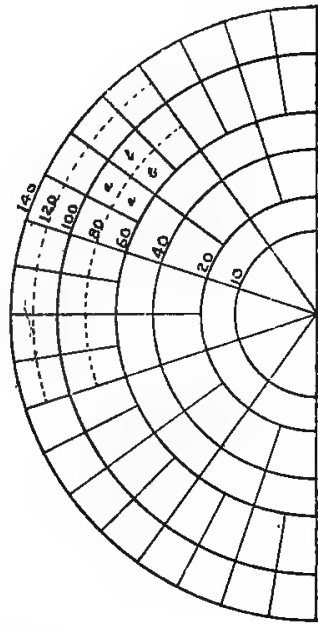

b

FIG. 58. $a$-Lafar's counter; $b$-Jeffer's modification. .

It is sometimes desirable to have an approximate idea of the number of colonies on a Petri dish, when their number is so great as to render the preceding method impracticable. In such cases Buchner, Neisser and others determine the average number of colonies present in the field of a microscope. A low power objective (No. 3 with No. I ocular) should be used for this purpose. The diameter of the microscopic field must be known. This can be ascertained by means of a stage micrometer (p. 127) or in a manner similar to that employed in the measurement of objects (p. 128). The field is projected on the table and the diameter measured. This divided by the magnifying power employed will give the diameter of the actual field under the microscope. Since circles are to each other as the squares of their radii, the area of the large circle or Petri disl can be easily obtained. Thus, if the radius of the microscopic field is . 65 mm.; that of the Petri dish $46 \mathrm{~mm}$.: and the average number of colonies in the microscopic field is 76 ; then,

$$
\mathrm{r}^{2}: \mathrm{R}^{2}:: 76: \mathrm{x}
$$$$
.4225: 2116:: 76: x \quad x=380,000 \text { colonies. }
$$ 
The number of colonies in 30 fields should be counted in order to obtain a good average number. With 1,500 or more colonies on a plate it is preferable to count with a microscope. This is especially true in water examinations and the like where different species are present. some of which grow rapidly and form large colonies whereas others grow slowly and give rise to very small ones. A cross-wire ocular micrometer should be used when the number of colonies in a field is large. The number of colonies, as calculated for the whole plate, is approximate but, if the work is properly done, the error need not exceed 12 or 15 per cent.

In case the number of bacteria is exceedingly great, the ThomaZeiss apparatus for counting blood corpuscles can be used to determine the number of bacteria present. The result thus obtained will be higher than that obtained by plate cultivation.

To obtain accurate results, however, it would be necessary to dilute a given volume of the water with a known volume of sterile water and then plate portions of this mixture. Thus, 1 c.c. of the water might be added to 99 c.c. of sterile water, and portions of $1,0.5,0.25$ and 0.1 c.c. of this diluted material could then be plated in the manner described.

The number of colonies counted on a plate are taken to represent the number of bacteria present in the water. In reality, this represents the minimum and not the actual number of bacteria present. A colony may be derived from several cells. Thus, a bacillus growing in pairs or in short threads, or a diplococcus or streptococcus may be the starting point of the colony. Again, the conditions of cultivation may not be such as to cause the development of all the bacteria that may be present. As pointed out, heretofore, the reaction and composition of the gelatin, as well as the prevailing temperature will influence the number of colonies that develop. Some bacteria may be present that will develop only at the temperature of the body. Moreover, the method takes into account only the aerobic bacteria. The anaerobic bacteria present will not develop. Consequently, the number of bacteria present in a water, as ascertained by the method given; is merely an approximate numper and serves to roughly indicate the amount of organic matter present. 
The more organic matter held in solution in a water, the more suitable it is, as a culture medium for bacteria. Such a water will not only permit the multiplication of common bacteria but will also favor any pathogenic germ, like the typhoid bacillus, if it should chance to be introduced. The same interpretation is to be given to a large number of bacteria in a water as is given to a large amount of organic matter determined by chemical analysis.

The counting of bacteria possesses especial-value in controlling, from time to time, the purity of a water-supply. A large and persistent increase in the number of bacteria should lead to an investigation of the cause. Where the water-supply is filtered, the daily counting of the bacteria in the filtered water will give the best indications as to the proper working of the plant.

The number and kind of species. - Apart from the recognition of specific, pathogenic bacteria very little need be said regarding the common water bacteria present. 'In general, a water should contain but a few, different species. When, for instance, 10 or more different species are present; especially if each is represented by an appreciable number, it would serve to indicate that the water contains considerable organic matter and is, therefore, a good medium for the growth of bacteria. It cannot be said to prove the existence of pollution, but it does show that there are conditions favorable to bacterial growth, and hence, favorable to pathogenic bacteria should they be introduced.

Considerable scientific interest is attached to the study of the different species of water bacteria, but apart from this they require no attention in the ordinary routine of water analysis. The various characteristics of a given species may be determined by making a microscopical and cultural study of the organism in accordance with the methods of study pursued heretofore. 
The detection of the typhoid fever and cholera bacilli has been discussed in the preceding pages and need, therefore, receive no further attention at this place. In addition to these organisms, the water may contain bacteria which are highly pathogenic and these, consequently, deserve especial consideration.

Pathogenic bacteria.-The chief object of the bacteriological examination of water is to determine the presence or absence of pathogenic or toxicogenic bacteria. In the method as ordinarily carried out, this is done by recognizing the colony of the specific organism sought for. When the pathogenic bacteria, as the cholera or typhoid fever bacillus for example, are present in large numbers, and this is very rarely the case, the identification can perhaps be easily done. On the other hand a few pathogenic bacteria in the presence of a large number of saprophytic organisms can be easily overlooked, and in such cases their recognition becomes well-nigh impossible. In view of these facts the following method was devised by Vaughan, and has been used in this laboratory since 1888. It is based upon the fact that a large number of the bacteria present in water are common saprophytes. which grow at the ordinary temperature, cannot grow at the temperatare of the body, and cannot, therefore, produce toxic or pathogenic effects. The bacteria which can develop at the temperature of the body may, or may not, be pathogenic. This can only be decided by an animal experiment. The method employed is as follows:

By means of a sterile pipette 1 c.c., 0.5 c.c. and 1 drop of the water are added, respectively, to each of three tubes of bouillon. These are set aside in the incubator at $39^{\circ}$ for 24 hours. If no growth occurs at this temperature it is at once sufficient evidence that the water is free from diseaseproducing organisms. On the other hand, if a growth does develop, injections of 1 c.c. of the culture are made intra- 
peritoneally into white rats or guinea-pigs by means of a sterile syringe. The recovery of the animal indicates the absence of pathogenic bacteria.

If death occurs it may be due to the typhoid fever bacillus, but as a rule, it is due to other pathogenic bacteria. It is necessary, therefore, to identify, if possible, the noxious organism. For this purpose, gelatin and agar plate cultivations are made from the heart blood. To still further test the pathogenic action of the organism 1 c.c. of a pure culture in bouillon is injected subcutaneously into guineapigs. The colon bacillus is not fatal under these conditions. If death does result it is frequently due to typhoidlike bacteria and the presence of such organisms should at once condemn the water. The addition of even a fraction of a drop of a virulent bouillon typhoid culture to a liter of water can be detected in this way.

Aerogenic bacteria, such as the colon bacillus, can be readily detected by employing a fermentation tube. Several forms have been devised but that of Einhorn will be found very convenient. The tube is filled with glucose bouillon, sterilized and inoculated with the suspected water. If the colon bacillus or other aerogenic organism is present, gas will be given off and will accumulate in the closed tube. The amount of carbonic acid in this gas can be roughly determined by filling the tube with 2 per cent. $\mathrm{NaOH}$. On carefully shaking the contents of the tube, the gas is brought into contact with the alkali. The difference in the volume of the gas, after absorption, is due to carbonic acid. The residual gas can be tested, qualitatively, for hydrogen. For this purpose the tube is partially inverted to allow the gas to pass into the bulb portion. A lighted match introduced into the tube will cause a slight explosion (Smith).

The bacteriological examination of snow or ice is of scientific and, at times, of practical interest. The material is melted in a sterile dish or flask and the water, thus obtained, is examined as above.

The air contains a large number of bacteria as dry, 'finely divided, suspended matter or dust. The precipita tion of rain or snow mechanically drags down a considera- 
ble number of these bacteria. Consequently, the air is purified to a marked extent by washing, as it were, with rain or snow.

The melted water from freshly fallen snow, collected at the ordinary low altitude, may contain from a few to as many as 500 bacteria per c.c. In the higher altitudes, the air contains only a few organisms and hence the precipitation in such places will contain a smaller number than in the previous case. The snow deposited at an altitude of 6,000 feet is not sterile, but very nearly so. Usually less than five bacteria per c.c. of melted snow are found. The rain-water at Paris has been found to contain from 5 to 20 bacteria per c.c.

It follows therefore that all surface waters, beginning even with the glacier brook, will contain bacteria. As the temperature of the water and the amount of organic matter increases, multiplication of the bacteria will take place. The number is increased by contact with the soil, dustladen winds and above all by animal excreta and wastematter.

The lakes in mountainous countries, as in Switzerland, because of their high altitude and the source of their water contain relatively very few bacteria. The surface water of these lakes usually contains less than 50 and only occasionally over 100 bacteria per c.c. Water, however, taken at some depth below the surface of these lakes may contain as many as 600 bacteria per c.c. This is undoubtedly due to a gradual sedimentation of the suspended organisms.

In some lakes, especially at low altitudes, this is not always the case and, indeed, the conditions may be reversed. Thus, the surface water may contain thousands of bacteria per c.c., whereas that near the bottom may contain but a few hundred. This difference may be due to the fact that these lakes are fed by deep springs; and, hence, while the water at the bottom is very cold, that on the surface is warmed by the sun and offers conditions which are favor- 
able to the growth of bacteria. In a given time, a much larger number of bacteria will be formed on the surface by multiplication then will be deposited by sedimentation. Moreover, the currents of the cold spring-water from be- . low will tend to keep the suspended matter in the surface layers.

The mountain lakes, on the other hand, are primarily fed by the cold, crystal-clear water derived from glacier streams. The water, whether on or below the surface, has a minimum low temperature, and, moreover, the rapid onward and downward flow prevents the warming of that on the surface. Sedimentation in such cases may occur, whereas the reverse, so far as numbers are concerned, will be met with at lower altitudes.

River waters, according to the conditions mentioned above, will necessarily vary greatly in the number of bacteria which they contain. Thus, the river Seine as it enters Paris has only about 300 bacteria in a c.c., whereas when it reaches its suburb $\mathrm{St}$. Denis, after receiving the sewage of Paris, it contains 200,000 per c.c. This number in itself is small owing to the rapid flow of the water and the consequent enormous dilution of the sewage. A low altitude, warm climate, abundance of organic matter and a slow current will necessarily favor the multiplication of bacteria in such river-water. The number of bacteria in river-water under these conditions may easily rise to 100,000 bacteria or more per c.c. The water of rapidly flowing rivers may, as a rule, be said to contain less than 500 bacteria in a c.c.

The ice formed on rivers and lakes will necessarily contain, like the water itself, a variable number of bacteria. The surface "snow-ice" will always contain a larger number than what is found in the clear ice. Thus, Prudden found the clear ice obtained from the Hudson river, a few miles below Albany, to yield 398 bacteria; whereas, the snow-ice gave 9,187 bacteria per c.c. of the water obtained 
by melting the ice. The ice supplied in cities, when melted; may contain as many as 25,000 bacteria per c.c. of water.

The rain-water and snow bring down from the air a large number of organisms. The water as it penetrates the ground is filtered, and, in this way, all the bacteria are retained by the upper layer of the soil. It follows, therefore, that water coming from the deeper layers of the earth is practically germ-free. This is often the case with springwater. As a rule, however, a small number of bacteria from the surface soil re-enter the water as it reaches the surface. Hence, spring-water usually contains less than 50 bacteria per c.c.

The water of artesian or tubular wells, for reasons just given, will likewise be free from bacteria. A small number of these are usually present, but this is due to contamination at or near the surface.

Ordinary wells, as might be expected, give the greatest known variation in the number of bacteria. The well water may contain practically no bacteria and on the other hand they may be almost innumerable. Thus, a number of wells have been found to contain as many as 800,000 bacteria per c.c. As a rule, a well-water, especially when it is very cold, will contain about 1,000 bacteria per c.c.

In sea-water, there is less variation in the number and kind of bacteria present, as well as in their distribution from the shore or from the bottom than is usually met with in ordinary waters. Russell has shown that the ocean surface watter contains from a few to as many as 120 bacteria per c.c. This number, however, may at times be considerably increased and may even reach 28,000 . In the deeper layers of the water they are no more abundant than on the surface. The slime at the bottom of the sea, at Naples, contained about 300,000 while at Wood's Holl only about 17,000 bacteria were present in.a c.c.' The enormous difference between the number of bacteria in the slime and in the 
overlying water is largely due to the fact that certain bacteria can live and multiply in the slime. Fully one-third of the bacteria present in the slime belonged to three species which were met with only in this material. Certain species of slime bacteria found at Naples at a depth of 3,500 feet were also met with at Wood's Holl, near the coast as well as at a distance of 100 miles. It is of interest to note that Fischer obtained no bacteria from slime gathered at a depth of 1-3 miles.

Sewage water, necessarily, is very rich in bacteria. Usually. a drop will be found to contain several hundred thousand. The sewage water in large cities like Paris and London is known to contain as many as 6 or 8 millions of bacteria per c.c. The water in the public washing stations which float in the Seine at Paris was found to contain from 12 to 40 millions of bacteria in a c.c.

\section{Soil.}

The upper layers of the soil contain enormous numbers tof bacteria which play a most important part in the economy of nature. As indicated in Chapter V (p. 112), the dead animal and vegetable matter deposited upon the surface of the earth is sooner or later broken up into the simplest of inorganic compounds,-carbonic acid, water, ammonia, nitrous and nitric acids, hydrogen sulphide, etc. This is done through the agency of the bacteria in the soil. Without their presence and activity, the dead matter would remain as such, and would accumulate as layer on layer. The nitrogen of the protein matter which is contained in the protoplasm of animal and plant cells is derrived from the in organic nitrogen of the soil. Were it not for the bacterial activity carried on in the soil this supply of inorganic nitrogen would in time become exhausted, and, as pointed out by Pasteur, life would soon cease to exist. The dead plant and 
animal, composed of the most complex chemical compounds, serve as food for this microscopic world which in turn converts the elements present into compounds which are suitable for the maintenance, of higher plant, and thus of higher animal life.

The bacterial changes going on in the soil are usually designated as those of fermentation or putrefaction. Either term implies the tearing down by bacteria or other organisms, of complex matter and transforming this into simpler forms. While a small number of bacteria may be considered as the most relentless foes of man, animals and even of plants, yet the vast army of these organisms constitute man's best friend.

The changes induced by bacteria, as usually observed, are analytic or reducing in character. Some of the organisms in the soil, however, give rise to important oxidation changes, such as is seen in the conversion of ammonia into nitrous and nitric acids. The so-called nitrifying bacteria are found widely distributed in the soil and in water. Under certain special conditions their action is so pronounced as to give rise to vast quantities of their characteristic product. The salt-peter of India or potassium nitrate, and the Chili salt-peter or sodium nitrate, as indicated heretofore, are most valuable commercial products which result from the action of certain bacteria upon animal excreta.

Another interesting group of soil bacteria, already referred to ( $p .110$ ), is met with in the characteristic nodules on the roots of leguminous plants. These bacteria have undoubtedly the power of assimilating the free nitrogen of the air and of transmitting it to the growing plant. Strange to say, the higher plant in this case is practically dependent upon these parasitic bacteria for its existence. It may grow in sterile soil but the growth in that case is poor and dwarfed, and presents a striking contrast to theplant growing in soil to which these organisms have been. added. 
- The soil may be considered as the natural habitat of certain pathogenic bacteria, notably, -tetanus, malignant edema, anthrax, pus-producing cocci and symptomatic anthrax. The latter has 'not been isolated from the soil but the others have been repeatedly found there. This is true especially of tetanus and of malignant edema which seem to be distributed in the soil over the entire surface of the globe.

Owing to the enormous numbers of common saprophytic bacteria, the tetanus or malignant edema bacilli cannot be isolated direct from the soil, by the ordinary plate method. It is necessary to resort to an animal experiment in order to effect their isolation. For this purpose, an incision is made through the skin of a rabbit or guineapig and a small pocket is made into the subcutaneous tissue (see p. 262). A small amount of the suspected soil is then introduced into this pouch. Most of the common bacteria are unable to grow in the body and are soon destroyed. The tetanus or malignant edema germ, if present, is favored by these saprophytic bacteria and is thus enabled to multiply and to produce the poison which soon destroys the animal. From the local abscess or from the tissues and serous surfaces of the animal, anaerobic cultures (p. 311) can then be made and the organism isolated in pure culture.

The above method is usually employed when attempting to isolate the organisms from the pus of a wound in a case of the disease. The direct isolation of the tetanus bacillus by the plate method was accomplished once, in this laboratory, from a case which originated by infection from a tooth.

The typhoid bacillus, as mentioned on p. 427 , has been isolated from the soil. The cholera vibrio may at times occur in the soil, but as yet it has not been found there. A non-virulent and even a virulent pest bacillus has been found in the earth.

Some idea of the enormous number of bacteria present may be given when it is said that 1 c.c. of the surface soil usually contains several hundred thousand bacteria. Indeed some observers have reported as many as 50 to 80 millions of these organisms.

The rain and snow bring down a great many bacteria from the air but, as soon as the soil dries and becomes pul- 
verized to dust, these and 'many others return to the atmosphere as a result of the action of wind or of other agencies. The bacteria brought down from the air do not penetrate the deeper layers of the soil. They are retained in the surface layers inasmuch as the earth is a good mechanical filter. For this reason, the number of bacteria in the earth diminishes with the depth. At a depth of about 6 feet the number of bacteria has greatly decreased. Sometimes the soil will be sterile; at other times, only a few hundred bacteria will be present. At a depth of 9 to 12 feet the soil is practically sterile: Not only is it impossible for the bacteria to penetrate from the surface downwards, for any great distance, but it is also impossible for many organisms to survive for any length of time under such conditions. This fact was unconsciously recognized by man, ages ago. Thus, in times of epidemics, as that of the Black Plague in London, in 1665, bodies were ordered to be buried at a depth of not less than 6 feet.

Burial experiments made with cadavers of hogs and other animals, into which large quantities of pure cultures were injected or in which diseased tissue was placed, have shown that at a depth of 3 to $4 \frac{1}{2}$ feet many bacteria are destroyed within a month. This was the case with pure cultures of the typhoid, cholera and Friedländer germs. In a typhoid spleen the Eberth bacillus was found alive on the 96th day. The tubercle bacillus' was alive and virulent on the 95th day but was dead in 123 days. The tetanus spores died out between the 8th and 12th month, and anthrax spores remained virulent at the end of 11 months. The hog erysipelas bacillus was alive after 8 months. The bacillus of green pus and the Micrococcus tetragenus died between the 1st and 4th month. In only two instances could the pathogenic germ' be detected outside of the cadaver. The disease-producing organisms, present in a cadaver, are destroyed in time when buried in the soil. 
Moreover, the buried organism, owing to the filtering power of the soil, is not to be considered as a source of danger, especially if surrounded by dry earth.

Method of analysis. -The collection of samples of earth from various depths can be readily accomplished by means of Fraenkel's earth-borer. For each culture experiment a definite quantity of the soil should be weighred out, or a measured volume taken. The latter is the simpler procedure, and can be done with a small platinum spoon which has a capacity of $\frac{1}{\delta}$ c.c.

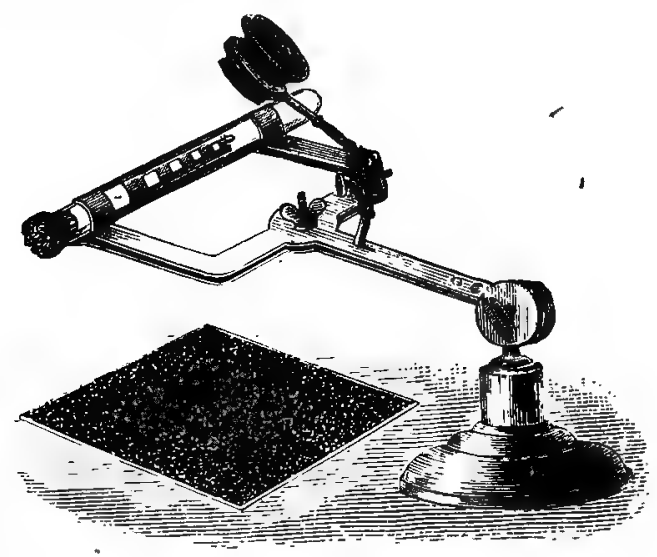

FIG, 59. Esmarch's apparatus for counting colonies in roll-tubes. -

With the above sterile instrument one spoonful of the earth is transferred to a tube of liquid gelatin. The contents of the tube are then mixed thoroughly, with a sterilized platinum wire, and an Esmarch roll-tube is made. The soil and the organisms present are thus brought into perfect contact with the gelatin, and after a lapse of a few days colonies develop.

The colonies can be readily counted, either direct, or by the aid of an Esmarch roll-tube counter. This apparatus is shown in Fig. 59. The roll-tube is placed in the holder and the average number of 
colonies in a square $\mathrm{cm}$. is ascertained in the same manner as described under water analysis. The area of the gelatin cylinder is then calculated from the formula $\pi \mathrm{D} a$; in which $\pi$ is $3.1416, \mathrm{D}$ the diameter of the tube and $a$ the length of the gelatin cylinder. The total number of colonies can then be determined. The kind of organ isms present-bacteria, moulds, etc.-can be determined by a study of the colonies, and by further culture and examination.

In this way it is easy to determine approximately the number and kind of organisms present. Unfortunately, this method is not adapted for the detection of anaerobic bacteria which are apparently widely distributed in the earth, and are represented by the well-known bacilli of tetanus, malignant edema and symptomatic anthrax. These have thus far been obtained from the soil only by the indirect method mentioned on p. 446 .

Air.

The bacteria present in the air are derived almost entirely from the soil. When the surface of the earth becomes dry, the fine particles of dust are readily taken up by gusts of wind and may then be carried upward to a considerable altitude. To a slight extent, germs may enter the air from the water. This can happen, however, only when the water is violently thrown into a spray as in the neighborhood of a water-fall or in the case of storm-lashed waves. The extremely fine particles of water are carried into the air and eventually may dry up leaving the solid organisms behind.

It should be clearly understood that germs do not and cannot enter the air of themselves. They must always be torn by force from the soil or from the water. Moreover, what is equally important, they are not removed from moist surfaces or from water by ordinary currents of air. The most pathogenic organism growing on the ordinary culture 
media cannot leave the moist surface: For the same rea: son, the breath of a person is practically free from bacteria; or, at all events, it does not take up and carry out the organisms that may be present in the air-passages. The breath of a consumptive, therefore, is free from the specific germ of this disease. It is only in case of violent expulsion of air, as in a fit of coughing, that particles of moisture with the contained organisms may be forcibly ejected from the air passages.

When air is passed over a moist surface, instead of taking up bacteria, it is usually deprived of those that are present. The suspended particles, whether mere grains of dust or the much smaller bacterial cells, when once brought into contact with a moist surface become fixed to that surface. Hence it is, that the farther one goes away from the shore the less numerous the air germs become. The air at sea, at some distance from the land, may be said to be practically free from organisms.

The suspended particles in the air are made up, in the first place, of the relatively heavy grains of dust. The surface of these dust particles is covered with such organisms as chanced to dry down. To a less extent the sun - motes can be considered as the carriers of bacteria. The free organism, either single or, more often in short threads or groups, constitutes the finest particle in the air.

The atmospheric germs, by no means, belong exclusively to the group of bacteria. While this form is quite common, yet the yeast is frequently met with and the spores of moulds are very abundant. Inasmuch as these several forms of living matter exist in the air as dried particles it is evident that they do not multiply. On the contrary, desiccation, when prolonged, tends to destroy many organisms and this unfavorable action is still further accentuated by the direct germicidal effect of sun-light. 
- The particles of dust and the free organisms suspended in the atmosphere are specifically heavier than the air and hence tend to settle. This sedimentation is very marked even in a few hours, in a room where currents of air are absent. To a certain extent this takes place in the open air, but the purification of the out-door atmosphere is chiefly accomplished by the precipitation of rain and snow, and by the washing of the air as it passes over the surface of lakes and seas. For the same reason expired air will contain but a very small fraction of the organisms present in that which was inspired. Thus, when the former contained 20,000 germs per cubic meter, the latter contained in a like volume only 40. It is evident, therefore, that air which contains the fewest organisms is to be met with at high altitudes, as on the tops of mountains and glaciers, and in mid-ocean.

The actual number of organisms present in the air is greatly over-estimated. This is indicated in the marked freedom from contamination by air germs; of the nutrient media, in ordinary routine bacteriological work. Naturally, the air of a recently swept room will contain more germs than that outside of the house. Likewise, the air of cities will be relatively. rich as compared with that of the open country. Moreover, the number of organisms in the air during the winter season will be less, nearly one-half, than in the spring or summer.

The following table will be of interest showing the :variation in the number of bacteria and moulds in different seasons and in country and city air. The figures represent the average numbers obtained by Miquel, who made monthly examinations extending over a period of 10 years. The results in the first half of the table were obtained at the Mont-souris observatory, situated in a park at the southern edge of Paris; whereas, the results in the other half of the table were obtained in the square before the City Hall, only about 2 miles distant from the former place. The figures 
give the number of organisms present in a cubic meter (1000 liters) of air.

\begin{tabular}{|c|c|c|c|c|}
\hline & \multicolumn{2}{|c|}{ MONT-SOURIS. } & \multicolumn{2}{|c|}{ CITY HALL. } \\
\hline & BACTERIA. & MOULDS. & BACTERIA. & MOULDS. \\
\hline 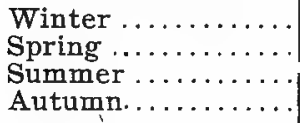 & $\begin{array}{l}170 \\
295 \\
345 \\
195\end{array}$ & $\begin{array}{l}145 \\
195 \\
245 \\
230\end{array}$ & $\begin{array}{l}4305 \\
8080 \\
9845 \\
5665\end{array}$ & $\begin{array}{l}1345 \\
2275 \\
2500 \\
2185\end{array}$ \\
\hline A verage........ & 250 & 205 & 6975 & 2705 \\
\hline
\end{tabular}

On an average, it may be said that the country air contains from 1 to 5 germs in 10 liters. Not infrequently the air of private rooms and hospitals, especially after sweeping, may contain 20 to 50 thousand organisms. In the absence of currents of air these suspended germs rapidly settle and are not removed by ordinary ventilation. Dry sweeping and dusting should, therefore, be avoided, especially 'in rooms which are occupied by persons afflicted with consumpton or with other infectious diseases.

The vast majority of the bacteria present in the air are harmless saprophytes. Pathogenic bacłeria are present, relatively, in very small numbers and hence their detection is extremely difficult. The pus-producing micrococci have been isolated direct from the air. The tubercle bacillus enters the body through the air and yet direct examinations, for the reasons given, have thus far been negative. It has been detected, however, in the dust of rooms,- - that is to say in the sediment deposited from the air.

Method of analysis. -It was pointed out in connection with the analysis of water (p. 437) that the number of bacteria present could not be determined absolutely. The same 
is true of the various methods employed in the determination of the number of atmospheric germs. If, for instance, all the organisms present in a given volume of air are transferred to water it does not follow that they will all develop upon the gelatin plate which is made from such water. Many of these may be anaerobic germs, others may require the temperature of the body, and again a large number may fail to grow because the medium is not a suitable one. Consequently, the results obtained in the examination of air are to be considered as merely relative in value.

The earliest method of examining air, that of Pasteur, consisted in breaking open, in the desired locality, a sterile flask from which the air had been previously expelled by boiling. The air rushing into the vacuum flask carried with it the suspended organisms which then developed in the nutrient medium present. This method demonstrated that atmospheric germs were not as numerous as had been supposed, and, furthermore, that they were not evenly distributed throughout the air. Koch endeavored to determine the number and kind of bacteria present in a given volume of air by making use of the gelatin plate method. Sterile gelatin, for example, was placed in an open Esmarch dish within a plugged glass cylinder of known volume. The apparatus was sterilized and then taken to the desired locality where the cotton plug was removed. The cylinder was now allowed to remain open for some minutes to allow the germ-laden air to enter. The plug was then replaced and the apparatus was set aside. The organisms present in this confined volume of air soon settled upon the surface of the gelatin where they developed forming colonies. The method, though simple, is extremely imperfect and fully as good results can be obtained by exposing an ordinary gelatin plate direct to the air for 10 to 30 minutes.

Very fair quantitative results can be obtained by means of Hesse's apparatus. This consists of a glass-tube $70 \mathrm{~cm}$. long and 3 or $4 \mathrm{~cm}$. in diameter. One end of the tube is closed with a stopper through which passes a short glass tube. This is connected by means of rubber tubing with an aspirating bottle of known volume. The other end of the tube is closed with two rubber caps, the inner one of which has a small central opening. The tube is first sterilized and then about 50 c.c. of gelatin are introduced. The gelatin is then solidified in a thin film over the inner wall of the tube thus making a large Esmarch roll-tube. The apparatus is placed in the room or locality 
where the air is to be examined; the outer cap is removed and the water: in the aspirating bottle is then allowed to escape. Any desired volume of air can thus be drawn through the tube. The bacteria present in the air, coming into contact with the gelatin, become fixed and after the tube is set aside they develop. The colonies can then be counted, and, inasmuch as the volume of air drawn through the apparatus is known, the average number of germs per liter of air can be ascertained.

A more exact method, requiring however very expensive apparatus, is that of Petri. In this method the air is

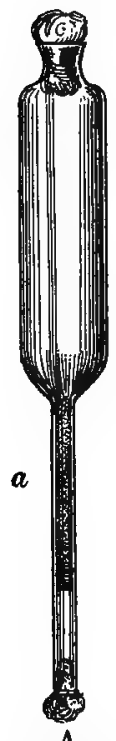

A

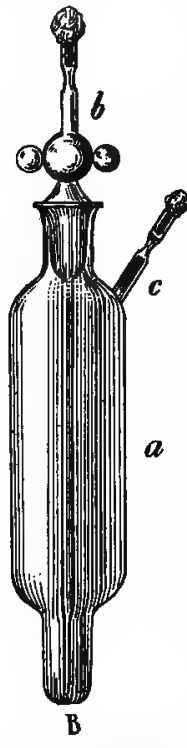

FIG. 6o. Apparatus for examination of air. $A-$ SedgwickTucker's; $B$-Straus-Wurtz's. drawn through a sterile sand filter Fig. $66 d$, p. 469: The suspended particles, dust and organisms, are held back by the well-packed, fine grained sand. The contents of the filter are then distributed into a number of Petri dishes, gelatin is added and after thorough mixing it is allowed to solidify. The colonies that now develop can be examined and counted with the same' ease as in water analysis. In this method it is possible for a large number of bacteria to adhere to a single grain of sand and when development takes place the result will be but one colony. The objection can be overcome, in part, by substituting a soluble compound for the sand. Thus; powdered sodium sulphate, or better, cane-sugar may be employed. Filters containing sugar have been employed by Miquel, and by Sedgwick and Tucker (Fig. $60 a$ ). On the addition of gelatin, the sugar dissolves leaving the germs present in a fine state of division. The number of colonies, thus obtained, represents quite closely the number of cells originally present in the air. 
A third method of procedure consists in aspirating the air through sterile water or gelatin. The bubbles of air are thus washed and the germs present are retained by the liquid. A very useful and cheap apparatus for this purpose is that employed by Straus and Wurtz, and is shown in Fig. $60 \mathrm{~b}$. It consists of a glass cylinder $a$ in the neck of which is fitted a ground pipette or tube $b$, the upper end of which is plugged with cotton.

The side-arm $c$ is provided with two cotton plugs one above and one below the middle constriction. The apparatus is sterilized in the dry-heat oven; 10 c.c. of gelatin are then introduced and a drop of sterile oil is added to prevent subsequent foaming. The plug is removed from the end of the pipette $b$ while the side-tube $c$ is connected with an aspirator. The air is drawn rapidly through the gela. tin, and, as a result, it is deprived of most of the suspended organisms. The remainder are held back by the cotton plug on the inside of the constriction in the side-tube $c$. At the close of the operation this plug is pushed down into the cylinder and thoroughly agitated with the gelatin in order to bring the adhering organisms into suspension. The gelatin can be solidified on the inside of the cylinder, thus forming an Esmarch roll-tube; or, definite portions of the gelatin ( 0.5 and 0.1 c.c.) can be transferred to gelatin tubes and Petri plates can then be made as in water analysis.

Laboratory work.-The student will examine two samples of water, one drawn from the tap and the other from a well. The number and kind of colonies are to be reported. A specimen of milk will also be plated and examined in the same manner as in water analysis. Esmarch roll-tubes will be made with three samples of soil. 
CHAPTER XIV.

SPECIAL METHODS OF WORK.

Pipettes.

Sterile, drawn-out tube pipettes, as employed by the Pasteur school, are invaluable in bacteriological work. They can be readily prepared and a good supply should be kept on hand. The method of preparing pipettes is as follows:

Glass-tubing having a diameter of about $8 \mathrm{~mm}$. is cut up into lengths of about $35 \mathrm{~cm}$. (14 in.). A slight constriction is made at a distance of about $6 \mathrm{~cm} .\left(2 \frac{1}{2} \mathrm{in}\right.$.) from each end. This is not necessary, but it serves to prevent the cotton plug from descending. Moreover, the tube can be readily sealed at this place whenever it is desirable to do so. The ends are then rounded in the blast-lamp. A piece of cotton is pushed into each end of the tube by means of a drawn-out piece of glass tubing. The tubes, thus equipped (Fig. 61 a)-are placed in a horizontal position in a dry-heat oven and sterilized (p. 160). The sterilized tubes should be kept in stock and from these the pipettes can be made in a few minutes whenever desíred.

To make the pipette proper, the plugged sterile tube $(a)$ is heated at the middle in a blast-lamp. The broad flame should be used in order to soften as long a piece of the tubing as possible $(3-5 \mathrm{~cm}$.). When the middle is thoroughly softened the two halves should be slowly drawn apart. A relatively wide, thick-walled capillary is thus obtained; whereas, if the tube is drawn out rapidly the resulting capillary will be very narrow and thin walled. The 
drawn-out portion should be about $40 \mathrm{~cm}$. (16 in.) long. This is then heated in the middle, and, as a result, two sealed pipettes are thus obtained (Fig. 61, $c$ or $d$ ).

Frequently, it is desirable to transfer a liquid or a suspension from one tube to another. For small quantities the ordinary pipette ( $c$ or $d$ ) can be used direct. For larger quantities a bulb should be blown into pipettes $c$ or $d$, thus giving pipette $d$. While it requires a great deal of practice to blow a perfect bulb, still a serviceable one can be made without muc difficulty.

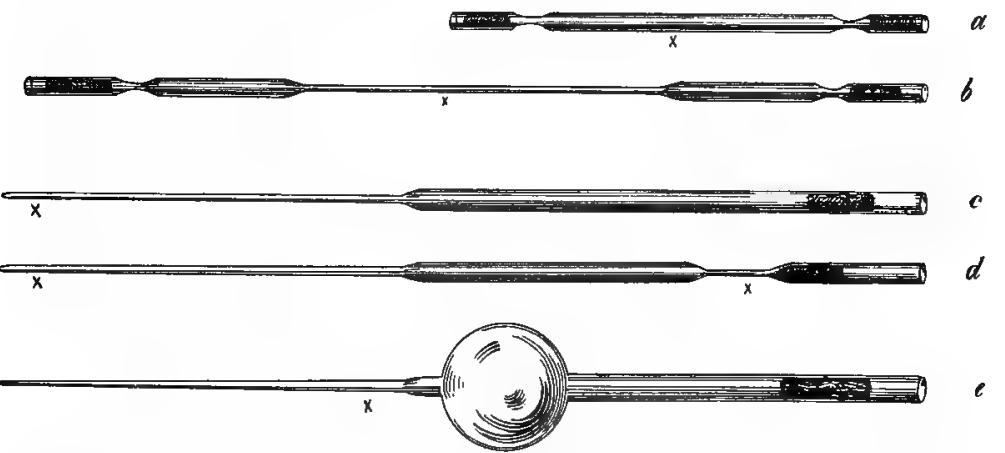

FIg. GI. Drawn-out tube pipettes of Pasteur. a-Plugged, sterile tube as kept in -stock; $b$-The same heated at $x$ in blast-lamp, drawn out; then sealed at $x ; c$ and $d$-completed pipettes; $e-$ The same with bulb.

A narrow flame of the blast-lamp is directed at the tube a short distance above the point where it begins to narrow. The tube is slowly turned until the glass softens; whereupon the ends are slightly pushed together so as to form a thick ring of glass. One or two additional rings are made in this way. A large flame is then turned on so as to melt the thickened zones of glass. When the glass is perfectly soft the end is brought rapidly to the mouth and the bulb is blown. The glass tuhe should be turned, slowly and steadily, while heating and also while the bulb. is being blown. The bulb pipettes can often be used to better advantage if the capillary is bent at right angles just below the bulb.

When a pipette is to be used the mouth end should be heated in the flame for a few moments. This is to prevent possible infection. 'The lower end of the capillary tube is then scratched with a file or glass-cutting knife and the sealed end removed. The end may be 
broken off by slightly bending it between the fingers. The open capillary end should now be passed several times through the flame in. order to sterilize the exterior. By blowing through the tube, directing the stream of air against the back of the hand, one can ascertain when the tube has cooled sufficiently for use. The pipette is then inserted into the test-tube or other container and the liquid is drawn up by suction. The end of the tube in the mouth is closed by the tongue or finger, and the pipette is then withdrawn. The liquid can now be transferred to another tube or to a series of tubes or flasks.

The pipette is very useful in removing the contents of a pus cavity in an animal, in collecting an exhdate (p. 277) or in drawing blood from the heart (p. 279). It can be used to remove liquid cultures or surface cultures from agar, potato, etc. In the latter case, sterile water or bouillon is transferred to the tube by means of the pipette. The growth is whipped up to make a fine suspension which then can be drawn up into the pipette. The pipette is extremely useful when it is desirable to inoculate a large number of tubes; in the tests for agglutination and in the preparation of sacs. It is even more useful than the plati. num wire.

The material can be kept in the tube by sealing the capillary end. 'If at the same time the tube is sealed below the cotton plug, the culture or material can thus be kept perfectly free from contamination or desiccation. It is frequently desirable to preserve the heart-blood of an animal, and thus keep the organism that may be present in its full virulence. This may be done in the manner described or by the following slightly modified procedure.

A pipette is heated about a half an inch beyond where it begins to narrow. When the glass has softened it is drawn out about $15 \mathrm{~cm}$. (6 in.). Fig. 62 a. shows such a pipette. The bulb and capillary is then filled to the $x$ mark according to the directions given above. The capillary is then sealed at the end and also at the $x$ mark. The growth or blood is thus sealed in a bulb tube about $15 \mathrm{~cm}$. 
long. The same pipette can be utilized for preparing a number of such sealed tubes.

In testing the action of moist heat on bacteria it is advisable to draw up the liquid into the capillary to a height of $8-10 \mathrm{~cm}$. $(3-4 \mathrm{in}$.) as shown in Fig. 62 c. The capillary in then sealed at the end and at $x$ just above the liquid. The used pipette can then be drawn out again as in Fig. $62 a$. By cutting off the tube above the bulb portion, the capillary portion can be filled and sealed in the manner just described. The same pipette can thus be used for the preparation of a large number of sealed capillary tubes. When it is desired to remove the liquid from the narrow, sealed, capillary tube, one end should be cut off and sterilized by flaming. This end is then introduced into the culture tube which is to be inoculated, while the sealed end is gradually brought into a fame. The vapor thus produced will drive out the bacterial liquid.
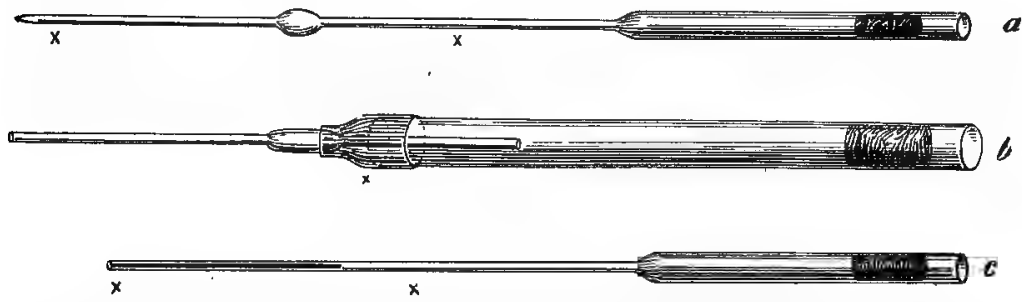

Fig. 62. Sealing of cultures in capillaries, a-Pipette $c$ Fig. 6r drawn out. When filled with liquid it is sealed at $x$. $b$-The method of removing the contents from a capillary bulb tube. A piece of rubber tubing $x$-slips over the end of the large sterile tube; $c-$ Filling and sealing a capillary for thermal death-point determination.

The removal of the liquid from the bulb tube can be accomplished in the manner indicated; or, by means of the arrangement shown in Fig. 62 b. One end of the narrow tube is opened and sterilized by flaming. The other end is then opened and inserted into a sterile glass-tube, the end of which is provided with a short piece of rubber tubing. By gently blowing into the tube the contents of the capillary can be expelled into a test-tube or other receiver.

The ordinary chemical, graduated pipettes ${ }^{1}$ are frequently made use of. The short $18 \mathrm{~cm} ., 1$ c.c. pipettes, as use $d$ in water analysis, are sterilized in a sheet-iron box

${ }^{1}$ A set of three pipettes of excellent construction, with a capapacity of $\frac{1}{T^{2}}$, rod and $\frac{1}{\text { rod }}$ c.c. respectively, can be obtained of Ruelle, Paris, 6 Rue Chouin. 
similar to that used for sterilizing plates. The larger pipettes must be protected in a different way against subsequent contamination from the air. The simplest procedure is to push a short cotton plug into the mouth end of the pipette. The delivery end is inserted through a cotton plug into a test-tube. The pipettes, thus protected, are then sterilized in the usual way.

\section{Drawing of Blood,}

In studying agglutination and in many other experi. ments it is necessary to draw a small quantity of blood from

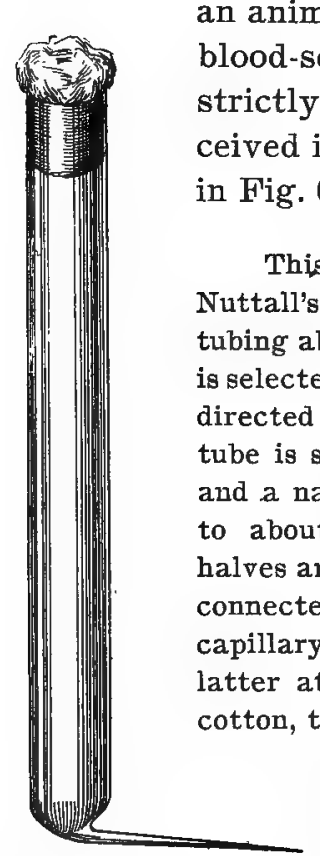

Fig. 63. Pipette for drawing blood to obtain sterile serum.

an animal. When it is desired to obtain sterile -serum the blood must be drawn under strictly aseptic conditions. It should be received in a sterile pipette similar to that shown

This pipette, which is a slight modification of Nuttall's, can be readily prepared. A piece of glass tubing about $2.5 \mathrm{~cm}$. in diameter and $50 \mathrm{~cm}$. in length is selected. A medium flame from a blast-lamp is directed against the middle of the tube. As the tube is slowly rotated and heated the glass softens and a narrow constriction results. When constricted to about one-third the original diameter, the two halves are drawn apart so that the parallel tubes are connected at an angle of about $120^{\circ}$ by the resulting capillary. They are finally separated by sealing the latter at the middle in a flame. After plugging with The capillary tip on each tube should not exceed 7 or $8 \mathrm{~cm}$. Moreover, it is important that the capillary should not be too narrow. The tip should be about 1.5 to $2 \mathrm{~mm}$. in diameter in order to obtain the best yield of blood.

In the absence of the wide glass-tubing indicated above, an excellent pipette can be made out of a large wide test-tube. The 
bottom of this and of a small test-tube should be heated in the blastlamp and then fused together. A narrow or medium flame should then be directed against the large test-tube at about 2 or $3 \mathrm{~cm}$. from the end. On slow rotation and careful heating a thickened constriction results. The two portions are then drawn apart and the capillary sealed as above.

The carotid artery of the anesthetized animal is exposed and a sterile silk thread is slipped under the vessel. A pair of pression forceps are applied to the artery as far up the neck as possible; or the artery may be tied in this place. A second pair are then applied about $3 \mathrm{~cm}$. below this point. The distended artery is grasped with an ordinary wide-tipped forceps, just below the upper clamp, and slightly stretched. It is then nicked with a pair of very fine scissors. After inserting the blades of a narrow-pointed pair of forceps the vessel can be distended so as to readily admit the introduction of the open end of a pipette. The end of the pipette, which should not be too narrow, is broken off, and flamed to fuse the sharp edges; when cool it is inserted as far as possible into the vessel. The ligature is then tied over the glass tip; or this may be held between the thumb and fore-finger. When the lower artery forceps are opened, the blood rapidly fills the sterile pipette. If necessary, suction can be applied to the end of the tube. When enough blood has been drawn the artery is again clamped, and the pipette is then removed and sealed in a blast-lamp. The artery should then be tied above and below the wound. After the blood-serum separates from the clot. it can be transferred to sterile tubes by means of a bulb pipette (Fig. 61 e).

When only a small quantity of blood is desired it can be obtained more easily from the jugular vein. Small bulb pipettes (Fig. $61 \mathrm{e}$ ) can be employed in the manner indicated above. Moreover, a syringe can be used to advantage in drawing blood from a vein. Several c.c. of blood can thus be drawn in a few moments from the external jugular or from the ear vein of a rabbit. 
Small quantities of human blood can be obtained in the following manner: A piece of rubber tubing is tied above the elbow in order to compress the surface veins. On strongly flexing the arm, the superficial veins on the extensor surface will stand out prominently. The median cephalic, or basilic veins can also be used. The surface of the skin over one of these large veins should be thoroughly washed and disinfected. 'The hypodermic syringe (2 c.c.), provided with a wide needle, is sterilized by boiling in water for 15 minutes. When cold, the needle is inserted into the vein without the slightest difficulty. The piston is then slowly withdrawn and the syringe fills with blood. As the needle is withdrawn the opening is closed with the finger and eventually an antiseptic compress and bandage is applied.

Scarcely any pain is experienced by the subject and the operation is borne a great deal better than if a lance were used. A quantity of sterile blood can thus be obtained in a few minutes.

In order to obtain serum for testing the agglutinating power, or for other purposes the blood is at once forced out into a centrifuge tube. It should be thoroughly whipped with a narrow glass rod and finally centrifugated: The clear serum is then taken up in a pipette and tested.

The blood of the horse can be obtained readily, and in large quantity, by introducing a trochar into the jugular vein. This method of bleeding is followed in the preparation of antitoxin. The operation is carried out under aseptic conditions and hence the blood-serum; thus obtained, is sterile. The method of procedure is essentially that given on p. 268. The trochar is connected by means of a sterile rubber tube with a short glass tube. This is passed into the sterile cylinder or battery jar. The latter should be covered with a double layer of paper before sterilization. The outer paper is then removed and thè glass tube is punched through the inner paper cover. From 4 to 6 liters of blood can be drawn from a horse at one bleeding. 
Oxalate blood or plasma.-As is well known, the coagulation of blood can be prevented by the addition of a small quantity of potassium oxalate. The author has utilized this fact in order to obtain fluid blood or unaltered plasma for culture purposes.

A 6 per cent. potassium oxalate solution is prepared. The capacity of the pipette (Fig. 63) is ascertained and the necessary amount of oxalate solution is added so that the collected blood will have $0.1-0.2$ per cent. of potassium oxalate. Thus, if the pipette can hold 25 c.c. of blood, then 0.8 c.c. of the oxalate solution should be added in order that the resulting mixture shall contain 0.2 per cent. of oxalate. The tube and contents are then sterilized in the autoclave.

Befbre inserting the tip of the pipette into the artery, the oxalate solution should be rolled so as to moisten the inner wall of the pipette. This is then filled with blood and sealed according to the directions given above.

'The pipettes can be placed in an ice-chest, in which case the corpuscles will subside in 2 or 3 days, and, as a result, a perfectly clear plasma can be obtained. This may be transferred by means of a bulb pipette to sterile tubes. It can be used as such, or can be solidified as in the case of blood-serum.

Similarly, the fluid blood itself may be placed in tubes and coagulated at about $70^{\circ}$. The addition of 1 part of oxalate blood to 2 parts of melted agar at $50^{\circ}$ yields a bright red medium which can be solidified in an inclined position. This blood-agar is an excellent medium for streptococci and other organisms.

\section{Blood-Serum.}

The serum from the blood of the ox is frequently employed for cultivation purposes. The blood as it is ordinarily collected in the slaughter house is not sterile. If, however, it is received into sterile vessels and kept covered at a low temperature the few organisms that are present will not multiply, and hence, a large proportion of the tubes filled with such serum will remain sterile.

The blood should be received in battery jars which have been covered with paper and sterilized." When the blood has formed a solid clot, it is transferred to the ice-chest. 
A considerable amount of serum separates in from 24-48 hours. It should be drawn off by means of a volume pipette and, if not perfectly clear, it should be set aside again for a day to allow the corpuscles to settle. The serum thus obtained can be sterilized by one of the follow. ing methods.

Sterilization of serum by filtration.-Clear, thoroughly sedimented blood-serum is very desirable in order to obtain rapid filtration. The serum is filtered through an unglazed, porcelain Pasteur-Chamberland, or through a Berkefeld infusorial earth bougie. The Pasteur filters differ greatly in their flow and this is due chiefly to the variable thickness of the wall. In most bougies the wall has a thickness of 2.5 to $2.8 \mathrm{~mm}$. and through such, blood-serum can be filtered with great difficulty. Occasionally a bougie is met with in which the wall is less than $2 \mathrm{~mm}$. thick, and through these the blood-serum can be filtered with ease.

The Berkefeld filter is considerably more porous than that of Pasteur and hence can be used to advantage in the filtration of blood-serum. A pressure of at least $75 \mathrm{lbs}$. to the square inch should be employed. 'Obviously, the less porous the filter the higher the pressure that must be employed. When a liquid contains protein substances it should always be filtered under as high a pressure as possible.

The filtering apparatus (Fig. 66, p. 469), permits the use of either the Pasteur or the Berkefeld filter, and can be used with or without pressure. The filters are sterilized according to the directions given. The filtered serum may be received in a sterile Erlenmeyer vacuum flask; or, in a globe receiver such as is shown in Fig. $68 \mathrm{~B}$. In the latter case the filtrate can be transferred to tubes or flasks, with a minimum risk of contamination.

Fractional sterilization of serum at $58^{\circ}$. -Blood-serum coagulates at about $70^{\circ}$ to an opaque, white mass. When, therefore, it is desired to obtain a sterile, liquid serum, or a solid, transparent serum it is necessary to resort, either to the procedure just given, or to fractional sterilization, at a 
lower temperature than that mentioned. A temperature of $58^{\circ}$ maintained for an hour will destroy, as a rule, actively growing bacteria. The spores, of course, are not affected by this heat. They must be given an opportunity to germinate, in which case the resistant spore is converted into a relatively weak, vegetating form. When this takes place the latter promptly yields to the temperature employed. It should be remembered, however, that there are bacteria which, far from being killed, actually thrive at this tem. perature (p. 72). If these should happen to be present it will be impossible to sterilize the serum by this method.

The sterilization of the serum tubes can best be accomplished by the use of a Roux water-bath. This exceedingly useful apparatus is shown in Fig. 64, and as shown, it is provided with a Roux metallic thermo-regulator (R). A wire basket (D) is immersed in the water and is provided with an adjustable bottom (E). In this way the tubes can be immersed in water to any desirable depth. The cover (C) is double-walled and filled with water. Through the opening on the top a thermometer is inserted into the liquid.

The wire basket, as ordinarily supplied with this apparatus, is provided with a movable bottom which is clamped to the central tube. The latter takes up desirable space and it is advisable, therefore, to alter the basket so that the bottom can be clamped, on the under side, to runners fastened on the inner surface of the wire basket. The thermometer and mercury thermo-regulator (Fig. 37, p. 246), can be suspended to advantage in the side compartment which is intended for the metallic regulator.

The serum tubes are sterilized in this apparatus by heating at $58^{\circ}$ for one hour on each of six consecutive days. The interval between heating may be shortened to 10 hours if the tubes, after each heating, are placed for $2-3$ hours at $37^{\circ}$. This will assist the germination of the spores present. The tubes are then kept at the ordinary room temperature for about eight hours, after which they are submitted again to a heat of $58^{\circ}$. 
A temperature of $58^{\circ}$, maintained continuously for 6-8 hours, will cause the coagulation of blood-serum. Löfler's serum and glycerin serum are not as readily affected.

Fractional sterilization of serum at $75-80^{\circ}$.-At this temperature ordinary blood-serum, and even Loffler's serum will

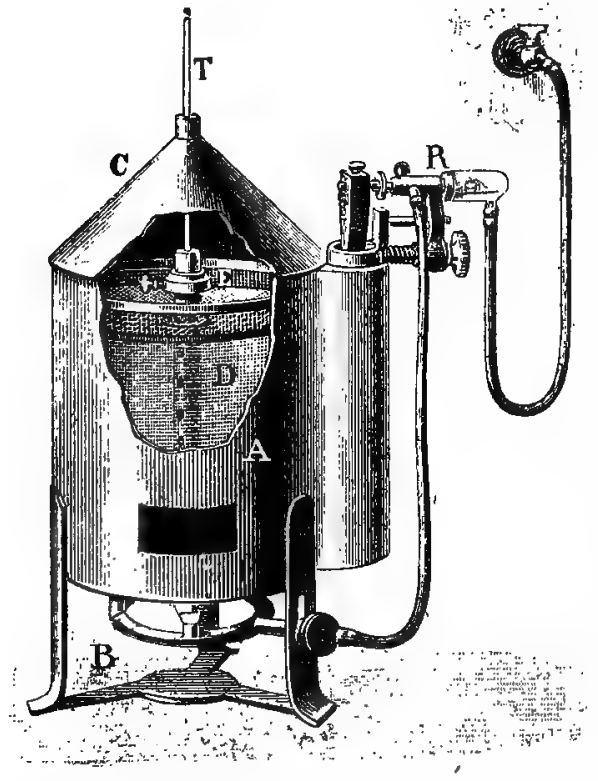

FIG. 64. The Roux water-bath for serum sterilization. $\mathrm{D}-$ Wirc basket with adjustable bottom $\mathrm{E} ; \mathrm{R}-\mathrm{Metallic}$ regulator of Roux. coagulate. Consequently, only solidified serum will be obtained by this procedure. The method takes less time than the preceding. It yields an almost perfectly transparent yellow medium which is a decided advantage over the method to be described next. The cotton plugs of the serum tubes should be cut off close to the end of the tube which should then be turned in a flame till the cotton begins to change color. The tubes are then sealed with sterile, rubber caps. This precaution insures the sterilization of the cotton plug, and hence, prevents subsequent contamination by moulds. Moreover, the serum when once solidified will not dry down during the subsequent exposures to heat.

The serum tubes, sealed as above, are inclined in what is known as Koch's serum sterilizer (Fig. 65). The inner compartment is surrounded by a water-jacket. A thermometer should be placed on the inside of the apparatus and another one is suspended in the water. A 
thermo-regulator is employed to secure a constant temperature. Heat is applied till a temperature of about $75^{\circ}$ is reached and this is then maintained for 1 hour. This is repeated on each of three or four successive days., The time necessary to secure sterilization can be shortened by transferring the tubes, for a few hours after each heat, to an incubator at $37^{\circ}$ as described in connection with the preceding method.

Sterilization of serum at $100^{\circ}$.- Serum sterilized at this temperature is opaque white. This, however, does not interfere with its value as a nutrient medium. The loss of transparency is counterbalanced by the ease with which it can be prepared in large quantities. If inclined blood-serum is heated rapidly to $100^{\circ}$ it will solidify, but the mass becomes torn up by gas bubbles due to the expul. sion of carbonic acid.

The formation of

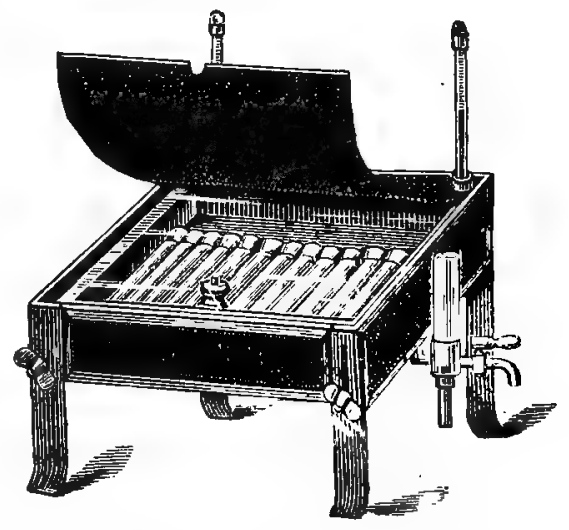

F1G. 65. Koch's serum sterilizer.

these bubbles or spaces can be avoided if the serum is first maintained at a temperature of about $80^{\circ}$ for some time. For this purpose, they can be inclined in an ordinary air. bath (Fig. 25), or in the apparatus shown in Fig. 65. After keeping the serum at $80^{\circ}$ for some time, the temperature should be gradually raised till that of $100^{\circ}$ is reached. The tubes are then steamed for 30 minutes on each of 3 successive days.

Solidification of serum.-The sterile, fluid serum can be used as such, but more often it is coagulated in an inclined position and employed for streak cultures. The fluid serum should be kept in sealed tubes to prevent evaporation (see 
Chapter XV). When inclined serum is wanted the tubes are placed in the coagulating apparatus (Fig. 65), and heated for about 1 hour at $70^{\circ}$. They are then ready for use. Loffler's blood-serum and glycerin serum must be heated to about $75^{\circ}$ in order to insure perfect coagulation. Obviously, the blood-serum tubes may be inclined in the ordinary dryheat sterilizer (Fig. 25) and solidified at $70^{\circ}$, or at a higher temperature.

Löffer's blood-serum.-This medium is chiefly employed for cultivating the diphtheria bacillus. It is prepared by adding to three parts of blood-serum, one part of ordinary bouillon to which 1 per cent. of glucose has been added. The mixture is then sterilized according to one of the methods outlined above. It is finally coagulated in an inclined position. It should be remembered that Loffler's serum requires more time and a higher temperature to secure coagulation than does ordinary serum.

Glycerin blood-serum.--This is prepared by merely adding 5-6 per cent. of glycerin to the blood-serum. This mixture is then sterilized and inclined according to the directions given above.

Boiled non-coagulated serum.-When serum is diluted with 5 to 10 parts of distilled water it can be sterilized by steaming without undergoing coagulation. This albuminous liquid-can be utilized to advantage in some cases.

Oxalate blood or plasma. - The preparation of this medium is described on p. 4 4 .

\section{Filtration of Bacterial Liquids.}

A liquid may be deprived of the bacteria which are present by filtration through unglazed porcelain. The Pasteur-Chamberland bougie is by far the most reliable for this purpose. As stated on p. 464 the walls of the bougies 
vary somewhat in thickness, and hence the rate of flow is variable. Highly albuminous fluids cannot be filtered unless the wall of the filter is relatively thin or unless extremely high pressure is employed to force the soluble proteins through the pores of the filter. With insufficient pressure these remain on the filter and only an aqueous liquid will pass through.

The liquid to be filtered should contain as little suspended matter as possible. For this reason, blood-serum should be centrifugated or allowed to settle until free from corpuscles. Bouillon cultures should first be filtered through several thicknesses of paper: The hardened, parchment-like paper (No. 575) of Schleicher and Schïll is well adapted for removing the mass of bacteria from a liquid.

A large number of filters have been devised, but most of these are far from being satisfactory for ordinary laboratory work. One of

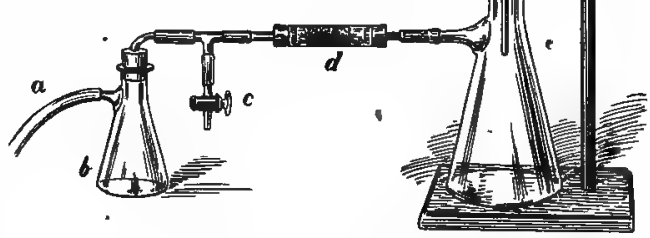

FIG. 66. Apparatus for filtering bacterial liquids (F. G. N.). the best filters is that of Martin, but it can be used only with negative pressure, - that is to say by producing a vacuum in the receiving flask. The author has described a filter ${ }^{1}$ which has been found to be very useful for laboratory work. It can be connected with an air pump, or additional pressure may be obtained by connecting with a cylinder of compressed air. It can be used for filtering large or small quantities of liquid.

The apparatus shown in Fig. 66 consists essentially of a glass cylinder $(g) 3 \mathrm{~cm}$. in diameter and $20 \mathrm{~cm}$. in length. The upper end is

${ }^{1}$ Centralblatt für Bakteriologie 22, p. 337, 1897. 
provided with a globe or reservoir having a capacity of 250,500 or 1000 c.c. The lower end is provided with a flange $(f)$ which is $2 \mathrm{~cm}$. wide and about $1 / 2 \mathrm{~cm}$. thick. The flange is ground on the lower surface. The upper surface of the flange, in the position shown in Fig. 66, should be parallel with the ground surface and not sloping. This is necessary in order to prevent the clamps from slipping off. The ground surface of the flange must form a perfect right angle with the inner wall of the cylinder (see Fig. 67). This is necessary in order to give a proper support to the shoulder of the bougie.

The manner of obtaining a perfectly tight joint is shown in Fig. 67. Before proceeding to make the connections the cylinder should

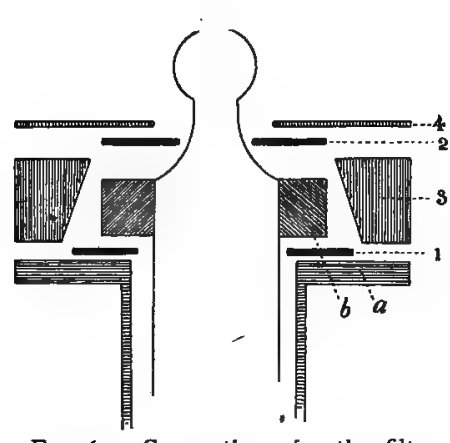

Fig. 6\%. Connections for the filter. filter; 1,2 and $3-$ Rubber rings; $4-$ Metal plater

(2) be inverted so that the globe rests on a small ring of a retort stand, with the flange uppermost. A rubber ring (Fig. 67,1 ) is slipped over the bougie and brought up against the shoulder. This 3 ring should not be made of very soft rubber inasmuch as it would expand laterally and crush the bougie when the clamps are applied. Ordinary cloth-covered rubber, from which the cloth has been torn will answer the purpose very well. The rubber ring is $2-3 \mathrm{~mm}$. thick and $5 \mathrm{~cm}$. in diameter. The circular opening should be cut so as to allow the ring to slip easily over the bougie (about $2.7 \mathrm{~cm}$. in diameter). The bougie provided with the rubber ring is then inserted into the cylinder.

Another rubber ring (Fig. 67, 2) is now slipped over the mouth of the bougie. This ring is $11 / 2-2 \mathrm{~mm}$. thick, $4 \mathrm{~cm}$. in diameter and has a central opening which is $1.3 \mathrm{~cm}$. in diameter. The thick rubber ring (Fig. 67, 3) is then placed in position. This should be $14-15 \mathrm{~mm}$. thick and should be $7 \mathrm{~cm}$. in diameter. The central opening is cut slanting so that the upper diameter is $4 \frac{1}{2} \mathrm{~cm}$, while that below is $5 \frac{1}{2} \mathrm{~cm}$. A brass or iron plate about $2 \mathrm{~mm}$. in thickness is then placed on top. This plate has the same diameter $(7 \mathrm{~cm}$.) as the flange, and the central opening is $2.2 \mathrm{~cm}$. in diameter.

Three clamps such as are employed in connection with the author's anaerobic apparatus (p. 313), are then applied. These are sufficient for vacuum filtration, but in case the liquid is to be subjected to a positive pressure of 60 or 80 pounds, it is well to add a fourth clamp. 
The mouth of the sterile bougie is now flamed and connected with the sterile glass tube which passes through the rubber stopper. A glass globe similar to that of Martin can be used in place of an Erlenmeyer flask to receive the filtrate (Fig. $68 \mathrm{~B}$ ). The cylinder is now inverted and connected with the receiver (Fig. $66 e$ ).

Ordinary liquids can be filtered by the aid of a Chapman aspirator. If the liquid under these conditions filters slowly, the neck of the globe (Fig. $66 \mathrm{~h}$ ) should be connected with a tank containing compressed air. When pressure is applied care must be taken to prevent the stopper or the glass tube from being blown out of place. The end of the glass tube after it has been slipped through the rubber stopper is softened in the flame and then flanged by means of a piece of charcoal or a nail. The stopper, when inserted, should be wired securely in place. A brass ring (h), placed on the upper side of the stopper, will prevent it from being cut by the wires.

The compressed air is contained in a small steel cylinder such as is used by physicians for atomizing purposes. A cheap substitute can be made by connecting a domestic, hot-water tank with a pressure gauge and a bicycle pump.

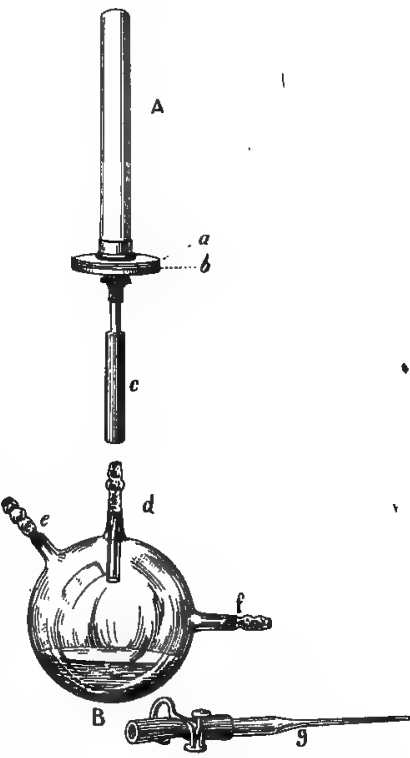

The Berkefeld filter can be attached Fig. 68. A Berkefeld filter showing to the glass cylinder in a somewhat simi- connections; $a$-Rubber disc; $b$ - Iron lar manner. An iron plate (Fig. 68 b) 5-6

$\mathrm{mm}$. thick and $7 \mathrm{~cm}$. in diameter is provided with a central opening $(1.2 \mathrm{~cm}$. diameter) just sufficient to allow the nozzle of the filter to pass through. A rubber ring (Fig. $68 \mathrm{a}$ ), $7 \mathrm{~cm}$. in diameter and $2 \mathrm{~mm}$. thick, with a central opening $3 \mathrm{~cm}$. in diameter, is placed between the glass flange and the iron plate. The whole is then clamped securely.

In place of the Erlenmeyer vacuum flask (Fig. $66 e$ ), a glass globe. like that shown in Fig. $68 \mathrm{~B}$ can be employed to advantage. The three tubes $(d, e, f)$ are plugged with cotton and the globe is then sterilized in the dry-heat oven. The tube $d$, after removal of the plug, is connected with the bougie by means of the sterile rubber tube $c$. The tube $f$ is connected in like manner with a rubber tube to the drawn- 
out glass tube $g$. The receiver and bougie are now heated in an autoclave to insure sterilization. Finally, the tube $e$, with the cotton plug in place, is connected with the pump in the manner indicated in Fig. 66. After filtration, the tube $d$ is disconnected and plugged with sterile cotton taken from an ordinary sterile tube. The tube $g$ is drawn out to a narrow capillary and sealed at the end. In order to remove the liquid from the receiver, the end of the tube $g$ is broken, flamed and inserted into the flask or tube to be filled.

The bougies, connecting tubes and receiving flasks must be perfectly sterile. The porcelain bougies should be scoured with sandpaper, dried and then sterilized either by the direct heat of a flame, or in a dry heat sterilizer. It is better to sterilize the moist bougie in an autoclave at $120^{\circ}$ for half an hour. The mouth of the bougie should be plugged with cotton and the entire bougie should be wrapped in paper. The Berkefeld filters are sterilized by boiling in water or bysteaming in an autoclave. After filtration, the entire apparatus should be sterilized by steam.

The receiving flask (Fig. $66 e$ ) is connected with a tube (d) filled with sterile sand. This serves to prevent bacteria from entering the flask when air is admitted through the glass stopcock $c$. The flask $b$ serves to collect any back-flow that may come from the aspirator.

\section{Tuberculin.}

Tuberculin contains essentially the soluble products of the tubercle bacillus. The organism growing in a suitable medium gives rise to chemical products, some of which pass out into the surrounding liquid. The bacterial cells are removed by filtration and the clear liquid, after concentration, is known as tuberculin. It can be prepared according to the following directions:

Ordinary bouillon is prepared from beef or veal, and 5 per cent. of glycerin is added. This medium is then filled into small Erlenmeyer flasks to a depth of about 1 inch. A broad, low flask provided with a loosely fitting, glass cap is very useful. The flasks should be provided with very firm cotton plugs. The flasks of bouillon are sterilized in steam in the usual way. 
They are then inoculated with the tubercle bacillus in such a manner that the material planted will remain floating on the surface of the liquid. This can be done by means of a looped platinum wire which should be bent at right angles, a short distance from the loop. A large piece of the dryish growth is loosely picked up on the loop which, as it is passed into the liquid, leaves the mass behind on the surface. A thin piece of cork about $1 \mathrm{~cm}$. :square may be placed in the bouillon and, after sterilization, the tubercle bacillus may be transferred to this by means of a Roux spatula (Fig. $49 a$, p. 278). The cotton plugs of the flasks are then charred and covered with caps of filter-paper. The flasks are carefully placed in an incubator at $39^{\circ}$ and they remain there for 4 or 5 weeks or more. The first indications of a growth will be seen in about two weeks. After that the growth is more rapid, and, about the third week, a thin folded, whitish scum will cover the entire surface. A yeast-like odor will pervade the incubator. Eventually, the growth which spreads over the surface forms a thick, folded, yellowish, dry mass.

The flasks are then steamed for 1 hour, after which the contents are filtered through paper (p. 469). The yellowish liquid may then be concentrated on the water-bath to about toth the original volume, or it may be filtered at once through a Pasteur or Berkefeld filter and placed in sterile bottles. One half per cent. of carbolic acid may be added as a preservative.

A more convenient procedure is to cultivate the tubercle bacilIus on glycerin potatoes in large Roux tubes. These should not be tightly sealed with rubber $\dot{c}$ aps or with wax, but should be closed with a cork stopper which is provided with a drawn-out glass tube (Fig. 54, p. 315). The lower portion of the potato should be in contact with 5 per cent. aqueous glycerin. Every two or three days the liquid should be agitated so as to moisten the potato. A very rich growth is thus obtained which can then be stirred into the dilute glycerin and finally removed by means of a drawn-out bulb pipette(Fig. 61 $e, \mathrm{p} .457$ ). The bacterial suspension is then steamed, filtered and concentrated as abovie. 
The tuberculin thus prepared should be tested upon tuberculous guinea-pigs. The fatal dose should be ascertained and the effect on the temperature of the animals should be observed.

\section{Diphtheria Toxin.}

The diphtheria bacillus when it grows in bouillon produces a powerful toxin. The ordinary bouillon which is alkaline at the time of inoculation becomes acid in about 24 hours and then gradually, in about 4 or 5 days, it returns to an alkaline condition. The toxin is produced especially during the alkaline stage. By passing a current of air over the liquid, the acid stage can be shortened and, hence, the toxicity of the culture is increased. If, however, the aeration is continued for more than 5 days it will cause an oxidation of the toxin, and, as a result, the liquid decreases in toxicity.

Bouillon made out of commercial meat extract does not give rise to acid products, and hence such media have been used for preparing toxins. The commercial peptons vary a great deal in their composition, and while some give a bouillon which does not change in reaction, others will give rise to acid products. The production of a temporary acid reaction in bouillon made out of meat is undoubtedly due to the presence of sugar. This can be removed by allowing the meat to ferment.

The bouillon best adapted for the cultivation of the diphtheria bacillus is prepared as follows:

1. $-500 \mathrm{~g}$. of chopped beef are added to 1000 c.c. of water; the mass is thoroughly mixed and set aside for 20 hours at $35^{\circ}$. The digestion at this temperature serves to destroy the sugar that may be present. The liquid is then strained through well washed muslin. $5 \mathrm{~g}$.of common salt and $20 \mathrm{~g}$. of Witte's pepton ( 2 per cent.) are added to 1 liter of the filtrate. The liquid is then titrated with $\mathrm{NaOH}$ in the 
manner described on p. 155 . To the amount of normal alkali necessary to neutralize the liquid an excess of alkali, corresponding to 7 c.c. of $\mathrm{N} \mathrm{NaOH}$ per liter, is added. The mixture is then heated to $70^{\circ}$ and filtered through paper, and finally through porcelain. The sterile filtrate is then transferred to sterile wide flasks, and inoculated with the culture.

The bouillon may be boiled, filtered and then sterilized by steaming on each of 3 successive days, or by heating in the autoclave at $110^{\circ}$. Heating, however, tends to alter the reaction of the medium, and hence diminishes the toxicity of the liquid. Nevertheless, sterilization by heat is more convenient and hence is resorted to most often.

2.-Instead of adding commercial pepton which, as indicated above, is liable to vary in composition, Martin adds an equal volume of a pepton solution obtained by digesting the stomach of a pig.

This is prepared as follows: A clean pig's stomach is freed from fat, cut up into small pieces which are then passed through an Enterprise sausage machine. To $200 \mathrm{~g}$. of the finely divided tissue, 1 liter of water and 10 c.c. of concentrated $\mathrm{HCl}$ are added, and the mixture is set aside at $50^{\circ}$ for about 12 hours.

The digested liquid is then carefully decanted to a filter of absorbent cotton (Fig. 35, p. 237). Inasmuch as the filtrate is intensely acid it is advisable to neutralize the excess of acid by the addition of a strong alkali. This can be done by adding 25 c.c. of a 16 per cent. $\mathrm{NaOH}$ solution. The liquid is then thoroughly mixed and the residual acidity is determined by titration (see p. 155). To the amount of $\mathrm{N}$ alkali necessary to neutralize the liquid, an excess of 7 c.c. per liter should be added in order to impart the most favorable alkalinity.

The mixture is then boiled or heated at $120^{\circ}$ for 10 minutes. When the precipitate settles, the liquid can be filtered through paper. The filtrate will be perfectly clear, if the neutralization was done in the cold. It may be used at once, or it may be placed in flasks and sterilized by steam; or at $115^{\circ}$ for 15 minutes.

In the pepton solution thus prepared, the diphtheria bacillus grows without producing any acidity. To make the culture medium proper, Martin adds to this pepton solution an equal volume of the fermented meat extract (p. 474) to which salt, but no Witte's pepton, has been added. The mixture is then titrated and given the alkalinity mentioned above. It is then heated, filtered, filled into flasks and sterilized by filtration through porcelain or by steam. This medium. as stated does not yield acid products, and it can give rise to a toxin such that 0.002 c.c. ( $\frac{1}{1}$ б C.c.) will kill a $500 \mathrm{~g}$. guinea-pig. 
The bouillon prepared as above under (1), or the mixed medium described under (2) is inoculated with the diphtheria bacillus and set aside at $37^{\circ}$. A scum or pellicle forms on the surface within 24 hours, and, if this is shaken down, another one will form. The absence of a pellicle in- . dicates a weak culture or an unfavorable medium. The maximum toxicity is reached on about the 5 th to the 7 th day. The culture should then be filtered, first through paper, then through porcelain. This should be done under pressure (p. 469). After the 10th day the toxin gradually diminishes. The filtered toxin can be transferred to sterile bottles, preferably of dark-brown glass; or, it may be drawn up into sterile bulb pipettes (p. 457) which are then sealed in the flame. Inasmuch as air tends to alter the toxin it is advisable to fill the bottle or pipette. Moreover, the toxin should be kept in a cool, dark place. A half per cent. of carbolic acid or. of toluol can be added as a preservative. The strength of the toxin must now be ascertained by inoculating a series of animals.

Determination of the minimum fatal dose.-For this purpose a number of guinea-pigs are selected having about the same weight (250 g.). Four of these animals should be injected subcutaneously with $\frac{1}{10}, \frac{1}{50}, \frac{1}{100}$, and gơ c.c. of the filtered toxin, respectively. The small quantities are measured as indicated on p. 479. The animal may die in a few hours or not until after the lapse of two or three weeks. In the latter case, post-diphtheritic paralysis of the extremities is likely to be observed for some days previous to death. The animal may be considered as having recovered, when it increases in weight and has a normal temperature.

The first series of guinea-pigs serves to establish approximately the fatal dose. Thus, if the guinea-pig that received to c.c. of toxin died whereas the one that received Ito $\mathrm{c}$ c. survived, it is evident that the minimum fatal dose, 
which is the amount just sufficient to kill, lies between these two limits. Another series of guinea-pigs is then taken and injected with $\frac{1}{60}, \frac{1}{70}, \frac{1}{80}$ and $\frac{1}{20}$ c.c. respectively of the filtered toxin. If $\frac{1}{80}$ of a c.c. still kills, whereas $\frac{1}{80}$ of a c.c., although it sickens the animal, does not kill, it follows that the former is to be considered as the minimum fatal dose.

The minimum fatal dose of a filtered diphtheria culture will vary with the duration of culture, the medium used, the temperature of incubation and the virulence of the bacillus employed. It has been obtained as low as 0.001 c.c., but as a rule it is above 0.01 c.c.

In accurate work it is desirable to inoculate three or six guineapigs with the estabiished minimum fatal dose. The individual animals vary more or less in resistance and it is, therefore, necessary to make certain that the minimum dose is surely fatal, not merely to one but to a number of animals. In a physiological experiment of this nature it must not be expected to obtain an accuracy comparable to that of a quantitative chemical analysis. Sometimes a guineapig will not die when inoculated with what is presumed to be several times the minimum fatal dose. Hence, it may happen that out of a dozen animals, inoculated with the minimum fatal dose, perhaps one or two may eventually recover. The resistance of the animal body is not a constant factor and for that reason the minimum fatal dose cannot be considered as an absolute result. Moreover, since the strength of an antitoxin is determined by testing against a known amount of the toxin it follows that the former result, expressed in immunity units, is approximate and not exact.

Recognizing the difficulties mentioned above, it is customary to define a "minimal fatal dose" as that amount of toxin which will usually kill guinea-pigs, weighing $250 \mathrm{~g}$., on the 4 th day, or at most on the 5th. This amount may kill a very susceptible animal in $1 \% / 2-2$, days. If, however, a number of animals die in less than two days it is evident that the quantity employed contains more than one minimum fatal dose. The animal should be weighed and the fatal dose per $250 \mathrm{~g}$. of body weight calculated.' Thus, if 
it is desired to ascertain the dose which is to be taken for an animal weighing $300 \mathrm{~g}$. on the basis of 0.05 c.c. per 250 g., then:

$$
300: x:: 250: 0.05 \quad x=0.06 \text {. }
$$

Testing of Antitoxin.

The strength of an antitoxic serum is expressed in immunity units. An immunity unit may be defined as the amount of antitoxin which is present in 10 times the amount of serum that is just sufficient to protect a $250 \mathrm{~g}$. guinea-pig against 10 times the minimal fatal dose of toxin. In other words, an immunity unit will theoretically protect against 100 times the minimum fatal dose. The protection extends not merely to saving the life of the animal but must also prevent local swelling, as well as variation in temperature and in body-weight. If, for ęample, 0.1 c.c. of serum protects a guinea pig, then 1 c.c. of that serum is said to contain 1 immunity unit. Again, if 0.001 c.c. protects then 1 c.c. of such serum contains 100 immunity units. Serum can be prepared of such strength that the astonishingly small amount of $0.000,05$ c.c. suffices to protect a guineapig. This serum, therefore, contains 2000 immunity units in 1 c.c.

The usual method of testing antitoxin is to inject subcutaneously into several guinea-pigs, each weighing about $250 \mathrm{~g}$.; mixtures of 10 times the minimum fatal dose of toxin and variable amounts of antitoxin. The several mixtures are made up to the same volume by the addition of sterile physiological salt solution $(0.75$ per cent. $\mathrm{NaCl})$. The amount of antitoxin $(a)$ which is just sufficient to prevent any ill effects, even local edema, represents $\frac{1}{10}$ of an immunity unit. Hẹce, $\frac{I}{10 a}$ represents the number of immunity units present in 1 c.c. of the serum. 
The method of determining the strength of an antitoxic serum can be shown by the following example:

The minimum fatal dose of the particular toxin employed was found to be 0.02 c.c. Hence, 0.2 c.c. of this toxin, when injected into each guinea-pig, per $250 \mathrm{~g}$. body-weight, represents 10 times the minimum fatal dose. In order to measure out this amount it is advisable to add 1 c.c. of the toxin to 4 c.c. of the $\mathrm{NaCl}$ solution. 1 c.c. of this dilute toxin (A) represents 0.2 c.c. of the original poison.

1 c.c. of toxin A corresponds to 10 times the minimum fatal dose for a guinea-pig weighing $250 \mathrm{~g}$. It is not always possible to obtain animals that will have exactly the same weight. They should, however, weigh as close to this amount as is possible; and, a corresponding correction for such variation should be made when measuring out the toxin and antitoxin. Thus, for an animal weighing $30 \mathrm{vg}$. the dose of toxin $\mathrm{A}$ is ascertained from the proportion:

$$
300: x:: 250: 1 \quad x=1.2 \text { c.c. toxin } \mathrm{A} \text {. }
$$

The calculated amounts of toxin A should then be measured out into small, sterile Esmarch dishes. Eventually, the calculated quantities of serum are added and each mixture is then injected into the proper guínea-pig.

If it is desired to ascertain whether the antitoxin passesses the strength claimed, the contents of the bottle should be drained into a sterile Esmarch dish. By means of an accurate pipette the voluma of the serum can be readily determined. If the bottle is said to contain $2000 \mathrm{I}$. U. and 7 c.c. are present it is evident that each c.c. of the serum should contain at least $286 \mathrm{I}$. U.

Suitable dilutions of the serum are now made in order to facilitate the measuring out of small quantities. Thus, 1.1 c.c. of the serum is added to 9.9 c.c. of the $\mathrm{NaCl}$ solution = dilution A. 0.1 c.c. of this dilution represents 0.001 c.c. of the original serum.

A second dilution may be prepared by adding 0.5 c.c. of dilution A to 4.5 c.c. of the $\mathrm{NaCl}$ solution = dilution B. 0.1 c.c. of this dilution represents 0.0001 c.c. of the original serum. This amount, according to the definition of an immunity unit, corresponds to 1000 I. U. Hence,

0.25 c.c. of dilution $B(0.00025$ c.c. serum) represents $400 \mathrm{I}$. U.

0.29 c.c. of dilution $B(0.00029$ c.c. serum $)$ represents $345 \mathrm{I}$. U.

0.33 c.c. of dilution $B(0.00033$ c.c. ser̂m) represents $303 \mathrm{I}$. U.

0.35 c.c. of dilution B (0.00035 c.c. serum) represents $286 \mathrm{I}$. U.

The above amounts, it will be understood, refer to a guinea-pig weighing $250 \mathrm{~g}$. Corrections for variations from this weight should be 
made as in the case of the toxin. Thus, if the $250 \mathrm{~g}$. animal is to receive 0.25 c.c. of serum $B$, but the one taken weighs $300 \mathrm{~g}$., then,

$$
300: x:: 250: 0.25 \quad x=0.30 \text { c.c. serum } B \text {. }
$$

The corrected amount of serum $B$ is measured out for each guinea-pig and added to the similarly corrected amount of toxin A. The mixture is then injected subcutaneously. The temperature and weight should be taken, and the local effects should be observed. If a given injection produces no ill effect it is evident that the amount of serum injected contains at least the corresponding number of immunity units. It may, of course, contain a larger number. On the other hand, if the animal dies, it is evident that the serum does not contain as many I. U. as are represented by the dose taken. If only a local swelling, or a slight scar results it indicates that the serum is nearly strong enough to neutralize the toxin injected.

The testing of an antitoxin will be rendered more clear by means of the following table:

\begin{tabular}{|c|c|c|c|c|c|c|c|}
\hline \multirow{2}{*}{ No. } & \multirow{2}{*}{ 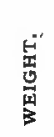 } & \multicolumn{2}{|c|}{$\begin{array}{l}\text { CORRECTED DOSE FOR } \\
\text { ACTUAL WEIGHT. }\end{array}$} & \multicolumn{2}{|c|}{$\begin{array}{l}\text { DOSE FOR } 250 \text { G. } \\
\text { BODY-WEIGHT. }\end{array}$} & \multirow{2}{*}{ 窇室 } & \multirow{2}{*}{ RESUIT. } \\
\hline & & $\begin{array}{l}\text { TOXIN A. } \\
\text { C.C. }\end{array}$ & $\begin{array}{l}\text { SERUM B. } \\
\text { C.C. }\end{array}$ & $\begin{array}{c}\text { Toxin } A . \\
\text { C.C. }\end{array}$ & $\underset{\text { C.C. }}{\text { SERUM B. }}$ & & \\
\hline 1 & 300 & 1.2 & 0.30 & 1.0 & 0.25 & 400 & Died. \\
\hline 2 & 240 & 0.96 & 0.278 & 1.0 & 0.29 & 345 & Slight scar. \\
\hline 3 & 220 & 0.88 & 0.281 & 1.0 & 0.33 & 303 & No scar; slight \\
\hline 4 & 260 & 1.04 & 0.364 & 1.0 & 0.35 & 286 & Marked gain \\
\hline
\end{tabular}

It is evident from the above that the serum contains more than 286 I. U., the strength claimed. The death of No. 1 indicates that the serum has less than 400 I. U. per'c.c. The actual amount lies between 303 and 345 .

It should be borne in mind that toxins of different origin and of different age have unequal neutralizing power. That is to say, the amount of serum, containing one immunity unit, which has been found sufficient to neutralize 100 times the minimum fatal dose of a given toxin, will not necessarily neutralize 100 times the minimum fatal dose of another toxin. This difference is more pronounced the older the culture. Thus, a culture several weeks old 
may yield a toxin of which an amount representing 20 or 30 times the minimum fatal dose is sufficient to neutralize one immunity unit. Hence in testing antitoxins, the toxin obtained from cultures which are but about a week old should be employed.

The method described recently by Ehrlich is to be considered as a distinct improvement upon the ordinary method of determining the strength of an antitoxin. It is based upon the fact, mentioned above, that the neutralizing power of a toxin with reference to a serum does not depend upon the number of minimum fatal doses present. $\mathrm{Al}$ though, theoretically, 1 I. U. should neutralize 100 minimum fatal doses yet it has actually been found to neutralize anywhere from 16 to 108 doses. This great variation is due to differences in the composition of various toxins. According to Ehrlich the pure toxin becomes converted, to a greater or less extent, into "toxoids" which, while they are no longer poisonous, yet possess the same neutralizing power as the original toxin.

Hence, the amount of toxin employed, when testing an antitoxin, should depend not upon the minimum fatal dose, but upon its neutralizing power with reference to 1 I. U. of a standard test-serum. Such a standard serum, prepared according to Ehrlich's directions, if available, would be invaluable for comparisons and for the determination of the actual strength of an antitoxin. This test-serum is dried in vacuo in the presence of phosphoric anhydride, and is then preserved in vacuum tubes. The strength of the serum is said to remain unaltered, owing to the absence of air and of moisture.

The contents $(2 \mathrm{~g}$.) of one of these tubes of test-serum is dissolved in 200 c.c. of a mixture of equal parts of glycerin and 10 per cent. $\mathrm{NaCl}$ solution. Inasmuch as the original serum had a strength of $1700 \mathrm{I}$. U. it follows that 1 c.c. of this solution represents $17 \mathrm{I}$. U.

The test-dose of toxin is to be ascertained by means of $1 \mathrm{I}$. U. of this standard serum. By the test-dose of toxin is understood the amount which, mixed with $1 \mathrm{I}$. U. and injected subcutaneously into a 250 g. guinea-pig, will cause death on about the 4 th day. The testdose can be readily and accurately ascertained by adding to portions of the dilute serum, each containing $1 \mathrm{I}$. U., variable amounts of the toxin (30, 50, 70, 100 times the minimum fatal dose) and then injecting these mixtures into guinea-pigs.

To determine whether a given serum has an advertised strength a portion of it should be diluted so that presumably $1 \mathrm{I}$. U. is contained 
in 4 c.c. The test-dose of toxin is added to this amount of serum and the mixture is then injected subcutaneously into a $250 \mathrm{~g}$. guinea-pig. If the trial serum actually does contain $1 \mathrm{I}$. U. death will not result till, as in the above determination of the test-dose, on about the 4 th day. If the animal dies on the $2 n d$ or $3 r d$ day it is evident that the amount of serum taken contains less than 1 I. U. and that the original serum is of less strength than advertised. If, on the other hand, the animal does not die till about the 6 th day, or even recovers, it follows that the amount of'serum taken contains more than 1 I. U.

In the latter case a series of guinea-pigs can be injected with mixtures of the test-dose of toxin and variable amounts of the dilute serum. Eventually a dilution of the serum will be reached which will correspond, as indicated above, to $1 \mathrm{I}$. U. The number of I. U. in 1 c.c. of the undiluted serum can then be readily calculated.

\section{Immunization against Diphtheria.}

The diphtheria toxin prepared according to the directions given can be employed for immunizing several rabbits. The animals should first be weighed and their temperature taken. For the first injection they should not receive more than what corresponds to the minimum fatal dose for guineapigs. Indeed, this amount of toxin may be injurious and it is advisable to add to the toxin a drop of diluted iodine solution (p. 288). The animals can tolerate the toxin, thus modified, better than if it were used direct. The temperature and weight of the animals are taken on each following day. When the condition of the animals has become normal, a second injection is made and this is repeated until the animals cease to appreciably react. The dose of toxin is then doubled and this amount with a little iodine is repeatedly injected. A new injection should not be given until the animals have fully recovered from the preceding one. When the animals shows no marked results, variation in temperature or loss in weight, after the injection of this amount of toxin then 4 times the minimum fatal dose may be used, mixed as before with a little iodine. Eventually 
about 10 times the minimal dose mixed with iodine should be administered.

The animals have now acquired a certain resistance to the toxin and the addition of iodine should be omitted. The dose of pure toxin should be small for the first injection, preferably the minimum fatal dose. Several injections of this amount should be given and later the dose may be doubled or quadrupled. The injections are repeated with the same or gradually increasing doses as the health of the animals will permit. The best results in immunization are always obtained by proceeding slowly. The animals should be given plenty of time to recover from the ill effects of the last injection.

Eventually, large doses of toxin can be tolerated by the experimental animals. The fact that a condition of immunity exists can be easily demonstrated by.injecting a new untreated rabbit with the same amount of toxin as is given to the treated animals.

When the animals have been immunized to such an extent that they can bear a large dose of toxin, say 50 or 100 times the minimum fatal dose, a small amount of blood may be drawn and its antitoxic properties tested. The best procedure, in this case, will be to draw about 3 c.c. of blood from the jugular vein by means of a syringe (p. 461), and to inject this at once, subcutaneously, into asguinea-pig. The latter, as well as a control animal should then be given an injection of 5 or 10 times the minimum fatal dose. The animal should survive, and, if the amount of antitoxin is sufficient, it will show no induration, swelling or scar at the point of inoculation.

The injections should always be made subcutaneously. The toxin should be made up to a convenient volume ( 1 or 2 c.c.) by the addition of physiological salt solution. It is hardly necessary to add that all the instruments and glassware employed must be sterile. 


\section{Anti-Infectious Serum.}

The serum of an animal which has /been immunized by repeated injections of a soluble toxin, as in the case of diphtheria, possesses antitoxic properties. That is to say, it contains a substance, antitoxin, which in some way neutralizes or renders inert the soluble poison when introduced into the body. This antitoxic serum should be sharply distinguished from the anti-infectious serum which results when an animal is immunized by repeated injections of certain living or dead bacteria. The defense of the body is carried out either by destroying the soluble poison that is being made in the body, or by destroying the bacteria themselves. There are, therefore, two distinct agencies at work.

By repeated injections of dead or living bacteria the cells are taught to take up, or otherwise destroy, the solid invading organisms: The serum of an animal thus immunized possesses the property of stimulating or causing the destruction of bacteria. It may incidentally possess, more or less, antitoxic action. If, for instance, the pest bacillus is injected subcutaneously into the horse the organism is localized at the point of injection and may grow at that point for some time. In so doing, it elaborates poisonous products which now induce the same reaction in the body as if they were injected separately. For the same reason, the blood of a horse immunized with living diphtheria bacilli is antitoxic rather than anti-infectious.

The anti-infectious serum has been especially studied in connection with cholera, typhoid fever, pest and rouget. It is evident from what has been said that a given organism may give rise to serums entirely distinct in action according as the soluble poison or the solid cell is injected. Thus, the serum of an animal vaccinated with the living or dead cholera vibrio will protect, in even extremely minute amount, against inoculation with the living germ, but 
it will not protect against the injection of the soluble toxin.

The antitoxic serum can be used as a preventive, or as a curative agent whereas the anti-infectious serum has a preventive value only. Even this action is manifest only under certain experimental conditions. Thus, the anti-infectious cholera serum, which protects perfectly against subcutaneous or intraperitoneal injections of the cholera vibrio, is of no value if the organism is introduced into the intestinal canal. The soluble poison elaborated by the vibrio in the intestines is absorbed and can be counteracted or rendered inert only by an antitoxic serum.

An animal that has recovered from an attack of a disease or has been rendered immune by treatment with' a germ or a toxin is said to have acquired active immunity. The blood of an actively immunized animal is capable, even in a small dose, of conferring a temporary exemption from that disease. This is designated as passive immunity. Thus, the blood of an animal actively immunized against the soluble diphtheria toxin will, if injected, confer passive immunity to man or animals. In other words, the soluble toxin or the virulent germ induces active immunity, whereas the antitoxic, or anti-infectious serum obtained from such actively immunized animal induces a condition of passive immunity.

The organism employed for immunizing purposes should possess a maximum degree of virulence. This can be obtained by repeated passage from animal to animal (p. 278), or by cultivation in collodium sacs (p. 496), arternating with passage through animals. The cultures should be grown on agar and should not be more than 20 or 24 hours old.

When it is desired to obtain a large surface growth of bacteria the Roux flask shown in Fig. 69 will be found extremely useful. 100 c.c. of agar or more are placed in the flask and sterilized. On placing the flask in a horizontal position the agar solidifies and a large surface is thus obtained. It can be inoculated by introducing a few drops of the culture suspended in bouillon, or better by swabbing the 
culture over the entire surface. The growth, as a rule, can easily be removed by adding sterile water, and then gently agitating the liquid. The thick suspension can then be poured out into sterile flasks or drawn up into sterile bulb pipettes (p. 457).

To measure out a dose of the growth Pfeiffer employs a small loop (Oëse) that will hold about $2 \mathrm{mg}$. of the material. This would correspond to a loop about $1 \mathrm{~mm}$. in diameter. A loopful of the growth is stirred up into 1 c.c. of bouillon. A fraction of a loop, as $1 / 4$, is obtained by transferring 1 loopful to 4 c.c. of

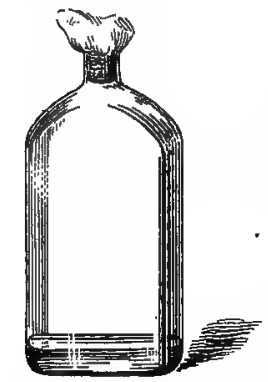

Fig. 69. Roux flask for surface cultures. bouillon and then taking 1 c.c. of the suspension. For the preliminary injections, it is advisable to employ dead cultures. The suspension can be heated for 1 hour at $58-65^{\circ}$, or it may be shaken up with a few drops of chloroform. If living cultures are employed, the dose for the first injection must be a very small fraction of the minimum fatal dose. A record must be kept of the temperature and weight of the animal, and, at no time, must an injection be repeated before the temperature and weight have returned to the normal. The first effect of an injection is to cause a slight elevation of temperature which is followed by a depression. The temperature may fall to $30^{\circ}$ or lower, before death occurs. The animal likewise loses considerably in weight.

Cholera.-Pfeiffer immunized guinea-pigs by injecting into the peritoneal cavity one-third of an agar culture, sterilized by chloroform or by heating at $65^{\circ}$. Seven to ten days later, about half a loopful of the living culture is injected in the same manner. After a like interval, one loopful of the living culture is injected; then, when the animal recovers two loopsful can be given. In this way the dose can be progressively increased till lasting immunity is obtained. Guinea-pigs can thus be immunized so as to withstand 50 or 75 times the fatal dose.

When the guinea-pig has acquired considerable resistance a large dose of the cholera vibrios should be injected into the peritoneal cavity. A few minutes later, a drop of liquid should be removed from the cavity by means of a drawn-out capillary tube (Fig. 62 c), and examined at once 
in the hanging-drop. Many of the cells will be seen to be perfectly motionless, while others are breaking up into granules. A drop of liquid should be drawn from the peritoneal cavity at the end of 10,20 and 30 minutes after the injection. The cells are first immobilized; they then break up into fragments, become granular and finally, in about 30 minutes, they disappear completely "like a piece of sugar in water." This is known as Pfeiffer's reaction. Exactly the same condition will be observed if a mixture of the serum of this animal and of the virulent cholera vibrios is injected into a normal guinea-pig. If the vibrios injected do not dissolve and disappear, it is conclusive evidence that they belong to a different species. Furthermore, it may be stated that the cholera vibrio will not disappear if injected by itself into a guinea-pig, whereas harmless vibrios will dissolve.

The serum of the animal thus vaccinated, as stated above, is anti-infectious. It will protect against the living germ but not against the soluble poison. If the cholera vibrio is planted in such serum it is not destroyed; it may become agglutinated, but Pfeiffer's phenomenon will not be seen. In other words, the serum is neither germicidal nor antitoxic.

The anti-infectious serum as indicated is not germicidal in vitro, nor is it antitoxic; and yet, a small amount is sufficient to save an animal from many times the fatal dose of the living culture. The organisms in this case may be totally destroyed in less than 30 minutes. It may be, as Pfeiffer believes, that this small amount of serum is converted in the body into a germicidal substance but it is more probable that this serum acts by stimulating the white blood cells and other phagocytic elements, which then give off the peculiar products that induce the Pfeiffer phenomenon.

It may be incidentally stated that the antitoxic serum likewise possesses no germicidal properties. It is supposed 
to neutralize the toxin in much the same way that an acid and alkali unite and neutralize one another. While this is a convenient way of explaining the action of antitoxin, it is probably no more correct than it would be to assume that the anti-infectious serum is germicidal. Antitoxic serum, like anti-infectious serum, does not act directly but it stimulates the cellular defenses of the body to activity. The cells of the body by preliminary training, such as repeated poisoning with a toxin, can take up such poison or give off products which will destroy it. If, as in the cholera experiment above, living cells are injected then the phagocytes are brought into action either directly, or by their germicidal products. In either case immunity always depends, directly or indirectly, upon the cellular elements of the body.

Pfeiffer's phenomenon is not limited to the cholera vibrio but will be.met with, under like conditions, when the Eberth bacillus or other organism is employed for immunization. It is of great value as a means of differentiating the cholera vibrio from other similar vibrios; or the Eberth bacillus from the colon and other Eberth-like bacilli. The reaction is specific. That is to say, the anti-infectious cholera serum will cause the destruction of cholera germs inside the peritoneal cavity of an animal. It will not protect against other vibrios or against the Eberth bacillus. Similarly, the anti-infectious typhoid serum will protect against the Eberth bacillus but not against the colon or pseudotyphoid bacteria.

In employing this test it should be remembered that normal serum may protect, in 1 c.c. dose, against the minimal fatal dose of the cholera or typhoid bacteria. Hence, several times the fatal dose should be employed and a control test should be made with a like amount of normal serum. The serum of convalescents from typhoid fever is not antitoxic but is anti-infectious and can, therefore, be 
used in testing the genuineness of a suspected Eberth 'bacillus. A few hundredths of a c.c. of such serum, injected into the peritoneal cavity, will protect a guinea-pig against several times the fatal dose of Eberth bacilli, whereas 0.5 c.c. of such serum will not protect against the colon bacillus.

Typhoid fever.-The Eberth bacillus, like the cholera vibrio, colon bacillus, etc., does not give rise to very poisonous soluble products when grown on ordinary bouillon. A soluble toxin has, however, been recently obtained by cultivating the highly virulent germ on an alkaline medium prepared by digesting the spleen with pepsin. With such a toxin Chantemesse has been able to obtain, although with difficulty, an antitoxic serum.

When, however, living or dead Eberth bacilli are injected subcutaneously or intraperitoneally, a condition of immunity is established and the serum in this case is antiinfectious. The method of immunizing the guinea-pig or rabbit is essentially the same as that given above under cholera. The serum of a guinea-pig immunized in this way has about the same preventive action as that of a convalescent from typhoid fever. The rabbit is more sensitive. The virulence of the Eberth bacilli will vary greatly, thus, some will produce death in a guinea-pig when $\frac{1}{50}$ of a loopful is injected into the peritoneal cavity. U'sually, however, $5-\frac{1}{2}$ a loopful is necessary. 2-3 loopsful of a culture, which has been killed by exposure to chloroform, or to a temperature of $65^{\circ}$, will be fatal to a $100 \mathrm{~g}$. guinea-pig in 24 hours.

The defibrinated blood of an animal that has died of an infectious disease may be employed to confer immunity. Thus, Toussaint injected into sheep 3 c.c. of defibrinated anthrax blood, previously heated to $55^{\circ}$ 'for 10 minutes, and obtained immunity. The heart-blood of rabbits dead of swine-plague, when heated to $54-58^{\circ}$, will protect rabbits in 
a dose of 1.5 c.c. introduced subcutaneously or intraperitoneally (Selander). In the latter case, Metchnikoff has. shown that the serum of the immunized animal has neither antitoxic, germicidal nor attenuating action. It will not protect against the soluble toxin. The serum, therefore, is anti-infectious and stimulates the phagocytes to destroy the bacterial cells.

\section{Elsner's Medium.}

Elsner's medium ${ }^{1}$ is a potato gelatin to which 1 per cent. of KI has been added. It is prepared according to the method of Holz. ${ }^{2}$ The details of preparation as given by these authors are very meager and unsatisfactory. The method of preparation as adhered to in this laboratory follows as closely as possible the original data.

1,000 g. of potatóes are weighed out. The potatoes are brushed clean under the tap and cut up into lumps which are then placed in an Enterprise fruit-press (No. 34). The potato comes through in a finely mashed condition. A sausage machine is not as useful since it cuts up the potato into small lumps, and when in this condition less juice can be obtained on subsequent squeezing. The finely mashed potatoes are placed in muslin and squeezed as much as possible. The dark colored juice is saved. The potato mass, wrapped in muslin, is then placed in a press and pressure applied. The liquid thus obtained is combined with the former. About 400 c.c. of a dark liquid is thus expressed out of $1 \mathrm{~kg}$. of potatoes.

The liquid is set aside in the ice-chest for 24 hours, after which it should be filtered. Owing to the presence of extremely small granules, the liquid can not be readily filtered through paper. It is advisable, therefore, to filter

' Zeitschrift für Hygiene 21, p. 29, 1896.

${ }^{2}$ Ibid, 8, p. 159, 1890. 
through absorbent cotton according to the procedure described on p. 237. When this filter clogs the surface layer of muslin should be carefully removed or scraped when thefiltration will recommence.

10 per cent. of gelatin and 1 per cent. of $\mathrm{KI}$ are added to the dark liquid which is gently warmed at about $40^{\circ}$ till the former has dissolved. Portions of 10 c.c. of the liquid are then titrated with $\mathrm{NaOH}$ (p. 155). 10 c.c. of the potato extract usually requires about 1.6 c.c. of $\frac{\mathrm{r}}{\mathrm{T}} \mathrm{NaOH}$ for neutralization. It may, however, require as much as 3.2 c.c. The addition of gelatin still further increases the, acidity. The gelatin should be acid, but not too much so. The acidity of 10 c.c. of the gelatin should not require more than 2.0 c.c. of $\frac{\pi}{10} \mathrm{NaOH}$. If it requires more, the excess over 2 c.c. (p. 354) should be neutralized by adding the corresponding amount of $\mathrm{N} \mathrm{NaOH}$ to the remaining gelatin. For example, if $10 \mathrm{c} . \mathrm{c}$. of the gelatin requires 3.2 c.c. $\mathrm{NaOH}$, then the excess acidity, above 2.0, corresponds to 1.2 c.c $\frac{\mathrm{N}}{\mathrm{N}} \mathrm{NaOH}$. Now, if the volume of the gelatin measures 350 c.c. this amount will require 42 c.c. of $\frac{\pi}{10} \mathrm{NaOH}=$ 4.2 c.c. of $\mathrm{N} \mathrm{NaOH}$, in order to reduce the acidity to the proper point.

$$
10: 1.2:: 350: x \quad x=42 \text { c.c. of } \frac{\pi}{10} \mathrm{NaOH} \text {. }
$$

The gelatin, having now the proper degree of acidity, is immersed in a boiling water-bath for about $3 / 4$ of an hour. The soluble proteins coagulate and clarify the liquid. This. is then filtered, filled into tubes, and sterilized by steaming. for 15 minutes on each of three successive days. As a rule, a slight precipitate forms in the tubes, during sterilization, but this does not interfere with the usefulness of the medium.

On this medium the Eberth bacillus yields very finely granular, small, bright droplets resembling water. On the other hand, the colon bacillus gives rise to colonies which: are large, spreading, more strongly granular and brown in appearance. A constant temperature of $15-18^{\circ}$ should be- 
maintained (p. 179). The acidity of this gelatin is such as to inhibit the development of the B. icteroides.

It hàs been proposed to utilize the fact that the typhoid colony tends to give off delicate fibrils from its periphery, whereas the border of the colon bacillus is sharply defined. To normal urine which has been allowed to stand for a couple of days till the reaction is alkaline, 0.5 per cent: of pepton and 3-5 per cent. of gelatin are added. The mixture is heated in the water-bath for an hour, filtered, filled into tubes and sterilized. This is done by steaming for 15 and 10 minutes on the first and second days, respectively.

Within 24 hours at $22^{\circ}$ the colon colonies appear as round, yellowish, finely granular and sharp bordered bodies, whereas the typhoid colonies are surrounded by whorls of threads. In this way, it is said to be possible to readily detect the typhoid bacillus in the urine or feces of the patient.

\section{Stoddart's Medium.}

The Eberth bacillus, as a rule, is more motile than the colon bacillus. This fact may be used to good advantage in distinguishing between these organisms. Direct microscopical examination or the staining of flagella will not give a satisfactory indication of the motility. If, however, a soft medium is used, the motile organism will rapidly diffuse throughout the medium or over its surface, whereas the non-motile organism forms a thick, white, non-spreading growth.

A liter of meat extract is prepared in the usual way (p. 153); $10 \mathrm{~g}$. of pepton and $5 \mathrm{~g}$. of $\mathrm{NaCl}$ are added, and the mixture slightly warmed till solution results. It is then divided into two equal portions.

To one portion, 10 per cent. of gelatin is added, and, when this has been dissolved, the liquid is titrated with Io $\mathrm{NaOH}$. An excess of 10 c.c. of $\mathrm{N} \mathrm{NaOH}$ per liter is added to impart the desired alkalinity. This and the subsequent procedure is exactly the same as that described on p. 155 . 
The other portion of 500 c.c. of meat extract is also titrated and an excess of 10 c.c. of $\mathrm{N} \mathrm{NaOH}$ per liter is added. The liquid is weighed; then boiled and filtered. To the clear bouillon, thus obtained, $5 \mathrm{~g}$. of finely cut agar areadded. The liquid is gently boiled till the agar has completely dissolved, when it is weighed. The loss in weight is compensated by the addition of a corresponding amount of distilled water. An equal volume of the 10 per cent. gelatin, prepared as above, is then added and the mixture is sedimented, as in the case of agar (p. 236), at $50^{\circ}$ for 1-2. hours. It is then filtered through the absorbent cotton filter (p. 237), and, if desired, it can then be passed through a filter paper.

The preparation of this medium can be simplified by heating the liter of meat extract till the soluble proteins coagulate. To the clear filtrate $5 \mathrm{~g}$. of agar are then added and the liquid is boiled till this. has dissolved. $50 \mathrm{~g}$. of gelatin are then dissolved in this agar solution and the liquid is titrated. To the amount of normal alkali necessary to neutralize the medium an excess of 10 c.c. per liter is added to impart the desired alkalinity. The liquid is then heated in a waterbath for about 30 minutes, allowed to sediment at $50^{\circ}$, and finally is filtered as above.

'It is evident that Stoddart's medium is a gelatin-agar which contains:

Gelatin, 5 per cent.

Pepton, 1.0 per cent.
Agar, 0.5 per cent. Salt, 0.5 per cent.

The medium is filled into large test-tubes, in portions. of about 10 c.c., and sterilized by exposure to steam for 15 minutes on each of three successive days.

The method of using the medium is as follows: It is poured out into wide ( $7 \mathrm{~cm}$.) Esmarch dishes, steamed and allowed to solidify in a horizontal position. The organism to be tested is touched, by means of a platinum wire, to the center of the surface of the medium. The dishes thus prepared are placed over some water in a moist chamber. The latter is placed in a horizontal position, in the incubator at $35^{\circ}$. In about 18 hours; the Eberth (and Sanarelli) bacilli will spread over the 
entire surface forming a transparent scarcely visible growth. The non-motile colon bacilli (Emmerich, Havelburg), will form a small white colony on the surface without any diffusion. The motile colon bacilli will diffuse, sometimes as rapidly as the Eberth bacilli, but unlike these the growth will be opaque and easily visible.

\section{Hiss' Tube Medium.}

This is similar in composition to the above and contains in addition 1 per cent of glucose. $5 \mathrm{~g}$. of Liebig's meat extract, $5 \mathrm{~g}$. of $\mathrm{NaCl}$ and $5 \mathrm{~g}$. of agar are added to 1000 c.c. of water. The mixture is heated till the agar is dissolved. "The water lost by evaporation is then replaced and 8 per cent. of gelatin is added. When this has dissolved the solution is titrated. It is left acid but the acidity is reduced by addition of $\mathrm{N} \mathrm{NaOH}$, so that it corresponds to 15 c.c. of normal acid per liter. The liquid is then cooled to $60^{\prime}$ and cleared by adding the white of an egrg, previously beaten in about 25 c.c. of water. The liquid is finally hoiled for a few minutes and $10 \mathrm{~g}$. of glucose are added. It is then sedimented at $50^{\circ}$, filtered through absorbent cotton, and then through paper if desired. The clear filtrate is finally filled into tubes and sterilized.

Stab cultures of the organisms to be tested, are made in this medium. The colon bacilli and $B$. icteroides give rise to gas bubbles, whereas the Eberth bacillus doeş not produce gas. Gas formation will be observed most satisfactorily in about 8 or 10 hours after the tubes have been placed in the incubator. . The Eberth and the motile colon bacilli diffuse through the medium, whereas the growth of the non-motile colon and Eberth bacilli will be confined to the line of inoculation.

The difference in the transparency of the diffused growths of the Eberth and colon bacilli as observed on Stoddart's medium is not seen in this tube medium. It is a convenient method, however, of demonstrating whether an 
organism can produce gas and a diffused growth. The latter is an excellent indication of the presence of motion.

The Hiss medium can be modified by substituting 1 per cent. of lactose for the glucose, and by rendering the medium alkaline and then adding litmus. The medium thus modified will indicate acid and gas production as well as diffusion.

\section{Uschinsky's Medium.}

As seen from the formula, this is an artificial medium made up of simple chemical compounds and wholly free from protein matter. The organisms planted on this medium are therefore obliged to build up their protoplasm from simple organic and inorganic bodies. The medium has been of great value in the study of the products produced by the bacterial cell. It possesses the following composition:

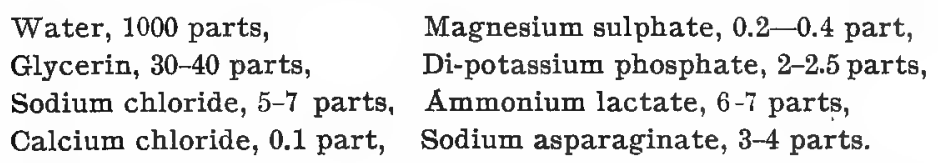

On the medium prepared as above, contrary to Uschinsky, the typhoid (and Sanarelli) bacillus will not grow, whereas the colon bacillus yields an excellent growth. The medium, therefore, can be used to good advantage in differentiating between these organisms.

Fraenkel has simplified the above solution by eliminating those constituents which are not necessary for the growth of bacteria. This modified solution contains $5 \mathrm{~g}$. of $\mathrm{NaCl}, 2 \mathrm{~g}$. of potassium di-phosphate, $6 \mathrm{~g}$. of ammonium lactate and $4 \mathrm{~g}$. of sodium asparaginate. Asparagin may be substituted for the latter and the neutral phosphate of sodium, for the phosphate of potassium, whereas the sodium chloride may be omitted. Fraenkel observed that in this medium the Eberth bacillus scarcely developed, whereas the colon bacillus gave a rapid, strong growth. 
A 1 per cent. solution of ammonium chloride and of glycerin, according to Fischer, likewise differentiates the colon and typhoid bacilli. The former can live on nitrogen in the form of ammonia, whereas the latter is unable to do so: The typhoid bacillus may give a slight growth in solutions which contain nitrogen in the amido combination, but it is especially dependent upon the nitrogen of protein matter (pepton).

\section{Collodium Sacs.}

The usual method of increasing the virulence of an organism is by successive passage through animals as indicated on p. 278. This, indeed, has been the only procedure until the recent introduction of the collodium sal method by Metch. nikoff, Roux and Salimbeni. In the hands of the French investigators this method has yielded remarkable results, notably in the study of cholera, pest, pleuro-pneumonia and tuberculosis.

The principle of the method consists in. planting the organism in a small collodium sac, which is then sealed hermetically and placed within the peritoneal cavity of an animal. Under these conditions diffusion takes place. The bacterial products pass out, whereas the albuminous, highly nutritive fluids of the living body pass into the sac. The organism is thus enabled to grow in the fluids of the living body unaffected, however, by plagocytes. As a result, it grows luxuriantly and is markedly increased in virulence. The growth is milky in appearance and the bacteria are vastly more a'bundant than in ordinary liquid media.

The method involves the most delicate bacteriological technique, and, inasmuch as the details of the process have not been heretofore published, the several steps will be described as minutely as possible.

The collodium on the market varies in composition according to the kind of nitro-cellulose employed. Hence every collodium cannot 
be used in making sacs. This is notably true of celloidin solutions. The film of collodium must possess a certain degree of elasticity, otherwise it cannot be slipped off the tube on which it is deposited.

The tubes should be preferably made of yellow glass and should be about $320 \mathrm{~mm}$. long. The end should be perfectly rounded off, like that of a test-tube. These tubes can be obtained having a. diameter of $14,18,25$ or $30 \mathrm{~mm}$. The $18 \mathrm{~mm}$. tube is the most convenient one for ordinary purposes.

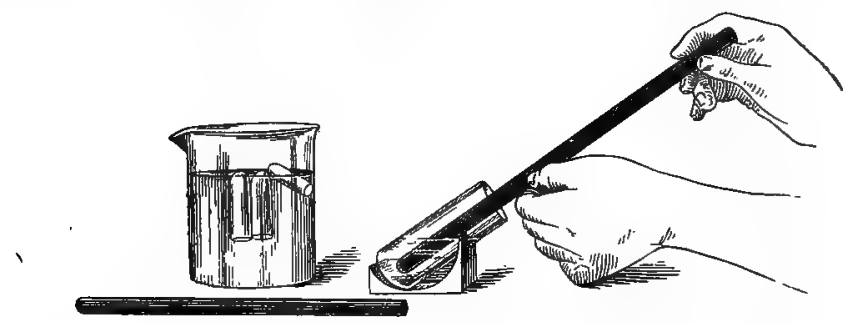

Frg. 7o. The rolling of collodium sacs. The beaker contains three sacs.

The collodium is placed in a small glass cylinder, about $4 \mathrm{~cm}$. in diameter and $9 \mathrm{~cm}$. high. This is inclined in the manner indicated in Fig. 70. The clean, dry glass tube is inserted into the collodium and and slowly rotated. Care must be taken to avoid touching the walls of the cylinder. The tube may be rested, at the desired angle, on the lower jaw of a retort clamp. From time to time, the tube is withdrawn almost completely out of the collodium and rolled in the air. When the tube is returned to the liquid another coat of collodium is deposited. By repeating this several times a good layer of collodium is deposited on the glass. It may happen that while the collodium is of the proper thickness above, where it has been repeatedly exposed to the air, yet only a very thin layer will cover the rounded end of the tube since this has remained continuously in the liquid. This difficulty can be overcome by completely withdrawing the tube out of the collodium, rotating a few moments in the air and then returning it slowly to the liquid. This should be done slowly and at as much of an angle as possible to prevent the formation of air-bubbles in the wall of the sac. Minute air-bubbles do not impair the efficiency of the sac but large ones are liable to burst during the subsequent process of sterilization. After the tube has been taken out and returned to the collodium three times, it will usually have formed upon its surface a sufficiently thick layer of collodium.

The tube is then rotated slowly in the air to allow the collodium to partially set. By proper rotation the liquid can be prevented from. 
gathering in droplets. The turning in the air is continued till the collodium layer is no longer sticky and is not too soft. It must not be exposed to the air till it becomes too dry, inasmuch as it would then be impossible to remove it from the glass tube. A little practice will enable one to tell, by the touch of the finger, when the exposure to air has been sufficient.

The coated end of the tube is then immersed in a beaker of distilled water and rotated in this for several minutes. The touch of a finger will again indicate when the desired hardness has been obtained. The tube is then withdrawn and the sac is ready to be peeled. The collodium layer is cut circularly, near the upper end, and the irregular border is removed. With a pair of forceps or with the finger-nail the edge of the collodium layer is bent back on itself. When the upper portion of the collodium tube has been turned "inside out", the sac can be drawn off the tube like a glove off a finger. The tube is grasped over the turned portion of the sac between the thumb and first two fingers. By gently drawing on this portion the sac is slowly everted

The sac thus prepared should be sufficiently firm so as not to collapse. It is then filled with distilled water and placed in the beaker of water for 15 minutes or longer.

The next step is to prepare a glass-tube which will enable one to hermetically seal the sac. The author employs for this purpose testtubes which are 2 or $3 \mathrm{~mm}$. less in diameter than the sac itself. The horizontal flame of the blast-lamp is directed against the test-tube at a point about $4 \mathrm{~cm}$. from the bottom. The tube is slowly rotated in the flame and as the glass softens a constriction results. When cool 'the rounded end of the tube must be cut off. A scratch is made with a file at about $1.5 \mathrm{~cm}$. below the constriction. A piece of burning charcoal is then applied to the end of the scratch. By gently breathing on the charcoal this can be kept aglow till the glass cracks. Usually the crack extends completely around the tube, but if it does not the crack can be led around by holding the glowing coal before it. A hot glass rod can be used instead of the charcoal. The cut end of the tube is then heated in the flame till the border is rendered perfectly smooth. The tube thus prepared has the appearance shown in Fig. 70 a. A number of these tubes should be prepared and kept on hand.

When a large sac is to be used it is liable to break after it is placed in the abdominal cavity. This defect is overcome by placing the sac in a wide tube which is freely perforated. The following modification made by the author can be employed to advantage in place of the usual method. In this procedure openings are blown into 
a test-tube and this is slipped inside of the sac. The first opening should be made in the bottom of the test-tube. A small narrow flame is directed against the bottom, and, when the glass has softened, it is touched with a piece of drawn-out glass-tubing which is then withdrawn. The glass adheres and-is drawn out into a thin capillary. This is then broken off at about $0.5 \mathrm{~cm}$. from the test-tube by a gentle tap with the glass-tube. The flame of the blast-lamp is then directed against the opening thus made. The broken edges sink to the level of the t ube and a round opening results. In this way a large number of holes can be blown into the lower end of the tube which is then constricted in the manner already described. The finished perforated tube has the appearance shown in Fig. $71 e$.

The next step is to attach the collodium sac to either of these constricted tubes. The open end of the sac is trimmed square with scissors. The sac is then placed between filter-paper and dried by the application of gentle pressure. It is especially desirable to have the inside of the neck of the sac perfectly dry. The constricted tube is then inserted into the sac up to where the constriction begins. This tube and sac are now, carefully and cautiously, rotated in a horizontal

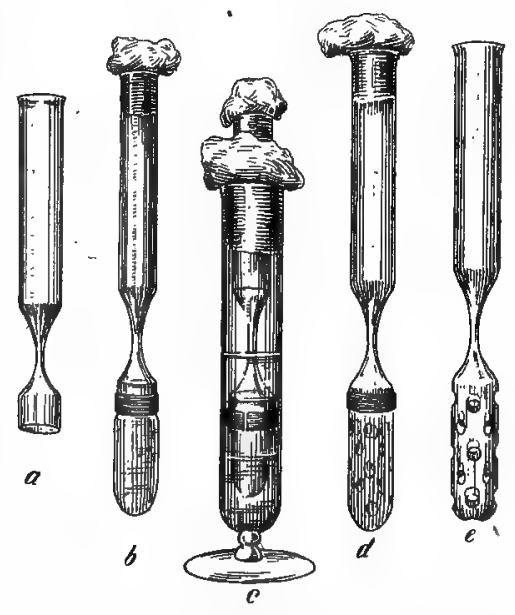

FIG. 7I. The preparation of collodium sacs. $a-$ Test-tube, constricted and cut; $b$-The sac attached to preceding and filled with water $c$-Same in test-tube on foot, in water, and sterilized; $e-$ Test-tube, constricted and perforated; $d-$ The same covered with sac.

position over a very small narrow flame. The modified Bunsen burner provided with a pilot light is especially useful for this purpose. The tube and sac are held at a distance of 3-5 cm. above the narrow flame. The collodium contracts down upon the glass and forms a perfect connection. To make this absolutely tight the collodium, over the glass, should be rubbed with a heated rod. In the absence of the burner mentioned, a hot glass rod passed repeatedly over the end of the sac will cause this to adhere to the glass.

A silk thread is then wrapped over this portion of the sac; and, finally it is thoroughly coated with collodium and allowed to dry. The object of the silk thread is to enable one, later on, to firmly hold the sac in a pair of forceps. The sac is now filled with distilled water and the upper end of the tube is closed with cotton. "The tubes thus 
prepared $(b$ and $d)$ are then placed in distilled water in a wide test-tube on foot (Fig. $71 \mathrm{c}$ ). The sacs are now sterilized by exposure to steam for 15 minutes, on each of three successive days; or, they may be sterilized in an autoclave at $115^{\circ}$ for 15 minutes. In this condition the sacs can be preserved for several weeks.

The filling of the sac with water or bouillon, and the withdrawal of that present, is done by means of a bulb pipette (Fig. $61 \mathrm{e}$ ). This should be bent at right angles, just below the bulb. The tip of the pipette is cut off and the end is flamed. Special care must be taken to heat the cut end of the pipette till the sharp edges are rounded off.

The sac is now ready for the final steps-inoculation, sealing and placing in the abdominal cavity. The cotton is removed and the mouth of the test-tube is then flamed. The narrowed end of the sterile pipette, prepared as just mentioned, is inserted through the constricted portion of the test-tube into the sac. The water is now carefully drawn up into the bulb. The pipette is then withdrawn and the cotton plug replaced. The water is blown out of the pipette which is then filled with the bouillon that has been inoculated with the organism to be tested. The pipette is again inserted into the sac and this is now filled up to the threaded portion, with the inoculated bouillon.

Nothing now remains but to seal the sac. For this purpose, it is taken out of the wide test-tube and the constricted portion is wiped dry with sterile filter-paper. The sac is then wrapped in a piece of sterile paper which should not project above the silk thread. One can thus grasp the glass end of the sac without causing contamination. A very narrow flame is then directed against the constricted tube till it is sealed. The sac is held in the sterile paper, avoiding unnecessary pressure, until the glass cools. The glass end is then coated several times with collodium, and, when this has dried, the sac is picked up by a pair of sterile forceps and placed in a sterile dish (Fig. 43, p. 265).. It is now ready to be inserted into an animal.

The guinea-pig or rabbit is usually used for sac experiments, although other animals may be employed. The animal is placed on a holder (Fig. 45, p. 268), and the hair over the abdomen is removed with a pair of curved scissors. The surface is then soaped and shaved, after which it is washed with alcohol and mercuric chloride. A piece of filter-paper soaked in' mercuric chloride is then spread over the prepared surface. The animal is now anesthetized with ether. An incision, about $3-5 \mathrm{~cm}$. long, is made through the skin of the upper part of the abdomen. A similar incision is made on one 
side of the linea alba, through the abdominal wall to the peritoneum. A grooved sound is then introduced into the cavity and the peritoneum is opened. The abdominal wall on each side of the incision is seized with a pair of pressure forceps, so that by crossing the forceps the cavity can be closed.

By means of a pair of forceps, the sac is picked up, at the threaded portion, and inserted into the abdominal cavity. The abdominal wall is then sewed by means of a curved needle. An assistant should hold the forceps so as to bring the cut edges together and have the walls on a stretch. When the abdominal wall has been closed the ends of the silk thread are cut off and the wound disinfected with a piece of filter-paper soaked in mercuric chloride. The skin is then sewed in like manner. The surface about the wound is disinfected, then washed with absolute alcohol and dried with sterile filter-paper. A few tufts of absorbent cotton are spread over the incision, after which collodium is applied and smoothed down with forceps or knife. When this has thoroughly dried the animal can be returned to its cage.

The sac is allowed to remain in the animal for from 3 to 5 days although, in special cases, it may remain for several months. When it is desired to remove the sac the animal is placed under a bell-jar and ether or illuminating gas is introduced. When the animal is dead it is spread out on a board (see p. 276) and opened. The sac will usually be found buried among the intestines and surrounded by adhesions. It should be carefully removed and the fibrinous covering stripped off. It is then placed in the sterile test-glass (Fig. 43), with the glass end down. A hot glass rod or searing iron (Fig. $48 a$, p. 275), is then applied to the end of the sac and an opening is thus burned through. The milky contents of the sac are then drawn up into a sterile bulb pipette. Transplantations can be made to the various nutrient media or another sac may be inoculated. The end of the pipette can then be sealed and the liquid preserved for subsequent examinations.

- It is understood, of course, that all the instruments, etc., used in the operation must be sterile. They are sterilized by boiling in a saturated solution of borax (Fig. $48 b$, p. 275). The hands of the operator should be thoroughly scrubbed with soap and water and then immersed for some minutes in mercuric chloride solution. 


\section{Inoculation for Rabies.}

The sac method of culture, as described, is not only useful in increasing the virulence of an organism, but it possibly affords a means of growing organisms that have hitherto resisted all known methods of cultivation. It has already enabled the isolation of the microbe of pleuro-pneumoniaan organism which is considerably smaller than any known representative of the group of bacteria. Undoubtedly, the cause of other diseases such as small-pox, scarlet fever, rabies, etc., will be found to belong to this class of extremely minute microbes.

The cause of rabies has not as yet been discovered, but it is known to reside especially in the central nervous system. It becomes important, at times, to confirm the diagnosis of rabies by injecting a portion of the spinal cord of the suspected animal under the dura mater of a rabbit. For this reason, the following method of inoculation, as practised at the Pasteur Institute is given:

The rabbit is fastened, lying on its abdomen, to a holder or to a table by means of leather thongs or stout cords, and is anesthetized with ether. The head of the animal should be turned toward a window. The excess of hair is removed from the surface of the skull which is then moistened with carbolic acid or with lysol. An incision about $3 \mathrm{~cm}$. in length is then made. One flap of the skin is raised by a pair of forceps and a jaw of a wire speculum is inserted. This is done, likewise, to the other side and the speculum is then distended. A trephine is applied to the exposed skull on one side of the median line. The button, about 6-8 $\mathrm{mm}$. in diameter is picked up and removed by means of a hooked needle or tenaculum.

A portion of the suspected medulla or cord has previously been rubbed up by means of a glass-rod with water or bouillon in a testglass (Fig. 43, p. 265). The suspension is drawn up into a syringe and 1 or more drops are injected under the dura mater. A few drops of 5 per cent. carbolic acid solution are applied to the surface and are then carefully removed with sterile filter-paper. This is repeated several times, and finally, after drying the surface thoroughly, the speculum 
is removed. The wound in the skin is then closed by a couple of silk sutures, for which purpose the Reverdin needle is usually employed. The incision is finally covered with cotton and collodium (p. 501).

In the case of rabies, the rabbit will usually die in about 14 to 20 days. By repeated successive passage the virus can be increased in virulence so that it will kill invariably in 10. days. The symptoms of rabies are manifested after about the 6 th day. The animal becomes apathetic, the temperature falls considerably below the normal, and the respiration is decreased. The loss of coordination of the hind legs is characteristic for paralytic rabies.

The cord and brain of the dead animal should be exposed and examined by staining, and by the culture method for bacteria. Similar examinations should be made of the heart-blood and internal organs. The absence of pathogenic bacteria must be established in order to justify the conclusion that the death was due to rabies.

The post-mortem in this case is carried on as follows: The animal is fastened on a tray or board and an incision is made with a pair of scissors from the top of the skull to the root of the tail. The lower end of the incision is prolonged towards each hind extremity. By means of a knife the skin is then wholly removed from the back and side of the animal, and also from over the skull, the ears being cut off close to the bone. Beginning at the base of the skull the muscles of the neck are then removed by a pair of dull-pointed scissors. After removing the scapulæ by means of bone forceps the muscles are removed from both sides of the vertebral column.

The skull is then grasped firmly in a pair of strong bone pincers (Farabœuf's), and the upper portion is removed by means of a pair of bone forceps. The tops of the vertebræ are removed in like manner, piece by piece, exposing thus, the entire brain and spinal cord. Care must be taken to avoid injury to the brain or spinal cord. The lower end of the cord is then held by forceps and all connections with the vertebral canal are cut. The cord is then laid back in the canal. The cord is used for the preparation of Pasteur's anti-rabic vaccine. The several steps taken are herewith indicated.

A noose of sterile silk thread is slipped over the end of the cord and drawn tight. The cord is then cut about $6 \mathrm{~cm}$. from the end and suspended from the neck of a sterile bottle. The latter contains some solid caustic potash or soda on the bottom. It is plugged with cotton and is marked C. A second slip-knot is then placed over the cut end of the cord and any connections of the latter with the canal are severed and finally the cord is cut, leaving a piece about $6 \mathrm{~cm}$. long attached to the thread. This is suspended like the former in a 
bottle marked $B$. In like manner the upper third of the cord is tied. with a silk thread, the connections cut loose, and lastly the cord is severed below the pons. This section of the cord is suspended in a bottle marked A.

The bottles containing the cords are set aside at a constant temperature of about $23^{\circ}$. The cords thus undergo desiccation, and, as a result, the virus gradually loses its virulence and becomes attenuated. From day to day, a portion of each cord is cut off and planted in bouillon. If bacteria develop it must be discarded. Suspensions in sterilized water or bouillon of the desiccated cords are employed in the preventive inoculations against rabies.

The brain and medulla are removed and placed in a sterile dish. A portion of the medulla about the size of a grain of wheat is placed in a sterilized test-glass (Fig. 43). By means of a sterile drawn tube pipette, a few drops of sterile water are added from time to time, and the tissue is reduced to a pulp by means of a sterile glass rod. When the insoluble matter has settled, the cloudy suspension, amounting to about 3 c.c. is drawn up into a sterile syringe. The needle is passed through a sterile paper and any air present is carefully expelled. A drop or two of this material can now be injected into another animal. The remainder of the material can be used to make the necessary bacteriological control examinations. In suspected cases the medulla, or brain should be placed in sterile glycerin. In this condition the dirus retains its virulence for many weeks. The material can thus be transported to a laboratory and examined in the manner indicated. 
CHAPTER XV.

SPECIAL METHODS OF WORK, Continued.

\section{Serum' Agglutination.}

The normal serum of the blood of various animals on contact with certain motile bacteria (Sanarelli, Eberth, Colon, etc.) will cause these to clump together or gather into masses. At the same time the individual cells lose their motion and become as it were paralyzed. This phenomenon is known as the agglutination reaction. If the normal serum is diluted the reaction will be less intense, and frequently will cease to be given when the serum is diluted with 10 parts of water. Occasionally, a normal serum will still agglutinate in a dilution of $1: 20$ or even $1: 30$.

In certain diseases, as in typhoid fever, the agglutinating substance is greatly increased in amount. The reaction then will be surely given with the Eberth bacillus when the serum is diluted $1: 30$, and may be given with a dilution of 1:50 or even with greater dilutions. It will be seen, therefore, that the reaction must be quantitative in character when applied to the blood of a suspected typhoid fever case. The following method permits an accurate estimation of the agglutinating power of a serum.

The blood is obtained by puncture of the finger and is allowed to clot. The clot can then be loosened with a wire and the mixture centrifugated. The clear serum is used for the test. An abundance of blood, and hence of serum, can be obtained from a vein in the manner described on p. 462. The serum and the diluting fluid should be measured by means of a drawn-out tube pipette (Fig. 61, p. 457). The 
drawn-out end of the pipette should be cut square. The dilution of the serum can be made with sterile water, physiological salt solution, or with bouillon.

A number of glass cups (Klötze) are placed on a mounting board (p. 279). By means of the pipette these receive 10 , $20,30,40$, and 50 drops, respectively, of the sterile water. The serum is then drawn up into the same pipette and one drop of it is placed in each of the dishes. The dilutions $1: 10,1: 20,1: 30,1: 40 ; 1: 50$ are then prepared. The liquids should be thoroughly mixed. This can be done expeditiously and thoroughly by means of the pipette used. This should be placed in a tumbler of water and the inside washed by repeatedly drawing in water. The pipette thus rinsed is now introduced into the weakest dilution (1:50) and the liquid is drawn up. It is then forced out and this operation repeated two or three times will bring about a thorough mixing of the serum and water. The pipette is again rinsed in clean water and the next dilution $(1: 40)$ is mixed in the same manner. In this way each dilution is rendered homogeneous.

A number of clean cover-glasses are laid on the board. A drop from each of the above dilutions is placed on the corresponding cover-glass. Each drop is then inoculated with a minute portion of an agar culture of the Eberth bacillus. The culture should be recent, preferably 12 to 18 hours old. A concave slide, ringed with vaselin, is brought over each cover-glass and the hanging-drops (p. 143), thus prepared, are ready for examination.

The specimens should be examined immediately, and at the end of $1 / 2,1$ and $1 \frac{1}{2}$ hours. If agglutination does not occur in this time it is unnecessary to prolong the observation. The undiluted serum should be tested at the same time as the others. Moreover, one or more control hangingdrops should be prepared in order to make certain that the organism does not exist already clumped in masses in the culture tube. In case the 1:50 dilution gives a marked ag. 
glutination, the test should be repeated with higher dilutions. Thus, 1 drop of $1: 10$ added to 10 drops of water will give 1: 100 .

Some workers prefer to make the dilutions with a young bouillon culture ( 6 to 8 hours at $37^{\circ}$ ) of the Eberth bacillus. This is intended to obviate the possible error of mistaking the clumps of bacteria as occasionally found in agar cultures for the real agglutinated masses.

- The reaction is applicable to dried blood and, indeed, this is perhaps the most convenient way of sending the material for an examination. The blood is allowed to fall, drop by drop, on different portions of a piece of filter-paper. When the blood has dried perfectly the paper may be folded and mailed. The blood-stained circles, each representing one drop of blood, are cut out and placed in dishes containing $10,20,30$, etc., drops of water, respectively. The solution of the blood constituents is facilitated by crushing the bit of paper with a glass rod. Hanging-drops are then prepared in the manner indicated above.

According to Johnston and MacTaggart pseudo-reactions are characterized by rapid clumping without the corresponding loss of motion which is so characteristic of the true reaction. After a few hours these clumps tend to break up. These pseudo-reactions can be avoided by using an attenuated culture which is transplanted at intervals, of about a month and grown at the room temperature. From such stock cultures a bouillon culture is made and developed at $37^{\circ}$ for 24 ours. This is then employed. By using such attenuated cultures the authors do not consider it necessary to make dilution tests. A large drop of water is placed on the blood stain and allowed to stand for a minute or two. A loopful of the solution thus obtained is taken from the top of the drop and mixed with a loopful of the bouillon culture. The reaction is usually given in this method in about 15 minutes.

\section{Poisonous Foods.}

The examination of food which is suspected to be the cause of illness is frequently demanded. Before the development of bacteriology these cases of poisoning were usually believed to be due to metallic poisons which were introduced, accidentally or otherwise, into the food. The 
possibility of the presence of injurious metals, such as tin or arsenic, should be conceded and a thorough examination of a poisonous food should involve tests for the recognition of such metals. On the other hand, the fact that a gram or two of cheese, or a tea-spoonful of ice-cream, at times, causes pronounced illness would indicate that the poison cannot belong to the group of metals.

In the examination of poisonous meat and sausage the possibility of the presence of trichinæ should be clearly borne in mind. A bacteriological examination will be of no value unless the presence of these parasites is definitely excluded. Repeated examinations should be made, and, if possible, these should be controlled by the examination of tissue known to contain trichinæ.

Having excluded the presence of metallic poisons and the presence of animal parasites, as trichinæ, then the poison-producing bacteria that may be present should receive attention. Food-poisoning from bacteria may originate in any one of the following ways: 1 .-The food is infected and the poison is generated exclusively before the food is taken. The organism in this case may be considered as a true saprophyte endowed, however, with the property of producing highly poisonous substances. Hence, the intoxication will be directly proportional to the amount of poison which is ready made in the food at the time this was taken. 2.-The infecting organism may begin the elaboration of its poisonous products outside of, and continue the same process inside the body. In this case the organism is able to grow in the intestines, and may even penetrate the organs and tissues. The illness has the general characteristics of an acute infectious disease. 3.- The illness may not result in the production of poisons until the food is taken into the body. As an illustration, milk or water infected with typhoid or cholera bacteria may give rise in the one case to typhoid fever, and in the other case to cholera. 
A proper bacteriological examination must take into account the several possibilities just mentioned. Inasmuch as anaerobic bacteria may be the cause of such food infection, plates should also be made in glucose gelatin and in glucose agar and allowed to develop in an anaerobic apparatus (p. 314). The gelatin plates are, of course, developed at ordinary room temperature, whereas the agar plates should be grown at $37^{\circ}$. The several varieties of colonies should be transplanted and the effects of the pure living cultures, and also of the filtered cultures, should be studied on animals. The animal employed should, if possible, be susceptible to the poisonous food itself. In many cases, the dog and cat are preferable to the ordinary experimental animals.

At the time the plate cultures are planted bouillon tubes should be inoculated and allowed to develop, some at $37^{\circ}$ and some at ordinary room temperature. The cultures, thus obtained, can be tested by subcutaneous injection into guinea-pigs or rabbits. In case of the death of the experimental animal, the serous fluid in the large cavities and especially the heart-blood should be used to obtain pure cultures of the pathogenic organism which is present.

\section{Purification of Litmus.}

The ordinary litmus cubes, as met with commercially, do not yield a pure blue solution of the pigment. 'This is due to the presence of various impurities, notably of a red pigment. A pure blue litmus solution can be readily prepared according to the following directions:

$25 \mathrm{~g}$. of litmus are placed in a beaker and 250 c.c. of distilled water are added. The solution is then heated on a boiling water-bath or in steam for about 30 minutes. The deep blue liquid is then decanted; water is added to the residue and the mixture heated as before, and finally the blue solution is again decanted. This washing out of the residue is repeated once or twice. The combined aqueous extracts are allowed to settle over night and are then filtered. The filtrate is concentrated in an evaporating dish, over a flame, to less than one-half. It is then 
filtered again and the concentration is continued till about 50 c.c. of liquid are left.

The concentrated blue liquid is then poured gradually, with constant stirring, into about 5 volumes of absolute alcohol. The blue pigment is thrown down as a sticky precipitate, whereas the red color remains in solution in the alcohol. The precipitate is allowed to settle thoroughIy; the alcoholic liquid is then decanted as much as possible or it is removed by filtration.

The sticky precipitate is then dissolved in about 50 c.c. of water and the blue pigment is again precipitated by pouring the solution, as directed above, into 5 volumes of strong alcohol. The alcoholic liquid is again decanted and is finally drained off as much as possible.

The mass now contains the purified blue pigment, - mixed with various lime salts. Inasmuch as the pigment may not possess the requisite degree of sensitiveness it is well to make it as sensitive as possible. For this purpose, about 250 c.c. of water should be added to the precipitate and the mixture warmed on the water-bath till complete solution has taken place place. Then dilute $\mathrm{H}_{2} \mathrm{SO}_{4}$ is added, drop by drop, till a portion of the liquid placed in a tube and diluted has a strong red color. The liquid is then boiled to expel $\mathrm{CO}_{2}$ and alcohol. It is then neutralized by adding saturated baryta water, drop by drop, and stirring well. This addition of baryta is continued till a few drops of the solution, placed in a test-tube and diluted with water, show a clear blue liquid without a tinge of red. If a drop or less of $\mathrm{HCl}$ is added to the tube it will promptly change to a deep red. The liquid is now boiled for a few minutes and set aside over night. The precipitate of barium and calcium salts is then removed by filtration. The purified, concentrated litmus solution is then placed in Billings' glass-stoppered flasks and sterilized by steam. Whenever it is desired to add the litmus to sterile lactose agar, glucose agar, glucose gelatin, milk, etc., it should be 
drawn up into a sterile bulb pipette (Fig. 61), and then transferred direct to these tubes. The addition of sterile litmus solution to sterile media is preferable to the ordinary method of adding the litmus to the medium before sterilization.

\section{Tubing of Media.}

In many experiments, as in the disinfection tests presently to be described, it is desirable to employ measured amounts of bouillon. This can be done expeditiously by means of the simple apparatus shown in Fig. 72. The lower end of a 100 c.c. burette is attached to a small glass T-tube which connects with the reservoir flask. The burette can thus be readily filled and exact amounts can be measured out into the tubes.

For rapid tubing of gelatin, bouillon, agar, etc., where it is not essential to employ a definite volume it is well to employ a funnel or glass globe which is connected by means of rubber tubing with a drawn-out glass tube. A Mohr's clamp is attached to the rubber tubing or in its stead a glass stop-cock may be used. The perforation in the latter should have a diameter of about $3 \mathrm{~mm}$. Such an apparatus is obtained by disconnecting the Fig. 72. Apparatus for long rubber tube at $a$ in Fig̀. 72. This of media. For ordinary long rubber tube at a in Fig. 72. This tubing disconnect at \& simple apparatus can, therefore, be em-

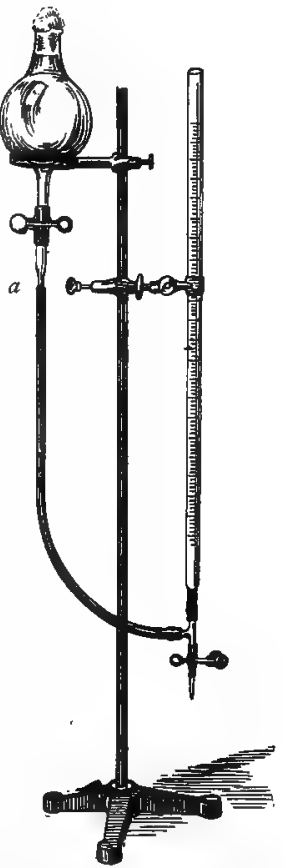
ployed for ordinary tubing of media, or for measuring out definite volumes. 


\section{The Sealing and Keeping of Cultures.}

The method of preserving cultures or specimens of blood, etc., in drawn-out bulb tubes has been touched upon (p. 458). In this connection it is desirable to indicate the methods employed in sealing the ordinary culture tubes. Three or four procedures are resorted to. The tubes may be sealed with rubber caps, corks, sealing-wax, or with paraffin. Thin sheets of rubber may also be used.

In either case, the cotton plug should be cut close to the end of the tube. It should then be drawn out slightly and rolled rapidly in the flame till the cotton is charred a trifle. The cotton is then pushed into the tube to prevent smioldering. If the tubes are sealed without this precaution, moulds are liable to develop starting from the cotton plug.

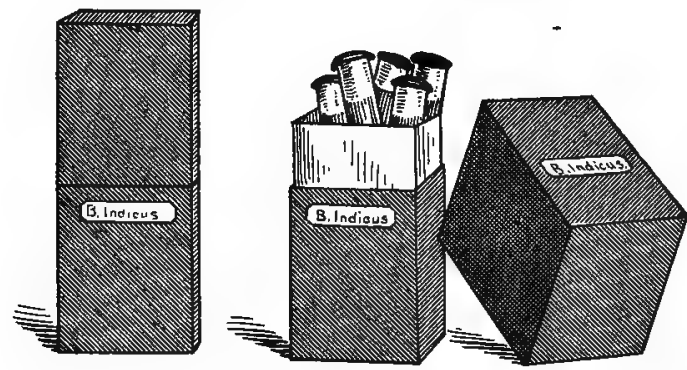

FIG. 73. The keeping of cultures in black, paper boxes (F. G. N.),

The corks or rubber caps should be immersed in mercuric chloride solution (1-1000) and steamed forr at least 15 minutes. They can then be used for sealing the tubes: The rubber caps, undoubtedly, are the most convenient for this purpose. They are, however, expensive and are likely to deteriorate on keeping. The corks are not only sterilized by the exposure to steam, but they are also' softened.' They can now be easily inserted into the tubes, and a perfectly tight closure can be obtained. When caps or corks 
are used the cotton plug should be pushed down into the tube $(1-1.5 \mathrm{~cm}$.) by means of sterile forceps. It , should not touch the rubber cap or cork.

A very cheap and satisfactory way of sealing tubes is to employ sealing-wax. The cotton plug should be firm and solid, and should not be pushed below the level of the mouth of the tube. The heated wax should be first applied to the edge of the cooled tube, and finally the center of the plug should be covered.

The tube cultures are usually kept in tumblers in a darkened case. The author employs for this purpose black, jacketed boxes shown in Fig. 73. The box is $6.5 \mathrm{~cm}$. wide, $10 \mathrm{~cm}$. deep and $18 \mathrm{~cm}$. high. The lid is $9 \mathrm{~cm}$. high, whereas the inner height of the lower portion is $12.5 \mathrm{~cm}$. A large number of boxes can thus be arranged like books on a shelf. The cultures are protected perfectly from dust and light.

\section{Thermal Death-point.}

As indicated heretofore, the vegetative and spore form of the same organism differ in their resistance to destruction. Thus, while the former is readily destroyed by a temperature of $60-80^{\circ}$, the latter requires an exposure to steam for some minutes. The vegetating forms of different species of bacteria likewise show different degrees of resistance. Some are killed readily at $55^{\circ}$ and others require 65 to $70^{\circ}$. Moreover, it should be borne in mind that several varieties of a given species may exist no two of which necessarily are equally resistant. Thus, we may have spores of anthrax which will be destroyed by a 5 per cent. solution of carbolic acid within 24 hours, while another yield of spores from a different variety of the same organism may resist this same disinfectant for 50 days or longer.

In studying the resistance of an organism it is essential to prepare as nearly a homogeneous suspension as possible. That is to say, 
each germ present should be single and wholly free from contact with other organisms. The massing of bacteria necessarily affords protection to the cells in the center of such groups. Because of this protection these few cells may survive, while all the others may be destroyed. The results obtained would conseguently be misleading. This error can be avoided by filtering the suspension through sterile absorbent cotton or glass-wool in the manner presently to be described.

When it is desired to test the resistance of the vegetative form of a given organism special care must be taken to exclude the presence of spores. Many

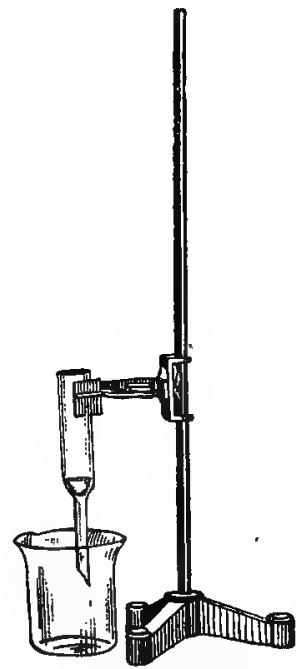
bacteria like those of typhoid fever, cholera, etc., do not form spores and hence such precautions are not observed when testing these organisms. The anthrax bacillus, however, does form spores, and hence in testing the resistance of the growing bacillus the spore form must be eliminated. This is accomplished by making so-called homogeneous cultures. These are obtained by making transplantations to bouillon every 6 or 8 hours. After several such transplantations only actively growing bacteria will be present.

The best procedure for studying the Fig. 74. Filter for bacterial action of moist heat on bacteria. is to suspensions.

place homogeneous suspensions in thin, straight capillary tubes. It is essential that the walls of the capillary tube shall be as thin as possible. They should not have a bulb of thick glass. When such sealed capillary tubes are immersed they rapidly acquire the temperature of the surrounding liquid.

Preparation of a bacterial suspension.-As a rule, it is best to employ young cultures on agar. Sterile bouillon is introduced into the test-tube by means of a drawn-out pipette (Fig. $61 e$ ) and the growth is thoroughly rubbed up. The suspension is now drawn up into the pipette and is transferred to a sterile filter (F'ig. 74), The filter-tube 
can be readily made by drawing out a test-tube. A layer of absorbent cotton should be placed on the bottom of the tube and then some glass-wool which acts as a weight. The filter thus prepared is plugged at both ends and sterilized. The filtered suspension should be examined under the microscope, and, if aggregations of bacteria are still present, it should be again filtered. The filtrate may be received in a large Esmarch dish, or in a sterile beaker which is covered with cotton or paper.

The suspension is drawn up into a sterile capillary pipette which is then sealed below and above the liquid (Fig. $62 c x$, p. 459). The liquid is now contained in a capillary tube about $10 \mathrm{~cm}$. long and $1-2 \mathrm{~mm}$. in diameter. A sufficient number of these sealed capillary tubes should be prepared (see p. 516) to meet the requirements of the experiment in view. They should be immersed in a test-tube containing mercuric chloride in order to disinfect the exterior. These tubes are used to test the action of moist heat. The same suspension is used for the preparation of silk threads, muslin squares and cover-glass specimens (p. 517).

\section{MOIST HEAT.}

The ordinary Hofmann iron water-bath will answer very well for these tests. It is filled with water and provided with a thermometer and a thermo-regulator which should be suspended so as not to touch the bottom or the sides (Fig. 75). The temperature of the water can thus be kept at any desired point. The water-bath of Roux, shown in Fig. 64, is very useful for these experiments. The capillary tubes should be placed on cross wires which are stretched over a syringe holder (Fig. 41 c, p. 263), and immersed in the water. . At the end of stated intervals, the holder is raised and a capillary tube is removed. The latter should be placed at once in a tube of cold water, to prevent the further action of heat. Each of the remaining capillary tubes, in like manner, is removed and placed in water at the end of the respective period of exposure. 
The contents of each of the capillary tubes are then inoculated into bouillon which is set aside at $37^{\circ}$ to allow the growth, if any, to develop. The contents are removed as follows: The tube is first wiped dry and then one end is slightly scratched with

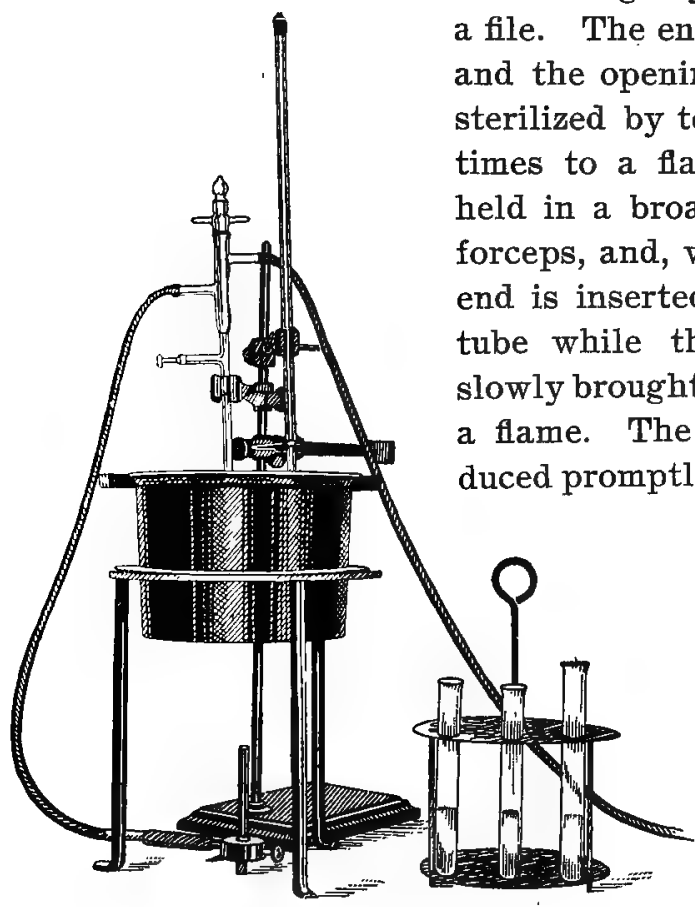
a file. The end is then removed and the opening of the tube is sterilized by touching it 2 or 3 times to a flame. The tube is broad-pointed pair of , when cool, the cut end is inserted into the culture tube while the closed end is anto contact with a flame. The vapor thus produced promptly expels the liquid from the capillary.

The student should make suspensions and test one or more of the following organisms, according to the directions given above, Cholera vibrio, Typhoid FIG. 75. Determination of the thermal death-point (F, G. N.). bacillus, Anthrax bacillus (homogeneous culture), Anthrax spores (p. 291) and Streptococcus pyogenes.

Action of moist heat at $58^{\circ}$.-The capillary tubes should be withdrawn at the end of $5,10,15,30$ and 60 minutes' exposure.

Action of moist heat at $70^{\circ}$.- The capillary tubes are withdrawn at the end of $1,3,5$ and 10 minutes. In addition to this the anthrax spore tubes should be exposed for 15,30 and 60 minutes.

Action of moist heat at $100^{\circ}$. - The capillary tubes are withdrawn at the end of 1, 2 and 3 minutes. Additional anthrax spore tubes are exposed for 5, 10 and 15 minutes. 
The ordinary dry heat sterilizer is employed in studying the action of this form of heat on bacteria. The bulb of the thermometer should be placed so as to be on a level with the objects exposed. It should not, however, rest on a metal surface. A thermometer is used to maintain a constant temperature.

The suspensions prepared as above cannot obviously be employed as such. It is customary to soak, in these suspensions, bits of sterile silk threads or small squares of muslin. These are then allowed to dry before they are used. Sterile cover-glasses are also smeared on one side with the suspended bacteria, and when dry they can be used in a similar manner.

The silk threads are prepared by cutting up some of the silk in lengths of about $1.5 \mathrm{~cm}$. These should be placed in a plugged test-tube and sterilized in the dry-heat oven. At the same time some muslin is cut up into squares of about $1 \mathrm{~cm}$. on a side. These are sterilized in a Petri dish. Thoroughly clean cover-glasses (p. 140), 18 to $20 \mathrm{~mm}$. square are cut into halves by means of a ruler and diamond. The oblong slips of glass are likewise placed in a Petri dish and sterilized.

The bacterial suspension prepared as above (p. 514) is transferred to a sterile wide Esmarch dish and the proper number of silk threads and muslin squares are then added and are allowed to soak thoroughly. One by one, they are then picked up by means of sterile forceps, and arranged in rows in a sterile Petri dish. A corresponding number of the sterile cover-glasses are likewise placed in the Petri dish and each one is covered with a large loopful of the suspension. This is spread over as much of the surface as possible and care is taken that the liquid does not run over the edge to the under side of the cover-glass. The silk-threads, muslin squares and cover-glasses are then allowed to dry 
at the ordinary temperature. The drying may be hastened by placing the dishes, slightly uncovered, in an incubator at $37^{\circ}$ for three hours.

When the temperature of the oven has reached the desired point, the proper number of silk threads, muslin squares or cover-glasses are placed in a sterile Petri dish which is then set within the oven on a level with the bulb of the thermometer. At the end of stated intervals a silk thread, muslin-square or cover-glass, is taken out as rapidly as possible, by means of sterile forceps, and is transferred to a tube of nutrient bouillon. This is labelled at once, and, eventually the entire set is placed in the incubator.

With infected silk threads, prepared as above, the student should test the action of dry-heat on the several organisms already studied. The results obtained in this, and in all other laboratory work, should be arranged in tabular form to facilitate comparison and review of results.

Action of $d r y$ heat at $70^{\circ}$.- The silk threads should be withdrawn at the end of 15, 30, 15 and 60 minutes' exposure.

Action of dry heat at $100^{\circ}$.-The specimens should be withdrawn at intervals as just given.

Action of dry heat at 120 $0^{\circ}$.-The specimens should be withdrawn at same intervals as above.

Action of dry heat at $150^{\circ}$. -The specimens should be withdrawn at intervals of $5,10,15,30,45^{\circ}$ and 60 ninutes.

\section{Testing of Disinfectants.}

In studying the action of physical and chemical agents on bacteria it is necessary to rigidly adhere to certain requirements without which the results would be of little value, if not wholly contradictory. The conditions which underly the testing of disinfectants may be summed up as follows: 
1.- Variable resistance of spores and of the vegetating forms of one and the same organism. It has been shown in recent years that considerable variation may exist in the resistance which an organism possesses to destruction. Thus, while there are some spores of anthrax which are readily destroyed by steam-heat $\left(100^{\circ}\right)$ others have been known to withstand this temperature for 10-12 minutes. Again, it was formerly stated that anthrax spores were destroyed by 5 per cent. carbolic acid in two days but the researches of Fraenkel have shown that spores of anthrax may be had which are not destroyed by an exposure of 30 to 40 days or even longer. In view of these facts several standards have been proposed. Thus, Fraenkel designates anthrax spores which are destroyed by 5 per cent. carbolic in less than 10 days as feebly resistant; in 10 to 20 days as of average resistance; in 20 to 30 days as very resistant; in 30 to 40 days as extremely resistant. Geppert's standard anthrax spores are those which are infectious after boiling for one minute 1 c.c. of a spore suspension which is added to 30 c.c. of boiling water. Esmarch has suggested as a standard, anthrax spores which when fixed on silk threads resist steam-heat of $100^{\circ}$ for 10 minutes.

2.-The influence of the medium in which the organism is tested. Thus, it has been shown that to destroy anthrax spores in bouillon it requires 20 times as much mercuric chloride (1-1000) than when they are suspended in water; and, 250 times as much are necessary when they are distributed in blood-serum.

3.-The temperature at which the disinfection is made. The higher the temperature at which the experiments are made the more rapid and energetic will be the action of the disinfectant. Cholera bacteria are not destroyed by mercuric chloride $(1-1000)$ in one hour at $-3^{\circ}$, whereas at $36^{\circ}$ they are killed in a few minutes. 
4.-Immediate and thorough contact with the disinfectant of all the organisms present. This can be done only with bacterial suspensions in which each organism is entirely free and separate from others. To obtain such a suspension, it is necessary to filter through glass-wool or absorbent cotton and then to agitate the liquid thorough$l y$, at a temperature of about $37^{\circ}$, until microscopical examination shows no aggregations of bacteria. Silk threads which have been soaked in bacterial suspensions and then dried are open to the objection that, on treatment with the disinfectant, the organisms are unequally exposed and some may even be protected by their position. The same objection, to a less degree, applies to cover-glasses on which a thin film of the suspension has been deposited.

5. - The number of bacteria in a given experiment. It can be shown readily that the greater the number of bacteria present the more slowly will disinfection take place. In order, therefore, that the results may be comparable, approximately the same number of organisms should be present in each experiment. This is readily ascertained by diluting a small portion of the bacterial suspension with 1000 parts of sterilized water and then making a gelatin plate with one drop of this dilution.

A more rapid procedure is to dilute 2 or 3 drops of the suspension to 100 c.c. Freshly distilled water containing 1 or 2 per cent. of formaldehyde should be used for this purpose. The suspension is transferred to a Thoma-Zeiss counter and the number of bacteria on the ruled square counted. Each of the small squares represents कơेo $\mathrm{cu}$. mm. and, since there are 400 of these, the total square corresponds to $\frac{1}{10} \mathrm{cu} . \mathrm{mm}$. Hence, the number of bacteria found under this square multiplied by 10,000 will give the number present in 1 c.c. of the diluted suspension. It is well to allow 20 or 30 minutes for the bacteria to settle before counting. 
6. -The amount of disinfectant which is carried over to each sub-culture. Thus, when the disinfectant is applied to the bacterial suspension and, at the end of stated intervals, transfers of 1-3 loopsful of the mixture are made to sterilized nutrient media, a sufficient amount of the disinfectant may be carried over to prevent the growth of the organism although it may still possess vitality. This has been a most serious source of error in the past. The error is more marked, the greater the antiseptic power of the disinfectant. It is, of course, less marked where the substance has weak antiseptic properties, and, where the transplantation occurs into relatively large amounts of the "nutrient medium (10 to 15 c.c.). It must be remembered that in all cases the first action of a disinfectant is to attenuate the organism, and, that when the latter is in this condition, a much smaller amount of the disinfectant will act as an antiseptic and prevent growth. This has been especially shown to be the case with reference to the action of mercuric chloride on anthrax spores. Formerly, it was supposed that these were killed by this substance in a strength of 1-1000 in one minute. If the mercury which is held fast by the silk thread, and which cannot be removed by mere washing, is rendered inert by the action of hydrogen sulphide it can be shown that the organism is alive and infectious even after an exposure of 4 hours. It may even possess vitality after an exposure of 24 hours. The first action of the disinfectant is to attenuate the organism, the growth of which is then prevented by mere traces of mercury. One part in two million according to Geppert suffices to produce this result.

When making transplantations in the subsequent work on disinfectants, the platinum wire should be provided with a large loop having about $2 \mathrm{~mm}$. clear diameter. The droplet of mercuric chloride (1-1000) adhering to this loop weighs about $10 \mathrm{mg}$. Hence, when transplanted to $10 \mathrm{c.c}$. of bouillon the latter will contain mercuric chloride in the 
proportion of 1 to $1,000,000$. It is evident, therefore, that in such work only one loopful should be carried over into. the bouillon, the volume of which should not be less than 10 c.c.

7.-Observation of the sub-cultures over a considerable length of time. The failure of tubes to develop within 24 . hours is not a positive indication that the organism has been destroyed by the disinfectant. In the attenuated condition the organism will grow more slowly than it would if it were normal and in passession of full vitality. Moreover, as stated already, traces of the disinfectant which are carried over in the experiment will still further tend to retard the growth. For these reasons the tubes should be kept under observation for at least one week before definite conclusions can be drawn.

8.-Temperature at which the sub-cultures are kept. The organism which has been exposed to the action of the disinfectant should be placed under conditions which are themost favorable to its growth. That is to say, the best nutrient medium and the most suitable temperature should be furnished. Transplantations made into gelatin and kept at ordinary room temperature frequently fail to grow while parallel bouillon and agar cultures, placed in the incubator, develop. It is, therefore, desirable to make the transplantation to the surface of inclined agar tubes or into bouillon and to keep the tubes under observation at a temperature of about $37^{\circ}$ for a week or more.

9. - Negative experiments with animals inoculated with organisms exposed to heat, or to the action of chemicals prove but little. The organism may be dead, or it may have become attenuated and is therefore without action. In the latter case it may still grow on artificial media. Thus, anthrax spores, which are exposed to the boiling temperature: 
for 2 minutes no longer kill guinea-pigs, but nevertheless they can grow in tubes, even after 5 minutes' exposure. On the other hand, positive results may be obtained by inoculating white mice or guinea-pigs with the mixture of bacteria and disinfectant; whereas, the same material transplanted to a nutrient medium may fail to grow owing to the antiseptic power of the disinfectant which is carried over.

10.- Control experiments.-A dozen or two of the uninoculated bouillon tubes should be placed in the incubator, together with the sub-cultures proper, in order to eliminate possible error. Moreover, as an additional control, at least one tube of bouillon should be inoculated with the original untreated culture, cover-glass or silk-thread.

Frequently, the sub-cultures yield doubtful results. The bouillon in that case is slightly clouded and the growth itself is rather uncertain. In this case, the tubes should be returned to, and kept in the incubator for about a week. Or, qne or two loopsful should be transplanted to freshly inclined agar.

If the growth that develops shows the slightest variation from the normal one, it should be examined under the microscope in order to exclude contaminations.

The action of a disinfectant on bacteria may be studied by bringing it into contact with infected silk-threads, muslin squares, cover-glasses, or by mixing direct with the bacterial suspension. The preparations of these specimens has been given on p. 517 .

Silk-threads.-This method was introduced by Koch and has been used extensively. Silk, linen or-cotton threads may be used. They are cut up into convenient lengths, placed in a plugged tube, sterilized and kept for future uṣe (p. 517).

To ascertain the disinfecting action of a solution, a dried thread, impregnated with the bacteria to be tested, is immersed in it for a given length of time, as for instance, 2 minutes. It is then removed with sterilized forceps and gently washed in sterilized water or in al- 
cohol, Finally, it is transferred to a tube of nutrient bouillon (10 c.c.) and then set aside in the incubator for a week or more. Similar tests with exposures of $5,10,30$ and 60 minutes are made.

The objections to this method are threefold and have already been incidentally mentioned. In the first place; the bacteria on the thread may not be evenly exposed to the action of the disinfectant; secondly, the disinfectant itself may. be transferred to the nutrient medium; and lastly, a small amount of the disinfectant may remain in chemical combination with the silk thread and by its presence inhibit the development of the already attenuated organism. The attempt is made to obviate the second objection by washing the threads. This may be successful in some cases, but in others it fails. The third objection is especially true of mercuric chloride which is apparently held fast by the fiber and can only be removed by the action of hydrogen sulphide (Geppert).

2.-Muslin squares.-The preparation of these specimens is given on p. 517. They are used chiefly as test-objects in experiments on the disinfection of rooms.

3.-Cover-glasses. This method was introduced by Geppert and has been used by Spirig and others. To test a disinfectant a dry cover-glass, streaked with the organism in the manner described on p. 517 , is immersed in it for a given length of time as in the case of the silk threads. It is then removed with sterilized forceps and washed in a large volume of sterilized water for about $\frac{1}{4}-\frac{1}{2}$ hour. The cover-glass is then placed in sterilized bouillon which is' set aside in the incubator.

The advantages of this method are (1) that a thin film of evenly spread bacteria is employed; and, (2) that the cover-glass does not unite with the disinfectant, as is the case with the silk threads. It is open to the objection, which holds true also for the silk threads, that the process of desiccation tends to lower the vitality of the organism. Furthermore, it may be urged that the disinfectant has not free access to all sides of the bacteria.

4.-Bacterial suspensions. - This method in some of its modifications is the one which is commonly employed, and, if used with proper precautions, it will yield perfectly reliable results. The first essential is to secure a suitable suspension of the organism to be tested. Directions for doing this are given on p. 514.

In general the procedure consists in adding to a given volume of the suspension an equal volume of the disinfectant of double the 
strength to be tested. At stated intervals $(1,2,5,10$ minutes, etc.) one large loopful of the mixture is transferred to nutrient bouillon (10 c.c.), or to agar and these tubes are then set aside in the incubator.

The method as given is open to the objection that an appreciable amount of the disinfectant is transferred each time to the culture tubes and that it may prevent growth. This is specially true with substances which possess marked antiseptic properties, as mercuric chloride. Whenever possible, the disinfectant should be rendered inert. Thus, traces of mercuric chloride can be removed by precipitation with hydrogen sulphide. With other substances the error is not so marked and is partly counterbalanced by keeping the tubes in the incubator for many days. The important point is to inoculate into a large volume of bouillon, not less than 10 c.c., and when this is done the results with mercury disinfectants are as good, if not. better, then when hydrogen sulphide is used.

Laboratory work.-The student will test the action of mercuric chloride (1-1000), carbolic acid (5 per cent.), lysol (5 per cent.) and formaldehyde ( 5 per cent.) on the following organisms: Typhoid bacillus, Staphylococcus pyogenes aureus, and Anthrax spores. The results. are to be tabulated.

Action of mercuric chloride. -A solution of mercuric chloride (1-500) in distilled-water is prepared. It is well to steam for a few minutes all freshly prepared disinfectants in order to insure freedom from error.

a.-By means of a sterile pipette 10 c.c. of this disinfectant solution are placed in a small, sterile Erlenmeyer flask or in an Esmarch dish, and an equal volume of the bacterial suspension (p. 514) is added, likewise by means of a sterile pipette. The liquid is mixed at once. At intervals of $5,10,15,30,60$ and 120 minutes, a large loopful of the mixture is transplanted to at least 10 c.c. of sterile bouillon. The loop used should have a clear diameter of $2 \mathrm{~mm}$. The set of tubes, properly labeled, are then placed in the incubator.

b.-In the above method the injurious effects of the mercury carried over in the transplantation are largely counteracted by the large volume of bouillon employed. The effects of the mercury can be almost wholly done away with by passing $\mathrm{H}_{2} \mathrm{~S}$ through the suspension. In this case, however, care must be taken to avoid two possible errors. As a result of the passage of the gas, $\mathrm{HCl}$ is liberated and, unless it is promptly neutralized, it may affect the test-organism. Again, the precipitate of mercury sulphide tends to drag down the 
suspended bacteria, and, as a result, the liquid may contain few or none. A loopful of the liquid, transplanted to bouillon, may therefore give no growth, although living organisms may be present in the tube. Constant results in "this method can only be obtained by transplanting several large drops by means of a drawn-out tube pipette.

The Liborius tube is admirably adapted for testing by this method. A small amount of dry $\mathrm{Na}_{2} \mathrm{CO}_{3}$ on the point of a knife (about $25 \mathrm{mg}$.) is placed in each tube. These are then sterilized in the dry-heat sterilizer.

To 20 c.c. of the bacterial suspension an equal volume of the mercury solution is added observing the same precautions as under $a$. At the end of 5, 10, 15, 30, 60 and 120 minutes about 2.5 c.c. of the mixture are transferred to a Liborius tube which is connected at once with a $\mathrm{H}_{2} \mathrm{~S}$ generator. The gas should be passed for 1 or 2 minutes, and the tube is then set aside for the mercury precipitate to subside. When this has taken place $2-3$ drops of the clear liquid are transferred by means of a sterile drawn-out pipette (p.457) to a tube of nutrient bouillon, which is eventually set aside in the incubator. Obviously, the same pipette can be used in transferring the several portions of the original mixture to the Liborius tubes, but a separate pipette must be used for each inoculation made from the latter.

When the mixture undergoing examination contains a very small amount of mercury (1-5000), or when gelatin or soap is present the mercury sulphide will not precipitate but will remain in solution. In such cases, it can be thrown out of solution by adding an equal volume of sterile saturated $\mathrm{NaCl}$ solution to the mixture in the Liborius tube before the $\mathrm{H}_{2} \mathrm{~S}$ is passed.'

Action of carbolic acid.-A 10 per cent. solution of carbolic acid is first prepared, by the aid of gentle heat. 5 c.c. of the bacterial suspension are placed in a sterile test-tube, Esmarch dish or small Erlenmeyer flask, and an equal volume of the slightly warmed, perfectly clear, 10 per cent. solution of carbolic acid is added. The liquid is at once thoroughly mixed and cooled. At intervals of 1, 3, 5, 10, 15, and 30 minutes a loopful of the mixture is transferred to a tube of bouillon which is labelled and eventually placed in the incubator.

In the case of anthrax spores it will be well to inoculate bouillon tubes with the mixture at the end of $1,3,6,24$, and 48 hours. The liquid should be thoroughly mixed just before making each transplantation.

Action of lysol.-The mixture of disinfectant and suspension is made according to the directions just given. The tests are likewise carried out in the same way. 
Action of formaldehyde.-The commercial formaldehyde containg approximately 40 per cent. of the active constituent. The 10 per cent. solution is prepared by adding 10 c.c. of formalin to 30 c.c. of sterile water. 5 c.c. of this solution are placed in a sterile Esmarch dish or test-tube and an equal volume of the suspension is then added. At intervals of $1,3,5,10,15,30$ and 60 minutes a loopful of the mixture is transplanted to bouillon.

In the above method of testing no account is taken of the numerical decrease of the organisms present. This, however, can readily be done by making agar Petri dishes at the intervals given above. The plates are placed in the incubator and the colonies that develop can then be counted.

\section{. Testing of Antiseptics.}

The first action of a chemical substance, when added to a recently inoculated culture medium, is to inhibit the growth of the organism. If the chemical substance is highly poisonous and is present in sufficient amount it will eventually kill the bacteria present. On the other hand, many weak substances, commonly designated as preservatives, will prevent the development of bacteria, but are not able to destroy them. It is necessary, therefore, to clearly understand the distinction between an antiseptic and a germicide. The latter kills a growth, whereas the former prevents its further development. A germicide, when sufficiently diluted, may act as an antiseptic. When an agar tube, inocu'lated with the spores of the hay bacillus, is exposed to an atmosphere of sulphur dioxide it becomes cloudy, and if it is then placed in the incubator no growth will result. Apparently the organism has been killed by the sulphur dioxide. If, however, a platinum wire is passed over the surface of the cloudy agar and is then rubbed over a fresh agar tube, the latter will show in a few hours an' abundant growth. Enough sulphur dioxide was dissolved in the first agar to prevent the growth of the organisms on its surface. It was not strong enough, however, to destroy them. 
The student should test the antiseptic action of several chemicals, according to the following directions, on one of the bacteria employed in the disinfection experiments.

Antiseptic action of mercuric chloride.-A bacterial suspension is made by adding a few drops of a fresh bouillon culture of the germ to about 200 c.c. of sterile bouillon. The 1:500 mercuric chloride solution is also used. 4 large sterile tubes are numbered consecutively, and equipped as follows:

No. $1-1$ c.c. of the $\mathrm{HgCl}_{2}+9$ c.c. of the suspension $=1: 5,000$.

No. $2-0.5$ c.c. of the $\mathrm{HgCl}_{2}+9.5$ c.c. of the suspension $=1: 10,000$.

No. $3-0.25$ c.c. of the $\mathrm{HgCl}_{2}+9.75$ c.c. of the suspension $=1: 20,000$. No. 4-0.1 c.c. of the $\mathrm{HgCl}_{2}+9.9$ c.c. of the suspension $=1: 50,000$.

The tubes are then placed in the incubator and examined at the end of 24 hours. From the cultures that show no growth, or at most a very faint cloudiness, a few drops should be transplanted to sterile bouillon tubes. The two sets should be returned to the incubator and examined on the following day. Hanging-drop preparations of the two sets should be made. Involution forms may be expected. The results should be tabulated.

Antiseptic action of carbolic acid.-An aqueous 1 per cent. solution of phenol is placed in a stoppered flask or bottle and steamed for a few minutes. The same bacterial suspension is used as above. 5 sterile tubes are numbered and equipped as follows:

No. $1-2$ c.c. of the phenol +8 c.c. of the suspension $=1: 500$.

No. $2-1$ c.c. of the phenol +9 c.c. of the suspension $=1: 1,000$.

No. $3-0.5$ c.c. of the phenol +9.5 c.c. of the suspension $=1: 2,000$.

No. $4-0.25$ c.c. of the phenol +9.75 c.c. of the suspension $=1: 4,000$.

No. $5-0.1$ c.c. of the phenol +9.9 c.c. of the suspension $=1: 10,000$.

The tubes are then placed in the incubator and examined as in the preceding experiment.

Antiseptic action of formaldehyde. -2.5 c.c. of the commercial 40 per cent. solution of formaldehyde are diluted to 100 c.c. with sterile water (= 1 per cent.). A series of 5 dilutions is then made employing the same quantities as in the above experiments.

Antiseptic action of sodium benzoate-An aqueous 1 per cent. solution of this salt is sterilized by exposure to steam. The 5 dilutions are prepared and tested in exactly the same manner as in the case of carbolic acid. 
Repeated cultivation of an organism, generation after generation, in the presence of an antiseptic such as carbolic acid will result in a more or less profound alteration of the physiological properties of the organism. Thus, when the anthrax bacillus is grown at $32^{\circ}$ in veal bouillon, containing variable amounts of phenol (from 1: 500 to 1: 5,000 ) and transplantations are made every 8-10 days, the sporeless or asporogenic modification will be obtained. If a similar culti. vation is carried on at $42.5^{\circ}$ not only is the sporeless variety obtained, but this is also deprived of its virulence. In other words, the antiseptic acting at a higher temperature induces more marked alterations than at a lower temperature and hence give rise to sporeless, attenuated cultures. The asporogenic characteristic is permanent on ordinary media but the spore production is said to return after cultivation on peptonless agar.

Similar modifications are obtained by growing motile bacteria in media containing carbolic acid. Thus, the Eberth and colon bacillus will lose their motility, when carried through several generations at $39^{\circ}$, in bouillon containing about 1 : 3000 of carbolic acid.

\section{Room Disinfection.}

In this laboratory a special room is provided having a capacity of $1,016 \mathrm{cu}$. feet ( $28.8 \mathrm{cu}$. m.) in which gaseous disinfectants may be tested. The gaseous compounds com. monly in use at the present time are sulphur dioxide and formaldehyde.

The sulphur, either in the form of flowers or in rolls, is placed in an iron water-bath which is set over a large pan of water. 3 pounds of sulphur per $1,000 \mathrm{cu}$. feet are ordinarily employed. To this amount, 50 c.c. or more of alcohol are added and this is then set on fire. The specimens have previously been arranged in the room, under the desired experimental conditions. All cracks about the door and win- 
dows are securely caulked with strips of cloth, or better with putty. The usual time of exposure is 20 hours.

The formaldehyde experiments may be carried out, either by sprinkling large sheets of muslin or filter-paper

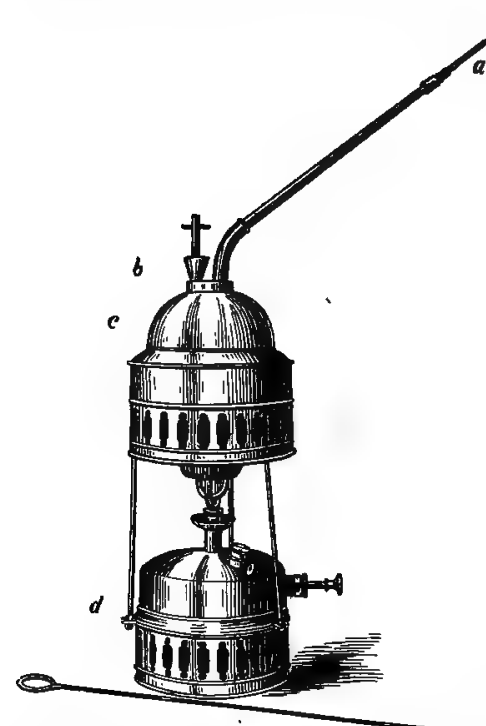

FIG, 76. The author's formaldehyde apparatus for room disinfection. $a-$ Narrow paratus for room disinfection, $a-$ Narrow tube provided with solid stopper; $c$-Reservoir; d-Kerosene burner. with the necessary amount of the 40 per cent. solution and then hanging these up in the room, or by distilling the formaldehyde into the room through a tube inserted into the key-hole. The author's apparatus ${ }^{i}$ shown in Fig. 76 has been especially designed for practical room disinfection. The copper vessel can be heated with a Bunsen burner or with a Primus kerosene lamp. 150 c.c. of the 40 per cent. formaldehyde solution are used for each $1,000 \mathrm{cu}$. ft. of air space.

The bacterial suspensions are prepared according to the directions given on p. 514; and sterile silk-threads, muslin squares and cover-glasses are then infected. The action of the disinfectant should be studied, at the same time, on moist and on dry specimens. The moist specimens can be kept in this condition, for hours if need be, by placing them over water in a large moistchamber. The dried specimens are obtained by placing the freshly prepared set in an incubator at $39^{\circ}$ for $2-3$ hours, the cover of the dish being slightly ajar. When the specimens have become dry they should be loosened from the bottom by means of sterile forceps.

${ }^{1}$ Teacher's Sanitary Bulletin, No. 3, Michigan State Board of Health; Medical News, May, 1898, p. 641. 
The wet and dry specimens are exposed in open Esmarch dishes in the room during the disinfection process. At the close of the period of disinfection, 5,10 , or 20 hours, the room is entered and the covers promptly replaced. Each specimen is then taken up by a pair of forceps and transferred to a tube of bouillon. The forceps must be sterilized in the flame before making each transfer.

The student will make experiments in the manner indicated with anthrax spores, diphtheria and typhoid fever bacilli, and with staphylococci. The results should be carefully controlled as indicated on p. 523 .

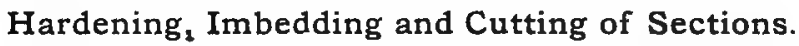

The direct microscopical examination of streak preparations made from the organs and tissues of infected animals, as well as cultural experiments will, as a rule, reveal the presence of micro-organisms. In order to ascertain the presence and especially the distribution of organisms within the tissues and organs it is necessary to harden these, then to cut sections and finally to stain the sections by suitable methods.

The tissue to be hardened must be cut up into small pieces, about 5-8 mm. in thickness. In special cases even thinner pieces must be used. These are then placed in the fixing and hardening fluid. It is always advisable to place the pieces of tissue on a piece of filter-paper or on some absorbent cotton. The liquid thus has free access to all parts of the tissue. The fixing and hardening of tissue, which is to be stained for bacteria, is usually done in alcohol, mercuric chloride or in a formaldehyde solution. Small widemouth bottles should be employed.

Alcohol. - The pieces of tissue, supported on filter-paper or cotton, are placed direct in 95 per cent. alcohol. They are allowed to remain in this alcohol for 3 or 4 days, after 
which they are transferred to absolute alcohol for 1 to 2 days. In case the pieces of tissue are large it will be well to make a second transfer to absolute alcohol.

The tissues may be placed in alcohol containing 2.5 per cent. of the commercial formaldehyde $(1$ per cent. of the gas). This may be especially desirable when the tissue contains highly virulent organisms.

Mercuric chloride. - A saturated aqueous solution of this salt is used. An addition of 5 per cent. of glacial acetic acid may be made. The tissue is fixed in this solution in from 4 to 12 hours. It may, however, be left in the liquid for 24 hours. The tissue must then be thoroughly washed in running water. This is done by placing the bottle, the mouth of which is covered with a piece of wire gauze, under a hydrant for 12 to 24 hours. The pieces of tissue are then placed in 70 per cent. alcohol for 24 hours, after which they are transferred for a like period of time to 95 per cent. and finally to absolute alcohol.

A serious draw-back to the use of mercuric chloride is its tendency to deposit a black, granular or semi-crystalline precipitate in the tissue. The granules should not be confounded with micrococci.

Formaldehyde.-A 4 per cent. solution of formaldehyde can be used for hardening tissue. This is prepared by adding one part $(10$ c.c.) of the commercial 40 per cent. solution of formaldehyde to 9 parts (90 c.c.) of distilled water. The tissue is allowed to remain in this solution for 4 to 12 hours. 'It is then transferred to 70 per cent. alcohol for 24 hours, after which it is placed for a like period of time in 95 per cent. and finally into absolute alcohol.

It will be seen that no matter what solution is used for fixing, eventually the tissues are placed in absolute alcohol. When thoroughly dehydrated the material is now ready for cutting direct, or for imbedding and subsequent cutting. If the tissue is to be kept for 
some time it is advisable to preserve it in a dilute alcohol of about 70 per cent. strength. Many bacteria, howerer, are affected by prolonged sojourn in alcohol to such an extent that they will not readily stain. This is notably true of the leprosy and tubercle bacilli. The author prefers, therefore, to imbed the tissue in paraffin and keep it in this form. The paraffin with the tissue may be kept in bottles rather than be put up into blocks.

Imbedding in paraffin.-The first step toward imbedding in paraffin is to place the tissue from absolute alcohol into toluol, for 24 hours, then for a like period into a strong solution of paraffin in toluol. Xylol, chloroform or turpentine may be used in place of toluol.

The paraffin necessary for the next step is kept in a . melted condition in a suitable oven. An ordinary air-bath may be used, although it is better to employ one with a water-jacket. The temperature of the oven should be about $50^{\circ}$, and is controlled by a thermo-regulator ( $p$. 246). Two wide-mouth bottles should contain the necessary soft and hard parafin. The soft paraffin melts at from 38 to $42^{\circ}$, whereas the hard paraffin melts at about $46^{\circ}$. The latter is usually prepared by bringing together equal parts 38 and $52^{\circ}$ paraffin. In very warm weather it will be necessary to use two parts of the latter to one of the former.

The tissue, after permeation with the toluol paraffin mixture, is placed in a small wide-mouth bottle, or better in the so-called tube vials. It is covered with melted soft paraffin and placed in the oven for 12 to 24 hours. The soft paraffin is then replaced by the melted hard paraffin which is also allowed to act for 12 to 24 hours. 'The tissue now thoroughly permeated with hard paraffin is ready to be blocked.

A rectangular trough, made by bringing together two glass L's on a zinc plate, is filled with melted hard paraffin. When the paraffin has slightly congealed on the bottom of trough, the piece of tissue is introduced into the liquid by means of a previously warmed pair of forceps. As soon as the paraffin has cooled, so as to become opaque, the plate is 
placed in cold water. The paraffin now sets thoroughly, and after a few minutes the L's can be removed. The block is now ready to be cut.

When a number of paraffin blocks are prepared, and especially if they are to be kept for some time, they should be labeled. This can be done by placing the label, with the written side turned down, in the bottom of the trough before pouring in the paraffin. The label then adheres to the paraffin block.

Imbedding in celloidin.-The tissue is transferred from absolute alcohol to a mixture of equal parts of absolute alcohol and ether for 24 hours. It is then placed in ether for 12 hours, and from this it is transferred to thin celloidin where it remains for 2 to 3 days or longer. The tissue is then placed in thick celloidin for an equal length of time after which it is ready to be blocked.

The end of a cork or similar block of wood is covered with thick celloidin. This is allowed to partially evaporate and the tissue is then placed on the block. After partial drying in the air the block is placed in 80 per cent. al. cohol for 24 hours. The material can then be sectioned or it may be preserved in this alcohol for future use.

Ordinary collodium may be used. The thick solution can be readily obtained by allowing some of the collodium to remain in an open wide-mouth bottle for some hours. The material can be thinned whenever necessary by the addition of a mixture of equal parts of absolute alcohol and ether.

Cutting sections.-A good sliding microtome should be on hand. This can be used for sectioning the material which has been frozen, hardened in alcohol, or imbedded in paraffin or celloidin.

- The tissue which has been fixed and hardened in alcohol can be cut direct without resorting to the imbedding process. The result, however, cannot be said to be as good. To do this a piece of the tissue is attached to a small cork by means of a glycerin gelatin mixture made by warm- 
ing 1 part of gelatin, 2 parts of water and 4 parts of glycerin. The cork is then securely clamped to the microtome and the sections are cut. The tissue and knife must be kept moistened with alcohol and the sections are transferred at once to alcohol by means of a camel-hair brush.

Frozen sections. - The tissue fixed and hardened in alcohol may be cut by means of the freezing microtome. It should not be more than 2 or $3 \mathrm{~mm}$. thick. The alcohol must first be removed from the hardened tissue by immersion in water. This can be accomplished in cold water in from 4 to 8 hours, depending on the size of the piece. If the water is warmed to $38^{\circ}$ the alcohol will be removed more rapidly, in from 1 to 2 hours. The tissue can then be frozen by the ether spray apparatus and sections cut. The knife is moistened with water and each section is at once transferred to water. This method is very useful in the staining of bacteria in tissues.

Paraffin sections. - The paraffin block is trimmed square and then firmly attached to the metal holder. When cutting paraffin sections the knife must be kept perfectly dry. Moreover, the edge of the knife should be parallel to that of the block. In other words', the knife is not fixed in a slanting position as in the case of alcohol hardened or celloidin imbedded objects.

This method of imbedding and cutting sections is easy of execution and thinner sections can be obtained than by any other procedure. It is to be preferred to the others for making bacteriological examinations of tissue. The paraffin sections are transferred from the knife by means of a brush or needle to a piece of clean filter-paper, which is covered by a bell-jar. Before staining the sections it is, as a rule, necessary to remove the paraffin. This can be readily done by means of toluol, xylol or turpentine. The sections, contained in an Esmarch dish, should be washed 
sereral times with the solvent to insure complete removal of the paraffin. The washed sections can then be bottled. in 70 per cent. alcohol.

The paraffin should not be removed from the sections in the manner described unless these are sufficiently thick to hold together. Very thin sections are liable to fall to pieces, and moreover, are difficult to handle in the subsequent process of staining. In such cases, it is advisable to fix the section to a cover-glass before removing the paraffin. This can be easily done by the following method:

The paraffin sections frequently become curled or folded. This difficulty can be readily. overcome by placing the sections in some tepid water contained in a large evaporating dish. The water must not be so warm as to melt the paraffin. The sections promptly spread out on the surface of the water. They are now ready to be taken up on coverglasses. For this purpose only perfectly clean coverglasses (p. 140) should be used.

A minute drop of the albumin fixative is placed on the cover-glass and spread out in a very thin layer. The coverglass, thus prepared, and held in a pair of forceps, is placed under the floating section. In this way the section can be raised and removed from the water. The section should be pressed out flat on the cover-glass by gently applying the tip of the finger. The cover-glasses, thus equipped with sections, are now set aside in the incubator at about $37^{\circ}$ for 24 hours in order that the sections may become firmly fixed to the glass. The paraffin can now be removed from the cover-glass by treatment with toluol, or other solvent, in the manner indicated above.

The albumin fixative is prepared by cutting up the white of an egg with a pair of scissors. The liquid is then strained through muslin. An equal volume of glycerin is then added to the filtrate and thoroughly mixed. A piece of camphor may be added to prevent the development of moulds. 
Celloidin sections. - In cutting sections from the celloidin block this, as well as the knife, should be kept moistened with dilute alcohol. The sections can be kept for a long time in 70 per cent. alcohol. On treatment with anilin dyes the celloidin becomes slightly stained and for that reason it has been suggested to dissolve out the celloidin, before staining, as in the case of paraffin. This, however, is wholly unnecessary in bacteriological work.

Inasmuch as oil of cloves dissolves celloidin, the sections should not be cleared in this oil but in oil of origanum.

\section{The Staining of Sections.}

It is very difficult at times to demonstrate the presence of organisms in sections, although they may be easily shown to be present in ordinary streak cover-glass preparations. This is frequently due to the absence of any sharp means of differentiating the organism from the surrounding tissue. The basic anilin dyes employed, it should be remembered, are nuclear as well as bacterial stains. The organism, therefore, has the same stain as the mass of tissue in which it lies imbedded. In many cases, however, a sharp differentiation can be obtained by double staining. either by Gram's method; or, as in the case of leprosy and tuberculosis, by the application of the usual process for staining these bacilli.

The student should begin the staining of sections made from the kidney, liver, spleen and lungs of a guinea-pig which died of anthrax. After acquiring the technique necessary for the successful staining of bacteria by the simple method and by Gram's process the other special stains can then be taken up. The concentration of the dye, time of exposure and the temperature of the liquid are important factors which must not be lost sight of by the operator. The concentration of the acid or alcohol which 
is employed in decoloration and the length of time that these are allowed to act on the stained section likewise affect the result. In the event of failure the student, therefore, should ascertain by systematic trial which of these factors is the one at fault. Success in staining sections requires an intelligent perseverance in, and a study of the method employed.

As a rule, a few sections should be transferred to some water in an Esmarch or Petri dish. Owing to the diffusion currents the sections spread out perfectly. The thin sections can then be transferred by means of a mounted needle, at times assisted by a spatula, to the filtered, staining fluid. The latter contains either fuchsin, gentian violet or methylene blue.

The fuchsin can be used in dilute aqueous solution (p. 147), such as is employed in the simple staining of coverglasses. Carbolic fuchsin (p. 292) is used for the simple and double staining of bacteria.

Gentian violet is employed in dilute aqueous solution, or as anilin-water gentian violet (p. 288). This dye stains rapidly and deeply and it should not, therefore, be allowed to act as long as the other dyes.

Methylene blue is a slow, weak stain and should be allowed more time to act than either of the preceding. It may be used in dilute aqueous solution or as Loffler's alkaline methylene blue solution ( $p .332$ ). Kühne's carbolic methylene blue, made by adding $1.5 \mathrm{~g}$. of methylene blue and $10 \mathrm{~g}$. of alcohol to $100 \mathrm{c}$.c. of 5 per cent. carbolic acid and heating till complete solution takes place, is very useful.

\section{ANTHRAX BACILLUS.}

Simple stain. -The section is transferred from water to the dilute anilin dye, and is allowed to remain there for from 5 to 15 minutes. It is then washed for 2 or 3 minutes 
in water in order to remove the excess of dye. After which it is placed in very dilute acetic acid $(1$ c.c. of the glacial acid to 1,000 c.c. of water) for $1 / 2-1$ minute. The treatment with acetic acid is not always necessary and should be avoided if possible. 'The section is now placed in strong alcohol for $1 / 2-1$ minute, and is then transferred to clean water. It is now taken up on a spatula, placed on a slide with a drop of water, covered, and examined with a No. 7 objective. This examination is made in order to orient oneself as to the condition of the specimen. The bacteria should be deeply stained and should be differentiated as much as possible from the surrounding tissue. If they are feebly stained it is unnecessary to proceed with the specimen. If the tissue is still deeply stained, thus masking the bacteria, it should be subjected again to decoloration with aceticwater and alcohol, and re-examined.

When the section shows the proper degree of differentiation, it should be placed in absolute alcohol, for a few seconds in order to thoroughly dehydrate it. Inasmuch as this treatment with alcohol removes additional dye, it is well to stop the decoloring process, as given above, while the specimen is still slightly over-stained. The treatment with alcohol, when dehydrating, will remove this slight excess of stain and thus complete the differentiation.

The section is then placed in oil of cloves, cedar or origanum for some minutes, after which it is transferred to xylol and then placed on a clean slide. The excess of xylol is removed by the application of a piece of filter-paper. A drop of Canada balsam is now applied, and a clean coverglass is placed in position. Gentle pressure, or slight warming will cause the balsam to.spread out evenly. A full history of the specimen should be recorded on the label, giving the name of the organism present, the animal and organ used, the date and method of preparation.

Oil of cloves can be used to good advantage in the clearing up of sections. It dissolves some of the stain and 
thus assists in the differentiation. This is especially true when it is used in Gram's method. All trace of the oil, however, must be removed from the section by washing in xylol. Some stains, like methylene blue, are readily dissolved by the essential oils, and in such instances the oil can be omitted. The dehydrated section in that case is placed direct in xylol.

In some instances the treatment with xylol is dispensed with. Thus, the section may be transferred direct from oil of origanum to a glass slide. After wiping off the excess of oil around the specimen, a piece of filter-paper is firmly pressed down on top of it. This is done several times until all the oil has been removed. The flattened section is now treated with balsam and covered.

When transferring the specimen on a spatula, care should be taken to keep the clean surface of the instrument evenly moistened with the liquid in which the section lies. The spatula is slipped under the section which is then drawn up on the blade by means of a needle. To remove the section from the spatula to a liquid requires no special care. When the section, however, is to be transferred to a slide a drop of the liquid, xylol or oil, should be first placed on the center of the slide. With the end of the spatula resting in the drop the section is drawn down by the needle, till a portion of it rests on the slide. By holding this portion of the section with the needle the spatula can now be easily withdrawn.

Instead of using the ordinary dilute anilin dyes, Loffler's or Kühne's methylene blue and dilute Ziehl's solution may be used. Thus, Pfeiffer stains the section for half an hour in dilute Ziehl solution, then transfers it to absolute alcohol which is very slightly acidulated with acetic acid. When the originally dark-red section changes to a peculiar reddish violet color, it is cleared up at once in xylol and mounted in balsam.

In staining sections it is advisable not to overstain too 
much. An excessive exposure to dye requires a corresponding exposure to the decolorizing agents, and, as a result, there is lack of differentiation. Hence, the section should remain in the dye for as few minutes as possible.

Double staining by Gram's method.-This is an extremely useful method but unfortunately it is not applicable to all bacteria. A list of those bacteria which can be stained by Gram's method is given on p. 289. The method is easy of execution and, when properly carried out, it will give clean, beautifully stained preparations.

Fresh solutions of anilin-water gentian violet and of iodine are prepared according to the directions given on p. 288. The stain may be slightly warmed, but this is not necessary. As stated above it is desirable not to overstain too much.

The section is placed in the stain for 10 to 15 minutes. It is then washed in water, or in anilin-water, to remove the excess of dye. In this way, the formation of unsightly deposits in the section, on subsequent contact with the iodine solution, is avoided. The section is then placed in the solution of iodine (Lugol's) for from 3 to 5 minutes. It is. now transferred to absolute alcohol, in which it is gently moved about till most of the stain is removed. 'The sections should not be completely decolored, but should still show a distinct violet color.

It is now placed in very dilute eosin for about $\mathrm{I} / 2 \mathrm{~min}$ ute. Over-staining with eosin will impart to the tissue a deep red color which will thus make an unfavorable contrast for the violet organism. It is preferable, therefore, to stain with eosin so that the tissue has a light pink color. Weigert's picrocarmin can be used instead of eosin. It should be allowed to act for from 3 to 5 minutes. The sections may be first stained with picrocarmin and then subjected to Gram's staining. 
The section is transferred from eosin to absolute alcohol for 1 or 2 minutes. When thoroughly dehydrated it is placed in oil of cloves, and should be allowed to remain in this oil till all the violet.color has been taken out of the section. The sections may remain in this oil over night without removing the stain from the bacteria. The section is then passed through two dishes of xylol, transferred to a slide and mounted in Canada balsam. The deep violet organism should stand out in bold relief against a light pink back-ground.

The section when placed in iodine tends to curl and easily breaks. Very thin sections, therefore, should be fixed on a cover-glass previous to the exposure to iodine.

The following summary of the simple and of the Gram method of staining will be useful:

\section{Simple Stain.}

Dil. anilin stain (5 to $15 \mathrm{~min}$.).

Wash in water (2 to 3 min.).

Acetic water $\left(\frac{1}{2}-\mathbf{~ m i n}\right.$.).

Strong alcohol ( $\frac{1}{2}-1$ min.)

Water and examine.

Ábsolute alcohol (few sec.).

Oil of cloves, or cedar.

Xylol.

Mount in Canada balsam.
Gram's Stain.

Anilin-water gentian violet, (10 to 15 min.).

Wash in water.

Iodine solution ( 3 to 5 min.).

Decolor in absolute alcohol.

Very dilute eosin ( $\left(\frac{1}{2}\right.$ min.).

Dehydrate in abs. alcohol,

(1 to 2 min.).

Oil of cloves (till decolored).

Xylol.

Mount in Canada balsam.

The process of simple staining is a general method which is appliable to nearly all bacteria. It must therefore be resorted to whenever the organism does not take the Gram's stain (p. 290).

In some instances, where Gram's method fails owing to the removal of the dye from the organism by the alcohol, the so-called Weigert's fibrin stain can be used to advantage. The cover-glass preparations or sections are stained for $\mathbf{5}$ minutes or more in anilin-water gentian violet, then rinsed 
in water and exposed to Lugol's iodine solution (p. 288), for 3 to 5 minutes. The specimens are then washed with water, dried with filter-paper, and transferred to a mixture of 2 parts of xylol and 1 part of anilin. They remain in this mixture till the color ceases to be given off, after which they are again dried with paper, covered with Canada balsam and examined.

\section{TUBERCLE BACILLUS.}

Thin sections made from tubercular human lung and from the spleen, liver and mesenteric tubercles of a guineapig which received an intraperitoneal injection of tubercular sputum, are stained according to the following method, which is a modification of the Ziehl-Neelsen process. The sections, if very thin, should be fixed on cover-glasses (p. 536).

It may be well, first of all, to call attention to two conditions which influence the staining of the tubercle bacillus. In the first place, the bacillus loses its specific staining power when the tissue has been kept in alcohol for some time. This is equally true of the leprosy bacillus. It is therefore advisable to use fresh tissues; or, as stated on $\mathrm{p}$. 533, to preserve it in paraffin. Again, Ziehl's carbolic fuchsin solution is not as permanent as it is commonly said to be. 'In time, a tarry deposit forms in the bottle and the staining power of the liquid is materially decreased. A fresh Ziehl solution should be prepared according to the directions given on p. 293.

The section is floated on cold, fresh carbolic fuchsin over night; or, for about $\frac{1}{2}$ hour orf a stain previously warmed to about $40^{\circ}$. The stain can be heated on an iron-plate as shown in Fig. 22. It is then poured into a warm Petri dish. The more deeply the section is stained the more difficult it will be to properly decolor it. 
The section is then transferred to a dish containing water in order to remove the excess of dye. It is now placed in 60 per cent. alcohol for 1-2 minutes, and then into Ebner's solution for $\frac{1}{2}$ minute, after which it is returned to 60 per cent. alcohol for another minute or two, or until it is almost wholly decolored. The light pink color of the section will be displaced on staining with methylene blue.

The almost decolored section is placed in Löffler's methylene blue for $\frac{1}{2}$ minute, after which it is washed in water. It is then placed in absolute alcohol for about 20 seconds in order to dehydrate. A longer exposure to alcohol will remove the blue stain. The section is drained by placing the edge of the spatula or of the cover-glass against some filter-paper; after which it is placed in xylol for 2 minutes. After removal of the excess of xylol by means of filter-paper the section is mounted in Canada Balsam.

Cedar or anise oil may be used, to clear the blue sections. On the other hand, oil of cloves should be avoided because it removes the blue color. A properly stained section will show deep red bacilli on a light blue back-ground.

Ebner's solution is ordinarily used for decalcifying purposes. The acid and alcohol present make it very useful for decolorizing sections, and it is to be preferred to the common procedure of treatment with nitric or sulphuric acids. It is prepared according to the following formula:

$\begin{array}{lr}\text { Sodium chloride, } & 0.5 \\ \text { Hydrochloric acid, } & 0.5 \\ \text { Distilled water, } & 30.0 \\ \text { Alcohol, } & 100.0\end{array}$

Instead of using Ebner's solution for decoloring the sections, a 2 per cent. aqueous solution of anilin hydrochloride can be employed with excellent results as it has little or no tendency to decolor the tubercle bacilli (Kühne, Borrel). The sections can be first stained in hematoxylin or hematein for about 2 minutes. After which they are 
washed in water till they acquire the characteristic bluish tint. They are now placed in Ziehl's solution for about 15 minutes, and then in the anilin hydrochloride solution for a few seconds. They are now washed in 60 per cent. alcohol till the color ceases to be given off, after which they are dehydrated in absolute alcohol, cleared in xylol, and mounted in balsam.

The carbolic fuchsin may be replaced by anilin-water fuchsin or gentian violet, as employed in the original method of Ehrlich.

The two methods for staining tubercle bacilli may be summarized as follows:

1st method.

Carbolic fuchsin (warm, 15-30

min.).

Wash in water.

60 per cent. alcohol (1-2 min.).

Ebner's solution ( $1 / 2 \mathrm{~min}$.).

60 per cent. alcohol (1-2 min.).

Löffler's methylene blue ( $1 / 2 \mathrm{~min}$ ).

Wash in water.

Absolute alcohol (20 sec.).

Xylol.

Canada balsam. 2nd method.

Hematein (2 min.).

Wash and develop in water.

Carbolic fuchsin (15 min.).

Anilin hydrochloride (few sec,).

60 per cent. alcohol.

Absolute alcohol.

Xylol.

Canada balsam.

LEPROSY BACILLUS.

In sections made from fresh tissue, the leprosy bacillus can be stained by the method employed for detecting the tubercle bacillus. But, as stated above, this staining peculiarity is lost, after a time, if the tissue is preserved in alcohol. Although the above method fails, yet the leprosy bacillus can be demonstrated easily in such tissue on staining by Gram's method (p. 541). Under these conditions, excellent results can be obtained by leaving the sections in anilin-water gentian violet over night.

The leprosy bacillus is distinguished from the tubercle bacillus by being easily stained with the ordinary dilute 
anilin dyes: The simple stain method (p. 538) can be applied to sections of the fresh tissue in order to differentiate between the two bacilli. The enormous number of the leprosy bacilli and their massing in the so-called leper cells will assist recognition.

\section{Eberth Bacillus.}

This organism, like that of glanders, cholera, etc., cannot be demonstrated in tissue by any process of double staining. In such cases the simple stain method must be used. The sections should be stained in Loffler's alkaline methylene blue for some hours, then washed slightly in water and placed in a 10 per cent. solution of tannic acid. The sections remain in this mordant for from 10 to $60 \mathrm{~min}$ utes, after which they are washed in water, dehydrated in alcohol, cleared in origanum, and mounted in balsam.

\section{Actinomyces.}

The pus from an abscess should be received in mercuric chloride where it soon hardens. The material after imbedding in paraffin can be cut into sections which should be fixed to cover-glasses. They can be stained by Gram's method or with hematoxylin.

In the latter case, the section should be floated in a Petri dish in water, to which a drop or two of Delafield's hematoxylin solution has been added. It should remain in this stain for an hour or more. Eventually, it is transferred to a large volume of water where it remains till it shows a clear blue color. The section is then dehydrated in absolute alcohol, cleared in oil of origanum, and mounted in Canada balsam. 


\section{LIST OF APPARATUS AND CHEMICALS.}

I Anaerobe tube apparatus, p. 3 I4

I Anaerobe tube apparatus, small

I Anaerobe plate apparatus, p. 3I4

I Anaerobe plate apparatus, vacuum

I Animal holder, Latapie's, p. 268

I Animal holder, Voges', p. 266

I Aspirator, Chapman's

I Autoclave $20 \mathrm{~cm}$. diam., p. 165

3 Battery jars for mędia, I2 2 I $2 \mathrm{~cm}$.

3 Battery jars for media, I $8 \times 24 \mathrm{~cm}$.

3 Battery jars, I5 x $24 \mathrm{~cm}$. p. 273

I Nest beakers

I Blast-lamp, Fletcher's

2 Boards, mounting, p. 279

2 Boards, post-mortem, p. 276

8 Bottles for stains ( $\mathrm{p} . . \mathrm{.}$ 5o), in stand

6 Bottles, 50 c.c.

6 Bottles, 50 c.c., yellow

4 Boxes for slides

I2 Boxes for cultures, p. 5 I 2

2 Burettes, 50 c.c. in to c.c., p. I55

I Burette, I00 c.c., p. 5 I I

2 Burette stands

Burners, Bunsen radial, p. 263

safety, p. 251

Camera lucida

I Colony counter, p. 434

6

- I ordinary

Corks

200 Cover-glasses, No. I, I $5 \mathrm{~mm}$. diam. 50 Cover-glasses, No. I, $20 \mathrm{~mm}$. diam.

I Cylinder, graduated, 25 c.c.

I Cylinder, graduated, 50 c.c.

I Cylinder, graduated, Ioo c.c.

I Cylinder, graduated, 500 c.c.

I Cylinder, graduated, rooo c.c.

I Disinfecting jar, with top, I5 $\times 20$ $\mathrm{cm}$. diam.

I Disinfecting jar, with top, for slides, $9 \times 10 \mathrm{~cm}$.

I Enamelled jar, 2 1., p. I 53

I Enamelled stew-pan, p. 263

I2 Esmarch dishes, $5 \mathrm{~cm}$. diam., p. I72

6

6

2 Files, triangular

$6 \mathrm{~cm}$. diam.

7 chen. diam.

Filter, Pasteur, p. 469 Berkefeld, p. 471

Filtering cylinder, $1 / 2$ 1., I 1., p. 469

Filter-paper, in sheets
Ioo 'Filter-paper, in circles, $20 \mathrm{~cm}$.

Ioo plaited, $32 \mathrm{~cm}$.

Ioo hardened, I $5 \mathrm{~cm}$.

Filter-plate, Witte's, Io cm., p. 237

Flasks, Erlenmeyer vacuum, I $1 / 21$.

Flasks, Erlenmeyer vacuum, $1 / 21$.

200 c.c., P. 469

200 c.c.

50 c.c.

round, 2 1. capácity

I $1 / 21$.

$1 / 21$.

Roux, p. 486

Funnels, $I_{5} \mathrm{~cm}$. diam.

$6 \mathrm{~cm}$. diam.

cylindrical, p. 5 I4

Forceps, broad-pointed, p. 46 I

cover-glass, P. I $4 \mathrm{I}$

narrow pointed, p. $46 \mathrm{r}, 10 \mathrm{~cm}$.

pressure, p. $46 \mathrm{I}$

long, p. 265

rat, p. 273

Formaldehyde generator, p. 530

Gas pressure regulator, p. 250

Glass benches, p. $\mathrm{I}_{72}$ cups with covers, p. 506

globe receivers, I l., p. 471

containers, I l., P. 5 I I

plates, P. 172

rods, $P \quad 172$

slides, white

concave well, p. I43

3

$500 \mathrm{~g}$.

66

tubing, $4 \mathrm{~mm}$. int. diam.; wall I

tubing, $6 \mathrm{~mm}$.

$8 \mathrm{~mm}$.

$22 \mathrm{~mm}$. " wall I.5

stop-cocks, pp. 308,469

vials $65 \times 13 ; 77 \times 26 \mathrm{~mm}$.

Hydrogen generator, Kipp's, I 1.

I Incubator, high temperature, p. 244

I low temperature, p. 179

I Instrument sterilizing case, p. 275

I Iron-box for pipettes

for plates, $5 \times 14.5 \times 17.5 \mathrm{~cm}$.

plate for heating, p. I50

water-bath, $18 \mathrm{~cm}$. diam. tripod, p. I50

roo Labels for slides, white

I2 Liborius tubes

4 L's, glass or metal, p. 533

I Micrometer, cross-wire ocular ocular

stage 
I Microscope, $2 / 3,1 / 8$, I $^{1}$ objectives; 2 eye-pieces; triple nose-piece, Abbe and iris diaphragm

\section{Microtome}

2 Moist chambers, p. 172

I Nuttall's needle, p. 278

I2 Petri dishes, p. I 72, I80

6 Pipettes, I c.c. in $\frac{1}{10}, 18 \mathrm{~cm}$.

3 I c.c. in 1 to

o.I c.c. in Ioro, p. 459

$2,5,10,25$ c.c.

Plate, ice apparatus for, p. I 76 water apparatus, p. 177

Platinum wires, p. I72

Porcelain evap. dishes, $1 / 21$., I 1.

Potato brush knives

Retort stands

Ioo Rubber caps

$2 \mathrm{~m}$. tubing, int. diam., $6 \mathrm{~mm}$.

$2 \mathrm{~m}$. tubing, int. diam., $8 \mathrm{~mm}$.

$2 \mathrm{~m}$. tubing, vacuum, int. diam.

Rubber stoppers Io $\mathrm{mm}$.; wall $4 \mathrm{~mm}$.

2 Scalpels

2. Scissors, $\mathrm{I}_{4} \mathrm{~cm}$.

curved on flat, $12 \mathrm{~cm}$.

fine pointed, Io $\mathrm{cm}$.

large, $25 \mathrm{~cm}$.

Searing iron, p. 275

Spatula for sections

Roux, p. 278

Speculum, p. 502

Sterilizer, dry heat, p. I60

serum, Roux, p. 466 serum, Koch, p. 467 steam, p. I64

3 Syringes, I, 2, 5 c.c., p. $26 \dot{2}$ holder for, p. 263

Test-glass, I $8 \mathrm{~cm}$. high 30 c.c., p. 265 50 c.c.

6

200 Test-tubes, I2 $\times 125 \mathrm{~mm}$.

$200 \quad I_{5} \times 150 \mathrm{~mm}$.

$20 \quad 20 \times 150 \mathrm{~mm}$.

I brush or swab

6 on foot, p. 489

Tubes for sacs, p. 497

2 Thermometer, clinical

Kappeler's, p. 252

$280^{\circ}$

$100^{\circ}$

$60^{\circ}$ in $1^{\circ}$

Thermo-regulators, p. 246

Trephine, Collin's

Tripods

6 Tumblers

I Wash-bottle, siphon

Waste crocks

6 Watch glasses, $5 \mathrm{~cm}$.
I Wax pencil, colored

2 Wire baskets, $24 \times 32 \mathrm{~cm}$. diam.

2 Wire-baskets, I $8 \times$ I $8 \times 24 \mathrm{~cm}$.

2 Wire-baskets, io $X$ i $2 \times$ i $8 \mathrm{~cm}$.

2 Wire cages, p, 274

3 gauze, $\mathrm{s} 6 \mathrm{~cm}$. sq.

Ioo g. Acetic acid, glacial

Ioo “c Agar agar

IOOO "s Alcohol

25 " Anilin hydrochloride

IOO " oil

25 " Bismarck brown

200 " Borax

20 " Canada balsam in tube

100 " Carbolic acid

50 "6" Celloidin

200 "Chloroform

209 " Collodium

25 "Eosin

200 " Ether

I00 " Extract of meat, Liebig's

50 " Ferrous sulphate

50 "Fuchsin

500 " Gelatin

$50^{\circ}$ Gentian violet

roo " Glucose

I00 " Glycerin

25 " Hematein

Ioo " Hematoxylin, Delafield's

100 " Hydrochloric acid

ro "6 Iodine

30 " Lactose

roo " L Litmus

6 sheets paper.

$200 \mathrm{~g}$. Mercuric chloride

5o "s Methyl violet

.50 " Methylene blue

roo " Nitric acid

50 " Oil of anise

50 " cedar

50 " cloves

50 "

I00 " each Paraffin, $40^{\circ}, 49^{\circ}, 52^{\circ}, 56^{\circ}$

2000 " liquid

200 " Pepton, Witte's

Ioo " Picrocarmin, Weigert's

20 " Potassium iodide

"50" Pyrogallic acid

200 "sealing wax

200 " Sodium carbonate

$200^{\prime \prime}$ - hydrate

I00 " Sulphuric acid

50 " Tannic acid

500 " Toluol

500 " Turpentine

100" Vaselin

500 " Xylol

I Kg. Zinc, granulated 


\section{INDEX.}

Abbe condenser, 133, I35

Abrin, 83

Abscess, 325, 336, 362, 364, 366, 370, 374,384

Accumulation method, 430

Acetic acid, 9o, 96-103 effect on growth, $8 \mathrm{I}$ fermentation, $195,96,98$

Acetification, I IO

Acetone, IOO

Achorion, 316, 392, 4I4

Achromatic condenser, 133 objective, I26

Acid, action of, 77 dyes, 146

products, 79, 90, 94, 24I

reaction, $90,24 \mathrm{I}$

resisting bacteria, 326-328

Actinomyces, $317,416,546$

Adjustment, coarse and fine, I 36

Aeration, action of, on toxins, 474

Aerobic bacteria, 68

Aerogenic bacteria, 92, 93

Agar-agar, 232

Agar, alkalization of, 238

colonies on, 239

filtration of, 236

gelatin, 492, 494

glycerin, 240

" inclined, 238

peptonless, 240, 284

plates, 285

preparation of, 235,243

roll-tubes, 239,

sedimentation of, 236

streak cultures, 238

Agglutination, 319, 505

Testing of, 500 .

Air, 58, 64, 449

action of, 76

analysis of, 452

bacteria in, $45 \mathrm{I}$

city and country, 45I, 452

moulds in, 450

yeasts in, $45^{\circ}$
Air, purification of, $45 \mathrm{I}$

Albumin, coagulation of, 72 fixative, 536

Albuminoid ammonia, 424

Albumose, 82, 83, 88

Alcohol, 79, 80, 9r, 96-100, 402, 406, 4ro

effect on growth, $8 \mathrm{I}$

hardening with, $53 \mathrm{I}$

effect on staining, 320,533

Alcoholic fermentation, 95i 96, IO2, I 20,387

Aldehyde, 97

Algæ, II 7

Alkali, 89

Alkaline reaction, 89-9I

Alkalization of media, I54, 234, 238

Alkaloids, 89

Amido-acids, 9I

Amines, 89-92, 94, II I , I I 2

Ammonia, 89, 94, 96, IO7-II2, 424

Ammoniacal fermentation, ro7, 424

Ammonium chloride, 496

Amoeba coli, 372

Amyl alcohol, 97

Amylolytic ferments, 86, 87

Anaerobic apparatus, $306-315$

bacilli, 70

bacteria, $29,35,38,68,73,92$, 103, $163,298-304$

culture of, 306

plate culture, 312

micrococci, 70

Analytic products, 82

Angina, 332, 340, 366, 384

Anilin dyes, 146

hydrochloride, 544

water, 287

fuchsin, 310

gentian violet, 287

Animal ferments, 87 parasites, 259

Animals, disposal of, 283

cultures from, 278, 282, 283

examination of, 274 
Animals, inoculation of, 260 observation of, 272

Anthrax, 296 immunity to, 489

Anthrax bacillus, 76, 194 poison of, 83 sections, 538 spores, $51,54,56,447$ work with, 283-294, 538

Anti-infectious serum, 484

Antiseptic, 74, 75, 81, 521, 527 action of, 527 effect on motion, 529 spores, 529 virulence, 529 testing of, 527

Antitoxin, 484 action of, 485,487 neutralizing value of, 480 preparation of, 483 testing of, 478,482

Aperture, angle of, 130 numerical, I $3 \mathrm{I}$

Appendicitis, 352

Arnold sterilizer, I63

Arrows, poisoned, 298, 300, 304

Arthrospores, 48

Artificial classification, 17

Ash of bacteria, 30, 59, 63

Ascitic fluid, 242, 364, 358, 384

Asparagin, 118, 495

Aspergillus, 389, 390 diastatic action of, 4 IO

flavescens, 395,403 fumigatus, 395,410 infection, 4 IO niger, 395,408

- oryzz, 4 I0

Asporogenic bacteria, $5 \mathrm{I}$

Attenuation, 23, 74, 76, 80, II5, II8, 257,529

Autoclave, 75,165 use of, 166

Bacillus, 17 aceticus, 99 acidi lactici, 226

aerogenes, 340,382

anthracis, 296

anthracis symptomatici, 298

Asiaticus, 38

butyricus, 228

choleŕæ gallinarum, 374 suis, 376

coli communis, 38,62 , 100, 352 , 382

immobilis, 382

conjunctividitis, 360 ,
Bacillus cyanogenus, ' 1 r6, 230

diphtheriæ, 332

fluorescens liquefaciens, 362 putidus, 204

icteroides, $38,356,382,492-495$

Indicus, 196

influenzæ, 360

lactis aerogenes, I00, 382

lepræ, 320,326

leptosporus, 54

mallei, 330

megaterium, 54, 216

mesentericus vulgatus, 214

murisepticus, 380

Neapolitanus, 352

cedematis maligni, 300

No. II, 302

ozcenx, 340, 342

Pasteurianus, 99

pestjs bubonicæ, 358,383

pneumoniæ, 340, 342

prodigiosus, I60, $170,173,183$, I94

psittacosis, 38

pyocyaneus, 362

pyogenes fotidus, 352

rabbit septicemia, 374

ramosus, 218

rhinoscleromatis, 340,342

rhusiopathiæe suis, 378

ruber Kiel, I98

rubidus, I7I, 200

subtilis, $54,56,2$ เ2

tetani, 304

tuberculosis, 322-328

typhosus, 38,354

violaceus, $116,171,202$

Bacteria, classification of, 17,48

contents $r f, 30,34$

defined, 24, 87

distribution, 63

ends, 19

examination of living, 142,286

stained, I49, 286.

form, 17,18

function, 93

liquefying, 86

multiplication of, $4 \mathrm{I}, 42,53,73,75$

organisms smaller than, 24, 259

oxygen requirements of, 68

relation to disease, 253,256

size, 18,24

staining of, 31, I45

structure of, 25

thermal death-point, $5 \mathrm{I} 3$

Bacterial ferments, 85

poisons, 83

proteins, $8 \mathrm{I}, 82$ 
Bacterial suspension, 514, 524

Bacteriopurpurin, 33, II4

Bacterium, I7, I8

aceti, 99

coli commune, 352

phosphorescens, II 8, 206

termo, I I 4, 220

Zopfii, 222

Bacteroids, 6I

Barley, 88

Basic dyes, 146 products, 84

Basidia, 391

Beef tea, see bouillon

Beer, 96-98, I04, 387

Beet-juice, Ioo

Beggiatoa, 30

Berkefeld filter, $464,47 \mathrm{X}$

Bi-polar stain, 32, 352, 354, 358, 374

Biscuit, slimy, I04 -shaped bacteria, I8

Bismarck brown, $\mathrm{I}_{4} 6$

Black death, 358,383

leg, 298

plague, 358,383

Blastomycetes, $\mathrm{I} 7,386$

Bleeding host, I 16 , I94

Blight, 392

Blood, 460,463 agar, $360,364,368,384,463$ plates, $327,364,366,383$ bacteria in, 65 coagulation of, 163,463 defibrinated, 489

drawing of, 460

media, 360

oxalate, 463

pipettes for, 458,460 plating of, 327,383

Blood-serum, 86, 463, 483, boiled, non-coagulated, 468 centrifugation of, 462

collection of, 463

filtration of, 464

fractional sterilization, $464-467$

glycerin, 468

Löffler's, 468

preparation of, 464

solidification, 467

see plasma

see serum

Bloody milk, II6, 194

Blue milk, 230

pus, 362

Boiling, 57 see moist heat

Borax, 275

Botkin's method for anaerobes, 309
Botrytis, 391, 392

Bouillon, preparation of, 75, 233, 234, 243
blood, 364
glueose, 243
glycerin, 240

Bread-flasks, I04, I I6, 394

Broncho-pneumonia, 336

Brownian motion, 34

Bubonic plague, 358,383

Buchner's method for anaerobes, 307

Budding fungi, I 7, 385

Bunt, 392

Burettes for neutralizing, I 55

Burners, 25 I, 263, 499

Butter, flavor of, I03, I06 fat, 88 , 106

Butyl alcohol, 9I, 97, I03

Butyric acid, 70, 90, 94, 102, 106, I21, bacteria, 228 .

fermentation, 68,95, I03

Cage for animals, 274

Calcium, 5 I

acetate, Io6

formate, 106

hydrate, 105,284

lactate, 103

pectate, IO4

Camera lucida, 440

Canada balsam, I5I

Cancer, 388

Cane-sugar, 88, 95, 98, 402, 406

Capillaries for thermal death-point, 459 emptying of, 459, 516

filling of, 459,515

Capsule, 28, 29, r04, 338, 340, 342, 370

false, 29,30

Carbohydrates, $33,59,69,90-92,94$, IOO, I03, I05, III, II2

Carbolic acid, 5I, 56, 8I, $9 \mathrm{I}$

antiseptic action, $5 \dot{2} 8$

disinfecting, 519,526

fuchsin, 292, 293, $54^{\circ}$

methylene blue, $53^{8}$

Carbon, 59, II2

monoxide, 92

Carbonic acid, $33,59,67,90,91,97^{-}$ IOI, I03, IO5-IO7, II, II3, 440

Caries of teeth, IOI

Casein, 102, 104, 106

Cedar oil, I30, I37, I45, 544

Cell division, $4 \mathrm{I}$

structure, 87

wall, 25-30

permeability, 27,28

Celloidin, imbedding in, 534 
Celloidin, sections 537

Cellulose, 25, 59, 92, 97, т03, II2

Central body, 31

Charbon, 296

Cheese, 88, 102, I04, II6, 391, 412 spirillum, 346

Chemistry of bacteria, 79

Chemotaxis, 78

Chicken cholera, 32, 350, 374 tuberculosis, 328

Chitin, 26

Chlorine, 77

Chlorophyll, 33, 59, IO9, II4

Cholera Asiatic, 39, 27, 83, 344 diagnosis, 346

immunity, 486

infantum, see diarrhea

infection, 344,422

nostras, 348

poison, 83

vibrio, $37,38,344$.

in discharges, 430

in soil, 447

in water, $422,430,432,433$

water relation to, 422

Chromatic aberration, I 25

Chromatin granules, 28

Chromogenic bacteria, 93, II4

Cider, 98

Cilia, 38

Clamps, 313, 470

Claviceps purpurea, 392

Cleavage products, 82

Clostrịdium, 50, IO3

Cloves, oil of, 537, 539, 542, 544

Cocco-bacillus, I7, 358

Cold, action of, 73

Collodium sacs, 496 inoculation of, 500

insertion of, 501

principle of, 496

rolling of, 497

sealing of, 499,500

Colon bacillus, 79, 91, 92, IOI, 102, $241,352,382$

distinctions from typhoid, 428 , 492-496

in water, 425,440

Colony, 169

deep, 187

examination of, 186

impression, preparation of, 222

isolation of, $175,186,189$

on agar plates, $238,239,285$ agar-blood plates; see blood gelatin plates, I7I, $180^{\circ}$ inclined agar, 239, 240 potato, 169,186
Colony, surface, 187 transplantation, 189

Columella, 390

Comma bacillus, I8, 46, 344

Compressed air, $47 \mathrm{I}$

Concave slide, 143

Conidia, 389, 39I, 393, 402

Correction collar, 127

Cotton plugs, $15^{8}$ spontaneous combustion, I2I

Counting of colonies, 434-437, 448, 449

Cover-glasses, 140 cleaning, I4I for disinfection, 517,524

Cover-glass forceps, I4I streaks from blood, 280 cultures, 147

Cream, ro6 tissues, 279

Crown-glass, I 30

Cultures, homogeneous, 5 I4 sealing and keeping, 512

Cutaneous inoculation, 26r

Czermak's holder, 267

Decalcification, Ior

Decalcifying liquid, 544

Deci-normal alkali, 155

Deep layer, cultures in, 306

Defining power, I 29

Deneke's vibrio, 346

De-nitrifying bacteria, I Io

Desiccation, 55, 58

Dextran, I05

Dextrin, 59, 87, I05

Dextrose, see glucose,

Diaphragm, iris, I34, I35

Diarrhea of infants, 100, 102, 352

Diastase, $87,88,98$

Diastatic ferments, 87 from moulds, $406-412$

Dilution cultures, I69 on agar, 238,239 on blood-agar, $36 \mathrm{I}$ on gelatin, 174 potato, $j 68$

Di-methylamin, go

Diphtheria, 52, 83, 332-336

bacillus, 332, 393

diagnosis, 334

immunization, 482

pseudo-, 335

streptococci in, 384

toxin, $83,84,88,474-476$

fatal dose of, $476-478$

test-dose, $48 \mathrm{I}$

Diplo-bacillus, 43 of Weeks, 360 
Diplo-coccus, 44

gonorrhœæ, 368

intracellularis meningitidis, 337

lanceolatus, 336,338

pneumoniæ, 29, 338,340

Dișease, action of bacteria in, 254 ,recognition of bacteria in, 255 relation of bacteria to, 253-259

Disease-producing bacteria, 66, 69, 7I, $76,83,93$

Disinfectants, action of, 527 errors in testing, 518-523 testing of, 519,525

Disinfection, factors involved, 5 10-523 of cover-glasses, I 45,524 of hands, 5 or of rooms, 529

Division of bacteria, 4I

Dough, rising of, 98

Drawing of objects, I 39

Draw-tube of microscope, I35

Drum-stick bacteria, 50, 103, 304

Dry heat, action of, 56 sterilization, 159,160 sterilizer, I 59

testing of, $5 \mathrm{I} 7$

Dunham's solution, 344, 431, 432

Dust, 73, 452

Dyspepsia, IOI

Diseases, bacterial, 258

fungous, 259,392

infectiouș, 259

microbic, 259

protozoal, 259

with unknown cause, 258

Eberth's bacillus, see typhoid bacillus Ebner's solution, 544

Egg albumin, 85

Electricity, 77

Elsner's medium, 490

Emmerich's bacillus, 352

Empusa muscre, 392

Empyema, 366

Endocarditis, 336, 340, 366, 368, 384

Endospore, 48

Ensilage, 100, 103, I21

Environment of bacteria, $5^{8}$ effect of, 20

Enzyme, 59, 85, 116, 396

Eosin, 146, 289

Equivalent focal distance, I28

Ergot, 392

Erysipelas, 364, 384

Escherich's bacillus, 352

Esmarch counter, 448

dish, 183

potato culture, 183
Esmarch counter, roll-tube culture, I81, 239

Ethyl alcohol, 9I; see alcohol

Eye-piece, I32; șee ocular compensation, 132 designation of, I 32

Facultative bacteria, 67, 69

Farcy, 330 in cattle, 393,420

Fat-splitting ferment, $85,87,88$, ro5

Fats, 30, 55, 59, 85, 90, 94, 98, 103 fermentation of, I05 rancidity of, 88, 105, 106

Fatty acids, 88, 90, 106

Favus, 316, 392, 4I4

Feces, 29, 427

Fermentation, 67, 70, 73, 80, 92, 93, $95, I_{13}, I_{14}, 120,121$

bacterial, 93

mould, 391

putrid, 94, II I

yeast, 387

Ferments, 59, 80-82, 85

Fibrin stain, 289, 542

Filamentous forms. 20

Filling of tubes, I58, $5 \mathrm{II}$

Filter, absorbent cotton, 237, 514

Berkefeld, 464, 47I cleaning and sterilizing of, 472

glass-wool, $5^{\mathrm{I} 4}$

plaited, 157

porcelain, 464,468

tube, 514

Filtering apparatus, $469-47 \mathrm{I}$ globe receiver for, 471

Filtration of bacterial liquids, 464, 468

Finkler-Prior's vibrio, 348

Fire-fly, II 7

Fish, II6 gelatin, 206

Fission fungi, 41

Fixation of nitrogen, $x 10$

Fixing of blond preparations, 280 cover-glasses, I48

Flagella, 27, 35 mordant for, 318

see giant-whips

stain for, 319

staining of, 316

Flax, retting of, IO4

Flies, disease of, $38_{3}, 392$

Flint glass, I 26

Fluorescing bacteria, I16, 204, 362

Fluorite lenses, I3 I

Foaming liver, 92

Focal distance, 128 
Focussing of object, I36, I44

Food epidemics, I I 6, 507 absorption of, 37

Forceps for cover-glasses, I4I mice, 265 pressure, 265, 46I rats, 273

Form, classification I8 typical, 20 variation in, 19,23

Formaldehyde, 75,527 antiseptic action, 528 apparatus, 530 disinfecting action, 527 room disinfection, 530 . hardening with, 532

Formalin, 527

Formed ferments, 86

Formic acid, 75, 90, roo

Formless ferments, 86

Fox-fire, II 7

Fraenkel's borer, 448

Fractional sterilization, I62, 464

Fragmentation, 393

Fränkel's diplococcus, 338

Freezing microtome, 535

Friedländer's bacillus, 97, 340, 447

Fruit-juice, fermentation of, 387

Fruit-organs, 17 of moulds, 389-39 I

Fuchsin, 146 anilin-water, 3 I9

aqueous, 3 I9

carbolic, 293

Fungous diseases, 259, 392

Fusel oil, 97

Fungi, 259, 385

Gas pressure regulator, 250

Gas production by bacteria, 70, 79, $9 \Gamma$, 93, $103,120,241$

Gastric juice, 85,88

Geese, disease of, 372

Gelatin, nutrient, 153, 178

agar, 492, 494

alkalization of, 155

disadvantage of, 232

filtration of, 157

inclined, I9o

liquefaction of, $86,88,232$

mineral, rog

plates, I 7 I

modified, I79

potato, $49 \mathrm{I}$

roll-tubes, $18 \mathrm{I}$

stab cultures, I8g

sterilization of, 161,162

urine, 492
Generation, 76, 257

Gentian violet, 146 anilin-water, 287

Germicides, action of, 74, 77, 527 see disinfectants

Giant-whips, 38, 208, 298, 304, 309, 352,356

in animal body, 39

in hanging-drop, 319

Glanders bacillus, 52, 59, 330 diagnosis of, 329

Globig's potato culture, 184

Globulin, 83

Glow-worm II7

Glucose, 69, 80, 87, 88, 90, 91, 94, 95, $98,100,101,103,105$

agar, 243, 315

bouillon, 243,315

gelatin, $9 \mathrm{I}$

media, $24 \mathrm{I}$

serum, 242

Glycerin, 88, 91, 96, 98, 105, 106, Iі8 media, 240, 243, 315, 472 mounting in, 395

Glycogen, 98,386

Glyco-proteids, 25

Golden pus producing coccus, 366

Gonococcus, 242, 368 isolation of, 384 media for, 384

'Gonorrhea, 66, 368

Gram's method, applicability of, 289 for cover-glasses، 287 for sections, $54 \mathrm{I}$

Granules, sporogenic, 3L, 32

Granulose reaction, 26, 30, 33, 59, 103.

Grape-juice, 387

Grape-sugar, see glucose

Green diarrhea, 204 pus, $37,362,447$ sputum; 204

Grippe, 360

Growth, condition of, 8 I

Gruber's tubes, 307

Guine2-pig cage, 274 holder for, 265-268 inoculation of, 262,265

Halving process, 95

Hanging-drop, I43 culture, 286 giant-whips in, 319

Hardening of tissues, $53 \mathrm{I}$

Hay bacillus, 56, 212 fermentation of, 121 spontaneous combustion of, I2I

Heart blood, cultures from, 289 examination of, 278,279 preservation of, 279 
Heart blood, streaks from, 280

Heat, action of, $56,74,85$

production, 120

Heating plate, 150

Hematein, 544

Hematoxylin, 544, 546

Hemp, retting of, ro4

Herring brine, 90

Herpes, 392, 4I4

Hesse's air apparatus, 453

Hiss' tube medinm, 494

Hog cholera, 376 erysipelas, 378,447

Homogeneous culture, 514 oil immersion lens, 130

Hops, fermentation of, 120

Horse, drawing of blood from, 462 inoculation of, $263,268,269$

Hospital gangrene, 39

Hot springs, 73

Human blood as medium, 364, 384 drawing of, 462

Hydration changes, 95

Hydrochloric acid, IOI

Hydrogen, 62, 90, 92, 94, 100, 103, 106, IJ 2

cultures in, 308

generation of, 3 I 2

peroxide, 76,77

sulphide, III

Hydrothionuria, I07

Hyphæ, 389

Hyphomycetes, 388

Hypochlorites, 77

Ice, 442

Ice apparatus for plates, I 75

Imbedding in celloidin, 534 paraffin, 533

Immunity, active, 495 cause of, 488

cholera, 486

defibrinated blood, 489

passive, 485

unit, definition, 478

determination, 479

neutralizing value, 480

Immunization to anthrax, 489

cholera, 486

diphtheria, 482

swine-plague, 489

typhoid fever, 489

Impression' preparations, 222

Incubator, high temperature, 71, 243 low temperature, I79 room, 245

Index of refraction, I30
Indigo, 110

Indol, 94, I I I

reaction, $79,344,428$

Infection, 254, 260

alimentary, 27I

cutaneous, 26I

ear, 272

eye, 270

intra-cranial, 270,502

intra-duodenal, 272

intra-peritoneal, 269, 500

intra-pleural, 270

intra-tracheal, $27 \mathrm{I}$

intravenous, 265

lymphatic, 270

mixed, 260

placental, 322

respiratory, 270

subcutaneous, 262

subdural, 502

Infectious disease, 254,259

Influenza, 360

Infusoria, II 2

Injection apparatus, 262, 264

Inoculation of tubes, I 73, I74 with pipettes, $45^{8}$

Insolation, effect of, 75, I I 5

Insoluble ferments, 86

Instruments, sterilization of, 167,275 sterilizer for, 275

Internal pressure, $\mathbf{2 6}$

Intestinal bacteria, $65,66,69,68,92$, 97, roo, 105, II I, 352

Intoxication, I22, 254

Intracellular changes, 81

Invert sugar, 98

Invertin, 98, 396

Inverting ferment, 87,88

Involution forms, 21, 99, 358, 383

Iodine, $28,33,386$ solution, 288

Iris diaphragm, 134

Isolation of bacteria, I69, I 7 I, I89, 232 , 253

Jequirity seed, 83

Kappeler's thermometer, 179

Kephir, 102

Kitasato's flasks, 309

Klatsch preparations, $222{ }^{\circ}$

Koch's dry-heat sterilizer, 159 plating apparatus, 176 safety burner, $25 \mathrm{I}$ serum sterilizer, 466 steam sterilizer, I63

Koumiss, IO2

Kühne's methylene blue, 538 
Lactic acid, 8r, 90, 94, 100-106, I2I bacteria, 226 dextro-rotatory, IOI fermentation, 95 , roo inactive, IOI levo-rotatory, Ior

Lactose, 80, 9I, IOI, 396 media, 24I.

Lafar's counter, 436

Lake water, 44I

Lanceolate bacteria, 18, 29

Latapie's holder, 267, 268

Leguminous plants, 6r, I Io

Leprosy, 66, 320, 326 sections of, 545

Leucin, 9I

Leuconostoc, 29, 105

Leuco-products, I I 5

Levulose, 95, 98

Liborius tubes, 306, 308, 526

Liehig's meat extract, 243, 474

Life history of bacteria, 4 I

Light, effect of on bacteria, 75, 85 on media, 75 producing bacteria, 93

Lipochrome, I1 6

Liquefaction, 88

Liquors, 96

Litmus as indicator, I 54 media, 9I, 96, 240, 24I, 310 purification of, 509 . sensitizing of, 5 ro

Liver, foaming, 92

Living bacteria, examination of, 142 ferments, 86

Lock-jaw, 304

Löffler's bacillus, 332 methylene blue, 332 mordant, 318 serum, 334,468

Low temperature apparatus, I 79 .

Lugol's solution, 288

Lumpy-jaw, 3I6, 393, 4I6, 546 .

Lupus, 322

Lysol as disinfectant, $\mathbf{5 2 6}$

Madura foot, 3I6, 393, 4 I8

Magnification, I28

linear, 129

measurement of, 128

superficial, 129

Malaria, 259, 372

Malignant cedema, 91, I94, 300, 446 No. II, 302 pustule, 296

Mallein, 329, 330

Malt, 87, 98

Maltose, $98,402,406$
Man, blood from, 462

Mannite, I05

Marsh-gas, 92, 103

Martin's filter, 469 pepton solution, 475

Mass culture, $x 68$

Measurement of objects, 137

Meat, II6, II7, I 53

Meat extract, Liebig's, 233, 243, 474 fermented, 474

Mechanical interference, $28 \mathrm{I}$

'Media, alteration by heat, I 66 light, 95

sterilization of, $\mathrm{r} 6 \mathrm{I}, 464$ tubing of, 5 I I

Meningitis, 336, 337

Mercaptans, 94, I I I

Mercuric chloride, antiseptic action of 528 .

germicidal action of, 525

hardening with, 532

and hydrogen sulphide, 525, 520 solution; $5 x, 167$

Mesentery, preparation from, 281

Metabolic products, 79

Methods of infection, 260

Methylamin, 90

Methyl violet, 146

Methylene blue, ${ }_{4} 6$

Kühne's, 538

Löffler's, 332

Mikulicz, cells of, 342

Mice, cage for, 273 inoculation of, 262, 265

Microbic association, $70,73,102,310$ diseases, 259

Micro-burners, $25 \mathrm{I}$

Micrococci, forms, of, 46 motility of, 35 multiplication of, 44

Micrococcus, 17 aceti, $99^{\circ}$ gonorrhœæ, 368 pneumoniæ crouposæ, 338 prodigiosus, I94 tetragenus, 370,447 ureæ, Ioy

Micrometer, ocular, 138 . cross-wire, 437

stage, 137

screw, 136 value, I 38

Micro-millimeter, see micron

Micron, 24, 138

Microscope, 123 care of, I 36

Microsporon furfur, $392,4 r_{4}$

Mildew, 392 
Milk, 65, 88, т03, I 16, 230 alteration by heat, I 66 coagulation of, 102,429 examination of, 455 fermented, IO2 media, 233, 239 souring of, IOO, IO2, IO4, 226 tubercle bacillus in, 325

Milk sugar, see lactose

Milzbrand, 296

Minimum fatal dose, $476-478$

Mixed culture, 152,3 IO infection, 261,340

Modified media, 240

Moist chamber, I67, I 76 heat, 56 testing of, 5 I 5

Moisture, 58

Molasses, fermentation of, I05

Molecular motion, 34

Monas prodigiosa, I 94

Monilia candida, 388, 392, 402

Mordant solution, $318,54^{\circ}$

Morve, 330

Mother of vinegar, 99

Motion, 33, 50

Moulds, 58, 63, 87, 93, 95, 98, I08, I I 2 , II 7, I 2 I, 388

culture of, 394

diseases due to, 392

examination of, 395

in air, 452 ...

- multiplication of, 16,389

structure of, 380

Mounting boards, 2;9

Mouse septicemia, 380

Mouth bacteria, $33,46,66,70,97,100$, I0 $4,109,336,364,370$

Mucor, 389,390 corymbifer, 393,404 fermentation by, 406 mucedo, 406

mycoses, 390, 404 racemosus, 406 rhizopodiformis, 395, 406

Muguet, 402

Muscardine, 391

Muslin squares, 5 I6, 524

Mycelium, 389

Mycetoma, 4 I 8

Mycoderma aceti, 99

Naphthalene monobromide, I4I

Natural classification, I 7, 48

Neisser's duuble stain, 336

Neutralization of media, I 54

Nitric acid, 96, I08, I12, 424

Nitrification, I08
Nitrifying bacteria, 33, 60, 67, 445

Nitrite agar, IO9

Nitro-bacteria, I Io

Nitrogen, 60, 92, 108, I I 2, I I3 fixation of, I IO, 445

Nitroso-bacteria, I IO

Nitrous acid, 96, 108, 112, 424

Non-living ferments, 86

Non-pathogenic bacteria, 71, 72, 193

Normal alkali, I 54

Nóse-piece, I 36

Nuclear matter, 3I stains, 3 I

Nuclein bases, 3 I

Nucleus in bacteria, 25, 3 I

Nuttall's needle, 278 pipette, modified, 460

Objectives, I 23 achromatic, I 26 apochromatic, 132,133 care of, 137, 145 designation of, I32 focussing of, I36, I44 micrometer value of, 138 oil-ímmersion, $r_{30}$

Obligative bacteria, 66,68

Observation of animals, 272

Ocean bacteria, 76, 443

Ocular, 132; see eye-piece micrometer, 138 screw micrometer, I39

Oedema, malignant, 300 No. II, malignant, 302

Oidia, 4r 4

Oidium, 389, 39I, 392 albicans, 402 lactis, 39I, 394, 400 Tuckeri, 400

Optimum temperature, 7 I

Orange sarcine, 208

Organized ferments, 86, 93

Origanum, oil of, 537

Osmotic pressure, 26

Osteomyelitis, 366

Otitis media, $336,340,366,384$

Oxalate blood or plasma, 463

Oxalic acid, 90

Oxidation changes, 95

Oxygen, 1 I 4 effect on growth, 65,76 , I I 5 light production, 1 I 8

' sporulation; $5 \mathbf{I}$ toxins, 85

Oysters, I 16

Ozone, 7 I

Palmitic acid, $9 c$ 
Pancreas, 85

Pancreatic secretion, 85, 87, 88, 105

Papain, 87

Paraffin bath, 533 imbedding in, 533

sections, 535

Para-lactic acid, roI

Parasitic bacteria, 66, 71

Pasteur filter, 464,468 pipette, 456

Pathogenic bacteria, 7I, 74, 76, 89, 93 , 95, ro6, I2I, 295

Penetrating power, I29, I3I

Penicillium, 389 , 39I glaucum, 395,412

Pepsin, 82, 88

Pepton, 51, 82, 83, 88 solution, Martin's, 475 Witte's, 153

Peptonizing bacteria, IOI ferments, 87

Peptonless agar, 240, 529

Pericarditis, $336,340,384$

Perithecia, 390

Peritoneal fluid, 277

Peritonitis, $336,340,366,368,370,384$

Permanent mounts, I50

Peronospora infestans, 392

Perpetuating form, 53

Pest, 358, 446

Petri, air apparatus of, 454

dish, I80 culture, 180

Pfeiffer's reaction, 487,488

Phagocytes, 293

Phenol, see carbolic acid

Phenol-phthalein, I54

Phosphorus, 92

Phosphorescence, I I6, 206

Phosphorescing animals, II 7 bacteria, 72, 93, I 7 7, 206 moulds, II 7

Photobacterium, I 18, 206

Photogenic bacteria, 93

Physical motion, 34

Pickles, 100

Picro-carmin, 289, 541

Pigment bacteria, 76, 93

primary, 224

production, 9, 23, II4

secondary, II 5

Pipette bottle, 147,150

for drawing blood, 460

Pasteur, 456

advantages of, $45^{8}$

sealing of cultures in, $45^{8}$

for thermal death-point, 459,516 use of, $277-279$
Pipette,

for water analysis, $433,+59$

graduated, 459

sterilization of, 460

Pityriasis, 392, 4I4

Plague, 358

Plant diseases, 259

ferments, 87

Plasma, oxalate, 463

Plasmodium, 372

Plasmolysis, 22, 27, 32, 36, 352

Plates, culture on, 17 I sterilization of, $17^{2}$

Platinum wires, $I 72$

Pleomorphism, 22, 432

Pleuritis, 336, 340, 384

Pleuro-pneumonia, 24

Pneumo-bacillus, 340

Pneumo-enteritis, 376

Pneumo-coccus, I $0,338,340$

Pneumonia, 97, 337, 338, 340, 384 diagnosis, 336,337

in pest, 383

secondary infection in, 384

Poison-producing bacteria, 93

Poisoned arrows, 298, 300, 304

Poisonous food, 507

bacterial products, $83,84,88$, 106

Poisons, relation to disease, 254

Polar bodies, 27, 32, 336

Pollution of water, 423-425

Post-mortem examination, 275

Potato bacillus, 214 spores of, 162

culture on, 167

dishes, Esmarch's, I 82

fermentation of, I2O

gelatin, $49^{\circ}$

glycerin, 240

tubes, 183

Precautions in laboratory, I 70, 282

Press, 490

Pressure, action of, 76

Primary products, 82

Propionic acid, go

Propyl alcohol, 97

Protamin, 3 I

Proteins, 26, 30, 37, 59, 81-83, 88, 90, $92,94,103,106-108$, I I I, I I 2

Protein free media, 495

Proteolytic ferments, $85-88$

Proteus vulgaris, 38,220

Protoplasm, 25, 30-33, 58, 6I, 73, 8I, I I2, I I 9

Protozoa, II 7

Protozoal diseases, 259

Pseudo-diphtheria, 384 bacillus, 335 
Pseudo,

flagella, 37

influenza bacillus, 360

spores, 52

tuberculosis, 326, 328, 393, 420

typhoid bacilli, 427

Ptomains, 84, 88, 9o, I 22

Puccinia, 392

Puerperal fever, 364, 384

Punk fire, II 7

Pure culture, isolation of, $169,253,257$

Putrefaction, 67, 93-95, III, II 3, I 2 I

Putrid fermentation, 94, I I I

Pyemia, 364, 366, 384

Pyocyanin, I 6,362

Pyrogallate method, 307, 312, 313

Quarter evil, 295

Ŕabbit cage, 274 holder, 267,268

inoculation of, 262, 265, 266 septicemia, 374

Rabies, diagnosis of, 502 preservation of virulence, 504 vaccine, $503^{\prime}$

Rag picker's disease, 296, 300

Rain, 65, 44I

Ranvier slide, $x_{43}$

Rat cage, 273

forceps for, 273

inoculation of, 262, 265

Rauschbrand, 298

Ray fungus, 416

Reaction of media, 63, 9I, I57 effect on color, II 5 phosphorescence, II8

Real image, I 23

Recurrent fever, 46, $37^{2}$

Red bacillus of water, 200

$$
\text { Kiel, I98 }
$$

Red color on foods, I94, 200, 208 milk, 208

sweat, 208

yeast, $338,-398$

Reduction changes, 96

Relapsing fever, 372

Rennet action, 87,88 , IO2

Reproduction, 47

Reproductive form, 47, 53

Resistance of bacteria, 55, I6I difference 'in, 56

of spores, 55 unequal, in same species, 519

Resolving power, I29, I3I

Resting form, see spore

Rhinoscleroma, 342

Ricin, 83
River-water, 77, 442

Rod-shaped bacteria, 17

Roll-culture, agar, 239 gelatin, I8I, 453, 455 . counting colonies in, 449

Rooms, disinfection of, 529 sweeping of, $45^{2}$

Root bacillus, 2 I8

Rouget, 378,380

Roux flask, 485

pipette, 307

spatula, 278

syringe, 262

thermo-regulator, 465

tube for potato, 185,315

water-bath, 465

Rules of Kógh, 255, 258

Saccharomyces, 97, 386

albicans, 402

cerevisia, 387,396

ellipsoideus, 387

glutinis, 388,398

niger, $39^{8}$

Pastorianus, 387

Safety burner, $25 \mathrm{I}$

Sakè, 4 Io

Saliva, bacteria in, $85,87,109,336-$ $338,366,370$

Salt, 27, I1 8,358

Salt-peter, 27, 108, 445

Salts, inorganic, 62

Sanarelli's bacillus, 356

Sang de rate, 296

Saprogenic bacteria, 93, 95, $\mathrm{Ir}_{3}$

Saprophytic bacteria, 66, 7I, 74

Sarcine, 44, 45 motile, $35,38,208$ orange, $r 7 x, 182,208$ ventriculi, 2 ro yellow, I82, 210

Sarco-lactic acid, IOI

Sarcoma, 388

Sauer-kraut, I00, I03, I2I

Sausage, I04

Scarlet fever, 384

Schizomycetes, $4 \mathrm{I}$

Scleratia, $39 r$

Screw-shaped bacteria, I 7

Sealing of tubes, 316,512

Searing iron, 275

Secondary infection, 256,384 products, 82, 96 spectrum, I 26

Sections, $53 \mathrm{I}$ cutting of, 534

fixing of, 536

Gram's stain, 54 I 
Sections,

simple stain, 538,540

tubercle stain, 543

Sedgwick-Tucker's air apparatus, 454

Seed, 20, 47, 87

Segmentation, 393

Septicemia, 368, 374, 382, 383

Septicémie, 68, 300

Serum media. 242

agar plates, 242

agglutination, $31 \mathrm{Ig}, 487,505$

anti-infectious, $344,484,487$

antitoxic, $344,478,483,484$

Löffler's, 242, 468

normal. 488,505

Pfeiffer's reaction, 487

see blood-serum

sterilizer, Koch's, 466

Roux, 465

Sewage, 444

Sheath, 30

Silk threads, 517,523 worm disease, 391

Skatol, 94, II I

Slimy beer, milk, etc., 29 fermentations, 104

Smegma bacillus, 326

Smut, corn, 392

Snake venom, 83

Snow, 65, 44I

Sodium benzoate, antiseptic action, 528 carbonate, I54, 275

hydrate, 154

$$
\text { deci-normal, I54 }
$$

Soil, 444 normal, 154

analysis, $44^{8}$

bacteria in, $65,69,70,73,108$

filtering action of, 443

number of bacteria, 446 pathogenic bacteria, 446 vitality of bacteria in, 447

Soluble ferments, 86

Soorpilz, 402

Species, identification of, I9 origin of, 23 variability of, 23

Spherical aberration, 124 bacteria, I7

Spirillum, 17, 46 anserini, 372

Obermeieri, 372

rubrum, II5, 224

see vibrió

undula, 27

Spirochæte, r8, 372

Splenic fever, 296

Spontaneous combustion, I2I

\section{Spontaneous,}

generation, 56, I6I

Sporangium, 389,390

Spores, characteristics of, 5 I

double stain, 290

feebly resistant, 393

formation of, 47-5I, $8 \mathrm{I}$

germination of, 52,75

of moulds, 389 .

of streptotrices, 392

of yeasts, 386

resistance of, $55,162,165,214,519$.

staining of, 55

structure of, 54

Sporogenic granules, 31, 32, 48

Sporulation, 48

Spring-water, 443

Sputum in influenza, 360

in leprosy, 320

in pest, 358

in pneumonia, 337

in tuberculosis, 324,325

septicemia, 338

Stab çulture, 189

Stage micrometer, I 37

Staining cover-glasses, Gram's, 287 simple, I45-I 5I, I9I, 287 tubercle bacillus, 324, 543

flagella, 316

glass-slides, 280

sections, Gram's, $54 \mathrm{I}$

simple, 538,540

tubercle bacillus, 543

- Stains, I46

bottle for, 147,150

heating of, I 50

preparation of, 146

Stand of microscope, 135

Staphylococcus, 44, 45

pyogenes albus, 366

aureus, 25, I I4, 366

citreus, 366

Starch, 86, 87, 98, II2, II5 splitting ferments, 85

Steam-heat, $56,16 \mathrm{I}$ testing action of, 516 under pressure, 56,165

Steam sterilizer, I 63 under pressure, I65 superheated, I67.

Stearic acid, 90

Sterigm $æ 390$

Sterile media, 155

Sterilization, I59 at $58^{\circ}$, fractional, 464 at $100^{\circ}$, fractional, I62, 465 by filtration, 464 failure of, $I 62$ 
Sterilizer, dry-heat, ${ }_{5} 5$ see steam

Stoddart's medium, 492

Stomach, 85, roo bacteria, 66, 92, I01, I02 sarcine, 210

Straus-Wurtz apparatus, 454

Streak culture, I86 on agar, 238 agar blood, 360 gelatin, Igo potato, 186 preparations, fixing of, 148, 28I of blood, 280 cultures, I47, 148 organs, 279 staining of, $148,28 \mathrm{I}, 282,290$

Streptococcus, 44, 45 erysipelatis, 364 in diphtheria, 334 pyogenes, 364,383

Streptothrix, 392 actinomyces, 416

1 farcinica; 420 Maduræ, 4I 8

Study, line of, I9I, Ig2

Sub-culture, 52I

Succinic acid, 90, 97

Sugar, 87, 97, 100, 103-105, II 2 see glucose, etc.

Sulphur compound's, 90, 92, 107, I I I dioxide as antiseptic, 527 room disinfection, 529

Summer complaint, see diarrhea

Sunlight, action of, 58,75, II 5

Suppuration, $\mathbf{8 2}$

Surface tension; 26

Suspensions, bacterial, 5I4, 524

Swabs, preparation of, 334

Swine plague, 376 immunity to, 489

Symptomatic anthrax, 38, 92, 298

Synthetic changes, 8I, 84

Syringe, 262-264

holder, 263 sterilization of, 263

Temperature, $7 \mathrm{r}$ constant, 178,245 effect on form, 2 I gelatin, I6I germination, 54 motion, 35 multiplication, 4r, 65, 7r phosphorescence, 118 pigment, 75, I I 5 size, $2 \mathrm{I}, 75$ sporulation, $5 \mathrm{I}$ for cultivation, $7 \dashv$ 36
Temperature, maximum and minimum, 71 of animals, 273 optimum, $7 \mathrm{I}$

Test-glass, 265

Test- tubes, $158,184,238$ cleaning of, 158 filling of, 158,458 plugging of, 158 sterilization of, 160

Tetanus bacillus, 70, 91, I02, 304, 446 447

Tetanus toxin, 84

Tetrads, 29, 44, 45

Thermal death-point, 5 I3

Thermogenic bacteria, I 20

Thermometer, 252 maximum and minimum, 179, 252

Thermophilic bacteria, 72

Thermo-regulator, 245 alcohol, 246 filling of, 248 mercury, 245 metallic, 265

Thermostat, 243

Thoma Zeiss apparatus, 437,520

Threads, 20, 43 for disinfection, 517,523

Thrush, 392, 402

Tilletia, 392

Tissue, normal, 65

1 cutting of, 534 hardening of, $53 \mathrm{I}$

imbedding of, 533

Tobacco, fermentation of, 120

Torula, $97,386,387$

Toxalbumin, 83

Toxalbumose, 83

Toxic, see poisonous

Toxicogenic bacteria, 93, I2 I

Toxin, 59, 8I, 84, 88 diphtheria, 474 immunizing with, 482

T'oxoids, 48r

Toxo-peption, 83

Trichina, 508

Tricophyton tonsurans, 392, 414

Tri-methylamin, go, I 20, 194

Trochar, 268, 269, 462

Trypsin, 88

Tubercle bacillus, $30,32,52,59,65$, $66,767.86$, I $29,24 \mathrm{I}, 256,3 \mathrm{I} 5$, 322-328, 393

action of, 325

agar culture, 315

bouillbn culture, 473

detection, 324

differentiation, 326, 327 
INDEX.

Tubercle bacillus,

in dust, 452

in milk, 325

in sections, 543

in soil, 447

in sputum, 324

in urine, 325

potato culture, 3 I5, 473 .

pseudo, 326-328

see tuberculosis

Tuberzulin, 322, 326, 472

Tuberculosis, aviary, 316,328

cattle, 324

fish, 328

mammalian, 328

pseudo, 328

secondary infection in, 384

Tubing of media, I 58, $5 \mathrm{II}$

Turnips, fermentation of, 120

Typhoid bacillus, 354,382

behavior in water, 426

detection in water, 426,428

distinction from colon bacillus, 428 , $489-496$

in sections, 546

in soil and feces, $427,44^{6}, 447$

in water, 427,428

toxin of, 489

Typhoid fever, 27, 32, 38, 52, 62, 73, $79,86,91,92,97$, IOI, IO2, $241,354,382,422$

immunity to, 489

infection, 382

secondary infection, 384

serum, action of, $488,505-507$

Typical form, 20

Tyrosin, 91

Under-correction, $\mathbf{1 2 7}$

Unorganized ferments, 86

Urea, 89, 95, 107

Urine, 75, 76, го4, 107, 108

ammoniacal fermentation, 107

bacteria in, $65,89,354$

hydrogen sulphide fermentation, ro6

smegma bacillus in, 327

tubercle bacillus in, 325

Uschinsky's medium, 495

Ustilago, 392

Vacuoles, 32, 386

Vacuum cultures, 307,313

Variability in form, 20

in species, 23

Vaughan's cage, 274

Vegetating form, 46, 72-75, I6I

Venom, 83

Vesuvin, 146
Vibrio, I8, 46

choleræ Asiaticæ, 69, 117,344

Deneké, 346

Finkler-Prior, 348

$\therefore \quad$ Metschnikovi, 350

Miller, 348

Nordhafen, 350

phosphorescing, I 7

proteus, 348

Vibrion butyrique, 68,228

septique, 300

Vine, disease of, 400

Vinegar, 98

Violet bacillus, 202

Virtual image, 123

Virulence, increase of, $278,485,496$ preservation of, $279,332,364,45^{8}$

Viscose, IO5

Viscous fermentation, I04

Vitality of cultures, 279

Voges holder, 265,266

Waste matter, I I 2

Waste products, $4 I$, 50, 79-8I, 89 of different species, 79 of weakened species, 80

Water, 30, 58, 62, 65, 73, 108 aerogenic bacteria in, 440 animal inoculation with, 440 cholera vibrio in, 429-433 colon bacillus in, 425,440 number of bacteria in, 433,437 number of species in, 439 organic matter in, 438 pathogenic bacteria in, 439 relation to disease, 422 typhoid bacillus in, 428 vibrios, 430

Water analysis, 422, 433 bácteriological, 424,433 chemical, 422-424 methods of, 433

Water-bath, Hoffmann's, I 50 Roux, 465

Water immersion objective, I30

Wax pencils, I 74

Weeks' diplo-bacillus, 360

Weigert's fibrin stain, 542

Well-water, I65, 443

Whips, 316

Widal's reaction, 505

Wild yeasts, 97,387

Wine, diseases of, $97,98,104,387$

Wool-sorter's disease, 296

Working distance, I 28

Wurzel bacillus, 218

X-rays, 78,80 
INDEX.

Ẋerosis bacillus, 335

Yeast, 385

baker's or brewer's, 394

black, 398

colonies, appearance, 386

culture of, 394

enzymes of, 97, 396

multiplication of, 385

red, 388,398

relation to bacteria, 385

moulds, 386

pathogenic action, 388
Yeast,

upper and lower, $3^{8} 7$

white, 398

wild, 97,387

Yellow fever, 356,383

sarcine, 2 Io

Ziehl-Neelsen method, 324, 543

Ziehl's solution, 292

Zooglea, 28, 99, 104

Zymase, 396

Zymogenic bacteria, 93, 95, I I 3

Zygospores, $39^{\circ}$ 



\section{ค LIST OF BOOKS}

\section{PUBLISHED BY}

\section{GEO. XT EIFR}

Publisher and Bookseller to The University of Michigan, ANN ARBOR.

Any book in this list will be sent, carriage free, to any address in the world on receipt of price named.

CHEEVER.- Select Method's in Inorganic Quantitative Analysis. By Byron W. Cheever, A.M., M.D., late Acting Professor of Metallurgy in the University of Michigan. Revised and enlarged by Frank Clemes Smith, Professor of Geology, Mining and Metallurgy in the State School of Mines, Rapid City, S. D. Parts I. and II. Third - edition. I 2 mo. \$I.75.

The first part of this brok, as indicated by the title, consists of Laboratory Notes for a Beginner's Course in Quantitative Analysis. It considers the subjects of Gravimetric and Volumetric Analysis, for beginners, by neans of the chemical analysis of a set of substances properly numbered. in each case giving the methods to be followed in such analysis; also the methods for calculatine and preparing volumetric standard solutions, generally following the course offered by Professor Cbeever to his students. It also considers the mechods for the determination of the specitic nravities of various liquids and solids.

Although a number of the aulyses contained in Part I. may be of only approximate accuracy, and of small cominercial value such are yet included with a special purpose, to wit:-thac they may supply the student with a wider range of work and a grrater diversity of chemical manipulation. This was Professor Cheever's idea, and it i certainly a good one, especially since, in most cases, the work of the "egin. ner simply serves to emphasize the necessity of careful scrutiny of details and methods for practical work in the future.

Part I. is offered, then, for the use of schools and colleges, and it is intended to supply a source of elementary information upon the subject of Quantrative Chemical Analysis rarely oftered in such form in works upon that subject - Preface

The author was for many years Professor of Metallurgy in the University of Michigan, and the methods here presented are chose mostiy offered by him to his students. As a beginner's book in quanticative analysis, it will be found eminenty practicd, and it can be honestly recommen.led to the student who desires a source of elementary information upon this branch of applied science. The book is divided into two parts, the first consisting of laboratory notes for beginners. The subjects of gravinetric and volmmetric analysis are considered by means of the chemisal analysis of a set of substances, properly numbered, in each case giving the inethods to be followed in such analysis, and also the methods of calculating and preparing volumetric standard soluticns, etc. Methods for the determination of specific gravities of various liquids and solids are also considered.

Part II. contains a number of select methods in inorganic quantitative analysis. such as the analysis of limestone, iron ores manganese ores. steel, the analysis of coal, water, mineral pliospliates, sinelting ores, Jeart'slags, copper arsenic, bismuth, etc. A cluapter on reagents concludes the work,-Pharmaceucal bra. 
DEWEY.-The Study of Ethics. A Syllabus. By John Dewey, Professor of Philosophy in the University of Chicago. Octavo. 144 pages. Cloth," \$I.25.

DOW.--Brief Outlines in European History. A Syllabus for the'Use of Students in History, Course I., in the University of Michigan. By Earl Wilbur Dow. 4I pages. Pamphlet, 35 cents.

Dow.-Brief Outlines in European History. A Syllabus for the Use of Students in History, Course II., in the University of Michigan. By Earl Wilbur Dow. 47 pages. Pamphlet, 35 cents.

DZIOBEK.-Mathematical Theories of Planetary Motions. By Dr. Otto Dziobek, Privatdocent in the Royal.Technical High School of Berlin, Charlottenburg. Translated by Mark W. Harrington, formerly Chief of the United States Weather Bureau, and Professor of Astronomy and Director of the Observatory at the the University of Michigan, President of the University of Washington, and Wm. J. Hussey, Assistant Professor of Astronomy in the Leland Stanford, Jr. University. 8vo. 294 pages. \$3.50.

The determination of the motions of the heavenly bodies is an important problem in and for itself, and also on account of the influence it has exerted on the development of mathematics It has engaged the attention of the greatest mathematicians, and, in the course of their not altogether successful attempts to solve it, they have displayed unsurpassed ingenuity. The methods devisen by them have proved useful, not only in this problem, but have also largely determined the course of advance in other branches of mathematics. A nalytical mechanics, beginning with Newton, and receiving a finished clearness from Lagrange, is especially indebted to this problem, and in turn, analytical mechanics has been so suggestive in method as to determine largely both the direction and rapidity of the advancement of mathematical science.

Hence, when it is desired to illustrate the abstract theories of analytical mechanics, the profundity of the mathematics of the problem of the motions of the heavenly bodies, its powerful influence on the historical development of this science, and finally the dignity of its object, all point to it as most suitable for this purpose.

This work is intended nor merely as an introduction to the special study of astronomy, but rather for the student of matheinatics who desires an insight into the creations of his masters in this field. The lack of a text-book, giving, within moderate limits and in a sirictly scientific manner, the principles of mathematical astromomy in their present remarkably simple and lucid form, is undoubiedly the reason why so many mathematicians extend their knowledge of the solar system but little beyond Kepler's law. The autbor has endeavored to meet this need, and at the same cime to produce a book which shall be so near the present state of the science as to include recent investigations and to indicate unsettled questions.

FORD.-The Cranial Nerves. I2 pairs. By C. L. Ford, M.D., late Professor of Anatomy and Physiology in University of Michigan. Chart, 25 cents.

FORD.-Classification of the Most Important Muscles of the Human Body, With Qrigin Insertion, Nervous Supply and Principal Action of Each. By C. L. Ford, M.D., late Professcr of Anatomy and Physiology in the University of Michigan. Chart, 50 cents.

FRANCOIS.-Les Aventures Du Dernier Abencerage Par Chateaubriand, Edited with Notes and Vocabulary. By Victor E. Francois, Instructor in Freuch in the University of Michigan. Pamphlet, 35 cents. 


\section{GRAY.-Outine of Anatomy. A Guide to the Dissection of the Human}

Body, Based on Gray's Anatomy. 54 pages. - Boards, 60 cents.

The objects of the outline are to inform the students what structures are found in each region and where the description of each structure is found in Gray's Anatomy. - 1 hirteenth edilion, datea 1897.

GREENE. - The Action of Materials Under Stress, or Structural Mechanits. With examples and problems. By Charles E. Greene, A.M., M.E., Professor of Civil Engineering in the University of Michigan. Consulting Engipeer. Octavo. Cloth, \$3.0o.

Contents.-Action of a Piece under Direct Force. Materials. Beams. Torsion. Moments of Inertia: Flexure and Defmction of Simple Beams. Restrained Beams: Continuous Beams, Pieces under Tension. Coupression Pieces:-Columns, Posts and struts. Safe Working Stresses. Internal Stress: Change of Form. Rivets: Pins, Envelopes: Boilers, Pipes, Dome. Plate Girder. Earth Pressure: Retaining Wall: Springs: Plates. Details in Wood and Iron.

HERDMAN-NAGLER.-A Laboratory Manual of Electrotherapeutics. By William James Herdman, Ph.B., M.D., Professor of Diseases of the Nervous System and Electrotherapeutics, University of Michigan, and Frank W. Nagler, B.S., Instructor in Electrotherapeutics, Uni'versity of Michigan. Octavo. Cloth. I63 pages. 55 illustrations. $\$$ I. 50 .

It has been our experience that the knowledge required by the student of medicine concerning electricity and its relation to animal economy is best acquired by the laboratory method. By that method of instruction each principle is impressed upon the mind through several separate paths of the sense perception and a manual doxterity is acquired which is essential to success in the therapeutic applications.

This has been the plan adopted for teaching electrotherapeutics at the University of Michigan. Every form of electric modality that has any distinctive physiological or therapeutical effect is studied in the laboratory as to its methods of generation, control and application to the pattent. We believe this to be the only practicable way for imparting the kind of instruction required for the practice of electrotherapeutics, but in our attempt to develop a naturally progressive and at the same time complete and consistent course of laboratory instruction we have found it a thing of slow growih.

This laboratory manual is the final result of our various trials and experiences, and while we do not claim for it either perfection in the arrangement of matter or completeness in detail, we feel that the time has come for putting our plans in a form that will permit for $1 \mathrm{t}$ a wider usefulness as well as gain for it in the intelligent criticism of the experienced workers to the field which it seeks to cultivate.-Hrom Preface.

HOWELL.-Directions for Laboratory Work in Physiology for the Use of Medical Classes. By W. H. Howell, Ph.D., M.D., Professor of Physiology and Histology. Pamphlet. 62 pages. 65 cents.

HUBER.-Directions for Work in the Histological Laboratory. By G. Carl Huber, M.D., Assistant Professor of Histology and Embryology, University of Michigan. Second edition, revised and enlarged. Octavo. I9I pages. Cloth, \$I.50.

It is adapted fcr classes in medical schools and elsewhere where it is desired to furnish the class with material already prepared for the demonstration of structure rather than to give instruction in the technique of the laboratory Provision for the latter is made, however, by the addition of a section of about 50 pages on the methods for laboratory work. This section includes methods of macerating, hardening and fixing, decalcifying. impregnation, injecting, embedding, czaining, and methods for preparing and staining blood preparations. The last is accompanied by an excellent plate of blcod elements. The selection of methods has in the main been judicious. The expositions are both clear and concise.-Journal of Comparative Neurology. 
In this little book Dr. Huber has given us a model manual of milcroscopical tech nique in the laboratory study of histology. The subject matter is divided into convement chapters, commencing with the cell and sell division (karyokinesis) in plant and animal life, and gradually developing, by easy stages, the most complex tissues of the animal and vegetable organism. Between each lesson biank pages are inter leaved, to be used by the student for diawing the objects seen by him with a percil or crayon-a most excellent plan as nothing fixes the appearance aud characteristıcs of objects more firmly on the mind iban drawing them, either free-hand or with a came! a lucida (the former being prefer able, as it educates the hald and eye). With each subject is given the source and origin, the best methods for obtaining and preparing it, and attention is called to the most noteworthy or characteristic points for examination.

The second part of the book is devoted to methods for laboratory work: soften ing, hardening, decalcification, etc., of the matter $1 \mathrm{n}$ gross; embedding, sectioning, staining and mounting, elc. The best stains, with methods of preparin the same, and, in short, a general formulary for the various reagents, etc., covcludes the work, which is intended, as stated, as an aide memoire supplementary to a course of lectures on histology

We congratulate Dr. Huber on the skill with which lie has developed the idea, and the didactic methods which he has employed. Such a book cannot but prove a Rrtat welp to both student and teacher, and it should be more widely known $-\boldsymbol{S t}$. Luis Medical and Surgeon's Juurnal.

JOHNSON.-Elements of the Law of Negotiable Contracts. By E. F. Johnson, B.S., LL.M., Professor of low in the Department of Law of the University of Michigan. 8vo., 735 pages. Full law sheep binding. $\$ 3.75$.

Several years of experience as an instructor has taught the author that the best method of impressing a principle upon the mind of the student is to show him a practical application of it. To remember absts act propositions, without knowin their. application, is indeed difficult for the average student. But when the ptimary principle is once associated in his mind with particular facts illustratıng its application, it is more easily relanned and more rapidly applied to analo ous cases.

It is deemed advisable that the student in the law sh suld be required, during his course, to master in connection with each gener al branch of the law, a few well-selected cases which are illustı ative of the philosophy of that subject. To seguire each student to do this in the larger law schools bas been found to be impracticable. owing to a lack of a sufficienc number of copies of individual cases. The only solution of this difficulty seems to be 10 place in the hands of each student a volume containing the desired cases, In the table of cases will be found many leading cases printed in black type.-From Preface.

LEVI-FRANCOIS.-A French Reader for Beginners, with Noles and Vocabulary. By Moritz Levi, Assistant Professor of French, University of Michigan, and Victor E. Francois, Instructor in French, University of Michigan. I2 mo. 26I pages. \$1.00.

This reader differs from its numerous predecessors in several respects. First, being aware that students and teachers in the French as well as in the German departments of high schools and colleges are becoming tired of translating over and over again the same old fairy tales, the editors have avoided them and selected some interesting and easy short stories. They have also suppressed this poetic selections which are never translated in the class room. Fillally, they have exercised the greatest care in the gradation of the passages chosen and in the prepalation of the vocabulary, every French word being followed not only by its primitive or ordinary meaning, but also by the different English equivalents which the text requires. After careful examination, we consider this reader as one of the best on the American mat ket.

IYMAN-HALL-GODDARD.-Algebra. By Elmer A. Lyman, A.B., Edwin C. Goddard, Ph.B., and Arthur G. Hall, B.S., Instructor in Mathematics, University of Michigan. Octavo. 75 pages. Cloth, 90 cents. 
MATTHEWS.-Syllabus of Lectures on Pharmacology and Therapeu. tics in the University of Michigan. Arranged Especially for the Use of the Classes Taking the Work in Pharmacology and Therapeutics at the University of Michigan. By S. A. Matthews, M.D., Assistant in Pharmacy and Thorapeutice, University of Michigan. I2mo. II 4 pages. \$1.00.

MEADER.-Clironological Outline of Roman Literature. By C. L. Meader, A.B., Instructor in Latin in University of Michigan. Chart, 25 cents.

MICHIGAN BOOK.-The U. of $M$. Book. A Record of Student Life and Student Organizations in the University of Michtgan. Articles contributed by members of the Faculty and by prominent Alumi. $\$ \mathrm{I} .50$.

MONTGOMERY-SMITH.-Laboratory Manual of Elementary Chemistry. By Jabez Montgomery, Ph.D., Professor of Natural Science, Ann Arbor High School, and Roy B. Smith, Assistant Professor in Chemical Laboratory, Ann Arbor High School. I2 mo. 150 pages. Cloth, \$1.oo.

This Work is intended as a laboratory guide to be used in connection with a good text-book or course of lectures, and in its arrangement and scope is is based upon the practical experience of two instructors in the Ann Arbor High School. It is therefore restricted to snch work as may be done by the average high school pupil. The experiments which are dis ected are given more to enable the student to comprehend the methods of analytical chemisti y han to aequire particular pionciency in the work of chemical analysis. The work is charactelized by minuteness of explanation, a feature which will be appreciated by the beginner.-Pharmaceutical Lra.

NETTO.-The Theory of Sibstitutions and its Application to Algebra. By Dr. Eugene Netto, Professor of Mathematics in the University of Giessen. Kevised by the author and translated with his permission, by F. N. Cole, Ph.D., formerly Assistant Professor of Mathematics in the University of Michigan, Professor of Mathematics, Columbia University. 8 vo. 301 pages. Cloth. \$3.00.

NOVY.--Laboratory Work in Physiological Chemistry. By Frederick G. Novy, Sc.D., M.D., Junior Professor of Hygiene and Physiological Chemistry, University of Michigan. Second edition, revised and enlarged. With frontispiece and 24 illustrations. Octavo. Cloth, $\$ 2.00$.

This book is designed for directing laboratory work of medical students, and in slowing them how to study the physics and physiology of the digestive functions of the blood, the urine and other substances which the body contains normally, or which it speedily eliminates as effere material. The second edition has appeared within a very short time after the publication of the first. The first chapters deal with the facts, the carbohydrates and proteids. Then follow others upon the saliva, the gastric juice, the pancreatic secretion, the bile, blood, milk, and urine, while the closing chapter deals with a list of reagents.

While the book is manifestly designed for the use of Dr. Novy's own students. we doubt not that other teachers will tind it a valuable aid in their work. At the close of the volume are a number of illustrations of the various sedimentary substances found in the urine, taken from the work of von Jaksch. -The Th, rapentue wazerte

This book, although now in its second edition, is practically unknown to British readers. Up to the present, anyone wishing to find out how a particular analytical method in physiological chemistry ought to be carried out, had of necessity to refer 
to a German text-book. This comparatively small book-for it only covers some three hundred pages-gives as good a general. account of ordinary labor atory methods as any teacher or student cuuld desire. Although the author refers in his preface to help derived from the works of Salkowski, Hamma: sten and ockers, it is but fair to say that the book has undoubtedly been witten by one who lias worked out the methods and knows the importance of exact practical details-Edinburgh Med. Jour, Scotland,

Physiological chemistry is one of the most important studies of the medical curriculum. The cultivation of this field has until recently been possible to but few. The rapid development of this department of science within a few years past has thrown much and needed light upon physiological processes It is from this quarter and from bacteriological investigations that progress must chiefly be expected. The rapid growth of this branch of chemistry is attended by another result. It necessitates the frequent revision of text-books. The present editicn of Dr. Novy's valuable book is a most wholly rewritten It is representative of the present state of knowledge and is replete with information of value alike to student and practitioner. Few are better prepared to write such a book than Dr. Novy, who has himself done much original work in this field. -The Medical Bulletin, Hhilad l, hia.

This is a greatly enlarged edition of Dr. Novy's work on Physiological Chemistry, and contains a large amount of new material not found in the former edition. It is designed as a text-book and guide for students in experimental work in the laboratory, and does not therefore cover the same ground as the works of Gamgee, Lea, and, other authors of books on physiological chemistry. As a Iaboratory guide it should be adopted by our medical colleges throughour the country. because it is an American production, c:ntains only such directions and descriptions as have been verified by actual practice with students, and because it is clear, concise and definite in all its statements. Its trst ten chapters treat of fats, carbohydrates, proteins, saliva, gastric juice pancreatic secretion, bile, blood, milk, ald urine. Chapter xi. is devoted to the quantitative analysis of urine, milk, gastric juice, and blood, while chapter xii. gives tables for examination of urine and a list of reagents, $-A m$. Medico-surgical Bullefin, $N$. $\boldsymbol{Y}$.

NOVY.--Laboratory Work in Bacteriology. By Frederick G. Novy, Sc. I)., M.D., Junior Professor of Hygiene and Physiological Chenistry, University of Michigan. Second edition, entirely rewritten and enlarged, 520 pages. Quarto. \$3.00.

STRUMPELL.- Short Guide for the Clinical Examination of Patients. Compled for the Practical Students of the Clinic, by Professor Dr. Adolf Strümpell, Director of the Medica] Clinic in Erlangen. Translated by permission from the third German edition, by Jos, L. Abt. Cloth, 39 pages, 35 cents.

Preface to the Second Edition.-The second edition of this book has been improved by me in severdl parts, and particularly the sections treating of the examination of the stomach and nervous system have been slightly extended. The author trusts that the book may also fulfill its purpose in the future in assisting the studen to learn a systematic examination of the patient, and to impress on him the most important requisite means and methods.

WARTHIN.--Practical Pathology for Students and Physicians. A Manual of Laboratory and Post-Mortem Technic, Designed Especially for the Use of Junior and Senior Students in Pathology at the University of Michigan. By Aldred Scott Warthin, Ph.D., M. D., Instructor in Pathology, University of Michigan. Octavo. 234 pages. Cloth, \$I.50.

We have carefully examined this book, and our advice to every student and practitioner of medicine is-buy it. You will never regret having invested your money in it, and you will acquire such a large fund of information that the siudy of pathilogy will become a pleasure instead of the drudgery which it sc unfortunately seems to be in many cases.

Part I. of this book, embracing some so3 pages, deals with the materials. which includes the proper examination and notation of the gross changes which have 
occurred in every part of the body. In fact it is a complete expose of what a com. plete and accurate autopsy should be, the observance of which is oftener followed in the breach than in the actuality. Part II., which includes 134 payes, deals with the treatment of the material. This is a very important part of the work, as it gives explicit directions in regard to the instruments to use, stains and staining methods, drawing. the preservation of specimens, hardening methods, in fact, of all those technical points connected with practical pathological microscopy. The examination of fresh specimens, injections, methods fixing specimens as well as special staining methods are taken up. In fact, space forbids us to give the entire, which are most valuable in every detail.-St. Loun Med cal and surgical Journal.

WATSON. - Tables for the Calculation of Simple or Compound Interest and Discount and the Averaging of Accounts. The Values of Annuities, Leases, Interest in Estates and the Accumulations and Values of Investments at Simple or Compound Interest for all Rates and Periods; also Tables for the Conversion of Securities and Value of Stocks and Bonds. With full Explanation for Use. By James C. Watson, Ph.D., LI.D. Quarto. Cloth, \$2.50.

A book most valuable to bankers, brokers, trustees, guardians, judges, lawyers, accountants, and all concerned in the computation of interest, the division and settlement of estates, the negotiation of securities, or the borrowing and lending of money, is the above work of the late Professor James C. Warson, formerly Director of the Observatories and Professor of Astronomy at the Universities of Michigan and Wisconsin, and Actuary of the Michigan Mutual Life Insurance Company.

It contains, in addition to the usual tables for the calculation of simple or comprond interest and discount, many tables of remarkable value, not found elsewhere, for the averaging of accontn*, the values of annuities, leases, interests in estates. and the accumulations and values of investments; also tables for the conversion of securities, and the values of stocks and bonds.

There are also given very full and clear explanations of the principles involved in financial transactions, and a great variety of miscellaneous examples are worked out in detail to illustrate the problems arising in interest, discount, partial payments, averaging of accounts, present values, annuities of difterent kinds. annual payments for a future expectation (as in life insurance), or for a sinking fund, conversion of securities, values of gtocks and bonds, and life interesrs.

This book was issued from the press under the author's careful supervision. Professor Watson was noted for his clear insight into problems involving computations, and also for his wonderful ability in presenting the method of sclution of such problems in a plain and simple manner. The varied array of practical examples given in connect on with his "Table" shows these facts in a remarkable manner. This book provides, for those least expert in calculations, the means of avoiding mistakes likely to occur; and for the man engrossed in the cares of business, the means of making for himself, with entire accuracy, the calculation which he may need. at the moment when it is needed.

WREN TMORE-GOULDING.-A Text-Book of Elementary Mechanical Drazeing for Use in Office or School. By Clarence G. Wrentmore, B.S., C.E., and Herbert J. Goulding, B S., M.E., Instructors in Descriptive Geometry and Drawing at the University of Michigan. Quarto. Ioo pages and 165 cuts. \$1.00.

This book is intended for a beginners course in Elementary Mechanical Drawing for the office and school. Illustrations have not been spared, and the explanation have been made in a clear and concise manner for the purpose of bringing the student to the desired results by the shortest route consistent with the imparting of an accurate knowledge of the subject.

The first chapter is devoted to Materials and Instruments; the second chapter, Mechanical Construction; third chapter, Penciling. Inking, Tintıng; fourth chapter, Linear Perspective; fifth chapıer, Teeth of Grass.

WRENTMORE.-Plain Alphabets for Office and School. Selected by C. G. Wrentmore; B.S., C.E., Instructor in Descriptive Geometry and Drawing, University of Michigan. Oblong. I9 plates. Half leather, 75 cents. 
Souvenir of the University of Michigan, Ann Arbor. Containing 38 photo-gravures of President James B. Angell, prominent University Buildings, Fraternity Houses, Churches, Views of Ann Arbor, Etc., Etc. Done up in blue silk cloth binding. Price, 50 cents, postpaid.

Physical Laboratory Note Book.-'A Note Book for the Physical Laboratory. Designed to be used in connection with Chule's Physical Laboratory Manual. Contains full directions for keeping a Physical Laboratory Note Book. II 2 pages of excellent writing paper, ruled in cross sections, Metric System, size $7 \times 91 / 2$ inches. Bound in full catnvass, leather corners. Price, by mail, 30 cents. Special prices to Schools furnished on application.

Botanical Laboratory Note Book. - A Note Book for the Botanical Laboratory. Contains directions for Botanical Laboratory. 200 pages of best writing paper, ruled with top margins. Pocket on inside of front cover for drawing cards. Bound in substantial cloth cover and leather back. Size $6 \times 9 \%$. Price, by mail, 35 cents. Special prices to schools furnished on application. 





then

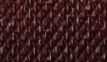

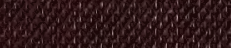

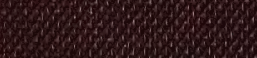

